\title{
Nanosilica functionalization for polypropylene-based dielectrics facilitating next generation HVDC transmission
}

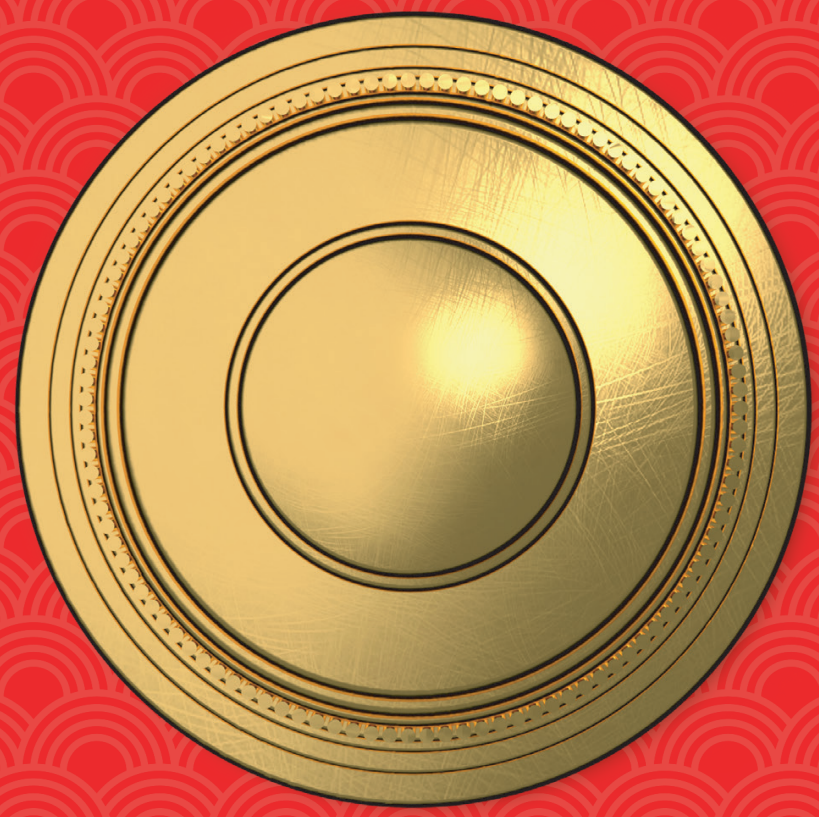





\section{NANOSILICA FUNCTIONALIZATION FOR POLYPROPYLENE-BASED DIELECTRICS \\ FACILITATING NEXT GENERATION HVDC TRANSMISSION}

Xiaozhen He 


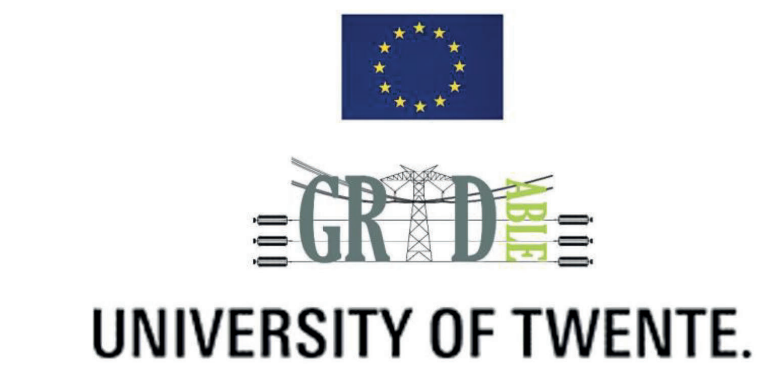

Elastomer Technology and Engineering stretching science

\section{Graduation Committee:}

\begin{tabular}{|c|c|c|}
\hline Chair / secretary: & Prof.dr.ir. H.F.J.M. Koopman & $\begin{array}{l}\text { University of Twente, ET, the } \\
\text { Netherlands }\end{array}$ \\
\hline Promoter: & Prof.dr.ir. W.K. Dierkes & $\begin{array}{l}\text { University of Twente, ET, the } \\
\text { Netherlands }\end{array}$ \\
\hline Co-supervisors: & Prof. dr. A. Blume & $\begin{array}{l}\text { University of Twente, ET, the } \\
\text { Netherlands }\end{array}$ \\
\hline \multirow{7}{*}{$\begin{array}{l}\text { Committee } \\
\text { Members: }\end{array}$} & Dr. R.P. Anyszka & $\begin{array}{l}\text { University of Twente, ET, the } \\
\text { Netherlands }\end{array}$ \\
\hline & Prof. dr. S. Luding & $\begin{array}{l}\text { University of Twente, ET, the } \\
\text { Netherlands }\end{array}$ \\
\hline & Prof. dr. A.J.A. Winnubst & $\begin{array}{l}\text { University of Science and Technology of } \\
\text { China (USTC), China \& University of } \\
\text { Twente, TNW, the Netherlands }\end{array}$ \\
\hline & Prof. dr. G. Chen & University of Southampton, UK \\
\hline & Prof. dr. R. Gerhard & University of Potsdam, Germany \\
\hline & Prof. dr. K. Lahti & Tampere university, Finland \\
\hline & Dr. E. Sarlin & Tampere university, Finland \\
\hline
\end{tabular}




\title{
NANOSILICA FUNCTIONALIZATION FOR POLYPROPYLENE-BASED DIELECTRICS \\ FACILITATING NEXT GENERATION HVDC TRANSMISSION
}

\author{
DISSERTATION \\ to obtain \\ the degree of doctor at the University of Twente, \\ on the authority of the rector magnificus, \\ Prof. dr. ir. A. Veldkamp, \\ on account of the decision of the Doctorate Board \\ to be publicly defended \\ on Friday $28^{\text {th }}$ of May 2021 at 12.45 hours
}

by

\author{
Xiaozhen He \\ born on the 21st of November, 1990 \\ in Laiyang, Shandong, China
}


This dissertation has been approved by:

Supervisor

Prof. dr. ir. W.K. Dierkes

Co-supervisors

Prof. dr. A. Blume

Dr. R.P. Anyszka

Cover design: Jakub Horna https://www.behance.net/JakubHorna

Printed by: Gildprint, Enschede https://www.gildeprint.nl/

Lay-out: Dr. Wei Ya

ISBN: 978-90-365-5186-1

DOI: $10.3990 / 1.9789036551861$

(C) 2021 Xiaozhen He, The Netherlands. All rights reserved. No parts of this thesis may be reproduced, stored in a retrieval system or transmitted in any form or by any means without permission of the author.

Alle rechten voorbehouden. Niets uit deze uitgave mag worden vermenigvuldigd, in enige vorm of op enige wijze, zonder voorafgaande schriftelijke toestemming van de auteur. 


\section{List of Chapters}

Chapter

Title

Page

1 Introduction

$2 \quad$ Literature Review 9

3 Summary--the Structure Behind $\mathbf{5 1}$

$4 \quad$ Paper I: Surface Modification of Fumed Silica by Plasma $\quad 67$

Polymerization of Acetylene for PP/POE Blends Dielectric

Nanocomposites

$5 \quad$ Paper II: Silica surface-modification for tailoring the charge trapping properties of PP/POE based dielectric nanocomposites for HVDC cable application

6 Paper III: The influence of nanosilica surface functionalization on PP/EOC cast thin film nanocomposites for HVDC cable application: micromorphology, dynamic mechanical and dielectric properties

7 Paper IV.: Dielectric performance of silica-filled nanocomposites based on miscible (PP/PP-HI) and immiscible (PP/EOC) polymer blends

8 Paper V: PP/PP-HI/Silica nanocomposites for HVDC cable insulation: Are silica clusters beneficial for space charge accumulation?

9 Paper VI: Joining good dispersion with tailored charge trapping in nanodielectrics by hybrid functionalization on silica

10 Summary

Samenvatting

List of Publications

Acknowledgement 


\section{Contents}

Chapter 1...

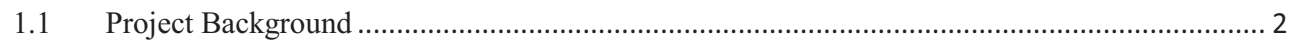

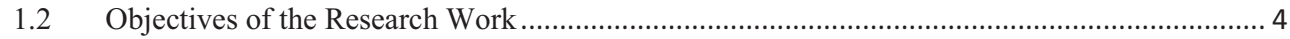

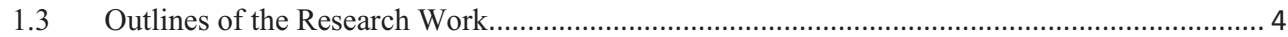

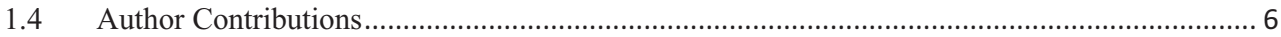

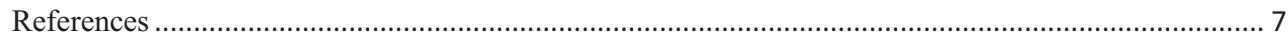

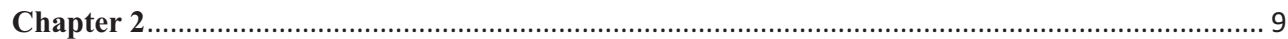

$2.1 \quad$ Problems and Challenges of Power Transmission .................................................................. 10

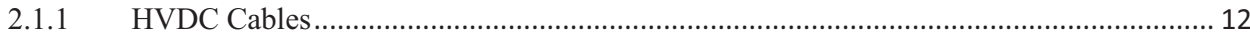

2.1.2 Some Critical Issues and Challenges of Extruded HVDC Cables..................................... 14

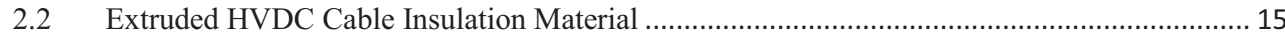

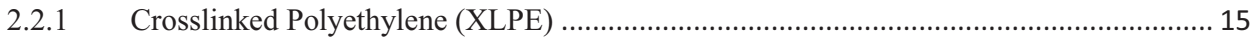

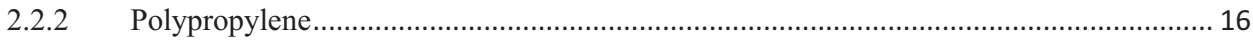

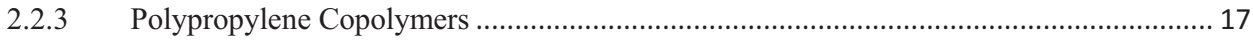

2.2.3.1 Melting and Crystallization Behaviour Influenced by Copolymerization ..................... 18

2.2.3.2 Influence of the Copolymer on the Morphology ......................................................... 19

2.2.3.3 Influence of the PP Copolymer on the Crystalline Structure …..................................... 20

2.2.3.4 Mechanical Properties as Influenced by the Copolymer.............................................. 21

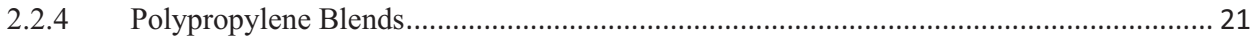

2.2.4.1 Micromorphology of PP blends and its Effect on Dielectric Performance ................... 25

2.2.4.2 Crystalline Morphology of PP Blends and its Effect on Dielectric Performance ......... 26

2.2.4.3 Space Charge Accumulation in PP Blends............................................................... 27

2.2.5 Dielectric Polymer Nanocomposites ......................................................................... 27

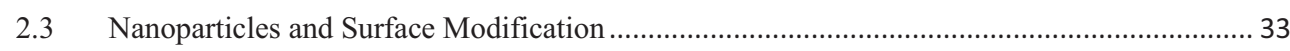

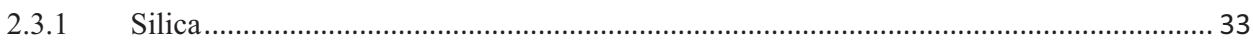

2.3.2 Surface Modification of Nanosilica.................................................................................. 35

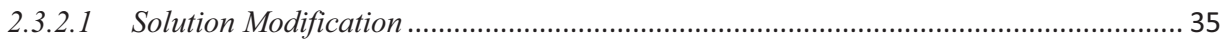

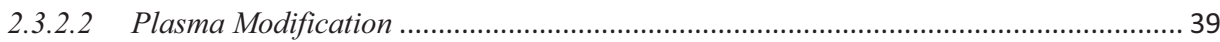

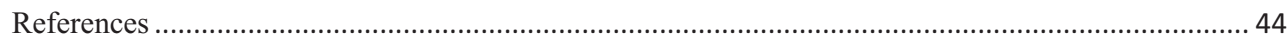

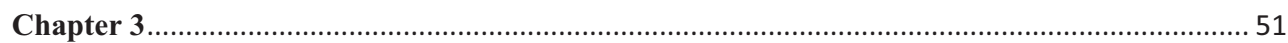

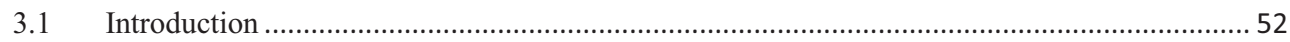

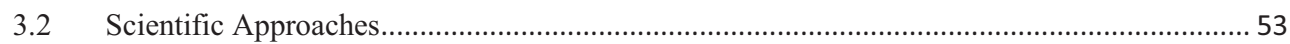

3.2.1 Surface Modification by Plasma Deposition of Acetylene................................................ 53

3.2.2 Surface Modification of Silica by a Series of Silanes ...................................................... 56

3.2.3 The Influence of Polarity of the Silica on the Properties of the Composites....................... 58 


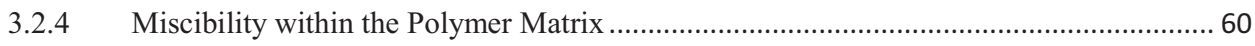

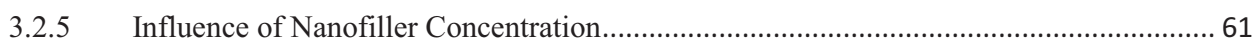

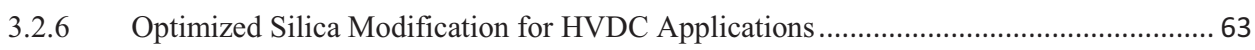

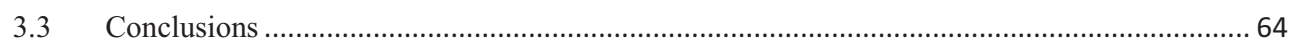

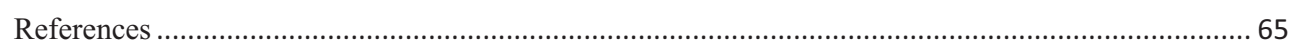

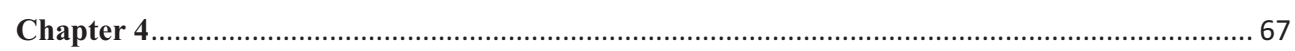

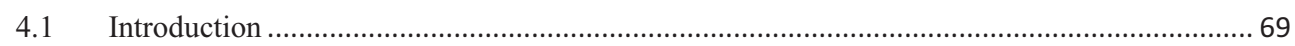

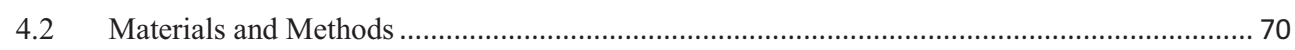

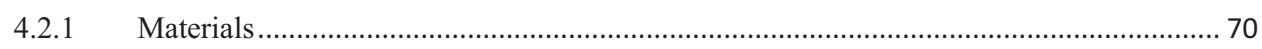

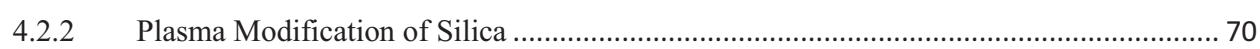

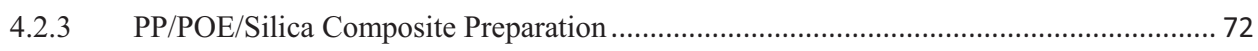

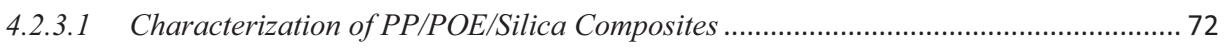

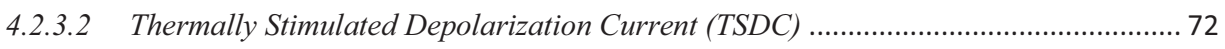

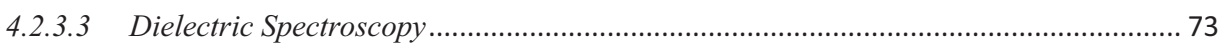

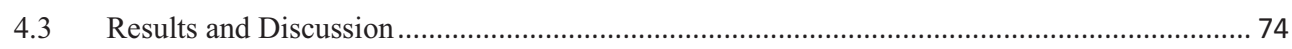

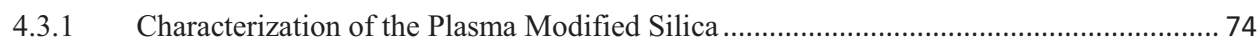

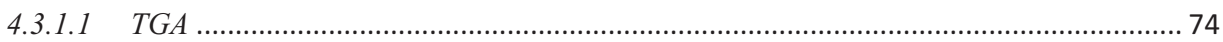

4.3.1.2 Analysis of the Polymer Deposit on the Silica Surface by XPS ..................................... 78

4.3.1.3 Analysis of the Polymer Deposit on the Silica Surface by STEM-EDX ........................ 79

4.3.2 Characterization of Silica Filled PP/POE Blends Nanocomposites ................................... 81

4.3.2.1 XRD Crystalline Structure Analysis ........................................................................... 81

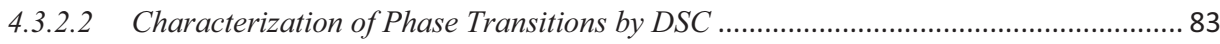

4.3.2.3 Morphology Analysis by SEM and Filler Dispersion Analysis by SEM-EDX Mapping87

4.3.2.4 Thermally Stimulated Depolarization Current (TSDC) ................................................ 89

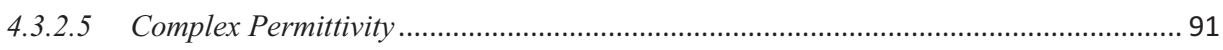

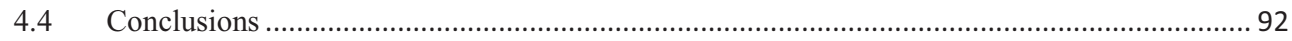

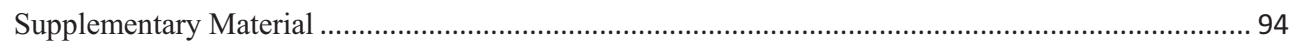

S1 Measurement of Isothermal Polarization and Thermally Stimulated Depolarization Current .. 94

S2 Determination of Trap Density Distribution from TSDC Data ............................................... 95

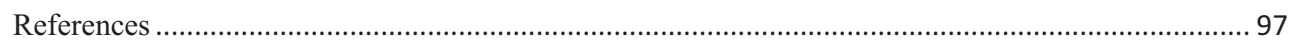

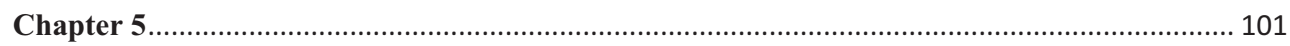

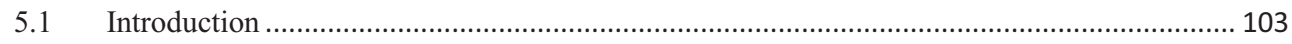

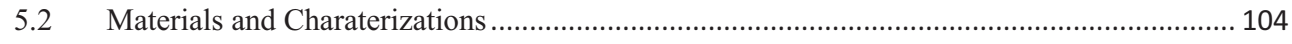

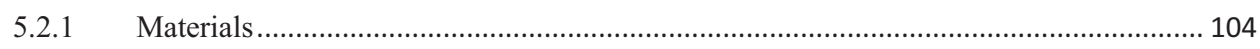

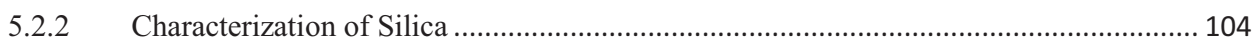

5.2.3 Characterization of Silica/PP/POE Nanocomposites ................................................. 105 


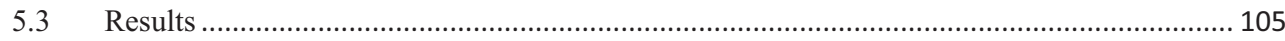

5.3.1 Solvent-free Modification of Silica by Silanes............................................................. 105

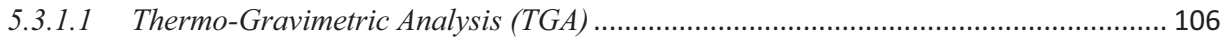

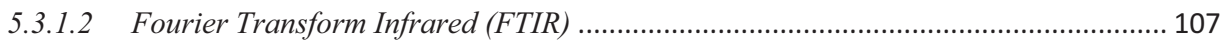

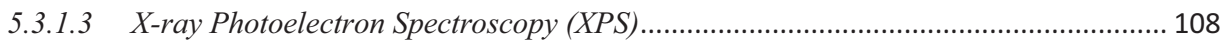

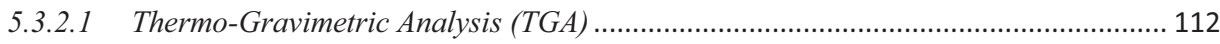

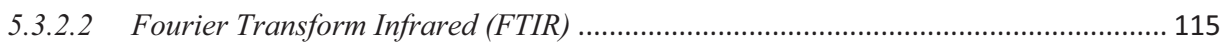

5.3.2.3 Transmission Electron Microscopy (TEM) with Elemental Mapping .......................... 115

5.3.3 Characterization of PP/POE of nanocomposites ............................................................ 117

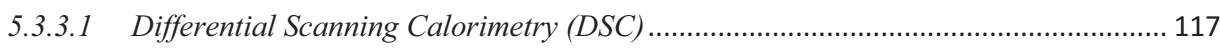

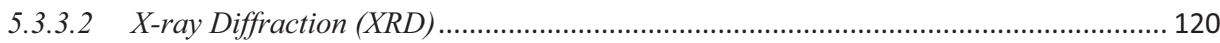

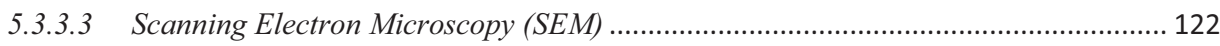

5.3.3.4 Thermally Stimulated Depolarization Current (TSDC) ............................................ 126

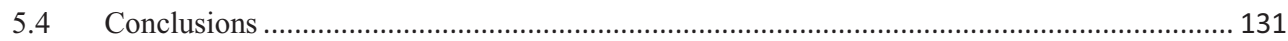

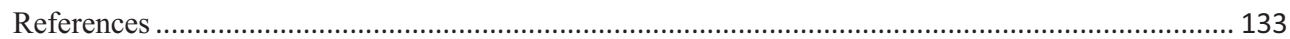

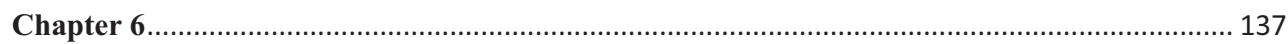

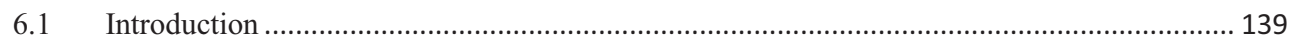

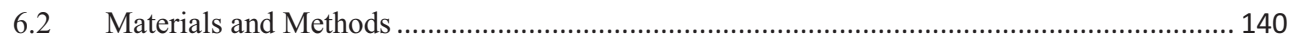

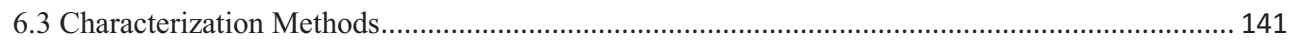

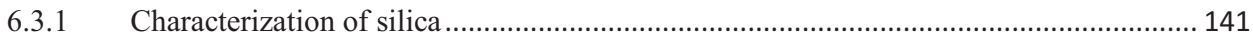

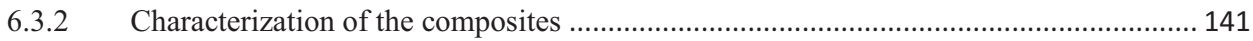

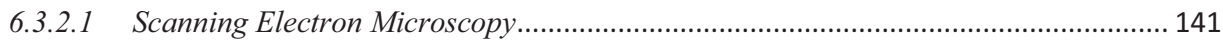

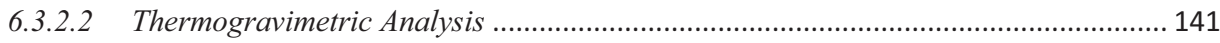

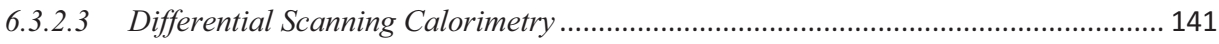

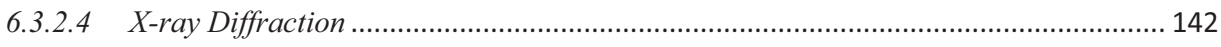

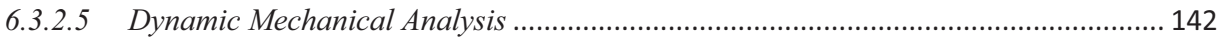

6.3.2.6 Thermally Stimulated Depolarization Current ………............................................. 142

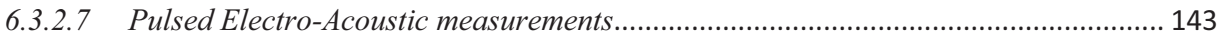

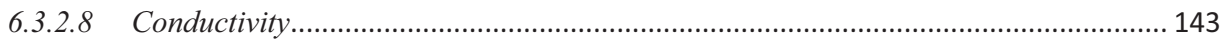

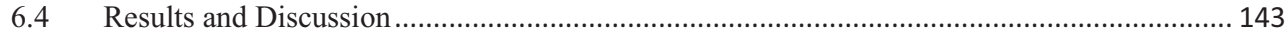

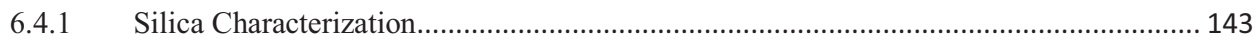

6.4.2 Nanocomposite Morphology and Silica Dispersion ..................................................... 145

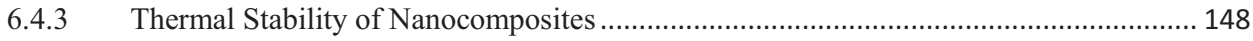

6.4.4 Crystallization Behaviour of the Nanocomposites ....................................................... 148

6.4.5 Chain Relaxation Behaviour of the Nanocomposites................................................... 150

6.4.6 Charge Trapping Properties of the Nanocomposites ................................................... 151 
6.4.7 Space Charge Behavior of the Nanocomposites............................................................ 156

6.4.8 Charging Current Density of the Nanocomposites......................................................... 159

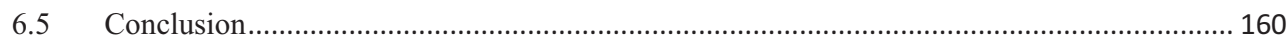

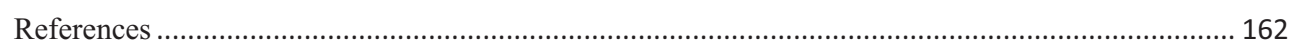

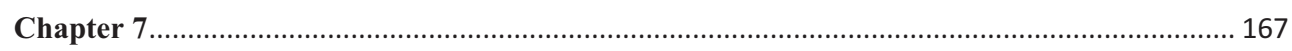

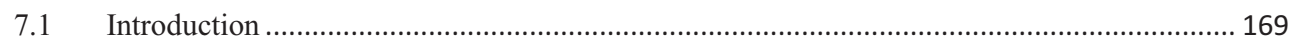

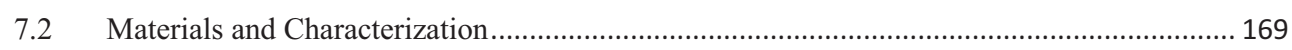

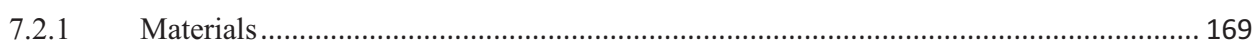

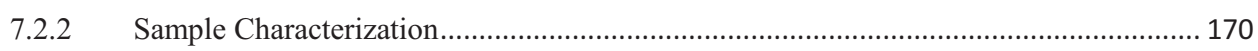

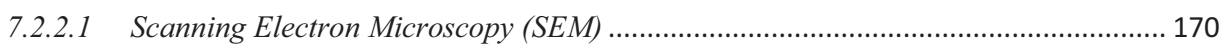

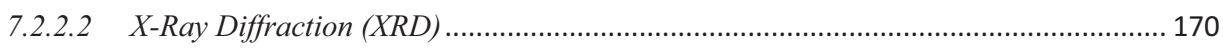

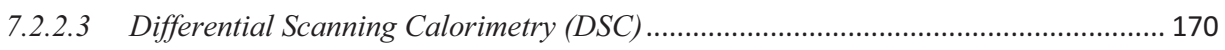

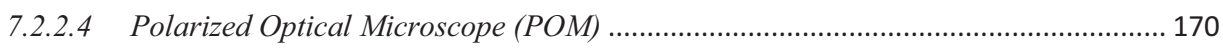

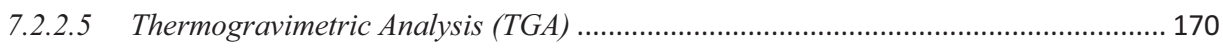

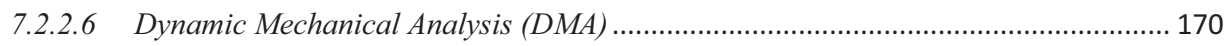

7.2.2.7 Thermally Stimulated Depolarization Current (TSDC) ............................................. 171

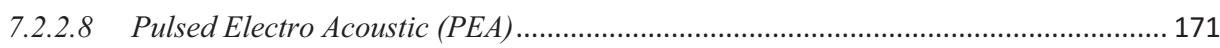

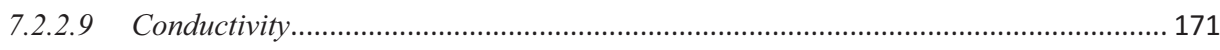

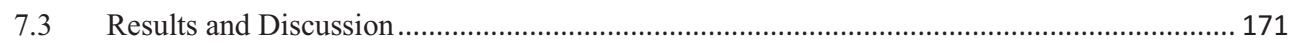

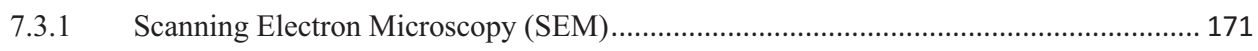

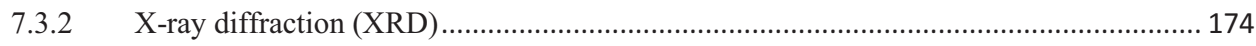

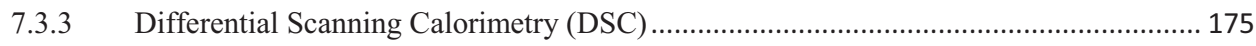

7.3.4 Polarized Optical Microscope (POM) ….................................................................... 177

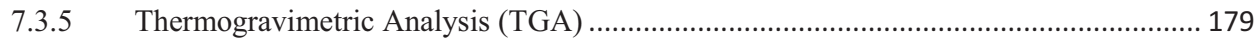

7.3.6 Dynamic Mechanical Analysis (DMA) ..................................................................... 180

7.3.7 Thermally Stimulated Depolarization Current (TSDC) ................................................ 182

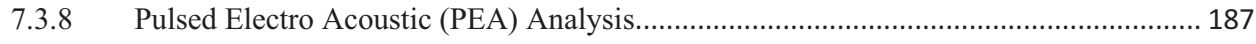

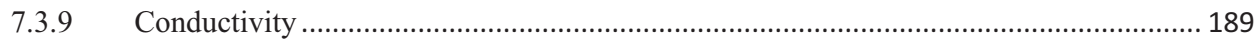

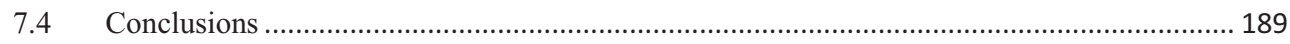

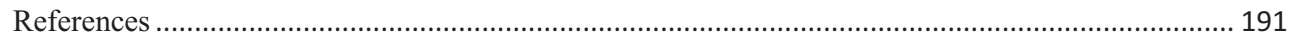

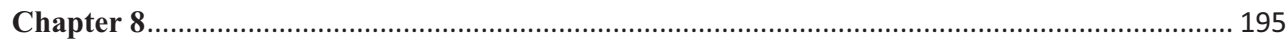

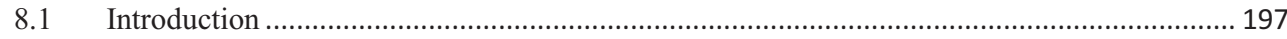

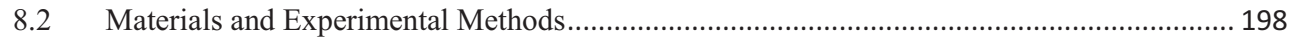

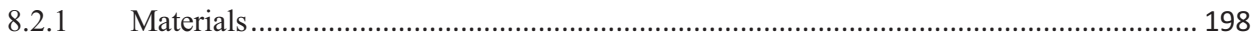

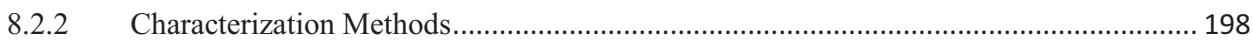

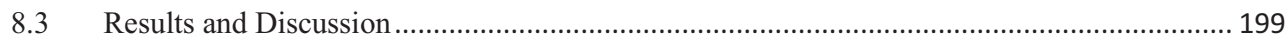




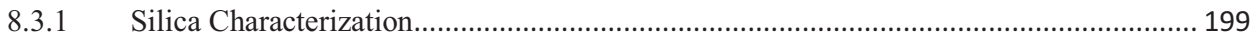

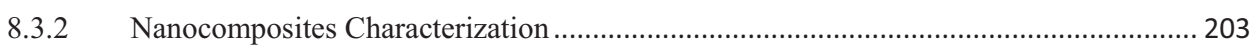

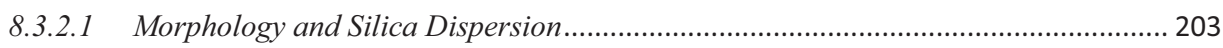

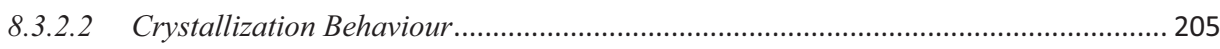

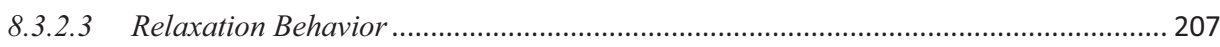

8.3.2.4 Space Charge Accumulation and Charging Current Density .................................... 209

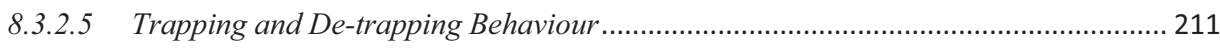

8.3.3 In-depth Discussion of the Dielectric Performance......................................................... 216

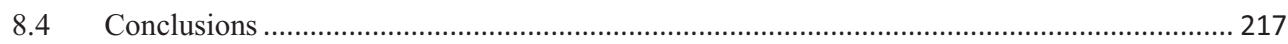

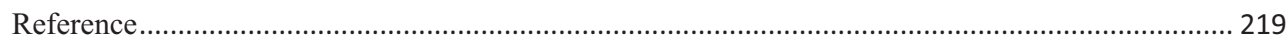

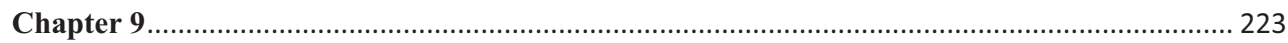

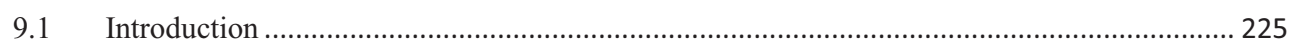

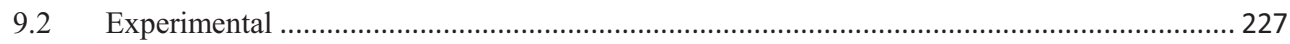

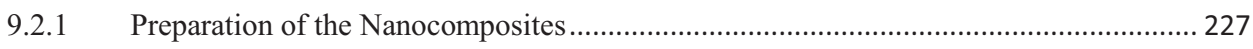

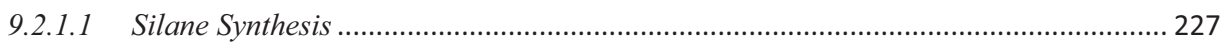

9.2.1.2 Reaction of 3-(Triethoxysilyl)propyl Isocyanate Silane (TEPI) with Alcohols ........... 227

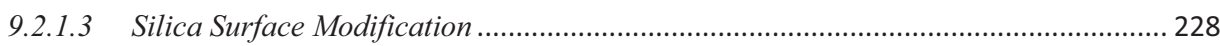

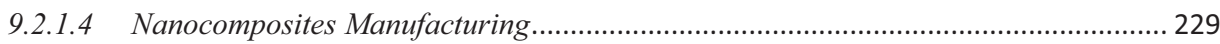

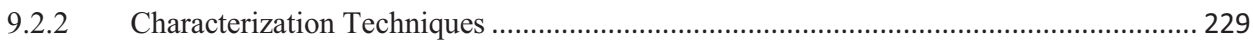

9.2.2.1 Fourier-Transform Infrared Spectroscopy of the Silanes ............................................ 229

9.2.2.2 Diffuse Reflectance Infrared Fourier-Transform Spectroscopy of the Silicas ............ 229

9.2.2.3 Thermogravimetric Analysis (TGA) of the Silicas ..................................................... 229

9.2.2.4 Scanning Electron Microscopy (SEM) of the Nanocomposites .................................. 229

9.2.2.5 Thermally Stimulated Depolarization Current of the Nanocomposites........................ 230

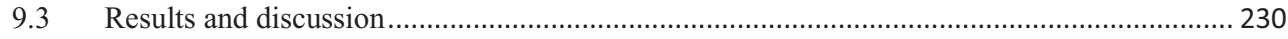

9.3.1 Fourier-Transform Infrared Spectroscopy (FTIR) of the Silanes .................................. 230

9.3.2 Diffuse reflectance infrared Fourier-Transform Spectroscopy (DRIFTS) of the Silicas 231

9.3.3 Thermogravimetrical Analysis (TGA) of Silicas .......................................................... 232

9.3.4 Scanning Electronic Microscopy (SEM) of the Nanocomposites .................................. 235

9.3.5 Thermally Stimulated Depolarization Current (TSDC) ............................................... 237

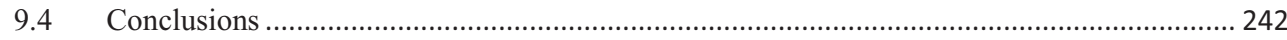

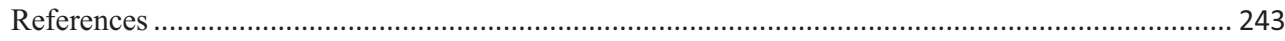

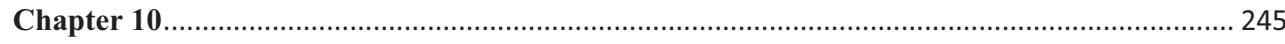

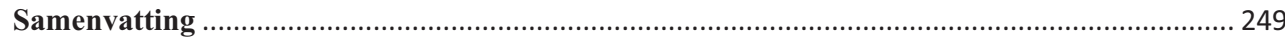

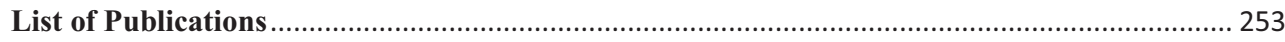

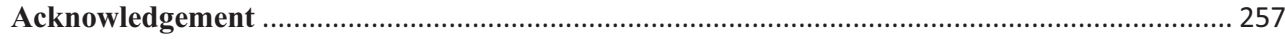


Chapter 1

Introduction 


\subsection{Project Background}

The rapidly developing world is facing an increasing demand for electricity. Electrical power is the backbone of modern economy, society and industry. Traditionally, electrical energy is transformed from mechanical energy, which is generated from water vapor convection heated up by burning fossil fuels (coal, petroleum and natural gas). The fossil fuels are extracted from beneath the earth's surface and transported to the demand region. They are consumed for generation of electricity since 1880s. Formation of the fossil fuels takes millions of years, and their consumption rate is much higher than the rate of formation. The impending scarcity of the fossil fuels makes it difficult to meet the increasing demand of electricity in the modern world. In addition, they consist mainly of carbon and hydrogen. When they are burned, carbon dioxide is produced. The increase of the concentration of carbon dioxide in the atmosphere causes heat trapping, which is the cause of the greenhouse effect. This in turn results in the climate change and global warming observed in the recent years. Therefore, solid steps are taken globally to integrate alternative renewable sources of energy in order to meet the growing electricity demand, to replace the existing fossil-based power production and to mitigate the negative effect on the climate. However, the most efficient renewable energy sources, e.g., offshore wind energy, desert solar energy and hydro power, are usually located in remote areas. To transport the "green" energy generated in remote locations to the demand regions for distribution to the end-users, efficient electricity transmission over long distances is needed (Figure 1.1).

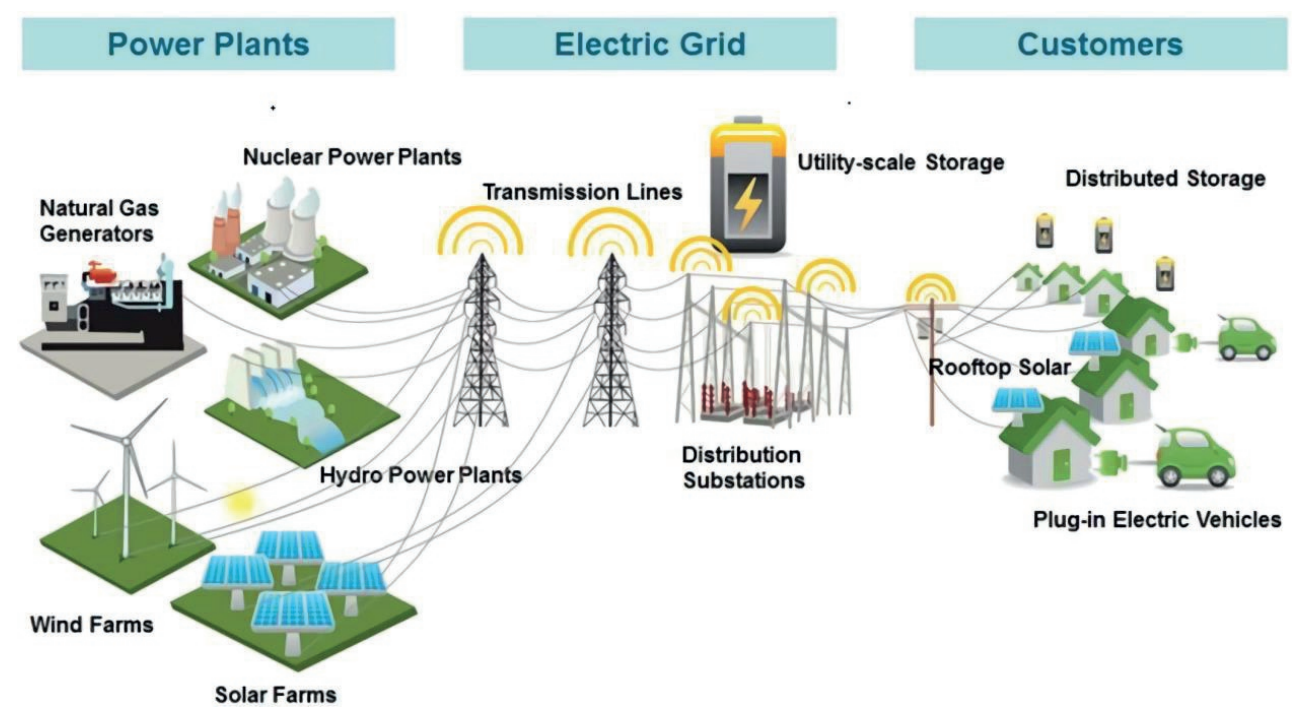

Figure 1.1 Transportation of alternative energy to the customers via the transmission lines $[1,2]$.

The power grids are becoming more and more inter-connected globally, meaning that the grids are hardly "national" anymore: electrical energy generated in one country (e.g., Norway) can be transmitted to and consumed in a completely separate location (e.g., in The Netherlands). The conventional Alternating Current (AC) transmission grids have originally not been planned from this point of view, and their transmission capability and efficiency are becoming challenged due to the increased (global) 
inter-connection of power grids. Gradually, High Voltage Direct Current (HVDC) transmission is getting a more and more important technology to transport electricity. It offers lower energy losses and is more compact in size than High Voltage Alternating Current (HVAC) transmission over long distances. In addition, another technical limitation for the AC system operation comes from the reactive (capacitive) power handling. Especially for the submarine AC cable, the length of the cable is technically limited due to high reactive power produced by the capacitance of the cable, which cannot in practice be compensated. As the limited fossil resources cannot meet the increasing power demand and the environmental issues need to be taken into consideration, the proportion of using renewable energy sources to generate electricity is getting higher and higher. Consequently, the necessity for implementing HVDC technology with advantages for long distance power transmission will increase day by day with the increase of renewable energy source utilization [3]. Moreover, HVDC is also the only way to directly connect two AC systems with different frequency, and it is also technically and practically the only solution for the long-distance submarine connections, due to the high reactive (capacitive) power of the cable in AC system.

HVDC transmission systems include many components and the most important ones are:

- converter terminal station, which is used to convert AC to DC (called rectifier terminal) or convert DC to AC (called inverter terminal);

- filters, which are used to minimize the harmonics; reactive power source, which is used to control the unstable voltage system;

- HVDC circuit breakers, which are used to interrupt the abnormal DC current, especially when a fault happens;

- DC overhead line or DC cable;

- accessories (e.g., joints).

Among all these indispensable parts of the HVDC transmission system, cable insulation material is one of the most important elements in HVDC cables. The performance of the cable insulation material can influence the overall transmission efficiency by limiting the voltage level of the cable. In principle, on the one hand, by increasing voltage, it can increase the power transfer. On the other hand, by increasing the current, it will also increase the energy transfer. However, that means thicker conductor made of expensive metal needs to be employed, which is not beneficial for the economical perspectives. Therefore, designing high quality and high performance HVDC cable insulation material which can endure high voltage is an important challenge. The current EU project GRIDABLE aims to reach a significant development in this area by designing new HVDC cable insulation materials: "the target of the project is to connect renewable energy sources to the energy grid in a more efficient way through innovative polypropylene (PP) based nanocomposites that aim to improve reliability at operating voltages in DC cable insulation and in power capacitors" [4]. -The "GRIDABLE" project is co-founded by the EU HORIZON 2020 program and conducted by 8 different partners located in 5 European countries. It is a consortium consisting of both, research groups from universities and companies covering the whole production and application chain, which makes this project very unique and focused on a real application. 
This PhD thesis is part of the GRIDABLE project and is focused on the design of the HVDC cable insulation material. Our research work focuses on the development of novel insulation nanocomposites. Driven by the environmental pressure, the green approach encourages us to find innovative solutions for the scientific and practical challenges in polymeric material development. In this research work, polypropylene is used as an insulation matrix due to its ease in recyclability and good dielectric properties in comparison to commonly used cross-linked polyethylene. The other focus point of this development is the use of nanoparticles. Nanodielectric polymer composites draw a lot of attention from both, industry and academia. It is reported that the interface between a nanofiller and polymer matrix plays an important role in reducing space charge accumulation and breakdown strength [5]. Hence, to perform nanofiller surface functionalization in order to tailor the interface properties becomes crucial. Plasma polymerization and solvent free modification were successfully applied for the modification of the nano-silica filler surface in order to improve the compatibility between the nanoparticles and polymeric matrix and to tailor the charge trap depth and distribution in the nanocomposites. The advantage of these modification methods is elimination of the negative impact of a solvent, which is commonly used in suspension type of modifications. This work can lay a foundation for future manufacturing and application of recyclable cables with improved dielectric performance for worldwide operation.

\subsection{Objectives of the Research Work}

The goal of this PhD study is to develop and optimize PP based insulation nanocomposites filled with tailored silica on laboratory scale for the next generation of HVDC cable insulation material.

In this research project, it also aims for:

1) Developing a green method of silica surface modification, which can be scaled up in order to supply a sufficient amount (approx. $20 \mathrm{~kg}$ ) of modified silica for a production trial of a cable compound.

2) Designing a new type of surface-modified silica reducing space charge accumulation in polypropylene based insulation nanocomposites.

3) Investigate the effect of surface modification of silica on charge trapping and space charge accumulation behaviour of the insulation material.

\subsection{Outlines of the Research Work}

Chapter 1 is the introduction of the project background and objectives of the thesis.

Chapter 2 is the literature review, in which the challenges and problems of power transmission are summarized and analysed. The state-of-the-art of the HVDC cable insulation material development, the introduction of nanosilica and surface modification methods are discussed.

Chapter $\mathbf{3}$ is a global overview of the research work. It introduces all topics and their interconnection to finally form the whole and complete research.

In Chapter 4 to 8 , the different research topics are reported as publications or manuscripts. 
Chapter 4 (published): "Surface modification of fumed silica by plasma polymerization of acetylene for PP/POE blends dielectric nanocomposites".

The goal of this study is twofold:

- Performing silica modification by plasma polymerization of acetylene, in order to improve the dispersion of the silica in the polymer matrix.

- Suppressing space charge accumulation of the composite via changing of the charge trapping properties of nanocomposites based on silica/PP/POE (polypropylene / ethylene-octene copolymer) blends.

The polymer/silica nanocomposites were produced via laboratory mini-scale compounding and injection moulding.

Chapter 5 (published): "Silica surface-modification for tailoring the charge trapping properties of PP/POE based dielectric nanocomposites for HVDC cable application".

In this chapter, an easy-to-scale up solvent-free silanization method was developed to tailor the silica surface properties. The goal is to investigate the influence of different silica surface modifications on the performance (crystallization, dispersibility and charge trapping dielectric behaviour) of PP/POE nanocomposites. As in Chapter 4, the nanocomposites were produced via laboratory mini-scale compounding and injection moulding.

Note: Starting from Chapter 6, the abbreviation of ethylene-octene copolymer was changed from POE into EOC.

Chapter 6 (under review): "The influence of nanosilica surface functionalization on PP/EOC cast thin film nanocomposites: micromorphology, dynamic and dielectric properties”.

In this chapter, the nanocomposites were prepared by pilot-scale compounding in a twin-screw extruder and cast film extrusion technique was used. Blends with modified polar and unpolar silicas were studied. The aim of this study is to compare the effect of unpolar and polar silica on the dielectric performance of the nanocomposite taking dispersion and compatibility with the polymers into consideration.

Chapter 7 (published): "Dielectric performance of silica-filled nanocomposites based on miscible (PP/PP-HI) and immiscible (PP/EOC) polymer blends".

For this study, nanocomposites were again prepared by pilot-scale compounding (twin-screw extruder) and cast film extrusion. The aim is to compare two polymer blends, one miscible ( $P P / P P-H I$ (propyleneethylene copolymer)) and the other one immiscible (PP/EOC (ethylene-octene copolymer)), in order to select the one with better mechanical and dielectric performance as polymer matrix for the nanocomposites. The effect of addition of modified silica on both polymer blends was also studied.

Chapter 8 (published): "PP/PP-HI/Silica nanocomposites for HVDC cable insulation: Are silica clusters beneficial for dielectric performance?"

In this chapter, modified silica with different concentrations were added to a PP/PP-HI (propyleneethylene copolymer) polymer blend. Besides filler amount, the effect of silica cluster morphology on the composite properties was investigated. 
Chapter 9 (under review): "Joining good dispersion with tailored charge trapping in nanodielectrics by hybrid functionalization on silica".

This part of the study focuses on bringing together all gained knowledge and experience for a newly designed and modified silica in order to improve the compatibility between the silica and polymer matrix while improving dielectric performance via surface-functionalization strategies developed in the earlier studies of this thesis.

Chapter $\mathbf{1 0}$ is the general summary of the whole thesis.

\subsection{Author Contributions}

This $\mathrm{PhD}$ work is based on a multidisciplinary approach comprising chemistry (nanoparticle surface functionalization), physics (plasma treatment, dielectric properties) and material engineering (nanoparticles, polymer blends, compounding, nanocomposites). It is a collection of various contributions especially from a dielectric properties perspective, nanosilica functionalization and nanocomposite compounding. The detailed author contributions to the experimental chapters (Chapter 4-9) are listed in this part.

The author was responsible for the nanosilica surface functionalization and characterization of the modified nanosilica. The author developed and conducted the solvent free method to perform the nanosilica surface functionalization. The author conceived and performed also the plasma modification of nanosilica.

The author did not contribute to the compound manufacturing, which was performed by the VTT Technical Research Centre of Finland, in particular Eetta Saarimäki, Ilkka Rytöluoto, and Mika Paajanen. VTT was one of the project partners.

All the dielectric characterizations (thermally stimulated depolarization current measurements (TSDC), dielectric spectroscopy, Pulsed Electro Acoustic (PEA) measurements, conductivity) were performed by the author and the project partners from the Tampere University (Ilkka Rytöluoto, Minna Niittymäki and Kari Lahti) and from the University of Bologna (Paolo Seri and Hadi Naderiallaf).

All remaining characterizations (X-ray diffraction (XRD), Differential Scanning Calorimetry (DSC), Thermogravimetric Analysis (TGA), Dynamic Mechanical Analysis (DMA)) were mainly done by the author at the University of Twente except for Scanning Electron Microscopy (SEM), which was performed by Mark Smithers at the MESA+ Institute at University of Twente. The Polarized Optical Microscopy (POM) was done by Ilkka Rytöluoto (VTT Technical Research Centre of Finland) at the Tampere University.

The author is the main contributor to the data analysis. Amirhossein Mahtabani, Rafal Anyszka and Wilma Dierkes (all from University of Twente) contributed to the discussion of the results. Paolo Seri contributed to the data analysis of PEA and conductivity. Ilkka Rytöluoto contributed to the data analysis of POM, dielectric spectroscopy and TSDC tests.

The author is also the main contributor to the manuscripts. Ilkka Rytöluoto, Paolo Seri, Kari Lahti, Rafal Anyszka and Wilma Dierkes commented on the manuscripts, and the author revised the manuscripts until submission. 


\section{References}

[1] Madani, V., Das, R., Aminifar, F., McDonald, J., Venkata, S.S., Novosel, D., Bose, A. and Shahidehpour, M., 2015. Distribution automation strategies challenges and opportunities in a changing landscape. IEEE Transactions on Smart Grid, 6(4), pp.2157-2165. DOI: 10.1109/TSG.2014.2368382

[2] Das, R. and Madani, V., 2013. Protection and control strategies for distribution automation in a smarter grid. Proc. IEEE Power Energy Soc. Innov. Smart Grid Technol. DOI: $10.13140 / R G \cdot 2 \cdot 2.22515 .02081$

[3] Keshri, J.P. and Tiwari, H., 2020. Fault Location Methods in HVDC Transmission System-A Review. Intelligent Computing Techniques for Smart Energy Systems, pp.411-419. ISBN: 978-981-150213-2. DOI: 10.1007/978-981-15-0214-9_45

[4] http://www.gridable.eu/index.htm, access date 27.08.2019.

[5] Tanaka, T. and Imai, T., 2013. Advances in nanodielectric materials over the past 50 years. IEEE Electrical Insulation Magazine, 29(1), pp.10-23. DOI: 10.1109/MEI.2013.6410535. DOI: 10.1109/MEI.2013.6410535 
8| Page 


\section{Chapter 2}

\section{Literature Review}




\subsection{Problems and Challenges of Power Transmission}

High Voltage Alternating Current (HVAC) and High Voltage Direct Current (HVDC) transmission as the two technologies to transport electricity play an important role in the modern economy and society. HVAC is very popular in industry and delivering power to domestic applications, mostly used to transfer power at relatively short distances. HVAC transmission benefits from the relatively low cost of the transformer, which can be used to easily step the voltage up when transmitting power (hence requiring lower currents, and smaller conductors as a consequence) and down, for all of the medium and low voltage users in the grid. However, HVAC transmission is affected by higher costs associated to conductors. Those costs arise mainly from the fact that with increasing transmission distance, also transmission losses increase, both due to the increased resistance of the conductor, but also caused by increased stray capacitance resulting in an additional loss of power towards the ground. When cables are underground or submarine, the associated capacitance (hence its losses) are greatly increased, so that unacceptable losses with AC transmission are encountered much sooner with this type of installations than with overhead lines. This is a phenomenon entirely absent in DC, where the increase in losses is only due to Joule heating of the inner conductor. However, HVDC transmission needs the usage of converters on each side of the transmission lines, in order to convert the alternating current of generators into the direct current of the grid and vice versa, to convert the DC power of the transmission lines into AC power for the users. Due to the high cost of those converter stations, HVDC transmission is not an economic option for short distances. However, over a certain long distance, the breakeven distance, the savings on the conductors equipment of HVDC offsets the costs of converters, which makes HVDC less expensive than HVAC, as shown in Figure 2.1 This breakeven distance is in the range of $500 \mathrm{~km} \sim 800$ $\mathrm{km}$ for overhead lines, depending to some extent on the power needed and the voltage level of the transmission [1], while it is only in the range of 35 $80 \mathrm{~km}$ for submarine or underground cables [2].

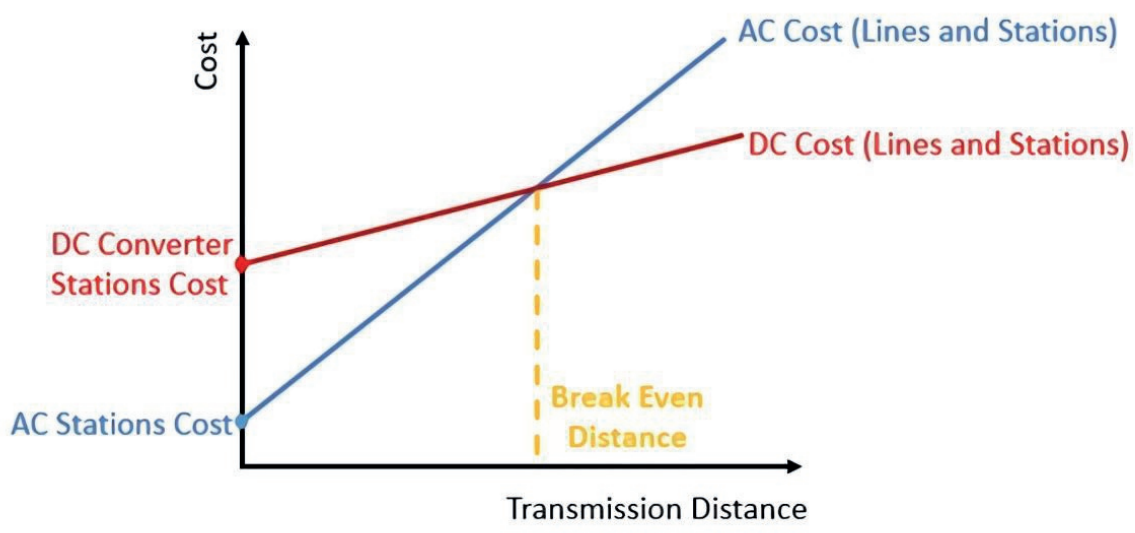

Figure 2.1 HVAC and HVDC transmission line costs [1, 2]. 
Another disadvantage of HVAC over HVDC transmission is the so-called skin effect, meaning that only a part of the conductor is actually used in HVAC: The skin effect is a phenomenon of the current flowing on the surface of the conductor being much higher than the flow in the core of the conductor. This results in a reduced effective cross-section of the conductor as shown in Figure 2.2 Since the resistance is inversely proportional to the cross-section of the conductor, the skin effect leads to an increased conductor resistance. Consequently, it results in higher loss. Moreover, longer distances of power transmission will obviously lead to significantly higher losses than short distances. The skin effect is highly dependent on the frequency. For HVDC, the frequency is zero, therefore no skin effect is observed. The loss in HVDC systems is much lower than in HVAC, especially for long distance transmissions. This also means that $\mathrm{AC}$ will require a larger amount of conductor and bigger transmission towers to transmit a certain power at a certain voltage.
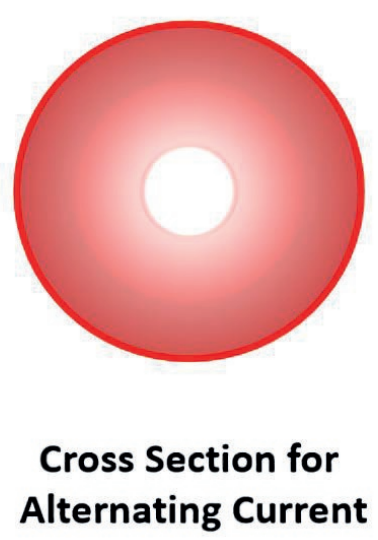

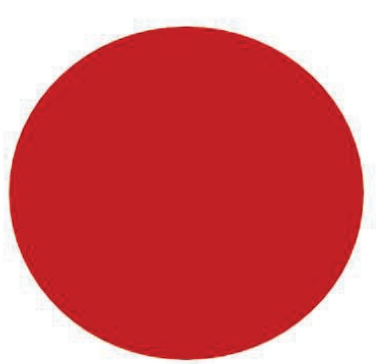

\section{Cross Section for Direct Current}

Figure 2.2 Scheme of current density in AC and DC conductors due to skin effect.

Summarized, the HVDC transmission technology offers lower energy losses and lower costs than HVAC transmission over long distances.

HVDC transmission is realized for many applications [3, 4], for example, long distance power transmission, offshore, underground and submarine cable transmission, and asynchronous ties. In general, there are two major types of HV transmission lines: overhead lines and cables. Overhead lines are still seen in our daily environment. The cable line is usually applied for submarine, offshore underground transmission [5, 6], and also in urban areas where the space needed for overhead lines is not available or acceptable. An HVDC cable is generally considered as the only solution for long distance sea power transmission and technically the only solution over a certain long distance power transmission. The application of HVDC underground cables can also solve the negative effect of power transmission on cityscape during urbanization, by replacing the overhead lines [7]. Moreover, underground cable lines are safer for humans and animals than overhead lines, for example if an overhead line breaks and fails due to severe weather conditions. In addition to this, one 'environmental' aspect is that people are typically strongly against building of new overhead lines especially in the 
vicinity of cities, making it difficult to get permissions for building new overhead lines. Therefore, as a contribution to the development of the HVDC technology, this work focuses on the development of a new dielectric composite for HVDC cable insulation layers.

\subsubsection{HVDC Cables}

In general, underground or submarine HVDC cables can be divided into the following categories:

- fluid filled cables,

- mass-impregnated cables (MI), and

- extruded cables.

Fluid filled cables are literally filled with an insulating fluid (low viscosity oil), and kraft papers (cellulose based paper with good insulation properties, high strength and a thickness of 50 to 125 microns) are used for insulation. The fluid filled cable (Figure 2.3) with properly designed polypropylene-laminated paper layers can reach up to $500-800 \mathrm{kV}[5,8,9]$. However, in order to keep the oil efficiently flowing in the channel, a pressurized feed system has to be applied, e.g. a pumping station with a working pressure of 16 bar [10], which limits the operational length of the oil filled cable to $30-60 \mathrm{~km}$. Additionally, there is also a risk of oil leakage [11], for which additional protective layers are also used, often rendering the cable heavy and difficult to install.

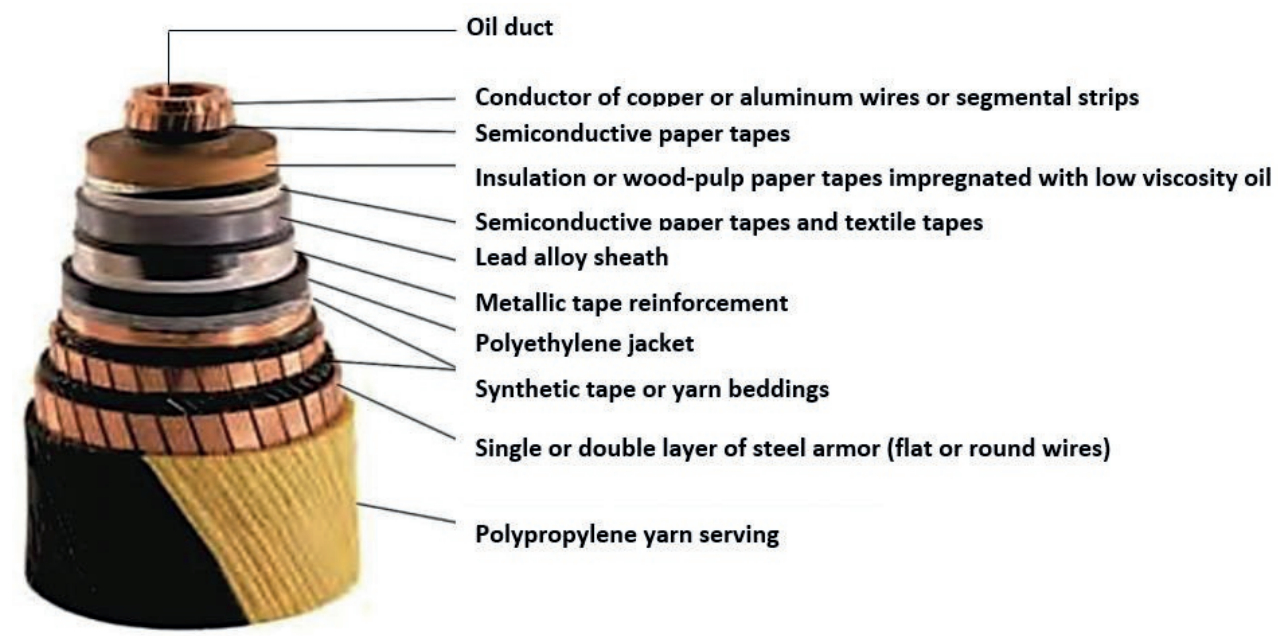

Figure 2.3 Oil-filled cable cross-section showing various functional layers [12].

The mass-impregnated cable (Figure 2.4) is a cable lapped with a high viscosity compound impregnated paper, and the high viscosity compound is usually a mineral oil with polyisobutylene additives [5]. In order to reach a high dielectric strength, high density kraft paper $\left(1000 \mathrm{~kg} / \mathrm{cm}^{3}\right)$ is usually used for 
insulation of the MI cable. The impregnated paper is lapped together in a clean processing facility, under controlled humidity, in order to produce a high-performance MI cable. During the lapping process, gap (also called butt gap) formation takes place due to the imperfect arrangement of adjacent paper layers because of their different winding angles [13]. A high viscosity compound is used to fill these gaps to avoid cavity formation. Additionally, there is no leakage from the MI cable, even if it is cut or broken. Therefore, it is a suitable option for submarine applications, and the service voltage can reach up to 500 $\mathrm{kV}$ [5]. However, the maximal working temperature of MI cables is about $50-55{ }^{\circ} \mathrm{C}$ in order to avoid migration of the high viscosity compound. Therefore, the power capacity of the traditional MI cable is limited due to the necessity of low operation temperature. With the application of the polypropylene laminated paper in the MI cable, the high viscosity compound migration can be avoided even at higher temperatures of $80-90{ }^{\circ} \mathrm{C}$. Consequently, the power capacity is expected to reach $1 \mathrm{GW}$, which is much more than $600 \mathrm{MW}$ of the traditional MI cable containing non-laminated kraft paper [14]. In principle, a MI cable has no length limitation from the insulation system point of view, since it does not need any oil pressure feeding systems. In practice, it is very hard to reliably manufacture this type of cables, which are very prone to inclusion of defects.

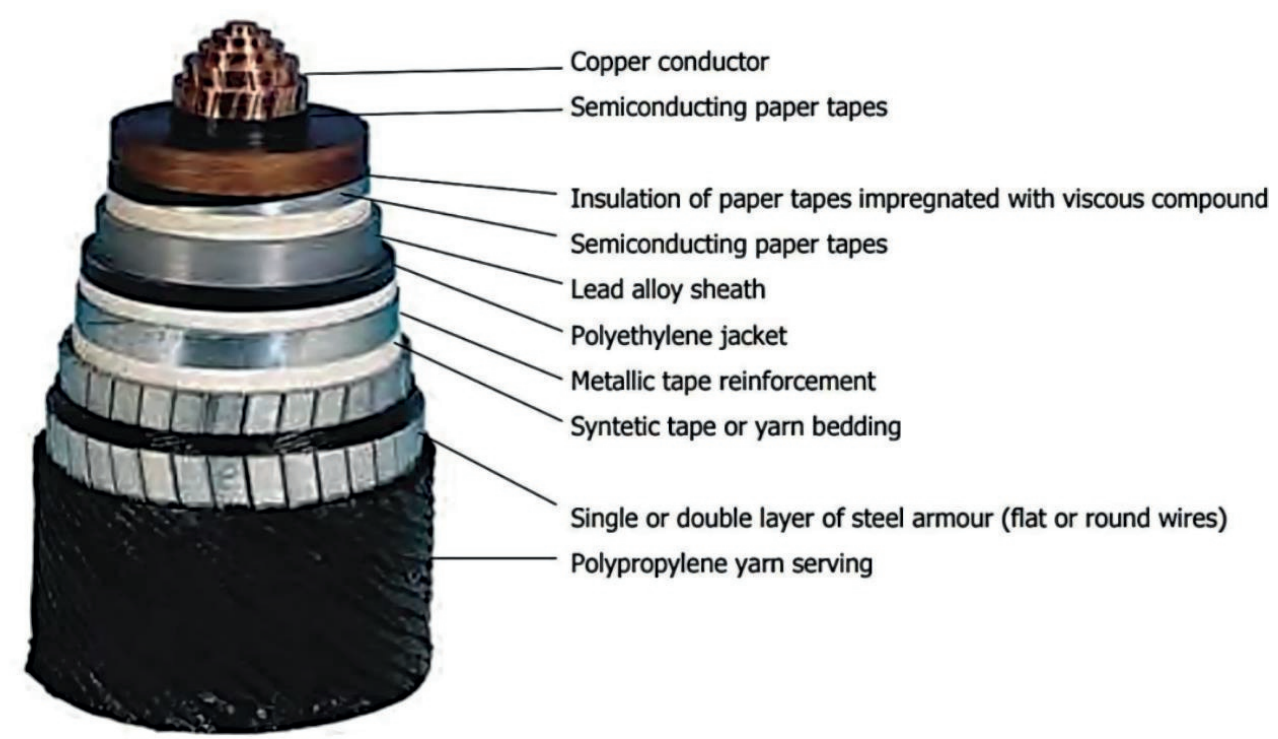

Figure 2.4 Mass-impregnated cable cross-sections showing various functional layers [12].

Another type of DC cable is the polymeric extruded cable (Figure 2.5), which contains a polymeric insulation material. This type of cable exhibits a number of beneficial features, such as a large capacitance and fast production, light weight, relatively simple joints and terminations and no oil leakage problems $[15,16]$. The world's first extruded HVDC cable was installed in 1997 by ABB and GEAB in Gotland, Sweden [17]. Due to the above mentioned advantages of the extruded DC cables over oilfilled and IM cables, a significant number of R\&D projects were performed on the extruded cable components $[7,15,18]$. 


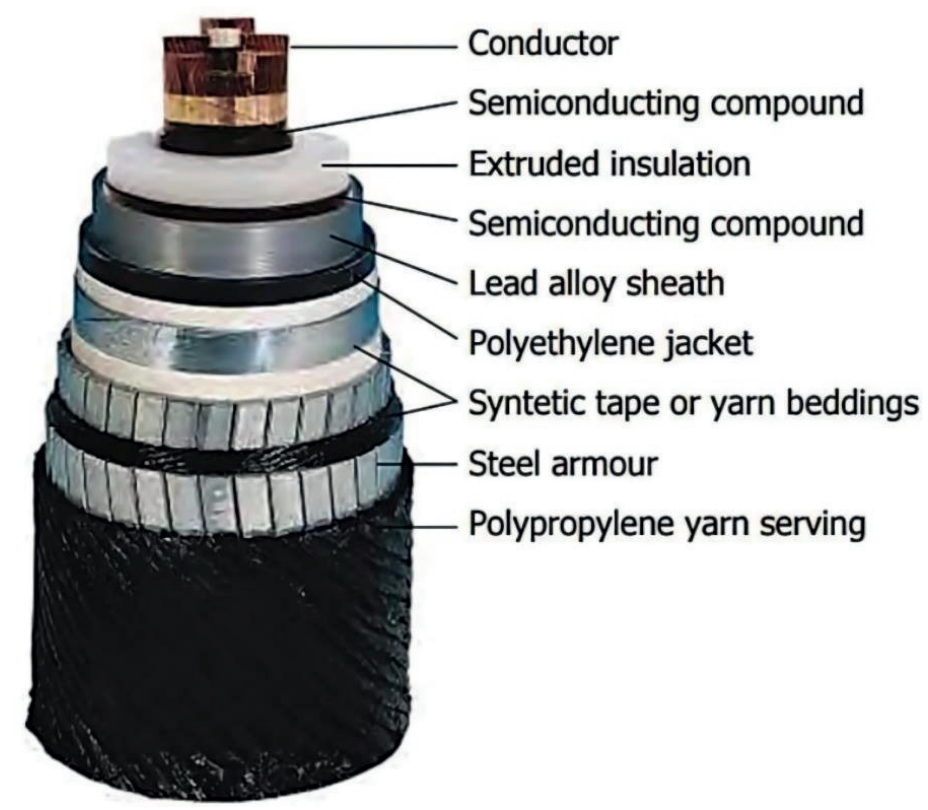

Figure 2.5 Extruded cable cross-sections showing various functional layers [12].

\subsubsection{Some Critical Issues and Challenges of Extruded HVDC Cables}

HVDC transmission features unique phenomena influencing the cable insulation behaviour. One of the critical issues of HVDC cables is the complex electric field distribution, which is different than HVAC cable with the "laplace", "capacitive" or "permittivity dependent" distribution [19]. The electric field distribution of HVDC cables is governed by the conductivity and permittivity of the insulation material [20]. The permittivity of the insulation can be assumed to be a constant within the operation temperature [19]. Differently, the conductivity of the insulation is influenced by the local temperature. As there is a gradient of temperature across the cable from the inner conductor to the outer sheath, it will result in a gradient of conductivity. Due to the varied ratio permittivity/conductivity with position under electric field, the space charge will be gradually build up. This space charge build up can also influence the electric field distribution and cause an electric field inversion [21]. Consequently, the extruded HVDC cable performance (e.g. dielectric loss, conductivity, breakdown strength, aging etc.) will be affected by this complex DC electric field distribution (including the effect of space charge build up and temperature related DC conductivity) in the insulation.

Space charge is considered as the amount of charge accumulated and stored in the insulation material. When an electric field is applied to an insulation material, space charge can be slowly injected into the material and accumulate inside the material, since there is enough time for slow field driven movements as the DC field is continuously in the same direction. This will cause a distortion of the electric field distribution from the theoretical distribution supposed during the design of the insulation system, resulting in accelerated local aging [22]. If for example the local electrical field happens to rise passing the the breakdown strength (the maximum electrical field an insulation material can withstand without failure) of the material, that portion of material will experience electrical failure. Or if the increased 
local electrical field is not high enough to cause an immediate breakdown, it can trigger a fast chain aging mechanism, eventually resulting in an aging degraded insulation, leading to the complete breakdown failure of the whole insulation. For example, during the cable insulation manufactory, the micro voids are inevitable. Hence, the final cable insulation consists of the polymer and the air-gap void. The space charge can trigger hot-electron avalanches via impact ionization of gas molecules [22] in the air-gap under an electrical field. These electrons will collide with polymers around the void, causing the damage of the adjacent polymer and initiating the aging of the polymer.

To control or limit space charge accumulation is one of the biggest challenges of HVDC cable insulation materials. There are two kinds of space charges present in an insulating material in the vicinity of the electrodes, when it is placed in an electrical field: homo-charges and hetero-charges. In general, homocharges are formed by charge accumulation near electrodes of the same polarity, and the hetero-charges are charges accumulated near electrodes with opposite polarity. There are also charge packets present in the bulk, which are charges moving from the electrode into the insulation bulk or charges generated by ionization of impurities (e.g. additives and catalyst residues) in the insulation composite. The space charge accumulation process highly depends on the distribution of charge trap levels in the materials, temperature, applied electric fields, and in general the local difference between the ratio of charge injection and extraction. In case of the homo-charge, when trapping is strong, it gives the smallest field distortion and can actually help preventing further charge injection since the field is reduced at the injection site (the electrode). In case of hetero-charge, when extraction of charges is prevented for some reason, this is the worst condition since the electrical field near the electrode is very high.

A charge trap is a location that can restrict charge movement in a dielectric material [23]. Release of restricted charges from the traps (detrapping) depends on the local electric field, temperature and also the trap depth. Depending on depth, traps can be divided into shallow or deep traps. The energy of shallow traps is normally in the range of $0.1-0.5 \mathrm{eV}$, while deep trap energy levels can equal a few electron volts [24]. The origins of shallow traps usually are physical defects of the insulation material, e.g., molecular conformational disorder, polymer chain folding, or intercrystalline links [25]. Deep traps are often introduced by chemical defects, e.g. impurities, broken chemical bonds, additives or byproducts of aging. In summary, the physical and chemical properties of the insulation materials have a significant influence on the trap distribution, both depth and density. The charge trap distribution is able to influence the charge mobility and trapping-detrapping process, hence space charge accumulation.

The relationship between space charge accumulation, trap distribution and the physical and chemical properties of the insulation material is very complex and not fully studied and reported in literature. Hence, it is important to have a systematic study on the relationship of the insulation material properties (e.g., morphology, crystallization behaviour, nanofiller addition and surface functionalization), trap distribution (trap depth and trap density) and space charge accumulation, in order to design a high performance HVDC extruded cable insulation material.

\subsection{Extruded HVDC Cable Insulation Material}

\subsubsection{Crosslinked Polyethylene (XLPE)}

Crosslinked polyethylene (XLPE) is widely used as HVDC cable insulation material owing to its high performance, e.g., low dielectric loss, good dielectric and thermo-mechanical properties. XLPE is 
produced by mixing of linear low density polyethylene (LDPE) with dicumylperoxide (DCP) during extrusion and crosslinking it in the next step. Due to the thermoset nature of XLPE, the thermomechanical properties of XLPE are enhanced in comparison to LDPE. The operation temperature of XLPE can reach up to $90^{\circ} \mathrm{C}$, which makes XLPE suitable for large power capacity cables. However, by-products are formed such as acetophenone, cumyl alcohol and $\alpha$-methylstyrene during the radical reaction of DCP as shown in Figure 2.6. It is widely accepted that the by-products can cause space charges in the polymeric matrix. This space charge can change the local electrical field distribution, which may cause insulation breakdown during service life [26]. Moreover, it is reported that cumyl alcohol, which is the main component of the total of by-products in XLPE (1.121 wt.\% of cumyl alcohol, 0.455 wt. $\%$ of acetophenone and 0.014 wt. $\%$ of $\alpha$-methylstyrene) promotes the accumulation of space charge more significantly than the other components [27]. This is explained by the changed charge trap distribution by the hydroxyl group of the cumyl alcohol [28]. Besides, XLPE is not easily recyclable due to the thermoset nature. With the increasing concern of environmental effects, the development of a recyclable and extrudable insulation material is of high interest.

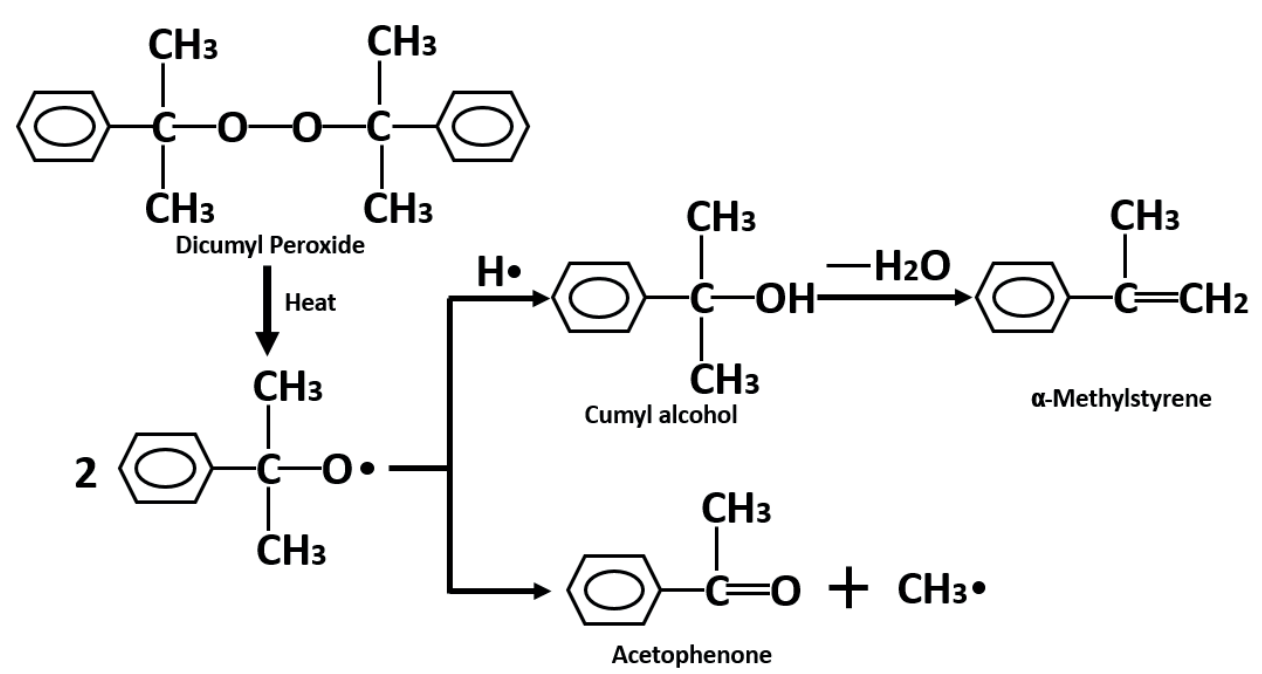

Figure 2.6 DCP radical dissociation and formation of by-products [29].

\subsubsection{Polypropylene}

Polypropylene (PP) is a promising candidate for a potential application in the next generation of HVDC cable insulation due to its recyclable nature, high melting temperature (reaching $170{ }^{\circ} \mathrm{C}$ depending on the type and grade of the PP), and excellent electrical characteristics (e.g. high breakdown strength of $300 \mathrm{kV} / \mathrm{mm}$ ). PP is a hydrophobic semi-crystalline thermoplastic and it hardly absorbs water. Consequently, the humidity of the surroundings does not influence its insulation properties. Additionally, due to the good mechanical properties and high melting temperature of PP, there is no need of crosslinking, which simplifies and accelerates the PP cable manufacturing avoiding crosslinking and degassing to remove the byproducts of the crosslinking process. 


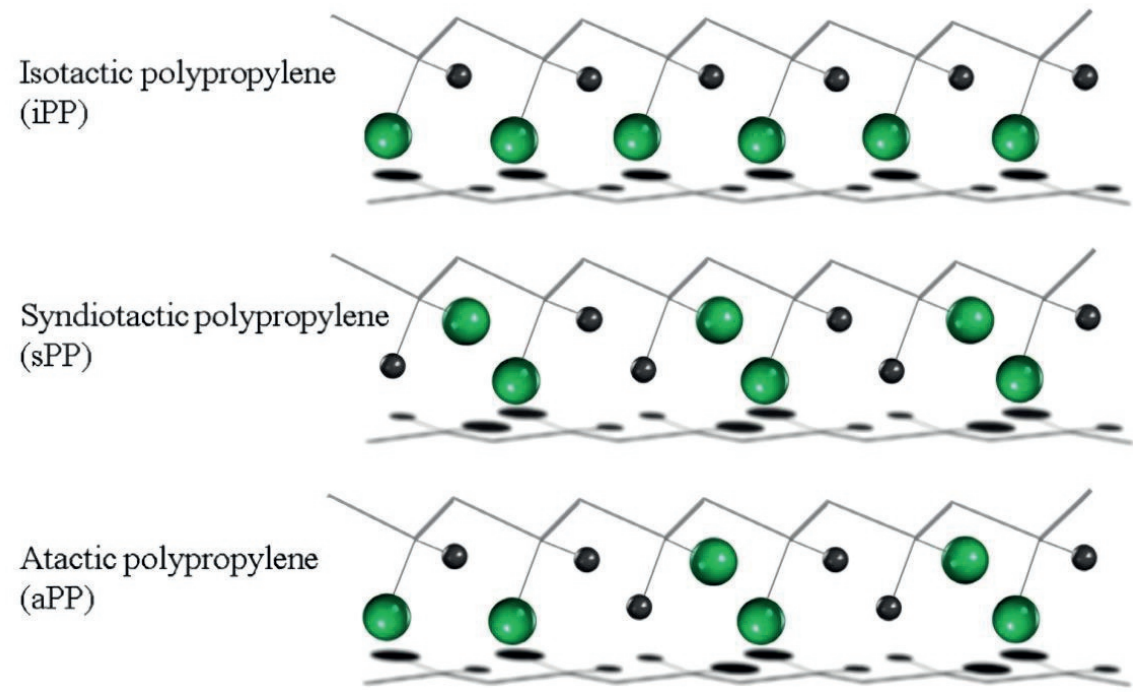

Key: Methyl $\left(\mathrm{CH}_{3}\right) \bigcirc \operatorname{Hydrogen}(\mathrm{H})$

Figure 2.7 Tacticity of polypropylene macromolecules [30].

Based on the methyl group position, PP can be divided into isotactic PP (iPP), syndiotactic PP (sPP) and atactic PP (aPP), as shown in Figure 2.7 Random positioning of the methyl group on the carbon backbone limits the crystallization process, which increases the amorphous phase in aPP. iPP and sPP are both semicrystalline polymers with melting temperatures of the crystalline phase of $170{ }^{\circ} \mathrm{C}$ and $150{ }^{\circ} \mathrm{C}$, respectively. Due to the arrangement of the methyl groups, which are located on one side of the carbon backbone in the iPP structure, a higher crystallinity (approx. $60 \%$ ) is present in comparison to sPP (approx. $30 \%$ ), in which the methyl groups are alternately on both sides. The high melting temperatures of iPP and sPP make both of them potential candidates for large capacity power cable application: iPP or sPP insulated cables can reach operation temperatures of more than $90{ }^{\circ} \mathrm{C}$. However, iPP lacks flexibility at room temperature and impact toughness at low temperature, resulting from its inherent chemical structure and high crystallinity. This is a major drawback of iPP, hindering its applicability for cable insulation. Hence, to overcome this problem, most studies on PP as an insulation material are nowadays focused on PP-copolymers [31] and PP/polyolefin blends that exhibit higher flexibility and low-temperature toughness [32,33].

\subsubsection{Polypropylene Copolymers}

In PP-copolymers, a second monomer (ethylene or another $\alpha$-olefin) is copolymerized with propylene. Based on the content and type of the co-monomer, PP-copolymers exhibit different morphologies and various thermal and mechanical properties, which also influence their dielectric performance. 


\subsubsection{Melting and Crystallization Behaviour Influenced by Copolymerization}

With increasing content of the co-monomer, the melting temperature (Tm) of the polypropylene copolymers is decreasing Figure 2.8. This occurs as the co-monomer acts as a molecular defect disturbing the crystallization process of the PP backbone, which also reduces the lamellae thickness (Figure 2.9) [34]. In addition, the melting temperature also varies with the type of comonomer: the most effective ones are octene- 1 and hexene- 1 and the butene- 1 shows the least effect on reducing the melting temperature. In order to design a high power capacity cable, a PP copolymer with a high melting temperature is needed.

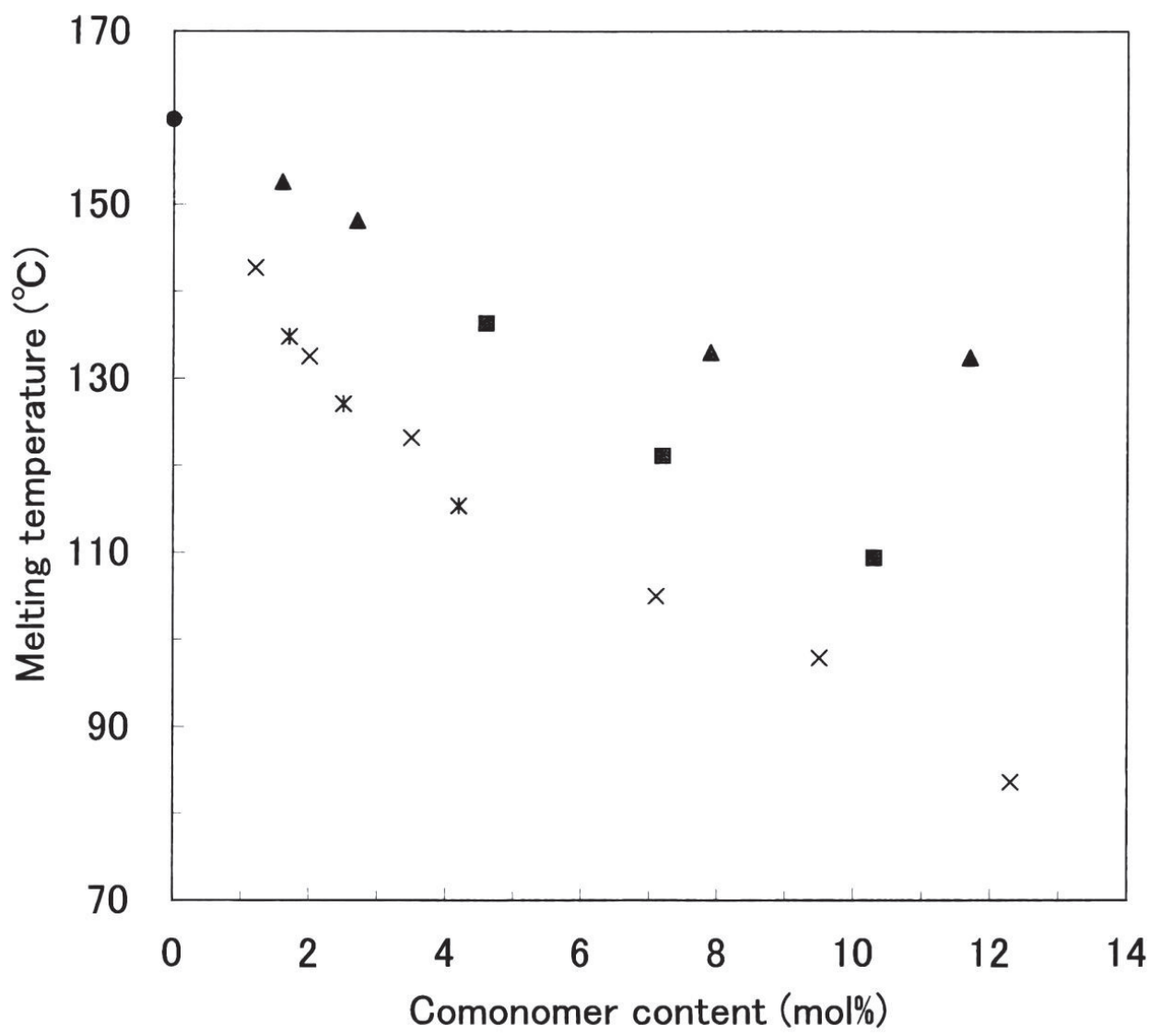

Figure 2.8 Change of melting temperature as a function of co-monomer content: • homo PP; copolymer: $\boldsymbol{\|}$ ethylene, $\boldsymbol{\Delta}$ butene, $\times$ hexene, $*$ octene [35]. 


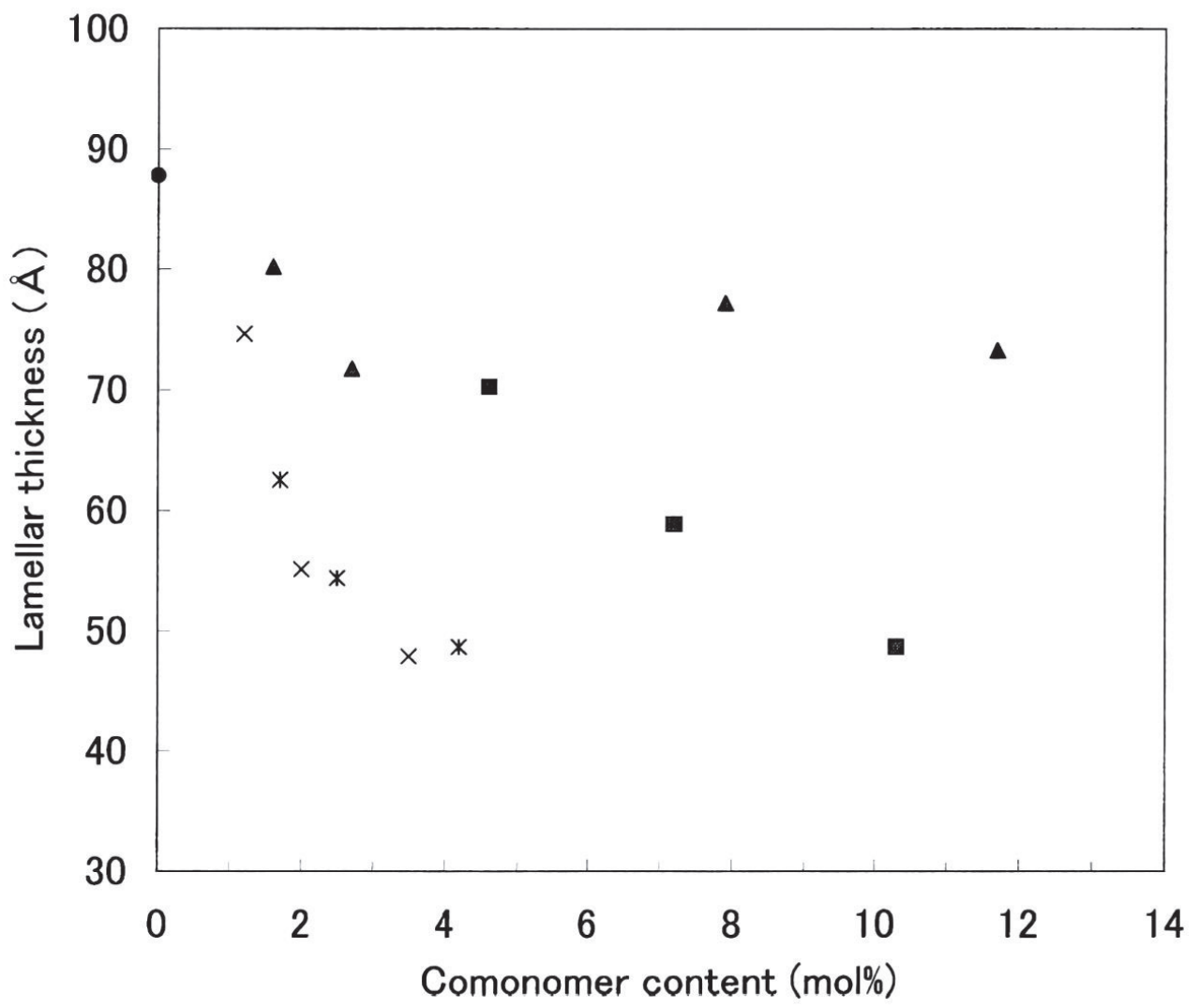

Figure 2.9 Change of lamellar thickness as a function of co-monomer content: • homo PP; comonomer: $\square$ ethylene, $\triangle$ butene, $\times$ hexane-, $*$ octane- $[35]$.

\subsubsection{Influence of the Copolymer on the Morphology}

The morphology of PP copolymers varies for random or block copolymers. Peng [36] compared the morphology between propylene-ethylene block copolymers (bc-PP with a high content of ethylene of 7-15\%), and PP-ethylene random copolymers (rc-PP, with a low content of ethylene of 1-4 \%). The results showed that a high content of ethylene results in formation of longer ethylene sequences in the bc-PP: ethylene-rich amorphous areas. These domains can be removed by heptane etching resulting in void formation in b-PP as shown in the SEM picture in Figure 2.10. Obviously, there are much smaller voids found in rc-PP due to the randomly distributed short ethylene sequences. Therefore, bc-PP contains clearly separated polymer domains, while rc-PP exhibits almost a fully homogenous morphology. The morphology of a polymer influences its electrical performance noticeably. In this case, the interface between the ethylene-rich area and PP will act as a weak area initiating the breakdown, or as trapping sites changing the charge trapping-detrapping process influencing space charge accumulation behaviour. 

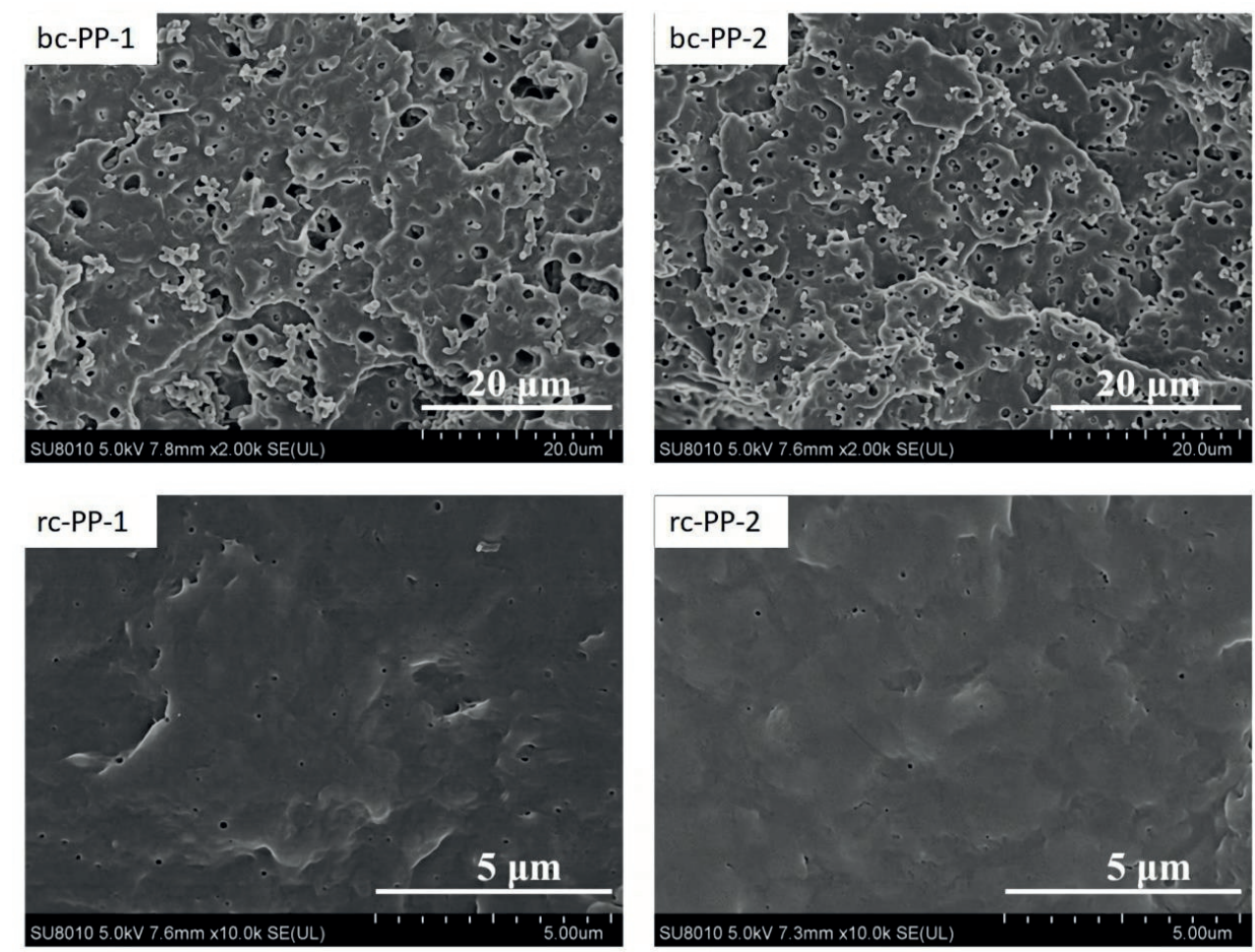

Figure 2.10 SEM images of PP copolymers (a) bc-PP-1 (b) bc-PP-2 (c) rc-PP-1 (d) rc-PP-2.

(b-PP-1 with $9 \%$ ethylene of a molecular weight (Mw) of $280000 \mathrm{~g} / \mathrm{mol}$; bc-PP-2 with $9.3 \%$ ethylene of Mw $410000 \mathrm{~g} / \mathrm{mol}$; rc-PP-1 with $2.8 \%$ ethylene of Mw $320000 \mathrm{Mw}$ g/mol; rc-PP-2 with $2.8 \%$ ethylene of Mw $380000 \mathrm{~g} / \mathrm{mol}$ ) [36].

\subsubsection{Influence of the PP Copolymer on the Crystalline Structure}

PP usually has three crystalline forms: monoclinic $(\alpha)$, trigonal $(\beta)$ and orthorhombic $(\gamma)$. The monoclinic $(\alpha)$ form is the most common one. The trigonal $(\beta)$ is formed mostly in presence of nucleating agents and is favourable for toughening of PP [37]. The orthorhombic $(\gamma)$ form is rare and increases with increasing crystallization temperature and concentration of stereo defects (heterotactic segments in an isotactic molecule) [34]

PP copolymers can form different crystalline phases depending on the microstructure of the polymer. For example, there is only the monoclinic $(\alpha)$ type present in iPP, while monoclinic $(\alpha)$ and trigonal $(\beta)$ forms are both present in PP-ethylene block copolymers (bc-PP), and monoclinic $(\alpha)$ and orthorhombic $(\gamma)$ can be seen in PP-ethylene random copolymers (rc-PP) (Figure 2.11). The presence of trigonal ( $\beta$ ) and orthorhombic $(\gamma)$ phases introduce deep charge traps, which capture the charge carriers and suppress the charge injection from the electrodes. As a consequence, the insulation performances of bc-PP and rc-PP are higher than the ones of iPP in terms of low conductivity and high DC breakdown strength [38]. 


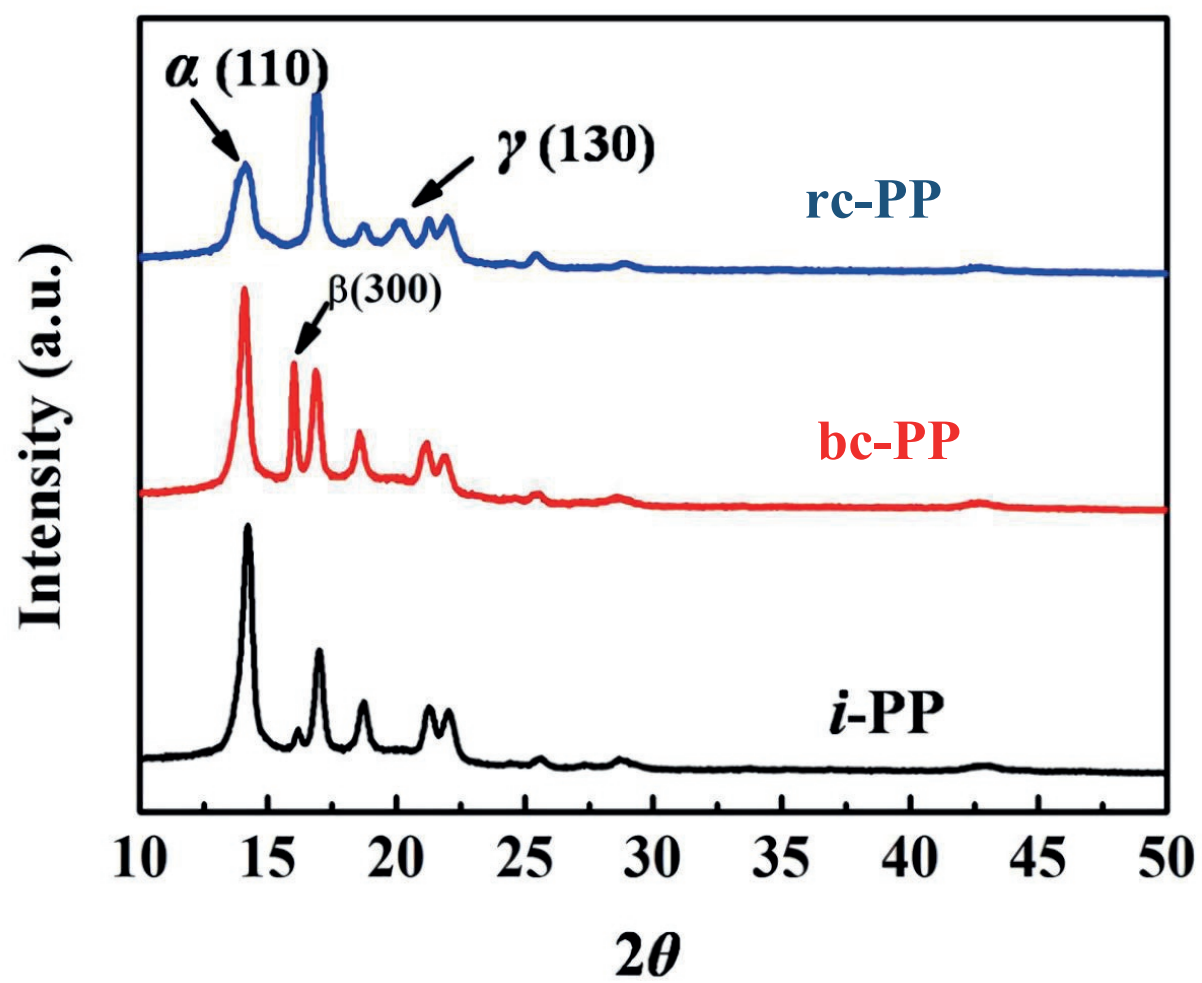

Figure 2.11 XRD patterns of a PP-ethylene block copolymer (bc-PP), a PP-ethylene random copolymer (rc-PP) and an isotactic PP (iPP) [38].

\subsubsection{Mechanical Properties as Influenced by the Copolymer}

The mechanical properties (e.g. tensile strength, elongation at break) also vary for different PPcopolymers. Firstly, PP copolymers show higher strain at break than iPP, which indicates that the PP copolymerization can increase its flexibility due to the differences in degree of crystallinity and crystal structure. In addition, the different PP copolymers also exhibit a variation in flexibility. For example, the random PP copolymer shows higher ductility than iPP and the PP block copolymer as shown in Figure 2.12. An HVDC cable needs certain flexibility: in order to facilitate easy transportation and installation, they need to withstand bending conditions. Therefore, PP random copolymers show a potential for HVDC cable applications due to the much higher flexibility than iPP.

\subsubsection{Polypropylene Blends}

Blending is an effective and generic way to obtain the desired properties based on commercially available polymers. In order to overcome the low flexibility of PP, blending with an elastomer or a polyolefin is performed. The first concern of effective polymer blending is miscibility of the polymers. 


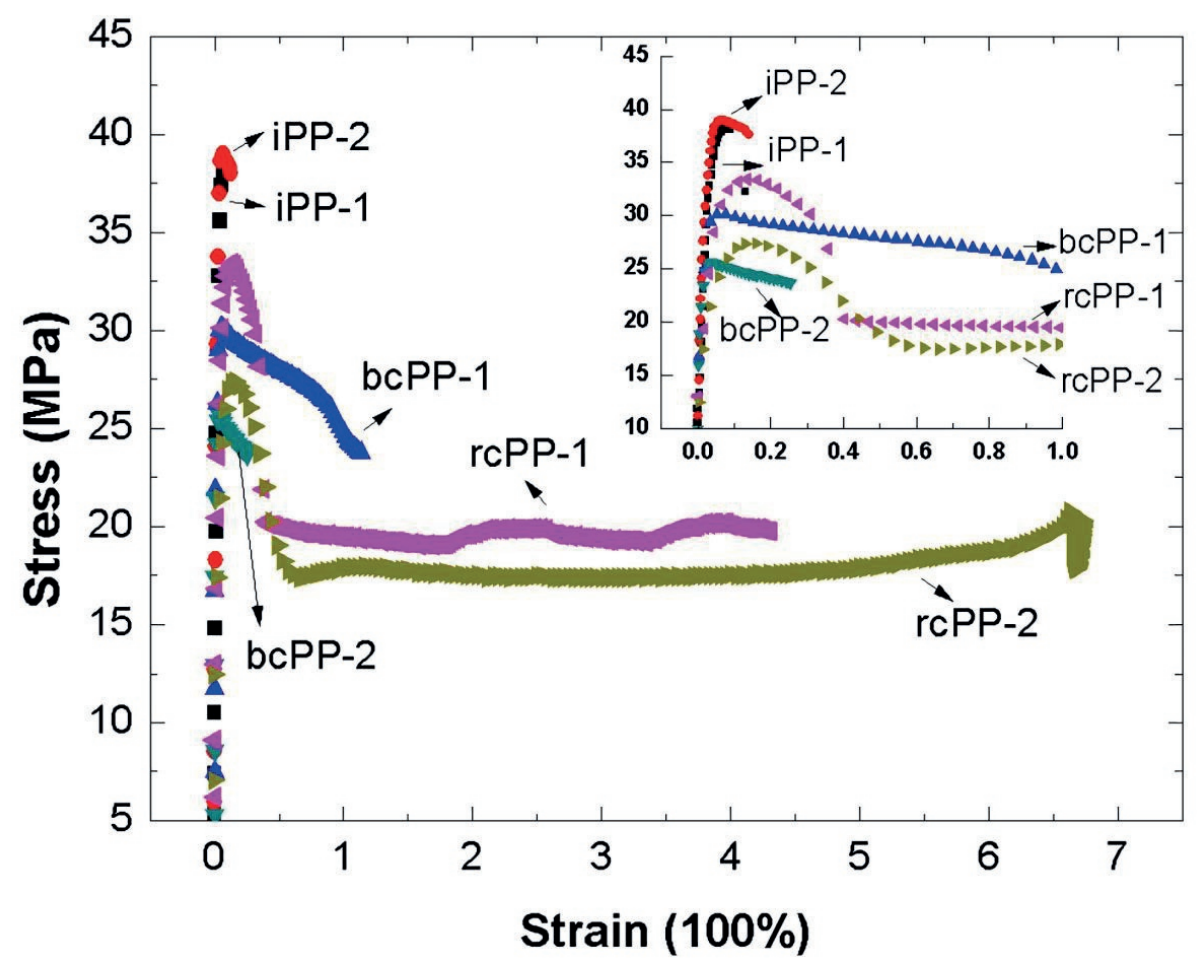

Figure 2.12 Tensile test results of PP block copolymers (bcPP), PP random copolymers (rcPP) and isotactic PP (iPP) [39].

In general, polymer blends can be divided into miscible (homogeneous) and immiscible (heterogeneous) ones. A miscible blend contains only one phase, while the immiscible blend shows phase separation. For a blend to be miscible it requires a negative Gibbs free energy $(\Delta \mathrm{G})$ defined in Equation (2.1):

$$
\Delta \mathrm{G}=\Delta \mathrm{H}-\mathrm{T} \Delta \mathrm{S}<0
$$

where: $\Delta \mathrm{G}$ is the Gibbs free energy; $\Delta \mathrm{H}$ is the enthalpy; $\Delta \mathrm{S}$ is the entropy; $\mathrm{T}$ is the mixing temperature.

For a polymer-polymer blend, the entropy (randomness of the molecular arrangement) is usually very low since the combinatorial entropy (the number of chain arrangements during mixing) of both polymers is low. To obtain a negative $\Delta \mathrm{G}$, high mixing temperatures or negative enthalpies are needed. A negative $\Delta \mathrm{H}$ requires formation of polymer specific interactions, e.g., hydrogen bonding or dipole-dipole interactions [34] during mixing. In a PP blend, there is usually no specific interaction due to its hydrophobic nature and lack of polar groups in the chemical structure. Additionally, the mixing temperature is generally around the polymer melting temperature, and a too high mixing temperature can degrade the polymer. Therefore, most PP blends are immiscible. 
In an immiscible polymer blend, phase separation takes place. In general, there are two types of polymer blend morphology:

1) One polymer forms the continuous phase, "encapsulating" another polymer which acts as the dispersed phase, as shown in Figure $2.13 \mathrm{a}$ and $\mathrm{b}$.

2) Two polymers act as co-continuous phases "interlocking" or "interpenetrating" each other, as shown in Figure $2.13 \mathrm{c}$.
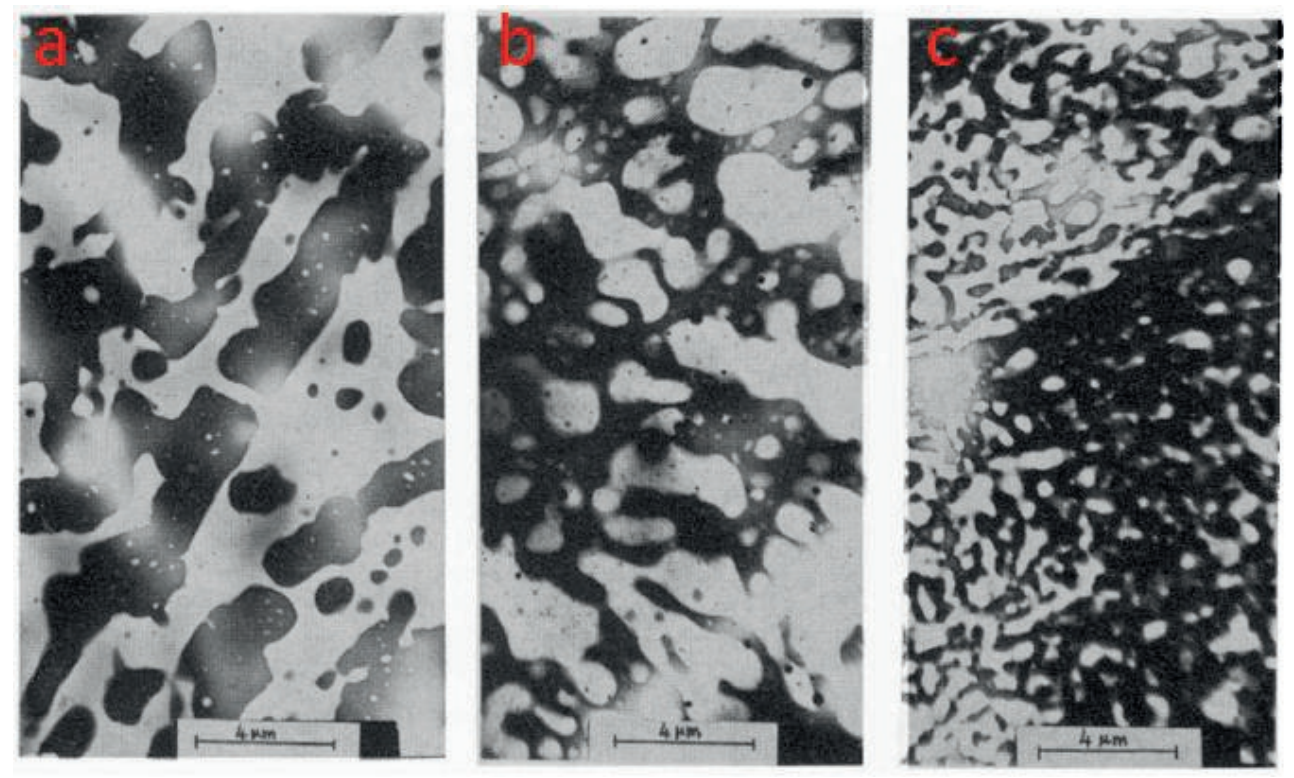

Figure 2.13. Morphology of EPDM/BR (50/50) blends. The white and black colour represent EPDM and $\mathrm{BR}$, respectively. $\mathrm{a}-\mathrm{EPDM}$ is the continuous phase; $\mathrm{b}-\mathrm{BR}$ is the continuous phase; $\mathrm{c}$ - both EPDM and BR are co-continuous phases [41].

Blend morphology is influenced by many factors, e.g., blending parameters (temperature, pressure, shear stress [40]), the weight ratio of the polymers and the molecular weight of the polymer [41]. An example is shown in Figure 2.13: for the same polymer blend, the morphology can be modified by changing mixing conditions to make either ethylene propylene diene rubber (EPDM) or polybutadiene rubber (BR) the continuous phase, or make both, EPDM and BR, co-continuous phases. The mixing conditions of these three blends are shown in Table 2.1. The varied mixing conditions (temperature, time and shear rate) result in different torque values indicating the mixing viscosity of the polymers. The results show that the low viscosity polymer becomes the continuous phase and encapsulates the high viscosity polymer (dispersed phase). With equal viscosity during mixing, both polymers form co-continuous phases. 
Table 2.1 Mixing conditions of the EPDM/BR blend

\begin{tabular}{|c|c|c|c|c|c|}
\hline \multirow[b]{2}{*}{ Blend system } & \multicolumn{3}{|c|}{ Mixing conditions } & \multirow{2}{*}{$\begin{array}{l}\text { Torque } \\
\text { EPDM/BR } \\
{[\mathrm{N} \cdot \mathrm{m}]}\end{array}$} & \multirow{2}{*}{$\begin{array}{l}\text { Continuous } \\
\text { phase }\end{array}$} \\
\hline & $\begin{array}{l}\text { Time } \\
{[\mathrm{min}]}\end{array}$ & $\begin{array}{l}\text { Rotor speed } \\
{[\mathrm{rpm}]}\end{array}$ & $\begin{array}{l}\text { Temperature } \\
{\left[{ }^{\circ} \mathrm{C}\right]}\end{array}$ & & \\
\hline A & 90 & 0.5 & 20 & $16.7 / 5.4$ & $\mathrm{BR}$ \\
\hline B & 1.8 & 50 & 112 & $16.7 / 41.7$ & EPDM \\
\hline $\mathrm{C}$ & 1.9 & 10 & 55 & $29.4 / 29.4$ & both \\
\hline
\end{tabular}
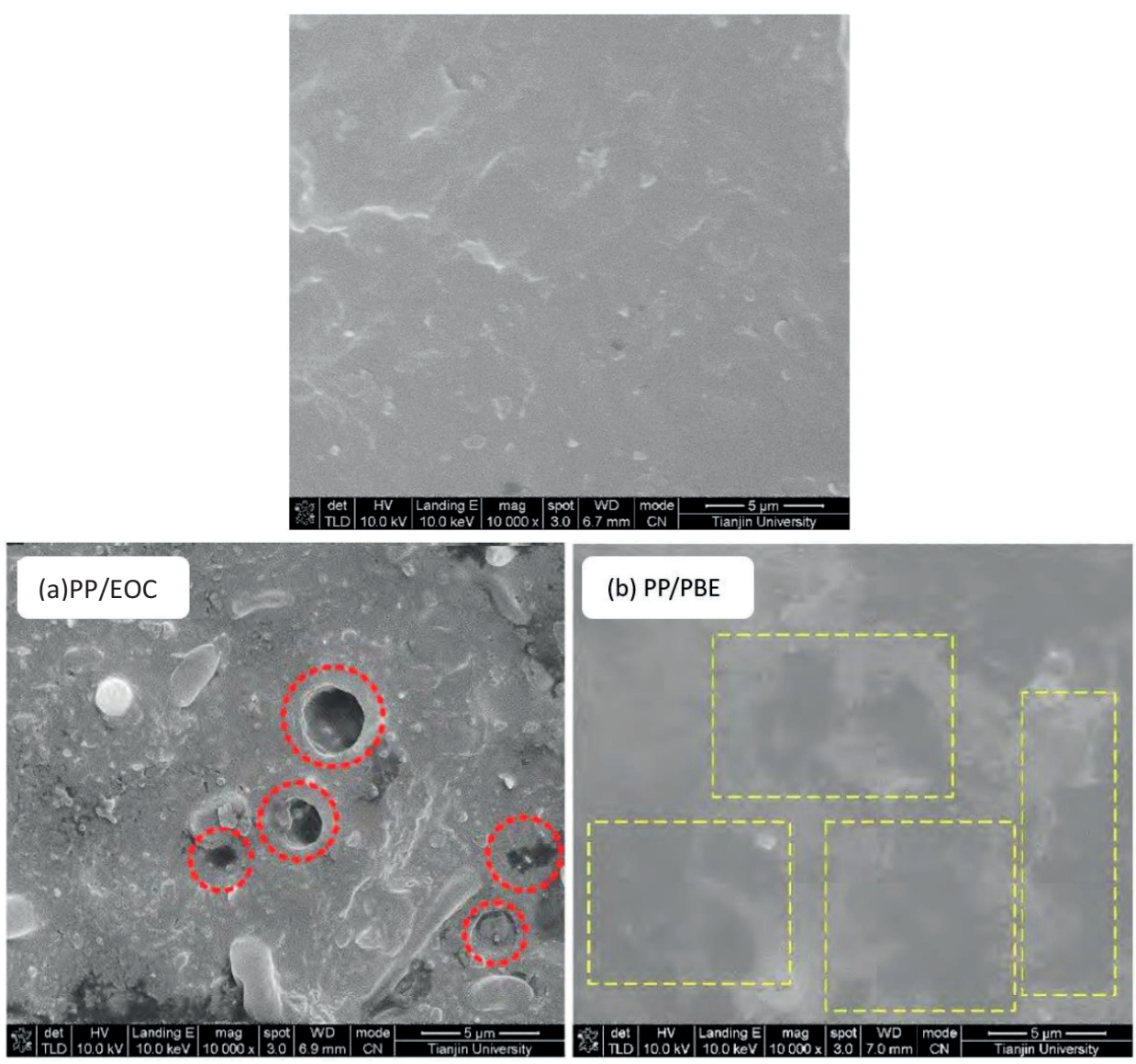

Figure 2.14 SEM images of PP/ethylene-octene copolymer (EOC) and PP/propylene-ethylene copolymer (PBE) blends. The red circles indicate detached EOC domains. The yellow rectangles indicate areas with both phases and the boundaries between PP and PBE. [33] 


\subsubsection{Micromorphology of PP blends and its Effect on Dielectric Performance}

For PP blends, morphology is the main factor influencing their dielectric performance. The composition of a polymer blend affects its miscibility and further influences charge transport, leading to different dielectric performance. The morphologies of two PP blends with ethylene-octene copolymer (EOC) (a) and propylene-ethylene copolymer (PBE) (b) are shown in Figure 2.14. For the PP/EOC blend, the cross section of the sample is rough, and the EOC forms isolated islands in the PP phase indicating immiscibility. In case of the PP/ PBE blend, the cross section is smooth. Although there is a visible boundary between PP and the PBE no isolated phases or cracks appear in the sample, indicating partial miscibility. This indicates that the compatibility between PP and PBE is higher than between PP and EOC. The authors found that PP/ PBE blends with good compatibility showed a higher breakdown strength than PP/EOC blends with low compatibility due to the different trap depths. The authors proposed that the polymer blend with good compatibility gives strong interaction between two polymers (tight interface), while the polymer blend with low compatibility has a weak interaction (loose boundary), as shown in Figure 2.15. They stated that the molecular chain disorder, as a defect, forms the traps that capture charges (e.g., electrons). For the PP/PBE blend, the tight interfaces cause the significant chain disorder. The molecular chain disorder is a chemical defect forming deep traps, from which the charges are not easily de-trapped, leading to a high breakdown strength of the blend. In case of PP/EOC, there is a loose interface acting as the physical defect, from which the trapped charges are easily released, leading to a low breakdown strength and increased conductivity.

Tight Interface

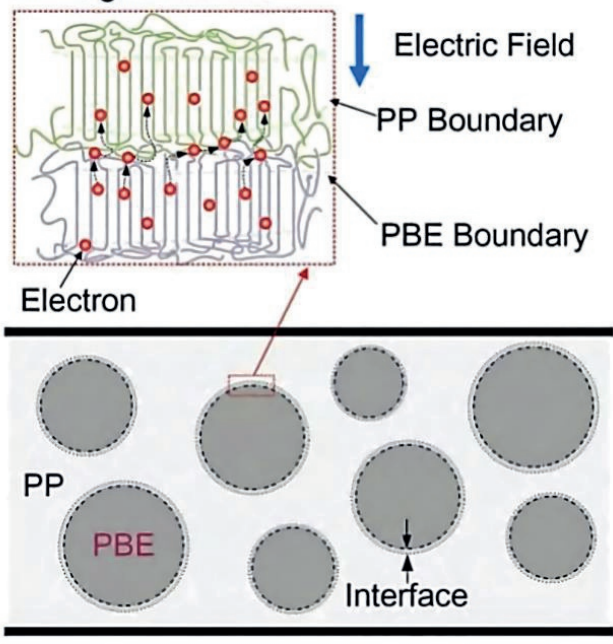

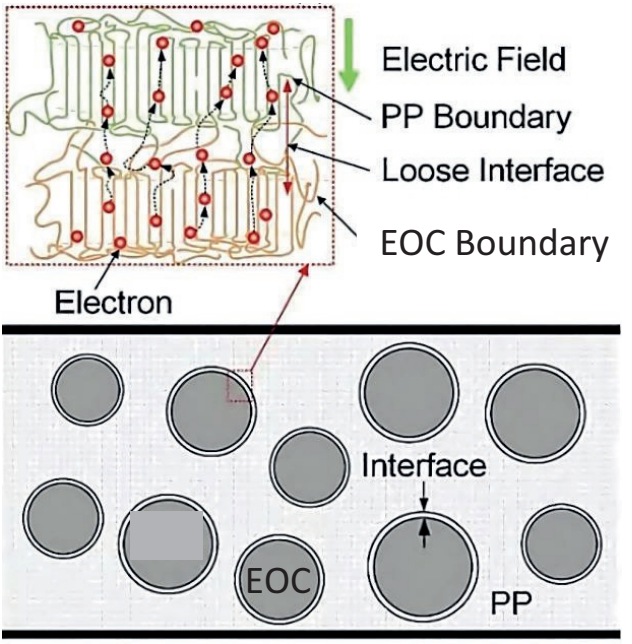

Figure 2.15 Schematic diagram of the microscopic morphology at the PP/PBE and the PP/EOC interfaces [33]. 


\subsubsection{Crystalline Morphology of PP Blends and its Effect on Dielectric Performance}

Another important feature of the PP blends influencing their dielectric properties is the crystallinity and crystalline morphology. Different PP blends exhibit different crystalline morphologies, as shown in Figure 2.16. Pure PP shows spherulites with a diameter of about 50-80 $\mu \mathrm{m}$. The PP/ propylene-ethylene copolymer (PBE) blend shows similar spherulites as the pure PP, while the PP/ ethylene-octene copolymer (EOC) blend exhibits a completely different crystalline morphology with a significantly reduced spherulite size and visibly increased spherulite boundaries resulting from the small size of the spherulite due to the nucleating effect of the EOC. The spherulite boundaries provide the paths of migration for charge carriers [42]. An increasing spherulite boundary area caused by small spherulites acting as the increased shallow traps (increased hopping sites) can improve the charge transportation, resulting in low space charge accumulation in the bulk of the material, where no space charge is present near the cathode and only very few hetero charges detected near the anode.
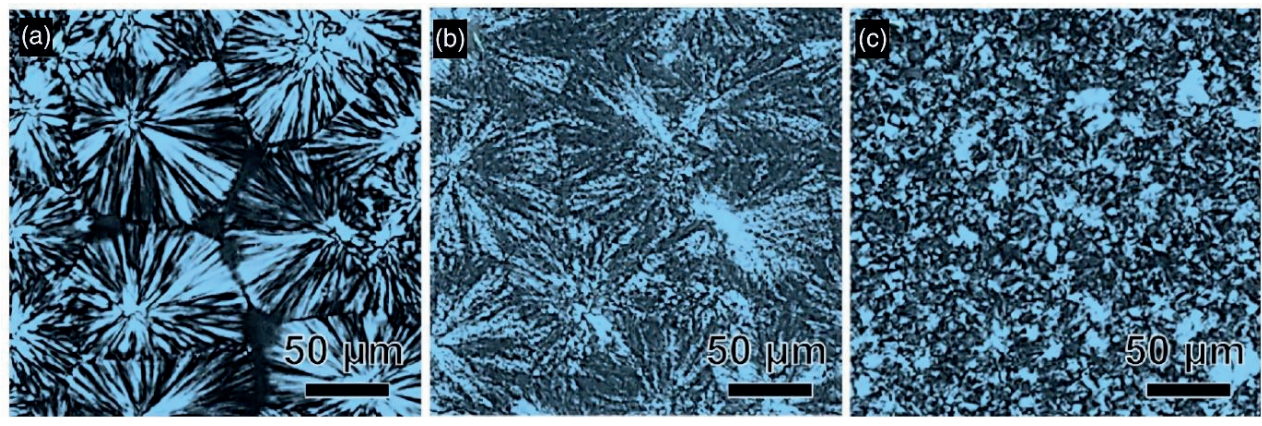

Figure 2.16 Polarized micrographs of PP (a), PP/ propylene-ethylene copolymer (PBE) (b) and PP/ ethylene-octene copolymer (EOC) (c) [44].

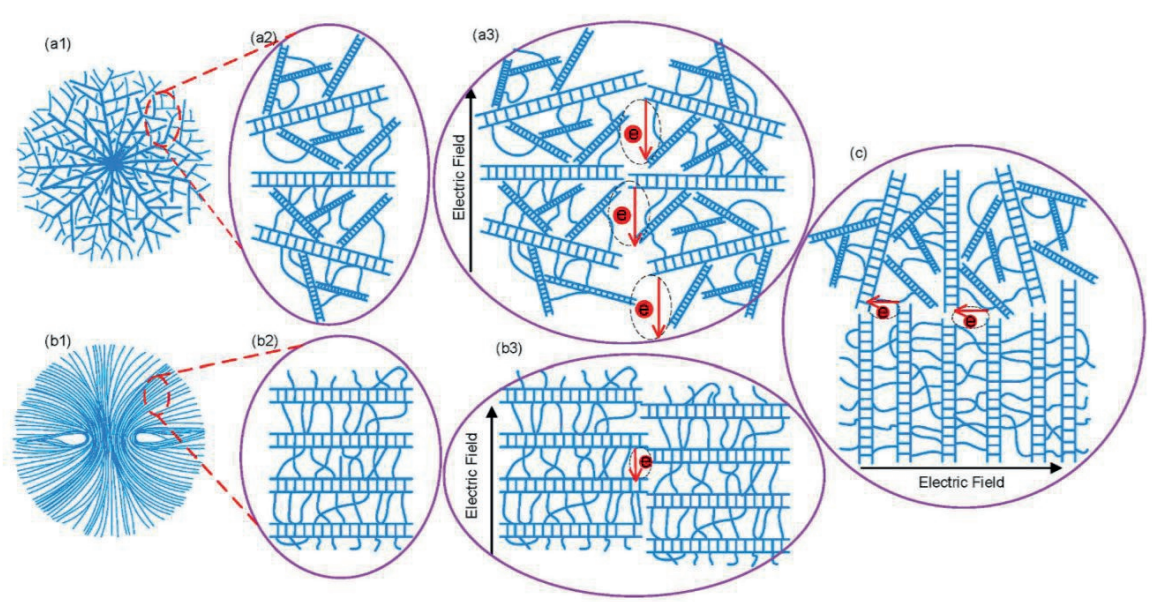

Figure 2.17 the $\alpha$ spherulites model (a1), $\beta$ spherulites model (b1), the $\alpha$ lamella crystal medel (a2), the $\beta$ lamella crystal model (b2), the $\alpha$ lamella crystal boundary model (a3), the $\beta$ and $\alpha$ lamella crystal boundary model (a3), the $\alpha$ and $\beta$ lamella crystal boundary (c) [43]. 
Besides, it is also reported that the type of the spherulite can also affect the dielectric performance of the polymer. As shown in Figure 2.17, the $\alpha$ spherulite lamellae grows radially from the center and appears bifurcations, while $\beta$ spherulite lamella seems like a bundle structure with the parallel or bend lamellae. Compared to the $\alpha$ spherulites, $\beta$ spherulites exhibit more molecular chains interactively with each other in the interfacial area, as shown in Figure 2.17. This results in a smaller free volume and limits the speed of electron traveling, contributing to a high breakdown strength in the polymer with $\beta$ spherulites rather than with $\alpha$ spherulites [43]. Besides, the interfacial molecular chains in these $\beta$ spherulite interphase regions generate deep traps in the PP film. These deep traps can reduce the charge carrier mobility, contributing to the low conductivity. It can also capture the injected charges and form an opposite electric field to the applied field, inhibiting the further charge injection and consequently leading to a low charge injection.

\subsubsection{Space Charge Accumulation in PP Blends}

In the HVDC research field, space charge accumulation is one of the main concerns, since it can cause distortion of the local electric field and thus lead to insulation failure [45, 46]. Homo- and hetero-charges can be accumulated in an insulating material when it is placed in a constant electric field. Homo-charges are formed by the charge injection from the electrodes (or conductor), and the hetero-charges are often generated by ionization of the impurities (e.g., additives and catalyst residues) in the polymer. The charge injection dominates over the ionization of the impurities for the PP blend for HVDC cable insulation due to the lower amount of impurities present in this polymer compared to XLPE, since there are no by-products from the crosslinking process. As the flexibility of PP is improved by blending with an elastomer or another copolymer, it is reported that space charge accumulation is higher in PP blends than in the pure polymer. For example [46], PP/ethylene-octene copolymer blends show higher space charge accumulation than pure PP, stemming from the large amount of trap sites introduced at the interface between PP and the ethylene-octene copolymer due to their low miscibility. In order to suppress space charge accumulation in the PP blends, the most common solution is the addition of a nanofiller. It is widely accepted that the interface introduced by the nanofiller plays an important role: the specific interactions between the nanofiller and the polymer matrix result in a tailored charge trap distribution and improved dielectric performance.

\subsubsection{Dielectric Polymer Nanocomposites}

A dielectric polymer nanocomposite comprises in general a polymer host and a guest of nano-sized inorganic particles (e.g., $\mathrm{ZnO}, \mathrm{MgO}, \mathrm{SiO}_{2}, \mathrm{TiO}_{2}$ ) when aiming for dielectric applications. When the nanoparticles are added to a polymer matrix, a new interface between the nanoparticles and the polymer matrix is introduced, see Figure 2.18 (a). In Figure 2.18 (b), it is shown that with decreasing particle size the volume of the interface region (the interface is composed of different layers) surrounding the particles becomes more and more significant. Consequently, the interface properties become crucial for the entire property profile of the nanocomposites. 


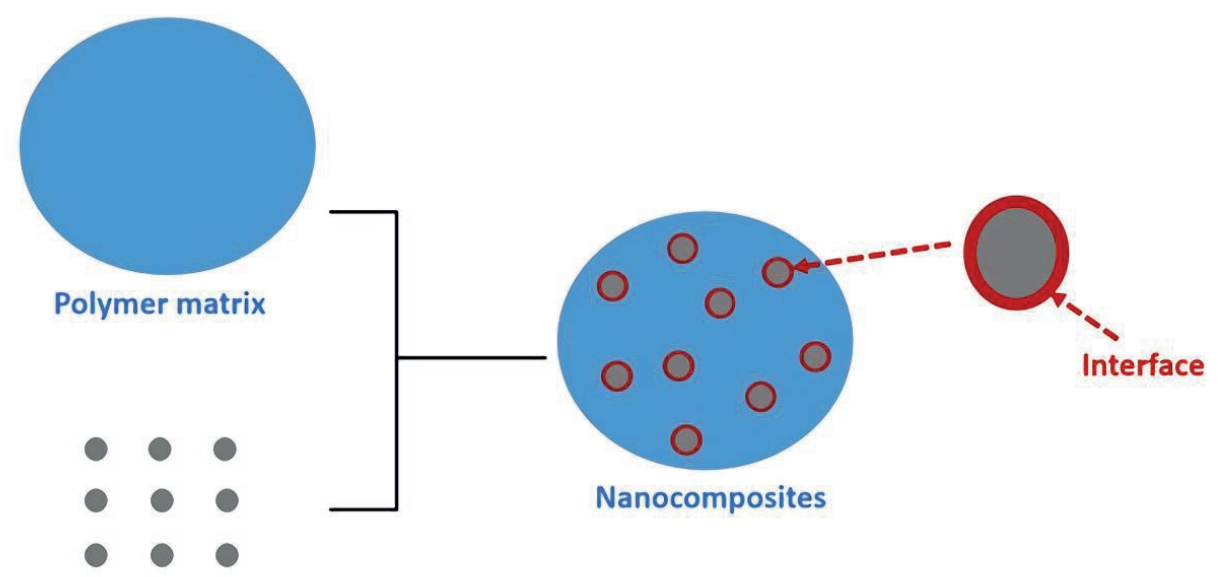

Nanoparticles

(a). Interface between nanoparticles and polymer matrix.

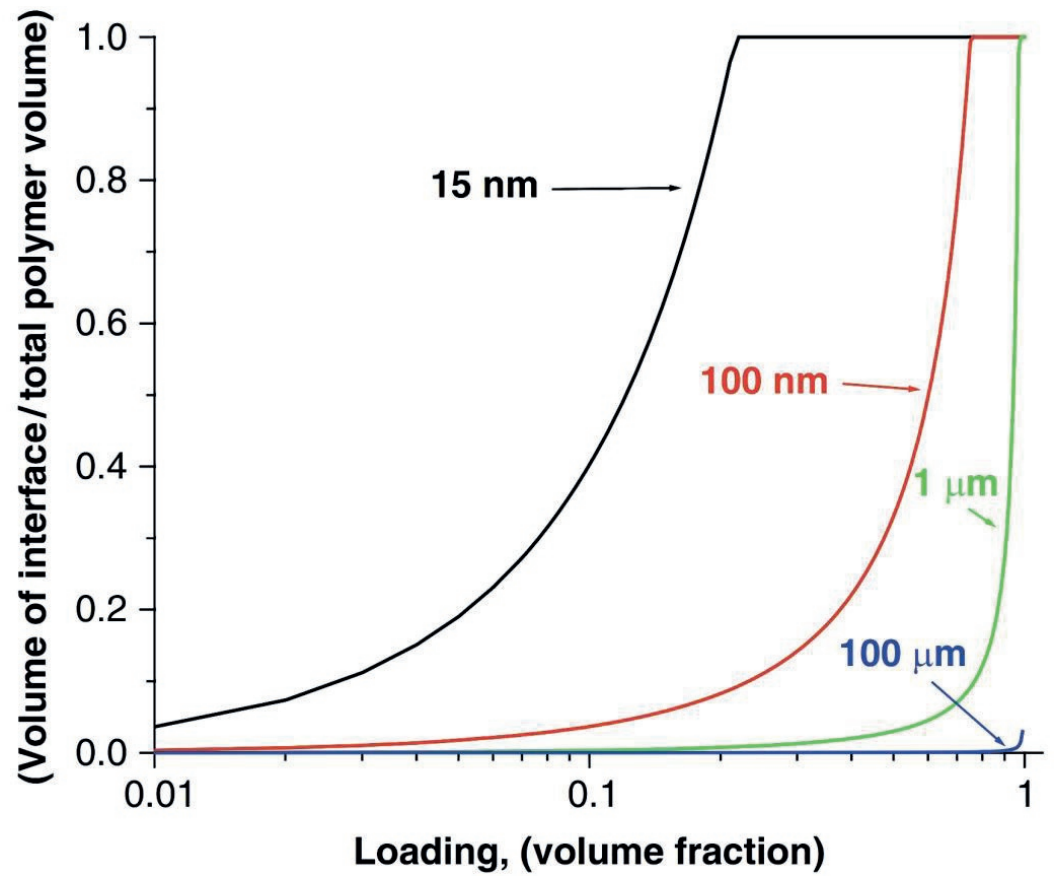

(b) Volume of interface versus total polymer volume of nanocomposites as a function of nanoparticle loading for different particle size.

Figure 2.18 (a) Interface between nanoparticles and polymer matrix and Figure 2.18 (b) Volume of interface versus total polymer volume of nanocomposites as a function of nanoparticle loading for different particle size [47] 
It is reported that the introduced large interfacial area between the nanoparticles and the polymeric matrix may act as charge scattering centres resulting in an improvement of breakdown strength and voltage endurance [48]. Besides, the large interface also brings many trap sites into the polymer matrix. These trap sites with different trap depths and density alter the charge carrier mobility and influence the charge-transport behaviour as well as the space charge accumulation of the nanocomposites [45]. It is also reported that the interface area around a nanofiller shows significant charge trapping capacities in nanocomposites. In high electric fields, free-moving charges are trapped by the introduced deep traps and increase the electrostatic barrier reducing further charge injection. Hence, the space charge accumulation is suppressed by addition of the selected nanofillers $[49,50]$.

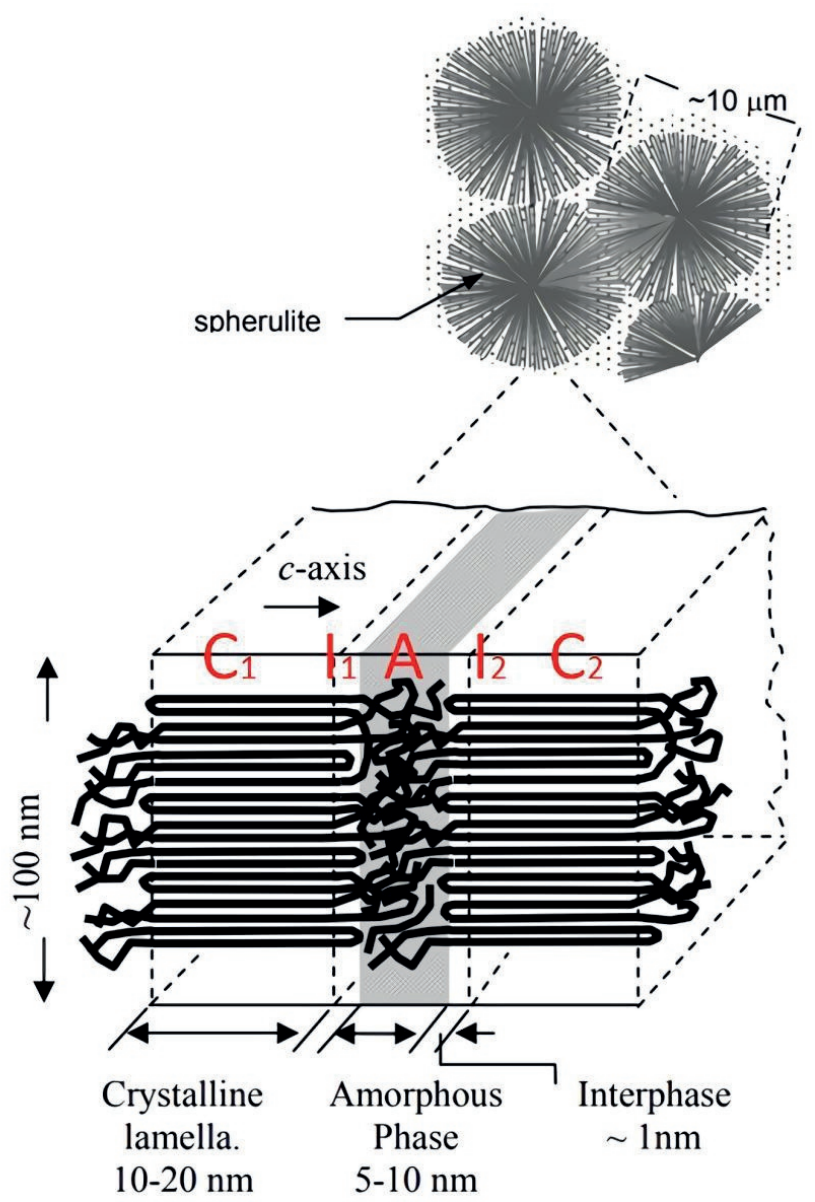

Figure 2.19 Morphology of semi-crystalline polyethylene to explain the tunneling effect [51].

Apart from the interface effect, there are also other factors which can affect the dielectric performance of nanodielectrics: 
1) A nanofiller with a certain band gap can also increase the hole (charge carrier) tunneling barrier in the amorphous phase of the polymer matrix and thus lead to a reduction in conductivity and space charge accumulation. Tunneling is a way of charge carrier movement; the mechanism is shown in Figure 2.19 [51]. Specifically, as shown in Figure 2.19, the crystalline lamella thickness and amorphous phase thickness is $10-20 \mathrm{~nm}$ and 5-10 nm, respectively, and the interface thickness is $1 \mathrm{~nm}$. The hole is moving (tunneling) through crystalline lamella chains $\left(\mathrm{C}_{1}\right)$ and is then trapped at the interface $\left(\mathrm{I}_{1}\right)$; the chain fold at the interface between the crystalline and amorphous phase forms the trap. The hole needs to overcome the energy barrier from the trap at the interface $\left(\mathrm{I}_{1}\right)$ in order to be released, to move to the amorphous region $(\mathrm{A})$ and to interact with the disordered chain. To further move to the neighbouring crystalline lamella region $\left(\mathrm{C}_{2}\right)$, the hole needs to overcome another energy barrier and will be released from the trap at the interface $\left(\mathrm{I}_{2}\right)$. The transportation of the hole from $\mathrm{I}_{1}$ through $\mathrm{A}$ to $\mathrm{I}_{2}$ is treated as a tunneling transition. When nanoparticles are added to the polymer, they are mainly present in the amorphous phase, and a new interface between nanoparticles and amorphous polymer is introduced. Consequently, this forms a new energy barrier to restrict the hole transportation. As a result, conductivity is reduced.

2) The nanoparticle (e.g., $\mathrm{MgO}$ with a dielectric constant $\varepsilon=9.8$ ) may also induce a potential well $(1.5-5 \mathrm{eV})$ to form a deep trap, which will trap and block the space charge under a high electrical field, resulting in suppressing space charge accumulation [52]

3) The concentration of nanoparticles also influences the dielectric performance. It is reported that with higher loadings of nanosilica ( 2 wt.\%, 5 wt.\%, 10 wt.\%), space charge accumulation increases, while a lower loading of $0.5 \mathrm{wt} . \%$ suppresses the space charge accumulation. With a loading of $0.5 \mathrm{wt} . \%$, there is a long distance between the adjacent traps (around the nanosilica) which prevents the tunneling process, in which the charge moves from one trap site to the adjacent trap [53]. With a high concentration of nanosilica, due to the short distance between adjacent traps, the charges can directly move to adjacent traps with no need to overcome the energy barrier (tunneling process or percolation paths or hopping). As a consequence, the charge is not trapped in the deep traps, but is tunneling through the adjacent traps, resulting in more charges being injected [54]. In a more detailed example as shown in Figure 2.20, the charges are injected by electrodes. With a high electric field $(50 \mathrm{kV} / \mathrm{mm})$, tunneling can occur in situation (a), where a large number of deep traps with a short distance is present. As a result, the charges keep being injected into the polymer leading to high space charge accumulation. Differently, in case (b), the distances between deep traps are long; as a consequence, tunneling cannot take place. Therefore, once the deep traps are occupied by the charge, no further charge can be injected resulting in a low space charge accumulation.

4) The dispersion and distribution of the nanoparticles also affect the charge transportation. As shown in Figure 2.21, the dispersion and distribution can be significantly different with the same concentration of nanoparticles, depending on the surface properties of the nanoparticles. The dispersion is the degree of aggregation, while the distribution is the evenly filling of the space. Figure 2.21 (a) shows a case of bad dispersion and distribution, while Figure 2.21 (d) shows the opposite: good dispersion and distribution. Figure 2.21 (b) presents good distribution but bad 
dispersion, while the Figure 2.21 (c) presents good dispersion but bad distribution. If we consider that the interface between the nanoparticles and polymer matrix create the traps, which is affected by the dispersion, and that the charge carrier move though the traps by tunneling, the tunneling distance will be influenced by distribution of the nanoparticles due to the differences in the distance between the nearest-neighbor particles or particle clusters.
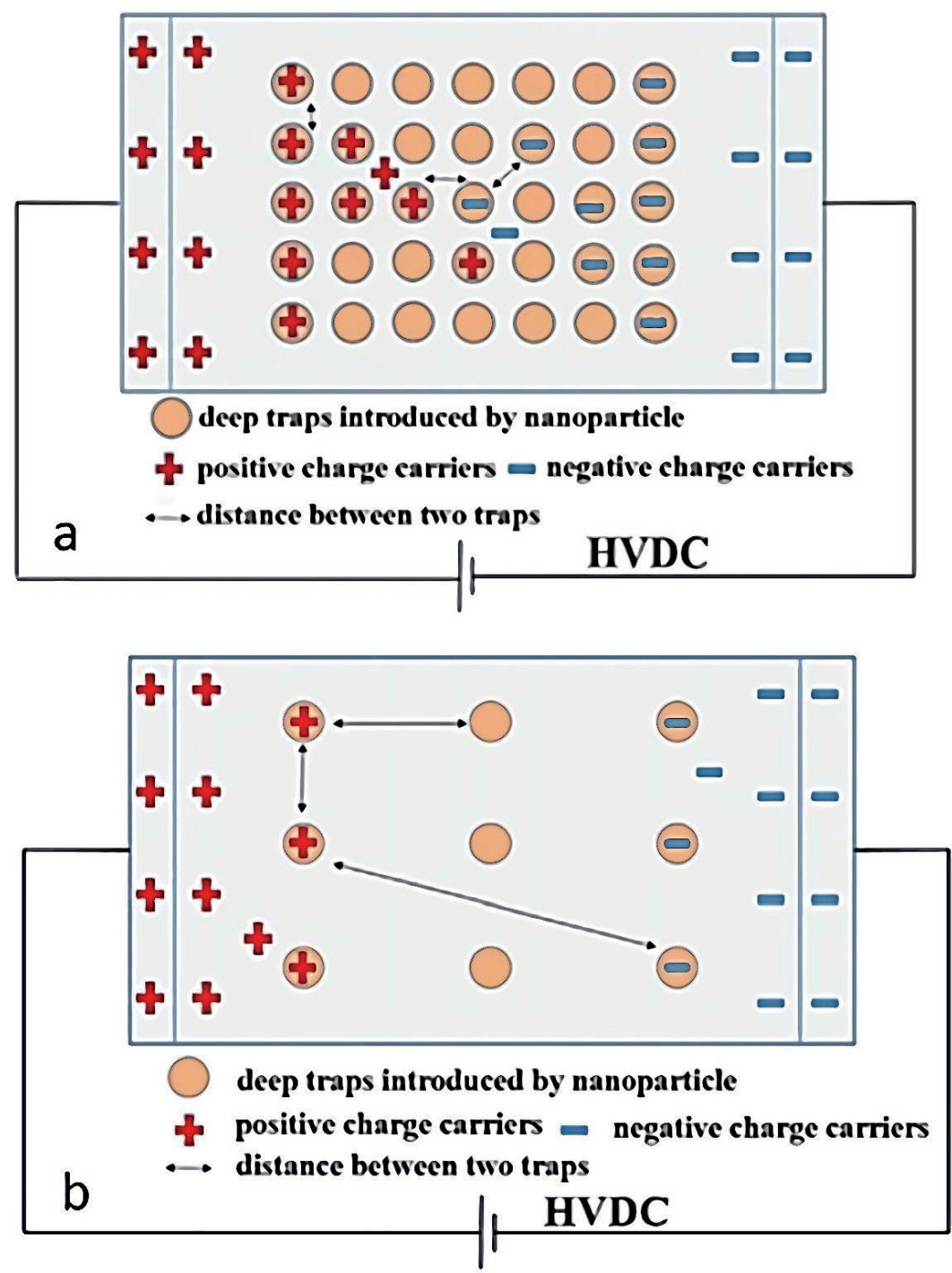

Figure 2.20 Scheme of charge distribution in nanodielectrics: (a) low concentration of nanoparticles (b) high concentration of nanoparticles [53]. 
5) The chemical surface properties of a nanofiller also affect the nanocomposite's dielectric performance: On the one hand, various surface modifications lead to differences in polarity of the nanofiller surface, which affect the compatibility between the nanofiller and the polymeric matrix influencing the nanofiller dispersion [55]; on the other hand, the chemical properties of a nanofiller surface influence the electrical characteristics of the interface with the polymeric matrix leading to different charge trap distributions, breakdown and leakage current performance [56].
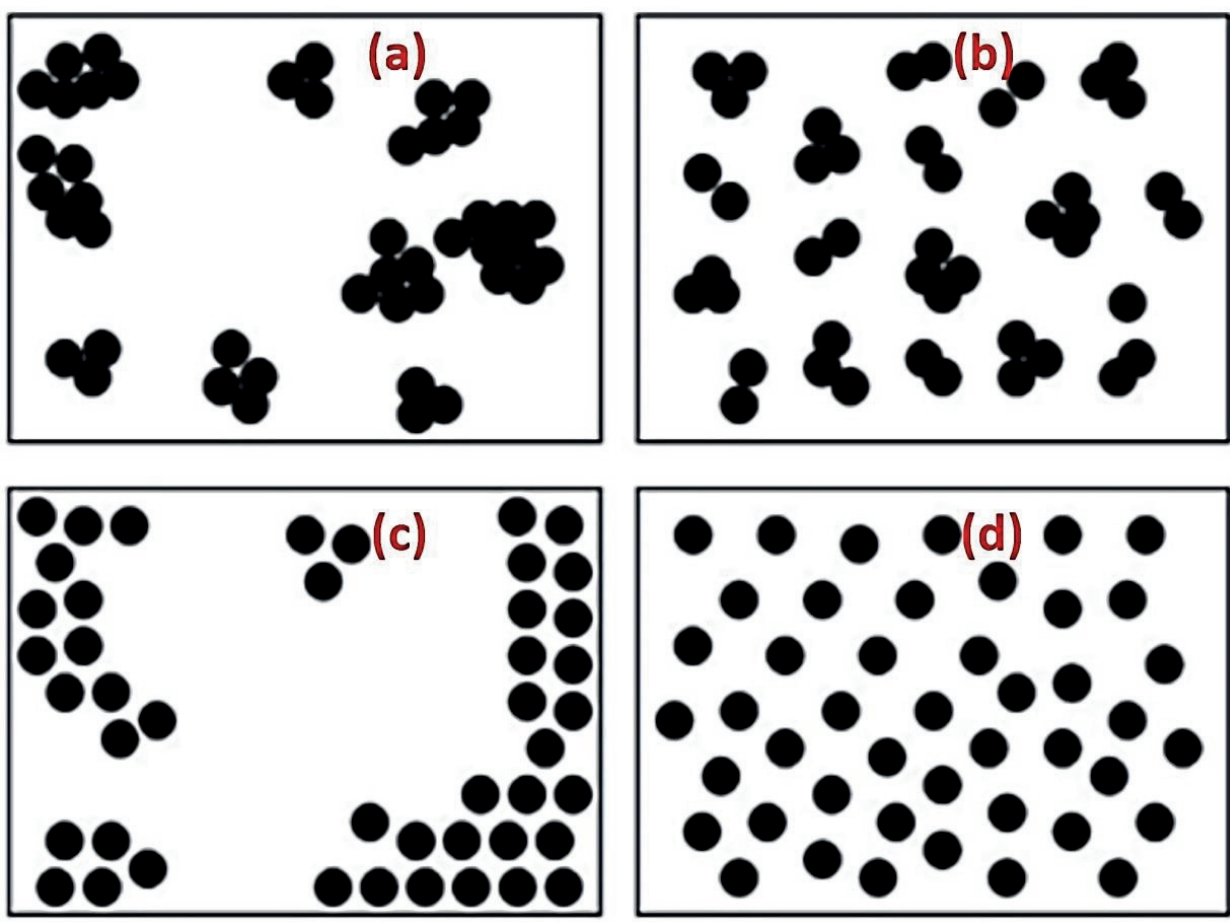

Figure 2.21 Scheme of possible dispersion and distribution states of nanoparticles in a polymer matrix

[54]

Summarized, the addition of nanofillers to dielectric materials can reduce the conductivity and the space charge accumulation and enhance the electrical breakdown strength. However, the overall dielectric performance of a nanocomposite is highly dependent on the nanofiller dispersion state, surface properties, concentration and type of nanoparticles. Nanoparticles with a polar surface are physicochemically not compatible with polypropylene of a rather unpolar nature making dispersion of unmodified nanoparticles in this matrix difficult. In such a case, nanoparticle agglomeration occurs, and it is reported to have a negative effect on the dielectric performance (e.g., high space charge accumulation and low breakdown strength). However, the polar moieties on the surface of the nanoparticles bring a positive effect in terms of introduction of deep traps, leading to suppressing space charge accumulation. 


\subsection{Nanoparticles and Surface Modification}

A nanoparticle is usually defined as a particle with at least one dimension between 1 and 100 nanometers (nm). The commonly used nanoparticles for nanodielectrics are $\mathrm{MgO}, \mathrm{SiO}_{2}, \mathrm{TiO}_{2}, \mathrm{ZnO}$ etc. Each of them with or without surface modification show a potential for an improved dielectric performance. Among all these nanoparticles, silica $\left(\mathrm{SiO}_{2}\right)$ with a low dielectric constant (as shown in Table 2.2) theoretically is a very promising nanofiller for electric insulation application.

Table 2.2 Dielectric constants of different nanoparticles

\begin{tabular}{|c|c|}
\hline Nanoparticle chemical composition & Dielectric constant at $1 \mathrm{MHz}$ \\
\hline $\mathrm{SiO}_{2}$ & 3.9 \\
\hline $\mathrm{ZnO}$ & 8.5 \\
\hline $\mathrm{MgO}$ & $9-10$ \\
\hline $\mathrm{Al}_{2} \mathrm{O}_{3}$ & 9 \\
\hline $\mathrm{TiO}_{2}$ & 85 \\
\hline
\end{tabular}

\subsubsection{Silica}

Silica $\left(\mathrm{SiO}_{2}\right)$, more specific amorphous silicon dioxide, is extensively used in industry due to its wide availability, chemical inertness and advantageous properties. The traditional industrial synthetic silicas include fumed and precipitated types, and the primary particle size is in a range of 5 to $100 \mathrm{~nm}$.

Fumed silica, also called pyrogenic silica, is obtained by high temperature (around $1800{ }^{\circ} \mathrm{C}$ ) processing in water vapor atmosphere. Silicon tetrachloride as the precursor is fed into the burner with hydrogen and dry air, and reacts in a flame to form silica, as shown in equations (2.2) and (2.3):

$$
\begin{aligned}
& 2 \mathrm{H}_{2}+\mathrm{O}_{2} \rightarrow 2 \mathrm{H}_{2} \mathrm{O} \\
& \mathrm{SiCl}_{4}+2 \mathrm{H}_{2} \mathrm{O} \rightarrow \mathrm{SiO}_{2}+4 \mathrm{HCl}
\end{aligned}
$$

The high process temperatures result in a low water content $(0.5 \sim 2.65$ wt. $\%$ moisture $)$ of the fumed silica grades. The specific surface area of fumed silica is in the range of 50 to $400 \mathrm{~m}^{2} / \mathrm{g}$, which can be controlled by the flame temperature and reactant ratios [57]. Due to the flame process, fumed silica forms chainlike clusters of branched morphology, as shown in Figure 2.22 (left). This results in a high surface area and very low bulk density powder.

Precipitated silica is produced by a reaction of alkali sodium silicate ("water glass") with a mineral acid (equation 2.4): 
After this reaction, precipitated silica is obtained by filtration, washing and drying. The moisture concentration in precipitated silica (3 7 wt.\%) is higher than in fumed silica. Several factors (remaining reactants, temperature, rate of addition) influence the properties (size, shape, density and specific surface area) of the final product. The morphology of precipitated silica is different from the one of fumed silica, as shown in Figure 2.22. Precipitated silica shows a more condensed structure than fumed silica. The different morphologies also result in a higher bulk density than fumed silica.

Chemically, silica consists of silicon and oxygen atoms forming an amorphous network. The surface of silica is covered by silanol groups ( $\mathrm{Si}-\mathrm{O}-\mathrm{H})$ or siloxane links ( $\mathrm{Si}-\mathrm{O}-\mathrm{Si})$. There are three types of silanol groups: isolated (free), vicinal and geminal, as shown in Figure 2.23. Due to the silanol groups on the silica surface, silica particles aggregate and adsorb water physically, which is one of the critical features of silica. The surface silanol groups offer hydrophilicity and create a weakly acidic surface. Silica has a high tendency to aggregate due to the very polar and active surface resulting in strong interparticular interactions. Hence, surface modification is a very important requirement for silica application in polymer composites, for which its surface activity has to be lowered in order to assure good dispersion in the polymer matrix. One method to achieve this is to graft organic groups onto the silica surface, and thereby change the hydrophilic character towards a more hydrophobic one, or to introduce specific functional groups [48].
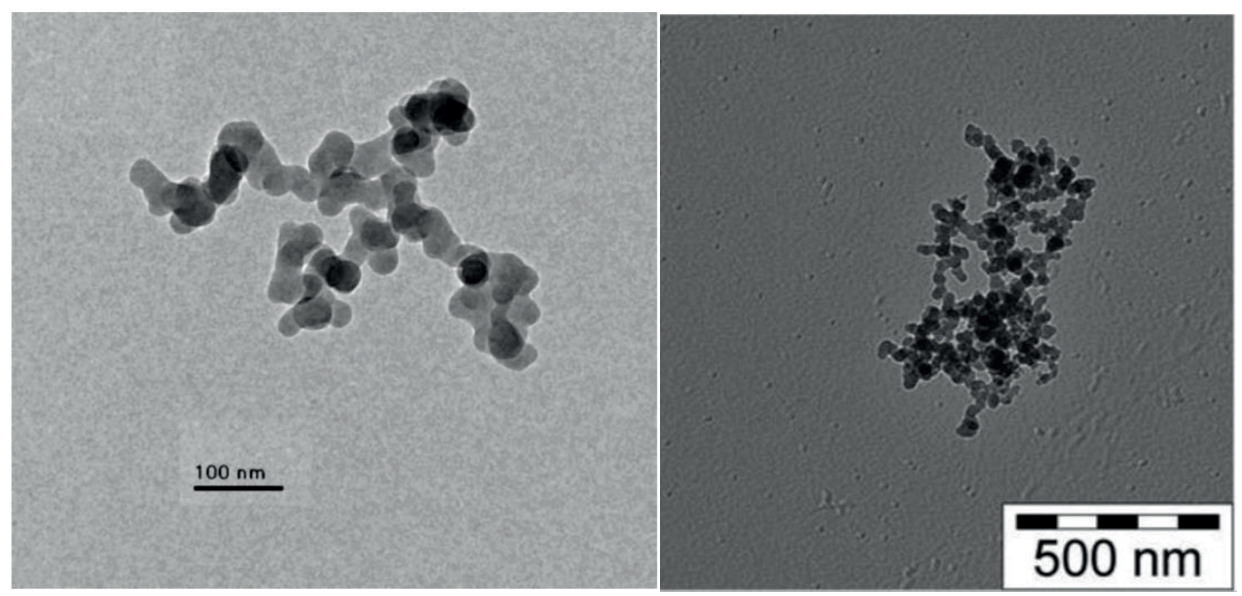

Figure 2.22 TEM images of fumed silica (left) [58] and precipitated silica (right) [59]. 

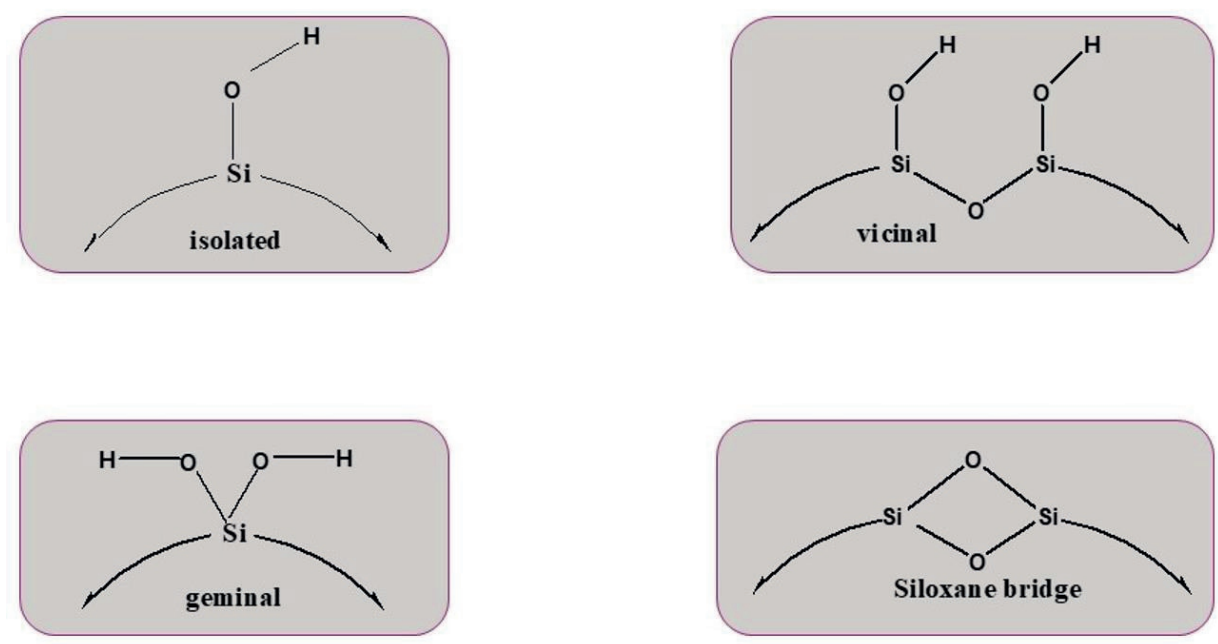

Figure 2.23 Chemical structure of the siloxane and silanol groups on the surface of silica [60].

\subsubsection{Surface Modification of Nanosilica}

\subsubsection{Solution Modification}

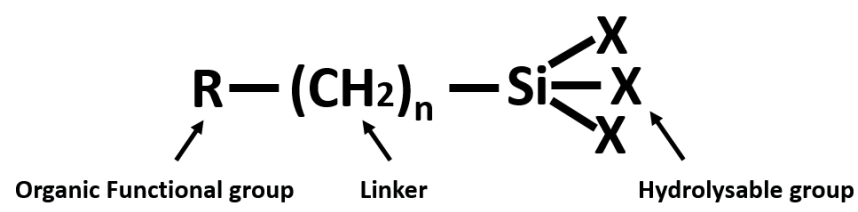

Figure 2.24 General structure of silanes.

Chemical modification in different liquid media is the most common way to modify nanoparticles. Silane coupling agents are widely used to modify silica surfaces. Silanes are chemical compounds that can react with inorganic substrates via strong covalent bonding. The general structure of silanes includes four parts (Figure 2.24): an organic functional group (R), a linker $\left(\mathrm{CH}_{2}\right)_{\mathrm{n}}$, the silicon atom and hydrolysable groups (X). The latter can be a halide atom or more often an alkoxy-group, which firstly form reactive silanol groups via hydrolysis. These silanols react with a silanol group on the surface of the silica particles to form a stable siloxane linkage via condensation. When 2 to 3 hydrolysable groups are available in the silane structure, condensation reactions between two or more silane molecules can also take place. The organic functional groups are non-hydrolysable and often possess specific functionalities which allow to change the surface properties of the substrate. The mechanism of silanization is shown in Figures 2.25 to 2.27. Firstly, silane hydrolysis takes place as a result of a reaction of water with the alkoxy groups resulting in silanol groups. The hydrolyzed silane can react with a substrate surface by 3 different mechanisms: 
1) The silane can directly react with the silanol group on the silica surface (Figure 2.25);

2) The silane molecules can condense and then react with the silanol group on the silica surface (Figure 2.26);

3) The silane molecules form an oligomer through a condensation reaction (Figure 2.27).
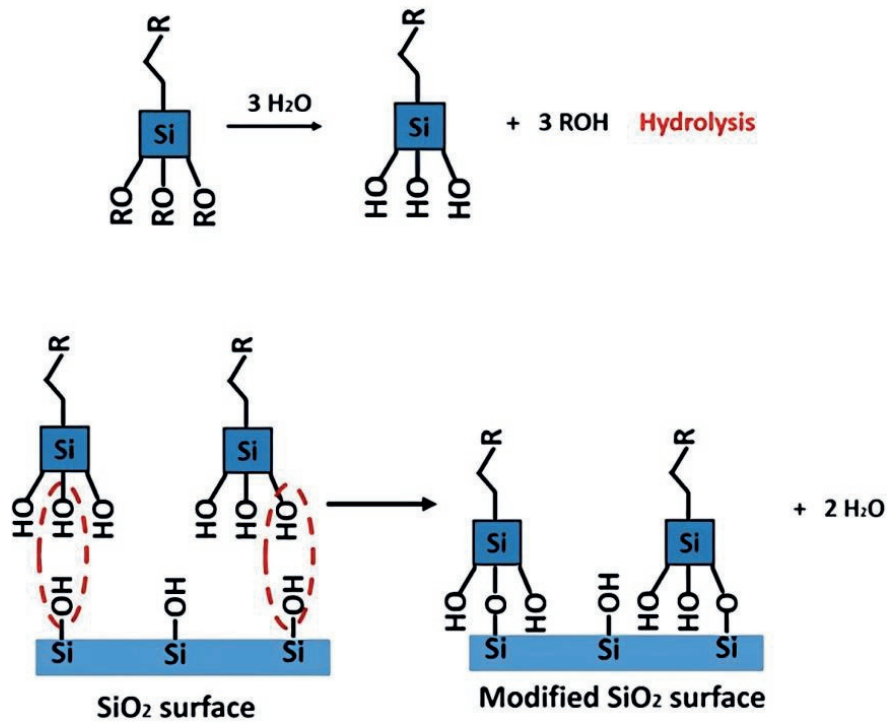

Figure 2.25 Silane reaction during silane-silica surface modification; Route 1: silane reacts directly with a silanol group on the silica surface.

Most commonly, a silane-silica reaction is performed in solution (e.g., toluene) with a certain $\mathrm{pH}$ value. The $\mathrm{pH}$ level of the solution significantly influences the yield of silane attachment. As already mentioned in Figure 2.25 to 2.27, there are three routes for the silane reaction, which might happen at the same time. However, the dominance of one route over the others differs depending on the $\mathrm{pH}$ value: [61] In an alkaline environment, condensation is faster than in an acidic one, Route 2 is dominating and the silane not only reacts with a silanol group on one silica particle, but can form a bridge between two or more silica particles resulting in formation of an aggregate. In an acidic environment, Route 1 is dominating and most of the silane reacts with silanol groups on the silica surface (Figure 2.28). This makes the morphology of the modified silica different, as shown in Figure 2.29. In Figure 2.29 (a) and (c), small aggregates of unmodified silica are shown. The aggregates tend to form bigger agglomerates due to strong hydrogen interactions formed by the silanol groups present on the silica surface. In an alkaline environment, the silica particles form large agglomerates and the silane condenses together, while under acidic conditions, no large agglomerates are present and the size of the modified silica cluster is smaller than for unmodified silica. It was evidenced that silanization performed in an acidic 
environment leads to a more uniform silane deposition on the silica surface, and with a decreased degree of oligomerization the tendency of silica agglomeration is reduced [61].
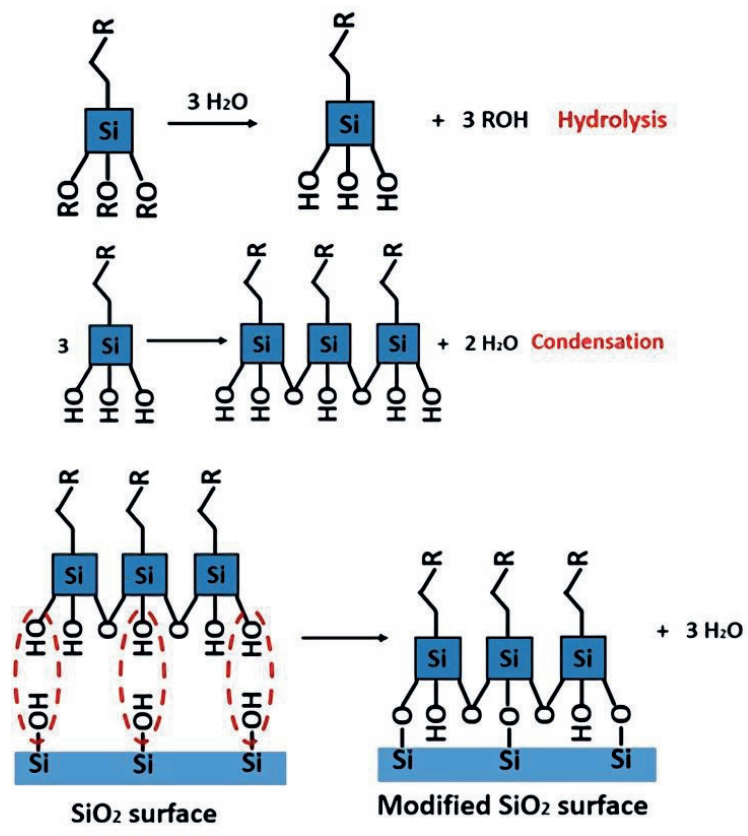

Figure 2.26 Silane reaction during silane-silica surface modification; Route 2: silane molecules condense and react with the silanol groups on a silica surface.
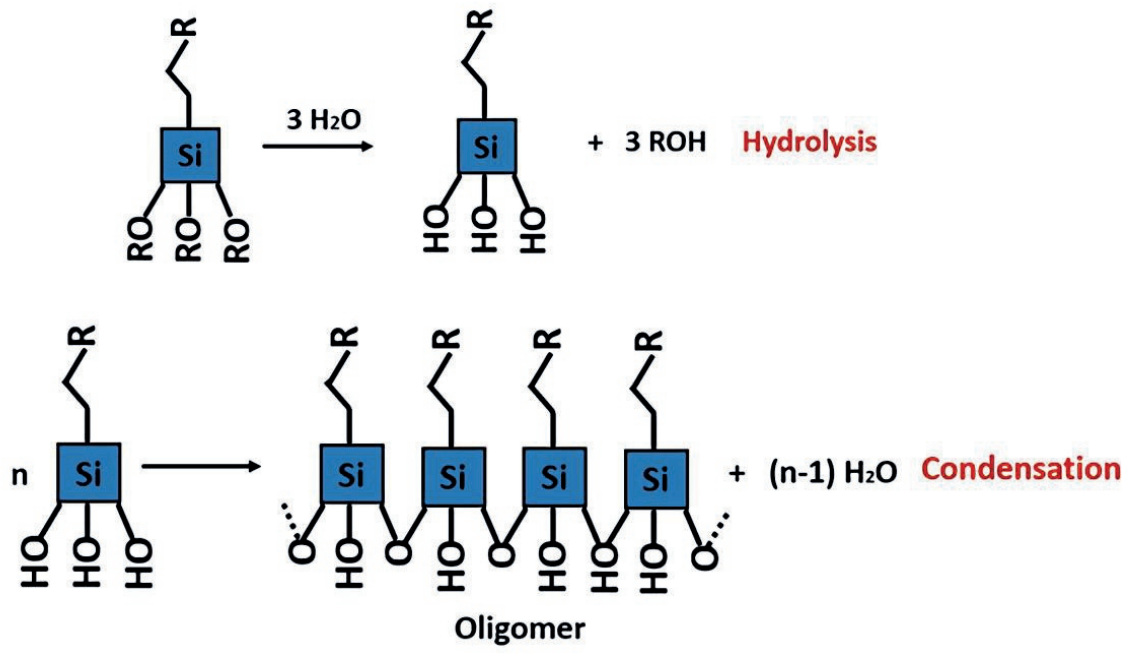

Figure 2.27 Silane reaction during silane-silica surface modification; Route 3: silane molecules form an oligomer through the condensation reaction. 


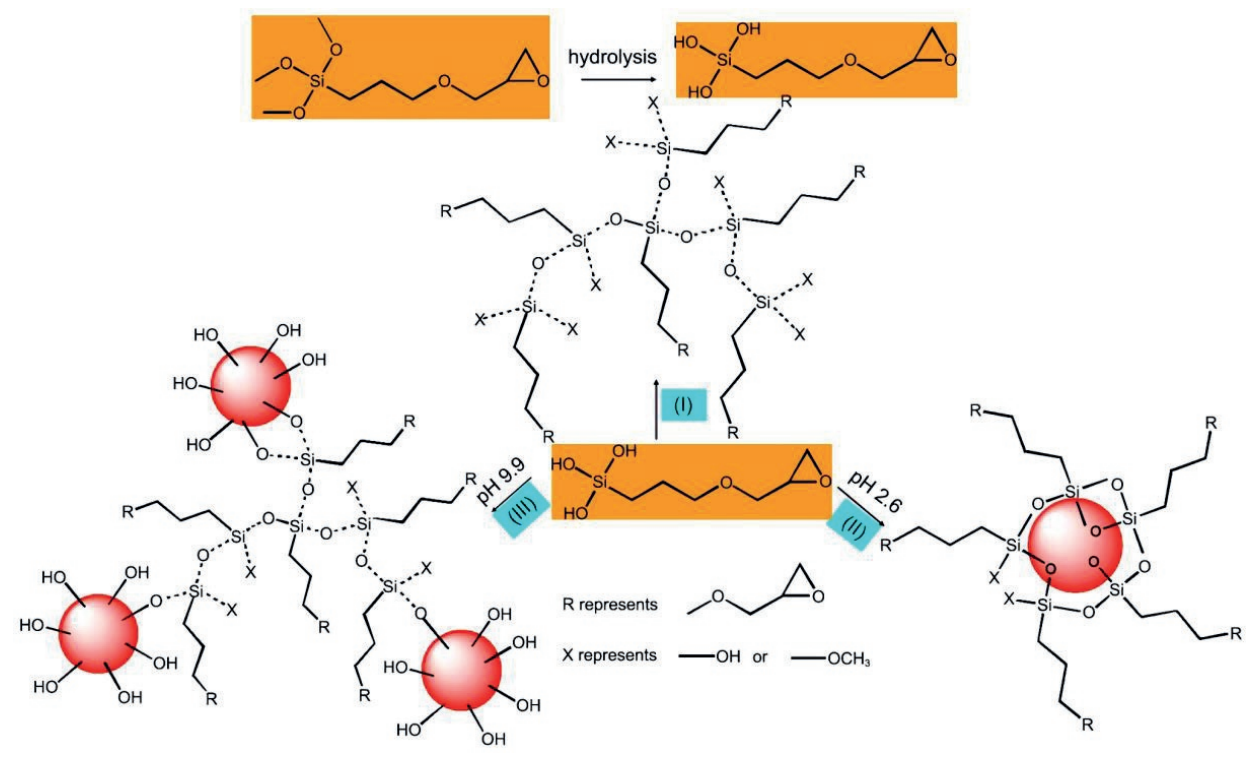

Figure 2.28 Possible grafting processes in acidic or alkaline environment [61].
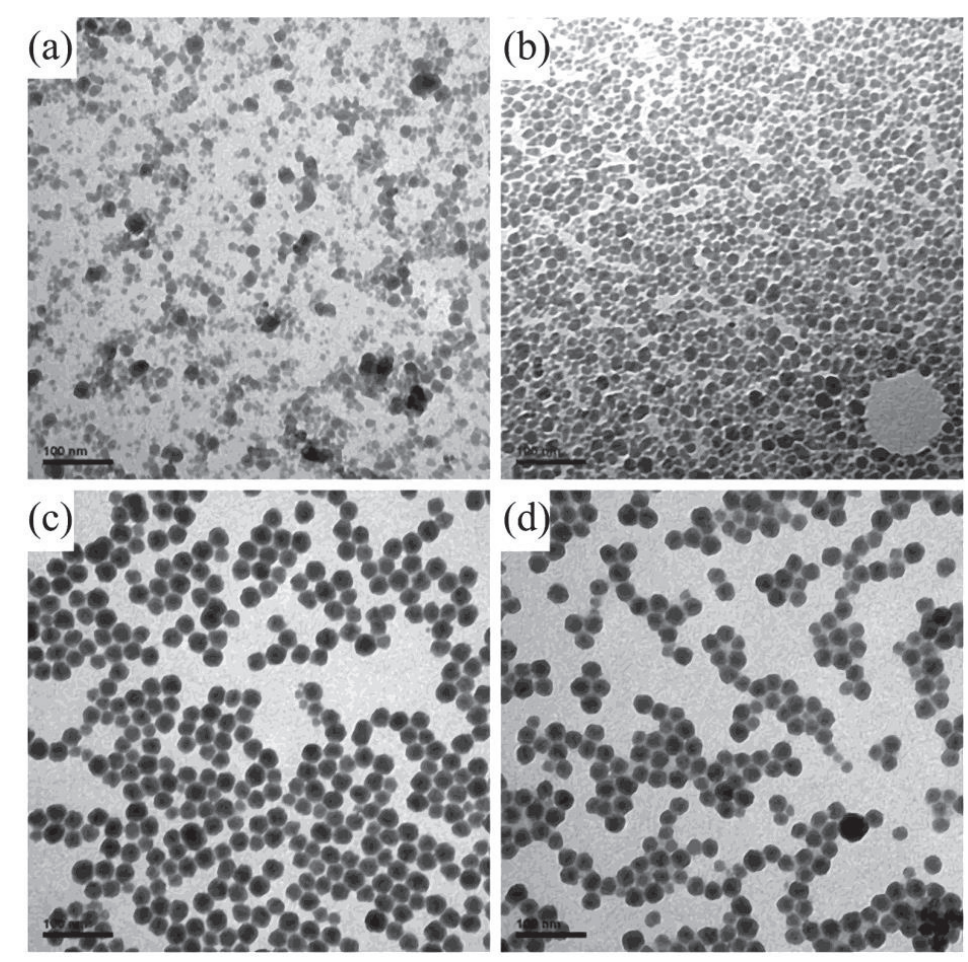

Figure 2.29 TEM micrographs of unmodified silica in acidic (a) or alkaline (c) suspension and the respective silane modified counterparts (b) and (d) [61]. 
Solution modification offers versatile surface treatment possibilities and is easy to control in terms of [62] e.g. reaction time, temperature and silane concentration, however, it consumes a lot of solvent (e.g., toluene). To modify $50 \mathrm{~g}$ of fumed silica, around 0.5 litter of solvent is needed. Additionally, in case of fire, organic solvents also pose a big threat.

In order to reduce to use the large amount of solvent, Evonik [63] developed two alternative methods of silica treatment with silanes. One approach is to perform the modification in vapor phase at temperatures of 50 to $800{ }^{\circ} \mathrm{C}$ for 0.1 to 6 hours under nitrogen as inert gas. The other approach is to perform the modification by spraying acidified water and silanes (in solution with a suitable solvents) onto the silica. After spraying, a post-mixing is still needed for 5 to 30 mins. Subsequently, the mixture needs to be heated to a temperature of 20 to $400{ }^{\circ} \mathrm{C}$ for 0.1 to 6 hours under nitrogen. Although in both approaches the use of a solvent is largely reduced, high temperatures and an inert gas are needed. Compared to the solvent-free modification, more energy is consumed and the modification setup is more complicated.

\subsubsection{Plasma Modification}

Plasma is the fourth state of matter; the others are solid, liquid and gas. Plasma can be defined as a collection of electrons and partially or completely ionized gas atoms [64]. In general, increasing the temperature of a chemical compound to a sufficient high level can generate a plasma. Figure 2.30 shows

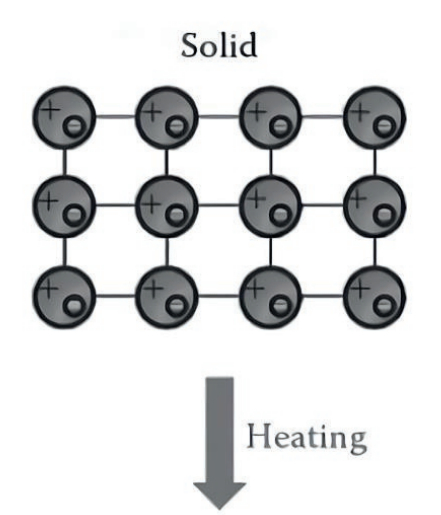

Liquid

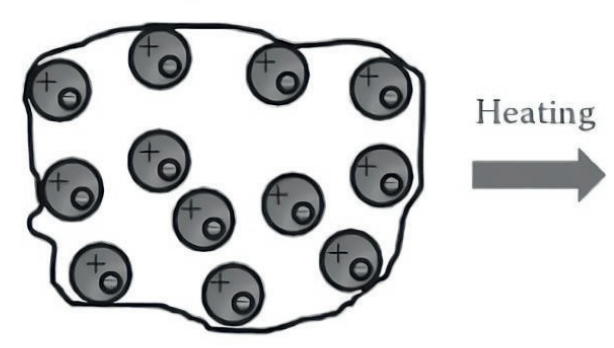

\section{Plasma}

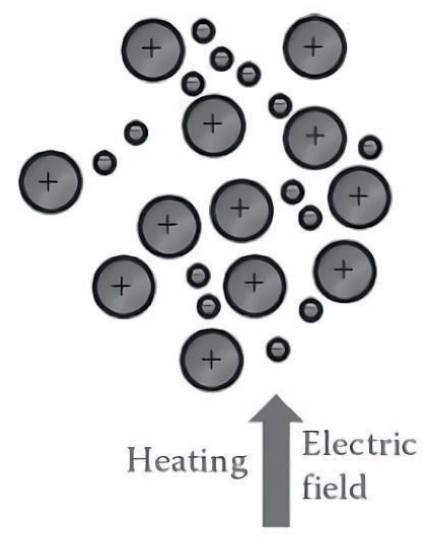

Gas

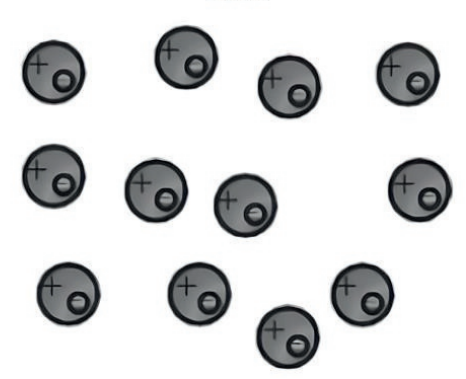

Figure 2.30 Schematic diagram of the state transition processes [67]. 
the change of the $\mathrm{H}_{2} \mathrm{O}$ states as an example [65]. At very low temperatures, $\mathrm{H}_{2} \mathrm{O}$ molecules arrange in a solid state with a crystal lattice (ice). As temperature increases above the melting point, the kinetic energy of the molecule increases leading to melting of the ice and formation of liquid water. At further increased temperature above the vapour point, the liquid water vaporizes into separate $\mathrm{H}_{2} \mathrm{O}$ molecules in the gas state. At even higher temperatures, collision between $\mathrm{H}_{2} \mathrm{O}$ molecules with very high kinetic energy can result in dissociation of $\mathrm{H}_{2} \mathrm{O}$ molecules and ionization of the atoms [66]. Hence, the $\mathrm{H}_{2} \mathrm{O}$ plasma state is reached.

Plasma exists in nature, but can also be artificially generated. In nature, the sun consists entirely of plasma due to its high temperature (approximately $5,000,000{ }^{\circ} \mathrm{C}$ ). When a lightning strikes, plasma is generated by electric discharge in air. In laboratories, plasma can be created through various methods such as electric discharge, heat, lasers and other energy-rich sources. The electrons gain energy from the external electric field. They can also lose energy by collision with particles and generate Joule heating. Based on the different temperatures of electrons (Te) and neutral particles (Tn), plasma is classified in hot (equilibrium) and cold (non-equilibrium) plasma:

hot (equilibrium) plasma: $\mathrm{Te}=\mathrm{Tn}$

cold (non-equilibrium) plasma: Te $>>\mathrm{Tn}$

In many non-equilibrium plasmas, the electron temperature is $\mathrm{Te} \sim 10,000 \mathrm{~K}(1 \mathrm{eV})$ and the gas particle temperature is around room temperature [68].

Natural lightning flashes and the hot sun core cause hot plasma, where very high temperatures (above $10,000 \mathrm{~K}$ ) are needed to generate plasma, and all plasma species are in a thermal equilibrium state at the same temperature as the local environment. Cold plasma can be generated by electrical discharge in a gas at certain frequencies, (e.g., radio frequency (RF) at $13.56 \mathrm{MHz}$ or microwaves (MW) at $2.45 \mathrm{GHz}$ ). The temperature of cold plasma is usually not higher than the surrounding temperature. Cold plasma is extremely useful and sometimes necessary when working with heat sensitive materials, since the working temperature of cold plasma is normally around room temperature. For most applications like "soft" surface modification (coating, etching and cleaning), the plasma temperature is limited. The modification is normally done in several hundred Angströms of the substrate surface, so the bulk properties of the treated material remain unchanged [69].

A scheme of a cold plasma reactor is shown in Figure 2.31. In general, it includes a plasma chamber (where the plasma occurs and also the substrate is located), a generator, a mass flow controller, a pressure gauge and a pump. Based on the pressure level during the plasma process, the cold plasma can be classified as low-pressure plasma or atmospheric-pressure plasma. In case of the low-pressure plasma, a vacuum in the reaction chamber is needed. Atmospheric-pressure plasma does not need a vacuum, decreasing the investment costs $[70,71,72]$. However, the disadvantage of the atmospheric plasma technology is its unstable discharge [73] and not very good control of the plasma coating layer. Therefore, in order to have a uniform deposition, low-pressure plasma is preferable.

In the cold plasma process, an external energy source is required. Most often electric power is used for the creation of the plasma. When the precursor is introduced into the plasma area, the molecules are excited and interact with the electrons and ions producing new activated species, e.g. single functional 
free radicals or double functional free radicals [75]. These radicals continue to be fragmented to form new radicals or recombine together in a disordered way to form polymeric matter with high molecular weight and a crosslinked structure [76]. The polymeric matter finally deposits on the substrate surface forming a thin layer. Due to the random recombination, the final plasma polymer is different from conventional polymerization products as shown in Figure 2.32. Therefore, the chemical structure of a polymer formed in a plasma is unpredictable and rather difficult to tailor.

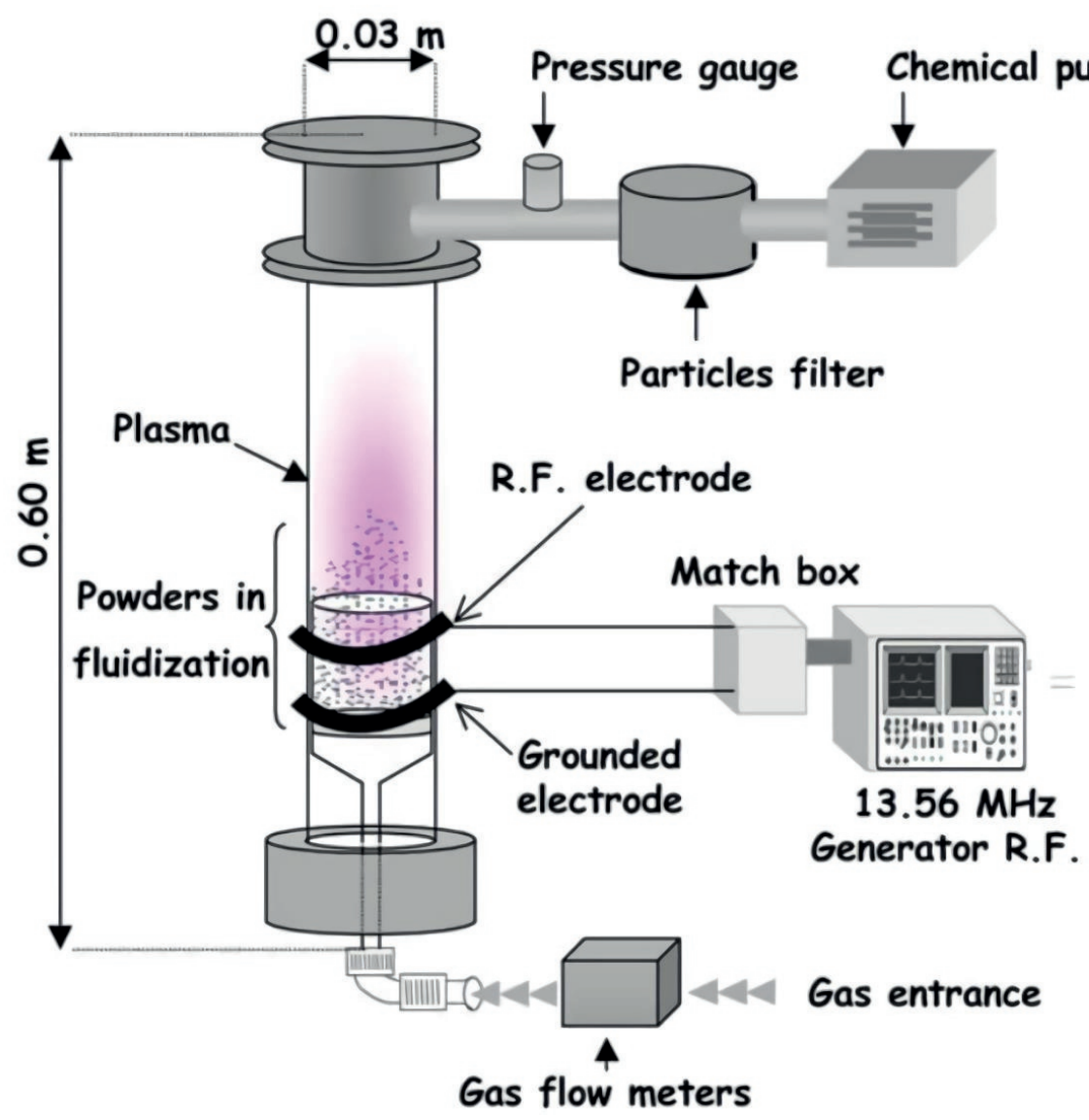

Figure 2.31 Scheme of a plasma reactor [74].

The model of acetylene plasma polymerization has been developed by S. Stoykov [77]. A high molecular weight polymer was formed through plasma polymerization: The precursor $\left(\mathrm{C}_{2} \mathrm{H}_{2}\right)$ was excited by an electron to produce radicals $\left(\mathrm{C}_{2} \mathrm{H} \bullet+\mathrm{H} \bullet\right)$. The precursor also interacts with the radical $\left(\mathrm{C}_{2} \mathrm{H} \bullet\right)$ to form new radicals $\left(\mathrm{C}_{4} \mathrm{H}_{3} \bullet\right)$. Continuously, all these molecules or activated radicals will undergo random recombination and finally form a high molecular weight polymer. 
Akhavan [78] applied plasma modification on silica with 1,7-octadiene as precursor for water purification systems. By this, a higher oil removal efficiency (ORE) could be realized, in which the modified silica can remove $99.0-99.5 \%$ of high viscosity motor oil within a treatment time of 10 minutes and adsorbs more than $99.5 \%$ of low viscosity crude oil in 30 seconds. This way, a high efficiency adsorbent was developed for water purification, as shown in Figure 2.33. Besides, María Guadalupe Neira-Vela'zquez [79] also proved that after ethylene plasma modification of nanoclay, the original polar material was becoming unpolar, and a uniform hydrocarbon layer was deposited onto the surface of the nanoclay with a thickness of $3 \mathrm{~nm}$.

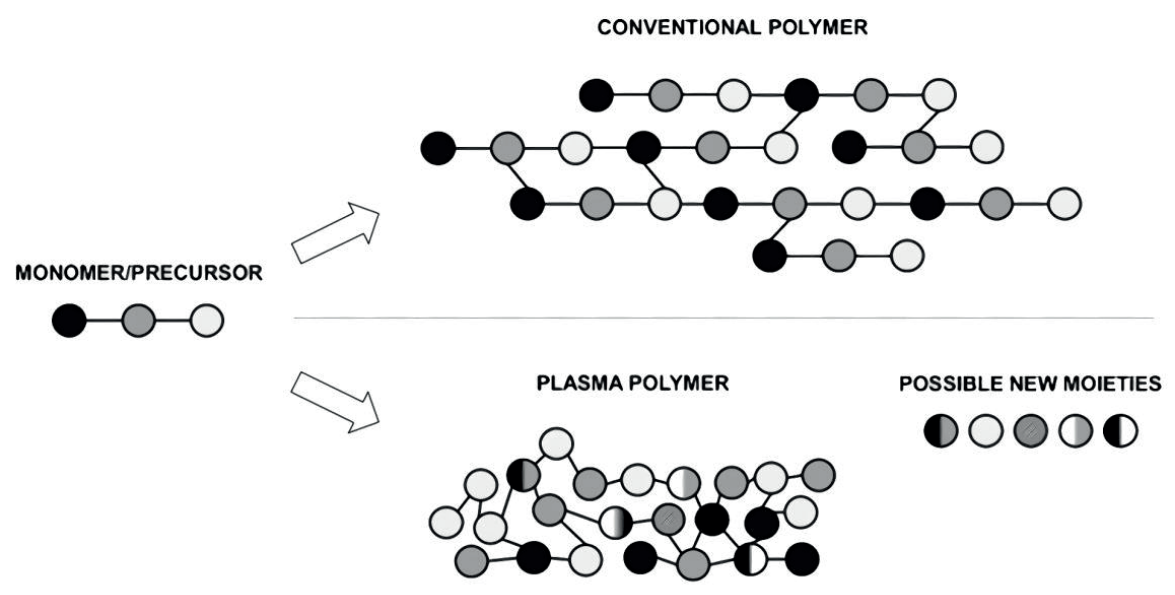

Figure 2.32 Scheme of conventional and plasma polymer formation from a given monomer (or precursor). [76].

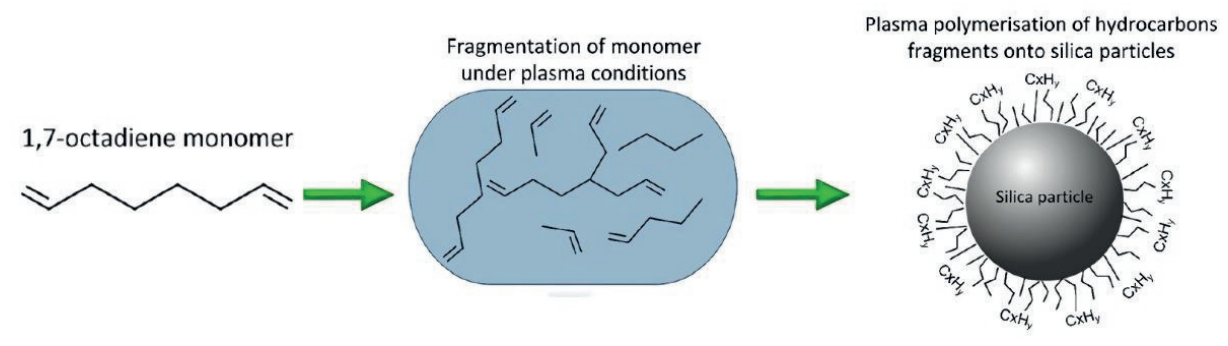

Figure 2.33 Schematic illustration of 1,7-octadiene plasma polymer deposition onto silica particles [78].

Apart from the hydrocarbon precursor, polar liquids can also be used as precursors to treat nanoparticles. It has been reported that diethylene glycol dimethyl ether was introduced as precursor to treat nanosilica by plasma modification. The nanosilica was finally coated with a poly(ethylene oxide)-like film, randomly crosslinked without repetitive molecular units [80]. Generally, there is no limitation in terms of the plasma precursor: in principle, all kind of gases and liquids can be used as precursor. 
Apart from the plasma precursor type, there are also other plasma parameters such as treatment time, flow rate, and input power, which heavily influence the properties of the deposited film. With increase of plasma treatment time, normally the degree of deposition on the surface of substrates will increase $[79,81]$. A. Ward et al. analysed the influence of plasma power $(\mathrm{W})$ and monomer flow rate $(\mathrm{F})$ on the retention of monomer functionality [82]. They suggested that a low W/F ratio contributes to a decrease in monomer fragmentation. As a result, more functional groups were preserved, less crosslinking occurred and a relatively low molecular weight plasma polymer was deposited. They introduced the term "mild plasma conditions", which allows to retain chemical functional groups to a high degree, and to deposit them onto the surface of a substrate during plasma processing under carefully controlled plasma conditions.

In recent years, cold plasma modification has attracted considerable interest as a very "smart" way to treat materials, which only changes the properties of the surface without compromising the bulk properties [69]. Generally, it is possible to deposit functional groups on the surface of metals, polymers, composites or nanofillers, by forming a thin and uniform layer. In comparison to the solution modification method, plasma modification is an environmentally friendly and "green" way of changing the surface chemistry by this one-step technology and virtually no waste production [83].

In summary, we can conclude that a nanofiller can be modified using the plasma technique with a proper precursor, to change the hydrophilic nature of the nanofiller or to introduce specific functional groups. This is another way to modify nanofillers in order to improve their miscibility with a polymer matrix or to tailor nanofiller-polymer interfacial properties. An example is an acetylene precursor, which changed the hydrophilic nature of silica into a more hydrophobic one by a uniform polyacetylene film deposited on the silica surface. The acetylene plasma modified silica also exhibited a better dispersion in ethylenepropylene-diene rubber, styrene-butadiene rubber and their blends [84].

However, there is not much published on plasma nanofiller modification for HVDC cable insulation material application, in particular not for the recyclable polypropylene based insulation nanocomposites. A study being close to the research in this thesis is silica surface treatment by plasma modification with a diethylene glycol dimethyl ether precursor. The aim of this work was to improve the interaction between silica and the epoxy matrix to reduce space charge accumulation. After the plasma treatment, a poly(ethylene oxide) (PEO)-like film was deposited on the silica surface. Unfortunately, the modified silica did not reduce the space charge accumulation of the epoxy-based composite. This was explained by the deposited PEO film containing oxygen making it highly polar and negatively charged, which caused the increased space charge accumulation [85]. Therefore, in order to improve the dielectric performance of the PP insulation nanocomposites, the precursor needs to be carefully selected for nanofiller surface modification. 


\section{References}

[1] Meah K, Ula S. Comparative evaluation of HVDC and HVAC transmission systems. In2007 IEEE Power Engineering Society General Meeting 2007 Jun 24, pp. 1-5. DOI: 10.1109/PES.2007.385993

[2] Van Eeckhout B, Van Hertem D, Reza M, Srivastava K, Belmans R. Economic comparison of VSC HVDC and HVAC as transmission system for a $300 \mathrm{MW}$ offshore wind farm. European Transactions on Electrical Power. 2010 Jul;20(5):661-71. DOI: https://doi.org/10.1002/etep.359

[3] Bahrman, M.P. and Johnson, B.K., 2007. The ABCs of HVDC transmission technologies. IEEE power and energy magazine, 5(2), pp.32-44. DOI: 10.1109/MPAE.2007.329194

[4] Okba, M.H., Saied, M.H., Mostafa, M.Z. and Abdel-Moneim, T.M., 2012, May. High voltage direct current transmission-A review, part I. In 2012 IEEE Energytech, pp. 1-7. DOI: $\underline{10.1109 / \text { EnergyTech.2012.6304650 }}$

[5] Worzyk, Thomas. Submarine power cables: design, installation, repair, environmental aspects. Springer Science \& Business Media, 2009. eBook ISBN: 978-3-642-01270-9. DOI: 10.1007/978-3-642$\underline{01270-9}$

[6] Purvins, A., Sereno, L., Ardelean, M., Covrig, C.F., Efthimiadis, T. and Minnebo, P., 2018. Submarine power cable between Europe and North America: A techno-economic analysis. Journal of Cleaner Production, 186, pp.131-145. DOI: https://doi.org/10.1016/j.jclepro.2018.03.095

[7] Zhou, Y., Peng, S., Hu, J. and He, J., 2017. Polymeric insulation materials for HVDC cables: Development, challenges and future perspective. IEEE Transactions on Dielectrics and Electrical Insulation, 24(3), pp.1308-1318. DOI: 10.1109/TDEI.2017.006205

[8] Fujimori, A., Fujii, K., Takashima, H., Suzuki, H., Mitani, M., Fujii, O., Shigetoshi, I. and Shimada, M., 1997. Development of $500 \mathrm{kV}$ dc PPLP-insulated oil-filled submarine cable. Electrical engineering in Japan, 120(3), pp.29-41. DOI: https://doi.org/10.1002/(SICI)1520-6416(199708)120:3<29::AIDEEJ4 $>3$.0.CO;2-T

[9] Liu, R., 2017, May. Progress of long-distance dc electrical power transmission. In 2017 1st International Conference on Electrical Materials and Power Equipment (ICEMPE), pp. 93-96. DOI: 10.1109/ICEMPE.2017.7982153

[10] Valenza, D. and Cipollini, G., 1995, November. HVDC submarine power cable systems-state of the art and future developments. In Proceedings 1995 International Conference on Energy Management and Power Delivery EMPD'95, 1, pp. 283-287. DOI: 10.1109/EMPD.1995.500739

[11] Chen, G., Hao, M., Xu, Z., Vaughan, A., Cao, J. and Wang, H., 2015. Review of high voltage direct current cables. CSEE Journal of Power and Energy Systems, 1(2), pp.9-21. DOI: $\underline{10.17775 / C S E E J P E S .2015 .00015}$

[12] Ardelean, M. and Minnebo, P., 2015. HVDC submarine power cables in the world. Joint Research Center.

[13] Håkonseth, G., Ildstad, E. and Furuheim, K.M., 2017, October. Local electric field in massimpregnated HVDC cables. In Proceedings of the Nordic Insulation Symposium (No. 25). DOI: https://doi.org/10.5324/nordis.v0i25.2351

[14] Hata, R., 2005. Solid DC Submarine Cable Insulated with PPLP (Polypropylene Laminated Paper). In IERE Southeastern Asia Forum S3-5. 
[15] Hanley, T.L., Burford, R.P., Fleming, R.J. and Barber, K.W., 2003. A general review of polymeric insulation for use in HVDC cables. IEEE Electrical Insulation Magazine, 19(1), pp.13-24. DOI: 10.1109/MEI.2003.1178104

[16] Mazzanti, G. and Marzinotto, M., 2013. Extruded cables for high-voltage direct-current transmission: advances in research and development, John Wiley \& Sons (No. 93). ISBN: 978-1-11809666-6. ISBN: 978-1-118-09666-6.

[17] Byggeth, M., Johannesson, K., Liljegren, C., Palmqvist, L., Axelsson, U., Jonsson, J. and Tornkvist, C., 1999, June. The development of an extruded HVDC cable system and its first application in the Gotland HVDC Light project. In Proceedings of Fifth International Conference on Insulated Power Cables, JICABLE, pp. 538-542.

[18] Murata, Y. and Kanaoka, M., 2006, June. Development history of HVDC extruded cable with nanocomposite material. In 2006 IEEE 8th International Conference on Properties \& applications of Dielectric Materials, pp. 460-463. DOI: 10.1109/ICPADM.2006.284214

[19] Mazzanti, G. and Marzinotto, M., 2013. Main principles of HVDC extruded cable design. ISBN: 978-1-118-09666-6.

[20] Ildstad, E., 2013. Challenges arising from use of HVDC. In Proceedings of the Nordic Insulation Symposium (No. 23). DOI: https://doi.org/10.5324/nordis.v0i23.2443

[21] Jeroense, M.J.P. and Morshuis, P.H.F., 1998. Electric fields in HVDC paper-insulated cables. IEEE Transactions on Dielectrics and Electrical Insulation, 5(2), pp.225-236. DOI: 10.1109/94.671940

[22] Mazzanti, G. and Montanari, G.C., 2005. Electrical aging and life models: the role of space charge. IEEE Transactions on Dielectrics and Electrical Insulation, 12(5), pp.876-890. DOI: 10.1109/TDEI.2005.1522183

[23] Britannica. https://www.britannica.com/science/trap-solid-state-physics. last accessed on 2021-0222.

[24] Meunier, M., Quirke, N. and Aslanides, A., 2001. Molecular modeling of electron traps in polymer insulators: Chemical defects and impurities. The Journal of Chemical Physics, 115(6), pp.2876-2881. DOI: https://doi.org/10.1063/1.1385160

[25] Meunier, M. and Quirke, N., 2000. Molecular modeling of electron trapping in polymer insulators. The Journal of Chemical Physics, 113(1), pp.369-376. DOI: https://doi.org/10.1063/1.481802

[26] Zhang, Y., Lewiner, J., Alquie, C. and Hampton, N., 1996. Evidence of strong correlation between space-charge buildup and breakdown in cable insulation. IEEE Transactions on Dielectrics and Electrical Insulation, 3(6), pp.778-783. DOI: 10.1109/94.556559

[27] Hussin, N. and Chen, G., 2012. Analysis of space charge formation in LDPE in the presence of crosslinking byproducts. IEEE Transactions on Dielectrics and Electrical Insulation, 19(1), pp.126-133. DOI: $10.1109 /$ TDEI.2012.6148510

[28] Hirai, N., Minami, R., Tanaka, T. and Ohki, Y., 2003. Chemical group in crosslinking byproducts responsible for charge trapping in polyethylene. IEEE Transactions on Dielectrics and Electrical insulation, 10(2), pp.320-330. DOI: 10.1109/TDEI.2003.1194118

[29] Hussin, N. and Chen, G., 2010, October. Space charge accumulation and conductivity of crosslinking byproducts soaked LDPE. In 2010 Annual Report Conference on Electrical Insulation and Dielectic Phenomena, pp. 1-4. DOI: 10.1109/CEIDP.2010.5724002 
[30] Ndiripo, A. and Pasch, H., 2020. Retention of polypropylene stereoisomers in solvent gradient interaction chromatography on porous graphitic carbon as influenced by temperature and mobile phase

composition. Journal of Chromatography A, 1618, pp.460865. DOI: https://doi.org/10.1016/j.chroma.2020.460865

[31] Hosier, I.L., Cozzarini, L., Vaughan, A.S. and Swingler, S.G., 2009, August. Propylene based systems for high voltage cable insulation applications. In Journal of Physics: Conference Series ,183(1), pp. 012015. DOI: $10.1088 / 1742-6596 / 183 / 1 / 012015$

[32] Green, C.D., Vaughan, A.S., Stevens, G.C., Pye, A., Sutton, S.J., Geussens, T. and Fairhurst, M.J., 2015. Thermoplastic cable insulation comprising a blend of isotactic polypropylene and a propyleneethylene copolymer. IEEE Transactions on Dielectrics and Electrical Insulation, 22(2), pp.639-648. DOI: 10.1109/TDEI.2015.7076758

[33] Gao, Y., Li, J., Yuan, Y., Huang, S. and Du, B., 2018. Trap distribution and dielectric breakdown of isotactic polypropylene/propylene based elastomer with improved flexibility for DC cable insulation. IEEE Access, 6, pp.58645-58661. DOI: 10.1109/ACCESS.2018.2874826

[34] Karger-Kocsis, J. and Bárány, T., 2019. Polypropylene handbook (Vol. 43). Springer International Publishing: Cham, Switzerland. ISBN 978-3-030-12903-3.

[35] Hosoda, S., Hori, H., Yada, K.I., Nakahara, S.Y. and Tsuji, M., 2002. Degree of comonomer inclusion into lamella crystal for propylene/olefin copolymers. Polymer, 43(26), pp.7451-7460. DOI: https://doi.org/10.1016/S0032-3861(02)00680-8

[36] Meng, P., Zhou, Y., Yuan, C., Li, Q., Liu, J., Wang, H., Hu, J. and He, J., 2019. Comparisons of different polypropylene copolymers as potential recyclable HVDC cable insulation materials. IEEE Transactions on Dielectrics and Electrical Insulation, 26(3), pp.674-680. DOI: 10.1109/TDEI.2018.007435

[37] Luo, F., Geng, C., Wang, K., Deng, H., Chen, F., Fu, Q. and Na, B., 2009. New understanding in tuning toughness of $\beta$-polypropylene: the role of $\beta$-nucleated crystalline morphology. Macromolecules, 42(23), pp.9325-9331. DOI: https://doi.org/10.1021/ma901651f

[38] Zha, J.W., Yan, H.D., Li, W.K. and Dang, Z.M., 2016. Morphology and crystalline-phasedependent electrical insulating properties in tailored polypropylene for HVDC cables. Applied Physics Letters, 109(22), pp.222902. DOI: https://doi.org/10.1063/1.4969063

[39] Huang, X., Fan, Y., Zhang, J. and Jiang, P., 2017. Polypropylene based thermoplastic polymers for potential recyclable HVDC cable insulation applications. IEEE Transactions on Dielectrics and Electrical Insulation, 24(3), pp.1446-1456. DOI: 10.1109/TDEI.2017.006230

[40] Chuang, H.K. and Han, C.D., 1984. Rheological behavior of polymer blends. Journal of applied polymer science, 29(6), pp.2205-2229. DOI: https://doi.org/10.1002/app.1984.070290625

[41] Avgeropoulos, G.N., Weissert, F.C., Biddison, P.H. and Bohm, G.G.A., 1976. Heterogeneous blends of polymers. Rheology and morphology. Rubber chemistry and technology, 49(1), pp.93-104. DOI: https://doi.org/10.5254/1.3534954

[42] Lin, Y., Du, W., Tu, D., Zhong, W. and Du, Q., 2005. Space charge distribution and crystalline structure in low density polyethylene (LDPE) blended with high density polyethylene (HDPE). Polymer international, 54(2), pp.465-470. DOI: https://doi.org/10.1002/pi.1727

[43] Xu, R.R., Du, B.X., Xiao, M., Li, J., Liu, H.L., Ran, Z.Y. and Xing, J.W., 2020. Dielectric properties dependent on crystalline morphology of PP film for HVDC capacitors application. Polymer, pp.123204. DOI: https://doi.org/10.1016/j.polymer.2020.123204 
[44] Dang, B., He, J., Hu, J. and Zhou, Y., 2016. Large improvement in trap level and space charge distribution of polypropylene by enhancing the crystalline- amorphous interface effect in blends. Polymer International, 65(4), pp.371-379. DOI: https://doi.org/10.1002/pi.5063

[45] Zhou, Y., He, J., Hu, J. and Dang, B., 2016. Surface-modified MgO nanoparticle enhances the mechanical and direct-current electrical characteristics of polypropylene/polyolefin elastomer nanodielectrics. Journal of Applied Polymer Science, 133(1). DOI: https://doi.org/10.1002/app.42863

[46] Du, B.X., Xu, H., Li, J. and Li, Z., 2016. Space charge behaviors of PP/POE/ZnO nanocomposites for HVDC cables. IEEE Transactions on Dielectrics and Electrical Insulation, 23(5), pp.3165-3174. DOI: 10.1109/TDEI.2016.7736882

[47] Nelson, J.K., 2010. Dielectric polymer nanocomposites. New York, NY, USA, Springer. DOI: ISBN 978-1-4419-1591-7

[48] Roy, M., Nelson, J.K., MacCrone, R.K. and Schadler, L.S., 2007. Candidate mechanisms controlling the electrical characteristics of silica/XLPE nanodielectrics. Journal of Materials Science, 42(11), pp.3789-3799. DOI: 10.1007/S10853-006-0413-0

[49] Han, B., Chang, J., Song, W., Sun, Z., Yin, C., Lv, P. and Wang, X., 2019. Study on Micro Interfacial Charge Motion of Polyethylene Nanocomposite Based on Electrostatic Force Microscope. Polymers, 11(12), pp.2035. DOI: https://doi.org/10.3390/polym11122035

[50] Zha, J.W., Wang, Y., Wang, S.J., Zheng, M.S., Bian, X. and Dang, Z.M., 2019. Space charge suppression in environment-friendly PP nanocomposites by employing freeze-dried $\mathrm{MgO}$ with foam nanostructure for high-voltage power cable insulation. Applied Physics Letters, 114(25), pp.252902. DOI: https://doi.org/10.1063/1.5110443

[51] Lewis, T.J., 2014. Charge transport in polyethylene nano dielectrics. IEEE transactions on dielectrics and electrical insulation, 21(2), pp.497-502. DOI: 10.1109/TDEI.2013.004173

[52] Takada, T., Hayase, Y., Tanaka, Y. and Okamoto, T., 2008. Space charge trapping in electrical potential well caused by permanent and induced dipoles for LDPE/MgO nanocomposite. IEEE Transactions on Dielectrics and Electrical Insulation, 15(1), pp.152-160. DOI: $10.1109 / \mathrm{T}$ DEI.2008.4446746

[53] Wang, Y., Qiang, D., Xu, Z., Chen, G. and Vaughan, A., 2018. The effect of loading ratios and electric field on charge dynamics in silica-based polyethylene nanocomposites. Journal of Physics D: Applied Physics, 51(39), pp.395302. DOI: 10.1088/1361-6463/aad7e8

[54] Chen, G., Li, S., \& Zhong, L. (2015, July). Space charge in nanodielectrics and its impact on electrical performance. In 2015 IEEE 11th International Conference on the Properties and Applications of Dielectric Materials (ICPADM), pp. 36-39. DOI: 10.1109/ICPADM.2015.7295202

[55] Lv, X.S., Han, B., Wang, J.Y., Chang, J.X., Hua, Y. and Sun, W.F., 2019. Modification mechanism of low-density polyethylene insulation by hydrophilic and hydrophobic porous $\mathrm{SiO} 2$ nanoparticles. Applied Nanoscience, pp.1-11. DOI:10.1007/s13204-019-01074-6

[56] Siddabattuni, S., Schuman, T.P. and Dogan, F., 2013. Dielectric properties of polymer-particle nanocomposites influenced by electronic nature of filler surfaces. ACS applied materials \& interfaces, 5(6), pp.1917-1927. DOI: https://doi.org/10.1021/am3030239

[57] Gun'Ko, V.M., Mironyuk, I.F., Zarko, V.I., Voronin, E.F., Turov, V.V., Pakhlov, E.M., Goncharuk, E.V., Nychiporuk, Y.M., Vlasova, N.N., Gorbik, P.P. and Mishchuk, O.A., 2005. Morphology and surface properties of fumed silicas. Journal of colloid and interface science, 289(2), pp.427-445. DOI: https://doi.org/10.1016/j.jcis.2005.05.051 
[58] Boldridge, D., 2010. Morphological characterization of fumed silica aggregates. Aerosol Science and Technology, 44(3), pp.182-186. DOI: https://doi.org/10.1080/02786820903499462

[59] Albers, P., Maier, M., Reisinger, M., Hannebauer, B. and Weinand, R., 2015. Physical boundaries within aggregates-differences between amorphous, para-crystalline, and crystalline Structures. Crystal Research and Technology, 50(11), pp.846-865. DOI: https://doi.org/10.1002/crat.201500040

[60] Blume, A., Gatti, L., Luginsland, H.D., Maschke, D., Moser, R., Nian, J.C., Röben, C. and Wehmeier, A., 2015. Silica and Silanes. Rubber Compounding: Chemistry and Applications, pp.251332. ISBN: 9781482235494.

[61] He, W., Wu, D., Li, J., Zhang, K., Xiang, Y., Long, L., Qin, S., Yu, J. and Zhang, Q., 2013. Surface modification of colloidal silica nanoparticles: Controlling the size and grafting process. Bulletin of the Korean Chemical Society, 34(9), pp.2747-2752. DOI: https://doi.org/10.5012/bkcs.2013.34.9.2747

[62] Mahtabani, A., Rytöluoto, I., Anyszka, R., He, X., Saarimäki, E., Lahti, K., Paajanen, M., Dierkes, W. and Blume, A., 2020. On the Silica Surface Modification and Its Effect on Charge Trapping and Transport in PP-Based Dielectric Nanocomposites. ACS Applied Polymer Materials, 2(8), pp.31483160. DOI: https://doi.org/10.1021/acsapm.0c00349

[63] Gruendau, M., Stockstadt, J., Hauau, H, Bruchkoebel, B. Evonik Degussa Gmbh, Essen(DE), Adhesives. United States patent US 2010/0305236 A1, 2008, May, 06.

[64] Ya, W., Konuk, A.R., Aarts, R., Pathiraj, B. and Huis, B., 2015. Spectroscopic monitoring of metallic bonding in laser metal deposition. Journal of materials processing technology, 220, pp.276-284. DOI: https://doi.org/10.1016/j.jmatprotec.2015.01.026

[65] Inagaki, N., Plasma surface modification and plasma polymerization. 1996: CRC Press. ISBN: 9781566763370 .

[66] van Os, M.T., 2000. Surface modification by plasma polymerization: film deposition, tailoring of surface properties and biocompatibility [PhD thesis]. Universiteit Twente, The Netherlands. ISBN: 9036515130 .

[67] Chu, P.K. and Lu, X. eds., 2013. Low temperature plasma technology: methods and applications. CRC Press. ISBN: 9780367576363.

[68] Fridman, A., 2008. Plasma chemistry. Cambridge university press. DOI: https://doi.org/10.1017/CBO9780511546075. ISBN: 9780511546075

[69] Chan, C.M., Ko, T.M. and Hiraoka, H., 1996. Polymer surface modification by plasmas and photons. Surface science reports, 24(1-2), pp.1-54. DOI: https://doi.org/10.1016/0167-5729(96)80003-3

[70] Cui, L., Ranade, A.N., Matos, M.A., Dubois, G. and Dauskardt, R.H., 2013. Improved adhesion of dense silica coatings on polymers by atmospheric plasma pretreatment. ACS applied materials \& interfaces, 5(17), pp.8495-8504. DOI: https://doi.org/10.1021/am401921k

[71] Wolf, R.A., 2007. Unique atmospheric plasma surface pretreatment approach for improving adhesion. Journal of Vinyl and Additive Technology, 13(2), pp.87-90. DOI: https://doi.org/10.1002/vnl.20107

[72] Araya, M., Yuji, T., Watanabe, T., Kashihara, J. and Sumida, Y., 2007. Application to cleaning of waste plastic surfaces using atmospheric non-thermal plasma jets. Thin Solid Films, 515(9), pp.43014307. DOI: https://doi.org/10.1016/j.tsf.2006.02.066 
[73] Desmet, T., Morent, R., De Geyter, N., Leys, C., Schacht, E. and Dubruel, P., 2009. Nonthermal plasma technology as a versatile strategy for polymeric biomaterials surface modification: a review. Biomacromolecules, 10(9), pp.2351-2378. DOI: https://doi.org/10.1021/bm900186s

[74] Bretagnol, F., Tatoulian, M., Arefi-Khonsari, F., Lorang, G. and Amouroux, J., 2004. Surface modification of polyethylene powder by nitrogen and ammonia low pressure plasma in a fluidized bed reactor. Reactive and functional polymers, 61(2), pp.221-232. DOI: https://doi.org/10.1016/j.reactfunctpolym.2004.06.003

[75] Yasuda, H. and Wang, C.R., 1985. Plasma polymerization investigated by the substrate temperature dependence. Journal of Polymer Science: Polymer Chemistry Edition, 23(1), pp.87-106. DOI: https://doi.org/10.1002/pol.1985.170230110

[76] Nisol, B. and Reniers, F., 2015. Challenges in the characterization of plasma polymers using XPS. Journal of Electron Spectroscopy and Related Phenomena, 200, pp.311-331. DOI: https://doi.org/10.1016/j.elspec.2015.05.002

[77] Stoykov, S., Eggs, C. and Kortshagen, U., 2001. Plasma chemistry and growth of nanosized particles in a $\mathrm{C} 2 \mathrm{H} 2$ RF discharge. Journal of Physics D: Applied Physics, 34(14), p.2160. DOI: https://doi.org/10.1088/0022-3727/34/14/312

[78] Akhavan, B., Jarvis, K. and Majewski, P., 2013. Hydrophobic plasma polymer coated silica particles for petroleum hydrocarbon removal. ACS applied materials \& interfaces, 5(17), pp.8563-8571. DOI: https://doi.org/10.1021/am4020154

[79] Neira-Velázquez, M.G., Ramos-deValle, L.F., Hernández-Hernández, E., Ponce-Pedraza, A., SolísRosales, S.G., Sánchez-Valdez, S., Bartolo-Pérez, P. and González-González, V.A., 2011. Surface modification of nanoclays by plasma polymerization of ethylene. Plasma Processes and Polymers, 8(9), pp.842-849. DOI: https://doi.org/10.1002/ppap.201000162

[80] Wu, C., Yan, W. and Phung, B.T., 2013, October. Influence of plasma-treated nanoparticles on space charge accumulation in epoxy resin insulation. In 2013 Annual Report Conference on Electrical Insulation and Dielectric Phenomena, pp. 784-787. DOI: 10.1109/CEIDP.2013.6748133.

[81] Befahy, S., Lipnik, P., Pardoen, T., Nascimento, C., Patris, B., Bertrand, P. and Yunus, S., 2010. Thickness and elastic modulus of plasma treated PDMS silica-like surface layer. Langmuir, 26(5), pp.3372-3375. DOI: https://doi.org/10.1021/la903154y

[82] Ward, A.J. and Short, R.D., 1993. A time-of-flight secondary ion mass spectrometry and X-ray photoelectron spectroscopy investigation of the structure of plasma polymers prepared from the methacrylate series of monomers. Polymer, 34(20), pp.4179-4185. DOI: https://doi.org/10.1016/0032$\underline{3861(93) 90174-9}$

[83] Yasuda, H. and Matsuzawa, Y., 2005. Economical Advantages of Low-Pressure Plasma Polymerization Coating. Plasma Processes and Polymers, 2(6), pp.507-512. DOI: https://doi.org/10.1002/ppap.200500002

[84] Tiwari, M., Mathew, T., Noordermeer, J.W., Dierkes, W.K., Datta, R.N., Talma, A. and van Ooij, W.J., 2008. Compatibilization of Elastomer-Filler Combinations by Plasma Polymerization. KGK. Kautschuk, Gummi, Kunststoffe, 61(10), pp.502-509.

[85] C. Wu, W. Yan and B. T. Phung, "Influence of plasma-treated nanoparticles on space charge accumulation in epoxy resin insulation," 2013 Annual Report Conference on Electrical Insulation and Dielectric Phenomena, Shenzhen, 2013, pp. 784-787, DOI: 10.1109/CEIDP.2013.6748133 
$50 \mid \mathrm{P}$ a g e 
Chapter 3

\section{Summary}

-the Structure Behind 


\subsection{Introduction}

The goal of this $\mathrm{PhD}$ study is focused on the development of silica surface functionalization and investigation of its influence on dielectric properties focusing on charge trapping distribution and space charge accumulation of PP based composites. This is part of an extensive R\&D project focused on the development and optimization of polypropylene/nanosilica based electric insulation nanocomposites for the next generation of high-voltage direct current (HVDC) cable applications.

The aim of this $\mathrm{PhD}$ research also includes:

1) Development of a sustainable material for HVDC cable application based on a nanosilica/polypropylene composite, including a "green" method of silica surface modification, and with the ability to be scaled up to supply a sufficient amount of the modified silica for a cable production trial.

2) Design of innovative modifications for silica to reduce space charge accumulation in polypropylene-based insulation nanocomposites under a high DC electric field.

3) Investigation of the effect of the surface modification of nanosilica on charge trapping, charge current density and space charge accumulation of the nanodielectrics, as well as other properties.

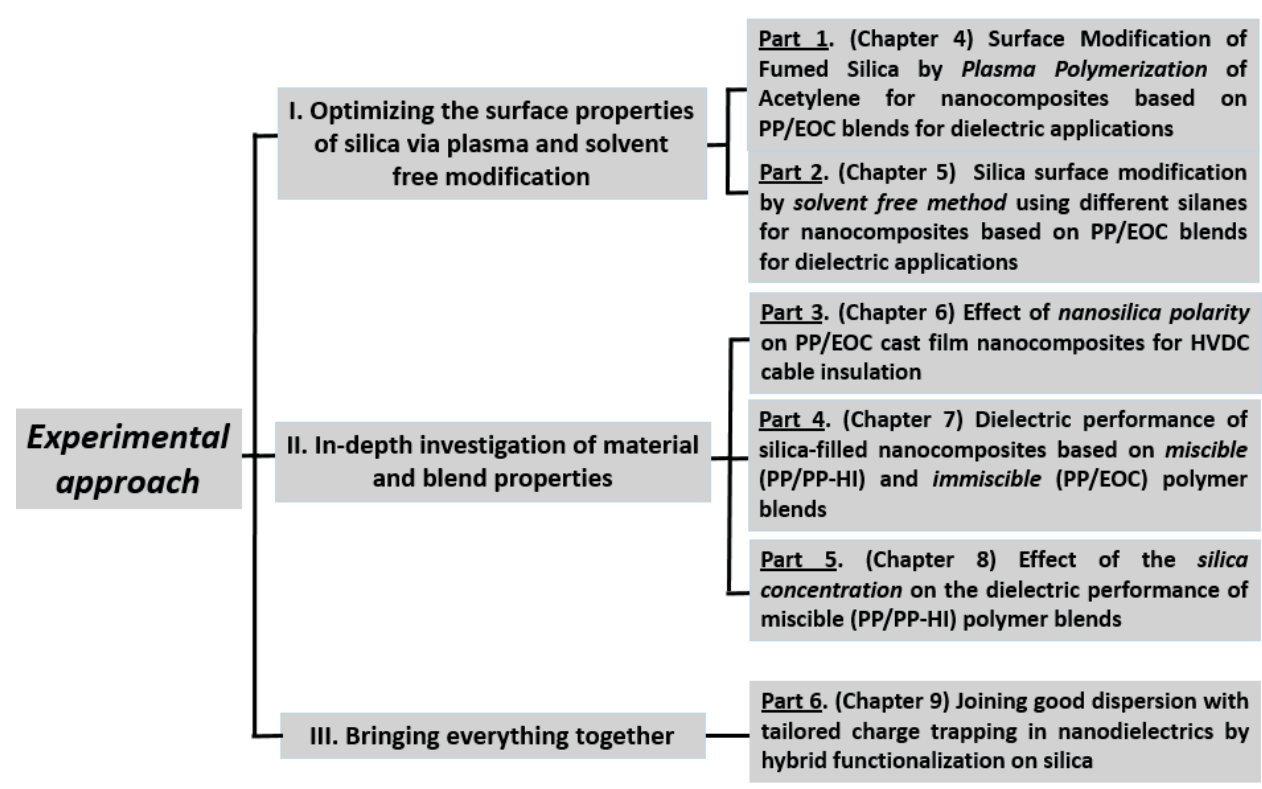

Figure 3.1 Approaches for optimizing the dielectric properties of the PP/silica composites.

In order to realize these research goals, two principal approaches to prepare compounds and film specimens filled with modified nanosilica were investigated: 
i) Mini-injection molding for small-scale (12 g) characterization in order to further optimize the most promising surface modification methods of silica, and

ii) Cast film extrusion for pilot-scale $(4 \mathrm{~kg})$ processing and studying the dielectric properties.

The selected fumed silica was modified through two environmentally friendly methods:

i) Plasma polymerization, and

ii) Solvent-free modification.

Figure 3.1 shows the approach used in this study to tailor the surface properties of silica and the interaction with the PP matrix, as well as to optimize the dielectric properties, focusing on charge trapping and transport of the PP/silica composite. All details of sample preparation, characterization methods and results are described in the corresponding thesis chapters.

\subsection{Scientific Approaches}

\subsubsection{Surface Modification by Plasma Deposition of Acetylene}

Regardless of the field of application (e.g., dielectric, transparent, flame-retarding nanocomposites), the dispersion of the nanoparticles and their compatibility with the polymer matrix affect the performance of the nanocomposites. Nanoparticles with a high specific surface area tend to aggregate together, leading to a poor dispersion in the polymer matrix. Consequently, initiation of electrical or mechanical failure (cracks or flaws) may occur around the clusters as shown in Figure 3.2. Therefore, improvement of the dispersion of the nanoparticles in the polymer matrix is one of the important challenges.

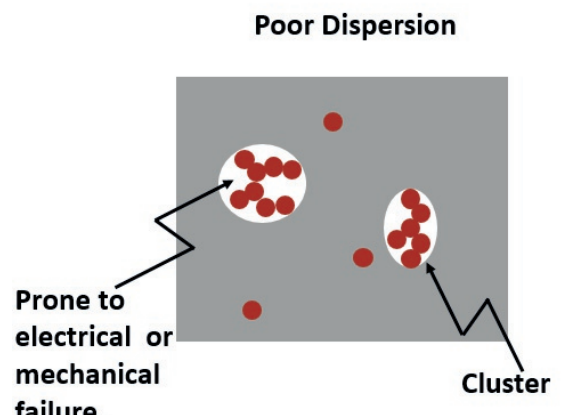

Ideal Dispersion

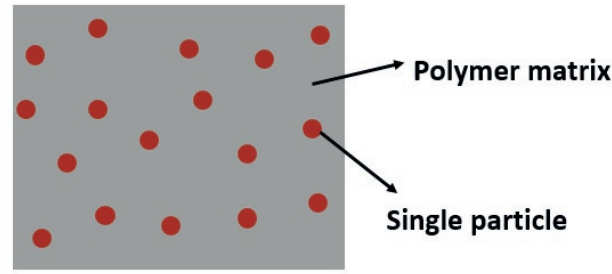

Figure 3.2 Dispersion of nanoparticles in polymer matrices (left: poor dispersion; right: ideal dispersion).

Specifically for dielectric nanocomposites, the addition of nanoparticles introduces a large interfacial region between the nanoparticle surface and polymer matrix, and it is generally accepted that this plays an important role for the dielectric performance. Several theories explain the role of the interfacial region: On one hand, the interfacial region around the nanoparticles can create a local conductive path or a 
quasi-conductive region [1]. It is considered to be more conductive than the bulk of the polymer matrix. Hence, it will lead to local charge redistribution and a reduction of space charge accumulation. On the other hand, the interfacial polymer chains present different local properties like changes in chain mobility or/and conformation, free volume, density, crystallinity etc. compared to the plain macromolecules. These changes may create a large number of trap sites with different depth, which may influence the mobility of charge carriers and their energy. Due to the large number of trap sites, the charge carriers are expected to be trapped more frequently resulting in energy reduction, which in turn results in less damage of the insulation layer and thus increased lifetime [2]

Nanoparticles can also act as scattering sites for electrons or charge carriers within the polymer matrix. Electrons accelerated by the applied electric field collide with the nanoparticles, losing their momentum consequently. As a result, the electrons cause electrical failure only at higher voltages, resulting in an improved breakdown strength. A good dispersion of the nanoparticles can create a myriad scattering sites, leading to an improved breakdown strength. In contrast to this, nanoparticles with poor dispersion and thus formation of clusters, which easily split up under high voltage and initiate crack formation in the polymeric matrix, lead to a reduced breakdown strength [3]. Moreover, nanoparticle addition can also reduce the space charge accumulation via the following mechanism: The nanoparticles introduce deep charge traps, which are able to capture the injected charges near the electrodes, thus inhibiting the transportation of the charges into the bulk of the insulation material via electrostatic repulsion. This is just one breakdown mechanism which is in the scope of this work; in actual practice other mechanisms are occurring as well.

In summary, nanoparticles as well as the interfacial region are both affecting the charge trapping behaviour, which in turn influences breakdown strength, life time and space charge accumulation of the nanocomposites. Furthermore, it is crucial to achieve a small particle cluster size meaning good dispersion, and thus increase the interfacial region area. The degree of dispersion of the nanoparticles allows to tailor the charge trapping distribution (e.g., to change the trap densities or introduce the deep traps) and to reduce the space charge accumulation, to finally improve the dielectric performance of the nanocomposites.

As introduced in Chapter 2, silica is a hydrophilic nanofiller and polypropylene (PP) is a hydrophobic polymer: these two components are not compatible. The first approach to increase the compatibility was to hydrophobize the silica surface.

Most commonly used silica surface modification methods are solvent-based, in which the solvent is e.g. toluene [4]. The chemical modification agent is dissolved in the liquid, in which the silica is dispersed as well, in order to enable surface modification in a controlled environment, e. g. temperature and time. However, this consumes a considerable amount of solvent, which has to be discarded after the treatment: To modify $50 \mathrm{~g}$ of fumed silica, around 0.5 liter of solvent is needed under laboratory conditions. On industrial scale, a corresponding large amount of solvent will be needed, and the same amount of waste will be generated. Considering environmental friendliness and sustainability, the aim of this thesis was to introduce a "greener" approach to perform silica surface functionalization.

In Chapter 4, a plasma surface functionalization method is used, which is not solvent-based. The detailed mechanism of the plasma surface functionalization is described in Chapter 2, Paragraph 3.2.2. Briefly, a monomer is introduced into the plasma chamber at low pressure. The monomer is excited, ionized or fragmented by electrons, resulting in radical or electron formation. The radicals recombine to form new 
chemical components, including polymers forming a two-dimensional film on a substrate. Simultaneously, the silica surface can also be excited by the high energy electrons. The newly formed chemical species react with the excited silica surface and form a hydrophobic layer in the case of an unpolar precursor.

A polypropylene macromolecule consists of hydrogen and carbon atoms. Therefore, the first approach to improve the compatibility of the silica with a polypropylene matrix was to deposit a hydrocarbon layer on the silica surface. The monomer used in Chapter 4 is acetylene $\left(\mathrm{C}_{2} \mathrm{H}_{2}\right)$, which is commonly used in industrial applications. A model of acetylene plasma polymerization is developed by S. Stoykov [5] and others [6]. In the plasma, acetylene molecules firstly dissociate into radicals $\left(\mathrm{C}_{2} \mathrm{H} \bullet\right.$ and $\left.\mathrm{H} \bullet\right)$ (equation 2.1). The radical $\left(\mathrm{C}_{2} \mathrm{H} \bullet\right)$ recombines afterwards with $\mathrm{C}_{2} \mathrm{H}_{2}$ to form another radical $\left(\mathrm{C}_{4} \mathrm{H}_{3} \bullet\right)$ (equation 2.2). All these species (including $\mathrm{C}_{2} \mathrm{H}_{2}$ and all different kinds of newly formed radicals) recombine together to form larger structures (equations 3.3, 3.4 and 3.5) through plasma polymerization:

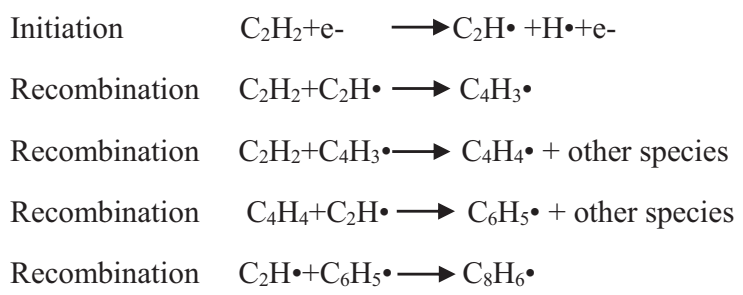

In previous studies, precipitated silica was successfully modified with acetylene [7]. The surface properties of the silica were changed by plasma modification from hydrophilic to a more hydrophobic nature due to deposition of a hydrocarbon layer onto the silica surface. This led to an improvement of the dispersion of silica in the elastomer compound. Being inspired by this successful work, and the expectation that polypropylene-like structures will be generated on the silica surface, acetylene was chosen as the monomer for the silica surface modification. This was expected to improve silica dispersion in the polypropylene matrix, and this way to suppress space charge accumulation, which can lead to early failure, by altering the charge trapping properties.

Plasma modification of a substrate is influenced by many parameters, e.g., monomer flow and radiofrequency $(\mathrm{RF})$ plasma generator power. It is important to optimize the modification conditions in order to achieve the most effective surface deposition. For the plasma setup in our laboratory as described in Chapter 4, the RF power range is $100-300 \mathrm{~W}$, and the monomer flow rate range is 2 to 24 $\mathrm{cm}^{3} / \mathrm{min}$ ( $\mathrm{sccm}$ ). In this part of the study, the plasma modification was optimized by using different acetylene flow rates $(2 / 4 / 6 / 8 / 12 / 18 \mathrm{sccm})$ at the same RF power $(150 \mathrm{~W})$, and different RF power level $(100 / 150 / 200 / 250 / 300 \mathrm{~W})$ using the same flow rate $\left(6 \mathrm{~cm}^{3} / \mathrm{min}\right)$. Finally, the silica modified in an acetylene plasma with a flow rate of $6 \mathrm{sccm}$ and a RF power of $150 \mathrm{~W}$ showed the highest deposition and was selected for the dielectric nanocomposite compounding. The main outcome from this study was that the dispersion of the acetylene plasma-modified silica was indeed improved, and that the composite charging current was slightly reduced: both positive effects. However, changes of the charging current and the trap level distribution were not very significant. The next step of this study would be to use different types of organic precursors and investigate their effect on the charge trapping properties and 
the charging current of the nanocomposites. However, this was not implemented within this project as the other modification method, the solvent-free one, turned out to be more promising.

\subsubsection{Surface Modification of Silica by a Series of Silanes}

Silanes are the most common modifying agents used for silica surface treatments, and there are various kinds of suitable silanes available in the market. The details of the silane silica reaction are presented in Chapter 2. In a short summary, the reaction consists of two major steps:

1) Hydrolysis of alkoxy group(s) of the silane into hydroxyl group(s).

2) Condensation of the silane, in which the hydrolyzed silanols react with each other or with a silanol moiety on the silica surface to form the Si-O-Si bridges.

Step 1 is the slowest step [8], which controls the reaction kinetics [9]. In general, hydrolysis reactions are of endothermic nature [10]: a higher reaction temperature increases the reaction rate. Besides, hydrolysis of the silane can occur at different $\mathrm{pH}$ values (acidic, alkaline or neutral) as shown in Figure $3.3[11,12]$ It is reported that the acidic hydrolysis is the fastest process [9], indicating that adjusting the $\mathrm{pH}$ can speed up the silane hydrolysis reaction.

\section{Neutral hydrolysis}

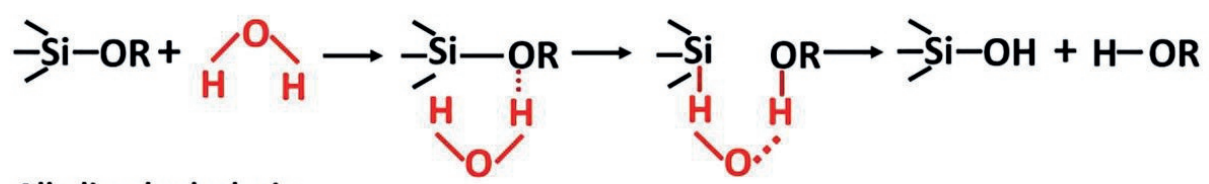

\section{Alkaline hydrolysis}

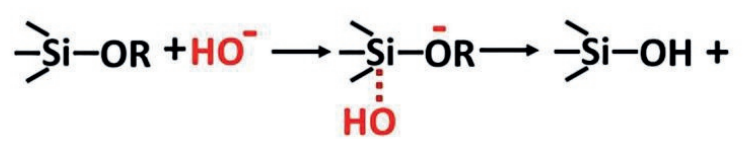

Acidic hydrolysis
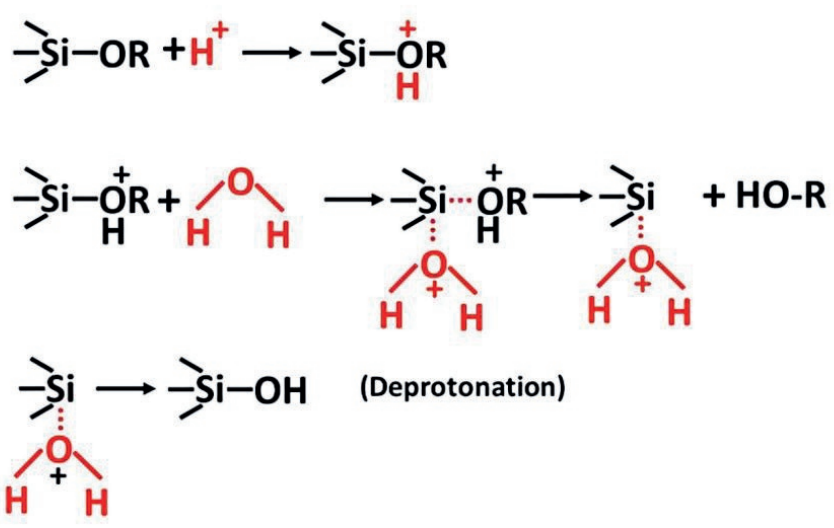

Figure 3.3 Schemes of the hydrolysis mechanism in neutral, alkaline or acidic environment [11-13]. 
In short, increasing the temperature or controlling the $\mathrm{pH}$ (e.g., using an acid or base as catalyst) are two ways to speed up the silanization reaction. However, working at ambient temperature allows utilization of a simpler reaction setup. Therefore, controlling the $\mathrm{pH}$ in order to accelerate the silane-silica reaction and a reaction at room temperature is used in this study as elaborated in Chapter 5. A new, solvent-free silane-silica modification method was developed with an easy to scale up reaction setup. Trifluoroacetic acid as catalyst successfully accelerates the silica surface modification with a silane in a dry environment and under vigorous mixing of the silica.
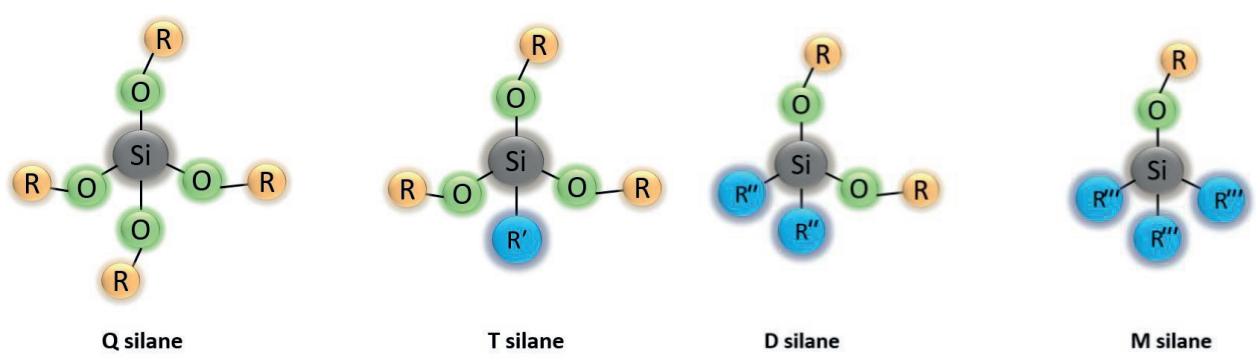

Figure 3.5 Scheme of the different kinds of silanes. Q silane is a silane with 4 alkoxy groups; T silane has 3 alkoxy groups; D silane carries 2 alkoxy groups; M silane just has 1 alkoxy group.

There are four types of silanes (Figure 3.5) differing in the number of reactive groups, for example alkoxy groups [9]. Q silanes contain 4 alkoxy groups and there is no additional non-hydrolysable group present. Therefore, it cannot introduce a functional group to a silica surface. Differently, T, D and M silanes all include at least one non-hydrolysable group $\left(\mathrm{R}^{\prime}\right)$, which gives the possibility to introduce various functional groups to the silica surface as well to control the polarity of the silica. 9 silanes (including T, D and M type of silanes) with different functional groups (polar and unpolar) are selected to modify the silica. The aims of this approach are:

1) to study whether the number of alkoxy groups on the silane has an effect on the degree of silica modification and further influence the dielectric performance of the nanocomposites;

2) to evaluate the effect of the polarity of the silica on the dielectric performance of the nanocomposites.

The result was that the solvent-free modification turned out to be an efficient method for silica surface treatment, as proven by the significant weight loss measured by TGA stemming from the decomposition of the chemically bound silanes ( 3 to $17 \mathrm{wt} . \%$ ). The differences in weight loss depend on the number of alkoxy groups and chemical structure of the silane. Besides, 11 PP-based nanocompounds (unfilled reference, 1 nanocomposite filled with the reference silica and 9 nanocomposites with modified silicas) were prepared via mini-scale compounding and injection moulding. SEM results show that the unpolar silicas exhibit a better dispersion than the polar silicas. All silicas increase the nucleation density in PP and increase the crystallization temperature of polypropylene. The obtained TSDC results indicate that the charge trap depth versus density distribution of the nanocomposites changed with surface 
modification. The nanocomposites filled with unpolar silicas had a higher charge trap density, while the polar silica increased the charge trap depth. The modified silicas containing a nitrogen atom in the functional group exhibited the strongest influence on the charge trapping behaviour, resulting in the lowest amount of injected charge. This indicates that these nanocomposites might have the lowest space charge accumulation, thus the most promising dielectric performance for direct current applications.

\subsubsection{The Influence of Polarity of the Silica on the Properties of the Composites}

The results of Chapter 5 show, that there are completely different effects on the charge trapping distribution properties of the compounds depending on the polarity of the silica. Therefore, in Chapter 6, two silicas (polar and unpolar) were selected to further evaluate their effect on micromorphology, dynamic and dielectric properties on pilot scale cast film nanocomposites. The unpolar silica was modified with trimethylethoxysilane, gaining the best dispersion in the polymer. The corresponding nanocomposite has the highest trap density and moderate trap depth. The polar silica was modified with 3-aminopropyltriethoxysilane reaching limited dispersion, and its nanocomposite has the lowest trap density and high trap depth. As discussed already in Paragraph 2.1, the dispersion of the nanofiller is one of the most important factors influencing the dielectric performance of a nanocomposite. However, it is also reported that the chemical surface properties of a nanofiller are another factor that effects the global performance of a nanocomposite: the chemical properties of a nanofiller's surface determines the polarity of a nanofiller, which in turn will affect the compatibility between the nanofiller and the polymeric matrix and influence the nanofiller dispersion [14]. Simultaneously, it influences the electrical characteristics of the interface with the polymeric matrix leading to different charge trapping properties and dielectric performance [15]. It turned out that space charge accumulation of the nanocomposite containing a polar silica carrying a $-\mathrm{NH}_{2}$ functionality is lower than that of the unpolar silica filled material. This can be explained by the polar silica introducing deep traps with lower trap density, which suppress the charge injection from both electrodes and reduce space charge accumulation within the bulk of the material. Hence, it can be concluded that the dielectric performance in terms of low space charge accumulation of the composite with polar silica is better, in spite of the fact that the dispersion of this silica is significantly lower, stemming from the $-\mathrm{NH}_{2}$ functional groups on the polar silica surface.

In Chapters 4 and 5, the compounds were prepared through mini-scale compounding and injection molding to screen the methods of silica surface modification, while in Chapters 6, 7 and 8, all compounds were prepared via pilot-scale compounding and cast film extrusion in order to further study the properties of the compounds; for these measurements, larger quantities of the composite were required. The reason for working on two different scales is bound to the nature of the GRIDABLE project, in which academia meets industry allowing for small-scale testing of many various nanocomposites, and stepwise scaling up of the most promising materials for further testing and applications studies. A comparative study of nanocomposites produced by mini injection molding and pilot scale cast film extrusion is published in [16], which is focused on the morphological and structural differences between the two sample types. A summary of the outcome is shown in Table 3.1.

In this study, indeed different properties of the samples are noticed (e.g morphological differences) between these two approaches. For example, for the samples prepared via mini-scale compounding and injection molding, a higher degree of crystallinity was observed compared to the extruded cast film 
samples. However, the results of Chapter 5 and 6 have shown that the trends of the changes in trap depth are consistent and depend mostly on the silica modification type, while for other properties they may be dissimilar. It was concluded that mini-scale injection molding can be a resource-efficient sample manufacturing method for facilitating early-stage screening of the best-performing material candidates, given that the morphological features are carefully taken into account [16].

Table 3.1 Comparison between mini-scale compounding and injection molding versus pilot-scale compounding and cast film extrusion techniques.

\begin{tabular}{|c|c|c|}
\hline & $\begin{array}{l}\text { Pilot scale compounding and cast film } \\
\text { extrusion }\end{array}$ & $\begin{array}{l}\text { Mini-scale compounding and } \\
\text { injection molding }\end{array}$ \\
\hline $\begin{array}{l}\text { Mixing } \\
\text { process }\end{array}$ & $\begin{array}{l}\text { 1) Pilot-scale compounding (batch size 1- } \\
80 \mathrm{~kg} \text { ) by a twin-screw extruder } \\
\text { (KraussMaffei Berstorff ZE 25/49D UTX). } \\
\text { 2) Extrusion of the granulated compounds } \\
\text { into a cast film by a single-screw extruder } \\
\text { (Brabender Plasticorder) equipped with a T- } \\
\text { die and a calendering system }\end{array}$ & $\begin{array}{l}\text { 1) Mini-scale compounding (batch } \\
\text { size } 12 \mathrm{~g} \text { ) by a mini-scale twin- } \\
\text { screw compounder (Haake MiniLab } \\
\text { Rheomex CTW5). } \\
\text { 2) Mini-injection molding (IM) into } \\
\text { thin film specimens (Haake MiniJet } \\
\text { Pro Piston Injection Moulding } \\
\text { System). }\end{array}$ \\
\hline Temperature & $195-230^{\circ} \mathrm{C}$ (cast film extrusion temperature) & $\begin{array}{l}230^{\circ} \mathrm{C} \text { (mini-compounding } \\
\text { temperature) }\end{array}$ \\
\hline Screw Speed & $85 \mathrm{rpm}$ (during the cast film extrusion) & $100 \mathrm{rpm}$ (during compounding) \\
\hline Time & $\begin{array}{l}\text { 3-5 mins (the residence time of the material } \\
\text { in the twin-screw extruder (compounding) } \\
\text { and single-screw extruder (cast film } \\
\text { extrusion) }\end{array}$ & $\begin{array}{l}4 \text { mins (during compounding in } \\
\text { mini-compounder) }\end{array}$ \\
\hline $\begin{array}{l}\text { Sample } \\
\text { thickness }\end{array}$ & $300-400 \mu \mathrm{m}$ & $500 \mu \mathrm{m}$ \\
\hline $\begin{array}{c}\text { Other } \\
\text { parameters }\end{array}$ & Calendering temperature $80^{\circ} \mathrm{C}$ & $\begin{array}{l}\text { Mold temperature } 60^{\circ} \mathrm{C} \\
\text { Pressure } 930-1000 \mathrm{bar} \\
\text { Hold time } 10 \mathrm{~s}+30 \mathrm{~s} \\
\text { Mold size } 26 \times 26 \mathrm{~mm}^{2}\end{array}$ \\
\hline
\end{tabular}




\subsubsection{Miscibility within the Polymer Matrix}

This part is focused on the influence of different polymer matrices on the nanocomposite properties. Background information on HVDC insulation polymers is included in Section 2.2 of Chapter 2. Crosslinked polyethylene is the most widely used insulation polymer. However, due to its crosslinked nature it is not recyclable, posing environmental problems. Polypropylene is one promising candidate with good dielectric performance and recyclable character. One of the concerns for using pure polypropylene as cable insulation material is its lack of mechanical flexibility. Hence, to improve the mechanical properties, the use of polypropylene copolymers or blends is utilized. In this study, a polypropylene copolymer and blend are used as polymeric matrices.

The polymeric matrix used in Chapters 4,5 and 6 is a blend of propylene-ethylene copolymer and ethylene-octene copolymer. Due to the fact that the ethylene content in this grade of the propyleneethylene copolymer is relatively low, it is abbreviated as PP. The abbreviation of the ethylene-octene copolymer is EOC.

PP and EOC are immiscible due to the different crystal structure and low intermolecular interactions, although they have similar chemical composition: they are both hydrocarbon polymers. This leads to a significant phase separation in the PP/EOC blend. The two phases are arranged in a layered cocontinuous structure. This phase separation also influences the silica dispersion and distribution. It was found that silica is located in the PP phase rather than in the EOC phase. This is due to the lower viscosity of PP (183 Pa·s @ the temperature of $195^{\circ}$ and speed with $100 \mathrm{~mm} / \mathrm{min}$ ) compared to EOC (395 Pa·s (a) the temperature of $195^{\circ}$ and speed with $100 \mathrm{~mm} / \mathrm{min}$ ), hence the PP chain with high molecular mobility can thus easier penetrate into the silica structure, resulting in an increased $\mathrm{PP} /$ silica interaction favouring and stabilizing silica location in the PP phase.
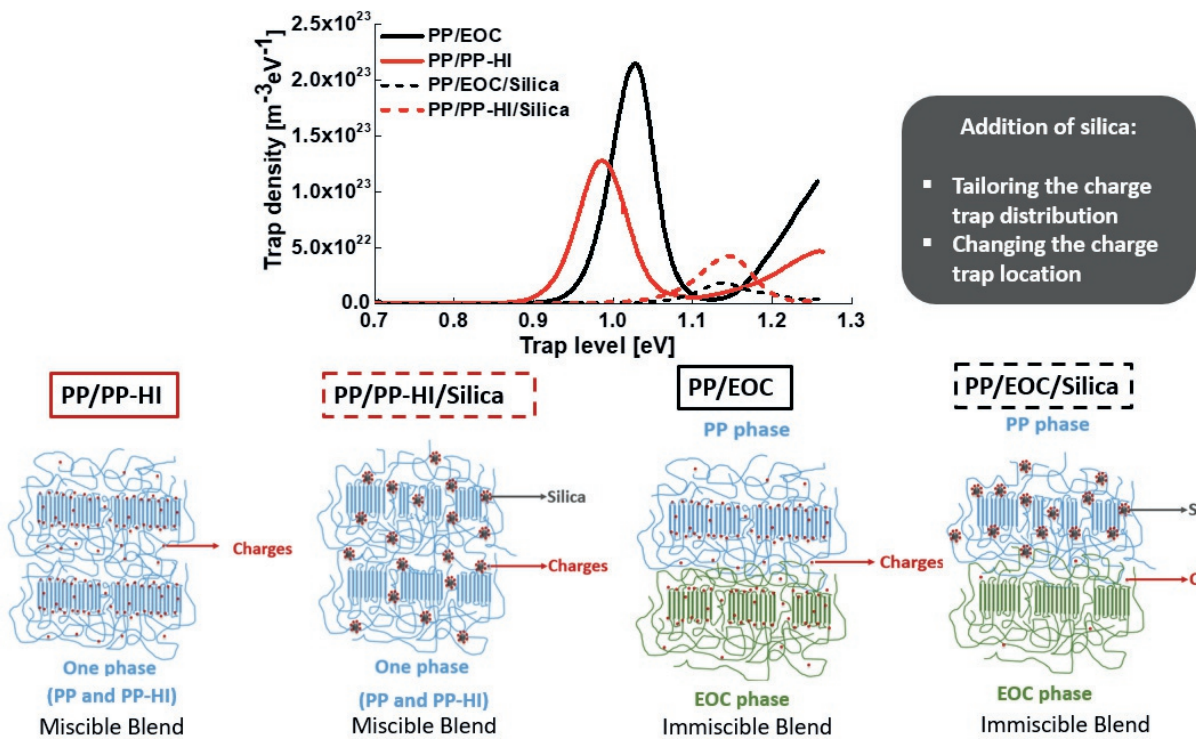

IPP/EOC/Silica I- - - - - -

Figure 3.6 Scheme of micromorphology and trap distribution of PP/PP-HI (propylene-ethylene copolymer) and PP/EOC (ethylene-octene copolymer) nanocomposites filled with polar silica (3aminopropyltriethoxysilane modified). 
In order to improve the silica dispersion and distribution in the polymeric matrix, a miscible polymer blend was used and the morphology as well as the dielectric performance of both blends were studied. Therefore, we used another grade of propylene-ethylene copolymer. The ethylene content (5-7 wt.\%) in this propylene-ethylene copolymer is higher than in the previous one (3 wt.\%). In order to make a difference between these two propylene-ethylene copolymers, the latter one is named PP-HI. This new grade PP-HI is found to be miscible with PP.

In Chapter 7, these two polymer blends are compared: immiscible PP/EOC and miscible PP/PP-HI, as shown in Figure 3.6. In addition, the effect of the silica on both polymer blends was studied. Besides, based on the results from Chapter 6, the polar silica, 3-aminopropyltriethoxysilane modified, shows the lowest space charge accumulation; therefore, this silica is used in this study in order to further optimize the insulation composite for HVDC cables.

The dispersion of the silica in the $\mathrm{PP} / \mathrm{EOC}$ and $\mathrm{PP} / \mathrm{PP}-\mathrm{HI}$ matrices is noticeably different. The silica is evenly distributed in the PP/PP-HI matrix, while it is located only in the PP phase in the PP/EOC matrix, as sketched Figure 3.6. Besides, the charge location in the sample is changed by adding silica: the charges are mostly located at amorphous-crystalline interfaces in unfilled blends, while for the nanocomposites, most of the charges are located at the silica-polymer interface, as also shown in Figure 3.6. In general, the PP/PP-HI blend exhibits better performance than the PP/EOC one in terms of low temperature flexibility and low space charge accumulation. The silica addition improves the thermal stability and tailors the charge trap distribution of both polymer blends. The filler can significantly decrease the space charge accumulation for both polymer blends due to the deep trap introduction. Finally, it is concluded that the PP/PP-HI/silica nanocomposite exhibits the best electrical (lowest space charge accumulation) and mechanical properties (the lowest modulus $2512 \mathrm{MPa}$ at $-93^{\circ} \mathrm{C}$ and $292 \mathrm{MPa}$ at $26^{\circ} \mathrm{C}$, good flexibility), showing promising properties for HVDC cable insulation applications.

\subsubsection{Influence of Nanofiller Concentration}

In the optimization of the insulation material for HVDC cables, the polar silica modified with 3aminopropyltriethoxysilane (APTES) showed the best performance, and the PP/PP-HI blend had better miscibility and lower space charge accumulation than the PP/EOC blend. Hence, the further investigation is focused on the PP/PP-HI blend, and using the polar silica modified with APTES. The aim of Chapter 8 is to investigate the concentration effect of this modified silica on the dielectric performance of the PP/PP-HI blend. Based on the literature [17], higher concentrations of the nanofiller (above 5 phr) cause increased space charge accumulation and severe electric field distortion, which could lead to reduced breakdown strength of nanodielectrics. High concentrations of nanofillers can cause their clustering and induce formation of void defects. Therefore, it is important to investigate the effect of silica concentration in the polymer matrix used in this study, though in rather low concentrations of a maximum of $2 \mathrm{wt} . \%$. This limitation is due to the challenging feeding of high amounts of nanoparticle powders due to its very low bulk density (approx. $50 \mathrm{~g} / \mathrm{l}$ ). The feeding rate of the nano-silica is very low in comparison to the polymers. Since this project is focused on industrially feasible applications, a comparative study of compounds filled with $1 \mathrm{wt} . \%$ and $2 \mathrm{wt} . \%$ of silica was done. 

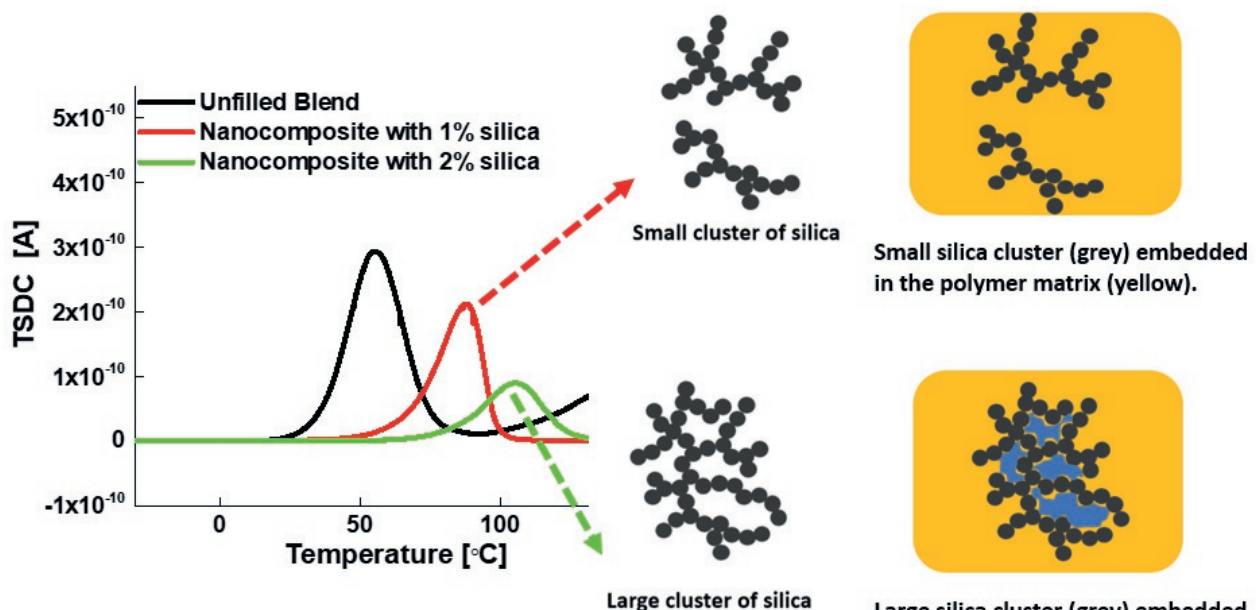
in the polymer matrix (yellow).

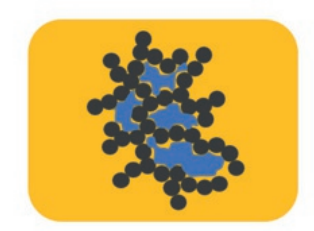
Large silica cluster (grey) embedded in the polymer matrix (yellow). The blue region indicates highly constrained polymer within the silica cluster.

Figure 3.7 Charge trap distribution of the unfilled PP/PP-HI blend and of the nanocomposites filled with 1 wt. $\%$ or 2 wt. $\%$ of silica.

The addition of 1 wt.\% or 2 wt. $\%$ of modified silica can influence the trap distribution of the nanocomposite, as shown in Figure 3.7, and it is clear that $2 \mathrm{wt} . \%$ of silica introduced deeper traps than $1 \mathrm{wt} . \%$. It was also noticed that relatively large silica aggregates (clusters with a particle size of $200 \mathrm{~nm}$ to $500 \mathrm{~nm}$ ) are present in the nanocomposite filled with $2 \mathrm{wt} \%$ of APTES modified silica. It is deduced that the deeper traps recorded for the nanocomposite with $2 \mathrm{wt} . \%$ of silica may stem from the highly constrained polymer chains inside the cluster, as shown in Figure 3.7. A highly constrained polymer chain is less mobile. When the charges are trapped at the interface between the highly constrained polymer and silica, which has a very high energy potential, the charge cannot move or de-trap easily. Furthermore, the immobile trapped charge might form another barrier based on the repelling force suppressing further charge injection, which results in low trap density, low space charge accumulation and also low conductivity. Another explanation for the higher trap level of the sample with $2 \%$ silica compared to the one with $1 \%$ might be the presence of amine-amine hydrogen bonding: Once there are more primary particles of silica clustered together, more hydrogen bonds are formed between amino groups on the silica surface. As a result, the electron cloud around nitrogen may induce deeper traps visible in the PP/PP-HI/S-2 (the nanocomposite with $2 \mathrm{wt} . \%$ of silica) peak in comparison to PP/PPHI/S-1 (the nanocomposite with 1 wt.\% of silica). Furthermore, if charges trapped around one amino group get enough energy to de-trap, it is highly likely that they will be easily trapped by a neighboring amino group. This leads to a lower mobility of the charges.

In summary, the addition of $2 \mathrm{wt} . \%$ of silica results in lower space charge accumulation (under 30 $\mathrm{kV} / \mathrm{mm}$ ) than the addition of only $1 \mathrm{wt} . \%$ of silica. This gives an indication that the presence of silica clusters with a proper surface modification can improve the dielectric properties in terms of reducing space charge accumulation. 


\subsubsection{Optimized Silica Modification for HVDC Applications}

It should be addressed that the conclusion of the benefit of the cluster in chapter 8 is based on the specific APTES surface treatment, it may not fit for other surface functionalized nanosilica. Therefore, in order to further optimize and to design the next generation of modified silica, all gained knowledge of the modification of silica for better dielectric properties of a composite with a PP matrix comes together: a surface structure with an inner layer and outer layer in order to improve the dispersion of the silica as well as the dielectric performance. Based on earlier results as well as literature $[4,18]$, it was concluded that the dielectric nanocomposite performance can be improved by

- better dispersion of the nanofiller in the polymeric matrix, and

- introduction of certain functional groups on the nanofiller surface.

After comparison of the above mentioned effects - dispersion and functional groups - as described in Chapters 5 and 6 , it was concluded that the introduction of functional groups on the nanosilica surface is more effective than a good dispersion. The effect of dispersion is related to two phenomena: the large surface area of single small particles in the matrix, and the presence of clusters with constrained polymer. Nonetheless, all the gained knowledge is brought together to further explore whether both can be achieved: good dispersion of the silica and reduced space charge accumulation. To study this, a series of newly designed and modified silicas were prepared via mini-scale compounding and injection molding as only small amounts of the silicas were available.

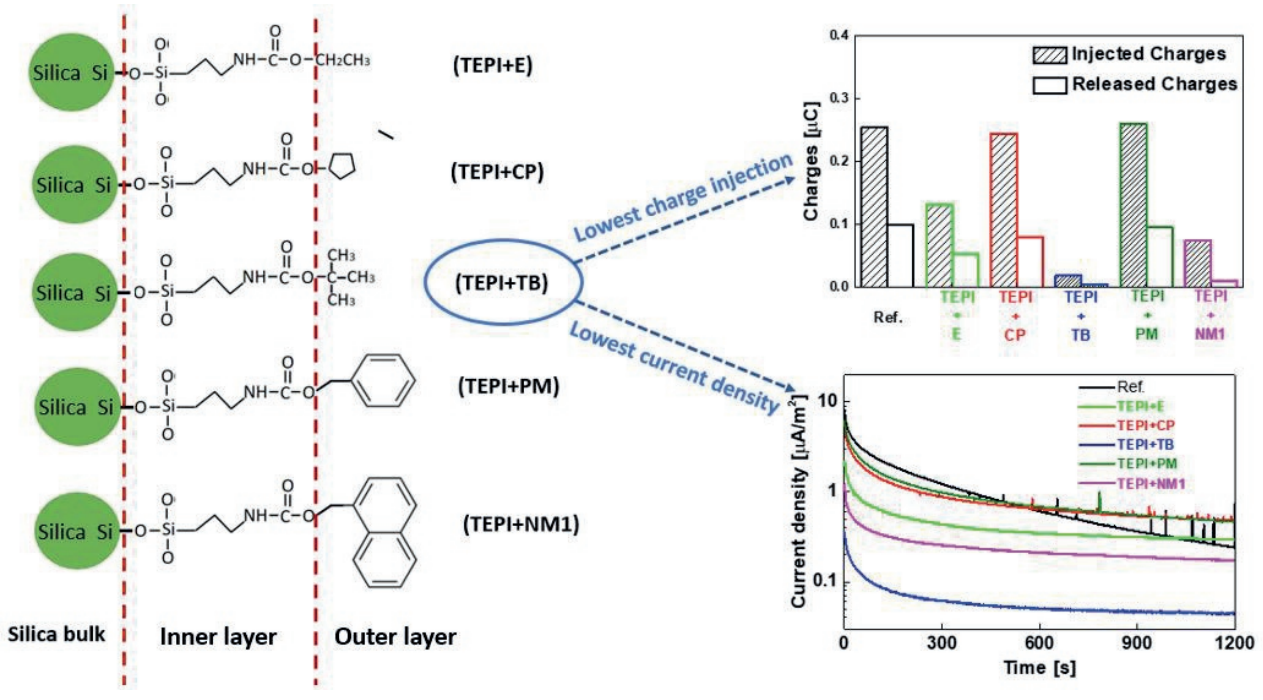

Figure 3.8 The newly designed and modified silica and its effect on the charge injection and current density of the composites. 
The newly designed and modified silicas contain a polar urethane group (with the purpose to tailor the charge trap behaviour) as the inner layer and an unpolar hydrocarbon moiety (with the purpose to improve the compatibility between the silica and polymeric matrix) as the outer layer. Five different newly designed and modified silicas were successfully prepared, as shown in Figure 3.8. The nanocomposites were produced using the PP/PP-HI polymer matrix. All designed silicas show better dispersion and introduce deeper charge traps than the unmodified silica. The silica with tert-butanol as precursor for the outer layer gives the most promising results with the lowest injected charges under the electrical field of $3 \mathrm{kV} / \mathrm{mm}$. This study shows that the properly designed silicas are able to disperse properly in the polymer matrix, but also facilitate a low level space charge injection into the nanocomposites.

\subsection{Conclusions}

Based on the aim given in the introduction, it can be concluded that:

A "green" method for silica surface modification via a solvent free method was developed.

Silica with an unpolar modification (hydrophobized) shows good dispersion in the polymer matrix, and shows a minor effect on the charge trapping behavior of the nanocomposite. However, it shows a severe negative effect on space charge accumulation. Modified silicas with various functional groups, and especially the ones with a $-\mathrm{NH}_{2}$ group on their surface, show insufficient dispersion, but at the same time a significant reduction of space charge accumulation.

Silica modified with 3-aminopropyltriethoxysilane showed the best performance: low space charge accumulation of its PP/PP-HI based nanocomposites. When further optimizing the silica modification, a newly designed silica with an inner-outer structure gave promising results of simultaneously improving dispersion and reducing charge injection.

In addition, a remark to short-circuit TSDC Measurement:

It should be stressed that the presented TSDC results come from the short circuit TSDC technique. The depolarization current in short circuit TSDC is only the apparent net value. Strictly speaking, it cannot represent all the charges in the samples. For example, during the depolarization process in short circuit TSDC, the charges with one polarity can move towards both electrodes. Hence, the total depolarization current only represents the non-symmetric part of the discharge current. In order to overcome this limitation, an open circuit TSDC can be performed, as it only has only a single ground-electrode metallization [19].

In addition, charge behavior inside the insulation martial is very complex phenomenon, which can be influenced by many factors, the external electric field, temperature, the polymer matrix, the location and the number of the trap site, the trap depth, the electrode. In the presented work, the TSDC has only been done under $3 \mathrm{kV} / \mathrm{mm}$ at $70{ }^{\circ} \mathrm{C}$. In order to fully evaluate the charge dynamics in the nanocomposite, a wide temperature range and various electric fields should be applied to assess the nanocomposites performance for the HVDC cable insulation application. 


\section{References}

[1] Ismail, N.H. and Mustapha, M., 2018. A review of thermoplastic elastomeric nanocomposites for high voltage insulation applications. Polymer Engineering \& Science, 58(S1), pp.E36-E63. DOI: https://doi.org/10.1002/pen.24822

[2] Smith, R.C., Liang, C., Landry, M., Nelson, J.K. and Schadler, L.S., 2008. The mechanisms leading to the useful electrical properties of polymer nanodielectrics. IEEE Transactions on Dielectrics and Electrical Insulation, 15(1), pp.187-196. DOI: 10.1109/T-DEI.2008.4446750.

[3] Mohanty, A. and Srivastava, V.K., 2013. Dielectric breakdown performance of alumina/epoxy resin nanocomposites under high voltage application. Materials \& Design, 47, pp.711-716. DOI: https://doi.org/10.1016/j.matdes.2012.12.052

[4] Mahtabani, A., Rytöluoto, I., Anyszka, R., He, X., Saarimäki, E., Lahti, K., Paajanen, M., Dierkes, W. and Blume, A., 2020. On the Silica Surface Modification and Its Effect on Charge Trapping and Transport in PP-Based Dielectric Nanocomposites. ACS Applied Polymer Materials, 2(8), pp.31483160. DOI: https://doi.org/10.1021/acsapm.0c00349

[5] Stoykov, S., Eggs C., and Kortshagen U., 2001. Plasma chemistry and growth of nanosized particles in a $\mathrm{C} 2 \mathrm{H} 2 \mathrm{RF}$ discharge. Journal of Physics D: Applied Physics, 34(14), pp. 2160 DOI: https://doi.org/10.1088/0022-3727/34/14/312

[6] Benedikt, J., Consoli, , A., Schulze, M. and Von Keudell, A., 2007. Time-resolved molecular beam mass spectrometry of the initial stage of particle formation in an $\mathrm{Ar} / \mathrm{He} / \mathrm{C} 2 \mathrm{H} 2$ plasma. The Journal of Physical Chemistry A, 111(42), pp.10453-10459. DOI: https://doi.org/10.1021/jp072892w

[7] Tiwari, M., 2010. Plasma coating of silica. A key to improved dispersion and properties of reinforced silica blends [PhD thesis]. University of Twente, The Netherlands. ISBN: 978-90-902-5850-8.

[8] Pohl, E.R. and Osterholtz, F.D., 1985. Kinetics and mechanism of aqueous hydrolysis and condensation of alkyltrialkoxysilanes. In Molecular Characterization of Composite Interfaces (pp. 157170). Springer, Berlin, Heidelberg. DOI: 10.1007/978-1-4899-2251-9. ISBN: 978-1-4899-2251-9

[9] Issa, A.A. and Luyt, A.S., 2019. Kinetics of alkoxysilanes and organoalkoxysilanes polymerization: a review. Polymers, 11(3), pp.537. DOI: https://doi.org/10.3390/polym11030537

[10] McIntosh, G.J., 2013. Theoretical investigations into the nucleation of silica growth in basic solution part I-ab Initio studies of the formation of trimers and tetramers. Physical Chemistry Chemical Physics, 15(9), pp.3155-3172. DOI: 10.1039/C3CP43399D

[11] Matinlinna, J.P., Lung, C.Y.K. and Tsoi, J.K.H., 2018. Silane adhesion mechanism in dental applications and surface treatments: A review. Dental Materials, 34(1), pp.13-28. DOI: https://doi.org/10.1016/j.dental.2017.09.002

[12] Cheng, X., Chen, D. and Liu, Y., 2012. Mechanisms of silicon alkoxide hydrolysis-oligomerization reactions: A DFT investigation. ChemPhysChem, 13(9), pp.2392-2404. DOI: https://doi.org/10.1002/cphc.201200115 
[13] Danks, A.E., Hall, S.R. and Schnepp, Z.J.M.H., 2016. The evolution of 'sol-gel' chemistry as a technique for materials synthesis. Materials Horizons, 3(2), pp.91-112. DOI: 10.1039/C5MH00260E

[14] Lv, X.S., Han, B., Wang, J.Y., Chang, J.X., Hua, Y. and Sun, W.F., 2019. Modification mechanism of low-density polyethylene insulation by hydrophilic and hydrophobic porous $\mathrm{SiO} 2$ nanoparticles. Applied Nanoscience, pp.1-11. DOI: https://doi.org/10.1007/s13204-019-01074-6

[15] Siddabattuni, S., Schuman, T. P., and Dogan, F., 2013. Dielectric properties of polymer-particle nanocomposites influenced by electronic nature of filler surfaces. ACS Applied Materials \& Interfaces. 5, pp. 1917-1927. DOI: https://doi.org/10.1021/am3030239

[16] Rytöluoto, I., Saarimäki, E., Pelto, J., Paajanen, M., He, X., Anyszka, R., Mahtabani, A., Dierkes, W., Seri, P., Naderiallaf, H. and Lahti, K., 2020. Feasibility of Mini-Scale Injection Molding for Resource-Efficient Screening of PP-Based Cable Insulation Nanocomposites. In 2020 IEEE 3rd International Conference on Dielectrics (ICD), pp.209-212. DOI: $\underline{10.1109 / I C D 46958.2020 .9341820}$

[17] Zhou, Y., Hu, J., Dang, B. and He, J., 2017. Effect of different nanoparticles on tuning electrical properties of polypropylene nanocomposites. IEEE Transactions on Dielectrics and Electrical Insulation, 24(3), pp.1380-1389. DOI: 10.1109/TDEI.2017.006183

[18] Zhou, Y., He, J., Hu, J. and Dang, B., 2016. Surface-modified MgO nanoparticle enhances the mechanical and direct-current electrical characteristics of polypropylene/polyolefin elastomer nanodielectrics. Journal of Applied Polymer Science, 133(1), pp. 42863. DOI: https://doi.org/10.1002/app. 42863

[19] Remke, R.L. and Von Seggern, H., 1983. Modeling of thermally stimulated currents in polytetrafluoroethylene. Journal of applied physics, 54(9), pp. 5262-5266. DOI: https://doi.org/10.1063/1.332702. 


\section{Chapter 4}

\section{Surface Modification of Fumed Silica by Plasma Polymerization of Acetylene for PP/POE Blends Dielectric Nanocomposites}

This chapter has been published in Polymers.

He, X., Rytöluoto, I., Anyszka, R., Mahtabani, A., Saarimäki, E., Lahti, K., Paajanen, M., Dierkes, W. and Blume, A., 2019. Surface modification of fumed silica by plasma polymerization of acetylene for PP/POE blends dielectric nanocomposites. Polymers, 11(12), pp.1957.

DOI: 10.3390/polym11121957 
Article

\title{
Surface Modification of Fumed Silica by Plasma Polymerization of Acetylene for PP/POE Blends Dielectric Nanocomposites
}

\author{
Xiaozhen $\mathrm{He}^{1, * \odot}$, Ilkka Rytöluoto ${ }^{2}$, Rafal Anyszka ${ }^{1, *} \odot$, Amirhossein Mahtabani ${ }^{1}$, \\ Eetta Saarimäki ${ }^{2}$, Kari Lahti ${ }^{3} \odot$, Mika Paajanen ${ }^{2}$, Wilma Dierkes ${ }^{1, * \odot}$ and Anke Blume ${ }^{1}$ \\ 1 Elastomer Technology and Engineering, Department of Mechanics of Solids, Surfaces \& Systems (MS3), \\ Faculty of Engineering Technology, University of Twente, P.O.box 217, 7500 AE, Enschede, The Netherlands: \\ a.mahtabani@utwente.nl (A.M.); a.blume@utwente.nl (A.B.) \\ 2 VTT Technical Research Centre of Finland Ltd., FI-33101 Tampere, Finland; Ilkka.Rytoluoto@vtt.fi (I.R.); \\ Eetta.Saarimaki@vtt.fi (E.S.); Mika.Paajanen@vtt.fi (M.P.) \\ 3 High Voltage Engineering, Tampere University, FI-33720 Tampere, Finland; kari.lahti@tuni.fi \\ - Correspondence: x.he@utwente.nl (X.H.); r.p.anyszka@utwente.nl (R.A.); w.k.dierkes@utwente.nl (W.D.)
}

Received: 13 September 2019; Accepted: 26 November 2019; Published: 28 November 2019

\begin{abstract}
Novel nanocomposites for dielectric applications-based polypropylene/poly(ethylene-co-octene) (PP/POE) blends filled with nano silica are developed in the framework of the European 'GRIDABLE' project. A tailor-made low-pressure-plasma reactor was applied in this study for an organic surface modification of silica. Acetylene gas was used as the monomer for plasma polymerization in order to deposit a hydrocarbon layer onto the silica surface. The aim of this modification is to increase the compatibility between silica and the PP/POE blends matrix in order to improve the dispersion of the filler in the polymer matrix and to suppress the space charge accumulation by altering the charge trapping properties of these silica/PP/POE blends composites. The conditions for the deposition of the acetylene plasma-polymer onto the silica surface were optimized by analyzing the modification in terms of weight loss by thermogravimetry (TGA). X-ray photoelectron spectroscopy (XPS) and energy-dispersive $\mathrm{X}$-ray fluorescence spectroscopy (EDX) measurements confirmed the presence of hydrocarbon compounds on the silica surface after plasma modification. The acetylene plasma modified silica with the highest deposition level was selected to be incorporated into the PP/POE blends matrix. X-ray diffraction (XRD) showed that there is no new crystal phase formation in the $\mathrm{PP} / \mathrm{POE}$ blends nanocomposites after addition of the acetylene plasma modified silica. Differential scanning calorimetry results (DSC) show two melting peaks and two crystallization peaks of the $\mathrm{PP} / \mathrm{POE}$ blends nanocomposites corresponding to the PP and POE domains. The improved dispersion of the silica after acetylene plasma modification in the PP/POE blends matrix was shown by means of SEM-EDX mapping. Thermally stimulated depolarization current (TSDC) measurements confirm that addition of the acetylene plasma modified silica affects the charge trapping density and decreases the amount of injected charges into PP/POE blends nanocomposites. This work shows that acetylene plasma modification of the silica surface is a promising route to tune charge trapping properties of $\mathrm{PP} / \mathrm{POE}$ blend-based nanocomposites.
\end{abstract}

Keywords: acetylene plasma modification; nano silica; dispersion; PP; POE; dielectric nanocomposites; TSDC

Note: In this chapter, POE is used as abbreviation for ethylene-octene copolymer. 


\subsection{Introduction}

Space charge accumulation is a major problem for insulation materials, particularly for high voltage direct current (HVDC) applications. Space charge can accumulate in specific regions in the bulk of the material resulting from the imbalance between the generation of charge carriers and their transit through the insulation material [1]. Under high DC voltages, the space charge accumulation can be linked to the propagation of electrical trees due to local electric field enhancement. Over time, this may finally cause an electric breakdown $[2,3]$. Therefore, suppressing space charge accumulation has become a key issue in HVDC cable insulation development [4].

Considering the concept of environmental protection and sustainable development, polypropylene as a recyclable polymer has shown great potential for new polymeric insulation materials. Due to the poor flexibility of polypropylene its blends with polyolefin elastomers (such as poly(ethylene-co-octene), POE, has drawn a lot of attention, especially for developing the next-generation thermoplastic insulation in HVDC cables. Although PP/POE blends exhibit improved flexibility in comparison to PP [5, 6] they are still facing the same problems of space charge accumulation [6]. The higher level of space charge accumulation contributes to the deformation of the electrical field. Based on one study the dependence is linear [7]. Therefore, it is important to suppress space charge accumulation.

There are many different methods to suppress space charge accumulation. It is well known that the cleaning process before compound mixing is crucial for lowering the amount of contaminations and residues which can cause increased internal charges. Moreover, designing a semi-conductive layer between the conductor and the main insulation layer allows to control the charge injection into the bulk of the insulating layer [1]. It is also reported that modification of the insulating polymer by tailoring its molecular structure can also suppress the space charge accumulation. For example, grafting maleic anhydride (MAH) with its polar carbonyl groups onto PP resulted in effective suppression of the space charge injection and accumulation [8]. Another approach is to incorporate nanoparticles into the polymer matrix [9-11]. Improved insulating properties (e.g., dielectric strength and voltage endurance) of these nanoparticle-filled polymers were reported $[10,12]$. The high- performance dielectric properties of polymer nanocomposites (PNCs) are explained by a large and evenly distributed polymer/filler interface area in well-dispersed nanoparticle-polymer systems. The large interfacial area may bring about changes in charge mobility and trapping properties, hence modifying the space charge accumulation of the bulk of a PNC insulation $[13,14]$.

However, the nanoparticles tend to cluster together due to their high polarity and tendency to form hydrogen bonds. A low degree of dispersion will decrease the performance of nano-dielectric composites. To overcome this problem, surface modification of the nanoparticles is crucial in order to increase the compatibility of the filler with the polymer matrix and to prevent filler aggregation and agglomeration [15]. The most widely used method of surface modification of nanoparticles is a suspension technique: The reaction takes place between the low molecular weight modifier in the solution and the active moieties on the surface of the suspended nanoparticles in various temperature ranges. However, this method is environmentally questionable as it is solvent-based. Alternatively, a plasma technique can be utilized for surface modification of nanoparticles. This is an environmentally friendly, physicochemical process of changing the surface characteristics by a one-step technology and it virtually generates no waste [16]. Plasma modification has attracted considerable interest as a very "smart" and flexible way to treat materials which changes only the surface properties of the substrate 
without compromising its bulk properties [17,18]. Generally, plasma treatment creates a very thin and uniform deposit, and thus allows chemical modification of many different surfaces such as metals [19], polymers [18], textiles [20], composites, ceramics [21], or powder nano-fillers [22].

In this study, a commercial, low moisture content fumed silica was selected to be incorporated into $\mathrm{PP} / \mathrm{POE}$ blends in order to enhance their electric properties. A custom-designed vertical plasma reactor was used to perform the low-pressure plasma modification of this silica. Acetylene was used as monomer to deposit a hydrocarbon layer on the silica surface in order to improve its compatibility with the PP/POE blends and therefore the dispersion of the silica in the polymer matrix. The goal of this modification is to suppress space charge accumulation of this composite via changing the charge trapping properties of silica/ $\mathrm{PP} / \mathrm{POE}$ blends nanocomposites.

\subsection{Materials and Methods}

\subsubsection{Materials}

Commercial low-moisture-content fumed silica was supplied by Evonik Industries AG, (Essen, Germany). Acetylene was purchased from Linde Gas Benelux BV (Schiedam, The Netherlands). A blend of two different types of polyolefin-based polymers, polypropylene (PP), and poly(ethylene-cooctene) (POE), was used as the polymer matrix. Common antioxidants were added to the compounds to protect them from thermo-oxidative degradation during melt-processing.

\subsubsection{Plasma Modification of Silica}

The custom-designed radio frequency $(13.56 \mathrm{MHz})$ vertical plasma reactor is shown in Figure 4.1. A schematic diagram is given in the left part of Figure 4.1 (a), and the actual reactor is shown in Figure 4.1 (b). This plasma reactor setup consists of four parts:

- Impedance matching unit;

- radio frequency $(\mathrm{RF})$ generator;

- magnetic stirrer;

- vertical glass reactor.

$2 \mathrm{~g}$ of the fumed silica were placed on the bottom of the vertical reactor, together with the magnetic stirrer bar to ensure homogenous modification. A vacuum of $0.33-0.34$ mbar was applied, necessary for the plasma to ignite, and the acetylene monomer was introduced during a reaction time of $1 \mathrm{~h}$ to enable the plasma polymerization. By analysing the results of thermogravimetric tests (TGA), the weight loss of the plasma modified samples is presented as a function of:

- Plasma power (100-300 Watt (W));

- gas flow rate $\left(3-18 \mathrm{~cm}^{3} / \mathrm{min}\right)$. 
The conditions for the acetylene plasma polymerization and deposition were optimized. After shutdown of the plasma polymerization acetylene was introduced into the reactor for another $5 \mathrm{~min}$ to avoid oxidation while taking out the modified sample.

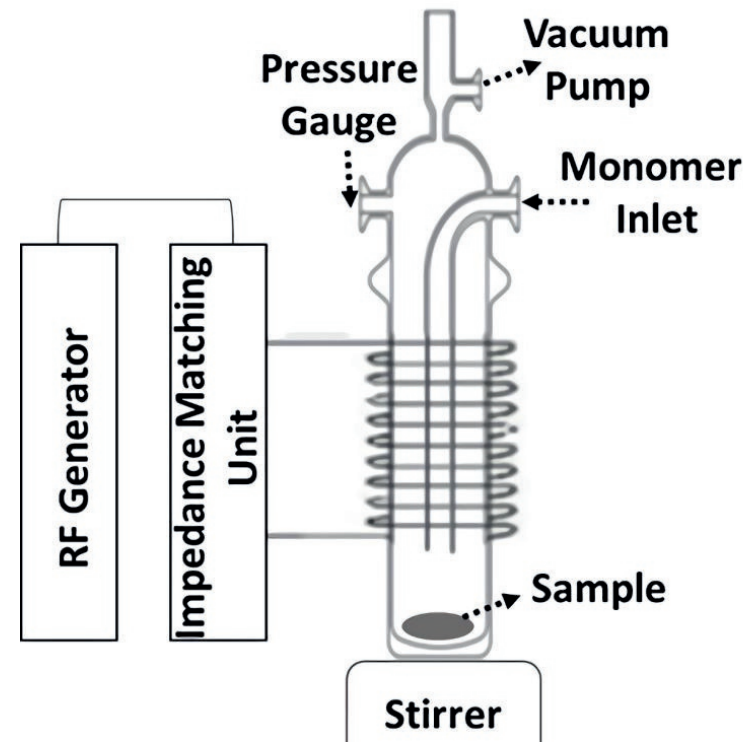

(a)

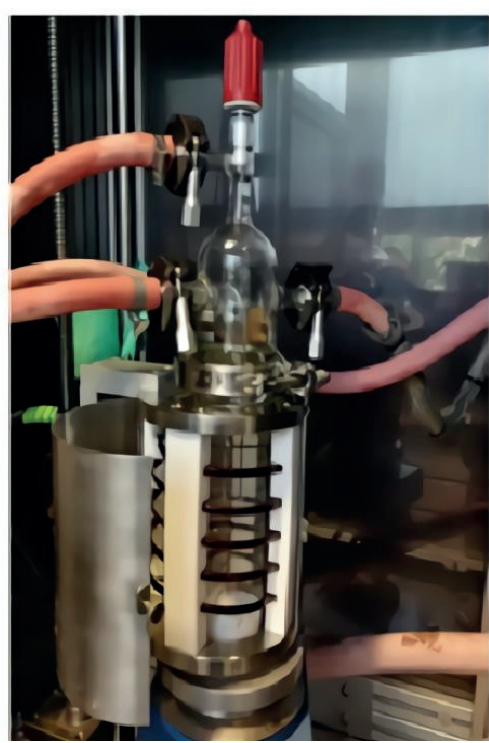

(b)

Figure 4.1 Schematic diagram of the plasma setup (a) and the actual reactor (b).

To evaluate the Acetylene Plasma Polymerization Silica Coating, thermogravimetric analysis (TGA) was performed using a Perkin-Elmer TGA-7 thermogravimetric analyzer (Waltham, Massachusetts, United States). This analysis was performed for the reference silica sample, as well as the plasmamodified silica samples. This characterization was done in a synthetic air atmosphere with a heating rate of $20^{\circ} \mathrm{C} / \mathrm{min}$ and a temperature range from ambient temperature to $850{ }^{\circ} \mathrm{C}$.

X-ray photoelectron spectroscopy (XPS) was conducted by means of a PHI Quantera scanning X-ray microscopy and X-ray photoelectron spectroscopy from Physical Electronics GmbH (in Munchen, Germany), It is based on irradiating a material with a beam of X-rays, while simultaneously measuring the kinetic energy and number of electrons that escape from the surface (up to $10 \mathrm{~nm}$ in depth) of the material being analyzed. In this way it is possible to measure the elemental composition in a parts per thousand (ppt) range.

The scanning electron microscopy (SEM) and energy-dispersive X-ray spectroscopy (EDX) were done by means of Zeiss MERLIN HR-SEM microscope (Oberkochen, Germany). 


\subsubsection{PP/POE/Silica Composite Preparation}

The different nanocomposite samples were prepared by melt-blending of the reference (unmodified) silica or plasma-modified silicas with the PP/POE polymer blend (PP/POE ratio of 55:45). The material formulations are shown in Table 4.1. Small batches of $12 \mathrm{~g}$ were compounded using a Haake MiniLab Rheomex CTW5 mini twin conical screw extruder (Thermo Fisher Scientific, Waltham, Massachusetts, USA) using a compounding temperature of $230{ }^{\circ} \mathrm{C}$, a screw speed of $100 \mathrm{rpm}$ and a mixing time of 4 min. After melt-blending the compounds were immediately transferred to a Hake MiniJet Pro Piston Injection Moulding System (Thermo Fisher Scientific, Waltham, Massachusetts, USA) and injectionmolded into thin sheets (size of $26 \times 26 \times 0.5 \mathrm{~mm}$ ). The injection mould temperature was $60^{\circ} \mathrm{C}$, injection temperature was $230{ }^{\circ} \mathrm{C}$, mean injection and holding pressure was $960 \mathrm{bar}$, and the total injection time (injection and holding) was $40 \mathrm{~s}$.

Table 4.1. Material formulations.

\begin{tabular}{cccc}
\hline & $\begin{array}{c}\text { PP/POE } \\
\text { Wt. } \%\end{array}$ & $\begin{array}{c}\text { PP/POE/reference silica } \\
\text { Wt. } \%\end{array}$ & $\begin{array}{c}\text { PP/POE/plasma silica } \\
\text { Wt. } \%\end{array}$ \\
\hline PP/POE blend (55:45) & 99.7 & 98.7 & 98.7 \\
\hline Antioxidants & 0.3 & 0.3 & 0.3 \\
\hline Reference Silica & - & 1 & - \\
\hline Plasma modified silica & - & - & 1 \\
\hline
\end{tabular}

\subsubsection{Characterization of PP/POE/Silica Composites}

Differential scanning calorimetry (DSC) was performed using a DSC Q2000 from TA Instruments (TA Instruments, New Castle, Delaware, USA). Disc-shaped samples weighing 12-14 mg were cut from the injection-molded thin sheets, placed in aluminum pans, and inserted into the DSC cell. The samples were first heated from ambient temperature to $230{ }^{\circ} \mathrm{C}$ at a rate of $10{ }^{\circ} \mathrm{C} / \mathrm{min}$ and maintained at this temperature for $5 \mathrm{~min}$ to erase any previous thermal history. The samples were then cooled down to -20 ${ }^{\circ} \mathrm{C}\left(40{ }^{\circ} \mathrm{C} / \mathrm{min}\right.$.) and heated again to $230{ }^{\circ} \mathrm{C}$ at a rate of $10^{\circ} \mathrm{C} / \mathrm{min}$.

X-ray diffraction (XRD) spectra were collected from the injection molded samples by means of a Philips X'Pert 1 X-ray diffractometer (Almelo, The Netherlands). The samples were scanned with $2 \theta$ values varying from 8 to $37^{\circ}$ with a scanning rate of $0.05 \% / 8 \mathrm{~s}$. Three samples were measured from each material.

SEM-EDX of the PO based composites was conducted by the Zeiss Merlin HR-SEM microscope (Oberkochen, Germany). The sample was first cut into a smaller piece and embedded in epoxy resin. After solidification of the resin, the sample was polished to expose the cross-sectional surface of the embedded sample, followed by sputter-coating of the polished surface with Au. The scanning electron microscopy (SEM) was done by Zeiss Merlin HR-SEM. The sample was prepared in liquid nitrogen without coating.

\subsubsection{Thermally Stimulated Depolarization Current (TSDC)}


The charge trapping properties of the composites were studied by the thermally stimulated depolarization current (TSDC) technique. Circular Au electrodes (diameter $16 \mathrm{~mm}$, thickness $100 \mathrm{~nm}$ ) were deposited on both sides of the sample sheets by electron-beam evaporation under high vacuum $(<$ $1 \times 10^{-6}$ mbar). The TSDC measurement system consisted of a liquid $\mathrm{N}_{2}$-based temperature control system (Novocool by Novocontrol Technologies, Montabaur, Germany), a high voltage DC power source (Keithley 2290E-5), and a sensitive electrometer (Keithley 6517B by Keithley Instruments, Cleveland, Ohio, USA). The measurements were performed using a shielded sample cell equipped with a PT100 temperature sensor (Novocontrol BDS1200HV by Novocontrol Technologies, Montabaur, Germany). The current measurement sensitivity of the shielded measurement system was better than 1 pA. During high voltage application, a series resistor $(100 \mathrm{k} \Omega)$ and a diode-based overload protection circuit were utilized to protect the electrometer in case of sample breakdown. The TSDC measurement procedure consisted of the following steps:

1. The sample was heated from room temperature to $70{ }^{\circ} \mathrm{C}$ and stabilized for $5 \mathrm{~min}$.

2. A DC poling field of $3 \mathrm{kV} / \mathrm{mm}$ was applied for $20 \mathrm{~min}$ under isothermal conditions at $70^{\circ} \mathrm{C}$.

3. The sample was rapidly cooled down to $-50{ }^{\circ} \mathrm{C}$ with the voltage still applied, and kept at this temperature for $5 \mathrm{~min}$ for stabilization.

4. The poling field was removed and the sample was short-circuited. The short-circuited sample was maintained at $-50{ }^{\circ} \mathrm{C}$ for $3 \mathrm{~min}$ to allow fast polarization to decay.

5. The sample was linearly heated up to $130{ }^{\circ} \mathrm{C}$ with a heating rate of $3{ }^{\circ} \mathrm{C} /$ min while measuring the thermally stimulated depolarization current.

In the above procedure, the electrometer was used to measure the current through the sample also during the isothermal polarization and cooling phases before the measurement of TSDC. Although the polarization time was too short to reach a steady-state DC conduction current [23], the isothermal charging current data nevertheless provided an indication of the transient electrical conductivity behaviour of the sample. Further details on the isothermal polarization/thermally stimulated depolarization current measurement are presented in Supplementary Material S1.

\subsubsection{Dielectric Spectroscopy}

Complex permittivity $\varepsilon_{\mathrm{r}}{ }^{*}$ of the injection molded composite samples was measured in the frequency range of $0.01 \mathrm{~Hz}$ to $1 \mathrm{MHz}$ using Novocontrol Alpha-A dielectric analyzer (Novocontrol Technologies, Montabaur, Germany) with a ZG4 test interface (two-wire mode). For the sample capacitance range considered in this study, the absolute loss factor ( $\tan \delta$ ) measurement accuracy was approximately \pm $10^{-4}$ (absolute phase accuracy of $6 \mathrm{~m}^{\circ}$ ). The measurements were performed at room temperature using an AC measurement voltage of 1 Vrms. The samples were the same as those used for TSDC; the permittivity measurements were performed right before and after the TSDC measurement from the same samples. 


\subsection{Results and Discussion}

\subsubsection{Characterization of the Plasma Modified Silica}

\subsubsection{TGA}

\section{Optimization of the Plasma RF Power via TGA Weight Loss Measurements}

The silica samples were treated at the same gas flow rate $\left(6 \mathrm{~cm}^{3} / \mathrm{min}\right)$ with different RF power settings for a duration of $1 \mathrm{~h}$. The TGA results indicated a two-step thermal degradation kinetics of the deposited organic coating. The first step starts around $300{ }^{\circ} \mathrm{C}$ and the second around $450{ }^{\circ} \mathrm{C}$. Further mass loss (above $600{ }^{\circ} \mathrm{C}$ ) can be explained by the remaining silanol group condensation similar to the reference silica sample. The two-step decomposition kinetics indicate the presence of various hydrocarbon species on the silica surface [24, 25]: Linear and branched polyacetylene chains, that degrade at lower temperatures, and highly cross-linked or possibly even carbonized structures [26] decomposing at higher temperatures. A schematic diagram of the plasma modification mechanism is shown in Figure 4.2. The cross-linked high molecular weight network is caused by small fragments, radicals, and atom formed in the plasma. These species recombine randomly together to form new irregular structures on silica surface.

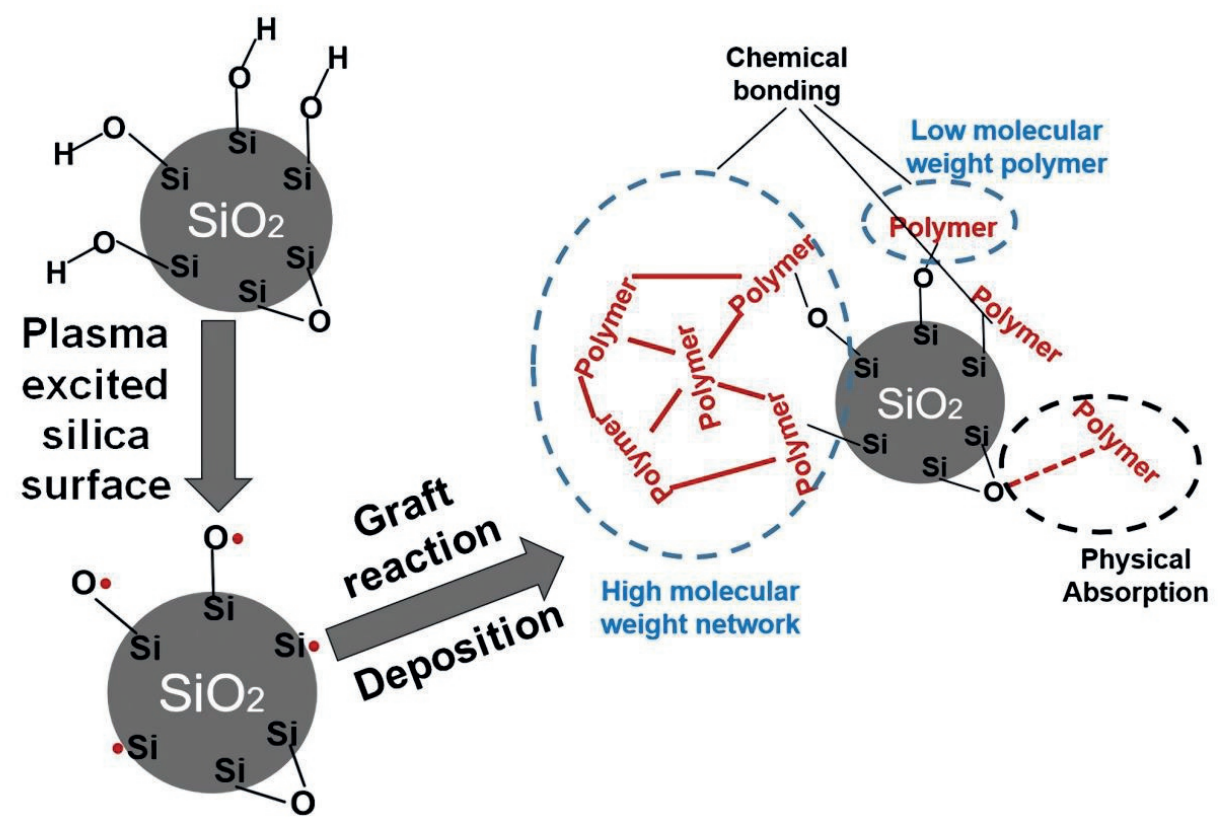

Figure 4.2 Schematic diagram of the mechanism of plasma modification of silica. 
Figure 4.3 shows the TGA curves of the reference sample and the plasma surface-modified samples: The weight loss percentage varies with the plasma RF power applied. Figure 4.4 shows a plot of the weight loss of the plasma modified silica samples in correlation to the RF power showing a non-linear relation between the applied RF power at $6 \mathrm{~cm}^{3} / \mathrm{min}$ flow rate and the maximum weight loss. The weight loss increases with increasing RF power up to a maximum at $150 \mathrm{~W}$ RF power; further increase of the plasma RF power results in a decrease of the weight loss. This non-linear correlation can be explained by an increasing electron concentration in the plasma with increasing RF power. As a consequence, the excitation and ionization reaction rates also increase which gives a higher polymerization efficiency. However, at higher levels of RF power the number of low-energy electrons becomes higher due to more frequent electron-electron and electron-neutral collisions. These collisions also lead to decomposition of larger fragments and formation of smaller and more stable fragments which suppress the degree of polymerization in the gas phase [27].

Based on these results, the RF power $150 \mathrm{~W}$ which gives the highest deposition level was used for the following steps.

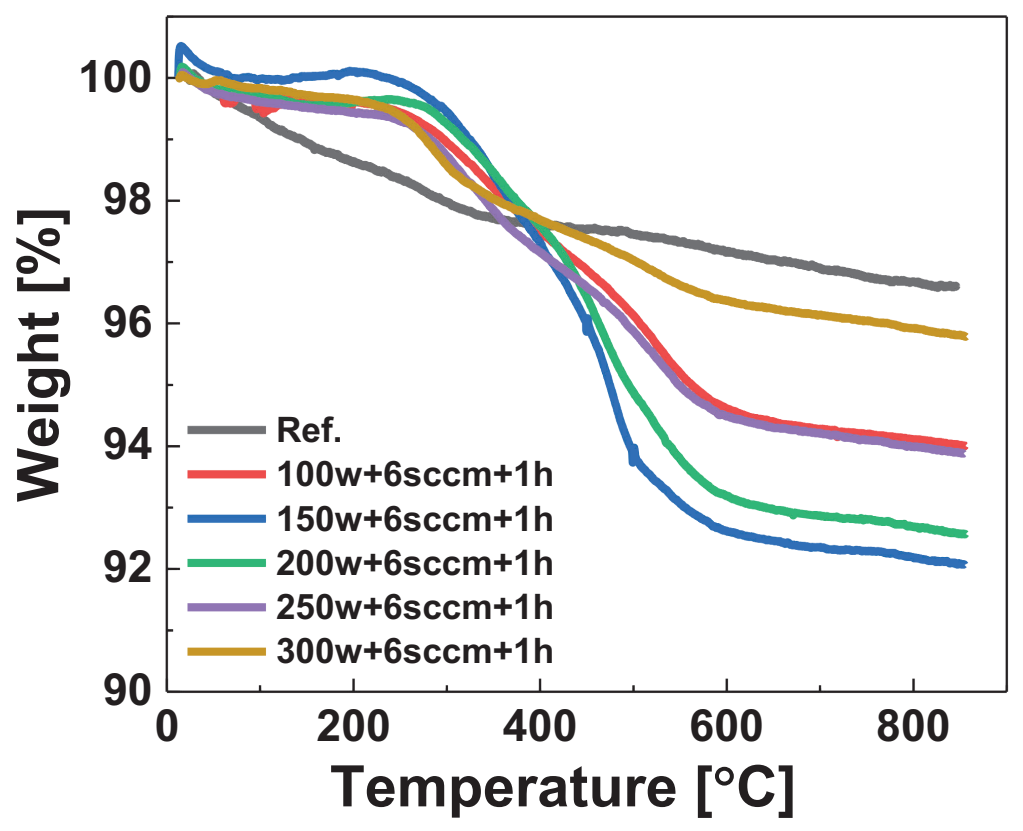

Figure 4.3 TGA results of the modified silica samples treated at different RF powers.

Figure 4.5 shows the TGA curves of the reference sample and the samples modified with acetylene plasma at various gas flow rates. The modified samples were treated at the same optimized RF power $(150 \mathrm{~W})$ with different gas flow rates and for a duration of $1 \mathrm{~h}$. The TGA results shown in Figure 4.5 depict an even more complex thermal decomposition behaviour of the modified samples than presented in Figure 4.3. Figure 4.6 shows the plot fitted to the weight loss percentage of plasma modified silica 
samples versus the gas flow rate. It shows a non-linear relation with the applied flow rates at $150 \mathrm{WRF}$ power, similar to the results presented in Figure 4.4 for different RF power values. The weight loss

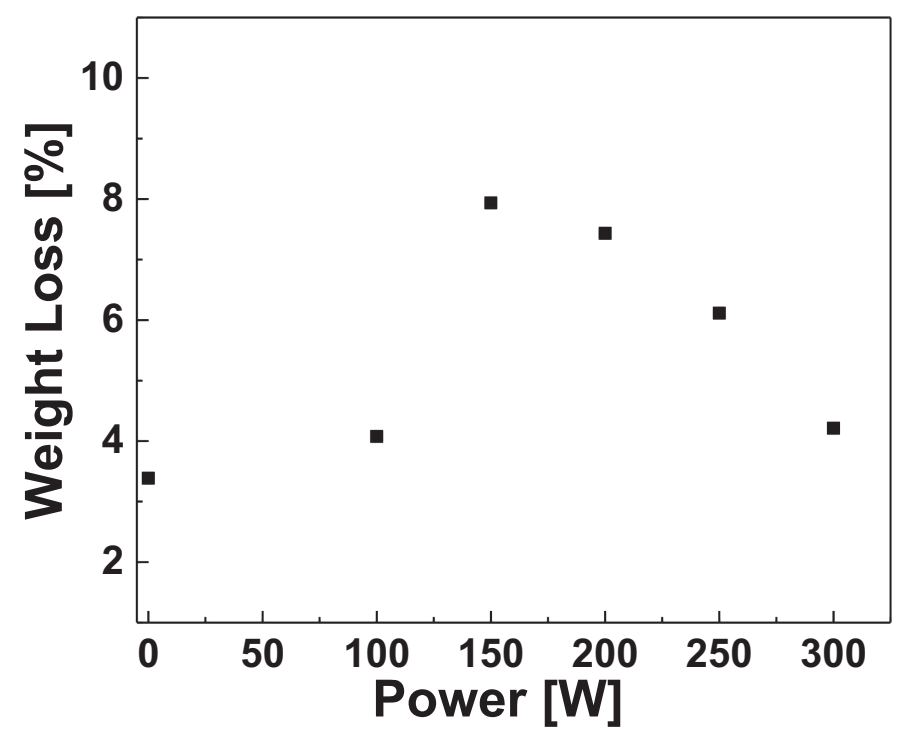

Figure 4.4 Amount of TGA weight loss correlated to radio frequency (RF) power.

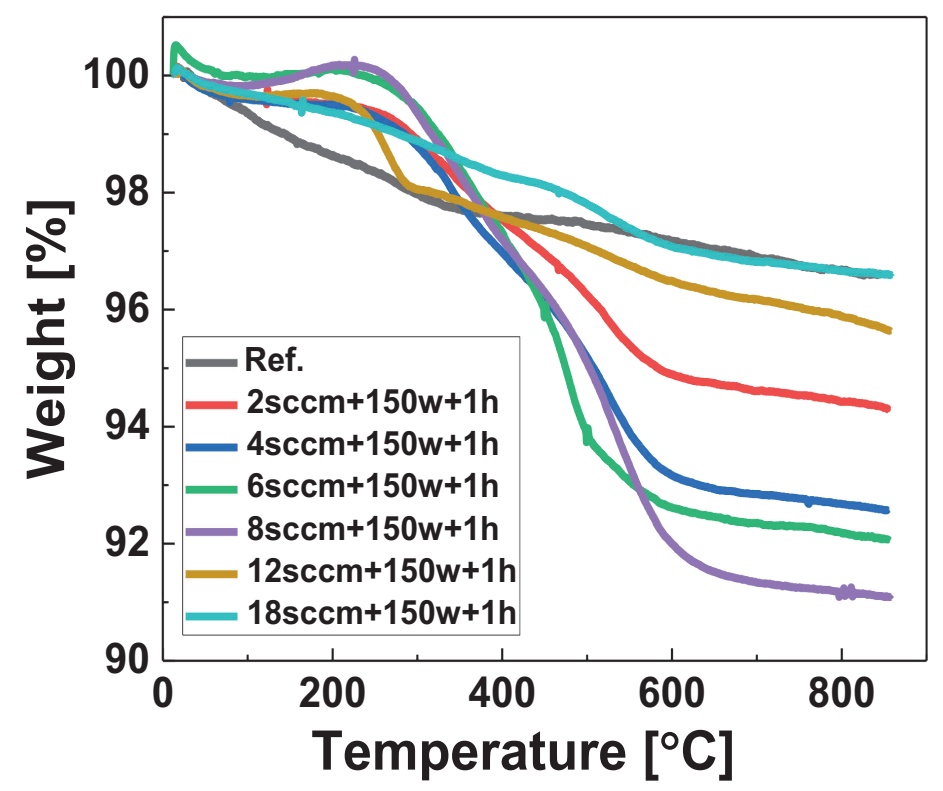

Figure 4.5 TGA results of the samples treated at different flow rates. 
increases firstly, reaches a maximum value at around $8 \mathrm{~cm}^{3} / \mathrm{min}$ and then decreases. This phenomenon can be explained by the mean free path theory equation (4.1) [28]:

$$
\lambda \approx \frac{1}{n \sigma_{c}(v)}
$$

where $\lambda$ is the mean free path (the distance traversed by an electron between two collisions), $n$ is the particles (atoms, electrons, ions, neutrals) density, and $\sigma_{c}(v)$ is the particles (atoms, electrons, ions, neutrals) average cross section (the subscript $c$ denotes collision).

\section{Optimization of the Gas Flow Rate via TGA Weight Loss Measurements}

The flow rates vary the amount of gas introduced into the plasma system per minute. The higher the applied flow rate, the higher the ionized gas particle density in the plasma system. As a consequence, length of the free path in the plasma system becomes shorter (based on the above Equation 1), likewise, when a lower flow rate is applied the mean free path is longer due to the lower particle density and the collision possibility between the partials (electrons, ions, neutrals) is very low. This leads to a lower ionization rate and a relatively low plasma polymerization rate. With increasing flow rate, the mean free path is decreasing, so the collision possibility is increasing. Therefore, the plasma polymerization rate is increasing. However, the increasingly more frequent collisions resulting from higher gas flow rates will lead to more energy dissipation of the plasma electrons or ionized particles, resulting in a decrease of gas particles ionization reaction.

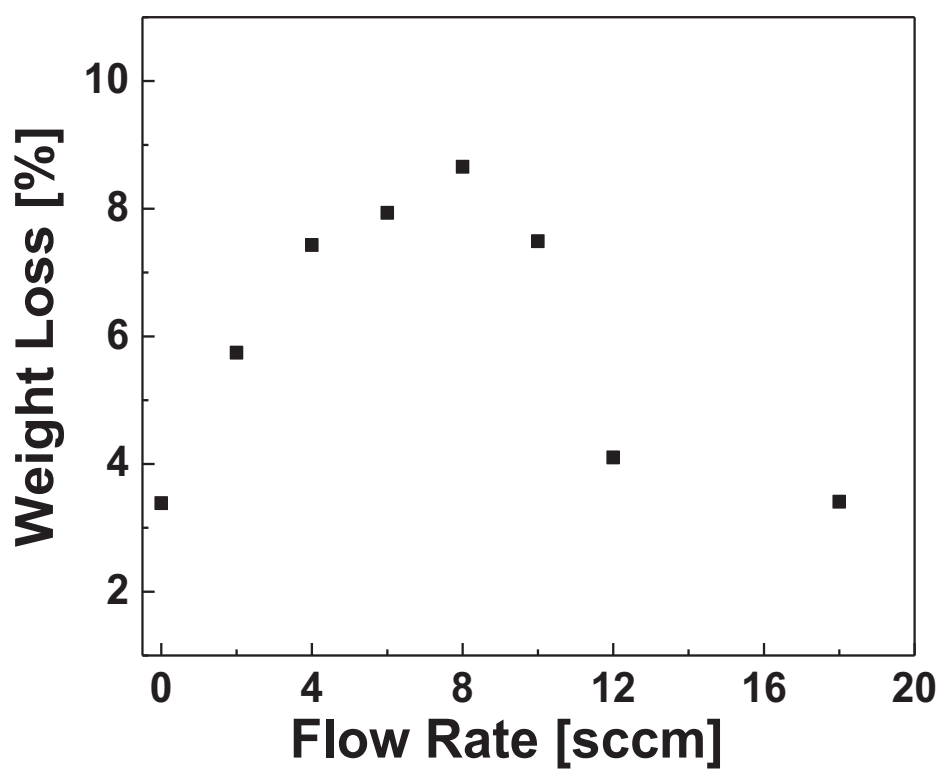

Figure 4.6 TGA weight loss compared to gas flow rates. 


\subsubsection{Analysis of the Polymer Deposit on the Silica Surface by XPS}

Figure 4.7 shows the XPS spectra of the reference silica and plasma modified silica. The strong $\mathrm{O} 1 \mathrm{~s}$ peak $(533.2 \mathrm{eV})$ and the $\mathrm{Si} 2 \mathrm{p}$ peak $(103.5 \mathrm{eV})$ are characteristic for silica. The energy range between

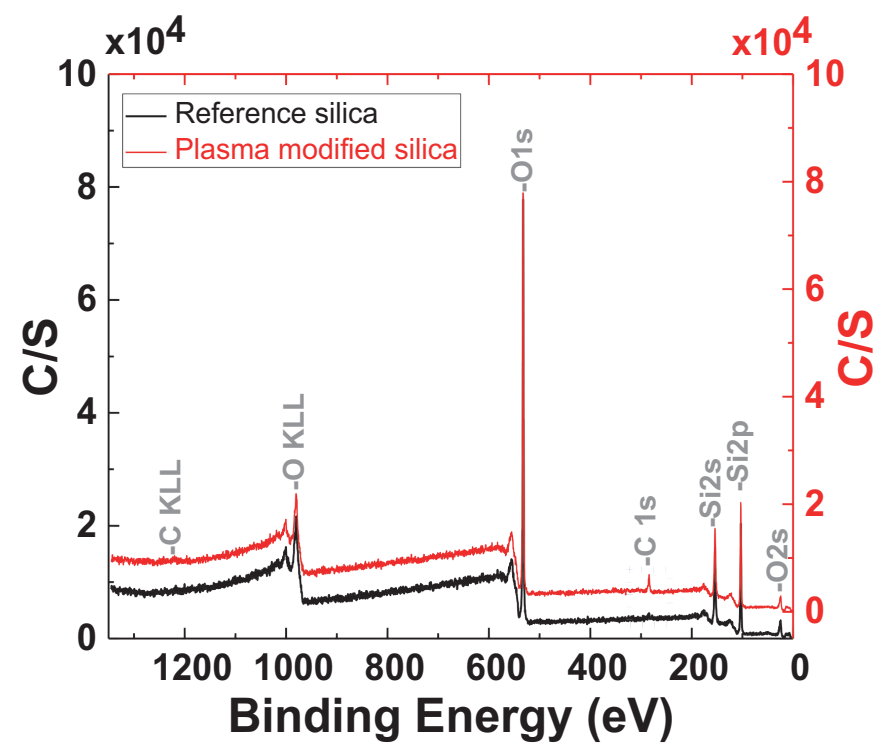

Figure 4.7 X-ray photoelectron spectroscopy (XPS) spectra of reference silica and plasmamodified silica (plasma settings: $8 \mathrm{sccm}, 150 \mathrm{~W}, 1 \mathrm{~h}$ ).

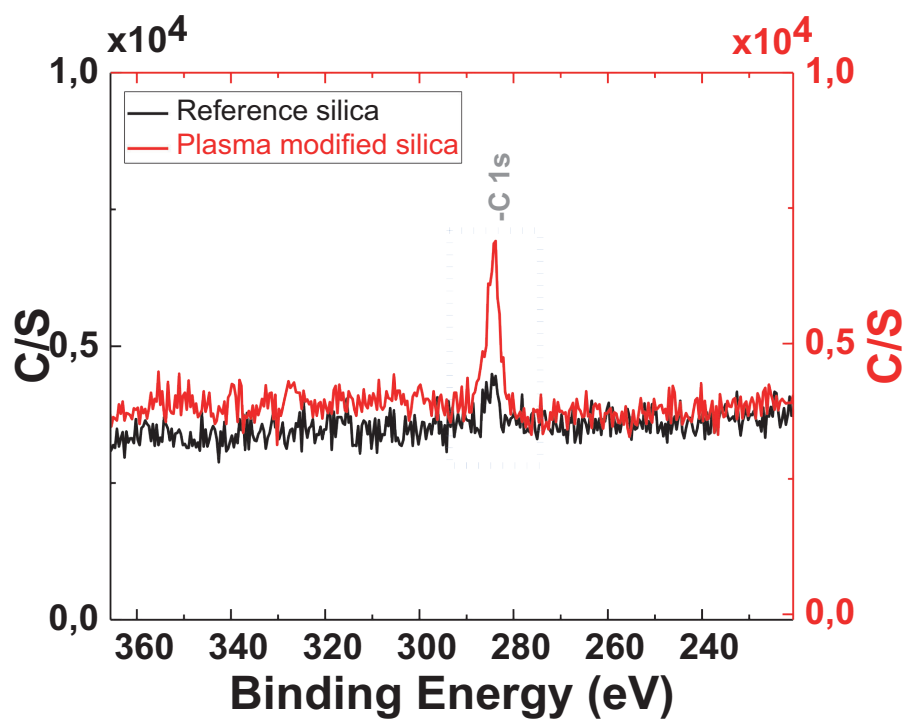

Figure 4.8 Spectra of reference silica and plasma-modified silica (plasma settings: $8 \mathrm{sccm}$, $150 \mathrm{~W}, 1 \mathrm{~h})$. 
25 and $104 \mathrm{eV}$ with one $\mathrm{Si}$ and several O peaks, can be considered a "fingerprint" of these materials. The reference silica also produces signals of C $1 \mathrm{~s}(283.8 \mathrm{eV})$ due to the remaining traces of hydrocarbon contaminants introduced during the XPS sample preparation.

From Figure 4.8, one can observe that the spectrum of the plasma modified silica shows a significant increase of the $\mathrm{C} 1 \mathrm{~s}$ signal, which indicates that organic groups are present on the plasma modified silica surface due to the polyacetylene deposition via plasma polymerization.

XPS analysis for the elemental surface analysis was performed on seven samples. Table 4.2 shows the XPS results of the reference sample and the samples treated at three different RF power settings. Table 4.3 shows the XPS results of the reference sample and the samples treated at three different gas flow rates. The results of these measurements are corresponding with the TGA results: The samples with the highest weight loss in the TGA analysis exhibit the highest content of elemental carbon. This proves that the chemical deposition on the silica surface is originating from the acetylene plasma polymerization.

Table 4.2 XPS results of the reference sample and the samples with different RF power settings.

\begin{tabular}{|c|c|c|c|}
\hline Sample & $\begin{array}{c}\mathrm{C} \\
{[\%]}\end{array}$ & $\begin{array}{c}\mathbf{O} \\
{[\%]}\end{array}$ & $\begin{array}{c}\mathbf{S i} \\
{[\%]}\end{array}$ \\
\hline Reference & 2.29 & 67.27 & 30.44 \\
\hline $150 \mathrm{~W}$ & 4.26 & 66.54 & 29.20 \\
\hline $200 \mathrm{~W}$ & 2.97 & 67.47 & 29.59 \\
\hline $300 \mathrm{~W}$ & 2.12 & 68.29 & 29.59 \\
\hline
\end{tabular}

Table 4.3 XPS results of the reference sample and the samples with different flow rate settings.

\begin{tabular}{cc|ccc}
\hline \multirow{2}{*}{ Sample } & Element & $\begin{array}{c}\text { C } \\
{[\%]}\end{array}$ & $\begin{array}{c}\text { O } \\
{[\%]}\end{array}$ & $\begin{array}{c}\text { Si } \\
{[\%]}\end{array}$ \\
\cline { 1 - 2 } & & 2.29 & 67.27 & 30.44 \\
& Reference & 4.44 & 66.57 & 29.05 \\
$4 \mathrm{~cm}^{3} / \mathrm{min}$ & 7.92 & 64.13 & 27.95 \\
$8 \mathrm{~cm}^{3} / \mathrm{min}$ & 2.70 & 67.45 & 29.84 \\
\hline
\end{tabular}

\subsubsection{Analysis of the Polymer Deposit on the Silica Surface by STEM-EDX}

To confirm the XPS results and to study the morphology of the silica, the reference sample and selected plasma modified samples with high degrees of modification were characterized by scanning electron microscopy (SEM) and at the same time to acquire the spectra of energy-dispersive X-ray fluorescence spectroscopy (EDX). Figure 4.9 shows the EDX spectrum with the same elements as identified in the 
XPS spectrum in Figure 4.7. The EDX spectra confirm the absence of carbon on the reference silica and the presence of carbon on the plasma modified silica due to the presence of the acetylene plasma polymer on the surface of silica.

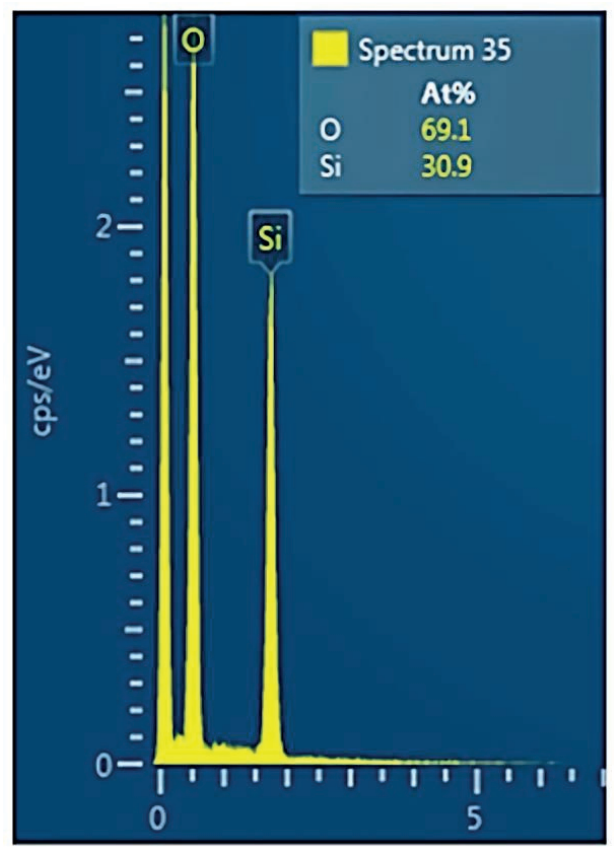

(a)

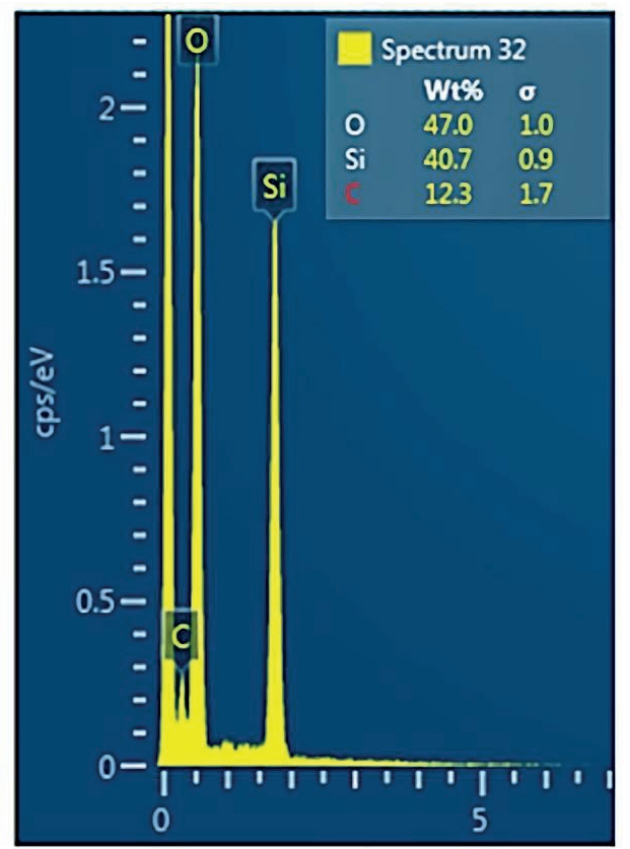

(b)

Figure 4.9 EDX spectrum of the reference silica (a) and plasma modified silica (b).

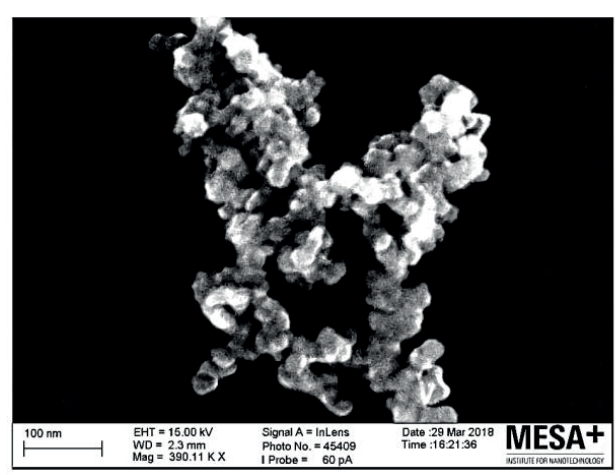

(a)

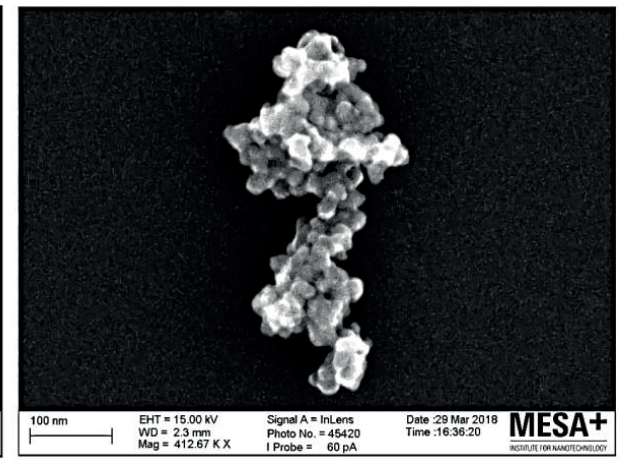

(b)

Figure 4.10 SEM image of the reference silica (a) and plasma modified silica (b). 
Figure 4.10 shows the morphology and microstructure of the reference silica and the plasma modified silicas. The typical branched structure of the reference silica aggregate is obvious [29]. Plasma modified silica shows a less branched structure and smaller dimensions. This change in structure can be explained as follows:

1. The electron or ionized particles in the plasma hit the silica and break weaker bonds in the silica aggregates during the plasma modification, which resulted in smaller dimensions of the plasma modified silica units.

2. The hydrocarbon layer deposited on the silica surface after modification prevents re-aggregation of the plasma modified silica.

In addition, this aggregate of silica is only one example, but it is a general picture which shows that the appearance of the aggregates is different: The modified silica shows the same degree of brightness over the whole surface of the aggregate. The reference silica exhibits a very bright area in the upper part (closer to the electron source) and is rather dark in the lower part. It is again a strong suggestion that there is indeed polyacetylene grafted on the plasma modified silica surface. The reference silica does not conduct electrons over its surface, so they accumulate on the upper part where the radiation is stronger, giving bright spots, whereas the lower part remains darker. When a conductive polyacetylene layer is deposited on the silica surface, the electrons are distributed evenly over the whole aggregate surface which may indicate some degree of conductivity originating from conjugated double bonds. It should be noted that the plasma polymerization of acetylene will not result in a very regular structure as described in [30] but rather in distributions of $\mathrm{sp}^{2}$ and $\mathrm{sp}^{3}$ bonds [31]. In the extreme case nano diamonds can be formed with plasma vapour deposition of $\mathrm{CH}_{4} / \mathrm{H}_{2} / \mathrm{N}_{2}$ [32]. Assuming a maximum of $10 \%$ polymer content of modified silica of $30 \mathrm{~nm}$ diameter the thickness of the polymer layer may be around $0.5 \mathrm{~nm}$.

\subsubsection{Characterization of Silica Filled PP/POE Blends Nanocomposites}

The plasma modified silica with the highest yield of polyacetylene surface deposition (P-silica) was incorporated into the $\mathrm{PP} / \mathrm{POE}$ blends matrix in order to compare its properties with the neat $\mathrm{PP} / \mathrm{POE}$ blends and the reference silica (R-silica)/ PP/POE blends nanocomposite.

\subsubsection{XRD Crystalline Structure Analysis}

Morphological variations arising from differences in the crystalline phase structure, crystallite size, and overall degree of crystallinity are expected to influence the dielectric properties of polymers. Thus, XRD analysis was performed to study the crystalline structure in detail. Representative XRD diffraction spectra of the injection moulded PP/POE-silica nanocomposites are shown in Figure 4.11 along with the unfilled PP/POE blend. Moreover, XRD spectra of the individual PP and POE components of the matrix polymer blend are also shown for reference (hot-pressed samples). The diffraction peaks at $2 \theta$ angles of $14.1,16.8,18.5$, and $25.4^{\circ}$ are characteristic of the thermodynamically stable $\alpha$-form PP and respectively correspond to (110), (040), (130), and (060) crystallographic planes. A small (300) diffraction peak at 


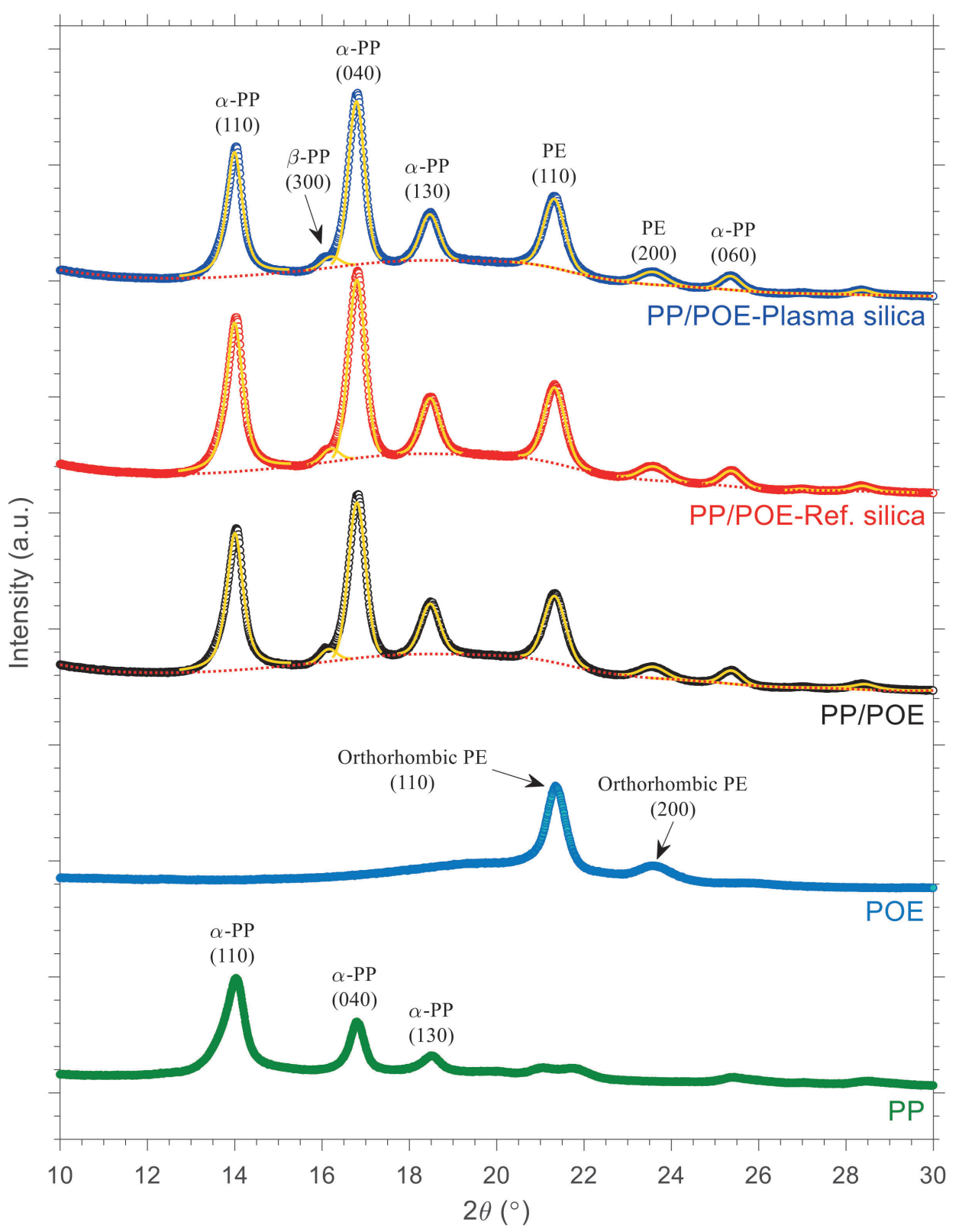

Figure 4.11 X-ray diffraction patterns of neat polypropylene/poly(ethylene-co-octene) (PP/POE) blends, reference silica filled PP/POE blends and plasma modified silica filled PP/POE blends. XRD diffraction patterns of PP and POE components are also shown (hotpressed samples). The solid yellow lines and dashed red lines show the fitted crystalline peaks (pseudo-Voigt) and amorphous background (cubic spline) from the multi-peak fitting procedure, respectively. The curves are shifted vertically for clarity and the predominant diffraction peaks are labelled. 
$16.1^{\circ}$ being attributable to $\beta$-form PP crystallites was also detected for all the samples [33]. In addition to the PP crystalline phase, orthorhombic PE crystals corresponding to (110) and (200) crystallographic planes were observed at $2 \theta$ angles of 21.4 and $23.4^{\circ}$, respectively, with these being attributable to the POE component of the polymer matrix [34].

The XRD diffraction spectra of the PP/POE composites were further analyzed by fitting and subtracting the amorphous background by cubic spline interpolation and thereafter fitting the remaining crystalline peaks using pseudo-Voigt profiles in MATLAB. The crystallinity index $X c$ was calculated by dividing the area under the crystalline curve by the total area under the original spectrum. Moreover, the apparent crystallite sizes were estimated by using Scherrer's equation (4.2):

$$
L=\frac{K \lambda}{\beta \cos \theta}
$$

where $L$ is the mean crystallite size, $K$ is a shape parameter $(\approx 0.89), \lambda$ is the $\mathrm{CuK} \alpha$ wavelength $(=1.5406$ $\AA), \beta$ is the full width at half maximum, and $\theta$ is the diffraction angle.

As shown in Table 4.4 the estimated crystallinity indices and crystallite sizes were, within experimental error, similar for all the PP/POE composites. Although nanosilica may bring about a mild increase in nucleation density (as will be discussed in the DSC section), the XRD data indicate no major differences in the crystalline phase structure or total crystallinity upon incorporation of neat or plasma modified nanosilica in the injection molded PP/POE composites. The relative $\beta$-form PP contents, approximated from the fitted crystalline peaks using the Turner-Jones $\beta$-form crystal index [35], were negligible $\left(k_{\beta}\right.$ $\sim 0.03$ ) and showed no clear dependence on nanosilica; while silica nanoparticles have been reported to increase $\beta$-form PP crystallinity in some polymer composite systems elsewhere [36], this is apparently not the case for the materials studied here.

Table 4.4 Estimated XRD crystallinity index and crystallite sizes.

\begin{tabular}{ccccccccc}
\hline \multirow{2}{*}{ Material } & \multirow{2}{*}{$\boldsymbol{X}_{\boldsymbol{c}}[\%]$} & $\alpha-\mathrm{PP}$ & $\alpha-\mathrm{PP}$ & $\alpha-\mathrm{PP}$ & $\alpha-\mathrm{PP}$ & $\beta-\mathrm{PP}$ & $\mathrm{PE}$ & $\mathrm{PE}$ \\
\cline { 5 - 10 } & & $(110)$ & $(040)$ & $(130)$ & $(060)$ & $(300)$ & $(110)$ & $(200)$ \\
\hline Neat PP/POE & $31.3 \pm 2.1$ & 17.9 & 17.6 & 15.2 & 14.6 & 16.4 & 13.7 & 11.7 \\
\hline PP/POE-Ref silica & $32.0 \pm 2.1$ & 18.0 & 17.4 & 15.4 & 14.3 & 18.1 & 13.9 & 11.5 \\
PP/POE-Plasma silica & $31.4 \pm 1.2$ & 18.5 & 17.8 & 15.6 & 14.6 & 17.0 & 14.0 & 11.4 \\
\hline
\end{tabular}

\subsubsection{Characterization of Phase Transitions by DSC}

In order to further characterize the crystallinity of the composites as found by XRD, DSC tests were performed. The melting and crystallization process of neat PP/POE blends, reference silica filled 
PP/POE blends (R-silica filled PP/POE), and plasma modified silica filled PP/POE blends (P-silica filled $\mathrm{PP} / \mathrm{POE}$ ) were studied. The DSC results are shown in Figures 4.12 and 4.13.

Based on the XRD results, it is clear that the crystalline phase consists of monoclinic $\alpha$-crystals, orthorhombic crystals, and small amounts of $\beta$-crystals The characteristic peaks originated from PP segments correspond to $\alpha(110)$ at $14.1^{\circ}, \alpha(040)$ at $16.9^{\circ}, \alpha(130)$ at $18.5^{\circ}$, and $\alpha(150) /(060)$ at $25.4^{\circ}$. The orthorhombic crystal structure is formed by PE segments giving peaks (110) at $21.4^{\circ}$ and (200) at $23.5^{\circ}$ [34]. The weak signal from $\beta$-crystals (300) is located at $16.1^{\circ}$. This makes the results of the DSC measurements very clear showing two melting and crystallization peaks belonging to the orthorhombic PE phase and monoclinic $\alpha$-PP phase shown in Figures 4.12 and 4.13. The results strongly suggests that the mutual miscibility of the PP/POE blends is limited, and that polymer/polymer phase separation is most probably very significant. This indicates the existence of a large PP/POE interphase area which influences charge mobility, trapping, and bulk dielectric properties.

Figure 4.12 shows the melting curves of the three compounds and neat polymer matrices (PP and POE). It clearly shows two melting peaks. One melting peak belongs to the orthorhombic PE phase in POE polymer and the other one belongs to the monoclinic $\alpha$-PP phase in PP polymer due to the consistent peak position compared to the neat polymer PP and POE matrix. All three samples show very similar melting curves with crystalline phase melting temperatures and comparable values of heat consumed during melting $(\Delta H)$ indicating a similar degree of crystallinity shown in Table 4.4 This is in line with the XRD measurement results showing similar crystallinity amount in the samples.

Figure 4.13 shows the cooling curves of the three compounds and the two neat polymer matrices (PP and POE). It also clearly shows the two cooling peaks belonging to POE and PP. The crystallization temperature of both crystalline phases of P-silica filled PP/POE are higher than that of R-silica filled $\mathrm{PP} / \mathrm{POE}$ and neat PP/POE as shown in Table 4.5. This is due to nucleation effect of silica, which is dispersed in both PP/POE phases. However, the results of DSC cooling curves suggest that the silica is predominantly located in the PP phase characterized by higher crystallization onset temperature. Plasma modified silica exhibits better nucleating performance most probably due to its higher surface compatibility with PP/POE matrices than the unmodified, highly polar silica.

Table 4.5 Calculated melting and cooling parameters.

\begin{tabular}{|c|c|c|c|c|c|c|c|c|}
\hline \multirow{2}{*}{ Material } & \multicolumn{4}{|c|}{ Melting } & \multicolumn{4}{|c|}{ Crystallization } \\
\hline & $T_{m I}\left({ }^{\circ} \mathrm{C}\right)$ & $\Delta H_{m 1}$ & $T_{m 2}$ & $\Delta H_{m 2}$ & $T_{c 1}$ & $\Delta H_{c l}$ & $T_{c 2}$ & $\Delta \boldsymbol{H}_{c 2}$ \\
\hline Neat PP & - & - & 142.5 & 83.0 & - & - & 100.5 & 84.7 \\
\hline Neat POE & 107.0 & 64.0 & - & - & 93.1 & 56.0 & - & - \\
\hline Neat PP/POE & 108.5 & 11.5 & 145.4 & 18.7 & 90.0 & 19.0 & 100.3 & 26.0 \\
\hline Reference silica filled PP/POE & 108.5 & 10.9 & 145.6 & 18.3 & 92.7 & 22.3 & 103.8 & 31.4 \\
\hline Plasma silica filled PP/POE & 107.7 & 11.6 & 144.6 & 18.1 & 93.4 & 25.2 & 107.0 & 34.0 \\
\hline
\end{tabular}




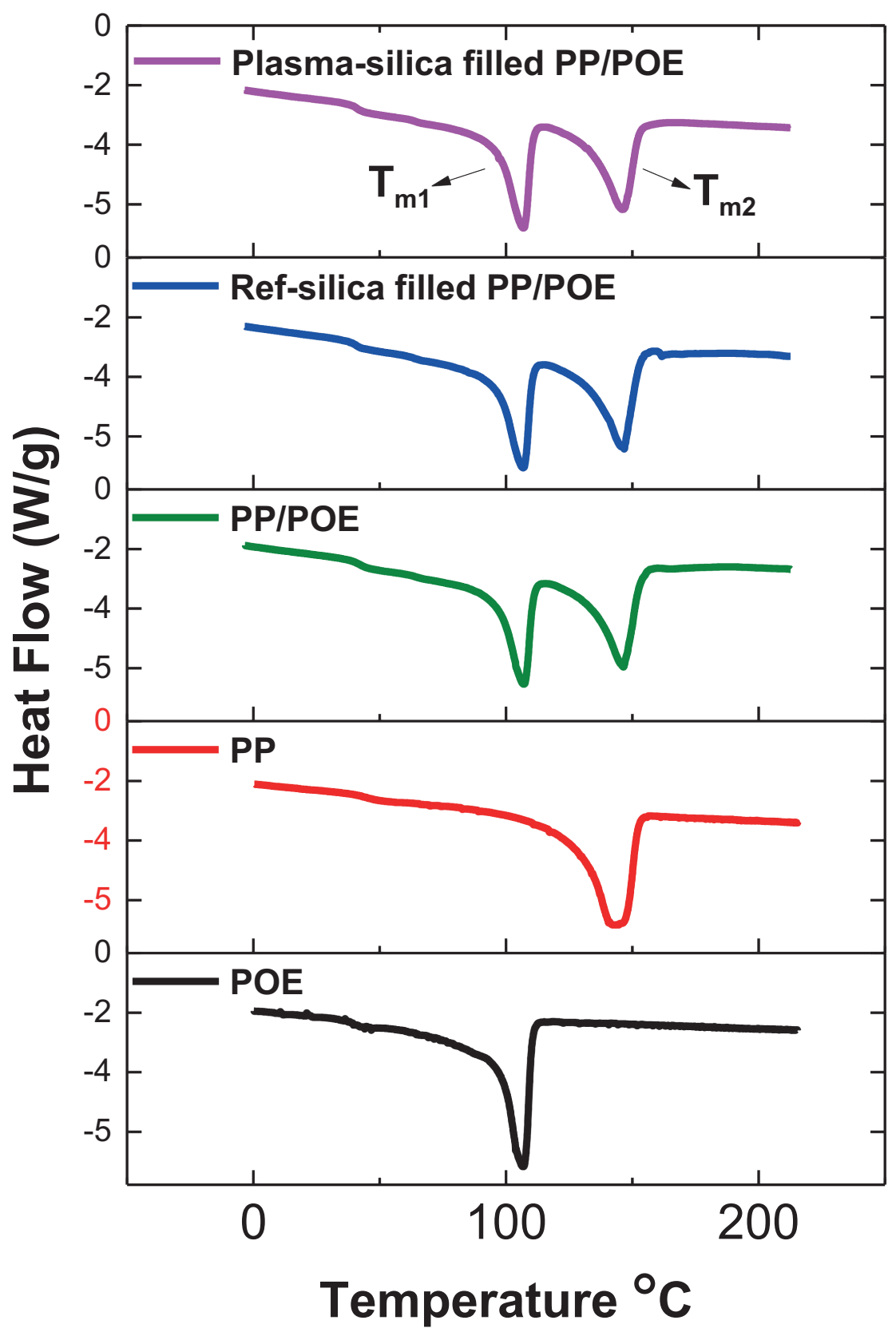

Figure 4.12 DSC melting curves. 


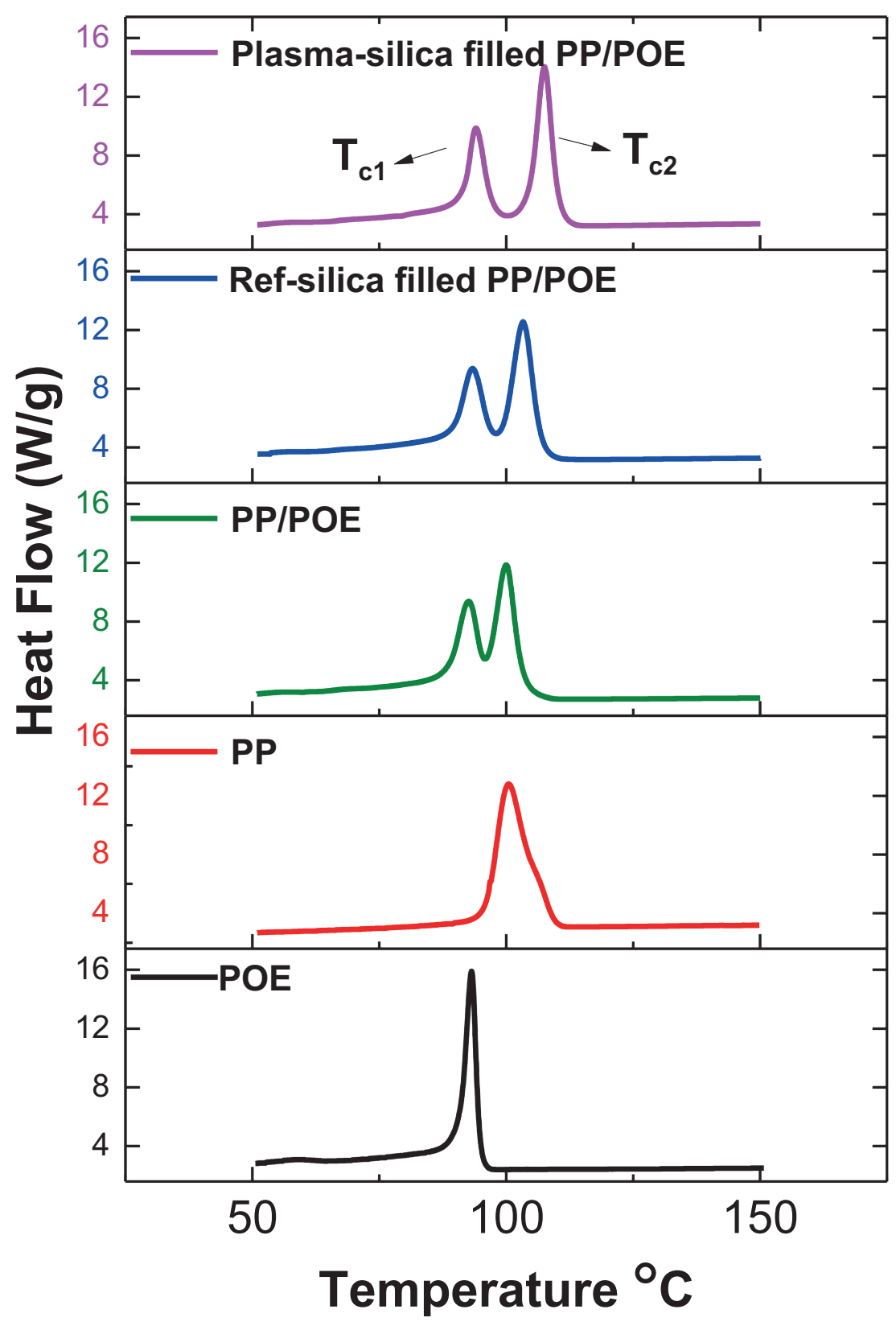

Figure 4.13 DSC cooling curves. 


\subsubsection{Morphology Analysis by SEM and Filler Dispersion Analysis by SEM-EDX Mapping}

SEM was applied to analyze the morphology of the composites shown in Figure 4.14 and SEM-EDX was applied to analyze the dispersion of the filler in the PP/POE blends matrix shown in Figure 4.15. For a clear investigation on the morphology of PP/POE matrix all the samples were prepared in the liquid nitrogen without any coating.

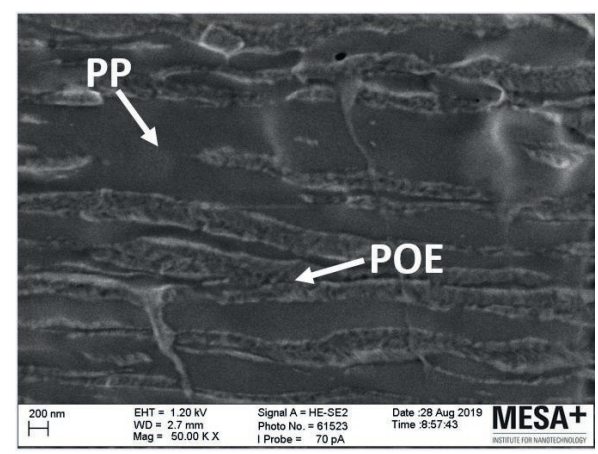

(a)

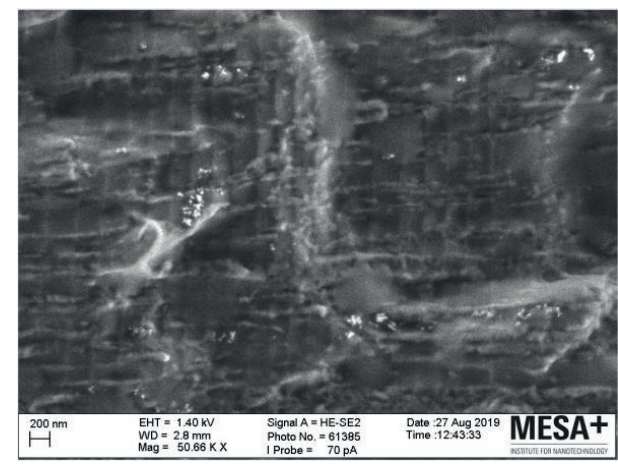

(b)

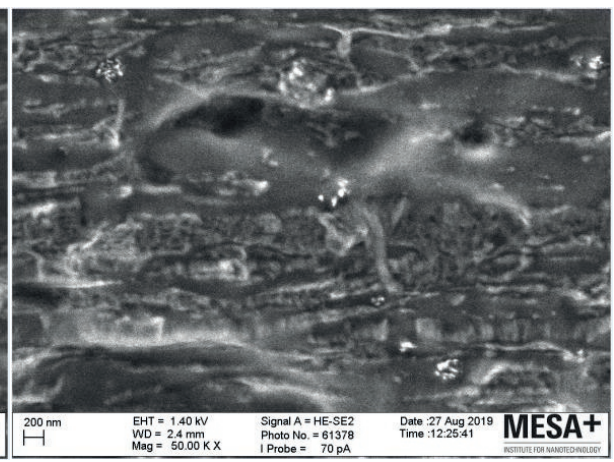

(c)

Figure 4.14 SEM images of (a) neat PP/POE, (b) reference silica filled PP/POE, and (c) plasma modified silica filled PP/POE.

Figure 4.14 shows the morphology of the three samples: Neat PP/POE (a), reference silica filled PP/POE (b), and plasma modified silica filled PP/POE (c). It is clearly shown that there are two separate polymer phases (one is smooth and the other one is rough) that form a layered structure. It is interesting to notice that the reference silica and plasma modified silica are most located in the smooth phase in Figure 4.14 (b) and (c). This is coherent with the DSC results, which exhibit that the silica has more pronounced influence on the crystallization of the PP phase. Therefore, it can be deducted that the smooth phase is the PP phase whereas the rough phase is the POE phase. 


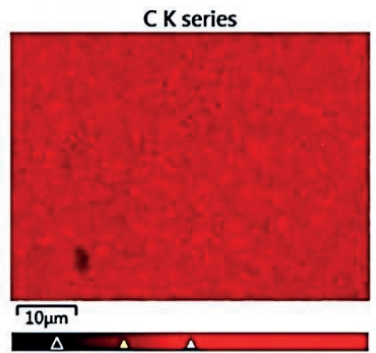

(a1)

O K series

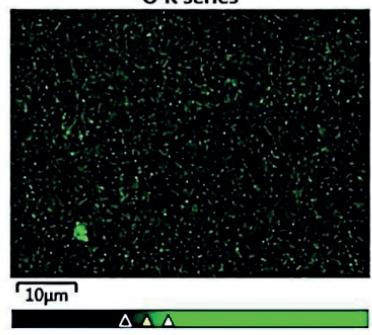

(a2)

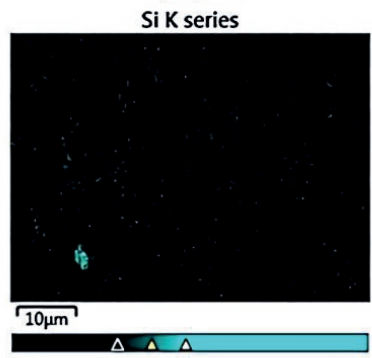

(a3)

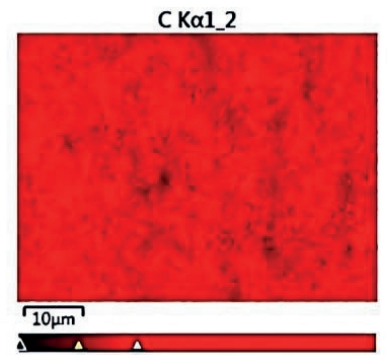

(b1)

o Ka1

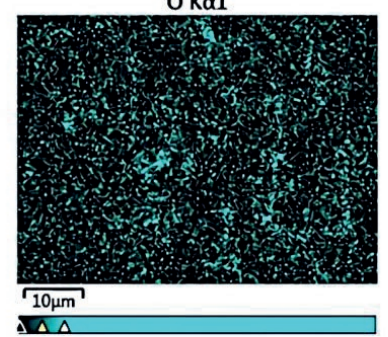

(b2)

Si Kal

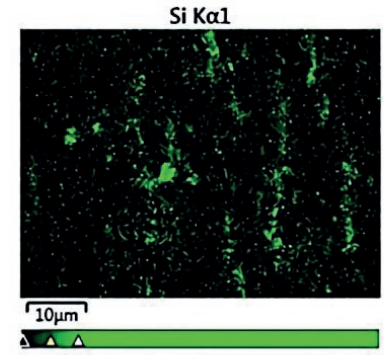

(b3)

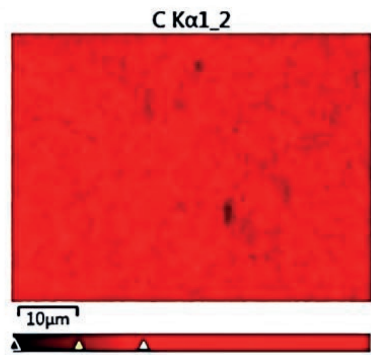

(c1)

O K $\alpha 1$

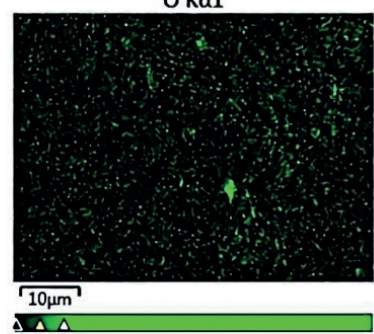

(c2)

Si $\mathrm{Kal}$

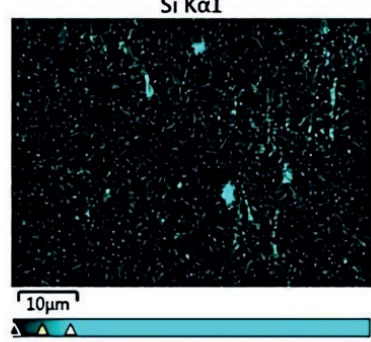

(c3)

Figure 4.15 SEM-EDX K $\alpha$ carbon (1), oxygen (2), and silicon (3) maps of the neat PP/POE (a), reference silica filled PP/POE (b) and plasma modified silica filled PP/POE (c).

To evaluate the dispersion of the unmodified and plasma modified silicas in the PP/POE polymer matrix SEM-EDX elemental mapping was applied (Figure 4.15). Figure 4.15 shows the distributions of carbon $(a 1, b 1, c 1)$, oxygen $(a 2, b 2, c 2)$, and silicon $(a 3, b 3, c 3)$, which were probed using SEM-EDX to measure the intensity of the $\mathrm{K} \alpha$ line across the three composite samples (a), (b), and (c). The silicon and oxygen mapping images show the increase of the dispersion level for plasma modified silica. This is an expected result confirming the positive effect of the plasma modification on the silica/PO compatibility. The signals from silicon visible in the neat PP/POE sample (a3) come most likely from a silica contamination. 


\subsubsection{Thermally Stimulated Depolarization Current (TSDC)}

Figure 4.16 shows the TSDC spectra of the studied injection molded PP/POE composites. In principle, for non-polar polymers, the TSDC above the glass transition temperature is mostly attributable to space charge relaxation, with the temperature at peak maximum and the peak intensity being related to the depth and density of the charge traps, respectively. Each composite exhibited a main TSDC peak at $\sim 75$ ${ }^{\circ} \mathrm{C}$ (Peak I), a small side peak at $\sim 108{ }^{\circ} \mathrm{C}$ (Peak II), and the onset of a third (incomplete) peak at $>120$ ${ }^{\circ} \mathrm{C}$ (Peak III). Compared to the DSC data as given in Table 4.4, the above TSDC peaks seem to be related to the onset of the melting of the POE phase (approximately $50{ }^{\circ} \mathrm{C}$ ), complete melting of this phase (approximately $110{ }^{\circ} \mathrm{C}$ ), and the onset of the melting of the PP phase (approximately $125{ }^{\circ} \mathrm{C}$ ), respectively. For calculating the trap depth and density distribution from the measured TSDC spectra, a numerical method allowing estimation of continuous trap density of states was applied [37], and the results are shown in the inset in Figure 4.16. The model assumes slow re-trapping conditions and that only electrons were injected in the sample during polarization. Further details on the numerical method are presented in the Supplementary Information S2.

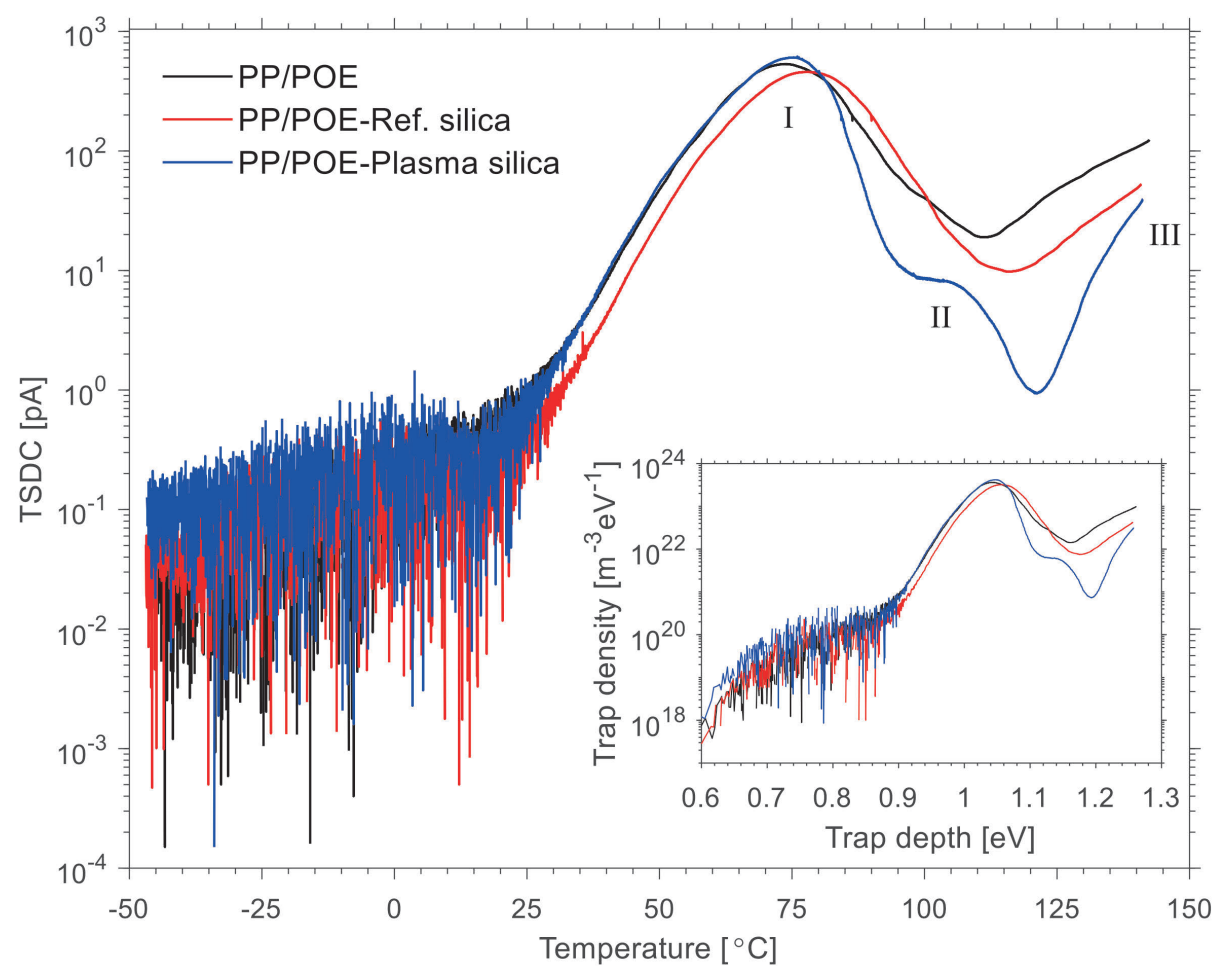

Figure 4.16 TSDC spectra of neat PP/POE, reference silica filled PP/POE, and plasma modified silica filled PP/POE. The inset shows the calculated trap depth versus density distributions. 
The main TSDC peak (Peak I) was located around $75^{\circ} \mathrm{C}$ for all samples, corresponding to a trap depth of $\sim 1.05 \mathrm{eV}$. The main TSDC peak temperature range was in good agreement with the onset of melting of the POE phase observed in DSC measurements, and can hence be attributed to the gradual relaxation of charge as the POE component softens and the POE crystals begin to melt at the PP/POE interphase. Slight differences in the TSDC peak intensity, and hence in the (apparent) trap density, were observed for the main peak (Peak I): For the sample filled with plasma modified silica, the TSDC peak intensity (trap density) was higher than that of the neat PP/POE composite, in contrast to the sample filled with the reference silica for which the peak intensity became lower than that of the neat PP/POE sample. Nevertheless, silica seems to have only a minor effect on the main peak characteristics which may indicate that the POE phase remains mostly unaffected by the silica. Larger differences between the TSDC spectra were however observed at higher temperatures in the $100-110^{\circ} \mathrm{C}$ region (Peak II) close to the DSC melting peak observed for POE at $108{ }^{\circ} \mathrm{C}$, with the plasma modified silica composite showing a significantly reduced trap density in this region. This indicates that silica is mostly contained in the PP phase, which is in line with the DSC results and SEM results, and that plasma modification of the silica reduced the deep trap density. Finally, each sample exhibited an initial rise portion of an incomplete TSDC peak at the highest temperatures close to $140^{\circ} \mathrm{C}$ (Peak III), which is close to the DSC melting peak observed for PP at $\sim 148{ }^{\circ} \mathrm{C}$ and may be attributed to relaxation of charges upon melting of PP crystallites.
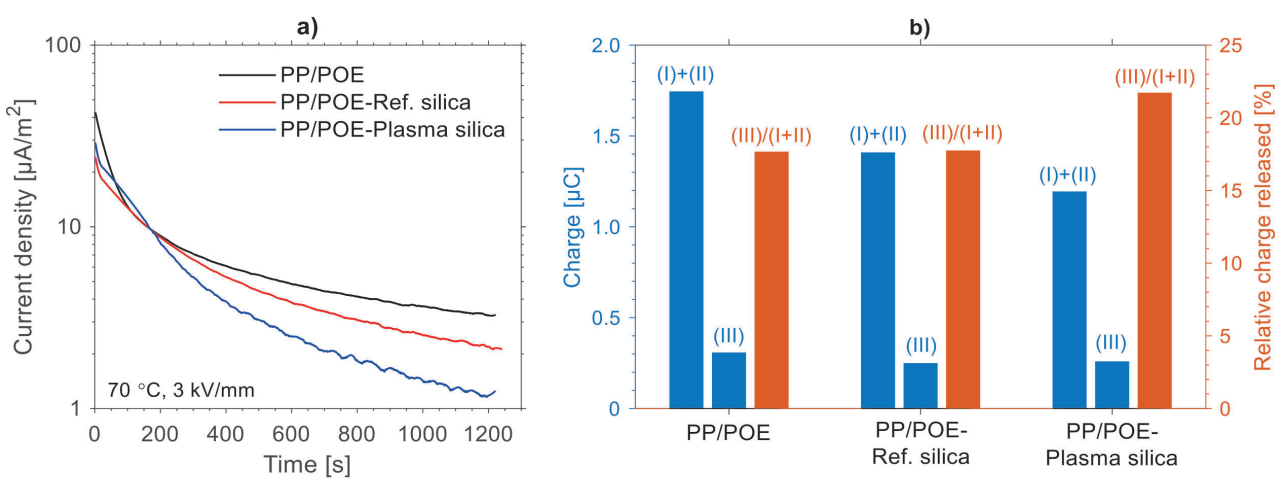

Figure 4.17 a) Isothermal charging current density during the polarization phase. b) Amount of charge injected (I+II) into or released (III) from the samples during TSDC measurements and their ratio (III)/(I+II).

Figure 4.17a) presents the charging current behavior during the isothermal poling phase for each sample. While it is clear that the polarization phase was too short to reach steady-state DC conduction current in any of the samples, the transient currents nevertheless indicate differences between the samples with the plasma modified silica composite showing the lowest charging current density, and hence apparent conductivity at the end of the polarization period $\left(1.1 \times 10^{-12} \mathrm{~S} / \mathrm{m}, 7.2 \times 10^{-13} \mathrm{~S} / \mathrm{m}\right.$, and $3.7 \times 10^{-13} \mathrm{~S} / \mathrm{m}$ for neat PP/POE, PP/POE-Reference silica, and PP/POE-Plasma silica, respectively). Furthermore, the integrated total charge at the end of the polarization phase (I+II) was compared to the total charge released during the TSDC (III) phase (Figure 4.17b). The amount of charge released during the thermally stimulated depolarization phase were found to be much lower for all samples than those injected during 
polarization. This behavior is quite typical, especially for relatively thick films [38], and can indicate that a large portion of the injected charge still remains trapped in the bulk of the composites. Nevertheless, the incorporation of plasma modified silica was found to increase the relative charge released during thermally stimulated depolarization, and this can be attributed to the reduced trap density in the high temperature region as seen in TSDC.

\subsubsection{Complex Permittivity}

Real and imaginary parts of the permittivity data of the injection molded samples are presented in Figure 4.18 as a function of frequency at room temperature. The pristine injection molded samples, measured right before the TSDC experiments, are considered first (Figure 4.18, left column). For the unfilled $\mathrm{PP} / \mathrm{POE}$ composite, the real part of the permittivity at $1 \mathrm{kHz}$ was 2.73 which is slightly higher than that of pure PP ( 2.25). A subtle increase in the real permittivity was observed upon incorporation of silica: The real permittivity at $1 \mathrm{kHz}$ increased to 2.74 and 2.75 for reference silica and plasma silica, respectively. Such a small difference in these three composites is however negligible when considering the instrumentation accuracy. All the composites showed ultra-low dielectric loss at higher frequencies, with $\tan \delta$ values in the $10^{-4}$ range which is typical for PP. However, at lower frequencies a considerable increase in the imaginary part of $\varepsilon_{r}^{*}$ was observed. When a material shows a non-negligible DC conductivity, the imaginary part of the complex permittivity contains both the polarization and conduction losses [39]:

$$
\varepsilon_{r}^{*}=\varepsilon_{r}-j \varepsilon_{r T}^{\prime}=\varepsilon_{r}-j\left(\varepsilon_{r}^{\prime}+\frac{\sigma}{\omega \varepsilon_{0}}\right)
$$

where $\varepsilon_{r}$ is the real permittivity, $\varepsilon_{r T}^{\prime}$ is the total loss factor, $\varepsilon_{r}^{\prime}$ is the polarization loss term, $\sigma$ is the conductivity, $\omega$ is the angular frequency, and $\varepsilon_{0}$ is the vacuum permittivity. The increase in $\varepsilon_{r T}^{\prime}$ at low frequencies is likely due to the contribution of interfacial polarization and DC conductivity arising from the heterophasic morphology of PP/POE. The ref. silica and plasma silica composites exhibited slightly lower total loss factors at low frequencies in comparison to the neat PP/POE, which, in agreement with the isothermal charging current data (Figure 4.17a), may be attributed to slightly reduced conductivity due to the silica-PP/POE interface.

From the permittivity data measured after TSDC (Figure 4.18, right column), a significant reduction in real permittivity is observed for all the composites in comparison to the pristine samples. This is likely related to the morphological changes, e.g., melting and recrystallization of the POE phase and lamellar thickening of PP crystallites, that have taken place during the high temperature (isothermal) polarization and (thermally stimulated) depolarization phases. The changes observed in the DSC analysis for the crystallization behavior of the silica-PP/POE composites seem to also be reflected as larger real permittivity values in comparison to the unfilled PP/POE. At lower frequencies, both $\varepsilon_{r}$ and $\varepsilon_{r T}^{\prime}$ show a considerable increase in comparison to the pristine samples measured before TSDC along with morphological changes. This may be attributed to a relatively large amount of space charge still being deeply trapped in the specimens after TSDC. 

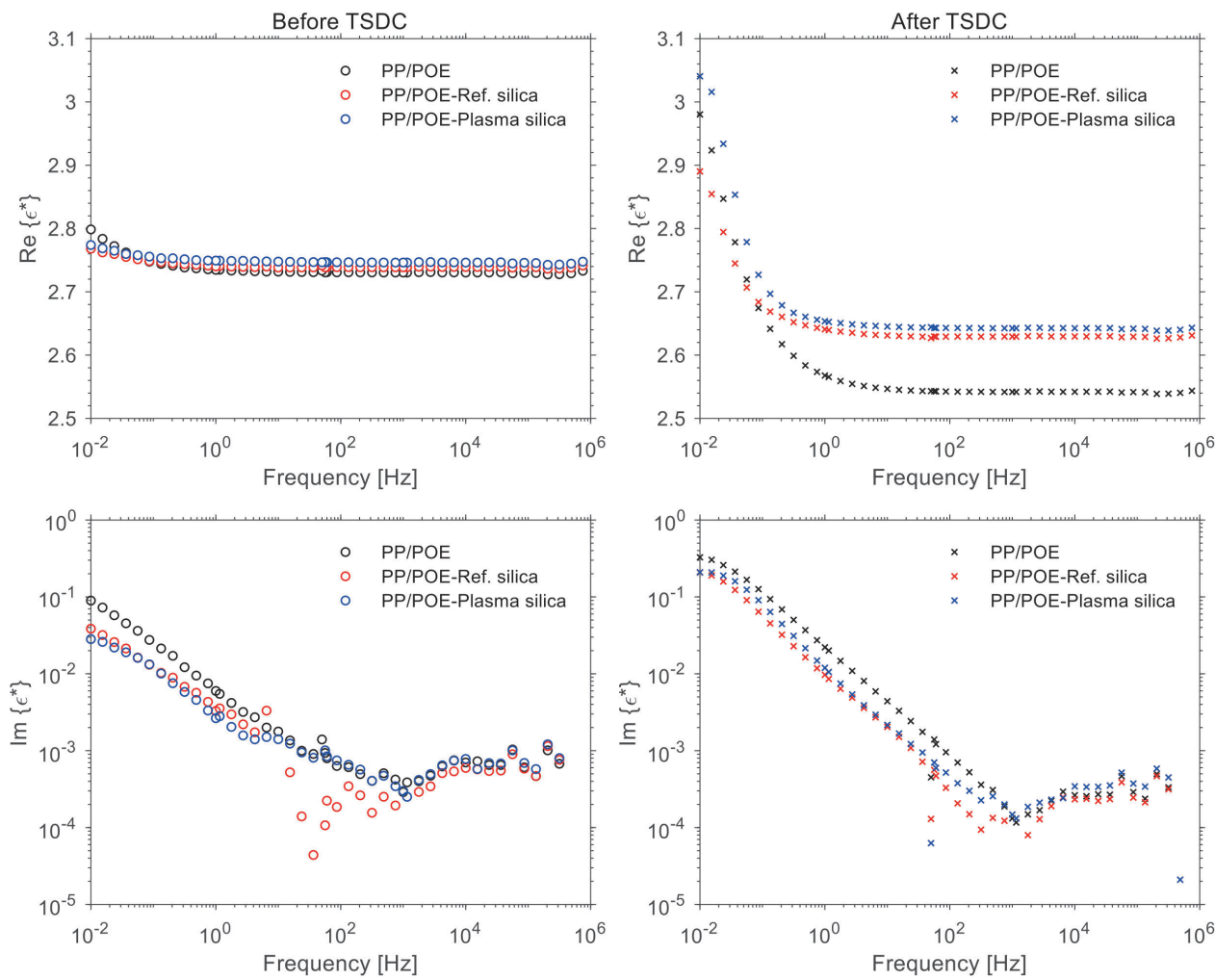

Figure 4.18 Real (top) and imaginary (bottom) parts of complex permittivity as a function of frequency before TSDC (left column) and after TSDC (right column).

\subsection{Conclusions}

Low-pressure plasma polymerization was successfully applied for surface modification of silica. A layer of hydrocarbon compounds deposited on the silica surface after acetylene plasma modification leads to improved dispersibility of silica nanoparticles in a PP/POE matrix.

Incorporation of the acetylene plasma modified silica into the PP/POE blend resulted in only slight changes of the polymer matrix crystallinity. However, the crystallization temperature of the blend containing acetylene plasma modified silica increased significantly indicating its higher nucleating properties. This effect is attributable to a higher degree of polymer-filler interaction caused by the presence of the hydrocarbon layer on the silica surface. The results also suggest that the silica is located mainly in the PP phase increasing its crystallization temperature to a higher extent than that of POE.

The charge trapping behavior of the PP/POE nanocomposites was studied by means of thermally stimulated depolarization current measurements under a moderate polarization field. TSDC results indicate that acetylene plasma modified silica changes the charge trapping properties and decreases the amount of injected charges into the PP/POE matrix and increases the percentage of released charges. This is caused by the acetylene plasma modification of the silica surface which assures better compatibility with the PP/POE matrix. Thus, the hydrocarbon layer on the silica surface after acetylene 
plasma modification changes the interface chemistry between silica and the polymer matrix and furthermore alters the nature of the charge trap sites. However, the permittivity measurement results showed that there might be still a relatively large amount of space charge still being deeply trapped in the specimens after TSDC. It might be the reason to explain why we see less charges being released and more charge being injected during TSDC measurements.

Acknowledgments: This project has received funding from the European Union's Horizon 2020 research and innovation program under grant agreement No 720858. The authors would like to thank the ECIU Researcher Mobility Fund for supporting this research work. The authors also would like to thank Evonik Industries for providing a free silica sample. 


\section{Supplementary Material}

\section{S1 Measurement of Isothermal Polarization and Thermally Stimulated Depolarization}

\section{Current}

Isothermal polarization current and thermally stimulated depolarization current (TSDC) measurements were carried out in order to study the transient electrical conductivity and charge trapping behavior of the nanocomposite samples (see Fig. S1). There are three main regions during the measurement:

1. Isothermal polarization;

2. Cooling (while still maintaining the electric field);

3. Thermally stimulated depolarization (no field applied).

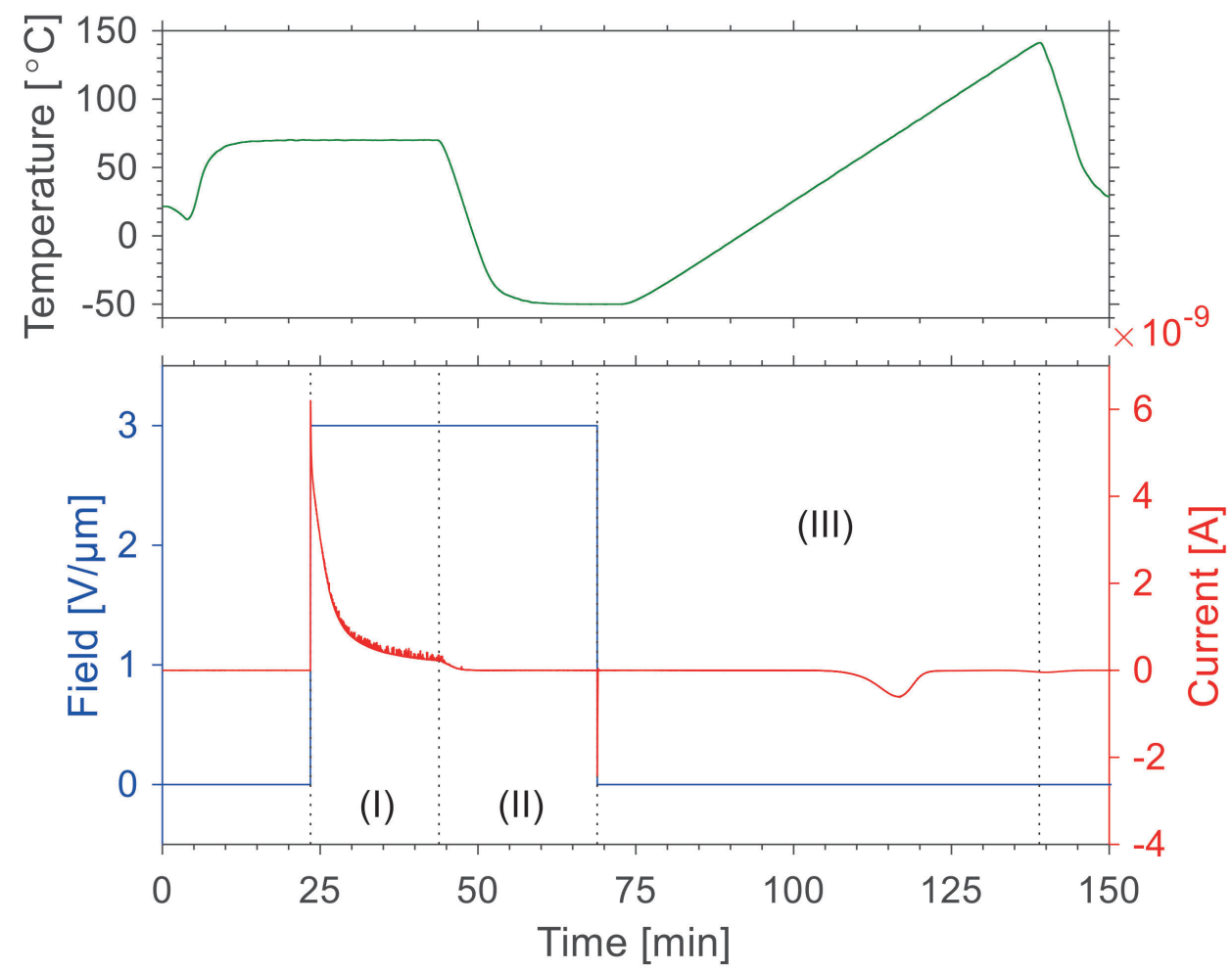

Figure S1. Exemplifying polarization/depolarization current of a plasma modified silica filled PP/POE composite. The annotated regions are (I): isothermal polarization, (II): cooling while still maintaining the electric field and (III): thermally stimulated depolarization.

The charges were injected into the sample during the poling phase and then kept for stabilization during freezing. During the polarization step, the measured isothermal charging current data provided an indication of the transient electrical conductivity behavior of the sample. During the subsequent 
depolarization step, when the sample was heated linearly under short-circuit conditions, gradual relaxation of (dipolar) polarization and trapped charge release occurs, giving rise to a thermally stimulated depolarization current (TSDC) in the external circuit. In principle, for non-polar polymers, the TSDC above the glass transition temperature is mostly attributable to space charge relaxation, with the temperature at peak maximum and the peak intensity being related to the depth and density of the charge traps, respectively.

\section{S2 Determination of Trap Density Distribution from TSDC Data}

Assuming that only electrons are injected into the sample during the high-field polarization, the induced thermally stimulated depolarization current density during linear heating can be expressed as $[37,40]$ :

$$
J(T)=\frac{q l^{2}}{2 d} \int_{E_{v}}^{E_{c}} f_{0}(E) N_{t}(E) e_{n}(E, T) \exp \left(-\frac{1}{\beta} \int_{T_{0}}^{T} e_{n}(E, T) d T\right) d E
$$

where,

$$
e_{n}(E, T)=v \exp \left(-E_{t} / k T\right)
$$

In (S.1) and (S.2), $q$ is the elementary charge, $l$ is the penetration depth, $d$ is the sample thickness, $f_{0}$ is the initial occupancy of electron traps, $N_{t}(E)$ is the trap level density distribution function, $E$ is the trap depth, $T$ is temperature, $\beta$ is the heating rate, $T_{0}$ is the cooling temperature, $e_{n}(E, T)$ is the rate of emission of electrons, $v$ is the attempt-to-escape frequency and $k$ is the Bolzmann constant. For the calculations it was assumed that the all the traps were initially filled $\left(f_{0}=1\right)$ and the penetration depth of the injected charge layer was $l=5 \mu \mathrm{m}$. The attempt-to-escape frequency was taken as $v=k T / h$ where $h$ is Planck's constant. Thus, within the experimental temperature range (from $-50{ }^{\circ} \mathrm{C}$ to $140{ }^{\circ} \mathrm{C}$ ), the attempt-to-escape frequency varies from $v=4.65 \times 10^{12} \mathrm{~s}^{-1}$ to $v=8.61 \times 10^{12} \mathrm{~s}^{-1}$ which is within the physically acceptable range.

As originally proposed by Simmons [40], a new function $G(E, T)$, appearing in the integrand of (1), can be defined as:

$$
G(E, T)=e_{n}(E, T) \exp \left(-\frac{1}{\beta} \int_{T_{0}}^{T} e_{n}(E, T) d T\right)
$$


The form of $G(E, T)$ is an asymmetrical bell-shaped curve which, according to the analyses provided by both Simmons [40] and Tian et al. [37], can be approximated as a $\delta$ function:

$$
G(E, T)=A\left(E_{m}\right) \delta\left(E-E_{m}\right)
$$

where $E_{m}$ corresponds to the trap level at peak maximum of $G(E, T)$ at temperature $T . A\left(E_{m}\right)$ at each temperature $T$ can be solved by numerical method provided by Tian et al. [2]. Finally, the trap density distribution $N_{t}(E)$ can be solved as:

$$
N_{t}\left(E_{m}\right)=\frac{2 d J(T)}{q l^{2} A\left(E_{m}\right) f_{0}}
$$




\section{References}

[1] Milliere, L.; Makasheva, K.; Laurent, C.; Despax, B.; Boudou, L.; Teyssedre, G. Effects of a modified interface by silver nanoparticles/SiOC: $\mathrm{H}$ barrier layer against space charge injection under HVDC. In Proceedings of the IEEE Conference on Electrical Insulation and Dielectric Phenomena (CEIDP), Des Moines, IA, USA, 19-22 October 2014; pp. 883-886.

[2] Lau, K.Y.; Vaughan, A.S.; Chen, G.; Hosier, I.L.; Holt, A.F.; Ching, K.Y. On the space charge and DC breakdown behavior of polyethylene/silica nanocomposites. IEEE Trans. Dielectr. Electr. Insul. 2014, 21, 340-351.

[3] Pitsa, D.; Danikas, M.G.; Vardakis, G.E.; Tanaka, T. Influence of homocharges and nanoparticles in electrical tree propagation under DC voltage application. Electr. Eng. 2012, 94, 81-88.

[4] Mazzanti, G.; Chen, G.; Fothergill, J.C.; Hozumi, N.; Li, J.; Marzinotto, M.; Wu, K.A. Protocol for space charge measurements in full-size HVDC extruded cables. IEEE Trans. Dielectr. Electr. Insul. $2015,22,21-34$.

[5] Zhou, Y.; He, J.; Hu, J.; Huang, X.; Jiang, P. Evaluation of polypropylene/polyolefin elastomer blends for potential recyclable HVDC cable insulation applications. IEEE Trans. Dielectr. Electr. Insul. 2015, 22, 673-681.

[6] Du, B.X.; Xu, H.; Li, J.; Li, Z. Space charge behaviors of PP/POE/ZnO nanocomposites for HVDC cables. IEEE Trans. Dielectr. Electr. Insul. 2016, 23, 3165-3174.

[7] Hornak, J.; Trnka, P.; Totzauer, P.; Gutten, M. The effect of space charge accumulation in high voltage insulation systems. In Proceedings of the IEEE 18th International Scientific Conference on Electric Power Engineering (EPE), Kouty nad Desnou, Czech Republic, 17-19 May 2017; pp. 15 .

[8] Zha, J.W.; Wu, Y.H.; Wang, S.J.; Wu, D.H.; Yan, H.D.; Dang, Z.M. Improvement of space charge suppression of polypropylene for potential application in HVDC cables. IEEE Trans. Dielectr. Electr. Insul. 2016, 23, 2337-2343.

[9] Wu, C.; Yan, W.; Phung, B.T. Influence of plasma-treated nanoparticles on space charge accumulation in epoxy resin insulation. In Proceedings of the IEEE Conference on Electrical Insulation and Dielectric Phenomena, Shenzhen, China, 20-23 October 2013; pp. 784-787.

[10] Han, B.; Wang, X.; Sun, Z.; Yang, J.; Lei, Q. Space charge suppression induced by deep traps in polyethylene/zeolite nanocomposite. Appl. Phys. Lett. 2013, 102, 012902.

[11] Rytöluoto, I.; Ritamäki, M.; Lahti, K.; Paajanen, M.; Karttunen, M.; Montanari, G.C.; Seri, P.; Naderiallaf, H. Compounding, structure and dielectric properties of silica-BOPP nanocomposite films. In Proceedings of the IEEE 2nd International Conference on Dielectrics (ICD), Budapest, Hungary,1-5 July 2018; pp. 1-4.

[12] Wang, S.J.; Zha, J.W.; Wu, Y.H.; Ren, L.; Dang, Z.M.; Wu, J. Preparation, microstructure and properties of polyethylene/alumina nanocomposites for HVDC insulation. IEEE Trans. Dielectr. Electr. Insul. 2015, 22, 3350-3356.

[13] Zhou, Y.; Hu, J.; Dang, B.; He, J. Titanium oxide nanoparticle increases shallow traps to suppress space charge accumulation in polypropylene dielectrics. RSC Adv. 2016, 6, 48720-48727.

[14] Roy, M.; Nelson, J.K.; MacCrone, R.K.; Schadler, L.S.; Reed, C.W.; Keefe, R. Polymer nanocomposite dielectrics-the role of the interface. IEEE Trans. Dielectr. Electr. Insul. 2005, 12, 629-643. 
[15] Reed, C.W. Functionalization of nanocomposite dielectrics. In Proceedings of the IEEE International Symposium in Electrical Insulation (ISEI), San Diego, CA, USA, 6-9 June 2010; pp. $1-4$.

[16] Yasuda, H.; Matsuzawa, Y. Economical advantages of low-pressure plasma polymerization coating. Plasma Process. Polym. 2005, 2, 507-512.

[17] Chan, C.M.; Ko, T.M.; Hiraoka, H. Polymer surface modification by plasmas and photons. Surf. Sci. Rep. 1996, 24, 1-54.

[18] Martinez, M.A.; Abenojar, J.; Lopez de Armentia, S. Environmentally friendly plasma activation of acrylonitrile-butadiene-styrene and polydimethylsiloxane surfaces to improve paint adhesion. Coatings 2018, 8, 428.

[19] Adachi, S.; Ueda, N. Wear and corrosion properties of cold-sprayed AISI 316L coatings treated by combined plasma carburizing and nitriding at low temperature. Coatings 2018, 8, 456.

[20] Ellinas, K.; Tserepi, A.; Gogolides, E. Superhydrophobic fabrics with mechanical durability prepared by a two-step plasma processing method. Coatings 2018, 8, 351.

[21] Tabari, K.; Hosseinpour, S.; Mohammad-Rahimi, H. The impact of plasma treatment of Cercon zirconia ceramics on adhesion to resin composite cements and surface properties. J. Lasers Med. Sci. 2017, 8 (Suppl. 1), 56-61.

[22] Sicinski, M.; Gozdek, T.; Bielinski, D.M.; Szymanowski, H.; Kleczewska, J.; Piatkowska, A. Plasma-modified graphene nanoplatelets and multiwalled carbon nanotubes as fillers for advanced rubber composites. IOP Conf. Ser. Mat. Sci. 2015, 87, 012012.

[23] Ghorbani, H.; Christen, T.; Carlen, M.; Logakis, E.; Herrmann, L.; Hillborg, H.; Petersson, L.; Viertel, J. Long-term conductivity decrease of polyethylene and polypropylene insulation materials. IEEE Trans. Dielectr. Electr. Insul. 2017, 24, 1485-1493.

[24] Tiwari, M.; Noordermeer, J.W.; van Ooij, W.J.; Dierkes, W.K. Plasma polymerization of acetylene onto silica: An approach to control the distribution of silica in single elastomers and immiscible blends. Polym. Advan. Technol. 2008, 19, 1672-1683.

[25] Mathew, T.; Datta, R.N.; Dierkes, W.K.; Noordermeer, J.W.; Van Ooij, W.J. Mechanistic investigations of surface modification of carbon black and silica by plasma polymerisation. Plasma Chem. Plasma Process. 2008, 28, 273-287.

[26] Salonen, J.; Laine, E.; Niinistö, L. Thermal carbonization of porous silicon surface by acetylene. J. Appl. Phys. 2002. 91, 456-461.

[27] Chen, G.; He, Z.; He, X.; Zhang, L.; Tang, Y. Effects of rf power on low-pressure inductively coupled plasma of hydrogen and trans-two-butane. High Power Laser Part. Beams. 2016, 28, 28092004.

[28] Franz, G. Low Pressure Plasmas and Microstructuring Technology; Springer: Berlin/Heidelberg, Germany, 2009.

[29] Vansant, E.F.; Van Der Voort, P.; Vrancken, K.C. Characterization and Chemical Modification of the Silica Surface; Elsevier: Amsterdam, The Netherlands,1995; p. 93.

[30] Wang, S.; Sun, Q.; Gröning, O.; Widmer, R.; Pignedoli, C.A.; Cai, L.; Zhu, J. On-surface synthesis and characterization of individual polyacetylene chains. Nat. Chem. 2019, 11, 924-930.

[31] Arias-Durán, A.; Giuliani, L.; D’Accorso, N.B.; Grondona, D.; Goyanes, S. Thin films of polymerized acetylene by RF discharge and its benzene absorption ability. Surf. Coat. Tech. 2013, 216, 185-190. 
[32] Sun, Q.; Wang, J.; Weng, J.; Liu, F. Surface structure and electric properties of nitrogen incorporated NCD films. Vacuum 2017, 137, 155-162.

[33] Wu, Y.H.; Zha, J.W.; Li, W.K.; Wang, S.J.; Dang, Z.M. A remarkable suppression on space charge in isotatic polypropylene by inducing the $\beta$-crystal formation. Appl. Phys. Lett. 2015, 107, 112901.

[34] Hölzer, S.; Menzel, M.; Zia, Q.; Schubert, U.S.; Beiner, M.; Weidisch, R. Blends of ethyleneoctene copolymers with different chain architectures-Morphology, thermal and mechanical behavior. Polymer 2013, 54, 5207-5213.

[35] Rytöluoto, I.; Gitsas, A.; Pasanen, S.; Lahti, K. Effect of film structure and morphology on the dielectric breakdown characteristics of cast and biaxially oriented polypropylene films. Eur. Polym. J. 2017, 95, 606-624.

[36] Chi, X.; Cheng, L.; Liu, W.; Zhang, X.; Li, S. Characterization of polypropylene modified by blending elastomer and nano-silica. Materials 2018, 11, 1321.

[37] Tian, F.; Bu, W.; Shi, L.; Yang, C.; Wang, Y.; Lei, Q. Theory of modified thermally stimulated current and direct determination of trap level distribution. J. Electrostat. 2011, 69, 7-10.

[38] Sessler, G.M. (Ed.) Electrets; Springer: Berlin/Heidelberg, Germany, 1987.

[39] Kao, K.C. Dielectric Phenomena in Solids: With Emphasis on Physical Concepts of Electronic Processes, 1st ed.; Elsevier Academic Press: San Diego, CA, USA, 2004.

[40] Simmons J.G.; Taylor G.W.; Tam M.C. Thermally Stimulated Currents in Semiconductors and Insulators Having Arbitrary Trap Distributions, Phys. Rev. B. 7 1973, 3714-3719. doi:10.1103/PhysRevB.7.3714. 
$100 \mid \mathrm{P}$ a g e 


\section{Chapter 5}

\section{Silica surface-modification for tailoring the charge trapping properties of $\mathrm{PP} / \mathrm{POE}$ based dielectric nanocomposites for HVDC cable application}

This chapter has been published in IEEE ACCESS.

He, X., Rytöluoto, I., Anyszka, R., Mahtabani, A., Saarimäki, E., Lahti, K., Paajanen, M., Dierkes, W. and Blume, A., 2020. Silica surface-modification for tailoring the charge trapping properties of PP/POE based dielectric nanocomposites for HVDC cable application. IEEE Access, 8, pp.8771987734.

DOI: $10.1109 / A C C E S S .2020 .2992859$. 


\title{
Silica Surface-Modification for Tailoring the Charge Trapping Properties of PP/POE Based Dielectric Nanocomposites for HVDC Cable Application
}

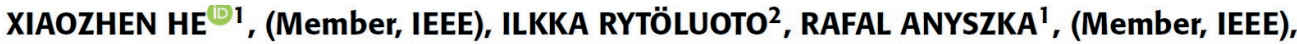 \\ AMIRHOSSEIN MAHTABANI ${ }^{1}$, (Member, IEEE), EETTA SAARIMÄKI ${ }^{2}$, KARI LAHTI ${ }^{3}$, \\ MIKA PAAJANEN ${ }^{2}$, WILMA DIERKES ${ }^{1}$, AND ANKE BLUME ${ }^{1}$ \\ ${ }^{1}$ Elastomer Technology and Engineering Group, Department of Mechanics of Solids, Surfaces and Systems (MS3), Faculty of Engineering Technology, \\ University of Twente, 7500 AE, Enschede, The Netherlands \\ ${ }^{2}$ VTT Technical Research Centre of Finland Ltd., 33101 Tampere, Finland \\ ${ }^{3}$ Research Group on High Voltage Engineering, Tampere University, 33720 Tampere, Finland \\ Corresponding authors: Wilma Dierkes (w.k.dierkes@utwente.nl), Xiaozhen He (x.he@utwente.nl), and Rafal Anyszka \\ (r.p.anyszka@utwente.nl)
}

This work was supported by the European Union's Horizon 2020 Research and Innovation Program under Grant 720858.

ABSTRACT This paper focuses on novel insulation polypropylene/poly(ethylene-co-octene) (PP/POE) nanocomposites for High Voltage Direct Current (HVDC) cable application. The composites contain silica modified by a solvent-free method using silanes differing in polarity and functional moieties. Thermogravimetric Analysis and Fourier Transform Infrared Spectroscopy showed that the solvent-free method is an effective way to modify silica by silanes. Silica/PP/POE nanocomposites were prepared in a mini twin-screw compounder, and the effect of silica on crystallization, dispersibility and dielectric properties of the samples was investigated. Differential Scanning Calorimetry results showed that the unmodified and modified silicas acted as nucleation agents and increased the onset of the crystallization temperature of the polymeric matrix. Scanning Electron Microscopy images showed that the silica is mostly located in the PP phase matrix. For the PP/POE nanocomposites filled with unpolar silica, a higher trap density (measured by Thermally Stimulated Depolarization Current, TSDC) was found; this might be caused by the larger interfacial area due to a better dispersion of the unpolar silica in the polymeric matrix. Polar silicas introduce deeper traps than the unpolar ones, which is most likely due to the hetero-atom introduction. Nitrogen atoms were found to have the strongest effect on the charge trapping properties. According to these results, amine-modified silica is a promising candidate for PP/POE nanocomposites for HVDC cable applications.

INDEX TERMS PP/POE, nanosilica, surface modification, dispersibility, crystallization behavior, trap distribution, HVDC cable.

Note: In this chapter, POE is used as abbreviation for ethylene-octene copolymer. 


\subsection{Introduction}

Thermoplastic dielectric composites containing nanofillers have attracted a lot of interest in the field of high voltage insulation field [1-3]. It is widely accepted that the large interface formed between a nanofiller and a polymer matrix plays a critical role in the high voltage dielectric properties of nanocomposites [4-6].

However, one of the general problems in the preparation of nanocomposite preparation is to achieve good dispersion of the nanofillers in the polymer matrix. For instance, it is reported that the dc conductivity of LDPE increases remarkably with higher nanofiller loading ( $>3 \mathrm{phr}$ ) than the one filled with a lower amount of nanofiller ( 1 to $3 \mathrm{phr}$ ) duo to extensive nanofiller agglomerate formation [7]. In principle, improving the performance of a nanodielectric requires good dispersion of the nanofiller, which results in larger interface area between the nanofiller and polymer.

Although a better dispersion of nanofillers is crucial, the type of surface functionalization of nanofillers is also very important from the point of view of dielectric properties. Incorporation of functional polar groups on the nanofiller surface can positively alter the dielectric properties of a nanocomposite. It is reported that the introduction of polar groups (amine) onto a nanoparticle surface improves the breakdown strength of polyethylene nanocomposites due to the modified electrical features (e.g. charge trapping) at the polymer-nanoparticle interface [8]. Modified silica containing polar chlorine atoms can suppress the space charge accumulation of in crosslinked polyethylene (XLPE) nanocomposites, due to a change in the spherulites size distribution [9]. Furthermore, nanofillers grafted with $\pi$-conjugated surface ligands can act as electron trap to alter the avalanche breakdown of silica-epoxy nanocomposites [10].

The dielectric performance of an insulation material is associated with charge trapping/detrapping phenomena. The temperature- and field-dependent transportation of charges in nanocomposites varies with the polarity and morphology of the polymeric matrix, the type, size and dispersion of the nanofillers, and and the tailored surface properties of the nanofillers [11, 12]. Therefore, the influence of modified nanofillers having different surface polarities on the charge trapping properties of the dielectric materials is studied. Previously, the introduction of different polar functional groups on the silica surface resulted in significant differences in charge trapping properties of the nanocomposites [13, 14]. Following up our previous study [14], the current research not only covers the phenomenon of charge trapping properties, but also includes various characterization methods to investigate the mechanism of it.

In general, silica-silane modification in most cases is performed in a solvent at various temperatures [15-17]. This enables utilization of various functional silanes and versatile conditions resulting in a good control of the silane deposition on the silica surface. However, this also causes an environmental burden by the solvent waste that needs to be properly recycled or disposed of. Therefore, this paper aims also to develop a new solvent-free method for silane modification of silica nanoparticles.

As one requirement of this new development was sustainability and recyclability, polypropylene (PP) and polyolefin (POE) based materials were chosen for the matrix. The currently used cross-linked polyethylene cannot be recycled and reused due to its polymer network. Switching to PP and POE allows to develop a recyclable HVDC cable insulation material with less environment impact. Blending PP with a polyolefin can improve the flexibility of the former polymer, and the addition of the nanofiller can result in a 
significant improvement of the thermoplastic dielectric properties. This can further increase the potential to be used as HVDC cable insulation material [1].

In summary, the objectives of this study are:

1) Developing an easy-to-upscale solvent-free method to tailor silica surface properties

2) Investigating the influence of a silica surface modification on the performance (crystallization, dispersibility and charge trapping dielectric behavior) in PP/POE nanocomposites.

\subsection{Materials and Charaterizations}

\subsubsection{Materials}

Fumed silica (Aerosil 200) was obtained from Evonik Industries Germany. The 3-glycidyloxypropyl trimethoxysilane, aminopropyltriethoxy silane and isocyanatopropyltriethoxy silane were purchased from Sigma-Aldrich, US. All other silanes (trimethylethoxysilane, dimethyldiethoxysilane, methyltriethoxysilane, vinyldimethylethoxysilane, phenyldimethylethoxysilane, mercaptopropyltrimethoxysilane) were purchased from Abcr GmbH, Germany, as were trifluoroacetic acid and ammonia. A blend of polypropylene (PP) and poly (ethylene-co-octene) (POE) was used as the polymeric matrix.

The silane modification of silica is described in Section 5.2.2. The different nanocomposites destined for high voltage DC (HVDC) cable insulation were prepared by melt-blending of $1 \mathrm{wt} . \%$ of the reference silica (unmodified) or the modified silicas with a PP/POE $=55: 45$ blend and $0.3 \mathrm{wt} . \%$ of antioxidant using a a twin-screw micro extruder, type Haake MiniLab Rheomix CTW5 (Thermo Fisher Scientific, Waltham, Massachusetts, USA). The compounding was performed at a temperature of $230^{\circ} \mathrm{C}$ using a screw speed of $100 \mathrm{rpm}$ for 4 minutes (min). After melt-blending, the nanocompounds were immediately transferred in molten-state to an injection moulding system Haake MiniJet Pro Piston Injection Moulding System (Thermo Fisher Scientific, Waltham, Massachusetts, USA) and moulded into thin sheets with dimensions of $26 \times 26 \times 0.5 \mathrm{~mm}$.

\subsubsection{Characterization of Silica}

Information on the chemical composition and structure of the surface of the unmodified and modified silicas was obtained by Fourier Transform Infrared Spectrometry (FTIR; Perkin Elmer - Spectrum 100 series) with the resolution of $0.1 \%$ in the range of 400 to $4000 \mathrm{~cm}^{-3}$. Thermogravimetric Analysis thermogravimetric analyzer TGA-7 (Perkin-Elmer, Waltham, Massachusetts, United States) was performed in order to investigate the extent of silanization of the silica. This characterization was done in a synthetic air atmosphere with a heating rate of $20^{\circ} \mathrm{C} / \mathrm{min}$. The temperature range was from ambient temperature up to $850{ }^{\circ} \mathrm{C}$. X-ray Photoelectron Spectroscopy (XPS) was conducted by means of a Scanning X-ray Microscope PHI Quantera (Physical Electronics GmbH, Munich, Germany) to check the chemical composition of the silicas. The silica particle morphology was evaluated by Transmission Electron Microscopy (TEM) and Energy Dispersive X-ray analysis (EDX) using a Transmission Electron Microscope CM300ST-FEG $300 \mathrm{kV}$ (Philips, Eindhoven, the Netherlands). 


\subsubsection{Characterization of Silica/PP/POE Nanocomposites}

The melting and crystallization properties of the nanocomposites were investigated by differential Scanning Calorimetry (DSC) using a DSC Q2000 (TA Instruments, New Castle, Delaware, USA). Samples with a weight of around 12-14 mg were placed in a standard aluminum pan. They were first heated from ambient temperature to $230^{\circ} \mathrm{C}$ at a rate of $10{ }^{\circ} \mathrm{C} / \mathrm{min}$, and kept at this temperature for $5 \mathrm{~min}$ to erase any previous thermal history. The samples were then cooled down to $-20{ }^{\circ} \mathrm{C}\left(40{ }^{\circ} \mathrm{C} / \mathrm{min}\right)$ and heated again to $230{ }^{\circ} \mathrm{C}$ at a rate of $10{ }^{\circ} \mathrm{C} / \mathrm{min}$ The crystalline structure was studied by X-ray Diffraction (XRD) spectra using a X'Pert 1 X-ray diffractometer (Philips, Almelo, The Netherlands). Diffraction spectra in the $2 \theta$ range from $5^{\circ}$ to $37^{\circ}$ with a scanning rate of $0.05 \% / 8 \mathrm{~s}$ were collected. Scanning Electron Microscopy (SEM) was done by means of a MERLIN HR-SEM (Zeiss, Oberkochen, Germany).The sample for SEM was prepared in liquid nitrogen without any coating.

The charge trapping properties of the PP/POE nanocomposites were studied by Thermally Stimulated Depolarization Current (TSDC). Circular gold electrodes (diameter $16 \mathrm{~mm}$, thickness $100 \mathrm{~nm}$ ) were deposited on both sides of the sample sheets by electron-beam evaporation under high vacuum $\left(<1 \times 10^{-6}\right.$ mbar). The TSDC measurement system consisted of a liquid nitrogen-based temperature control system Novocool (Novocontrol Technologies, Montabaur, Germany), a DC high voltage source 2290E-5 (Keithley Instruments, Cleveland, Ohio, USA) and a sensitive electrometer 6517B (Keithley Instruments, Cleveland, Ohio, USA ). The samples were placed in a shielded sample cell equipped with a PT100 temperature sensor (Novocontrol BDS1200HV). The TSDC measurement procedure consisted of the following steps:

1) The samples were heated up to $70^{\circ} \mathrm{C}$ and kept stable for $5 \mathrm{~min}$;

2) A DC electric field of $3 \mathrm{kV} / \mathrm{mm}$ was applied for 20 min under isothermal conditions at $70{ }^{\circ} \mathrm{C}$;

3) The samples were rapidly cooled down to $-50{ }^{\circ} \mathrm{C}$ with the voltage still applied, and kept at this temperature for $5 \mathrm{~min}$ for stabilization;

4) The electric field was removed and the samples were short-circuited through an electrometer. The shortcircuited samples were maintained at $-50{ }^{\circ} \mathrm{C}$ for $3 \mathrm{~min}$ to allow fast polarization to decay;

5) The samples were linearly heated up to $130{ }^{\circ} \mathrm{C}$ with a heating rate of $3{ }^{\circ} \mathrm{C} / \mathrm{min}$. Meanwhile, the depolarization current was recorded.

\subsection{Results}

\subsubsection{Solvent-free Modification of Silica by Silanes}

In this study, trimethylethoxysilane (Figure 5.1) was chosen as the starting modifying agent, as it has only one ethoxy group and therefore does not suffer from side reactions such as condensation. Two kinds of catalysts, ammonia and trifluoroacetic acid, were used to accelerate the silanization reaction to make it take place at room temperature.

The solvent-free modification method was chosen, as it is more environmentally friendly in comparison to solution methods. It was carried out in a sealed glass jar with a magnetic stirrer at room temperature for 24 hours. All the modification variants were performed with the same amount of fumed silica (10 g), 
trimethylethoxysilane $(0.98 \mathrm{~g})$, water $(0.3 \mathrm{~g})$ and fixed amounts of the catalysts (trifluoroacetic acid $(0.2$ g) or ammonia $(1 \mathrm{~g})$ ). After the modification, the product was extracted in a Soxhlet unit with ethanol for 24 hours to remove all contaminants, and afterwards placed in a vacuum oven at a temperature of $80^{\circ} \mathrm{C}$ for 24 hours to remove ethanol.

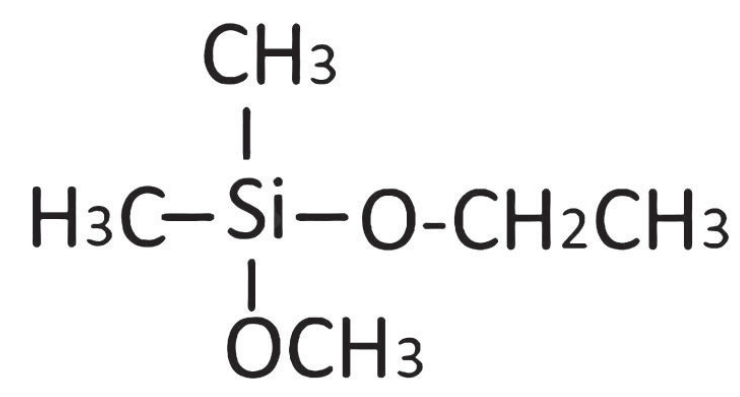

Figure 5.1 Chemical structure of Trimethylethoxysilane.

Five different silica modifications were selected:

$\mathrm{S}$ - unmodified silica;

SS - silica modified with the silane;

SSW - silica modified with the silane in presence of water;

SSWA - silica modified with the silane in presence water and trifluoroacetic acid;

SSWB - silica modified with the silane in presence water and ammonia (base).

\subsubsection{Thermo-Gravimetric Analysis (TGA)}

For quantitative analysis of the modification effects, TGA was performed. The results are depicted in Figure 5.2.

The weight loss of the pure silica (S) was 2 wt.\%; however, in the case of SS and SSW, comparable weight losses of $2.1 \mathrm{wt} . \%$ and $2.4 \mathrm{wt} . \%$ respectively were measured. This indicates that there are barely any organic groups chemically attached to the silica surface in case of SS and SSW silicas. After the functionalization in presence of the catalysts, the weight loss increased to $4.1 \mathrm{wt} . \%$ for SSWA and to 7.2 wt. $\%$ for SSWB. On the basis of the TGA curves, it can be concluded that solvent free silane modification of silica proceeds successfully only when a catalyst is present, regardles of its nature - acidic or basis. However, the kinetics of thermal decomposition of the samples modified in presence of the acid and of the base varies significantly. The modification performed in presence of ammonia results in a high amount of volatiles and relatively loosely bound surface-deposition; it shows a constant decrease of mass from ca. $100{ }^{\circ} \mathrm{C}$ to $500{ }^{\circ} \mathrm{C}$. This might be due to the physical adsorption of the base onto the silica surface through hydrogen bonds, which are not stable and will be decomposed at lower temperature. 


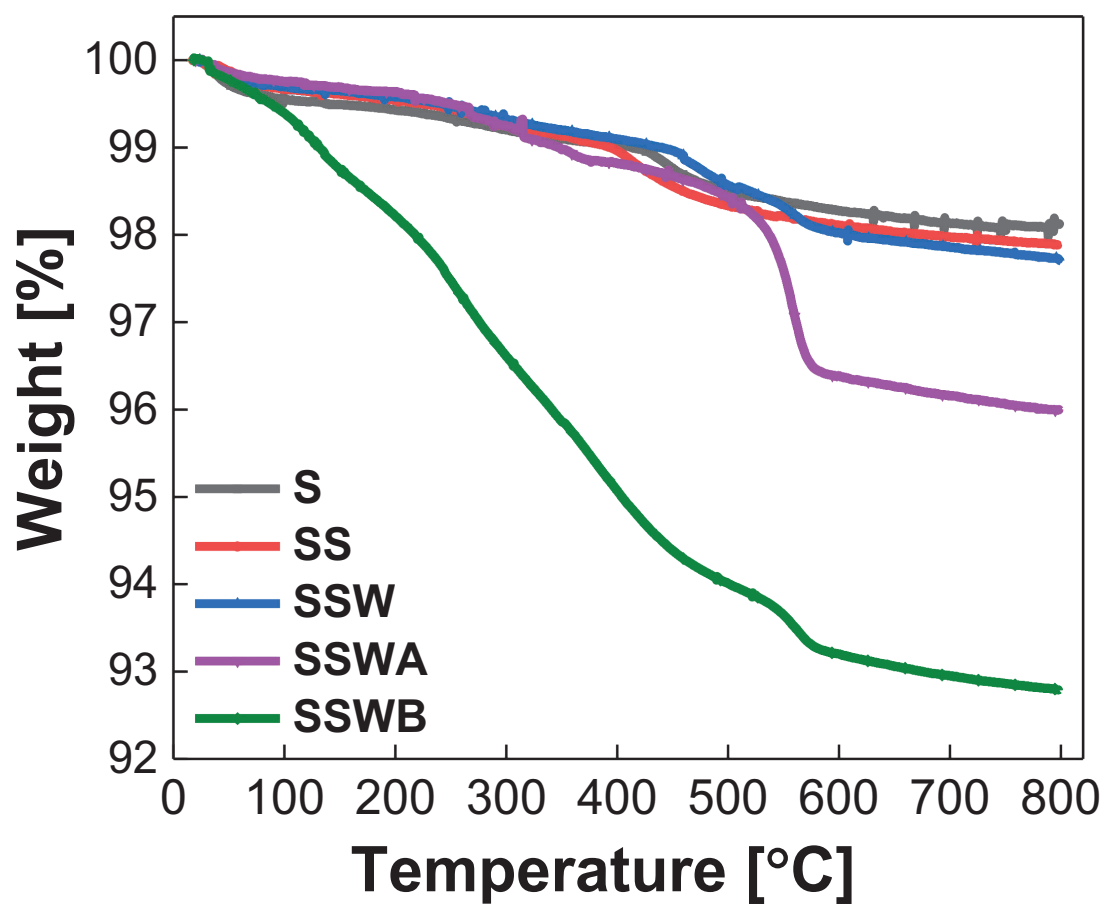

Figure 5.2 TGA results of the unmodified and modified silicas.

\subsubsection{Fourier Transform Infrared (FTIR)}

Fourier Transform Infrared (FTIR) analysis was performed on the unmodified silica and modified silica samples. This technique allows to identify organic groups by measuring the absorption of infrared radiation by the sample material within a certain spectrum of wavelengths. The infrared absorption bands are characteristic for certain molecular components and structures.

Figure 5.3 compares the spectra of unmodified silica (S) and modified silicas (SS, SSW, SSWA and SSWB). A new band in the region of $2963 \mathrm{~cm}^{-1}$ appeared after the silane modifications. This band represents the $\mathrm{C}-\mathrm{H}$ stretching [18] which comes from the trimethylethoxysilane. The stronger the band intensities, the higher the modification effect. As seen in Figure 5.3, the band became stronger with the addition of the catalysts. These results are in agreement with the results of the TGA analysis, indicating that there is indeed a C-H organic layer covering the silica surface after the solvent-free modification. The reaction mechanism of the silica-silane modification is shown in Figure 5.4. 


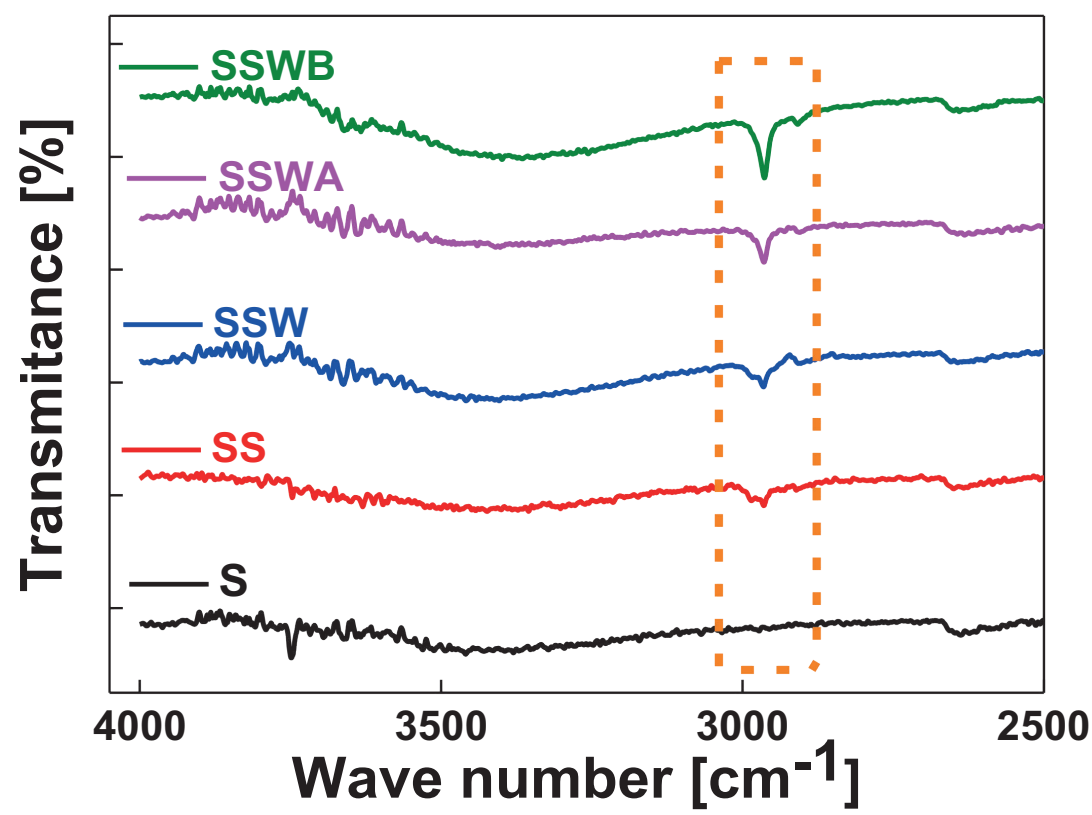

Figure 5.3 FTIR spectra of the unmodified and modified silicas.

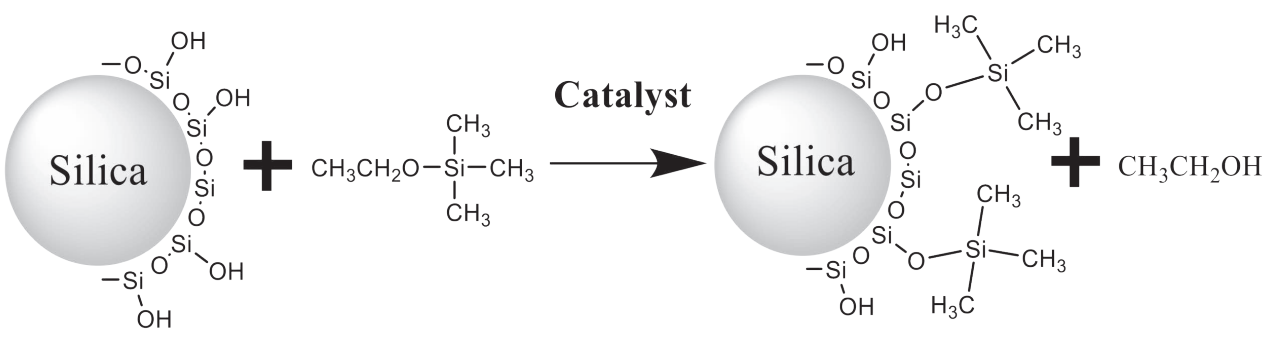

Figure 5.4 Reaction scheme of the solvent-free silica-silane modification.

\subsubsection{X-ray Photoelectron Spectroscopy (XPS)}

In order to further evaluate the modification of silica, X-ray Photoelectron Spectroscopy (XPS) was performed on the unmodified silica (S) and modified silicas (SSWA and SSWB). XPS works on the basis of irradiating a material with a beam of X-rays while simultaneously measuring the kinetic energy and number of electrons that escape from the surface $(0$ to $10 \mathrm{~nm})$ of the analyzed material. Thus, it is possible to measure the elemental composition of a very thin surface layer in a parts per thousand range. The XPS results are presented in Figure 5.5 and Table 5.1. The carbon, oxygen and silicon atom spectra and their content are shown. 

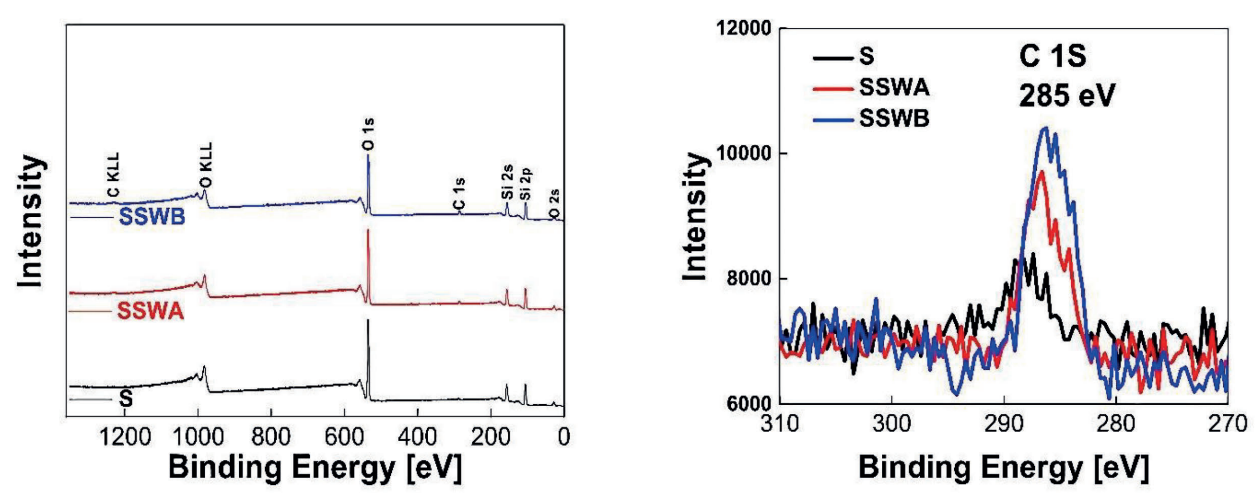

Figure 5.5 XPS spectra of the unmodified (S) and modified silicas (SSWA, SSWB).

Table 5.1 Chemical composition of the surface of unmodified and modified silicas by XPS.

\begin{tabular}{|l|c|c|c|}
\hline Element & C & O & Si \\
\hline S / atom\% & 1.8 & 69.2 & 28.9 \\
\hline SSWA / atom\% & 5.4 & 64.6 & 29.9 \\
\hline SSWB / atom\% & 8.8 & 61.7 & 29.4 \\
\hline
\end{tabular}

Both, SSWA and SSWB, exhibited an increased C1s signal at a binding energy of ca. $280 \mathrm{eV}$ in the XPS spectrum (Figure 5.5) demonstrating the presence of carbon from the silane molecules. This corresponds to the results obtained from FTIR and TGA measurements. The small carbon signal present in the reference spectra $(\mathrm{S})$ comes from atmospheric contaminations deposited on the silica during sample preparation. The oxygen signal (O1s) originates from the silica structure. The decreased O1s signal for the SSWA and SSWB samples versus the S sample at ca. $530 \mathrm{eV}$ also confirms a succesful surface modification. The decreased oxygen content observed in the silane modified silica samples is a result of the presence of the methyl groups, which are bond to a silicone atom in the silane molecule. This increases the amount of carbon in the surface layer of the modified samples covering the amorphous $\mathrm{SiO}_{2}$ particles.

The silicon signal ( $\mathrm{Si} 2 \mathrm{p}$ ) intensity is similar for unmodified and silane-coated silicas. This is an effect of two simultaneous processes: attachment of silicon atoms with the deposition of silane molecules and covering of the $\mathrm{SiO}_{2}$ particles. In summary, the effect of enriching the surface layer with silicone atoms seems to be more efficient resulting in a slight increase of the silicone amount (Table 5.1). Altogether, TGA, FTIR and XPS proved a successful silica-silane modification via the solvent-free modification.

In conclusion, the solvent-free method is an effective and sustainable approach to perform silica-silane modification using an acidic or alkaline catalyst. In general, the silica modified using the base catalyst (SSWB) exhibit a higher modification level in comparison with the acid catalyzed silica (SSWA). However, the modification performed on SSWB is significantly less thermally stable, based on the TGA results: the onset of the mass loss of SSWB starts from $100{ }^{\circ} \mathrm{C}$, while it is $500{ }^{\circ} \mathrm{C}$ for SSWA. Therefore, trifluoroacetic acid is selected as a catalyst for the silane-silica modification. 
5.3.2 Tailoring the silica surface with different silanes via the solvent-free method

The solvent-free method was performed on fumed silica using 8 different silanes. The investigated silanes were divided into three groups:

The above classification aims at verifying the effectiveness of the modification by the solvent-free method and at producing different silicas with varied surface properties in order to study their influence on dielectric properties of nanocomposites. For purification, the samples were put into a vacuum oven at $80{ }^{\circ} \mathrm{C}$ to remove the residues, instead of extraction and oven drying.

I. Aliphatic silanes with different numbers of alkoxy groups (Table 5.2).

Table 5.2 Silanes from Group I.

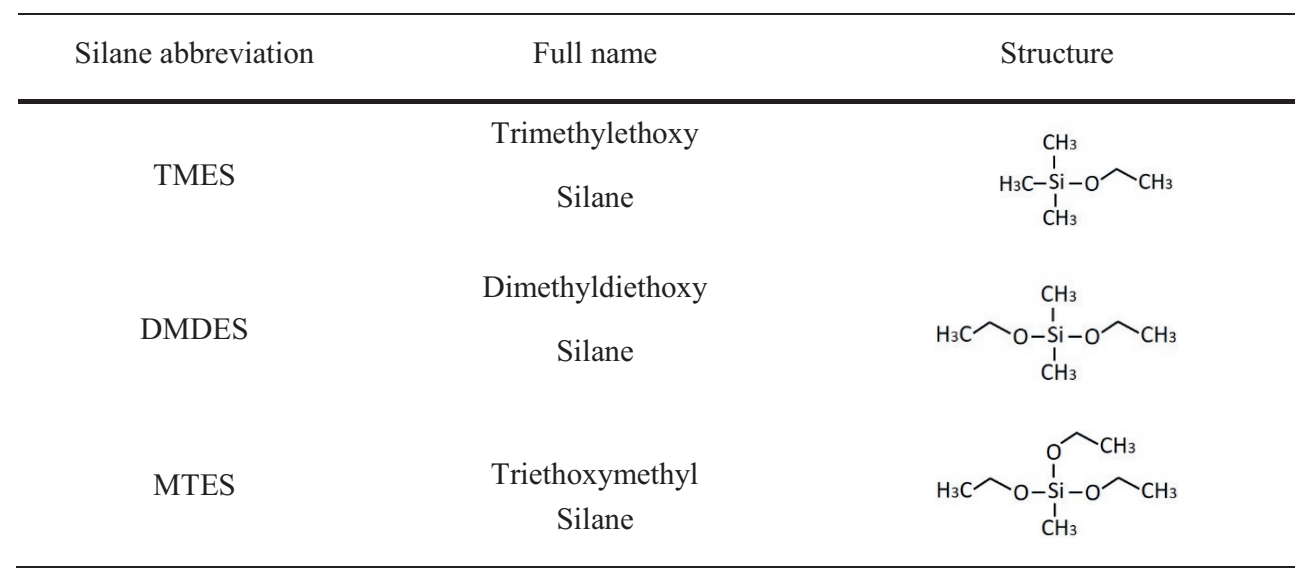

II. Hydrocarbon silanes containing delocalized electron clouds (Table 5.3).

Table 5.3 Silanes from Group II.

\begin{tabular}{|c|c|c|}
\hline Silane abbreviation & Full name & Structure \\
\hline VDMES & $\begin{array}{l}\text { Vinyldimethylethoxy } \\
\text { Silane }\end{array}$ & $\begin{array}{c}\mathrm{CH}_{3} \\
1 \\
\mathrm{H}_{3} \mathrm{C}-\mathrm{Si}-\mathrm{CH}=\mathrm{CH}_{2} \\
1 \\
\mathrm{O}_{2} \mathrm{CH}_{2} \mathrm{CH}_{3}\end{array}$ \\
\hline PDMES & $\begin{array}{l}\text { Phenyldimethylethoxy } \\
\text { Silane }\end{array}$ & 等3 \\
\hline
\end{tabular}


III. Polar silanes containing hetero elements (nitrogen, sulfur or oxygen) (Table 5.4).

Table 5.4 Silanes from Group III.

\begin{tabular}{|c|c|c|}
\hline Silane abbreviation & Full name & Structure \\
\hline HS-silane & $\begin{array}{l}\text { Mercaptopropyltrimethoxy } \\
\text { Silane }\end{array}$ & - \\
\hline Epoxy-silane & $\begin{array}{l}\text { 3-Glycidyloxypropyl } \\
\text { trimethoxysilane }\end{array}$ & $\mathrm{OCH}_{3}$ \\
\hline Amino-silane & $\begin{array}{c}\text { Aminopropyltriethoxy } \\
\text { Silane }\end{array}$ & 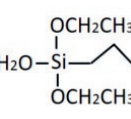 \\
\hline NCO-silane & $\begin{array}{l}\text { Isocyanatepropyltriethoxy } \\
\text { Silane }\end{array}$ & 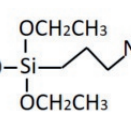 \\
\hline
\end{tabular}

The amount of the silane added for the modification is shown in Table 5.5. It is based on Equation (5.1):

$$
\mathrm{m}(\text { silane })=\mathrm{m}(\text { silica }) * \mathrm{~S}(\text { silica }) * \mathrm{~N}(\mathrm{Si}-\mathrm{OH}) * \mathrm{M}(\text { silane }) / \mathrm{NA}
$$

where:

$\mathrm{m}($ silane $)=$ amount of silane used for the solvent-free modification;

$\mathrm{m}($ silica $)=20 \mathrm{~g}$ is the amount of silica used for the solvent-free modification;

$\mathrm{S}($ silica $)=200 \mathrm{~m}^{2} / \mathrm{g}$, the specific surface area of the silica;

$\mathrm{N}(\mathrm{Si}-\mathrm{OH})=2.5 / \mathrm{nm}^{2}$, the number of silanol groups on the silica surface per $1 \mathrm{~nm}^{2}$;

$\mathrm{M}($ silane $)=$ molecular weight of a silane;

$\mathrm{NA}=6.022 \times 10^{23}$, Avogadro constant.

The calculation is based on two assumptions:

1. One silane molecule will react with one silanol group on the silica surface.

2. All the silanol groups on the silica surface will have reacted with the silane. 
Table 5.5 Recipe for silane-silica solvent free modification.

\begin{tabular}{|c|c|c|c|c|}
\hline & Silane/g & Silica/g & Water/g & Acid/g \\
\hline TMES & 1.96 & & & \\
\hline DMDES & 2.46 & & & \\
\hline MTES & 2.94 & & \multirow{2}{*}{20.60} & 0.40 \\
\hline VDMES & 2.15 & & & \\
\hline PDMES & 2.98 & & & \\
\hline Epoxy-silane & 3.92 & & & \\
\hline HS-silane & 3.25 & & & \\
\hline NCO-silane & 4.10 & & & \\
\hline Amino-silane & 3.67 & & &
\end{tabular}

\subsubsection{Thermo-Gravimetric Analysis (TGA)}

The TGA curves of the modified silicas are shown in Figure 5.6 (Group I (a), Group II (b) and Group III
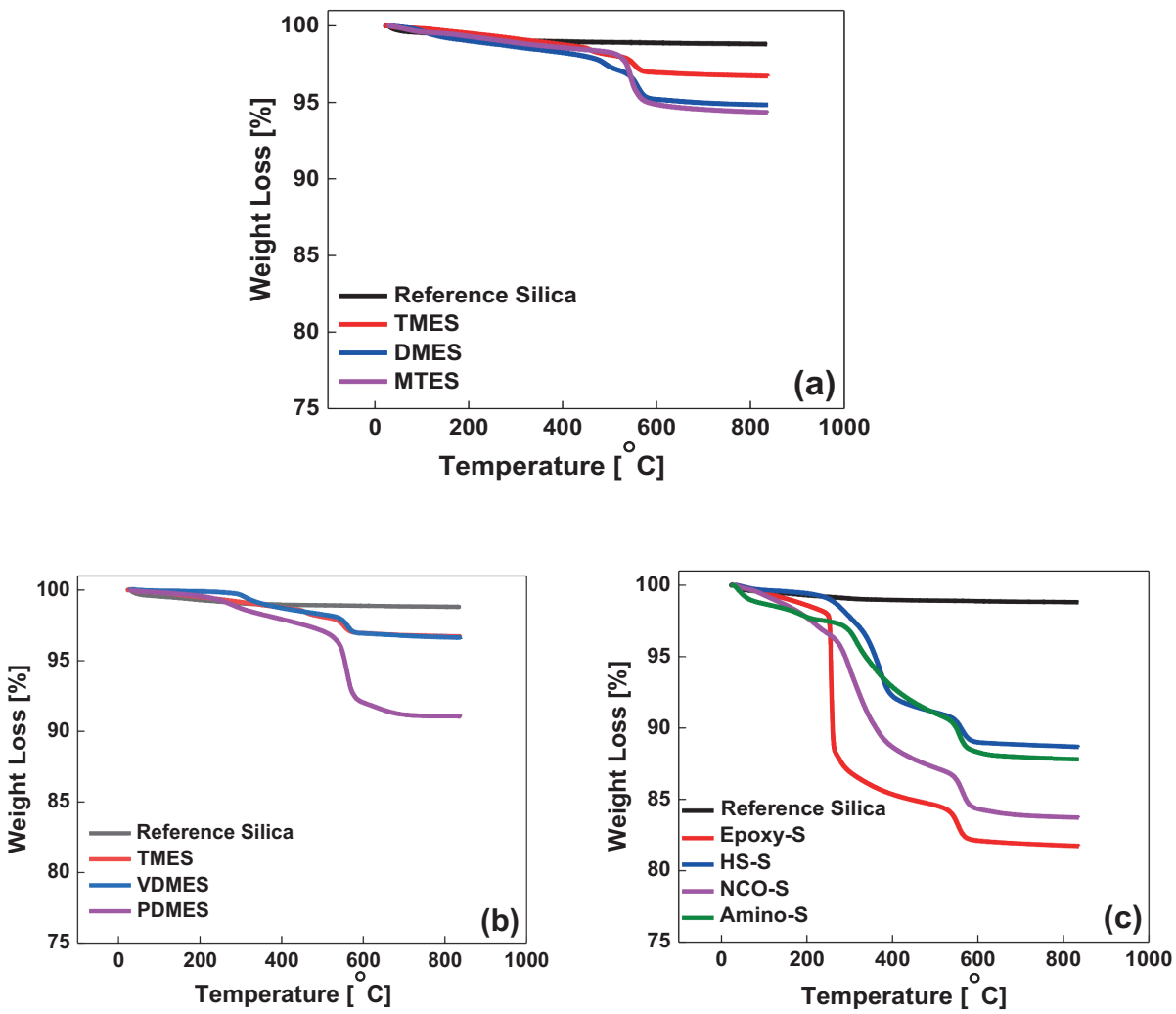

Figure 5.6 TGA curves of unmodified and modified silicas treated with silanes from different groups (Group I (a), Group II (b) and Group III (c)). 
(c)). The weight loss attributes to the removal of the molecules from the silica surface. The weight loss (c)). The weight loss attributes to the removal of the molecules from the silica surface. The weight loss below $100{ }^{\circ} \mathrm{C}$ results from water and unreacted alkoxy groups present on silica surface which undergo condensation [19]. The breakage of Si-C bonds mostly contributes to the weight loss between $300 \sim 450{ }^{\circ} \mathrm{C}$ [20]. With increasing temperature, the condensed organic polymer on silica surface starts degradation which leads to the weight loss at temperatures higher than $450{ }^{\circ} \mathrm{C}[20,21]$.

Comparing the weight loss of silica modified with the silanes from Group I, it is clear that using molecules containing more than one alkoxy group results in a higher degree of deposition. This is most likely due to a condensation reaction of the alkoxy groups leading to silane oligomerization, thus enhanced surface covering. In Group II, the silica modified with PMDES showed noticeably higher weight loss resulting from the higher molecular weight of the phenyl group of the silane. The silanes with different polar moieties in Group III all have three alkoxy groups and a slightly longer side chain than the one in Group I. This caused a much higher weight loss during the TGA tests as shown in Figure 5.6. It is worth noting that utilization of the 3-glycidyloxypropyltrimethoxy silane (epoxy-S) and isocyanatepropyltriethoxy silane (NCO-S) for modification resulted in a deposition exceeding $15 \%$ of the treated silica mass. This is an exceptional result in comparison to the state of the art methods presented in the literature, in which the deposition level usually does not exceed $10 \mathrm{wt} . \%$ [22]. Higher levels of silica modification usually require a complex, multi-step procedure [23].

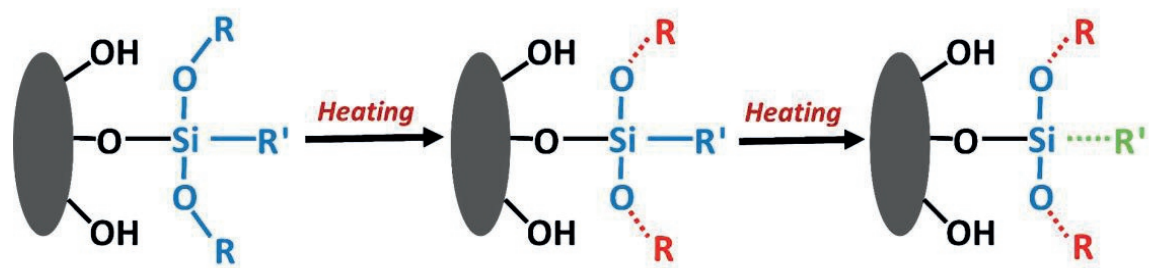

Figure 5.7 Mechanism of thermal degradation of a silane grafted on silica.

(During heating, the unreacted alkoxy groups marked in red will leave firstly; with further increasing temperature, the Si-R' bond of the side chain from the silane marked in green will be broken.)

To get further quantitative insight into the modification degree of the solvent-free method, the molar amount of grafted silane was calculated based on the dquation (5.2) [24]:

$$
\text { Grafted amount }(\mathrm{mmol} / \mathrm{g})=\frac{10^{3} \mathrm{~W}}{\mathrm{M}(100-\mathrm{W})}
$$

where:

$\mathrm{W}=$ weight difference between the weight at $100^{\circ} \mathrm{C}$ and $750{ }^{\circ} \mathrm{C}$;

$\mathrm{M}=$ molecular weight of a side chain of a silane. 
This equation was introduced by W. He et al. [24]. However, the whole molecular weight of a silane was considered to be equal to $\mathrm{M}$ by the authors. This is not accurate considering the mechanism of thermal detachment of the silane molecules from a silica surface (Figure 5.7). Silane molecules form strong covalent bonds ( $\mathrm{Si}-\mathrm{O}-\mathrm{Si}$ ) with the silica surface. This bond is very unlikely to break during a TGA test. Instead, at lower temperatures residual alkoxy groups are removed (C-O bond), whereas at higher temperatures breakage of Si-C bond occurs. During water and acid catalyzed silanization, we assume that all the alkoxy groups are hydrolyzed to form silanol groups (Si-O-H). Therefore, only the removal of a side chain of a silane contributes to the weight loss during TGA measurements. This gives a strong indication that the molecular weight of the silane side chain should be taken into consideration in the above equation rather than the whole silane molecular weight. This is also confirmed by other researchers [25].

The results of the calculated amount of deposited silanes are shown in Figure 5.8 (right). They are noticeably different than the TGA total weight losses shown in Figure 5.8 (left). While the TGA weight loss in Figure 5.8 (left) exhibited a correlation with the molecular weight of the side chain in the silane, the grafted amounts in Figure 5.8 (right) were more dependent on the number of reactive alkoxy groups.
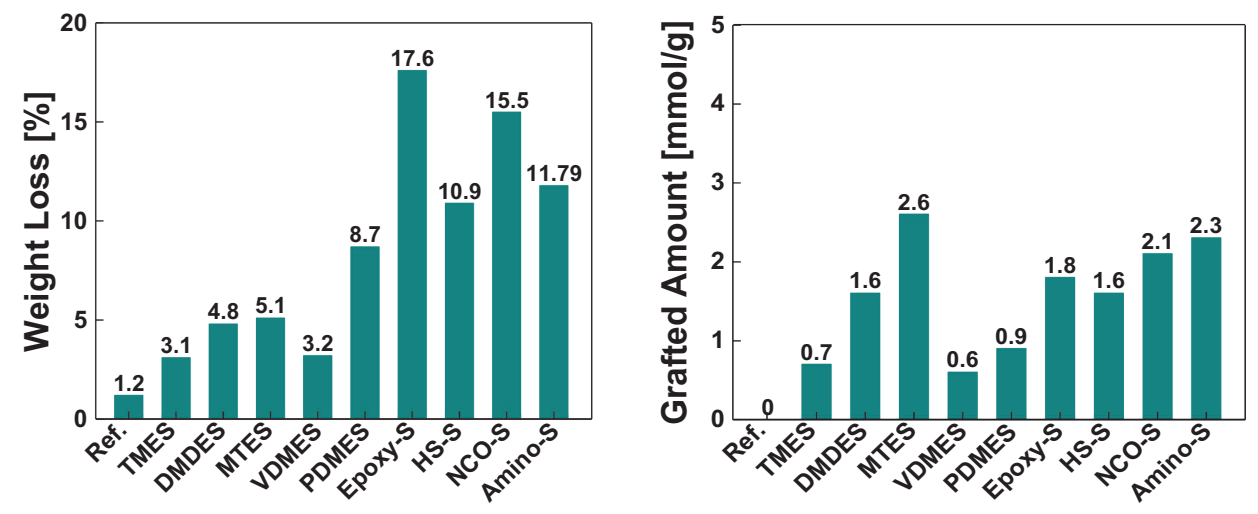

Figure 5.8 The weight loss of the modified silicas (left) and molar amount of grafted silanes on silica surface (right).

These results show that there is almost the same amount of silane (in mmol) grafted onto the silica surface when modified with a silane containing only one alkoxy group $(0.7 \mathrm{mmol} / \mathrm{g}$ of TMES, $0.6 \mathrm{mmol} / \mathrm{g}$ of VDMES, $0.9 \mathrm{mmol} / \mathrm{g}$ of PDMES). However, the total weight loss of PDMES modified silica gave a much higher value ( $8.7 \%$ ) than the silicas modified with the two other silanes (1.2 wt.\% of TMES and $3.2 \mathrm{wt} . \%$ of VDMES) due to the much higher molecular mass of the phenyl ring. Furthermore, regarding to the silanes containing three alkoxy groups (MTES, epoxy-silane, HS-silane, NCO-silane and amino-silane), the degree of modification was more or less on the same level (from $1.6 \mathrm{mmol} / \mathrm{g}$ to $2.6 \mathrm{mmol} / \mathrm{g}$ ), while there is a significant difference in the total weight loss results (from $5.1 \mathrm{wt} . \%$ to $17.6 \mathrm{wt} . \%$ ).

In summary, a higher TGA weight loss does not directly equal to a more effective silane modification on the silica surface. To compare the degree of modification, the grafted amount of silane in $\mathrm{mmol} / \mathrm{g}$ should 
be calculated. Nevertheless, all the above results show that the solvent-free method is an efficient way to perform silica-silane modification.

\subsubsection{Fourier Transform Infrared (FTIR)}

The FTIR spectra of unmodified and modified silicas are shown in Figure 5.9. The band at $2956 \mathrm{~cm}^{-1}$ is attributed to the stretching vibration of $\mathrm{C}-\mathrm{H}$ [26]. This proves that TMES, DMDES and MTES were successfully grafted onto silica surface.

The bands at $3100 \mathrm{~cm}^{-1}$ and $1429 \mathrm{~cm}^{-1}$ presented in Figure 5.9 (c) and (d) result from the aromatic ring of PMDES [26, 27]. The presence of the vinyl group can be proven by the absorption band at $1593 \mathrm{~cm}^{-1}$ [26].

The bands at 2989 2843 $\mathrm{cm}^{-1}$ corresponding to the $\mathrm{C}-\mathrm{H}$ [28] vibrations shown in Figure 5.9 (e) demonstrate, that all four polar silanes are grafted onto the silica surface. The intensive $\mathrm{C}-\mathrm{H}$ stretching band in Figure 5.9 (e) indicates the presence of the epoxy silane on the silica surface due to the high number of $\mathrm{CH}_{2}$ units in the chemical structure of this silane, although the bands from the epoxy ring group were not detected [29]. The broad band around $1631 \mathrm{~cm}^{-1}$ visible in Figure 5.9 (f) and assigned to N-H stretching belongs to the amine group of the amino silane [28]. Due to the sensitivity of the $\mathrm{N}=\mathrm{C}=\mathrm{O}$ group to water, it can be easily turned into a urethane group. Therefore, the bands at $1600 \sim 1500 \mathrm{~cm}^{-1}$ from the distortion oscillation of N-H and stretching oscillation of C-N, and the band at 1800-1600 $\mathrm{cm}^{-1}$ from stretching oscillation of $\mathrm{C}=\mathrm{O}$ represent the presence of urethane, which proves the $\mathrm{NCO}$-silane grafting onto the silica surface and its further reaction with water to form urethane groups.

It needs to be admitted that it is difficult to detect a S-H band at $2568 \mathrm{~cm}^{-1}$, because it gives a very weak response in FTIR [28]. Nevertheless, all above results can prove that all the silanes were successfully grafted onto the silica surface through solvent-free modification.

\subsubsection{Transmission Electron Microscopy (TEM) with Elemental Mapping}

To visualize the morphology of a silica surface with silane grafted onto it, TEM elemental mapping was carried out on the silica modified with amino-silane (Figure 5.10). It shows that the average size of the primary particles of the modified silica is around $20 \mathrm{~nm}$, which is more or less as same size as untreated fumed silica particles. Elemental mapping was performed in order to identify the distribution of carbon (Figure 5.10 (b)) and silicon (Figure 5.10 (c)) on the silica surface after modification. Figure 5.10 (d) reveals the silane layer depicted in green with a smooth surface and a thickness of approximately $1 \mathrm{~nm}$. 

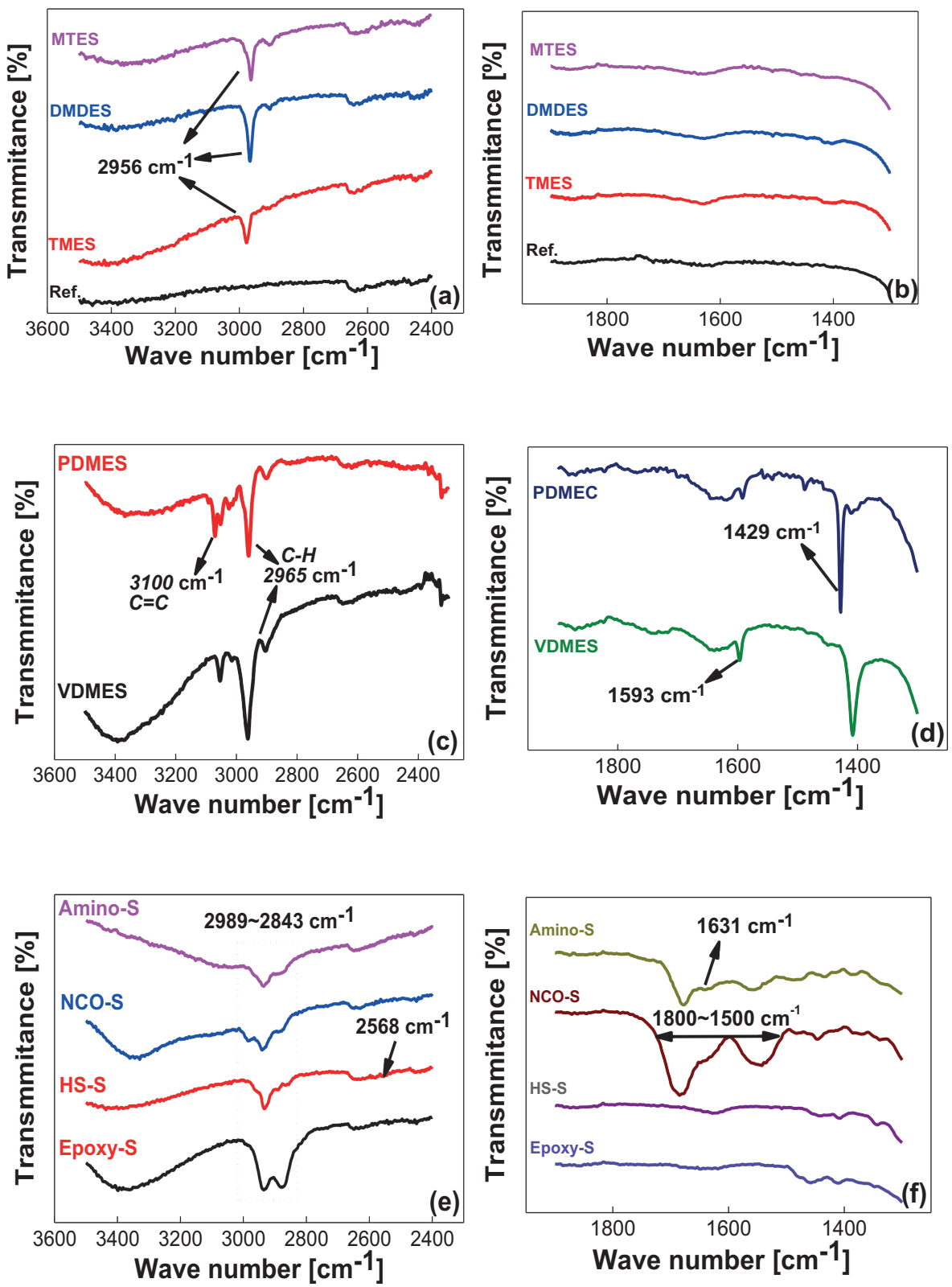

Figure 5.9 FTIR spectra of unmodified and modified silicas with the silanes belonging to Group I (a, b), Group II (c, d) and Group III (e, f). 

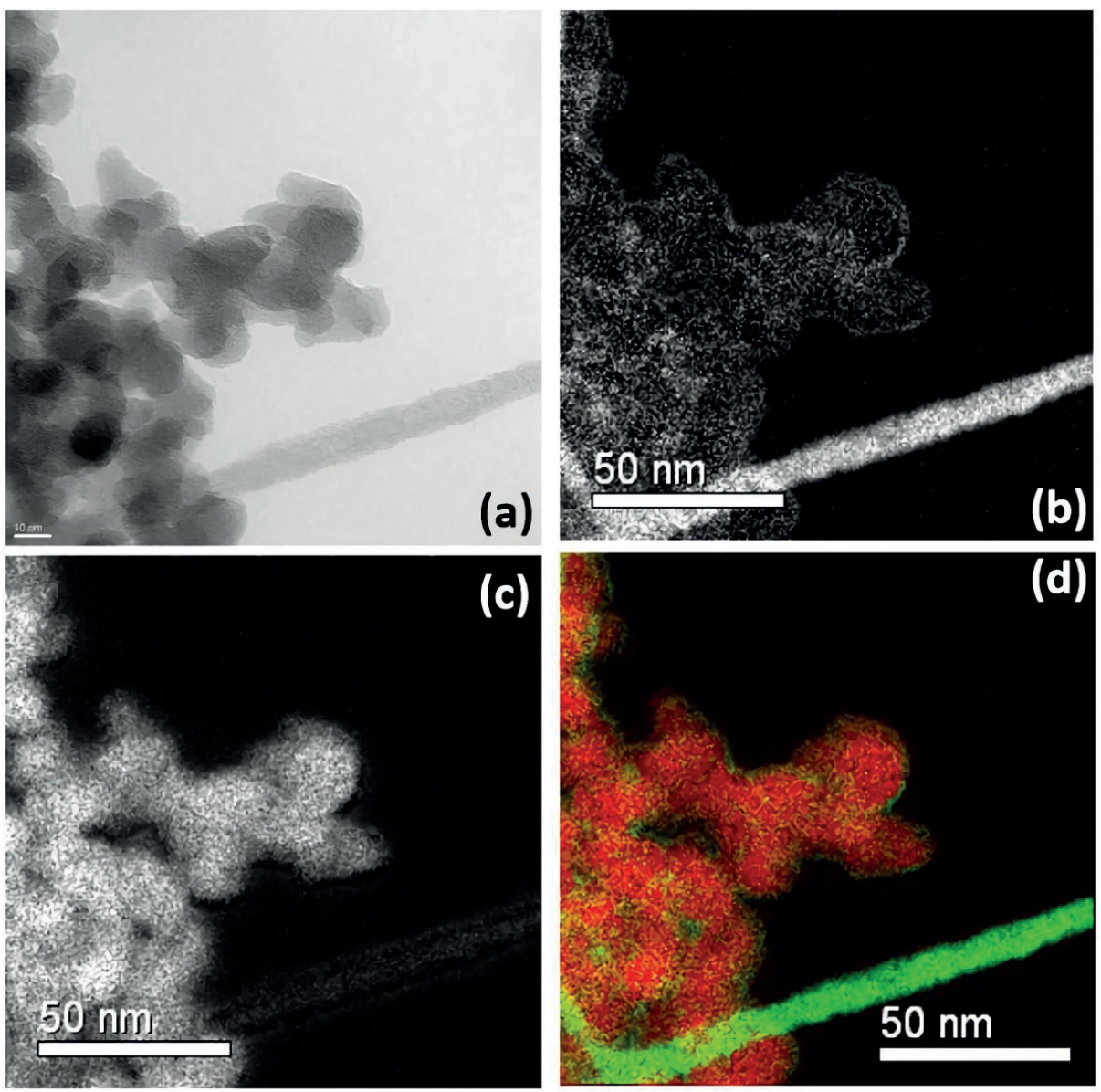

Figure 5.10 TEM elemental mapping image of silica modified with amino silane.

(a: TEM image of modified silica; b: carbon mapping; c: silicon mapping; $d$ : combined mapping images of carbon in green and silicone in red.))

\subsubsection{Characterization of PP/POE of nanocomposites}

\subsubsection{Differential Scanning Calorimetry (DSC)}

Figure 5.11 and Figure 5.12 represent the crystallization and melting behaviour of all PP/POE nanocomposites, respectively. The crystallization parameters are shown in Table 5.6. It is obvious that there are two melting and crystallization peaks corresponding to the two polymer phases. In accordance with the previous results [30], the first peak at the lower temperature originates from the POE phase, in which the crystalline PE domains melt (or crystallize), while the second peak at higher temperature comes from the PP phase.

In general, the modified silica has a more pronounced effect on the crystallization behaviour of both phases, PP and POE, than unmodified silica. The exothermic peak of the PP and POE phase is shifted to 
higher temperature values, suggesting a strong nucleating effect of the modified silicas [31,32]. This also corresponds to literature, stating that silica can act as a heterogeneous nucleating agent because of the high specific surface area [33]. According to literature, the new silica/polymer interface reduces the nuclei size needed for crystal growth. This is due to the creation of an interface between the polymer crystal and the substrate, which might be less hindered than the formation of the corresponding free polymer morphology [34].
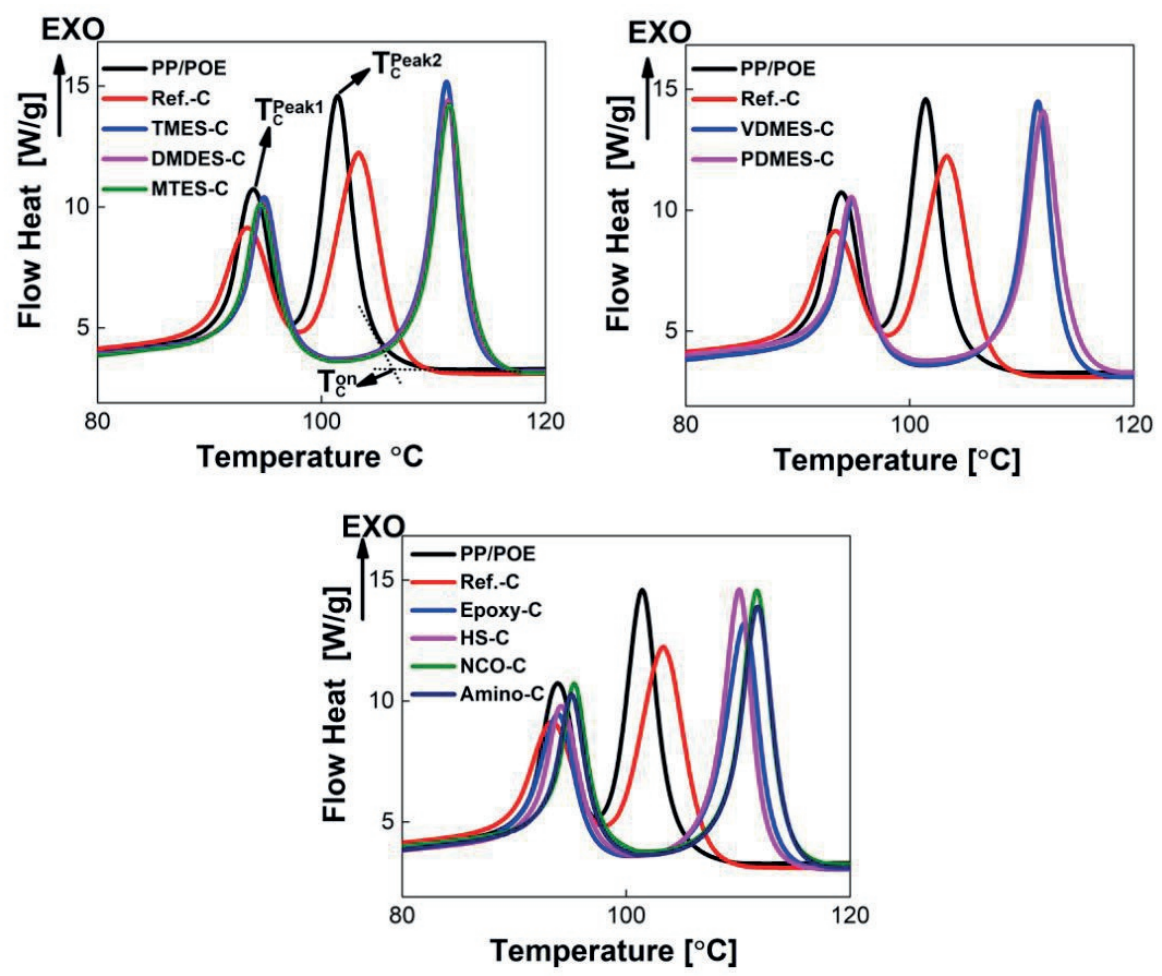

Figure 5.11 DSC crystallization curves for PP/POE nanocomposites with unmodified silica (Ref.-C) and silica modified with the silanes described in Tables 5.2-5.4.

However, the nucleating effect is different in the separate PP and POE phases. There is a significant shift of the crystallization onset temperature of approx. $10{ }^{\circ} \mathrm{C}$ with regard to the $\mathrm{T}_{\mathrm{C}}$ Peak 2 of the PP phase compared to a smaller shift of approx. $1{ }^{\circ} \mathrm{C}$ of $\mathrm{T}_{\mathrm{C}}{ }^{\text {Peak }} 1$ of the POE phase: this indicates that the silica exhibits a more pronounced effect on the PP phase than on the POE phase. One of the reasons for it is the favoured location of silica in the PP phase caused by the lower viscosity of the polymer compared to POE.

It is also worth noting that the silicas modified with the different types of silanes also exhibit different levels of impact on the crystallization behaviour of the PP/POE composites. The silicas modified with unpolar silanes (TMES, DMDES, MTES, VDMES and PDMES) exhibit a consistent shift on the exothermic peak shown in Table 5.6 and marked in red font, while the silicas modified with the polar 
silanes (HS-silane, epoxy-silane, NCO-silane and amino-silane) give various values of temperature shift. This is most likely due to the differences in silica/polymer compatibility. The silicas modified with unpolar silanes exhibit better compatibility with the polymer blend than the silicas modified with polar silanes. Moreover, the PP phase crystallization temperature also varies with polarity of the silane and their chemical nature.

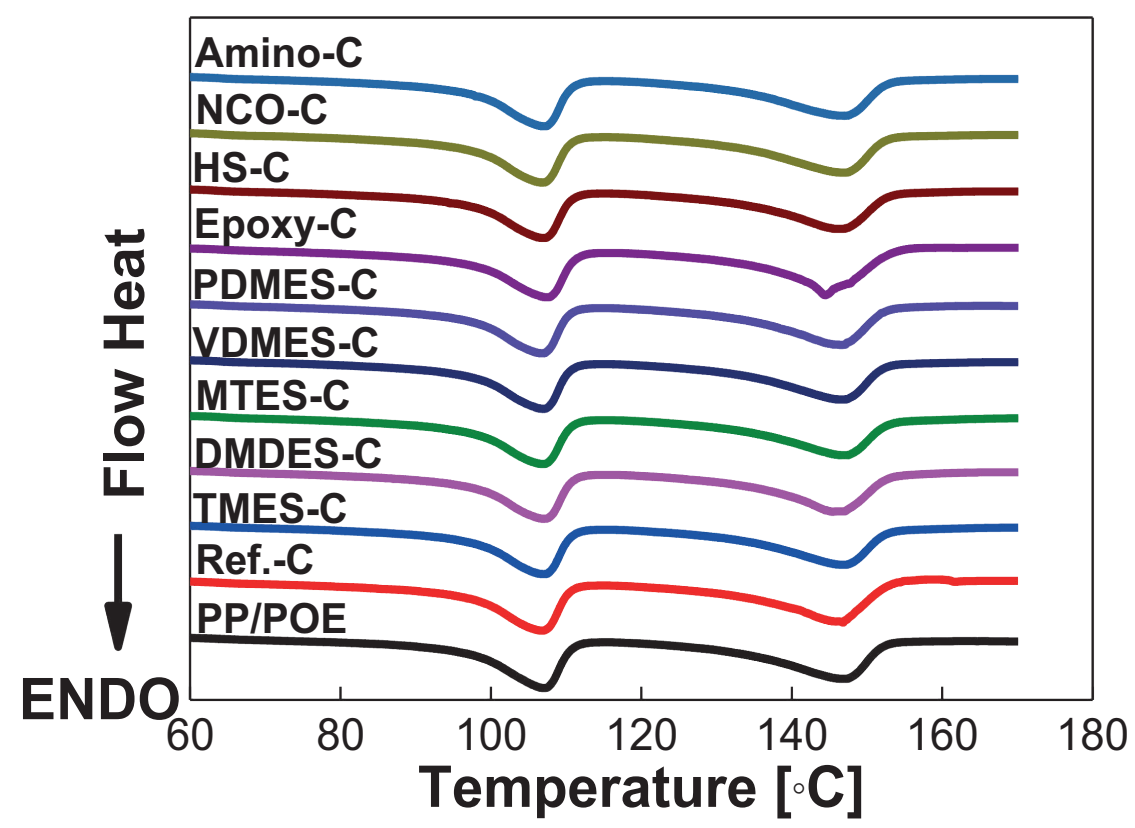

Figure 5.12 DSC melting curves for PP/POE nanocomposites.

Crystallinity was additionally calculated from DSC melting curves based on equation (5.3):

$$
\mathrm{X}_{\mathrm{C}}=\frac{\Delta \mathrm{H}_{\mathrm{C}}}{\mathrm{w} \Delta \mathrm{H}_{100}} 100
$$

where,

$\Delta \mathrm{H}_{\mathrm{C}}=$ heat of crystallization $(\mathrm{J} / \mathrm{g})$

$\mathrm{W}=$ weight percent of the polymer.

$\Delta \mathrm{H}_{100}$ is the heat of crystallization of the hypothetical $100 \%$ crystalline polymer. PP and POE blends are used in our study; POE is a copolymer containing PE as crystalline phase. In this equation, the $\Delta \mathrm{H}_{100}$ value of $209 \mathrm{~J} / \mathrm{g}$ for PP [35] and $293 \mathrm{~J} / \mathrm{g}$ for PE [36] were used. The results are presented in Table 5.6. Compared to the neat $\mathrm{PP} / \mathrm{POE}$, the crystallization energy of the PP/POE nanocomposites are rather similar, indicating that the degree of crystallinity of PP/POE was not changed. 
Table 5.6 DSC crystallization results for PP/POE nanocomposites.

\begin{tabular}{lccccc}
\hline Sample & $\mathrm{T}_{\mathrm{C}}{ }^{\text {peak1 }}\left({ }^{\circ} \mathrm{C}\right)$ & $\mathrm{T}_{\mathrm{C}}{ }^{\text {peak2 }}\left({ }^{\circ} \mathrm{C}\right)$ & $\mathrm{T}_{\mathrm{C}}{ }^{\text {on }}\left({ }^{\circ} \mathrm{C}\right)$ & $\Delta \mathrm{H}_{\mathrm{C}}(\mathrm{J} / \mathrm{g})$ & $\mathrm{X}_{\mathrm{C}}(\%)$ \\
\hline PP/POE & 93.8 & 101.4 & 104.1 & 71.1 & 28.5 \\
Ref-C & 93.3 & 103.3 & 107.0 & 70.6 & 28.3 \\
TMES-C & 94.9 & 111.1 & 113.2 & 69.9 & 28.0 \\
DMDES-C & 94.7 & 111.2 & 113.5 & 69.8 & 27.9 \\
MTES-C & 94.6 & 111.4 & 113.8 & 69.5 & 27.8 \\
VDMES-C & 94.9 & 111.4 & 113.7 & 69.2 & 27.7 \\
PDMES-C & 94.7 & 111.8 & 114.5 & 70.3 & 28.2 \\
Epoxy-C & 93.9 & 110.6 & 113.1 & 68.9 & 27.6 \\
HS-C & 94.0 & 110.0 & 112.3 & 69.2 & 27.7 \\
NCO-C & 95.4 & 111.7 & 114.3 & 68.8 & 27.6 \\
Amino-C & 95.1 & 111.7 & 114.1 & 70.2 & 28.1 \\
\hline
\end{tabular}

$\mathrm{T}_{\mathrm{C}}^{\text {peak1 }}:$ crystallization peak temperature of the POE phase;

$\mathrm{T}_{\mathrm{C}}{ }^{\text {peak2 }}$ : crystallization peak temperature of the PP phase;

$\mathrm{T}_{\mathrm{C}}$ : : onset of crystallization peak of the PP phase;

$\Delta \mathrm{H}_{\mathrm{C}}$ : total heat during the crystallization process;

$\mathrm{X}_{\mathrm{C}}$ : calculated crystallinity of $\mathrm{PP} / \mathrm{POE}$ nanocomposites.

Marked in red exothermic peak temperature of PP/POE filled with unpolar silica.

Marked in blue: exothermic peak temperature of PP/POE filled with the polar silica.

The melting curves of all samples are shown in Figure 5.12. Compared to the crystallization temperature, there is no significant difference of melting temperature between neat PP/POE and silica-filled PP/POE composites; this effect was also reported in the literature [37].

\subsubsection{X-ray Diffraction $(X R D)$}

In order to gain a better understanding of the influence of the modified silicas on the crystallization behaviour of the PP/POE nanocomposites, XRD measurements were conducted on all the samples. The results are shown in Figure 5.13. Three different crystal phases ( $\alpha$ and $\beta$ crystals of the PP phase and orthorhombic crystals from the PE phase) are detected, as have been reported in previous work [30]. It is important to notice that no obvious increase of the $\beta$ crystal content in the silica filled PP/POE composites was recorded (both untreated and treated silicas). These results are similar to our previous findings, while it is different from results reported in literature: nanofillers to act as nucleating agents increasing the amount of $\beta$ crystals under favourable crystallization conditions, [33]. Moreover, the degree of overall crystallinity and the PP phase crystallinity were calculated by dividing the total area of the crystalline peaks by the corresponding area of all peaks; and the results are shown in Table 5.7. There is no significant difference in the crystalline phase content for any of the nanocomposites, which is in line with the DSC results. In summary, $1 \mathrm{wt} . \%$ of silica does not significantly affect the degree of crystallinity; however, it influences strongly the crystallization behaviour of PP/POE composites with an emphasis on the PP phase, where it acts as a strong nucleating agent. 

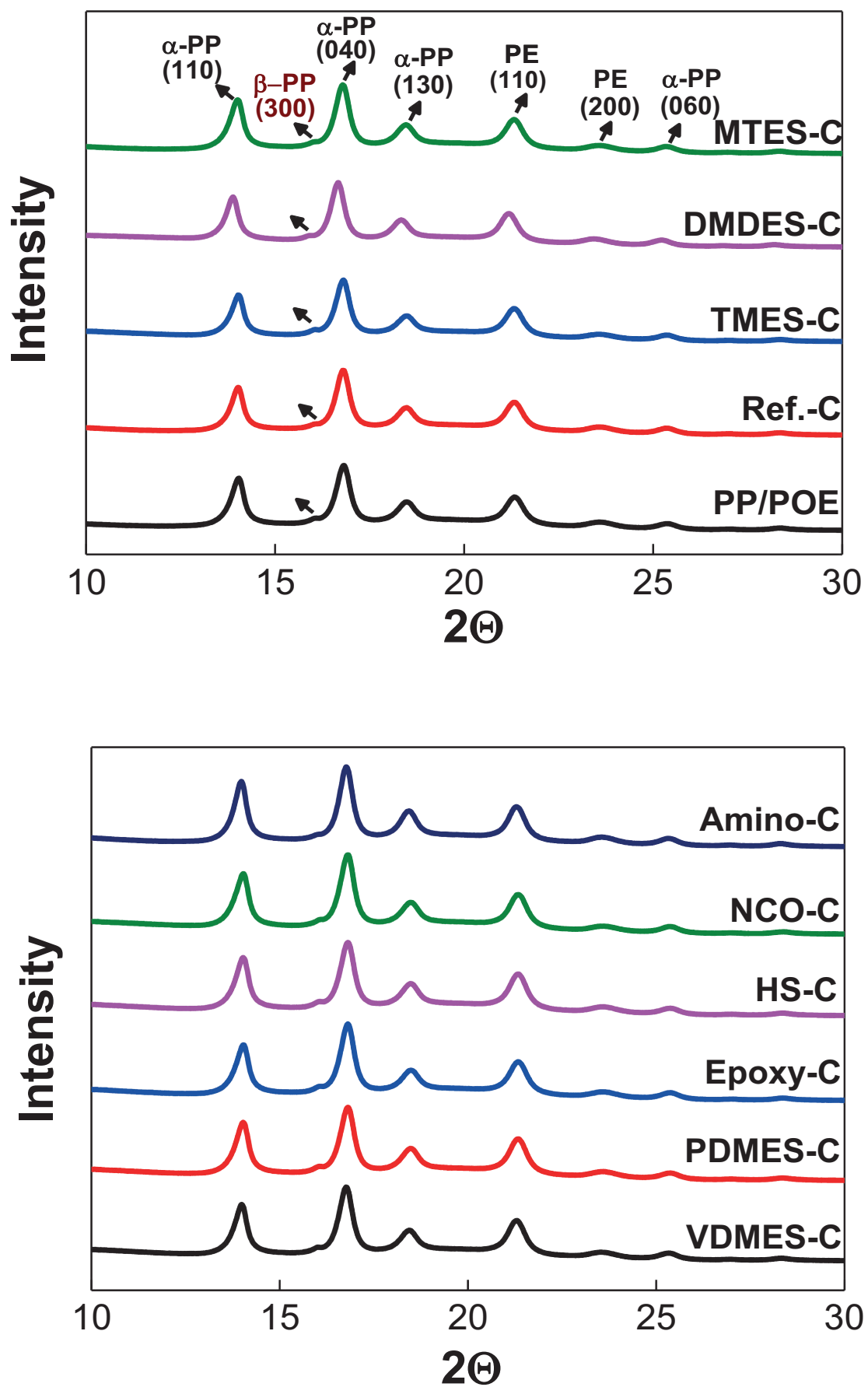

Figure 5.13 XRD curves of all PP/POE nanocomposites. 
Table 5.7 Calculated crystallinity based on XRD curves of all PP/POE nanocomposites.

\begin{tabular}{ccc}
\hline Composites & Overall Crystallinity (\%) & PP phase Crystallinity (\%) \\
\hline PP/POE & 44.6 & 32.3 \\
Ref-C & 43.7 & 31.6 \\
TMES-C & 43.2 & 31.2 \\
DMDES-C & 44.1 & 31.9 \\
MTES-C & 45.7 & 33.0 \\
VDMES-C & 44.6 & 32.2 \\
PDMES-C & 44.2 & 31.9 \\
Epoxy-C & 44.4 & 32.1 \\
HS-C & 44.0 & 31.8 \\
NCO-C & 45.1 & 32.6 \\
Amino-C & 47.3 & 34.2 \\
\hline
\end{tabular}

\subsubsection{Scanning Electron Microscopy (SEM)}

The morphology of the PP/POE nanocomposites was evaluated by SEM. The samples were broken after cooling in liquid $\mathrm{N}_{2}$ (at a temperature below the Tg's of the polymers) and analyzed without surface coating. Two images of each sample with magnification of 50K and 20K are shown in Figures 5.14, 5.15 and 5.16 .

The SEM pictures show clearly that there are two phases evenly mixed together layer by layer in the unfilled PP/POE composites in Figure 5.14 and in the silica filled PP/POE nanocomposites in Figures 5.15 and 5.16. One of the phase has a smooth surface, whereas the other one appears more rough/granular. Moreover, it is interesting that all the silicas, including the reference silica and the modified silicas, are by preference located in one of the phases, the smooth phase, which is coherent with the DSC results. From the DSC results, it could be concluded that the silica has a more pronounced influence on the PP phase by means of the crystallization peak shift. Therefore, we can deduct that the smooth phase shown in the SEM images is the PP phase, while the rough phase is POE. The preferential location of the silica in the PP phase can be explained by the lower viscosity of this phase. PP is a thermoplastic material, while POE exhibits elastomeric properties and therefore a higher viscosity.
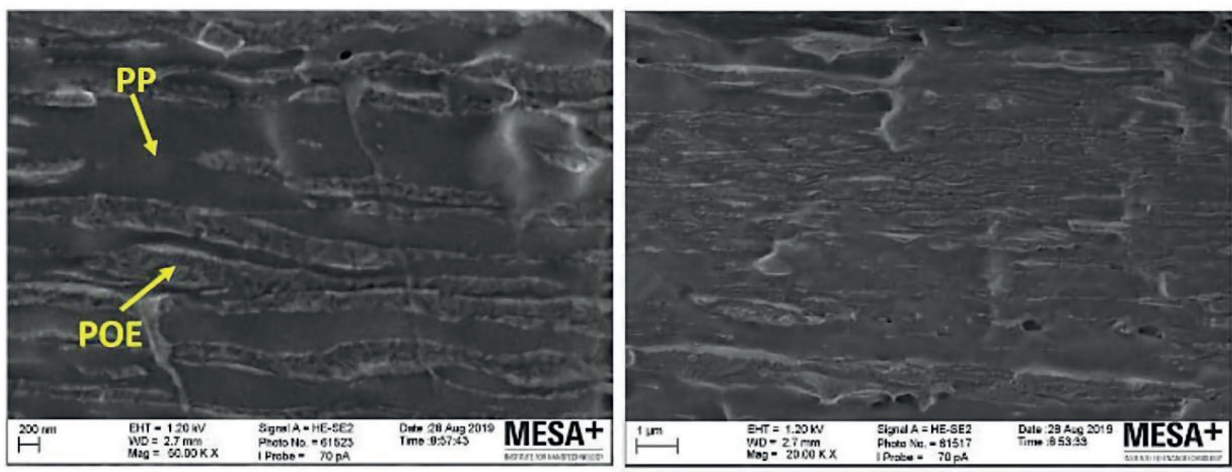

Figure 5.14 SEM image of unfilled PP/POE composites (Left 50K and Right 20K). 
Regarding the dispersion of silica, Figures 5.15 (A and a) shows some agglomerates in the reference silica filled PP/POE composites, and the silica is not evenly distributed in the polymer matrix. Whereas the silicas modified with the unpolar silanes (TMES, DMDES, MTES, VDMES or PDMES) exhibit better dispersion and distribution within the polymer matrix, which is shown in Figures 5.15 (B, C, D, E, F and $\mathrm{b}, \mathrm{c}, \mathrm{d}, \mathrm{e}, \mathrm{f}$ ). This is due to a higher compatibility between the modified hydrophobic silicas and the PP/POE polymer matrix.
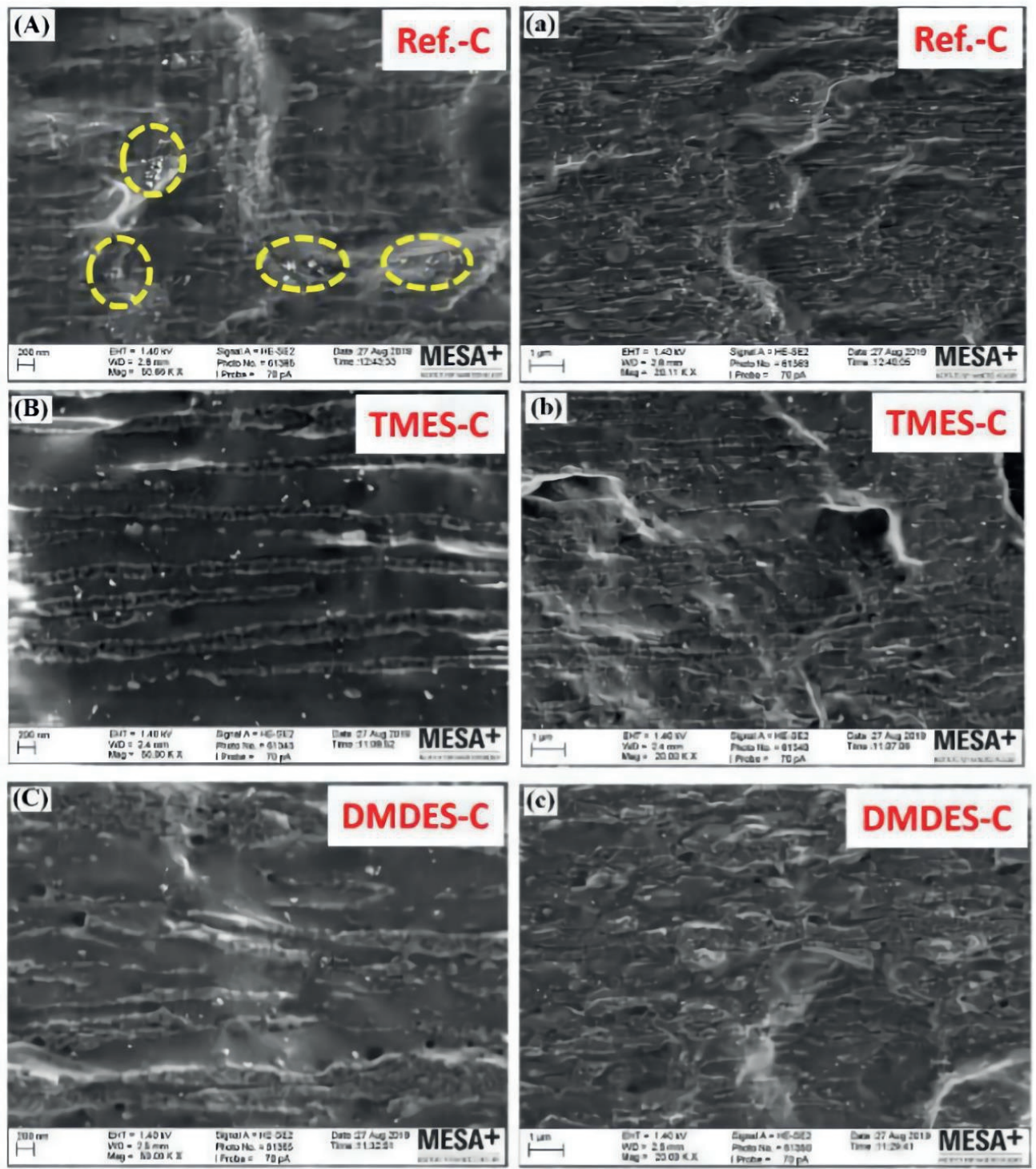
In Figure 5.16, all silicas modified with hydrophilic silanes are shown (HS-silane, epoxy-silane, NCOsilane or amino-silane). As all modified silicas shown in Figure 5.16 still retain their hydrophilic nature, they are less compatible with the unpolar polymer matrix. Consequently, these silicas are more likely to agglomerate and form clusters, which are strongly connected by polar-polar interactions, and therefore exhibit a lower degree of dispersion and distribution in the PP/POE polymer matrix.
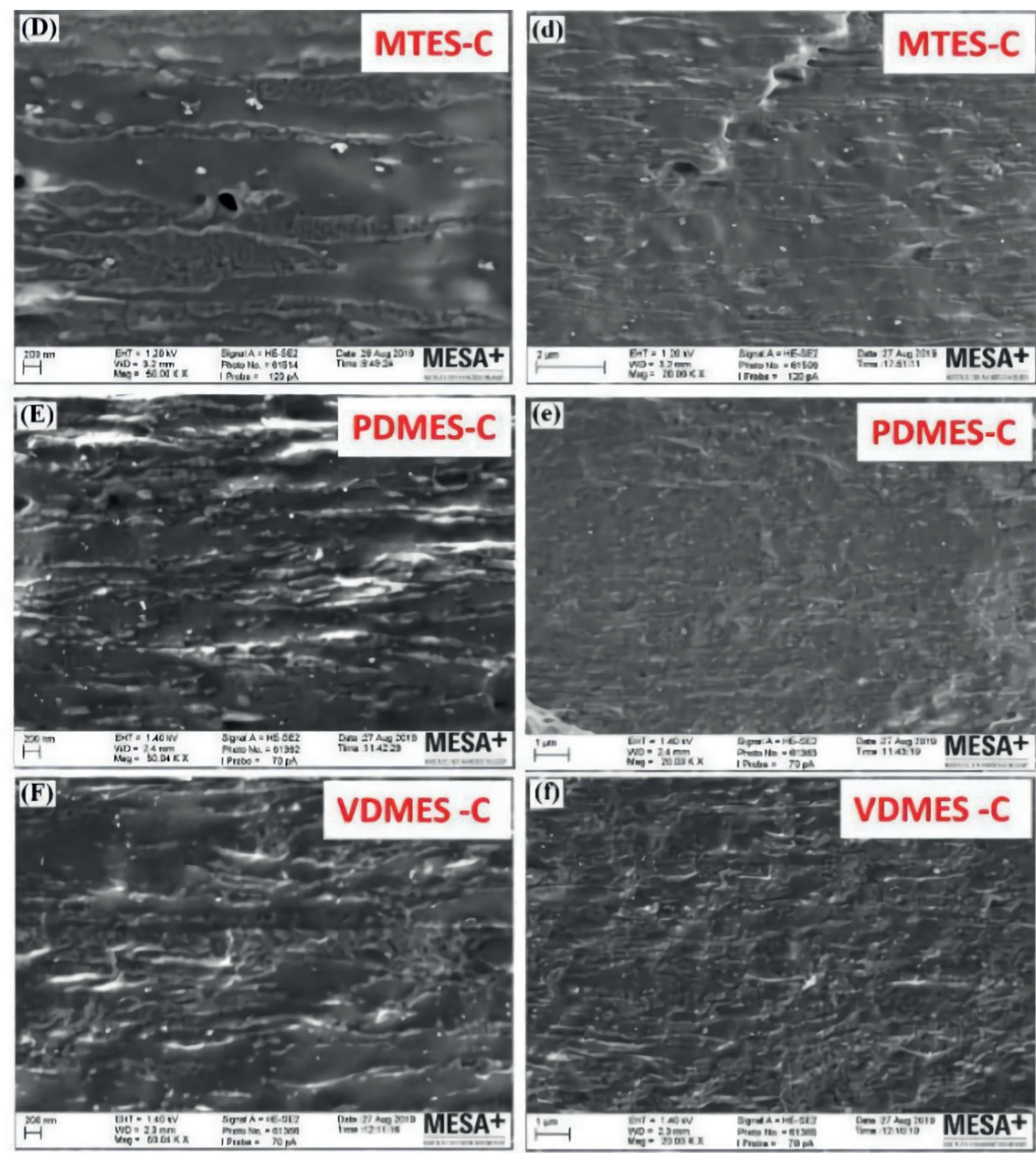

Figure 5.15 SEM images of PP/POE composites filled with the reference silica and modified hydrophobic silicas (Left 50K and Right 20K). 

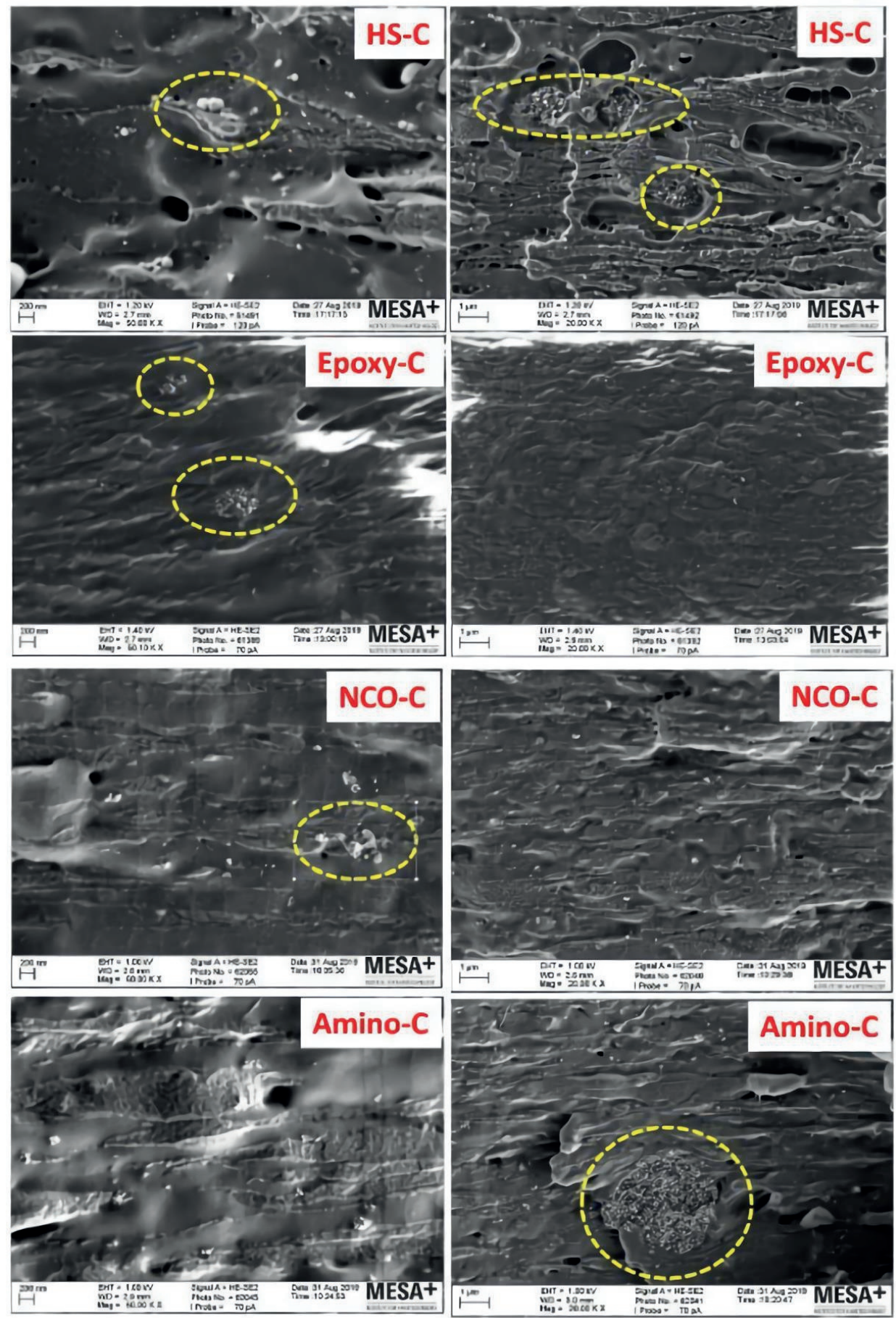

Figure 5.16 SEM images of PP/POE composites filled with the reference silica and modified hydrophilic silicas (Left 50K and Right 20K). 


\subsubsection{Thermally Stimulated Depolarization Current (TSDC)}

To investigate the influence of different surface-modified silicas on the charge trapping properties in PP/POE nanocomposites, TSDC measurements were performed. The measured TSDC spectra are shown in Figures $5.17 \mathrm{~A}, \mathrm{~B}$ and $\mathrm{C}$.
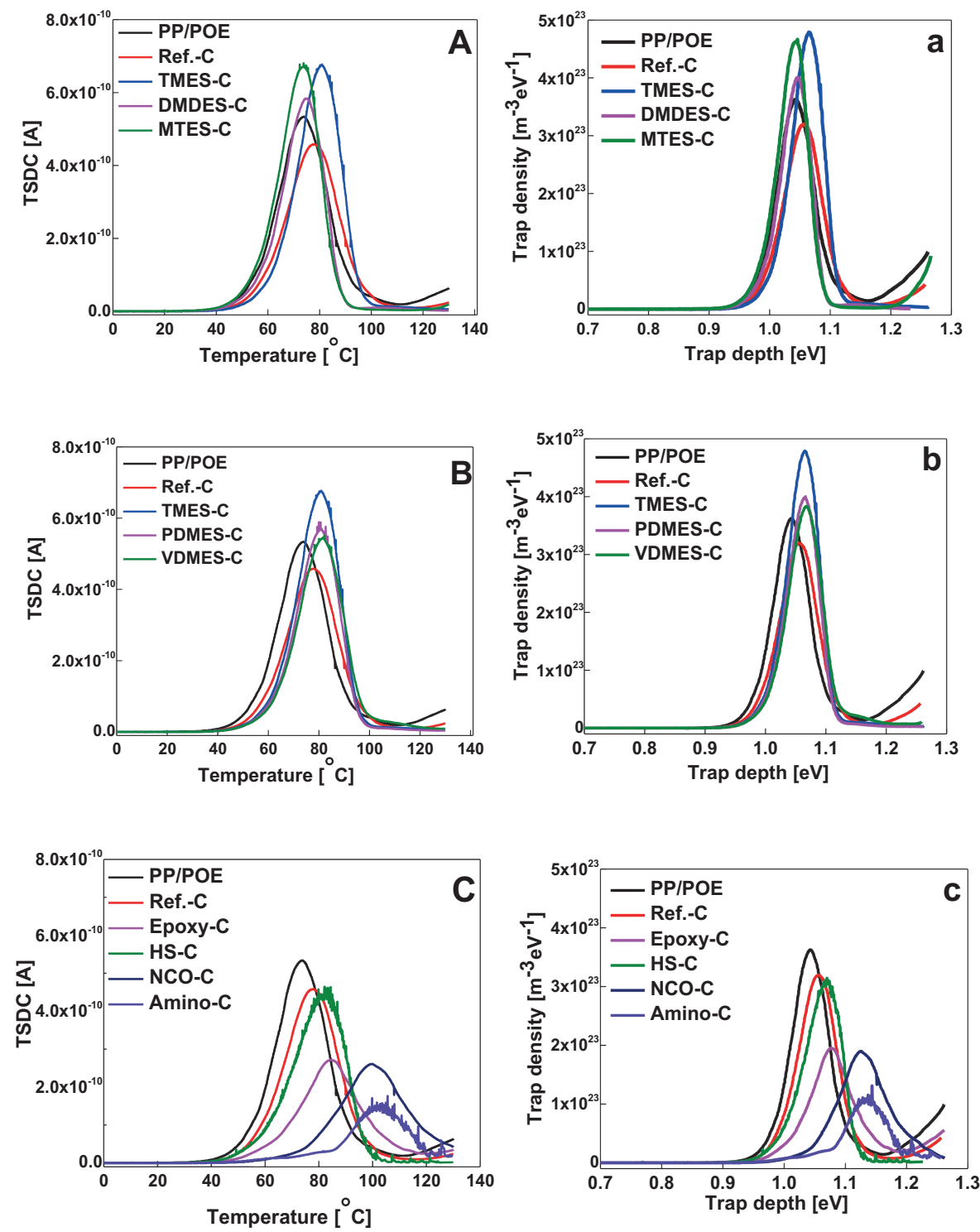

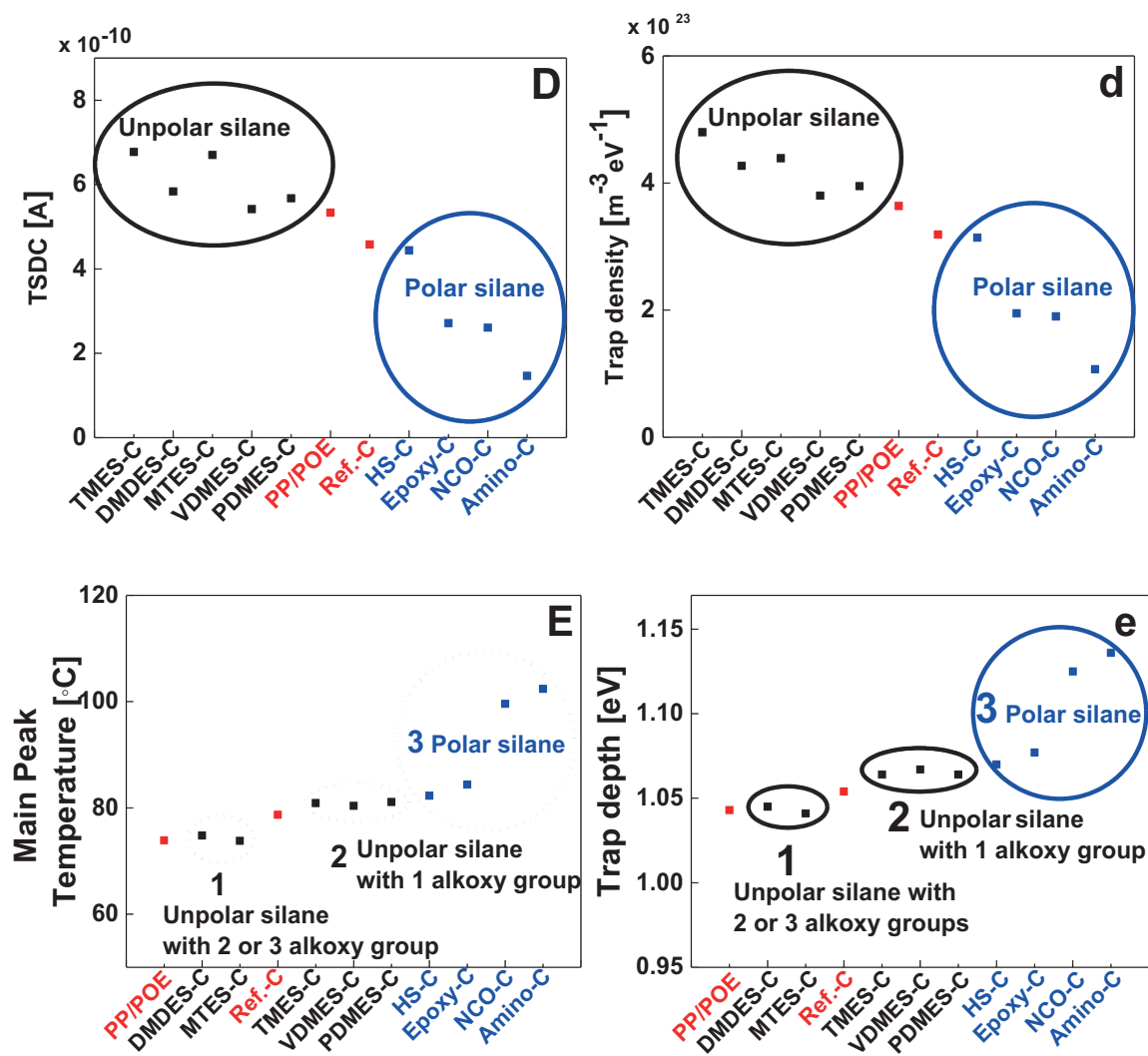

Figure 5.17 TSDC results of all composites and the calculated trap density and depth.

Region 1 is the main peak temperature (left) and the trap depth of the PP/POE composites filled with the silica modified with the unpolar silane with 2 or 3 alkoxy groups.

Region 2 is the main peak temperature (left) and the trap depth of the PP/POE composites filled with the silica modified with the unpolar silane with 1 alkoxy group.

Region 3 is the main peak temperature (left) and the trap depth of the PP/POE composites filled with the silica modified with the polar silane.

The current formed by the relaxation of trapped charges during the thermally stimulated depolarization process is related to the charge trap distribution in the nanocomposites. In principle, the TSDC current intensity is associated with the trap density, and the TSDC temperature is related to the trap depth. The calculated trap depth vs. density distributions, obtained from the TSDC spectra by using a numerical method presented in [38] are shown in Figures 5.17 a, b and c.

As shown in the Figure 5.17, the silicas treated with different silanes tailor the charge trapping properties of the PP/POE composites. With increasing temperature the TSDC current starts to rise from $30{ }^{\circ} \mathrm{C}$ onwards, corresponding to the onset of charge relaxation. The TSDC measurement range for all the $\mathrm{PP} / \mathrm{POE}$ samples is the same, from $50^{\circ} \mathrm{C}$ to $130^{\circ} \mathrm{C}$, limited by the melting temperature of the PP phase. 
The TSDC peak intensity (apparent trap density) and peak temperature (trap depth) are changing and varying for the PP/POE nanocomposites filled with different modified silica.

It is interesting to notice that the TSDC peak intensity (apparent trap density) is higher for the PP/POE nanocomposites filled with the silicas modified by unpolar silanes such as TMES, DMDES, MTES, VDMES and PDMES than for the silicas modified by polar silanes (HS-silane, epoxy-silane, NCO-silane, amino-silane), as shown in Figure 5.17 D. Since there is no significant difference in the degree of crystallinity for the silica filled PP/POE nanocomposites based on the DSC and XRD results, the differences in TSDC spectra are attributed to new trap sites arising from the chemical nature of the surface modification. These traps (associated with the silica surface functionalization) are then dispersed along with the silica throughout the matrix. In addition, the interface between a silica and polymer matrix plays an important role in the charge trapping properties. It has been reported that adding nanoparticles can introduce a large interfacial area [6] resulting in higher amount of traps formed in nanodielectrics [39].

In our study, we applied 9 silanes to be grafted onto silica surface, which lead to different compatibilities between the modified silicas and the polymer matrix. The silicas modified with unpolar silanes (TMES, DMDES, MTES, VDMES or PDMES) exhibit better compatibility with the PP/POE matrix. This resulted in better dispersion of the unpolar modified silicas in the PP/POE matrix and implied a larger interfacial area (Figure 5.18 left) between silica and PP/POE matrix, which is consistent with the SEM results presented in Figure 5.15. Consequently, a higher peak intensity (trap density) is measured as shown in Figures $5.17 \mathrm{~A}$, a and $5.17 \mathrm{~B}, \mathrm{~b}$. On the contrary, the unmodified silica and the silica modified with polar silanes (HS-silane, epoxy-silane, NCO-silane, amino-silane) still tend to agglomerate and have a lower compatibility towards the PP/POE matrix, which is also seen in the SEM images in Figure 5.16. As a result, compared to the unpolar silica less interface (Figure 5.18 right) is formed. This resulted in a lower TSDC peak intensity and apparent trap density as shown in Figures $5.17 \mathrm{C}$ and c.

The differences observed in TSDC peak intensity (apparent trap density) of the nanocomposites may also be linked to differences in their trap depth and charge mobility. During the isothermal polarization stage, the charges are injected and trapped into the samples, with the temperature- and field-dependent charge mobility and trapping phenomena dictating the total amount of accumulated charge. Subsequently, during the thermally stimulated depolarization stage, the previously accumulated space charge in the sample is relaxed (detrapped). Thus, the TSDC peak intensity is in principle related to the amount of injected charge (neglecting e.g. charge recombination inside the specimen). With increasing trap depth, the build-up of deeply trapped homocharge layer near the electrode-specimen interface reduces further charge injection, and thus higher temperature or electric field would be required during the polarization step to inject more charge. Therefore, under the same polarization temperature and electric field, less charge is accumulated in the sample with a higher trap depth, with this also resulting in lower TSDC current intensity and apparent trap density, which is line with our observations.

It is important to notice that the trap depth also varies for the composites containing differently modified silicas (Figure 5.17 e). It is obvious from Figure 5.17 E that there is a large peak temperature shift (approx. $10 \sim 30{ }^{\circ} \mathrm{C}$ ) to a higher level for the nanocomposites filled with the polar silane modified silicas. These polar silanes contain hetero-atoms $(\mathrm{S}, \mathrm{O}$ or $\mathrm{N})$ imposing the polar character of the functional moieties to the silica surface. Therefore, the silicas modified with polar silanes introduce deeper traps into the nanocomposites. This phenomenon is also described in literature, where studies or models indicate that polar groups or hetero-atoms of electronegativity higher than the one of carbon can introduce deep traps 
$[11,40-42]$. It is worth to note that the most pronounced one is the silica modified with NCO and amino silanes, which both contain a nitrogen atom.
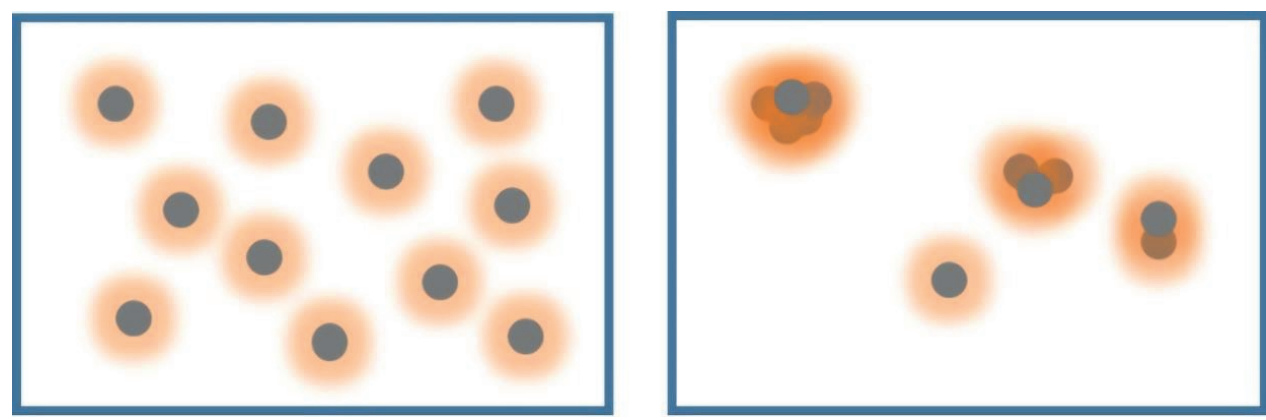

Figure 5.18 Schemes of the interface area between silica and matrix.

(Left: Unpolar silica, good dispersion; right: polar silica forming clusters.)

Regarding to the silicas modified with the unpolar silanes in Figures 5.17 A and B, the results are also very interesting. Although there is no significant shift of the peak temperature compared with the one modified with polar silane in Figure $5.17 \mathrm{C}$, a small variation of approximately $5^{\circ} \mathrm{C}$ is measured, see Figure $5.17 \mathrm{E}$. In order to explain, why silica modified with unpolar silanes give different results concerning the trap level, a hypothesis is depicted in Figure 5.19. As discussed above, there is an organic layer on silica surface after the modification. The efficiency of the silane shielding of the silica surface is dependent on the number of alkoxy groups in the silane molecule as shown in Figure 5.19. Therefore, the surface properties of silica are altered via the silane agents containing various numbers of alkoxy groups. Consequently, the interface between silica and the polymer matrix is also affected.

Due to condensation of silanes, the silica modified with the silane containing two or three alkoxy groups shield efficiently the silica surface based the calculated the amount of grafed silane on silica surface in Figure 5.8 right. As a result, all unreacted silanol or siloxane groups are covered by silane molecules as shown in Figures 5.19 (a) and (b). Therefore, the silicas modified with DMDES or MTES have their surface covered by a hydrocarbon layer, which exhibits a similar physicochemical character as the polymer matrix. As a result shown in Region 1 in Figure 5.17 E, the temperature of the main TSDC peak of the composites filled with DMDES or MTES modified silicas is more or less the same as for the unfilled PP/POE matrix. In conclusion, there is no new deep trap introduced due to a similar energy state of hydrocarbons at the interface between the modified silicas and polymer matrix.

However, if the silica was modified with a silane containing only one alkoxy group, there are still unreacted silanol or siloxane groups exposed on the silica surface (Figure 5.19 (c)). This inefficient shielding will influence the final interface properties between the silica and a polymer matrix [40]. As a result, the silica modified with TMES, VDMES or PDMES have still unreacted silanol or siloxane group exposed to the Polymer matrix. There are new deeper traps introduced by the O- or Si-atoms on the silica surface, which are located in the interface between silica and the polymer matrix, due to a different energy state of atoms $(\mathrm{O}, \mathrm{Si}, \mathrm{C}, \mathrm{H})$. Consequently, the maximum of the main TSDC peak temperature is shifted to a higher temperature as shown in Region 2 in Figure 5.17 E. 
(a)

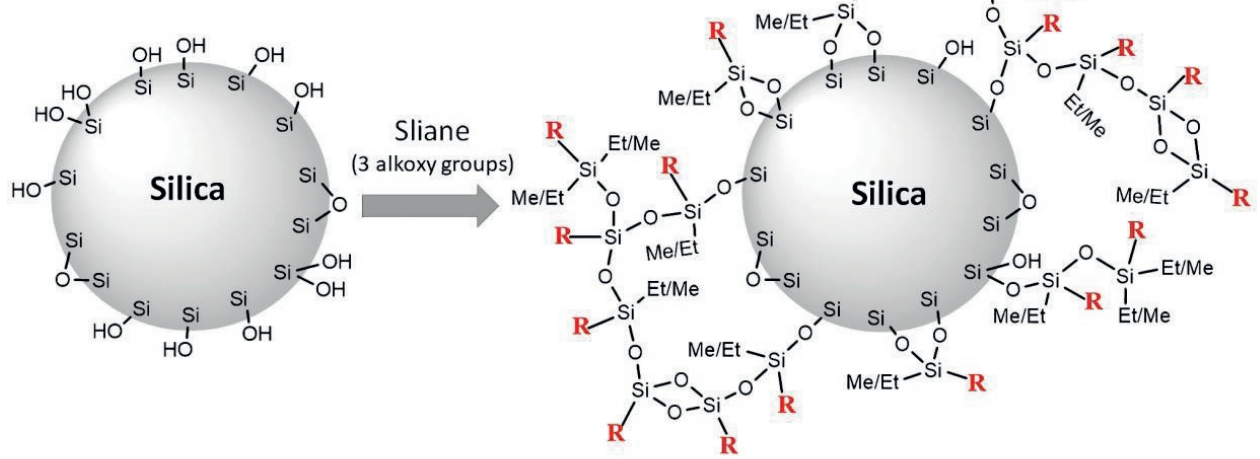

(b)

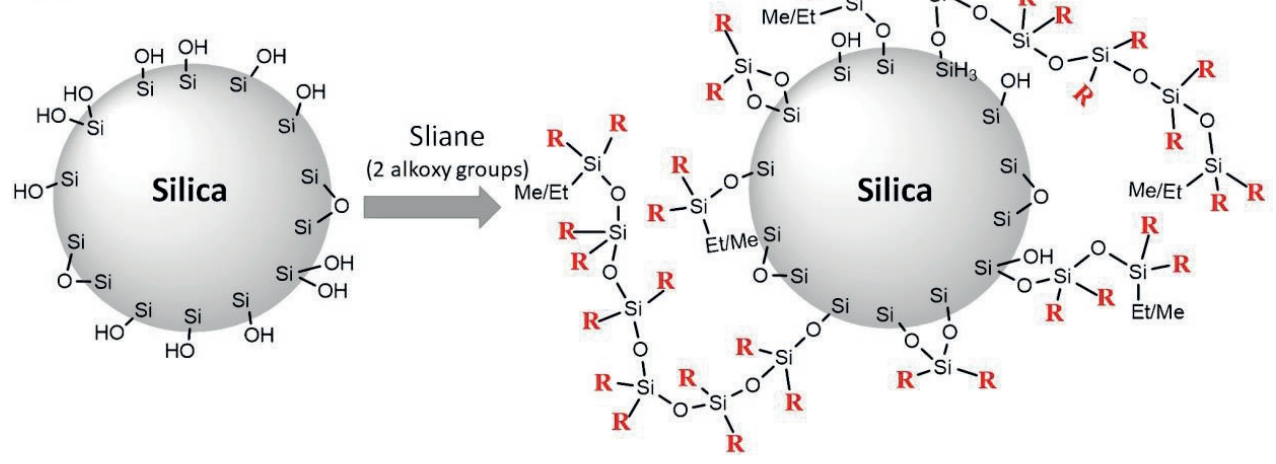

(c)

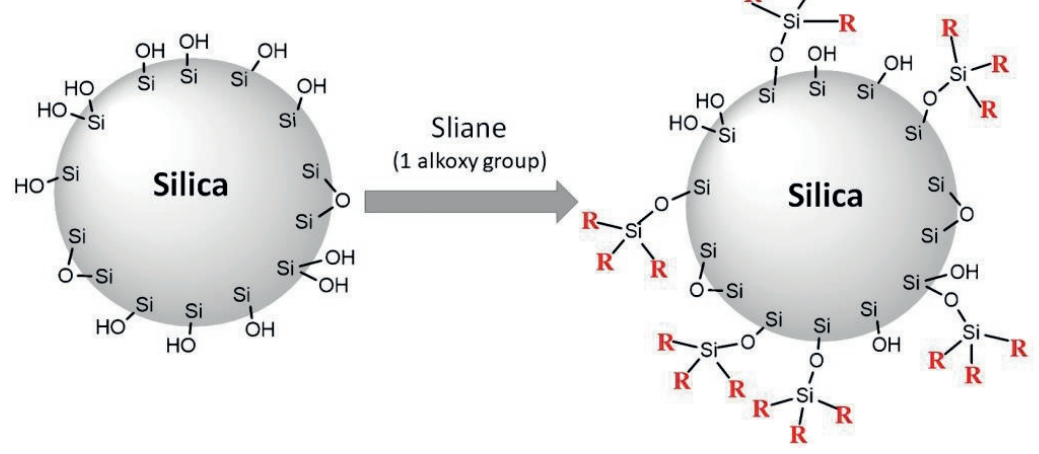

Figure 5.19 Schemes of silica surface modification and shielding effect by silanes having different number of alkoxy groups.

In order to further analyse the TSDC results, the amount of charge injected during the isothermal polarization and the amount of charge released from the samples during the TSDC measurement were calculated by integrating the current versus time curves during polarization and depolarization (Figure 5.20 left). In principle, for unpolar polymers, when the temperature is above the glass transition temperature, 
the TSDC current is mostly due to the space charge relaxation. This relaxation is influenced by the chemical and structural characteristics [43].

In comparison with the neat PP/POE matrix, the amount of charges injected into the samples during the polarization decreased for the samples filled with the reference silica and silane modified silicas, indicating lower charge mobility for all these nanocomposites. It is also noticeable that a much lower amount of charges was injected into the NCO silane and amino silane modified silica filled composites than into the composites containing silicas modified with other silanes.

The relative amount of released charges was also calculated and shown in Figure 5.20 right. In general, this value increased for all the nanocomposites containing the reference silica or modified silicas compared to the unfilled PP/POE matrix. However, there is a more significant rise when incorporating the silica modified by NCO and amino silane. This means that less charges are permanently trapped or dissipated in the NCO or amino silane modified-silica filled composites. In other words, incorporation of the NCO or amino silane modified silicas can significantly suppress the space charge accumulation effect of the $\mathrm{PP} / \mathrm{POE}$ composites. This might be due to the polar NCO or amino group, which can form large dipoles and introduce deeper traps [41]. The trap depth of these materials is large enough to block the movement of the charge carrier, leading to suppression of space charge formation [44].
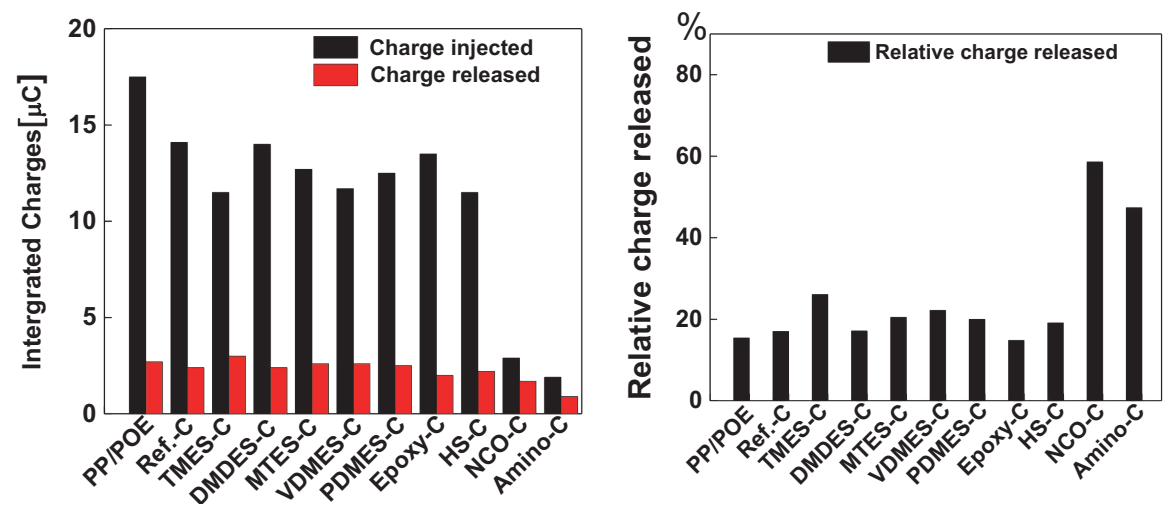

Figure 5.20 Calculated injected charge and relative released charge during TSDC.

\subsection{Conclusions}

Based on the investigations performed and presented in this paper, the following conclusions can be drawn:

- A new solvent-free silane-silica modification method was developed. A catalyst (acid or base) successfully accelerates the silica surface modification. The efficiency of this method was supported by TGA, FTIR, XPS and TEM elemental mapping measurements.

- Based on the TGA results, the modification of the silica surface assisted by an acid catalyst is more stable than the one achieved by a base catalyst. 
- Successful silica modification was achieved using 9 different silanes, which was proven by TGA and FTIR results.

- When blended with a PP/POE matrix, the silicas increased the onset of the crystallization temperature due to the nucleation effect. This effect was most prominent in the PP phase, where the silica particles were preferentially located. In terms of degree of crystallinity, DSC and XRD results both confirmed that there is barely any influence of adding these silicas.

- Based on SEM images, silica modified with unpolar silanes were easier to disperse in a PP/POE polymer matrix, while the ones modified with polar silanes agglomerated in the polymer matrix.

- Based on the TSDC results, the efficiency of covering the silica surface depends on the number of alkoxy groups in the silane. This influences the charge trapping properties noticeably.

- TSDC results have shown as well, that the PP/POE nanocomposites filled with an unpolar silane increased the charge trap density, while the ones filled with a polar silica increased the charge trap depth.

- Silica modified with the polar silane containing nitrogen atoms showed less injected charges, which might be an indication of suppressing the space charge accumulation by introducing nitrogen-rich polar functional groups.

Acknowledgments: This project has received funding from the European Union's Horizon 2020 research and innovation program under grant agreement No 720858 .

The authors would like to thank the ECIU Researcher Mobility Fund for supporting this research work.

The authors also would like to thank Evonik Industries for providing a free silica sample. 


\section{References}

[1] Y. Gao, J. Li, Y. Yuan, S. Huang, and B. Du, "Trap distribution and dielectric breakdown of isotactic polypropylene/propylene based elastomer with improved flexibility for DC cable insulation,"IEEE Access. vol. 6, pp. 58645-58661, 2018.

[2] I. Rytöluoto, M. Ritamäki, K. Lahti, M. Paajanen, M. Karttunen, G.C. Montanari and H. Naderiallaf, "Compounding, Structure and Dielectric Properties of Silica-BOPP Nanocomposite Films," Proceedings of IEEE 2nd International Conference on Dielectrics., Hungary, July, 2018, pp. 1-4.

[3] E. Helal, C. Pottier, E. David, M. Fréchette, and N.R. Demarquette, "Polyethylene/thermoplastic elastomer/Zinc Oxide nanocomposites for high voltage insulation applications: Dielectric, mechanical and rheological behavior, "European Polymer Journal, vol.100, pp. 258-269, 2018.

[4] A.M. Pourrahimi, R.T. Olsson and M.S. Hedenqvist, "The Role of Interfaces in Polyethylene/Metal-Oxide Nanocomposites for Ultrahigh-Voltage Insulating Materials," Advanced Materials, vol. 30, no. 4, pp. 1703624, 2018.

[5] L.K.H. Pallon, A.T. Hoang, A.M. Pourrahimi, M.S. Hedenqvist, F. Nilsson, S. Gubanski and R.T. Olsson, "The impact of $\mathrm{MgO}$ nanoparticle interface in ultra-insulating polyethylene nanocomposites for high voltage DC cables, "Journal of Materials Chemistry A, vol. 4, no. 22, pp. 8590-8601, 2016.

[6] M. Roy, J.K. Nelson, R.K. MacCrone, L.S. Schadler, C.W. Reed and R. Keefe, "Polymer nanocomposite dielectrics-the role of the interface, " IEEE transactions on Dielectrics and Electrical Insulation, vol. 12, no. 4, pp, 629-643, 2005.

[7] D. Liu, A.T. Hoang, A.M. Pourrahimi, L.H. Pallon, F. Nilsson, S.M. Gubanski, R.T. Olsson, M.S. Hedenqvist and U.W. Gedde, "Influence of nanoparticle surface coating on electrical conductivity of LDPE/A1 2 O 3 nanocomposites for HVDC cable insulations," IEEE Transactions on Dielectrics and Electrical Insulation, vol. 24, no. 3, pp.1396-1404, 2017.

[8] D. Ma, T.A. Hugener, R.W. Siegel, A. Christerson, E. Mårtensson, C. Önneby and L.S. Schadler, "Influence of nanoparticle surface modification on the electrical behaviour of polyethylene nanocomposites, " Nanotechnology, vol. 16, no. 6, pp. 724, 2005.

[9] X. Huang, P. Jiang and Y. Yin, "Nanoparticle surface modification induced space charge suppression in linear low density polyethylene,"Applied Physics Letters, vol. 95, no. 24, pp. 242905, 2009.

[10] M. Bell, T. Krentz, J.K. Nelson, L. Schadler, K. Wu, C. Breneman, S. Zhao, H. Hillborg and B. Benicewicz, "Investigation of dielectric breakdown in silica-epoxy nanocomposites using designed interfaces," Journal of Colloid and Interface Science, vol. 495, pp. 130-139, 2017.

[11] S. Li, D. Min, W. Wang and G. Chen, "Linking traps to dielectric breakdown through charge dynamics for polymer nanocomposites," IEEE Transactions on Dielectrics and Electrical Insulation, vol. 23, no. 5, pp. 2777-2785, 2016.

[12] S. Li, G. Yin, G. Chen, J. Li, S. Bai, L. Zhong, Y. Zhang and Q. Lei, "Short-term breakdown and long-term failure in nanodielectrics: a review, " IEEE Transactions on Dielectrics and Electrical Insulation, vol. 17, no. 5, pp. 1523-1535, 2010.

[13] A. Mahtabani, X. He, I. Rytöluoto, K. Lahti, M. Paajanen, E. Saarimäki, K. Lahti, M. Paajanen. R. Anyszka, W. Dierkes and A. Blume, "Effect of Silica Modification on Charge Trapping Behavior of PP blend/Silica Nanocomposites, ” In 2019 2nd International Conference on Electrical Materials and Power Equipment. China, April, pp. 241-245, 2019. 
[14] X. He, A. Mahtabani, I. Rytöluoto, E. Saarimäki, K. Lahti, M. Paajanen. R. Anyszka, W. Dierkes and A. Blume, "Surface Modification of Fumed Silica by Dry Silanization for PP-based Dielectric Nanocomposites," In 2019 2nd International Conference on Electrical Materials and Power Equipment, China, April, pp. 254-259, 2019.

[15] A. Mahtabani, X. He, I. Rytöluoto, K. Lahti, M. Paajanen, E. Saarimäki, K. Lahti, M. Paajanen. R. Anyszka, W. Dierkes and A. Blume, "Solution Modified Fumed Silica and Its Effect on Charge Trapping Behavior of PP/POE/Silica Nanodielectrics,". In Proceedings of the Nordic Insulation Symposium, Finland, August, pp. 129-133, 2019.

[16] A.C. Borges-Muñoz, D.P. Miller, E. Zurek and L.A. Colón, "Silanization of superficially porous silica particles with p-aminophenyltrimethoxysilane," Microchemical Journal, vol. 147, pp. 263 268, 2019.

[17] J.S. Sonn, J.Y. Lee, S.H. Jo, I.H. Yoon, C.H. Jung and J.C. Lim, "Effect of surface modification of silica nanoparticles by silane coupling agent on decontamination foam stability, "Annals of Nuclear Energy, vol. 114, pp. 11-18, 2018.

[18] H. Hemmatpour, V. Haddadi-Asl and H. Roghani-Mamaqani, "Synthesis of pH-sensitive poly (N, $\mathrm{N}$-dimethylaminoethyl methacrylate)-grafted halloysite nanotubes for adsorption and controlled release of DPH and DS drugs, " Polymer, vol. 65, pp. 143-153, 2015.

[19] Y.W. Ngeow, A.V. Chapman, J.Y. Heng, D.R. Williams, S. Mathys and C.D. Hull, "Characterization of silica modified with silanes by using thermogravimetric analysis combined with infrared detection," Rubber Chemistry and Technology, vol.92, no.2, pp.237-262, 2019.

[20] L.A. Prado, M. Sriyai, M. Ghislandi, A. Barros-Timmons and K. Schulte, "Surface modification of alumina nanoparticles with silane coupling agents," Journal of the Brazilian Chemical Society, vol. 21, no. 12, pp. 2238-2245, 2010.

[21] J.D. Rusmirović, T. Radoman, E.S. Džunuzović, J.V. Džunuzović, J. Markovski, P. Spasojević and A.D. Marinković, "Effect of the modified silica nanofiller on the mechanical properties of unsaturated polyester resins based on recycled polyethylene terephthalate," Polymer Composites, vol. 38, no. 3, pp. 538-554, 2017.

[22] Z. Tang, J. Huang, X. Wu, B. Guo, L. Zhang and F. Liu, "Interface engineering toward promoting silanization by ionic liquid for high-performance rubber/silica composites," Industrial \& Engineering Chemistry Research, vol. 54, no. 43, pp. 10747-10756, 2015.

[23] Y. Shin, D. Lee, K. Lee, K.H. Ahn and B. Kim, "Surface properties of silica nanoparticles modified with polymers for polymer nanocomposite applications," Journal of Industrial and Engineering Chemistry, vol. 14, no. 4, pp. 515-519, 2008.

[24] W. He, D. Wu, J. Li, K. Zhang, Y. Xiang, L. Long, and Q. Zhang, "Surface modification of colloidal silica nanoparticles: Controlling the size and grafting process," Bulletin of the Korean Chemical Society, vol. 34, no. 9, pp. 2747-2752, 2013.

[25] B. Qiao, T.J. Wang, H. Gao and Y. Jin, "High density silanization of nano-silica particles using $\gamma$ aminopropyltriethoxysilane (APTES)," Applied Surface Science, vol. 351, pp. 646-654, 2015.

[26] X. Yang, Q. Shao, L. Yang, X. Zhu, X. Hua, Q. Zheng and G. Lai, "Preparation and performance of high refractive index silicone resin-type materials for the packaging of light-emitting diodes, " Journal of Applied Polymer Science, vol. 127, no. 3, pp. 1717-1724, 2013.

[27] N.A. Negm, G.H. Sayed, F.Z. Yehia, O.I. Habib and E.A. Mohamed, "Biodiesel production from one-step heterogeneous catalyzed process of Castor oil and Jatropha oil using novel sulphonated phenyl silane montmorillonite catalyst, ”Journal of Molecular Liquids, vol. 234, pp. 157-163, 2017. 
[28] F. Zucchi, A. Frignani, V. Grassi, G. Trabanelli and M. DalColle, "The formation of a protective layer of 3-mercapto-propyl-trimethoxy-silane on copper," Corrosion Science, vol. 49, no. 3, pp.1570-1583, 2007.

[29] J.C. Hoepfne and S.H. Pezzin, "Functionalization of carbon nanotubes with (3-glycidyloxypropyl)trimethoxysilane: Effect of wrapping on epoxy matrix nanocomposites, ” Journal of Applied Polymer Science, vol. 133, no. 47, 2016.

[30] X. He, A. Mahtabani, I. Rytöluoto, E. Saarimäki, K. Lahti, M. Paajanen. R. Anyszka, W. Dierkes and A. Blume, "Surface Modification of Fumed Silica by Plasma Polymerization of Acetylene for PP/POE Blends Dielectric Nanocomposites,” Polymers, vol. 11, no. 12, pp. 1957, 2019.

[31] B. Dang, J. He, J. Hu and Y. Zhou, "Tailored sPP/silica nanocomposite for ecofriendly insulation of extruded HVDC cable," Journal of Nanomaterials, vol.16, no. 1, pp. 439, 2015.

[32] J. Qian, P. He and K. Nie, "Nonisothermal crystallization of PP/nano-SiO2 composites," Journal of Applied Polymer Science, vol. 91, no. 2, pp. 1013-1019, 2004.

[33] S. Borysiak, Ł. Klapiszewski, K. Bula and T. Jesionowski, "Nucleation ability of advanced functional silica/lignin hybrid fillers in polypropylene composites," Journal of Thermal Analysis and Calorimetry, vol. 126, no. 1, pp. 251-262, 2016.

[34] M. Mucha and Z. Królikowski, "Application of DSC to study crystallization kinetics of polypropylene containing fillers," Journal of Thermal Analysis and Calorimetry, vol. 74, no.2, pp. 549-557, 2003.

[35] Y. Zare and H. Garmabi, "Nonisothermal crystallization and melting behavior of $\mathrm{PP} /$ nanoclay/CaCO3 ternary nanocomposite," Journal of Applied Polymer Science, vol. 124, no. 2, pp. 1225-1233, 2012.

[36] F.M. Mirabella and A. Bafna, "Determination of the crystallinity of polyethylene/ $\alpha$-olefin copolymers by thermal analysis: Relationship of the heat of fusion of $100 \%$ polyethylene crystal and the density," Journal of Polymer Science Part B: Polymer Physics, vol. 40, no. 15, pp. 1637$1643,2002$.

[37] L. Huang, R. Zhan and Y. Lu, "Mechanical properties and crystallization behavior of polypropylene/nano-SiO2 composites," Journal of Reinforced Plastics and Composites, vol. 25, no. 9, pp. 1001-1012, 2006.

[38] F. Tian, W. Bu, L. Shi, C. Yang, Y. Wang and Q. Lei, "Theory of modified thermally stimulated current and direct determination of trap level distribution," Journal of Electrostatics, vol. 69, no. 1, pp. 7-10, 2011.

[39] B. Du, H. Xu, J. Li and Z. Li, "Space charge behaviors of PP/POE/ZnO nanocomposites for HVDC cables," IEEE Transactions on Dielectrics and Electrical Insulation, vol. 23, no. 5, pp.3165-3174, 2016.

[40] B. Du, J. Su, M. Tian, T. Han and J. Li, "Understanding trap effects on electrical treeing phenomena in EPDM/POSS composites,” Scientific Reports, vol. 8, no. 1, pp. 8481, 2018.

[41] M. Roy, J.K. Nelson, R.K. MacCrone and L.S. Schadler, "Candidate mechanisms controlling the electrical characteristics of silica/XLPE nanodielectrics," Journal of Materials Science, vol. 42, no. 11, pp. 3789-3799, 2007

[42] G. Teyssedre and C. Laurent, "Charge transport modeling in insulating polymers: from molecular to macroscopic scale," IEEE Transactions on Dielectrics and Electrical Insulation, vol. 12, no. 5, pp. 857-875, 2005. 
[43] V.M. Gun'Ko, V.I. Zarko, E.V. Goncharuk, L.S. Andriyko, V.V. Turov, Y.M. Nychiporuk, R. Leboda, J. Skubiszewska-Zieba, A.L. Gabchak, V.D. Osovskii, Y.G. Ptushinskii, G.R. Yurchenko, O.A. Mishchuk, P.P. Gorbik, P. Pissis and J.P. Blitz, "TSDC spectroscopy of relaxational and interfacial phenomena," Advances in Colloid and Interface science, vol. 131, no. 1-2, pp. 1-89, 2007.

[44] T. Takada, Y. Hayase, Y. Tanaka and T. Okamoto, "Space charge trapping in electrical potential well caused by permanent and induced dipoles for LDPE/MgO nanocomposite," IEEE Transactions on Dielectrics and Electrical Insulation, vol. 15, no. 1, pp. 152-160, 2008. 


\section{Chapter 6}

\section{The influence of nanosilica surface functionalization on PP/EOC cast thin film nanocomposites for HVDC cable application: micromorphology, dynamic mechanical and dielectric properties}

This chapter has been submitted to Surfaces and Interfaces.

He, X., Seri, P., Rytöluoto, I., Anyszka, R., Mahtabani, A., Naderiallaf, H., Niittymäki, M., Saarimäki, E., Mazel, C., Perego, G. and Lahti, K., Paajanen, M., Dierkes, W. and Blume, A., 2021. The influence of nanosilica surface functionalization on PP/EOC cast thin film nanocomposites for HVDC cable application: micromorphology, dynamic mechanical and dielectric properties 


\title{
The influence of nanosilica surface functionalization on PP/EOC cast thin film nanocomposites for HVDC cable application: micromorphology, dynamic mechanical and dielectric properties
}

\author{
Xiaozhen He ${ }^{1, *}$, Paolo Seri ${ }^{2}$, Ilkka Rytöluoto 3, Rafal Anyszka 1,*, Amirhossein Mahtabani ${ }^{1}$, \\ Hadi Naderiallaf ${ }^{2}$, Minna Niittymäki ${ }^{4}$, Eetta Saarimäki ${ }^{3}$, Christelle Mazel ${ }^{5}$, Gabriele Perego ${ }^{5}$, \\ Kari Lahti $^{4}$, Mika Paajanen ${ }^{3}$, Wilma Dierkes ${ }^{1, *}$ and Anke Blume ${ }^{1}$ \\ 1 University of Twente, Faculty of Engineering Technology, Department of Mechanics of Solids, \\ Surfaces \& Systems (MS3), Chair of Elastomer Technology and Engineering, Enschede, The \\ Netherlands \\ 2 University of Bologna, Department of Electrical, Electronic and Information Engineering \\ "Guglielmo Marconi", Bologna, Italy \\ 3 VTT Technical Research Centre of Finland Ltd, Tampere, Finland \\ 4 Tampere University, High Voltage Engineering, Tampere, Finland \\ 5 Nexans Research Center, 29 rue Pré Gaudry, 69007 Lyon, France \\ * Correspondence: w.k.dierkes@utwente.nl;+31 618513309 (W.K. Dierkes)
}

\begin{abstract}
:
This study focuses on the influence of fumed silica surface functionalization (polar and unpolar) on the micromorphological, thermal and dielectric properties of PP/EOC (polypropylene/poly(ethyleneco-octene)) nanocomposites. Scanning Electron Microscopy images show that phase separation occurs in the PP/EOC composites, that silica is mostly located in the PP phase and that the unpolar silica exhibited better dispersion in PP. Thermally stimulated depolarization current (TSDC) measurements show that the nanosilica addition changed the charge trapping properties of the composites: Both silicas introduce deep traps; however, polar silica introduced deeper charge traps than the unpolar one. The latter increased the apparent deep trap density, while the polar silica resulted in its decrease. Based on dynamic-mechanical analysis (DMA) and TSDC results we postulate that in the unfilled PP/EOC blend, the charge trap sites are mostly located at the crystalline-amorphous interfaces, while for the $\mathrm{PP} / \mathrm{EOC} /$ silica nanocomposites these charges are preferably accumulated in the silica-polymer interface, inside silica clusters or possibly also in the PP crystalline-amorphous interface. The latter is due to a significant modification of the PP crystallite micromorphology due to the nucleating effect of the silica. Therefore, both the crystalline-amorphous interfaces as well as the silica-polymer interfaces play a major role in charge trapping and transport in these composites. The PP/EOC/polar-silica nanocomposite exhibits a reduction in space charge accumulation and only slightly increased charging current density, thus showing a potential for the application in the main insulation layer for HVDC cables.
\end{abstract}

Keywords: PP/EOC; nanosilica; HVDC; charge trap distribution; space charge accumulation 


\subsection{Introduction}

The growing population and rapid industrial development on global scale requires an increased energy supply. This leads to higher carbon dioxide emissions by power plants, if they solely rely on the nonrenewable resources (fossil fuels like coal, oil or gas). To reduce the carbon dioxide emissions, a promising alternative for traditional energy sources are the renewables such as wind or solar energy. However, most of this renewable energy is generated at remote locations, and the electric power transmission to the urban and industrial areas becomes a challenge. In the long-distance electric power transmission, the traditional high voltage alternating current (HVAC) grid exhibits more energy loss and higher costs than the power transmission through the high voltage direct current (HVDC) grid [1,2]. Therefore, the HVDC grid is designed to be a solution for transporting electric power over long distances with higher efficiency, including power transmission across the seas. In the last decade, submarine HVDC and offshore cables have been intensively researched [3,4]. One of the challenges in HVDC cable systems is the space charge accumulation in the transmission cable main insulation. The space charge accumulation can distort the local electrical field and accelerate the cable ageing process, ultimately leading to premature breakdown [5]. To suppress the space charge accumulation under HVDC, new insulation materials of improved dielectric properties have to be developed.

Cross-linked polyethylene (XLPE) is the most common polymeric HVDC cable insulation polymer. However, the residues of the cross-linking agent in XLPE act as defects, resulting in higher space charge accumulation and uneven electric field distribution under high electro-thermal stress. In addition, it is not easy to recycle XLPE due to the cross-linked structure. In comparison to XLPE, thermoplastic polypropylene (PP) exhibits better thermal properties, processing behavior and recyclability. In addition, a higher breakdown strength performance of the latter makes it a promising substitute for XLPE as the main insulation for HVDC cable applications [6]. Many studies are focused on the PP HVDC insulation development $[6,7]$. However, the application of pure isotactic PP, the most common PP stereoisomer, is limited due to its low flexibility in the cable operating temperature range. Therefore, an elastomer (e.g., ethylene-octene [8], ethylene-propylene [9, 10], styrene-olefin copolymers [11]) are blended with pure PP in order to gain better elastic performance. However, it is reported that the space charge accumulation increases in PP/elastomer blends [12]. Suppressing space charge accumulation in such blends is essential.

It is reported in literature that nanofiller addition allows tailoring the charge trap distribution $[13,14]$ and suppressing the space charge accumulation due to the interfacial interactions between the nanofiller and a polymeric matrix [12, 15-17]. However, the charge trap distribution and space charge accumulation depend on different characteristics of the nanofiller including:

i) The chemical composition of the nanofiller such as $\mathrm{ZnO}$ [18], $\mathrm{MgO}$ [19], $\mathrm{SiO}_{2}$ [20] $\mathrm{TiO}_{2}$ [21]. Due to the different electrical resistivity of these nanofillers, the electrical performance of nanocomposites is expected to be different.

ii) Concentration of the nanofiller. It is reported that with higher loadings of nanosilica (2 wt.\%, 5 wt.\%, $10 \mathrm{wt} . \%$ ), space charge accumulation increases, while a lower loading of $0.5 \mathrm{wt} . \%$ suppresses the space charge accumulation [22] 
iii) The chemical surface properties of a nanofiller: 1) On the one hand, these properties lead to a different polarity of the nanofiller, which will affect the compatibility between the nanofiller and the polymeric matrix influencing the nanofiller dispersion [23]. It is reported that a proper surface functionalization can improve the dispersion of a nanofiller in a polymer matrix, which can result in an improvement of breakdown strength [24]. This is highly likely due to the lower free volume presented in the nanocomposites containing well-dispersed nanofiller. Better dispersion of the nanofiller results in a short distance between nano fillers, which causes the lower free volume as the free path for the electron to accelerate, consequently, increasing the breakdown strength [25]. Additionally, the nanofiller incorporation can introduce a local conductive path [26] at the nanofiller-polymer interfacial region. This local conducive path is considered to be more conductive than the polymer bulk, which contributes to redistribution the local charges and reduce the space charge accumulation. 2) On the other hand, the chemical properties of a nanofiller's surface will influence the electrical characteristics of the interface with the polymeric matrix leading to different charge trapping distributions and dielectric performances [27].

In this study, two types of chemical modifications were performed via a sustainable solvent free approach: with 3-aminopropyltriethoxysilane (APTES) for a rather polar surface, and with trimethylethoxysilane (TMES) for an unpolar one. The compounds were prepared in a twin-screw extruder, followed by a single screw extruder with a T-shaped die and a calendaring system in order to get the final thin cast films. A polypropylene/poly(ethylene-co-octene) (PP/EOC) blend was selected as the insulating polymeric matrix. The influence of these different nanofillers on microstructural, thermal, dynamic and dielectric properties of the PP/EOC nanocomposites was investigated, and finally, a potential insulation material for HVDC cable application in terms of reducing space charge accumulation was developed.

\subsection{Materials and Methods}

The nanocomposites were prepared in a KraussMaffei Berstorff ZE 25/49D UTX twin screw extruder by melt-blending of 1 wt. $\%$ silica (Aerosil 200 from Evonik) with a PP/EOC 55:45 wt.\% blend and 0.3 wt. $\%$ of commonly used antioxidants. Two types of antioxidants were used: $0.15 \mathrm{wt} . \%$ of a phenolic one for preventing thermal oxidation of PP at higher temperatures; and $0.15 \mathrm{wt} \%$ of a phosphite processing stabilizer. The extruded strands were cooled in a filtered water bath and granulated, and thereafter extruded into cast film specimens using a Brabender Plasticorder single-screw extruder. The average thickness of the cast film samples was approximately $400 \mu \mathrm{m}$.

Two types of polymers were used:

- PP: propylene/ethylene random copolymer (low content of ethylene),

- EOC: an ethylene-octene copolymer (extrusion grade), added to improve the flexibility of PP.

Two types of modified fumed silica were used: 
- polar silica (P-S): modified with 3-aminopropyltriethoxysilane (APTES),

- unpolar silica (Up-S): modified with trimethylethoxysilane (TMES).

The silica was surface functionalized according to the following procedure (a solvent free approach): 20 $\mathrm{g}$ of silica (without pre-treatment) were mixed with $3.6 \mathrm{~g}$ of APTES or $2.0 \mathrm{~g}$ of TMES, $0.4 \mathrm{~g}$ of trifluoroacetic acid and $0.6 \mathrm{ml}$ of deionized water in a sealed jar at room temperature for $24 \mathrm{~h}$. Afterwards, both modified silicas were put into a vacuum oven at $80{ }^{\circ} \mathrm{C}$ for another $24 \mathrm{~h}$ to remove the unreacted residuals. The more detailed introduction of this modification method has been published in [25].

\subsection{Characterization Methods}

\subsubsection{Characterization of silica}

The silica samples were characterized by Thermogravimetric Analysis (TGA) and Diffused Reflectance Infrared Fourier Transform (DRIFT) Spectroscopy. TGA was performed using a TA 550 thermogravimetric analyser (TA Instruments). The measurements were performed in an atmosphere of synthetic air with a heating rate of $10{ }^{\circ} \mathrm{C} / \mathrm{min}$. The temperature range was ramped from ambient temperature up to $850^{\circ} \mathrm{C}$. For the DRIFTS spectrum, $\mathrm{KBr}$ was used for diluting the silica and for the background record. The spectra were measured using a Perkin Elmer Spectrum 100 device with a diffuse reflectance mode recording spectrum in the range of $4000-400 \mathrm{~cm}^{-1}$ with $4.0 \mathrm{~cm}^{-1}$ of resolution and 124 scans.

\subsubsection{Characterization of the composites}

\subsubsection{Scanning Electron Microscopy}

Scanning Electron Microscopy (SEM) was done by means of a Zeiss MERLIN HR-SEM. The crosssection surfaces were prepared by breaking the samples in liquid nitrogen at a temperature below their glass transition. The surfaces obtained were not coated in order to preserve the delicate micromorphological features and to obtain high imaging accuracy at nanoscale level.

\subsubsection{Thermogravimetric Analysis}

Thermogravimetric Analysis (TGA) was performed using a TA 550 thermogravimetric analyser (TA Instruments). TGA measurements were done in an atmosphere of synthetic air with a heating rate of $10{ }^{\circ} \mathrm{C} / \mathrm{min}$. The temperature range was ambient temperature up to $600{ }^{\circ} \mathrm{C}$.

\subsubsection{Differential Scanning Calorimetry}

The melting and crystallization properties of the nanocomposites were investigated by Differential Scanning Calorimetry (DSC) using a DSC Q2000 from TA Instruments. The samples weighing 12-14 $\mathrm{mg}$ were enclosed in a standard aluminum pan. They were firstly cooling down from ambient temperature to $-50{ }^{\circ} \mathrm{C}$ at a rate of $3{ }^{\circ} \mathrm{C} / \mathrm{min}$ under nitrogen atmosphere, and maintained at this temperature for $5 \mathrm{~min}$, then the samples were then heating up to $230{ }^{\circ} \mathrm{C}\left(3{ }^{\circ} \mathrm{C} / \mathrm{min}\right)$ and maintained at 
this temperature for $5 \mathrm{~min}$. The melting characteristics was measured during this heating mode. After that, the sample was cooling down $\left(3^{\circ} \mathrm{C} / \mathrm{min}\right)$ again to room temperature. The crystallization characteristics was measured during this cooling mode.

The crystallinity (Xc) of all the composites was calculated by equation (6.1),

$$
X c=\frac{\Delta \mathrm{Hm}}{\mathrm{w} \Delta H_{100}} \times 100 \%
$$

where:

$\Delta \mathrm{Hm}$ - melting enthalpy of the sample

$\mathrm{W}$ - weight percent of the polymer

$\Delta \mathrm{H}_{100}$ - melting enthalpy of the pure polymer.

In this equation, $\Delta \mathrm{H}_{100}$ values of $209 \mathrm{~J} / \mathrm{g}$ for PP [29] and $293 \mathrm{~J} / \mathrm{g}$ for PE [30] were used.

\subsubsection{X-ray Diffraction}

The crystalline structure of the polymer blend and the composites were studied by X-ray diffraction (XRD). A Philips X'Pert 1 X-ray diffractometer was used, and the samples were scanned within $2 \theta$ diffraction angle varying from $5^{\circ}$ to $37^{\circ}$ with a scanning rate of $0.05 \%$ s.

\subsubsection{Dynamic Mechanical Analysis}

The viscoelastic and relaxation behavior of the composites were investigated using a DMA (GaboNetzsch, Eplexor) in tensile mode. The samples were heated from -100 to $140{ }^{\circ} \mathrm{C}$ with a heating rate of $2{ }^{\circ} \mathrm{C} / \mathrm{min}$. The applied frequency was $1 \mathrm{~Hz}$, whereas the static strain was $0.2 \%$ and the dynamic strain was $0.1 \%$.

\subsubsection{Thermally Stimulated Depolarization Current}

Thermally Stimulated Depolarization Current (TSDC) was applied on the PP/EOC composites to study the charge trapping properties. The cast film samples were firstly gold-coated by circular electrodes to a thickness of $100 \mathrm{~nm}$ and a diameter of $16 \mathrm{~mm}$ on both sides. The TSDC test was done by following steps:

1. The sample was heated up to $70{ }^{\circ} \mathrm{C}$ and then kept at this temperature for $5 \mathrm{~min}$.

2. A DC electric field $(3 \mathrm{kV} / \mathrm{mm})$ was applied for 20 mins.

3. The sample was quickly cooled down to $-50{ }^{\circ} \mathrm{C}$ under the DC electric field and stabilized for 5 mins.

4. The DC electric field was removed and the sample was short-circuited for 3 min at $-50{ }^{\circ} \mathrm{C}$. 
5. The sample was heated up to $130{ }^{\circ} \mathrm{C}$ in a linear mode $\left(3{ }^{\circ} \mathrm{C} / \mathrm{min}\right)$. At the same time, the setup recorded the depolarization current.

\subsubsection{Pulsed Electro-Acoustic measurements}

Pulsed Electro-Acoustic (PEA) tests were carried out on thermally pre-treated cast film samples (72 hours at $60{ }^{\circ} \mathrm{C}$ in vacuum) to remove volatile by-products and humidity, which can affect the interface between nanoparticles and host material and thus the measurement results [31]. Space charge measurements were done during $3 \mathrm{~h}$ testing time under polarization (DC voltage applied) and $1 \mathrm{~h}$ of depolarization (DC voltage is zero and the specimen is short-circuited). The tests were performed at a temperature of $60^{\circ} \mathrm{C}$ in a controlled environment under a poling field of $30 \mathrm{kV} / \mathrm{mm}$.

\subsubsection{Conductivity}

Conductivity tests were performed on specimens with gold sputtered electrodes with a diameter of 26 $\mathrm{mm}$ and a guard ring, under an electric field of $30 \mathrm{kV} / \mathrm{mm}$ and at a temperature of $60^{\circ} \mathrm{C}$. After sputtering of the electrodes, specimens were left short circuited to ground for 24 hours at $60{ }^{\circ} \mathrm{C}$ in order to drain any possible charge introduced in the process.

\subsection{Results and Discussion}

\subsubsection{Silica Characterization}

The silica samples were firstly characterized by DRIFTS in order to check whether it was successfully modified by the silane. The results (Figure 6.1(a)) show the C-H stretching band at $2979 \mathrm{~cm}^{-1}$ originating from methyl groups of TMES, indicating that TMES is grafted onto the silica surface. Similarly, the bands at $3300 \mathrm{~cm}^{-1}$ (N-H stretching) [32] and $2979 \mathrm{~cm}^{-1}$ (C-H stretching) originating from the APTES amino group and propyl spacer respectively, prove a successful reaction with the silica surface. The mechanism of the silica silane modification is an alkoxy-group condensation with a silanol group on the silica surface [33]. The progress of the silica surface modification can be tracked by the band from isolated silanol groups of the silica observed at a wavenumber of $3747 \mathrm{~cm}^{-1}$ [34]. Figure 6.1 (a) and (b) show the tall band of isolated silanol groups present at the reference silica, while the intensity of the band is decreased for the TMES and APTES modified silicas. However, the band for the APTES silica is much lower than for TMES silica. This indicates that both silanes chemically reacted with silica, but the grafting degree is higher for APTES than TMES. To further verify the above statement, TGA measurements were done. The reference silica exhibits $2.6 \%$ weight loss mainly resulting from the removal the physically adsorbed water on the silica surface [35]. However, for the modified silicas there are higher weight losses: $4.7 \%$ and $12.2 \%$ for TMES and APTES modification, respectively. The weight losses above $2.6 \%$ weight result from the organic groups present on the silica surface, originating from TMES or APTES grafting. Moreover, it is obvious that the extent of APTES grafting is significantly higher than the one of TMES, stemming from the different chemical structure of the silanes, as shown in Figure 6.2. There are 3 alkoxy groups in each APTES molecule, while there is only one group in the TMES structure. Hence, in case of APTES, the alkoxy groups not only react with the silanol groups on the silica surface, but also react with itself (APTES self-condensation). As a result, they form 

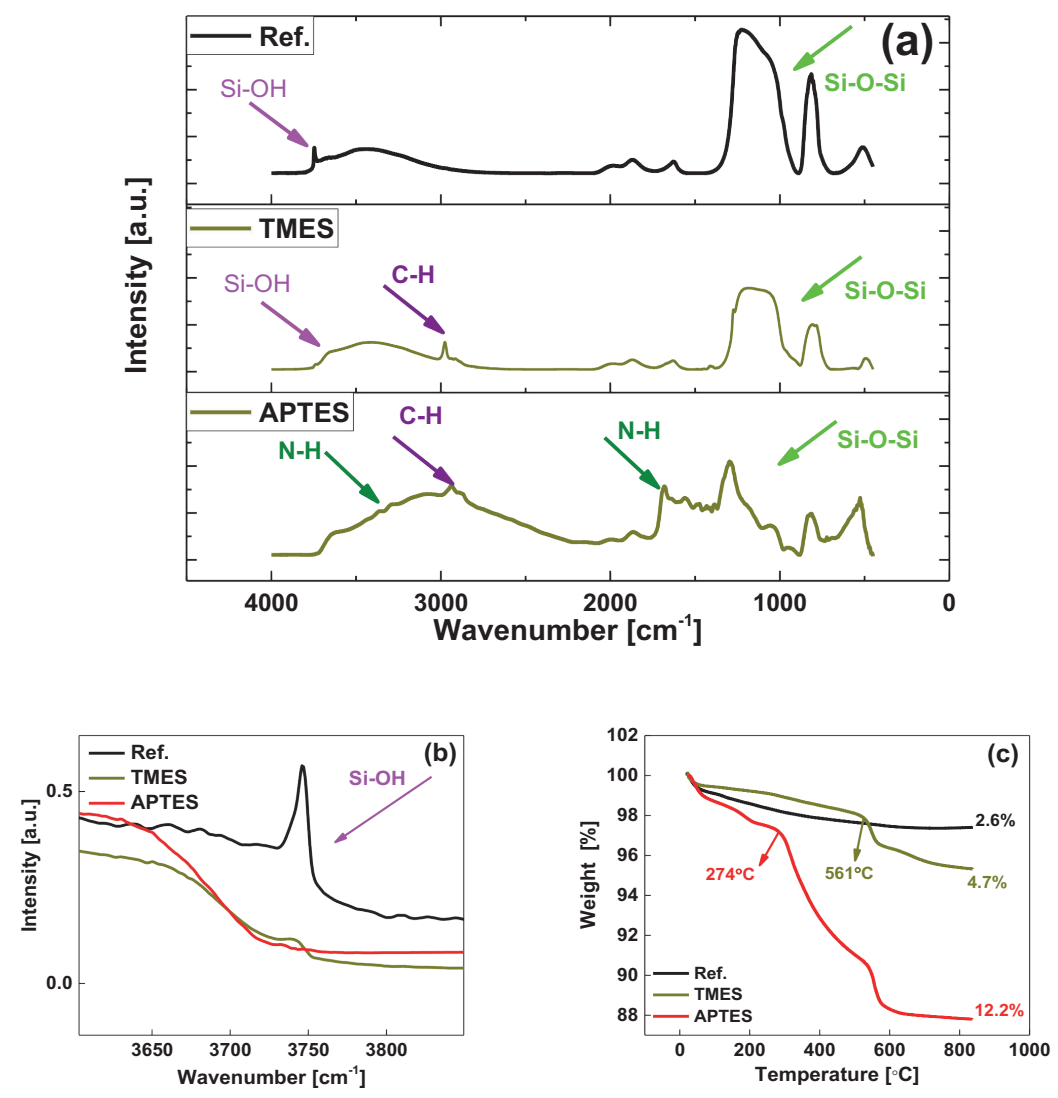

Figure 6.1 DRIFT curves (a and b) and TGA curves (c) of the reference and the modified silicas.

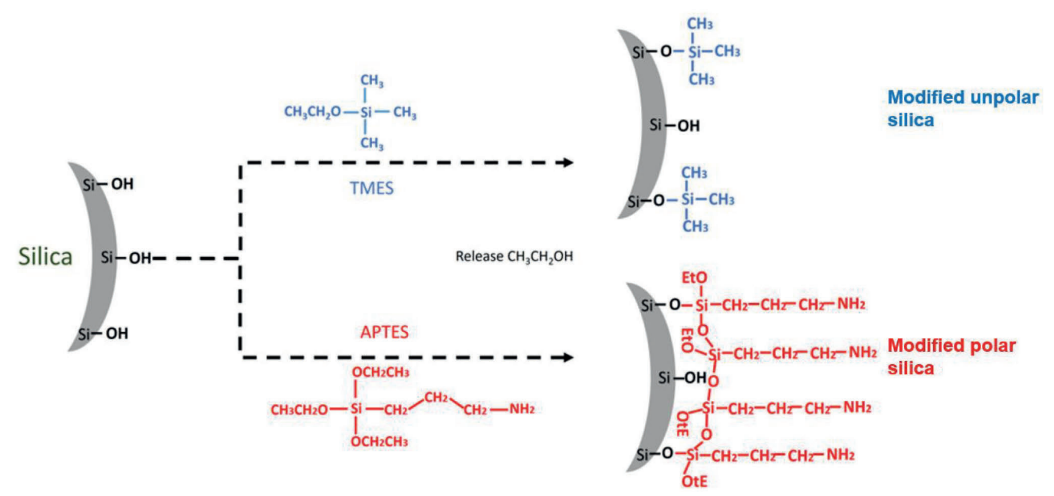

Figure 6.2 Possible reaction schemes of silica-silane surface modification. 
a layer of oligomerized APTES deposited on the silica surface (Figure 6.2). In contrast, TMES with only one alkoxy group does not undergo self-condensation, thus resulting in a less efficient deposition on the silica surface. Furthermore, the amine-functional group of APTES is a catalyst in the silanization reaction [36] and thus might contribute to the higher modification yield as well.

\subsubsection{Nanocomposite Morphology and Silica Dispersion}

Micromorphology and nanofiller dispersion play a major role in influencing the properties of nanocomposites. In order to have insight into the PP/EOC composite morphology, the microstructure of pure PP and EOC, of the PP/EOC blend and of the nanosilica-filled composites were studied by SEM. The cross-sectional microstructure of the pure PP (Figure 6.3 a) appears noticeably smoother than the microstructure of EOC (Figure $6.4 \mathrm{~b}$ ). This is most likely due to the thermoplastic nature of PP, for which the cracking of the sample during cryo-fracturing is brittle, whereas EOC can preserve a residual elastic behaviour even at low temperatures resulting in slight surface deformation while breaking, leading to a rougher appearance.

The microstructures of the PP/EOC blend and the composites are presented in Figure 6.4 (a) and (b). It is clearly observed that the biphasic structure consists of separate PP and EOC domains in a layered assembly. The EOC as the dispersed phase was elongated and broken into large domains distributed evenly in the low-viscosity PP. This is supported by the G.N. Avgeropoulos's statement that for a blend system, in which the mixing viscosities of the components are unequal, the low-viscosity component "encapsulates" the high-viscosity component and becomes the continuous phase [37].

It is also interesting to note that both, polar (Figure $6.4 \mathrm{c}$ and d) as well as unpolar silica (Figure 4e and f), are mostly located in the PP phase rather than in the EOC phase. This is again most likely related to the lower viscosity of PP compared to EOC, which allows more effective penetration of the PP macromolecules into the silica clusters and their adsorption onto the silica surface resulting in a higher compatibility of the two components. Apart from this, unpolar silica is in general better dispersed than polar silica, as seen in Figure 6.4 (c): relatively large clusters of polar silica can be observed in Figure 6.4 (d). This can be explained by the tendency of polar silica to cluster together in unpolar polymers caused by their incompatibility and high polar interactions between the silica particles. Polar silica can form agglomerates even larger than $1 \mu \mathrm{m}$. A better compatibility between the unpolar silica and the as well unpolar polymeric matrix originates from its low surface energy and dispersive interfacial interactions, ensuring a better dispersion of this nanofiller as shown in Figure 6.4 (e) and (f). 


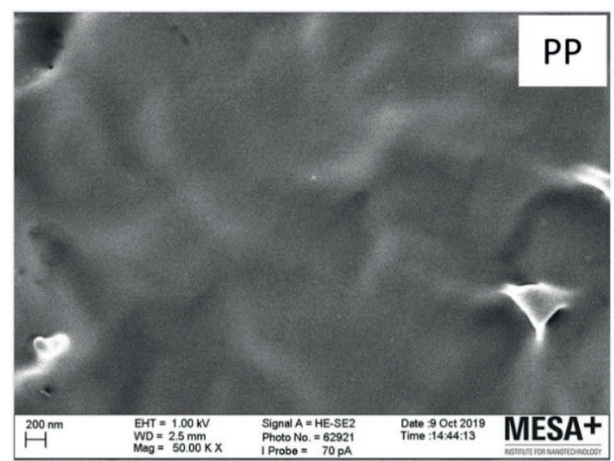

(a)

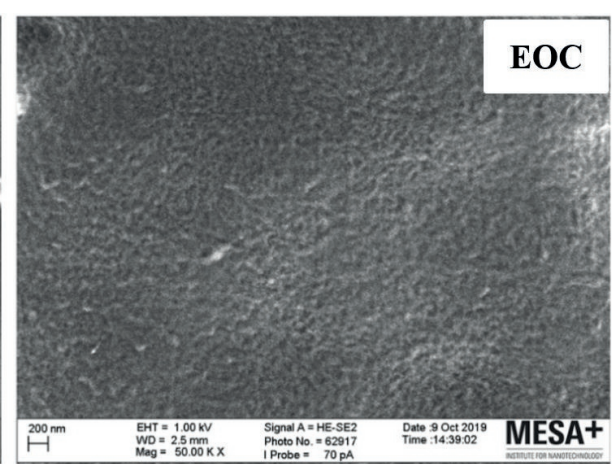

(b)

Figure 6.3 SEM images of unfilled PP (a) and EOC (b).

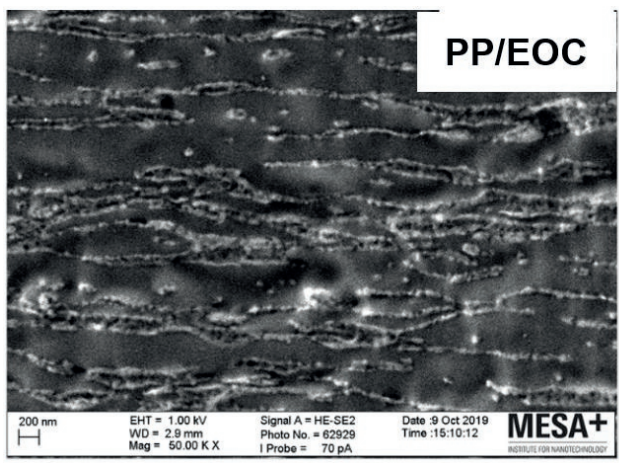

(a)

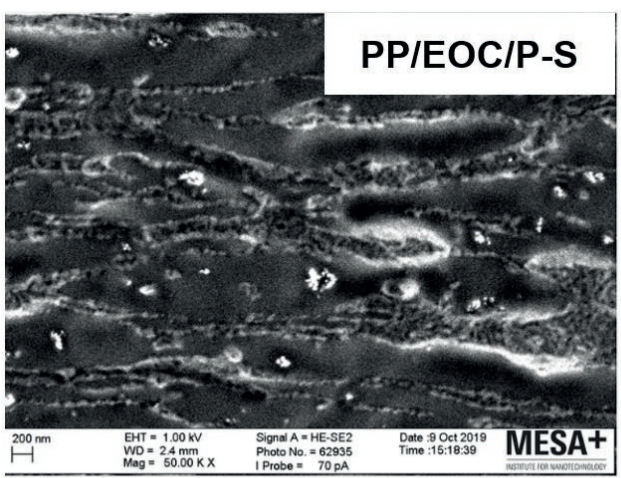

(c)

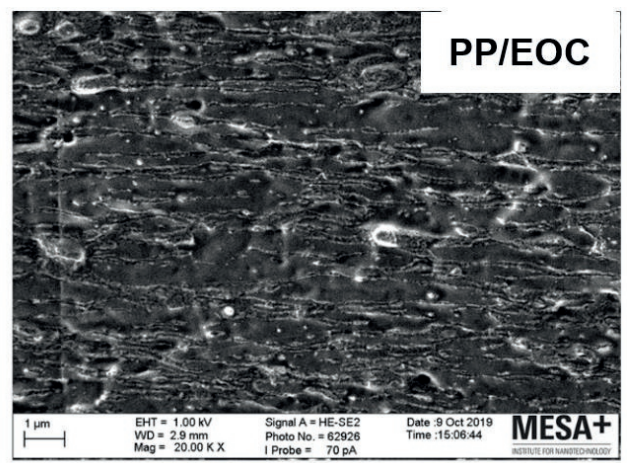

(b)

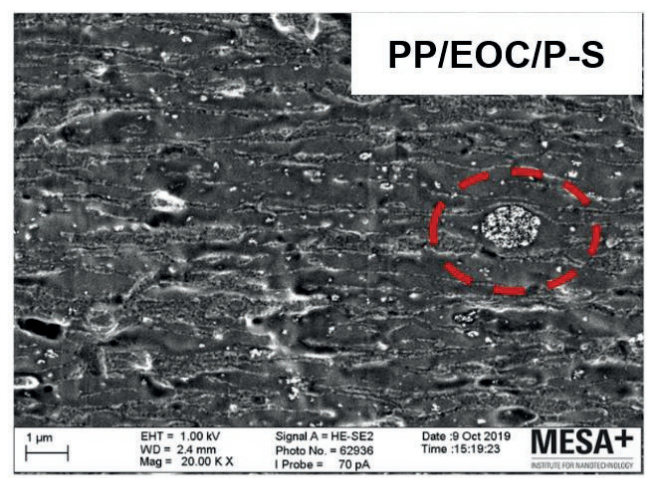

(d) 


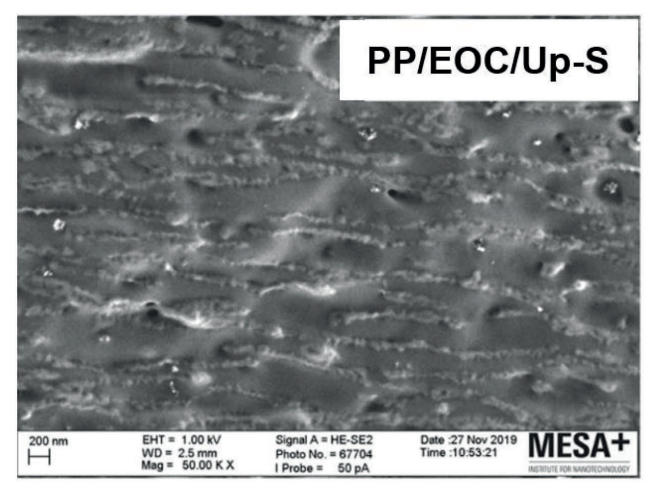

(e)

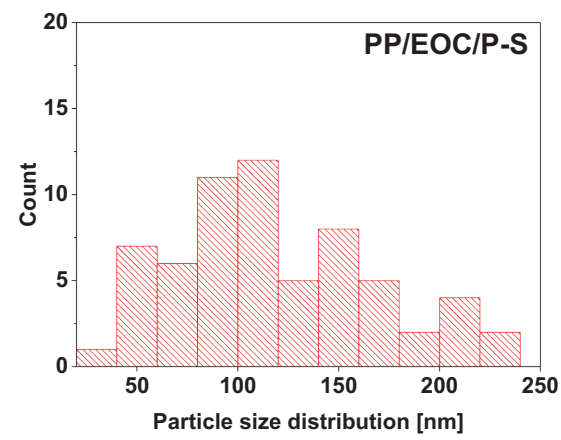

(G)

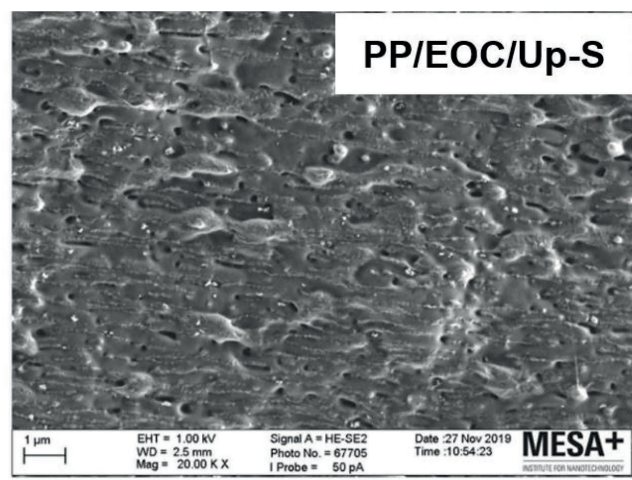

(f)

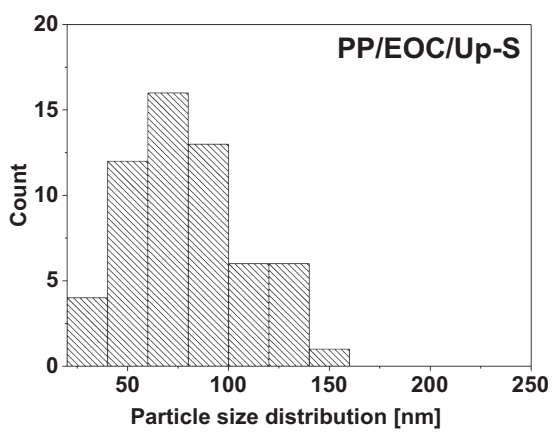

(H)

Figure 6.4 SEM micrographs of PP/EOC, PP/EOC/P-S, PP/EOC/Up-S and the particle size distributions (G and H) (Up-S: unpolar silica (TMES modified silica), P-S: polar silica (APTES modified silica)).

In order to qualitatively investigate the particle size difference between the polar and unpolar silica, the particle size distributions determined via ImageJ software are shown in Figure $6.4 \mathrm{G}$ and H. It is clearly shown that the polar silica exhibits a wider size distribution than the unpolar silica. All analysed unpolar silica particles exhibited diameters below $150 \mathrm{~nm}$ and the mean particle size is around 60-80 nm. For the polar silica, there is a significant portion of silica particles with diameters larger than $150 \mathrm{~nm}$, and the mean particle size is around $100-125 \mathrm{~nm}$. This indicates that the unpolar silica presents a better dispersion than polar silica. 


\subsubsection{Thermal Stability of Nanocomposites}

TGA measurements were performed on the nanocomposite samples to evaluate their thermal degradation characteristics. Figure 6.5 shows that the thermal degradation starts at the same temperature for all three samples. However, the kinetics of the thermal decomposition are significantly influenced by the presence of either the polar or the unpolar silica. The onset temperature of thermal degradation depends on the properties of the PP macromolecules, whereas the kinetics of degradation depend on the interfacial effects between the silica and the polymer blend. The variation in the degradation kinetics may result from the restricted motion of the polymer chains due to the presence of the nanosilica: Welldispersed nanoparticles create large interfaces with the polymeric matrix due to their high specific surface area. The interaction between the silica and the polymeric matrix reduces the polymer chain mobility at the interface. When the PP polymer chain is constrained by adding silica, the temperature at which anaerobic chain scission occurs will rise. Additionally, under oxidative conditions diffusion of air into the bulk is suppressed and thus a delay in the oxidation of polymer chains takes place [38].
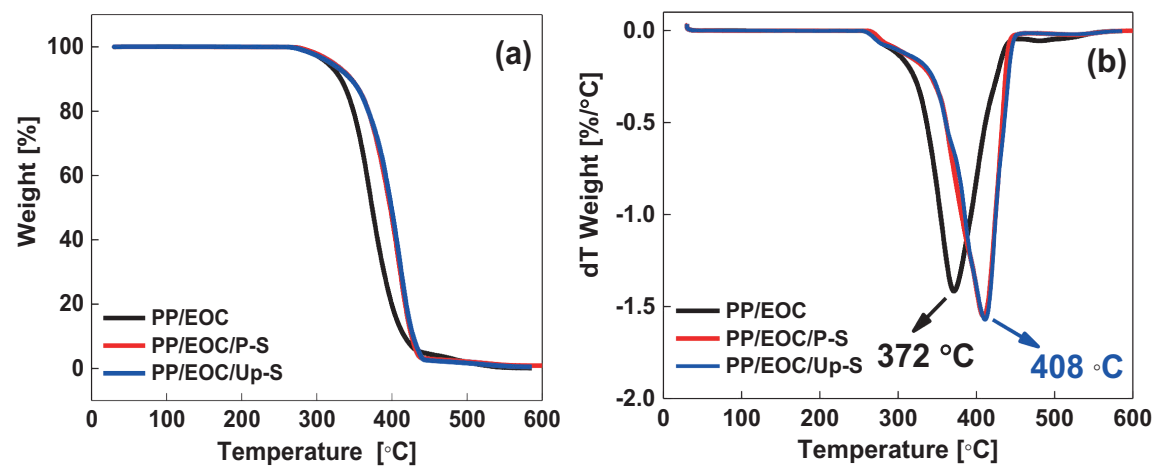

Figure 6.5 TGA measurements of the blend and the composites (a), and their derivatives (b).

\subsubsection{Crystallization Behaviour of the Nanocomposites}

In order to characterize the crystallization behaviour, DSC and XRD tests are performed. The results are shown in Figures 6.6 and 6.7, and the crystallization behaviour parameters are presented in Table 6.1. It should be noted that the Tg could not be identified in the DSC curve. This section is only focusing on the region of the melting and crystallization peaks, as shown in Figure 6.6 (a) and (b). Two separate crystallization peaks are seen in Figure 6.6 a, and two melting peaks in Figure 6b. In our previous work [39], we concluded that the lower temperature crystallization peak belongs to the crystalline polyethylene blocks from the EOC phase, while the other one belongs to the PP phase. This also supports our previous observation that PP and EOC are not miscible, as shown in the micrograph in Figure 6.4.

As seen in Figure 6.6 a and Table 6.1, the polymer filled with silica (polar or unpolar) feature a higher crystallization temperature (Tc peak 2), higher by about $7{ }^{\circ} \mathrm{C}$ than the Tc peak 2 of the unfilled polymer blend. This means that silica promotes the crystallization process by a nucleating effect $[40,41]$. Moreover, it is interesting to note that both silicas affect only the PP crystallization peak, but have barely 
any influence on the EOC crystallization peak (Tc peak 1). This is due to the preferential location of silica in the PP phase, in line with the SEM investigation results.

Figure $6.6 \mathrm{~b}$ and Table 6.1 show that there is no significant influence on the melting temperature (Tm peak 1 and Tm peak 2) of polymers by the addition of the nanosilica. The crystallinity of the studied composites is calculated and also reported in Table 6.1. It is clear that addition of silica decreased the crystallinity of the polymer blends. This might stem from two overlapping effects: crystallization nucleation and macromolecule confinement. The nanosilica acts as nucleating agent and promotes the onset of the crystallization process, but can also suppress the crystallization process by confining the polymer chain mobility [42]. Unpolar silica will exhibit stronger confinement than polar silica due to a higher compatibility and interfacial area between silica and polymer (better dispersion), resulting in lower crystallinity. This confinement obviously has a higher impact than the nucleating effect in both nanocomposite samples.

The XRD results are shown in Figure $6.6 \mathrm{c}$. All three composites show the same 6 peaks at the following diffraction angles: $14.5^{\circ}, 16.87^{\circ}, 18.48^{\circ}, 21.25^{\circ}, 23.64^{\circ}$ and $25.44^{\circ}$ respectively. The peaks appearing at $14.5^{\circ}, 16.87^{\circ}, 18.48^{\circ}$ and $25.44^{\circ}$ belong to the $\alpha$ crystal phase of PP. The peaks at $21.25^{\circ}$ and $23.64^{\circ}$ are the orthorhombic crystals from PE blocks in EOC, as reported earlier [8]. The obtained XRD results show that there are no new types of crystals formed as a result of the silica addition.
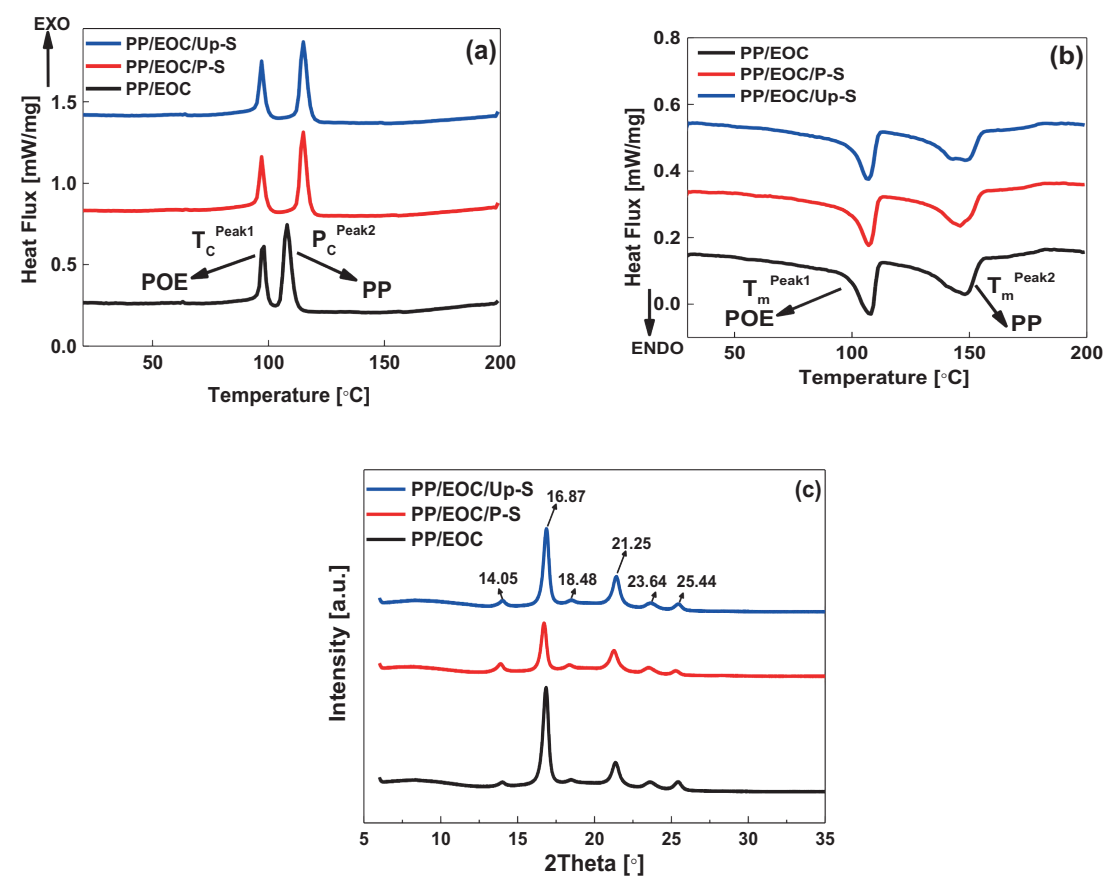

Figure 6.6 DSC measurements of the polymer composites: crystallization curves (a), melting curves (b), and XRD results (c). 
Table 6.1 Crystallization parameters.

\begin{tabular}{lcccccc}
\hline Sample & $\mathrm{T}_{\mathrm{C}}^{\text {peak1 }}\left({ }^{\circ} \mathrm{C}\right)$ & $\mathrm{T}_{\mathrm{C}}^{\text {peak2 }}\left({ }^{\circ} \mathrm{C}\right)$ & $\mathrm{T}_{\mathrm{m}}{ }^{\text {peak1 }}\left({ }^{\circ} \mathrm{C}\right)$ & $\mathrm{T}_{\mathrm{m}}{ }^{\text {peak2 }}\left({ }^{\circ} \mathrm{C}\right)$ & $\begin{array}{c}\Delta \mathrm{H}_{\mathrm{m}} \\
(\mathrm{J} / \mathrm{g})\end{array}$ & $\mathrm{X}_{\mathrm{C}}(\%)$ \\
\hline $\mathrm{PP} / \mathrm{EOC}$ & 97.3 & 108.2 & 107.5 & 148.4 & -99.0 & 39.8 \\
$\mathrm{PP} / \mathrm{EOC} / \mathrm{P}-\mathrm{S}$ & 96.9 & 115.3 & 107.0 & 146.4 & -91.0 & 36.2 \\
$\mathrm{PP} / \mathrm{EOC} / \mathrm{Up}-\mathrm{S}$ & 96.9 & 115.2 & 106.5 & 148.4 & -82.4 & 32.8 \\
\hline
\end{tabular}

$\mathrm{T}_{\mathrm{C}}^{\text {peak1 }}$ : crystallization peak temperature of the EOC phase;

$\mathrm{T}_{\mathrm{C}}{ }^{\text {peak2 }}$ : crystallization peak temperature of the PP phase;

$\mathrm{T}_{\mathrm{C}}{ }^{\text {on }}$ : onset of crystallization peak of the PP phase;

$\Delta \mathrm{H}_{\mathrm{m}}$ : total enthalpy during the crystallization process;

$\mathrm{X}_{\mathrm{C}}$ : calculated crystallinity of $\mathrm{PP} / \mathrm{EOC}$ nanocomposites.

\subsubsection{Chain Relaxation Behaviour of the Nanocomposites}

DMA measurements were performed in order to investigate the viscoelastic and relaxation behaviour of the composites, as measured by the storage modulus E' and the loss factor $(\tan \delta)$ vs temperature as presented in Figure 6.7. In semi-crystalline polymers, different responses from the amorphous (Tg) and crystalline phases (crystal movement and melting) are measured. Besides, silica addition might influence the polymer chain relaxation behaviour of the PP/EOC blend.

The storage modulus E' shows a descending trend with temperature for all composites as shown in Figure $6.7 \mathrm{a}$. When the temperature is lower than the glass transition temperature ( $\mathrm{Tg}$ ) of a polymer, its chains in the amorphous phase are in the glassy state and exhibit low mobility. When the temperature is higher than $\mathrm{Tg}$, the modulus of the polymer rapidly decreases due to the rise of segmental mobility, that contributes significantly to the overall dynamics of the polymer macromolecules.

The PP/EOC/P-S and PP/EOC/Up-S nanocomposites show lower E' values than the unfilled PP/EOC blend, Figure 6.7 a. This probably results from the reduction of the crystalline phase due to the balance between nucleating effect and chain confinement of silica on the macromolecules, as previously discussed (Table 6.1).

The results of the tan $\delta$ measurements are presented in Figure $6.7 \mathrm{~b}$. There are four peaks at temperatures around $-36{ }^{\circ} \mathrm{C},-6{ }^{\circ} \mathrm{C}, 66{ }^{\circ} \mathrm{C}$ and above $104{ }^{\circ} \mathrm{C}$ respectively. The small peak at $-36{ }^{\circ} \mathrm{C}(\beta$ (EOC)) corresponds to the $\mathrm{Tg}$ of the EOC phase, which is the primary amorphous chain relaxation (also called $\beta$ relaxation) of EOC According to literature, the glass transition temperature of the EOC amorphous phase is between $-50{ }^{\circ} \mathrm{C}$ and $-20^{\circ} \mathrm{C}$, depending on the different ratios of ethylene and octene monomers $[43,44,45]$. The peak ( $\beta(\mathrm{PP}))$ at $-6{ }^{\circ} \mathrm{C}$ is the $\mathrm{Tg}$ of the PP phase [46], which is the primary amorphous chain relaxation ( $\beta$ relaxation) of PP. The peak $(\alpha 1)$ starts from $30{ }^{\circ} \mathrm{C}$ and it is coherent with the small step around 30 to $70{ }^{\circ} \mathrm{C}$ in the inserted DSC figure. At this temperature, the crystals do not yet start melting. Hence, this peak $(\alpha 1)$ is associated with the relaxation of restricted amorphous chains by both PP and EOC crystalline phases $[47,48]$. The peak $(\alpha 2)$ starting from $104{ }^{\circ} \mathrm{C}$ is related to the melting 
process of both EOC and PP crystals, which is in agreement with the melting temperature of both $\left(\mathrm{T}_{\mathrm{m}}\right.$ EOC $=105^{\circ} \mathrm{C}$ and $\mathrm{T}_{\mathrm{mPP}}=148{ }^{\circ} \mathrm{C}$ ), as shown in the inserted graph in Figure $6.7 \mathrm{~b}$. It should be noted that due to the limited temperature range of the measurement, the melting temperature of PP cannot be clearly indicated.

No significant influence of silica on the relaxation behaviour of the PP/EOC composites could be detected. It is shown that no effect of the filler on Tg is shown in DMA. Regarding to the $\alpha$-relaxation, the DMA results are identical with DSC results, this indicates that silica does not affect the melting behaviour of the nanocomposites. It is highly probable that this is due to the low concentration of the filler (only $1 \mathrm{wt} . \%$ ). The effect of this small amount of filler on the $\alpha$-relaxation process is expected not to be detectable by DMA neither.
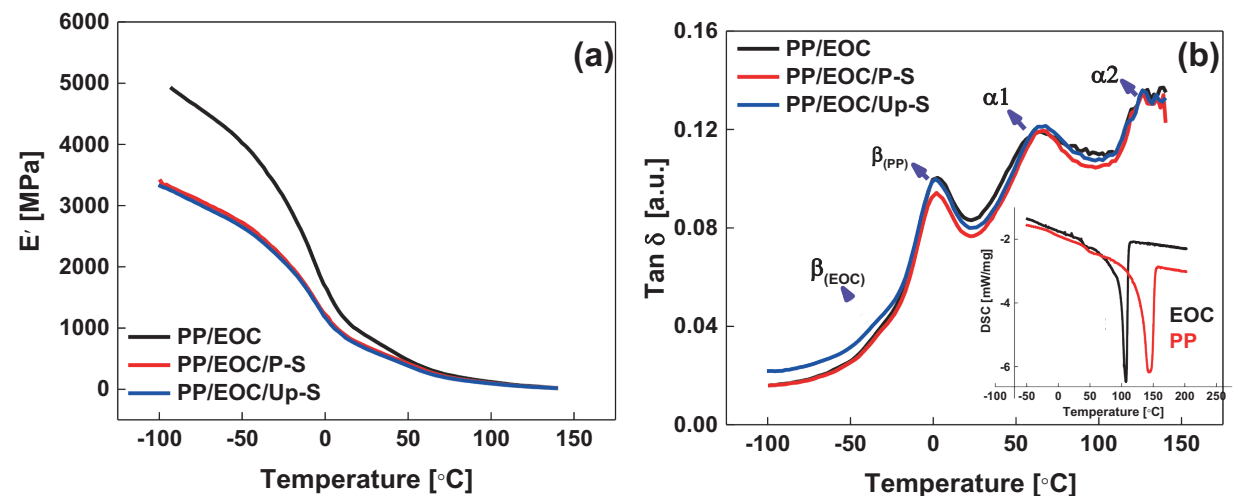

Figure 6.7 Storage modulus vs temperature (a), and loss factor vs temperature (b) of $\mathrm{PP} / \mathrm{EOC}, \mathrm{PP} / \mathrm{EOC} / \mathrm{P}-\mathrm{S}$ and PP/EOC/-Up-S samples. The insert shows the DSC melting curves of pure PP and EOC.

\subsubsection{Charge Trapping Properties of the Nanocomposites}

To investigate the influence of silica with different surface modifications on the charge trapping properties of the PP/EOC nanocomposites, TSDC measurements were performed. Charges were first injected into the sample during the high voltage poling phase at elevated temperature, after which the specimen was cooled down rapidly to effectively 'freeze' the trapped space charge. During the subsequent thermally stimulated depolarization step, during which the sample is heated linearly under short-circuit conditions, gradual relaxation of polarized species and trapped charge release occurs, giving rise to a thermally stimulated current in the external circuit. The measured thermally stimulated depolarization current as a function of temperature is plotted in Figure 6.8 a. Temperature and thermally stimulated current are related to the depth and density of charge traps respectively. The charge trap is a location restricting the mobility of charges with a certain energy (trap depth). The trap density of states versus trap depth distribution approximated from the TSDC spectra using a numerical method [49], is presented in Figure $6.8 \mathrm{~b}$. 

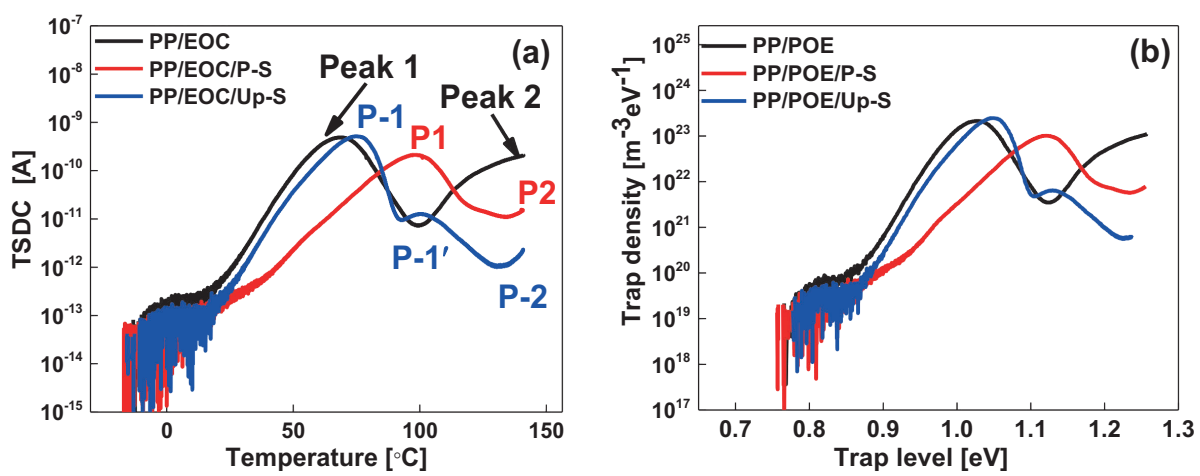

Figure 6.8 TSDC test results (a) and the calculated trap distribution (b) of PP/EOC, $\mathrm{PP} / \mathrm{EOC}-\mathrm{P}-\mathrm{S}$ and PP/EOC/Up-S nanocomposites.

For the unfilled PP/EOC blend, two peaks can be distinguished in Figure 6.8: one complete (Peak 1) and one incomplete (Peak 2) (the measurement was stopped before the complete melting temperature $150{ }^{\circ} \mathrm{C}$ ). In order to protect the TSDC equipment, the test had to be stopped at a temperature of $140{ }^{\circ} \mathrm{C}$ just before the melting temperature of the sample was reached. The temperature at the maximum of Peak 1 is $68.6{ }^{\circ} \mathrm{C}$, corresponding to a trap level of about $0.96 \mathrm{eV}$. The visible part of Peak 2 is in the range between $110^{\circ} \mathrm{C}$ and $140{ }^{\circ} \mathrm{C}$, corresponding to a much deeper trap level (above $1.1 \mathrm{eV}$ ). These two peaks are correlated to the relaxation behavior $\left(\alpha 1\right.$ at $65^{\circ} \mathrm{C}$ and $\alpha 2$ at $\left.133{ }^{\circ} \mathrm{C}\right)$ of the polymer, as shown in Figure 6.9, which is the motion of the amorphous chains constrained by crystals in the polymer matrix $(\alpha 1)[47,48]$ and the melting process of the crystallites $(\alpha 2)$, respectively. This gives an indication that the charge released from the trap sites associated with the molecular motion, the motion of the amorphous chains restricted by the crystals and the crystalline melting. Hence, it also supports the statement that the charge trap sites are associated with the crystalline-amorphous interfaces and the crystalline regions in the unfilled blend, originating from the defects within these areas [50], as shown in Figure 6.10 (a).

Similarly to the unfilled PP/EOC blend, there are also two peaks (P1 and P2) detected in the PP/EOC/P$\mathrm{S}$ (Figure 6.8). However, the charge traps P1 $(1.12 \mathrm{eV}$ ) and P2 (above $1.25 \mathrm{eV}$ ) in PP/EOC/P-S are significantly deeper than the traps in the unfilled PP/EOC blend. This indicates that polar silica introduces deeper traps in comparison to the unfilled PP/EOC blend. Moreover, there is no correlation between the TSDC depolarization current peak and DMA relaxation peak, as seen in Figure $6.8 \mathrm{~b}$. This suggests that the charge trap sites in the nanocomposite might not be located in the amorphous-crystal interfaces or in the crystalline region anymore. Thus the question arises where the charge trap sites are located in the nanocomposites. A number of reports state that the introduction of a nanofiller creates trap sites around the latter or in the nanofiller-polymer interface [51, 52, 53]. Therefore, the silica addition to the polymer is expected to modify the distribution of the charge traps within the polymer matrix, as new interfaces between silica and the polymeric matrix are introduced. It is very likely that the charges are mostly trapped around the silica, at the interface between silica and polymer matrix (Figure $6.10 \mathrm{~b}$ ). Therefore, the charge trap distribution in the nanocomposites might be more related to the silica itself and the filler-polymer interface, rather than to the crystal/crystal or crystal/amorphous 

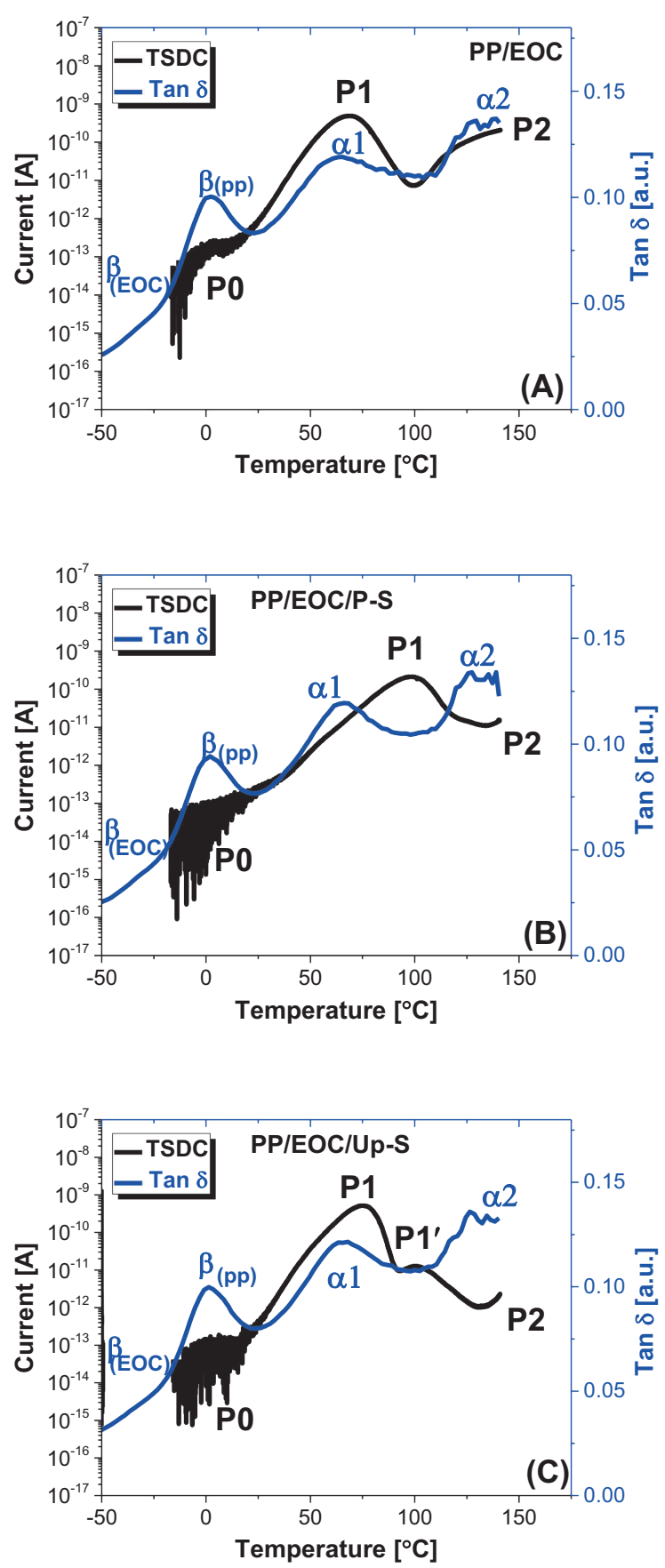

Figure 6.9 Relationship between TSDC depolarization current and DMA relaxation peak: unfilled PP/EOC blend (a); PP/EOC/P-S (b); PP/EOC/Up-S (c). 
interfaces. This interpretation is also consistent with the trap distribution of the PP/EOC/Up-S nanocomposite: here as well no clear correlation between the TSDC depolarization current peaks and DMA relaxation peaks is seen.

All phenomena mentioned above support the hypothesis that the charge carriers are strongly attracted to the silica surface. We assume that the driving force of this attraction is twofold: The polar character of the silica itself can attract ionic charge carriers. It is also reported that silanol groups and especially residual water molecules adsorbed on the silica surface exhibit strong attractive forces to electrons, withdrawing them from the polymer matrix [54]. Moreover, due to the different resistivity of silica and $\mathrm{PP}$, the silica-PP interface constitutes charge trapping sites distorting the local electrical field at the interface [55]. Since the silica itself - in particular the silica-polymer interface - plays an important role in terms of tailoring the charge trapping distribution, we can conclude that the silica surface functionalization and the nucleation effect of the silica also influences the charge trapping properties.

We have noticed that there are differences but also similarities in the introduced trap distribution between the PP/EOC/Up-S and PP/EOC/P-S. According to the experimental results shown in Figure 6.8, addition of the unpolar silica results in a higher trap density, while for the PP/EOC/P-S the trap density decreases. One possible reason is the dispersion of silica: The dispersion of the unpolar silica in the polymeric matrix is better due to a higher compatibility with the polymer matrix than the dispersion of the polar silica. This leads to an increase in interfacial area between unpolar silica and polymeric matrix. Consequently, the trap density is increased by adding unpolar silica, while it is reduced by adding polar silica, as its clustering (Figure 6.4) limits the number of trapping sites. Another reason is the effect of functional groups introduced by the addition of the modified silicas. In this study, the functional group is an amine group $\left(-\mathrm{NH}_{2}\right)$ of electronegative nature, which induces deeper traps in a material and reduces charge injection [12], resulting in a lower current (lower trap density) in the PP/EOC/P-S nanocomposite. In case of unpolar silica, the functional group is methyl $\left(-\mathrm{CH}_{3}\right)$. This moiety performs similarly to the hydrocarbon chains in the polymer blend.

Considering the trap depth differences, caused most likely by the different types of surface modification and homogeneity of the surface deposition of the modified silicas, (the relatively uniform deposition on the polar silica and inefficient deposition on the unpolar silica) as shown in Figure 6.2, different chemical and morphological effects of the modification introduce different energy states leading to the various trap levels. For P-S, we can expect a relatively even silane distribution with a layer of condensed APTES on the silica surface, while for the Up-S, it is likely to obtain an uneven TMES molecule distribution. The functional silane layer on the outer surface of the P-S contains amine-groups (-NH2), which makes its surface less hydrophobic than Up-S. Due to the specific surface chemistry, the amine moiety, a new interfacial energetic state is generated by addition of the polar silica. This results in a new interfacial energetic state based on the interaction between the -NH2 group and the polymer, which might cause the deep trap (P1) in the PP/EOC/P-S nanocomposite. Due to the limited extent of the silica surface modification with TMES, not the complete silica surface can be covered by TMES. Only isolated and geminal silanols are reactive towards ethoxy groups; vicinal groups will not react [56]. Moreover, TMES cannot oligomerize to shield the non-reactive sites on the silica surface, resulting in remaining silanol groups. Therefore, the vicinal silanols and siloxanes present on the silica surface are exposed at the interface between the silica and the polymeric matrix. Hence, on the outer surface of Up-S, $-\mathrm{CH}_{3}$ and $\mathrm{CH}_{2}$ groups are present, similar to the chemical structure of the polymer, and unreacted silanol (Si-OH) and siloxanes ( $\mathrm{Si}-\mathrm{O}-\mathrm{Si}$ ) groups. All these groups generate various and complex energy states resulting 
in the new traps $\left(\mathrm{P}-1\right.$ and $\left.\mathrm{P}-1^{\prime}\right)$ in the $\mathrm{PP} / \mathrm{EOC} / \mathrm{Up}-\mathrm{S}$ nanocomposites. To summarize, the $\mathrm{P} 1$ peak in $\mathrm{PP} / \mathrm{EOC} / \mathrm{P}-\mathrm{S}$ and the $\mathrm{P}-1$ and $\mathrm{P}-1$ 'in $\mathrm{PP} / \mathrm{EOC} / \mathrm{Up}-\mathrm{S}$ originate from the surface functionalization of the silica and the modification efficiency. This is in line with our previous observations [28].

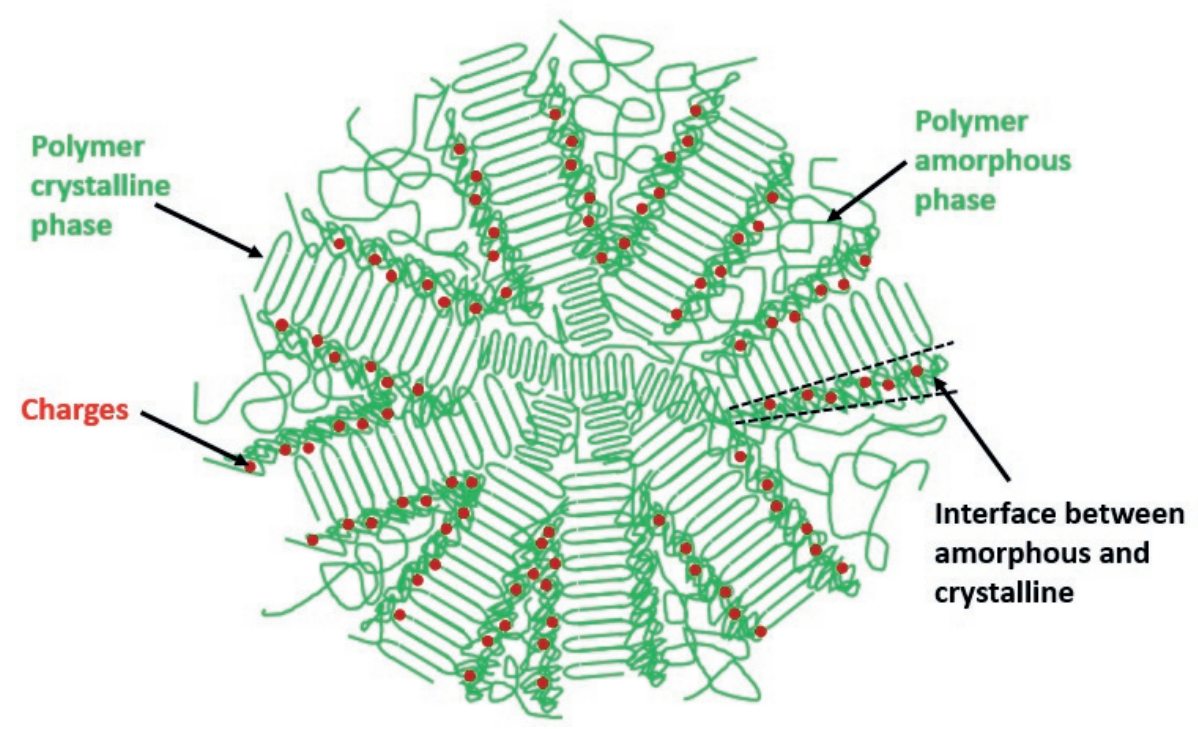

(a)

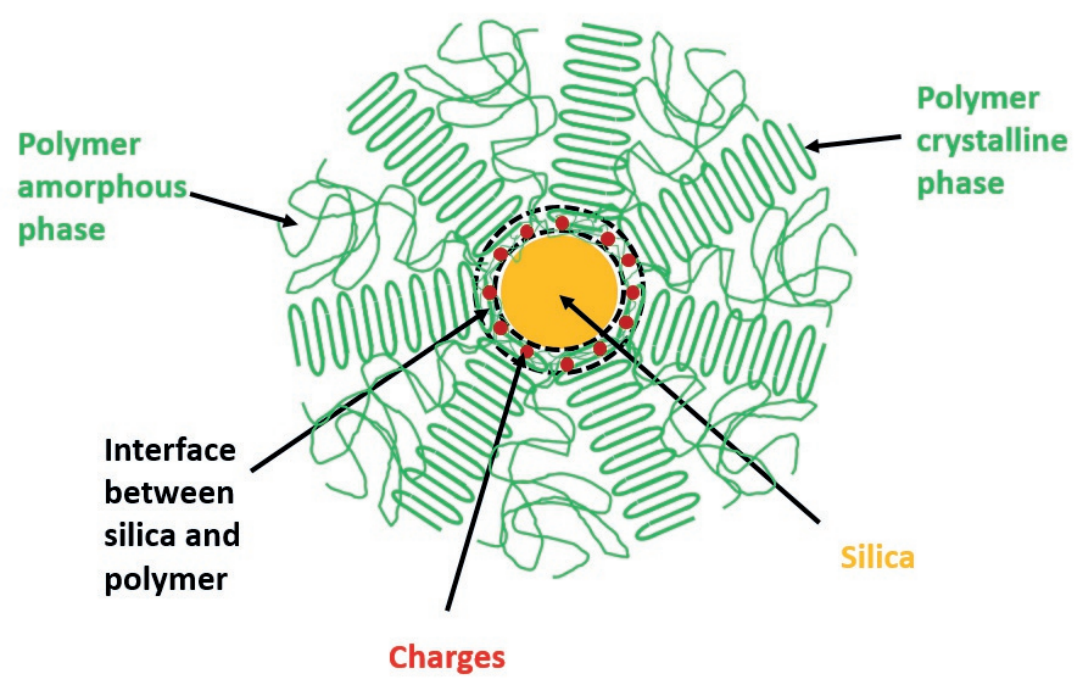

(b)

Figure 6.10 The scheme of the most of the charges possible location in the unfilled blend (a) and nanocomposites (b). 
Consequently, one should consider the origin of the deepest traps (P2) present in both, PP/EOC/P-S and $\mathrm{PP} / \mathrm{EOC} / \mathrm{Up}-\mathrm{S}$ nanocomposites. We observed that these deepest traps are located at a temperature of $140{ }^{\circ} \mathrm{C}$. Due to the fact that complete melting of the PP crystalline phase occurs at around $160{ }^{\circ} \mathrm{C}$, it was not feasible to continue the TSDC measurements above $140{ }^{\circ} \mathrm{C}$. Nevertheless, we found that there is a very deep trap peak (P2) present in the nanocomposites starting its rise at a temperature of $140{ }^{\circ} \mathrm{C}$. This was also observed in other studies [51]. It is reported that adding nanosilica introduces the deepest traps at high temperatures above $140{ }^{\circ} \mathrm{C}$ or $160{ }^{\circ} \mathrm{C}$. It was suggested that these trap sites originate from the nanosilica itself, since similar peaks were not noticed for the non-filled reference materials. Besides, in the present study, it is also found that the release of the charges around $140{ }^{\circ} \mathrm{C}$ correlates with the beginning of PP melting. Moreover, the silica is only located in the PP phase and increases the crystallization temperature of the PP phase due to the nucleating effect. Therefore, the deepest trap peak P2 might also be related to the interface between the silica and the induced PP crystallites independently from the surface modification.

1) Silica itself might act as the charge trapping site;

2) Nucleating effect of the silica alters the interface between the silica and induced PP crystallites leading to the $\mathrm{P} 2$ formation.

\subsubsection{Space Charge Behavior of the Nanocomposites}

To evaluate the amount of space charge accumulation within PP/EOC, PP/EOC/Up-S and PP/EOC/P-S, Pulsed Electro-Acoustic (PEA) tests were performed. The space charge profiles of the compounds are shown in Figure 6.11. The amount of stored charge measured after removing the electric field is presented in Figure 6.12, along with the peak trap densities originating from the TSDC measurements.

Figure 6.11 clearly displays the presence of slow charge packets crossing the samples for all the tested materials. Comparing the results of the filled PP/EOC/silica composites with the unfilled PP/EOC sample, it is noticed that the unpolar silica addition decreases the number of positive heterocharges trapped in the specimen near the ground electrode. Polar silica addition further decreases the amount of the heterocharge accumulation during the poling phase.

Considering the amount of charge stored during the polarization phase, the addition of polar silica significantly decreases the charge accumulation, while the presence of unpolar silica increases it. This is also in agreement with the TSDC data (Figure 6.9) concerning charge trap density of the samples at medium temperatures. A lower charge trap density reduces the charge storage in the material, while a higher charge trap density increases it.

Furthermore, charge depletion during the depolarization phase is very different in these three samples. The unfilled PP/EOC composite tends to quickly drain charges, while the addition of silica reduced this tendency. This was expected from the introduction of deeper traps by the nano-silica particles. Furthermore, the presence of the polar silica in the polymer matrix resulted in a much lower depletion speed than the presence of unpolar silica, which is in agreement with the obtained TSDC results showing increased trap depth and decreased density. This is another indication that the P-S introduces more deep traps than the Up-S. 


\section{PP/EOC}

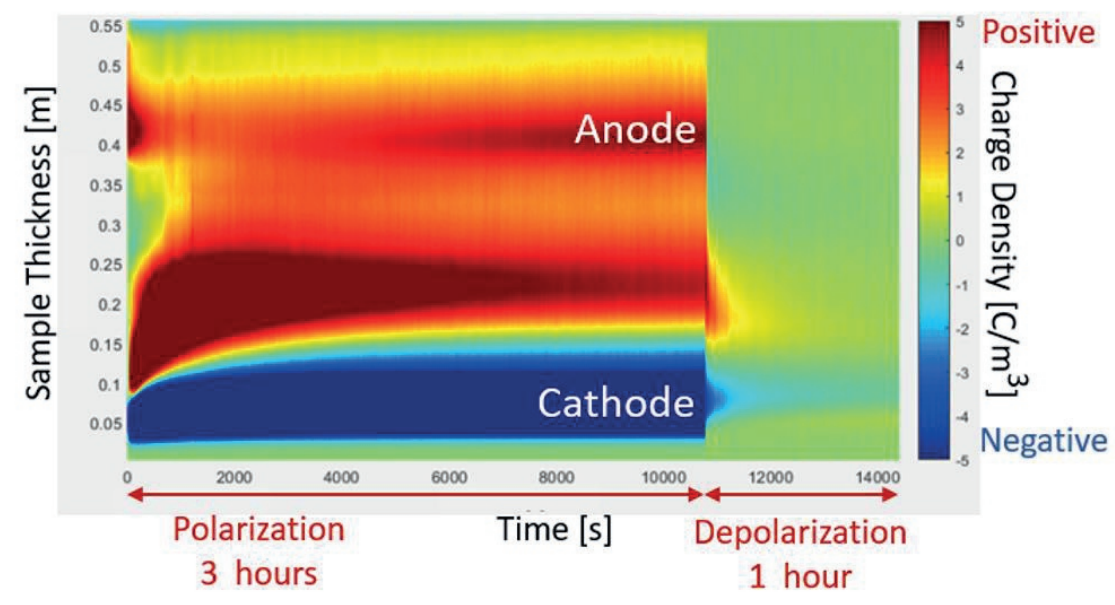

\section{PP/EOC/Up-S}

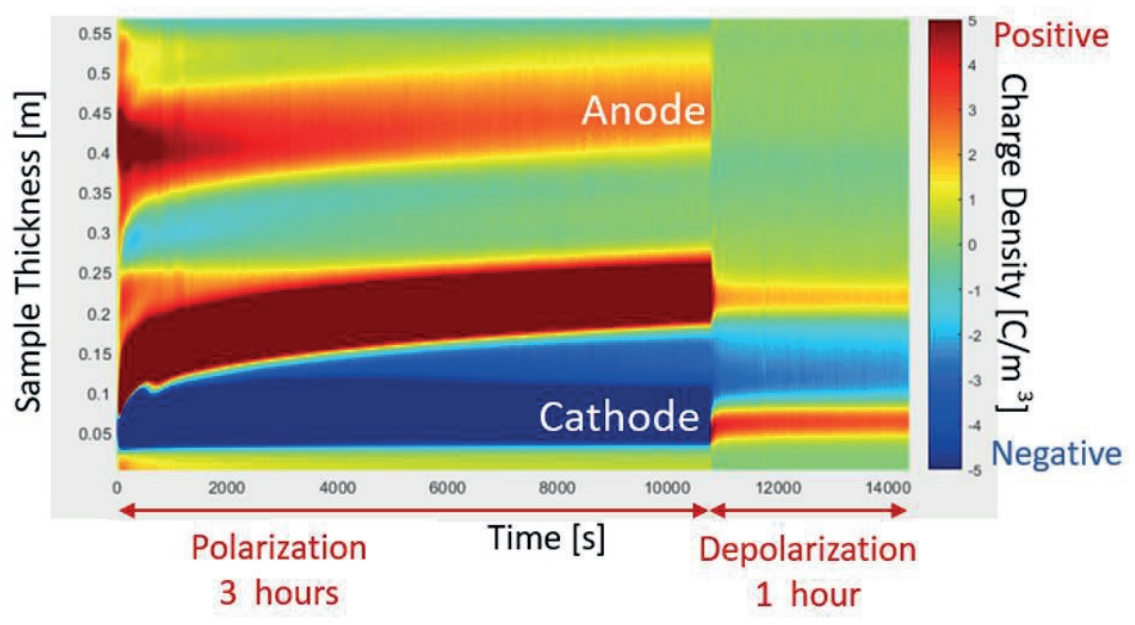




\section{PP/EOC/P-S}

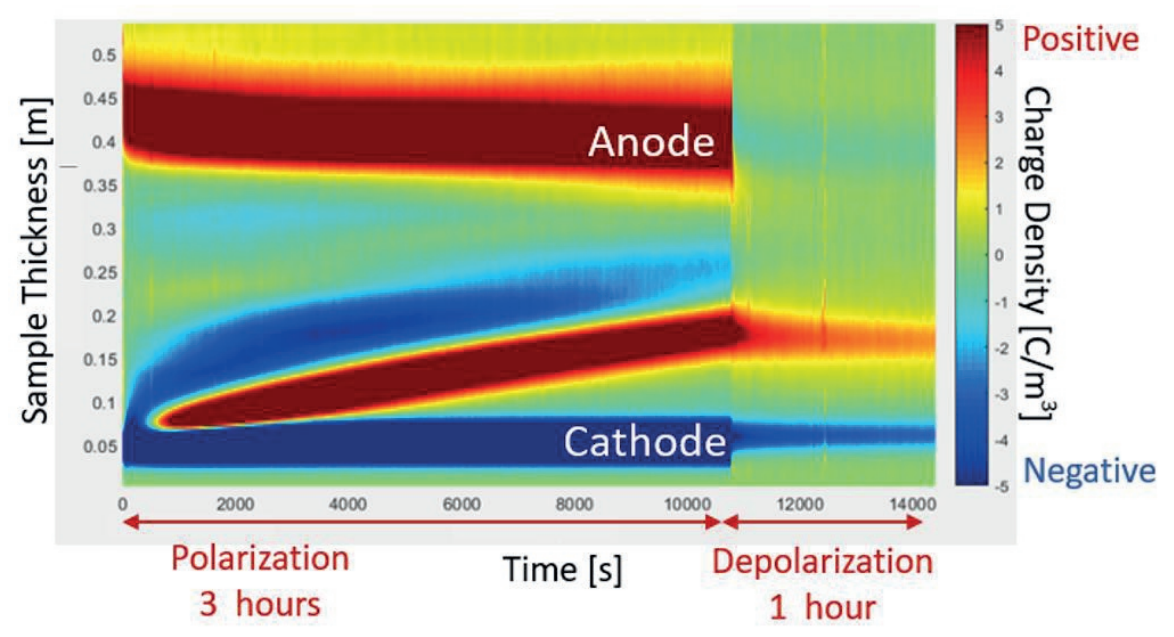

Figure 6.11 PEA space charge profiles of PP/EOC, PP/EOC/P-S and PP/EOC/Up-S nanocomposites.

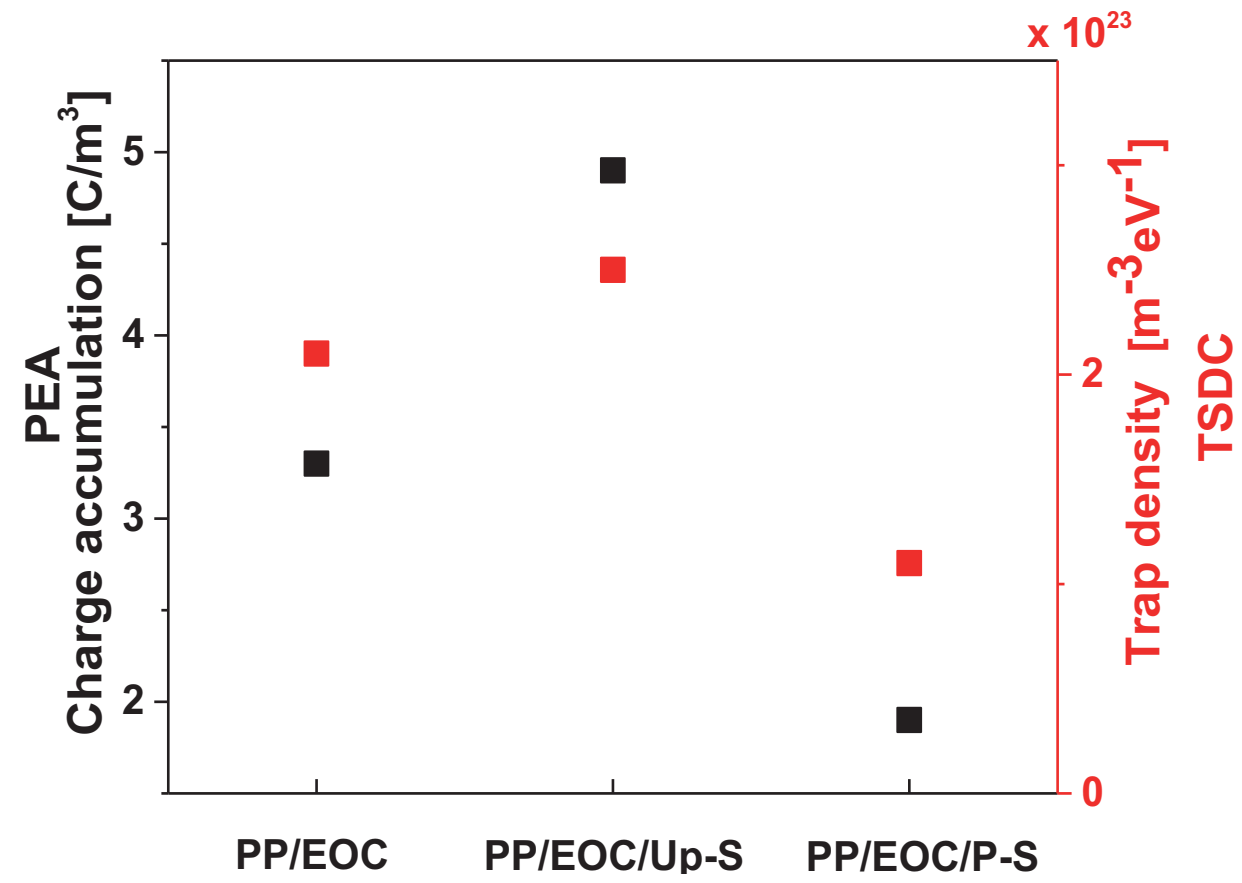

Figure 6.12 Charge accumulation after Volt off and trap density in the PP/EOC, PP/EOC-P$\mathrm{S}$ and PP/EOC-Up-S nanocomposites. 


\subsubsection{Charging Current Density of the Nanocomposites}

To further investigate the insulation properties of the materials, conductivity tests were performed. The charging current density versus time curve on a log-log scale is shown in Figure 6.13. Bulk conductivity was evaluated under steady state conditions (i.e., after the polarization phenomena were fully elapsed). On the one hand, taking into account the final charging current density at the end of the polarization period for these three samples, the addition of polar silica increases conductivity, and the sample containing unpolar silica features the highest resistivity. On the other hand, focusing on the beginning of the test it is noticed that the filled materials feature a decreased value for the initial polarization current. This could be due to the different space charge behavior described in the previous section, affecting the conductivity [57]. The detected current shift directly depends on the charge sign, speed and direction. In Figure 6.11, a stronger heterocharge migration near the cathode is detected in the neat sample. This will in turn increase the detected current and conductivity.

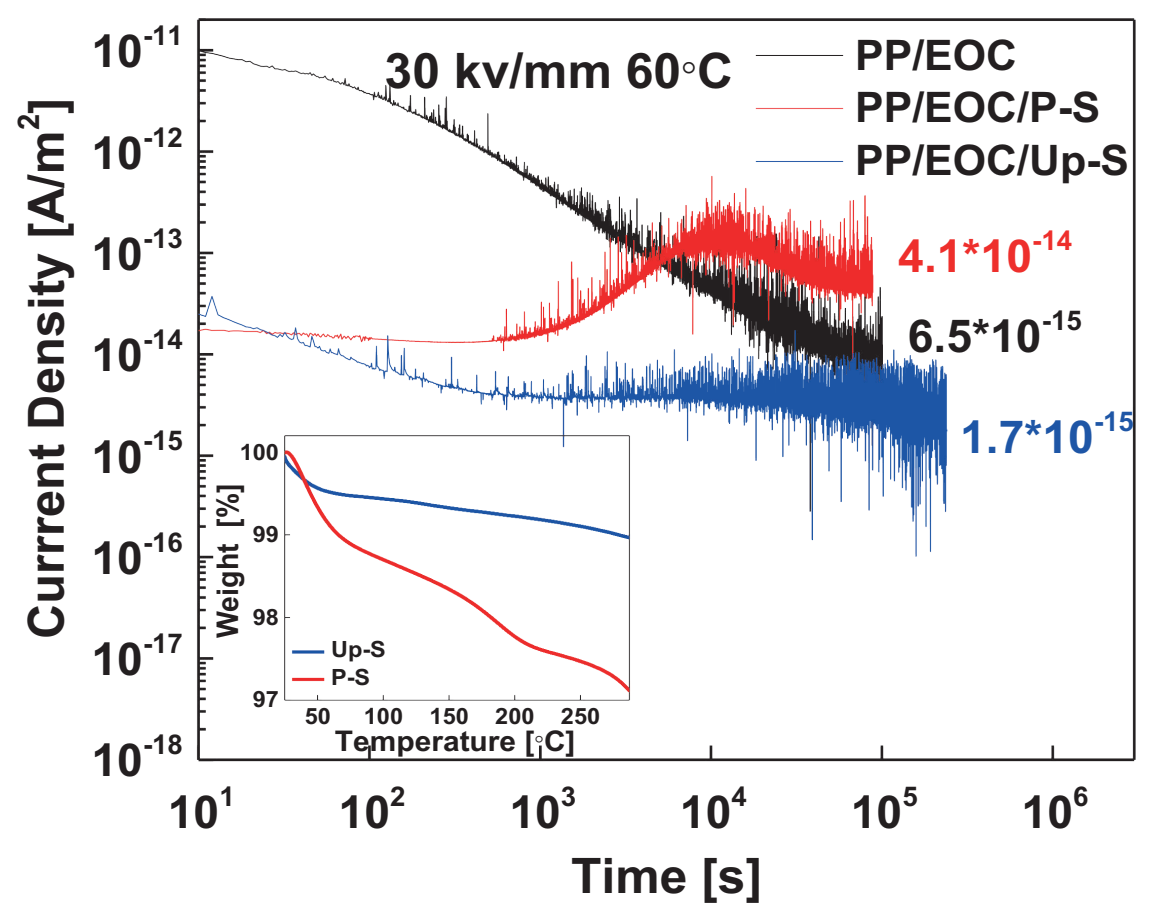

Figure 6.13 Charging current density of the PP/EOC, PP/EOC/P-S and PP/EOC/Up-S samples. The inserted TGA curve shows the weight loss temperature of both silicas.

The same mechanisms are also responsible for the temporary increase of charging current measured during the polarization period, as the current density peak is clearly affected in the PP/EOC/P-S blend as well as in the PP/EOC/Up-S blend with minor strength after $104 \mathrm{~s}$ of polarization time. The fact that (for longer times) a positive current density peak is noticeable in PP/EOC/P-S samples is justified in 
Figure 6.11: While PEA measurements show a constant drift of charges, for the other materials this effect is much milder, gradually reaching a stable condition.

Another explanation as suggested by M. Praeger et al. [58] is related to the effect of residual water on the charging current. In our case, the charging current of PP/EOC/P-S started increasing after approximately $600 \mathrm{~s}$, which seems to be related to the slowly travelling heterocharges near the ground electrode, as seen in the PEA results in Figure 6.11 (marked in red). The slowly travelling heterocharges in the PP/EOC/P-S sample might be formed due to the hygroscopic nature of the amine groups on the surface of the polar silica. Water adsorbed on the polar silica (P-S) by hydrogen bonds with the amine group, started to be released below a temperature of $100{ }^{\circ} \mathrm{C}$, as shown in the inserted TGA curve in Figure 6.13 and Figure 6.1 (c). The adsorbed water will generate ions in an electrical field and contribute more to the positive and negative ionic charges in bulk PP/EOC/P-S under the applied electric field. A higher amount of water would then cause an increased value of current density.

\subsection{Conclusion}

The aim of this study was to compare the effect of different surface functionalizations on micromorphological, thermal, dynamic and dielectric properties of PP/EOC nanocomposites for HVDC cable applications, with the purpose to decrease the space charge accumulation. Two types of modified silica, APTES-modified polar and TMES-modified unpolar silica, were successfully obtained through a solvent free modification. The modification efficiency is influenced by the structure of the silane (number of reactive ethoxy groups), resulting in a much higher degree of deposition of APTES containing three ethoxy groups allowing silica surface modification but also silane self-condensation and oligomerization.

Regarding the compound morphology, SEM results showed two co-continuous phases in the PP/EOC blend due to the immiscibility of PP and EOC. Both, polar and unpolar silica, are preferentially located in the PP phase. The unpolar silica exhibits better dispersion in the PP/EOC polymer matrix, while the polar silica forms relatively large clusters. Both silica types act as nucleating agents for crystallization, but decrease the total crystallinity, which results in lowering of the storage modulus value (E') at low temperatures. Thermal properties are slightly improved after addition of silica, while the dynamic mechanical properties of the PP/EOC blend are not directly influenced. The charge distribution of the $\mathrm{PP} / \mathrm{EOC}$ composites can be tailored by adding silica differing in surface functionalization and modification efficiency. Polar silica introduces much deeper traps than unpolar silica, but their density is lower. Although the conductivity of the $\mathrm{PP} / \mathrm{EOC} / \mathrm{P}-\mathrm{S}$ nanocomposite is slightly higher than the one of the $\mathrm{PP} / \mathrm{EOC} / \mathrm{Up}-\mathrm{S}$ blend, addition of the polar silica significantly decreased the space charge accumulation. In conclusion, the PP/EOC nanocomposite containing APTES modified silica with the lowest space charge accumulation and acceptable conductivity is preferable than the one filled with unpolar silica for HVDC cable application.

Author Contributions: Methodology: Xiaozhen He, Hadi Naderiallaf, Minna Niittymäki, Eetta Saarimäki; resources: Kari Lahti, Christelle Mazel ,Gabriele Perego; writing-original draft preparation: Xiaozhen He; analysis: Amirhossein Mahtabani, Paolo Seri, Ilkka Rytöluoto; writingreview and editing: Xiaozhen He, Paolo Seri, Ilkka Rytöluoto, Rafal Anyszka, Wilma Dierkes; 
supervision: Rafal Anyszka, Wilma Dierkes, Anke Blume; project administration: Mika Paajanen. All authors have read and agreed to the published version of the manuscript.

Funding: This research was funded by European Union's Horizon 2020 research and innovation program under grant agreement No 720858 .

Acknowledgments: The authors also would like to thank Evonik Industries for providing a free silica sample. 


\section{References}

[1] Van Eeckhout B, Van Hertem D, Reza M, Srivastava K, Belmans R. Economic comparison of VSC HVDC and HVAC as transmission system for a 300 MW offshore wind farm. European Transactions on Electrical Power. 2017,20, 661-671. DOI: https://doi.org/10.1002/etep.359

[2] Meah K., Ula S. Comparative evaluation of HVDC and HVAC transmission systems. Proceedings of the 2007 IEEE Power Engineering Society General Meeting. Tampa, FL. 2007,1-5. DOI: 10.1109/PES.2007.385993.

[3] Ardelean M, Minnebo P. HVDC submarine power cables in the world. Joint Research Centre, European Union. 2015.

[4] Ndreko M, Van der Meer A A, Gibescu M, Van der Meijden M A M M, Bos J A, Jansen K P J. Transient stability analysis of an onshore power system with multi-terminal offshore VSC-HVDC transmission: A case study for the Netherlands. Power \& Energy Society General Meeting, Vancouver, BC, 2013. 21-25 July. 1-5. DOI: 10.1109/PESMG.2013.6672663.

[5] Zhang Y, Lewiner J, Alquie C, Hampton N. Evidence of strong correlation between space-charge buildup and breakdown in cable insulation. IEEE Transactions on Dielectrics and Electrical Insulation. 1996, 3, 778-783. DOI: 10.1109/94.556559.

[6] Kurahashi K, Matsuda Y, Ueda A, Demura T, Miyashita Y, Yoshino K. The application of novel polypropylene to the insulation of electric power cable. IEEE/PES Transmission and Distribution Conference and Exhibition, Yokohama, Japan. 2002, 2, 1278-1283. DOI: 10.1109/TDC.2002.1177662.

[7] Li Z, Zhong Z, Du B. Dielectric relaxation and trap-modulated DC breakdown of polypropylene blend insulation. Polymer, 2019,185, 121935. DOI: https://doi.org/10.1016/j.polymer.2019.121935

[8] McNally T, McShane P, Nally G M, Murphy W R, Cook M, Miller A. Rheology, phase morphology, mechanical, impact and thermal properties of polypropylene/metallocene catalysed ethylene 1octene copolymer blends. Polymer. 2002, 43, 3785-3793. DOI: https://doi.org/10.1016/S00323861(02)00170-2

[9] Nitta K H, Shin Y W, Hashiguchi H, Tanimoto S, Terano M. Morphology and mechanical properties in the binary blends of isotactic polypropylene and novel propylene-co-olefin random copolymers with isotactic propylene sequence 1. Ethylene-propylene copolymers. Polymer. 2005, 46, 965-975. DOI: https://doi.org/10.1016/j.polymer.2004.11.033

[10] Zhou Y, Dang B, Wang H, Liu J, Li Q, Hu J, He J. Polypropylene-based ternary nanocomposites for recyclable high-voltage direct-current cable insulation. Composites Science and Technology. 2018, 165, 168-174. DOI: https://doi.org/10.1016/j.compscitech.2018.06.022

[11] Alanalp M B, Durmus A. Quantifying microstructural, thermal, mechanical and solid-state viscoelastic properties of polyolefin blend type thermoplastic elastomer compounds. Polymer. 2018, 142, 267-276. DOI: https://doi.org/10.1016/j.polymer.2018.03.054

[12] Du B X, Xu H, Li J, Li Z. Space charge behaviors of PP/POE/ZnO nanocomposites for HVDC cables. IEEE Transactions on Dielectrics and Electrical InsulationV, 2016, 23, 3165-3174. DOI: 10.1109/TDEI.2016.7736882.

[13] Gao Y, Huang X, Min D, Li S, Jiang P. Recyclable dielectric polymer nanocomposites with voltage stabilizer interface: toward new generation of high voltage direct current cable insulation. ACS 
Sustainable Chemistry \& Engineering. 2018, 7, 513-525. DOI: https://doi.org/10.1021/acssuschemeng.8b04070

[14] Mahtabani A, Rytoluoto I, Anyszka R, He X, Saarimaki E, Lahti K, Paajanen M, Dierkes W, Blume A. On the Silica Surface Modification and Its Effect on Charge Trapping and Transport in PP Based Dielectric Nanocomposites. ACS Applied Polymer Materials. 2020, 2(8), 3148-3160. DOI: https://doi.org/10.1021/acsapm.0c00349

[15] Rytöluoto I, Ritamäki M, Lahti K, Paajanen M, Karttunen M, Montanari G C, Seri P, Naderiallaf H. Compounding, structure and dielectric properties of silica-BOPP nanocomposite films. IEEE 2nd International Conference on Dielectrics, Budapest. 2018, 1-4. DOI: 10.1109/ICD.2018.8514775.

[16] Lewis $\mathrm{T}$ J. Interfaces are the dominant feature of dielectrics at the nanometric level. IEEE transactions on dielectrics and electrical insulation. 2004, 11, 739-753. DOI: 10.1109/TDEI.2004.1349779.

[17] Alhabill F N, Ayoob R, Andritsch T, Vaughan A S. Introducing particle interphase model for describing the electrical behaviour of nanodielectrics. Materials \& Design. 2018, 158, 62-73. DOI: https://doi.org/10.1016/j.matdes.2018.08.01

[18] Dang B, Li Q, Zhou Y, Hu J, He J. Suppression of elevated temperature space charge accumulation in polypropylene/elastomer blends by deep traps induced by surface-modified $\mathrm{ZnO}$ nanoparticles. Composites Science and Technology. 2017, 153, 103-110. DOI: https://doi.org/10.1016/j.compscitech.2017.10.005

[19] Pourrahimi A M. Polyethylene nanocomposites for the next generation of ultralow-transmissionloss HVDC cables: Insulation containing moisture-resistant $\mathrm{MgO}$ nanoparticles. ACS applied materials \& interfaces. 2016, 8, 14824-14835. DOI: https://doi.org/10.1021/acsami.6b04188

[20] Wang Y, Hao M, Xu Z, Qiang D, Chen G, Vaughan A. Experimental demonstration of deep traps in silica-based polyethylene nanocomposites by combined isothermal surface potential decay and pulsed electro-acoustic measurements. Applied Physics Letters. 2018, 113, 022904. DOI: https://doi.org/10.1063/1.5025633

[21] Li S, Zhao N, Nie Y, Wang X, Chen G, Teyssedre G. Space charge characteristics of LDPE nanocomposite/LDPE insulation system. IEEE Transactions on Dielectrics and Electrical Insulation. 2015, 22, 92-100. DOI: 10.1109/TDEI.2014.004524.

[22] Wang Y, Qiang D, Xu Z, Chen G, Vaughan A. The effect of loading ratios and electric field on charge dynamics in silica-based polyethylene nanocomposites. Journal of Physics D: Applied Physics. 2018, 51, 395302. DOI: 10.1088/1361-6463/aad7e8

[23] Lv X S, Han B, Wang J Y, Chang J X, Hua Y, Sun WF. Modification mechanism of low-density polyethylene insulation by hydrophilic and hydrophobic porous $\mathrm{SiO} 2$ nanoparticles. Applied Nanoscience. 2019, 1-11. DOI: https://doi.org/10.1007/s13204-019-01074-6

[24] Akram, S., Castellon, J., Agnel, S., Zhou, K., Habas, J.P. and Nazir, M.T.,. Multilayer polyimide nanocomposite films synthesis process optimization impact on nanoparticles dispersion and their dielectric performance. Journal of Applied Polymer Science, 2021, 138(4), 49715. DOI: https://doi.org/10.1002/app.49715

[25] Akram, S., Nazir, M.T., Castellon, J., Agnel, S., Zhou, K. and Bhutta, M.S.,. Preparation and distinguish dielectric properties of multi-layer nanoparticles-based polyimide films. Materials Research Express. 2019, 6(12), 125092.DOI: 10.1088/2053-1591/ab5c40. 
[26] Ismail, N.H. and Mustapha, M., 2018. A review of thermoplastic elastomeric nanocomposites for high voltage insulation applications. Polymer Engineering \& Science, 58(S1), pp.E36-E63.

[27] Siddabattuni S, Schuman T P, Dogan F. Dielectric properties of polymer-particle nanocomposites influenced by electronic nature of filler surfaces. ACS Applied Materials \& Interfaces. 2013, 5, 1917-1927. DOI: https://doi.org/10.1021/am3030239

[28] He X, Rytöluoto I, Anyszka R, Mahtabani A, Saarimäki E, Lahti K, Paajanen M, Dierkes W, Blume, A,. Silica surface-modification for tailoring the charge trapping properties of PP/POE based dielectric nanocomposites for HVDC cable application. IEEE Access. 2020, 8, 87719-87734. DOI: 10.1109/ACCESS.2020.2992859.

[29] Zare Y, Garmabi H. Nonisothermal crystallization and melting behavior of PP/nanoclay/CaCO3 ternary nanocomposite. J. Journal of Applied Polymer Science. 2012, 124, 12251233. DOI: https://doi.org/10.1002/app.35134

[30] Mirabella F M, Bafna A. Determination of the crystallinity of polyethylene/-olefin copolymers by thermal analysis: Relationship of the heat of fusion of $100 \%$ polyethylene crystal and the density. J. Polym. Sci. B, Journal of Polymer Science Part B: Polymer Physics, 2002, 40, 1637-1643. DOI: https://doi.org/10.1002/polb.10228

[31] Fleming R J, Henriksen M, Holboll J T. The influence of electrodes and conditioning on space charge accumulation in XLPE. IEEE Transactions on Dielectrics and Electrical Insulation. 2000, 7 , 561-571. DOI: 10.1109/94.868078.

[32] Wilfong W C, Srikanth C S, Chuang S S. In situ ATR and DRIFTS studies of the nature of adsorbed CO2 on tetraethylenepentamine films. ACS Applied Materials \& Interfaces. 2014, 6(16), 13617 13626. DOI: https://doi.org/10.1021/am5031006

[33] Blume A, Jin J, Mahtabani A, He X, Kim S, Andrzejewska Z. New Structure Proposal for Silane Modified Silica. International Rubber Conference, IRC . London. 2019. 3 - 5 Sep.

[34] McCool B, Murphy L, Tripp C P. A simple FTIR technique for estimating the surface area of silica powders and films. Journal of colloid and interface science. 2006, 295, 294-298. DOI: https://doi.org/10.1016/j.jcis.2005.08.010

[35] Zhuravlev L T. The surface chemistry of amorphous silica. Zhuravlev model. Colloids and Surfaces A: Physicochemical and Engineering Aspects. 2000, 173(1-3), 1-38

[36] Brochier Salon M C, Bayle P A, Abdelmouleh M, Boufi S, Naceur Belgacem M, . Kinetics of hydrolysis and self condensation reactions of silanes by NMR spectroscopy. Colloid. Surface. A. 2008, 312, 83-91. DOI: https://doi.org/10.1016/j.colsurfa.2007.06.028

[37] Avgeropoulos G N, Weissert F C, Biddison P H, Bohm G G. Heterogeneous blends of polymers. Rheology and morphology. Rubber chemistry and technology. 1976, 49, 93-104.

[38] Barranco-García R, Gómez-Elvira J M, Ressia JA, Quinzani L, Vallés E M, Pérez E, Cerrada M L. Effect of iPP molecular weight on its confinement within mesoporous SBA-15 silica in extruded iPP- SBA-15 nanocomposites. Microporous and Mesoporous Materials. 2020,294, 109945. DOI: https://doi.org/10.1016/j.micromeso.2019.109945

[39] He X, Rytöluoto I, Anyszka R, Mahtabani A, Saarimäki E, Lahti K, Paajanen M, Dierkes W, Blume A. Surface modification of fumed silica by plasma polymerization of acetylene for PP/POE blends dielectric nanocomposites. Polymers. 2019, 11(12), 1957. DOI: 10.3390/polym11121957

[40] Jankong S, Srikulkit K. Preparation of polypropylene/hydrophobic silica nanocomposites. J. Met. Mater. Miner. 2008,18, 43-146. 
[41] Jain S, Goossens J G P, Van Duin M. Synthesis, Characterization and Properties of (Vinyl Triethoxy Silane-grafted PP)/Silica Nanocomposites. In Macromolecular symposia. 2006, 233, 225-234. DOI: https://doi.org/10.1002/masy.200690022

[42] Zhao W, Su Y, Wen X, Wang D. Manipulating crystallization behavior of poly (ethylene oxide) by functionalized nanoparticle inclusion. Polymer. 2019, 165, 28-38. DOI: https://doi.org/10.1016/j.polymer.2019.01.019

[43] Samir M A, Chazeau L, Alloin F, Cavaillé J Y, Dufresne A,. Sanchez J Y. POE-based nanocomposite polymer electrolytes reinforced with cellulose whiskers. Electrochimica Acta. 2005, 50, 3897-3903. DOI: https://doi.org/10.1016/j.electacta.2005.02.065

[44] Gao Y, Li J, Yuan Y, Huang S, Du B. Trap distribution and dielectric breakdown of isotactic polypropylene/propylene based elastomer with improved flexibility for DC cable insulation. IEEE Access. 2018, 6, 58645-58661. DOI: 10.1109/ACCESS.2018.2874826.

[45] McNally T, McShane P, Nally G M, Murphy W R, Cook M, Miller A. Rheology, phase morphology, mechanical, impact and thermal properties of polypropylene/metallocene catalysed ethylene 1octene copolymer blends. Polymer. 2002, 43, 3785-3793. DOI: https://doi.org/10.1016/S00323861(02)00170-2

[46] Hassan A, Rahman N A, Yahya R. Extrusion and injection-molding of glass fiber/MAPP/polypropylene: effect of coupling agent on DSC, DMA, and mechanical properties. Journal of Reinforced Plastics and Composites. 2011,30, 1223-1232. DOI: https://doi.org/10.1177/0731684411417916

[47] Luo F, Xu C, Wang K, Deng H, Chen F, Fu Q. Exploring temperature dependence of the toughening behavior of $\beta$-nucleated impact polypropylene copolymer. Polymer. 2012, 53,1783-90. DOI: https://doi.org/10.1016/j.polymer.2012.02.024

[48] Read B E, Tomlins P E \& Dean G D. Physical ageing and short-term creep in amorphous and semicrystalline polymers. Polymer. 1990, 31, 1204-1215. DOI: https://doi.org/10.1016/00323861(90)90209-H

[49] Tian F, Bu W, Shi L, Yang C, Wang Y, Lei Q. Theory of modified thermally stimulated current and direct determination of trap level distribution. Electrostat. 2011, 69, 7-10. DOI: https://doi.org/10.1016/j.elstat.2010.10.001

[50] Ieda M. Electrical conduction and carrier traps in polymeric materials. IEEE transactions on electrical insulation. 1984, 162-78. DOI: 10.1109/TEI.1984.298741.

[51] Gao M, Yang J, Zhao H, He H, Hu M, Xie S. Preparation methods of polypropylene/nanosilica/styrene-ethylene-butylene-styrene composite and its effect on electrical properties. Polymers. 2019, 11, 797. DOI: https://doi.org/10.3390/polym11050797

[52] Huang Y, Wu K, Bell M, Oakes A, Ratcliff T, Lanzillo NA, Breneman C, Benicewicz BC, Schadler LS. The effects of nanoparticles and organic additives with controlled dispersion on dielectric properties of polymers: Charge trapping and impact excitation. Journal of Applied Physics. 2016,120, 055102. DOI: https://doi.org/10.1063/1.4959771

[53] Pourrahimi A M, Olsson R T, Hedenqvist M S. The Role of Interfaces in Polyethylene/Metal-Oxide Nanocomposites for Ultrahigh-Voltage Insulating Materials. Advanced Materials. 2018, 30,1703624. DOI: https://doi.org/10.1002/adma.201870025

[54] Saiz F, Quirke N. The excess electron in polymer nanocomposites. Physical Chemistry Chemical Physics. 2018,20,27528-27538. DOI: 10.1039/C8CP04741C 
[55] Ma D, Hugener T A, Siegel R. W, Christerson A, Mårtensson E, Önneby C \& Schadler L S. Influence of nanoparticle surface modification on the electrical behaviour of polyethylene nanocomposites. Nanotechnology. 2005, 16(6), 724. DOI: 10.1088/0957-4484/16/6/016

[56] Blume A, Janik M, Gallas J P, Thibault-Starzyk F, \&Vimont A. Operando infrared study of the reaction of triethoxypropylsilane with silica. Kgk-Kautschuk Gummi Kunststoffe. 2008, 61, 359362.

[57] Wintle H J. Reversals in electrical current and other anomalies in insulating polymers. IEEE Transactions on Electrical Insulation, 1986, 5, 747-762. DOI: 10.1109/TEI.1986.348923.

[58] Praeger M, Hosier I L, Holt A F, Vaughan A S \& Swingler S G. On the effect of functionalizer chain length and water content in polyethylene/silica nanocomposites: Part II-Charge transport. IEEE Transactions on Dielectrics and Electrical Insulation. 2017, 24(4), 2410-2420. DOI: 10.1109/TDEI.2017.005789. 


\section{Chapter 7 \\ Dielectric performance of silica-filled \\ nanocomposites based on miscible (PP/PP-HI) and immiscible (PP/EOC) polymer blends}

This chapter 7 has been published in IEEE ACCESS.

He, X., Rytöluoto, I., Seri, P., Anyszka, R., Mahtabani, A., Naderiallaf, H., Niittymäki, M., Saarimäki, E., Mazel, C., Perego, G. and Lahti, K., Paajanen, M., Dierkes, W. and Blume, A., 2020. Silica surface-modification for tailoring the charge trapping properties of $P P / P O E$ based dielectric

nanocomposites for HVDC cable application. IEEE Access, 8, pp.87719-87734.

DOI: $10.1109 / A C C E S S .2020 .2992859$. 


\title{
Dielectric Performance of Silica-Filled Nanocomposites Based on Miscible (PP/PP-HI) and Immiscible (PP/EOC) Polymer Blends
}

\author{
XIAOZHEN HE $^{\ominus}$, (Member, IEEE), PAOLO SERI ${ }^{2}$, (Member, IEEE), ILKKA RYTÖLUOTO ${ }^{3}$, \\ RAFAL ANYSZKA ${ }^{1}$, (Member, IEEE), AMIRHOSSEIN MAHTABANI' (Member, IEEE), \\ HADI NADERIALLAF 2 , (Member, IEEE), \\ MINNA NIITTYMÄKI ${ }^{4}$, (Graduate Student Member, IEEE), EETTA SAARIMÄKI ${ }^{3}$, \\ CHRISTELLE MAZEL 5 , GABRIELE PEREGO5 ${ }^{5}$, KARI LAHTI ${ }^{4}$, (Member, IEEE), \\ MIKA PAAJANEN ${ }^{3}$, WILMA DIERKES', AND ANKE BLUME I \\ 'Elastomer Technology and Enginecring Group. Depurtment of Mechanics of Solidx Surfaces \& Systems (MS3). Feculry of Enginecring Technolozy. Univensity \\ of Twente, 7522 NB Enschede. The Netherlands \\ "Department of Eectrical, Eectronic and Information Enginecring "Guglielmo Marconi," University of Bologna 40136 Bologna Italy \\ 'VTT Technical Research Center of Finland Lid, 33101 Tampere. Finland \\ ${ }^{4}$ High Voltage Enginecring. Tampere Univensity, 33720 Tampere, Finland \\ ${ }^{5}$ Nerans Researth Center. 69007 Lyon. France \\ Corresponding authors: Wilma Dierkes (w.k.dierkes@utwente.nl), Rafal Anyszka (r.p.anyska@utwente.nl), and Xiaozhen He \\ (x.he@ utwente.nI)
}

This work was supported by the European Union's Horizon 2020 Research and Innovation Program under Grant 720858.

\begin{abstract}
This study compares different polymer-nanofiller blends conceming their suitability for application as insulating thermoplastic composites for High Voltage Direct Current (HVDC) cable application. Two polymer blends, PP/EOC (polypropylene/ethylene-octene copolymer) and PP/PP-HI (polypropylene/ propylene - ethylene copolymer) and their nanocomposites filled with $2 \mathrm{wt} \%$ of fumed silica modified with 3-aminopropyltriethoxysilane were studied. Morphology, thermal stability, crystallization behavior dynamic relaxation, conductivity, charge trap distribution and space charge behavior were studied respectively. The results showed that the comprehensive performance of the PP/PP-HI composite is better than the one of the PP/EOC composite due to better polymer miscibility and flexibility, as well as lower charging current density and space charge accumulation. Nanosilica addition improves the thermal stability and dielectric properties of both polymer blends. The filler acts as nucleating agent increasing the crystallization temperature, but decreasing the degree of crystallinity. Dynamic mechanical analysis results revealed three polymer relaxation transitions: PP glass transition $(\beta)$, weak crystal reorientation $(\alpha 1)$ and melting $(\alpha 2)$. The nanosilica introduced deep traps in the polymer blends and suppressed space charge accumulation, but slightly increased the conductivity. A hypothesis for the correlation of charge trap distribution and polymer chain transition peaks is developed: In unfilled PP/EOC and PP/PP-HI matrices, charges are mostly located at the crystalline-amorphous interface, whereas in the filled PP/EOC/silica and PP/PP-HI /silica composites, charges are mostly located at the nanosilica-polymer interface. Overall, the PP/PP-HI (55/45) nanocomposite with $2 \mathrm{wt} . \%$ modified silica and $0.3 \mathrm{wt} . \%$ of antioxidants making it $a$ promising material for PP based HVDC cable insulation application with $a$ reduced space charge accumulation and good mechanical properties.
\end{abstract}

INDEX TERMS PP/EOC, PP/PP-HI, nanosilica, charge trap distribution, space charge accumulation, HVDC cable insulation. 


\subsection{Introduction}

High Voltage Direct Current (HVDC) technology shows advantages of lower dielectric losses and lower costs of long-distance power transmission in comparison to High Voltage Alternating Current (HVAC). The insulation material for cables plays an important role in the efficiency of power transmission in HVDC. Polypropylene (PP) as one of the possible insulation materials exhibits a relatively high melting temperature, recyclability and good dielectric properties. Therefore, it became an alternative insulation material to replace cross-linked polyethylene (XLPE) for HVDC cable applications recently [1-3]. However, PP exhibits low temperature brittleness, limiting its application for HVDC cables. To overcome this shortcoming, most studies for PP as insulation material nowadays are focused on PPcopolymers [4,5] and PP/polyolefin blends [2,6].

In PP-copolymers, a second monomer is copolymerized with propylene. It is reported that a polypropylene/polyethylene block copolymer is not a good choice for HVDC cable applications due to its brittleness and low elongation at break originating from the regular block-structure. Alternatively, a propylene/ethylene random copolymer exhibits superior low temperature elasticity, which are promising for HVDC applications [4].

Apart of PP-copolymerization, blending of PP with an elastomer can also improve the flexibility without compromising the electrical properties. Different types of polyolefins exhibiting elastomeric behaviour can be used for this purpose. Some of them show limited miscibility with PP, even though they have a similar polymer composition $[7,8]$. The type and content of the elastomer used for blending have been reported to influence morphology, crystallization, space charge accumulation and breakdown of the PP blend [6, 9]. As an example, C. D. Green and A. S. Vaughan [6] studied isotactic polypropylene/propylene-ethylene (with different ethylene content) blends. Polypropylene blends with 50 wt.\% propylene-ethylene copolymer with an ethylene concentration of $9 \mathrm{~mol} \%$ exhibited good electrical breakdown and mechanical properties on laboratory scale.

In the HVDC research field, space charge accumulation is one of the main concerns, since it can cause distortion of the local electric field resulting in partial discharge, and thus leading to insulation failure. [10] To solve this problem, PP based nanocomposites have drawn a lot of attention in both, industry and academia, due to the fact that a nanofiller can suppress space charge accumulation and charge trap distribution of an insulation material, which would enhance the electrical properties $[11,12]$. In this study, we compared the different properties (morphology, thermal properties and dielectric performance) of two polymer composites based on polypropylene/propylene-ethylene copolymer and polypropylene/ethylene-octene copolymer blends and their nanocomposites in order to develop a promising insulation thermoplastic composite for HVDC cable application.

\subsection{Materials and Characterization}

\subsubsection{Materials}

Fumed silica (Aerosil 200) was modified with 3-aminopropyltriethoxy silane (APTES) in a solvent-free reaction. $20 \mathrm{~g}$ of nanosilica was mixed with $3.6 \mathrm{~g}$ of APTES, $0.4 \mathrm{~g}$ of trifluoroacetic acid and $0.6 \mathrm{~g}$ of deionized water as catalysts in a sealed jar at room temperature for 24 hours. Then the modified silica was put into a vacuum oven at $80{ }^{\circ} \mathrm{C}$ for 24 hours in order to remove all unreacted residuals. 
The nanocomposite samples were prepared by a KraussMaffei Berstorff ZE 25/49D twin screw extruder by melt-blending of $2 \mathrm{wt} . \%$ of the silica with PP/EOC $=55: 45 \mathrm{wt} . \%$ or PP/PP-HI $=55: 45 \mathrm{wt} . \%$ and 0.3 wt. $\%$ of antioxidants. The extruder set temperature ranges from 195 to $230{ }^{\circ} \mathrm{C}$. The extruded compound was quenched in a water bath, granulated, and extruded into cast films by a single screw extruder (Brabender Plasticorder) equipped with a $\mathrm{T}$ die and a calendar system $\left(80^{\circ} \mathrm{C}\right)$. The average thickness of the cast film sample is around $400 \mu \mathrm{m}$.

\subsubsection{Sample Characterization}

\subsubsection{Scanning Electron Microscopy (SEM)}

Scanning Electron Microscopy (SEM) was performed on the samples in order to study the microstructure and silica dispersion using a Zeiss MERLIN HR-SEM (Oberkochen, Germany). The samples were firstly put into liquid nitrogen for $5 \mathrm{~min}$ and then broken into two parts. The cross section of these samples was selected for the SEM pictures without any surface treatment in order to preserve the surface morphology. The silica particle size distribution was obtained by ImageJ software by analysing 3 SEM images of each compound.

\subsubsection{X-Ray Diffraction (XRD)}

X-ray Diffraction (XRD) measurements were carried out on the plain samples with a Philips X'Pert $1 \mathrm{X}$ ray diffractometer (Almelo, The Netherlands). The samples were scanned from $2 \Theta=5^{\circ}$ to $35^{\circ}$ with a scanning rate of $0.05^{\circ} / 8$ seconds.

\subsubsection{Differential Scanning Calorimetry (DSC)}

Differential Scanning Calorimetry (DSC) measurements were performed on the samples with a weight of 12 to $14 \mathrm{mg}$ using a DSC NETZSCH DSC 214 Polyma (Germany). The samples were firstly cooled down to $-50{ }^{\circ} \mathrm{C}$ and then heated up to $250{ }^{\circ} \mathrm{C}$ with a heating rate of $3{ }^{\circ} \mathrm{C} / \mathrm{min}$.

\subsubsection{Polarized Optical Microscope (POM)}

Polarized light microscopy (POM, Meiji Techno ML8530 microscope) was done on the microtomed samples with a thickness of $30 \mu \mathrm{m}$.

\subsubsection{Thermogravimetric Analysis (TGA)}

The thermal stability of the studied samples was investigated by Thermogravimetric Analysis (TA Instruments 550, US). The samples were heated up from $30^{\circ} \mathrm{C}$ to $850^{\circ} \mathrm{C}$ with a heating rate of $10{ }^{\circ} \mathrm{C} / \mathrm{min}$ in synthetic air atmosphere.

\subsubsection{Dynamic Mechanical Analysis (DMA)}


Dynamic Mechanical Analysis (DMA) tests were performed on an Eplexor $2000 \mathrm{~N}$ (Gabo/Netzsch, Germany) in tensile mode with a frequency of $1 \mathrm{~Hz}$; the static strain was $0.2 \%$ and the dynamic strain was $0.1 \%$. The samples were firstly cooled down to $-100{ }^{\circ} \mathrm{C}$ and then heated up to $150{ }^{\circ} \mathrm{C}$ with a heating rate of $2{ }^{\circ} \mathrm{C} / \mathrm{min}$.

\subsubsection{Thermally Stimulated Depolarization Current (TSDC)}

The charge trap distribution was tested by Thermally Stimulated Depolarization Current (TSDC) measurements by using a custom made setup comprising of a high voltage DC source (Keithley 2290E5), an electrometer (Keithley 6517B) and a Novocool temperature control system equipped with a shielded sample cell and a PT100 temperature sensor (accuracy $\pm 0.1{ }^{\circ} \mathrm{C}$ ). A gold layer of 100 nanometers $(\mathrm{nm})$ was deposited on both sides of the specimen acting as circular electrodes. The samples were polarized at $70{ }^{\circ} \mathrm{C}$ for $20 \mathrm{~min}$ under a $3 \mathrm{kV} / \mathrm{mm}$ DC field. Then the samples were rapidly cooled down to $-50{ }^{\circ} \mathrm{C}$. Later on, the poling voltage was removed and the samples were short circuited. TSDC was then measured by heating the samples from $-50{ }^{\circ} \mathrm{C}$ to $140{ }^{\circ} \mathrm{C}$ with a linear heating rate of $3{ }^{\circ} \mathrm{C} / \mathrm{min}$.

\subsubsection{Pulsed Electro Acoustic (PEA)}

Space charge behaviour was investigated by a Pulsed Electro-Acoustic (PEA) method with a custom made setup comprising a high voltage DC source (FUG HCN 35 - 20000), a PEA cell built in-house and an oscilloscope (Tektronix 3032). The samples were firstly placed in a vacuum oven for 72 hours at $60{ }^{\circ} \mathrm{C}$ and then tested at $60^{\circ} \mathrm{C}$ under a $30 \mathrm{kV} / \mathrm{mm}$ electric field. The electric field was applied for 3 hours $(10800$ s) for poling, after which the depolarization phase was monitored for 1 hour.

\subsubsection{Conductivity}

The final conductivity test was done on the samples under an electric field of $30 \mathrm{kV} / \mathrm{mm}$ and at a temperature of $60{ }^{\circ} \mathrm{C}$ with a custom made setup comprising a high voltage DC source (FUG HCN $35-$ 35000), an electrometer (Keysight B2980A) and a conductivity measurement cell built in-house. The samples were pretreated with gold sputtered electrodes with a diameter of $26 \mathrm{~mm}$ and a guard ring.

\subsection{Results and Discussion}

\subsubsection{Scanning Electron Microscopy (SEM)}

The morphology of the polymer blends and nanocomposites was studied using SEM and is shown in Figure 7.1. PP and PP-HI are uniformly distributed with no noticeable phase separation, which indicates that PP and PP-HI are highly miscible. In contrast to this, there is a significant phase separation in the PP/EOC blend. These two phases are arranged in a layered structure with the smooth phase being PP and the rough phase EOC. This reveals that PP and EOC have a limited degree of miscibility, visibly lower than PP and $\mathrm{PP}-\mathrm{HI}$. This leads to formation of an interface between the two polymers in the PP/EOC blend due to phase separation. 

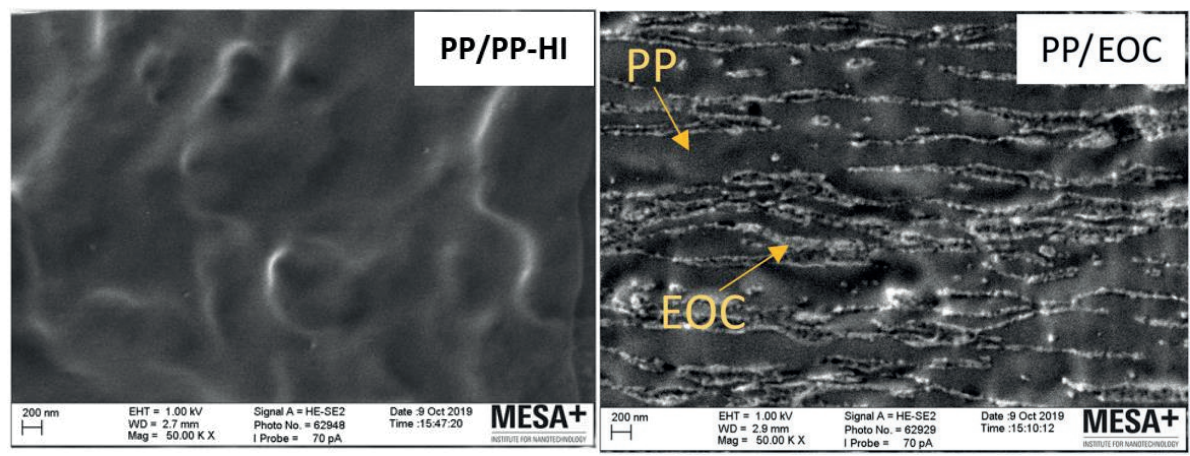

Figure 7.1 Morphology of the unfilled PP/EOC and PP/PP-HI composites.
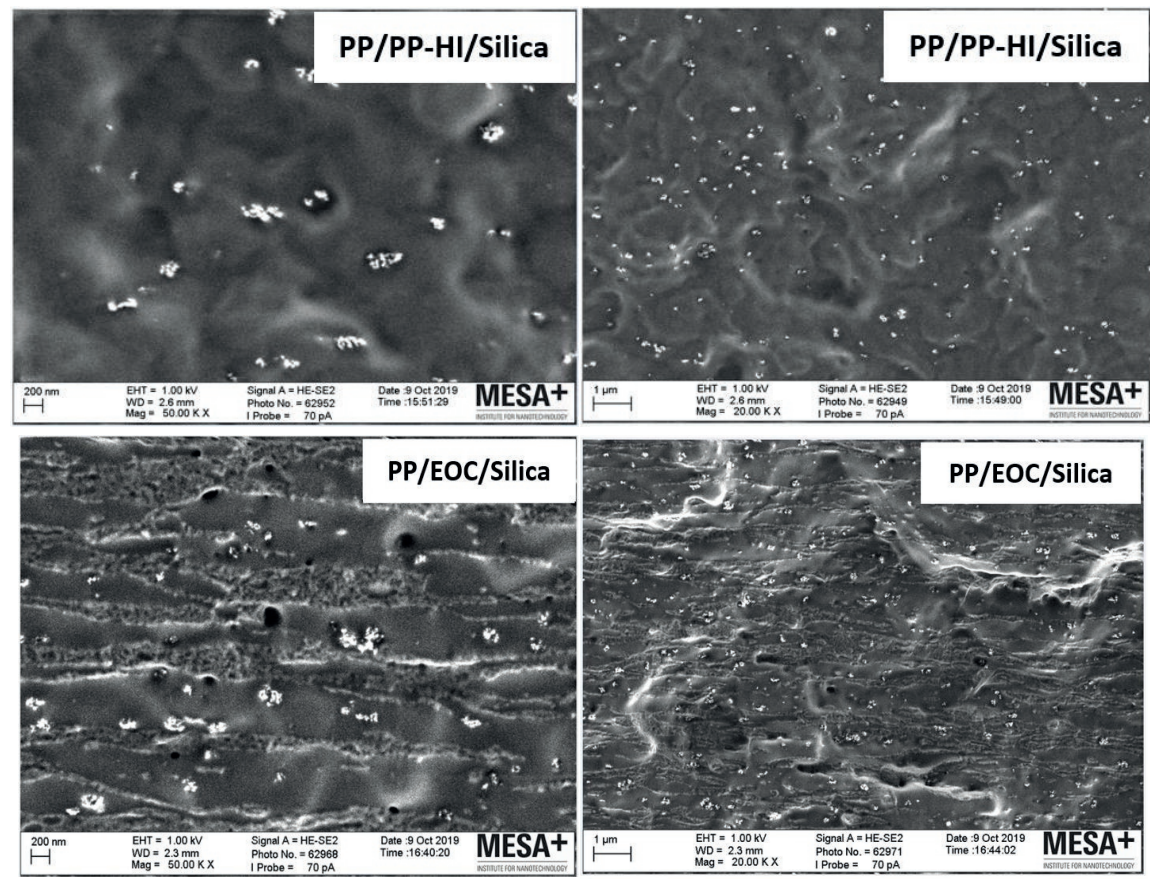

Figure 7.2 Morphology of silica-filled nanocomposites: PP/EOC/Silica and PP/PP-HI/Silica.

The dispersion of the silica in the PP/EOC and PP/PP-HI matrices is shown in Figure 7.2. It is noticeable that the silica is distributed differently in the PP/EOC and PP/PP-HI matrices. The silica is evenly distributed in both phases in the PP/PP-HI matrix, while the silica incorporated into the PP/EOC matrix is located only in the PP phase. This effect occurs most likely because of a significant difference in the viscosity of PP and EOC. The PP chains of lower molecular weight (lower viscosity) exhibit higher 
mobility and thus can penetrate the porous structure of silica better than EOC, and therefore increase the $\mathrm{PP} /$ silica interaction favouring and stabilizing silica location in the PP phase. The preferential location of the silica in the PP phase is also confirmed by DSC, which will be discussed later. Furthermore, the histogram of the silica size distribution in both nanocomposites is shown in Figure 7.3. It is clearly seen that there are clusters of silica (> $100 \mathrm{~nm}$ ) present in both, the PP/EOC and the PP/PP-HI matrix. This is due to the unpolar character of both polymer matrices, whereas the silica modified with 3aminopropyltriethoxysilane has a polar character, which limits its dispersibility in the polymer matrices. Besides, there are relatively more large size silica clusters $(>200 \mathrm{~nm}$ ) present in the PP/EOC than in the $\mathrm{PP} / \mathrm{PP}-\mathrm{HI}$ matrix. This is caused by the preferred location of the silica in the PP phase of the PP/EOC matrix, which reduces the average distance between smaller silica clusters and thus a higher degree of recombination.
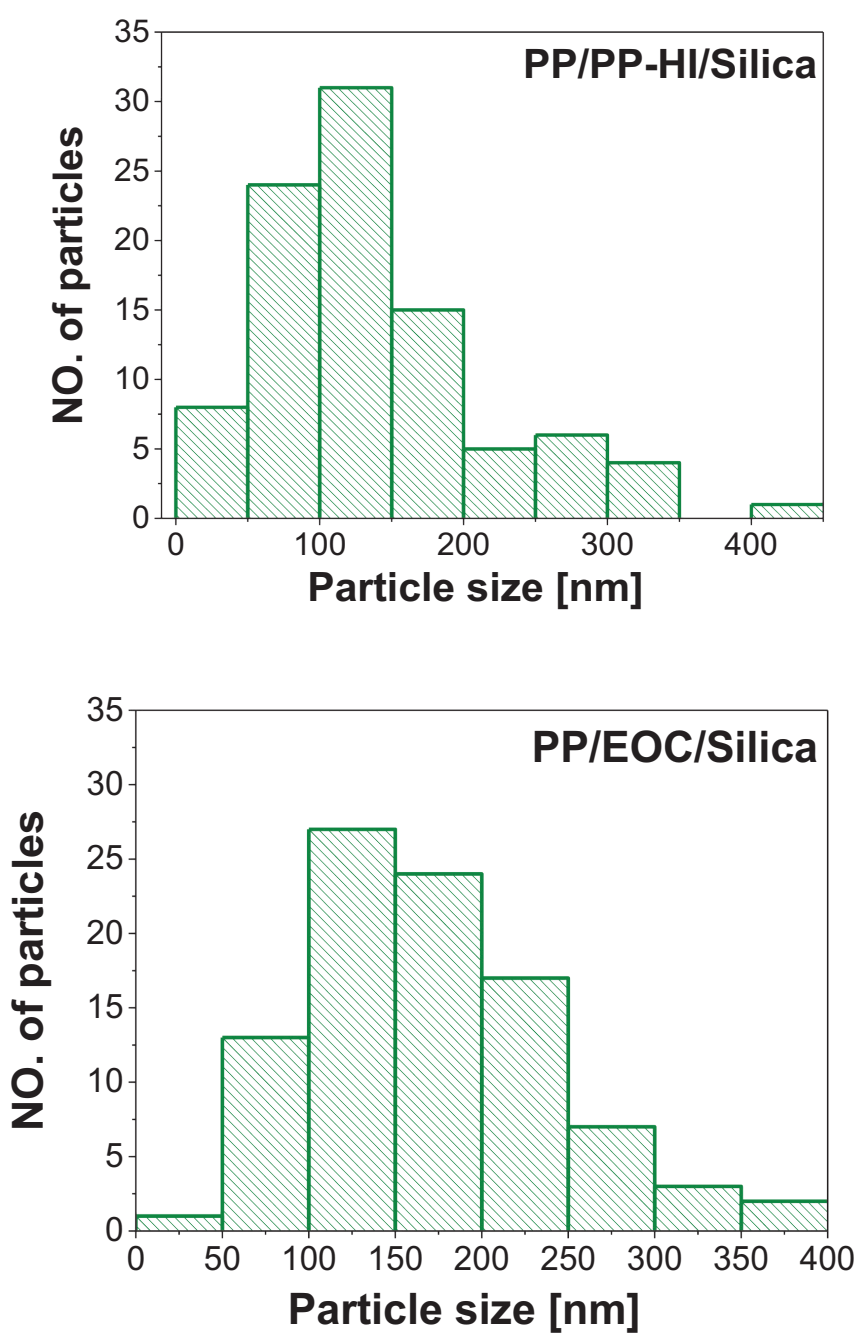

Figure 7.3 Silica particle size distribution in PP/EOC and PP/PP-HI matrix. 


\subsubsection{X-ray diffraction $(X R D)$}

In order to investigate the crystalline phase present in both blends and the nanocomposites, XRD was performed. The results are shown in Figure 7.4. In the left graph of Figure 7.4, only two peaks for pure EOC are found at $21.3^{\circ}$ and $23.5^{\circ}$, which are corresponding to the orthorhombic (110) and (200) crystal planes originating from polyethylene blocks present in the molecular structure of EOC, which are prone to crystallization. The same type of peaks for pure PP and pure PP-HI are found at similar positions corresponding to $\alpha$-crystals formed by the PP phase. Peaks for the $\alpha-(110), \alpha-(040), \alpha-(130), \alpha-(060)$ are located at $2 \Theta=14.1^{\circ}, 16.8^{\circ}, 18.5^{\circ}, 25.5^{\circ}$ for pure PP, and at $2 \Theta=14.5^{\circ}, 17.2^{\circ}, 18.8^{\circ}, 25.6^{\circ}$ for pure PP$\mathrm{HI}$, respectively. This indicates that PP and PP-HI have a similar crystalline structure, while PP and EOC have different ones. When blending PP and PP-HI, the crystal peaks stemming from pure PP and PP-HI are invariable and all show up in the spectrum of the PP/PP-HI samples in the right graph of Figure 7.4. All peaks indicate $\alpha$-crystals, from which the $\alpha-(040)$ structure is the most pronounced one. However, when mixing PP and EOC together, the $\alpha-(110)$ and $\alpha-(130)$ crystals almost disappear, while the $\alpha-(040)$ and $\alpha-(060)$ from the PP phase and the orthorhombic PE (110) and (200) from the EOC phase are found to be predominant. This shows that blending influences the morphology of the crystalline phase in both cases (PP/PP-HI and PP/EOC) promoting formation of the $\alpha-(040)$ structure over the $\alpha-(110)$ one.
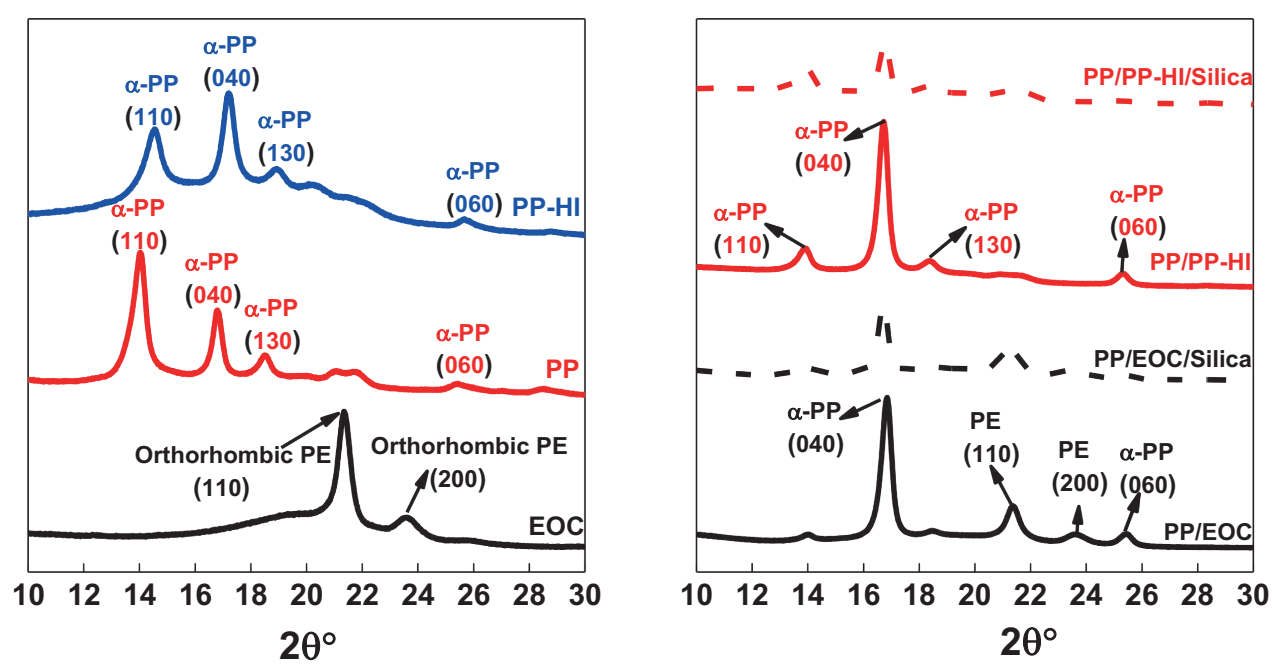

Figure 7.4 XRD results of pure PP, EOC and PP-HI (left), and PP/EOC, PP/PP-HI, PP/EOC/Silica, $\mathrm{PP} / \mathrm{PP}-\mathrm{HI} /$ Silica composites (right).

Both nanocomposites show the same type of crystals as the unfilled polymer blends. However, it is clearly seen that the intensity of the peak is considerably reduced by adding silica, compared to the unfilled blends, revealing that the number of crystals is decreased by adding silica. 


\subsubsection{Differential Scanning Calorimetry (DSC)}

The DSC thermograms (Figure 7.5) show the presence of two peaks of melting and crystallization of the $\mathrm{PE}$ and PP crystals in the unfilled PP/EOC blend and its nanocomposite. The crystallinity (Xc) was calculated and is presented in Table 7.1. The calculation is based on the Equation (7.1).

$$
X c=\frac{\Delta \mathrm{Hm}}{\mathrm{w} \Delta \mathrm{H}_{100}} \times 100 \%
$$

where:

$\Delta \mathrm{Hm}$ - melting enthalpy of the sample,

w - weight percent of the polymer.

$\Delta \mathrm{H}_{100}$ - melting enthalpy of the pure polymer.

In this equation, a $\Delta \mathrm{H}_{100}$ value of $209 \mathrm{~J} / \mathrm{g}$ is taken for PP [13] and $293 \mathrm{~J} / \mathrm{gram}$ for PE [14].

Table 7.1 Crystallization parameters.

\begin{tabular}{|c|c|c|c|c|c|c|c|c|c|}
\hline & $\begin{array}{c}\mathrm{T}_{\mathrm{m}}^{1} \\
\left({ }^{\circ} \mathrm{C}\right)\end{array}$ & $\begin{array}{c}\mathrm{T}_{\mathrm{m}}^{2} \\
\left({ }^{\circ} \mathrm{C}\right)\end{array}$ & $\begin{array}{c}\mathrm{T}_{\mathrm{m}} \\
\left({ }^{\circ} \mathrm{C}\right)\end{array}$ & $\begin{array}{c}\mathrm{T}_{\mathrm{c}}^{1} \\
\left({ }^{\circ} \mathrm{C}\right)\end{array}$ & $\begin{array}{c}\mathrm{T}_{\mathrm{c}}^{2} \\
\left({ }^{\circ} \mathrm{C}\right)\end{array}$ & $\begin{array}{c}\mathrm{T}_{\mathrm{c}} \\
\left({ }^{\circ} \mathrm{C}\right)\end{array}$ & $\begin{array}{c}\mathrm{T}_{\mathrm{c}}^{\mathrm{on}} \\
\left({ }^{\circ} \mathrm{C}\right)\end{array}$ & $\begin{array}{c}\Delta \mathrm{Hm} \\
(\mathrm{J} / \mathrm{g})\end{array}$ & $\begin{array}{c}\Delta \mathrm{X}_{\mathrm{C}} \\
(\%)\end{array}$ \\
\hline \begin{tabular}{c}
$\mathrm{PP} / \mathrm{EOC}$ \\
\hline PP/EOC \\
/Silica
\end{tabular} & 107.2 & 147.2 & - & 97.2 & 108.2 & - & 114.2 & -99.8 & 39.7 \\
\hline $\begin{array}{c}\mathrm{PP} / \mathrm{PP}- \\
\mathrm{HI}\end{array}$ & - & - & 146.1 & - & - & 105.5 & 112.0 & -42.7 & 20.4 \\
\hline $\begin{array}{c}\mathrm{PP} / \mathrm{PP}- \\
\mathrm{HI} \\
/ \text { Silica }\end{array}$ & - & - & 146.2 & - & - & 114.2 & 118.3 & -38.4 & 18.4 \\
\hline
\end{tabular}

$\mathrm{T}_{\mathrm{m}}^{1}$ : melting peak temperature of the EOC phase;

$\mathrm{T}_{\mathrm{m}}^{2}$ : melting peak temperature of the PP phase;

$\mathrm{T}_{\mathrm{m}}$ : melting peak temperature of the PP/PP-HI blend;

$\mathrm{T}_{\mathrm{c}}^{1}$ : crystallization peak temperature of the EOC phase;

$\mathrm{T}_{\mathrm{c}}^{2}$ : crystallization peak temperature of the PP phase;

$\mathrm{T}_{c}$ : crystallization peak temperature of the PP/PP-HI blend;

$\mathrm{T}_{\mathrm{c}}^{\mathrm{on}}$ : onset crystallization temperature;

$\Delta \mathrm{Hm}$ : melting enthalpy of the polymer;

$\Delta \mathrm{X}_{\mathrm{c}}$ : calculated crystallinity of the polymer. 

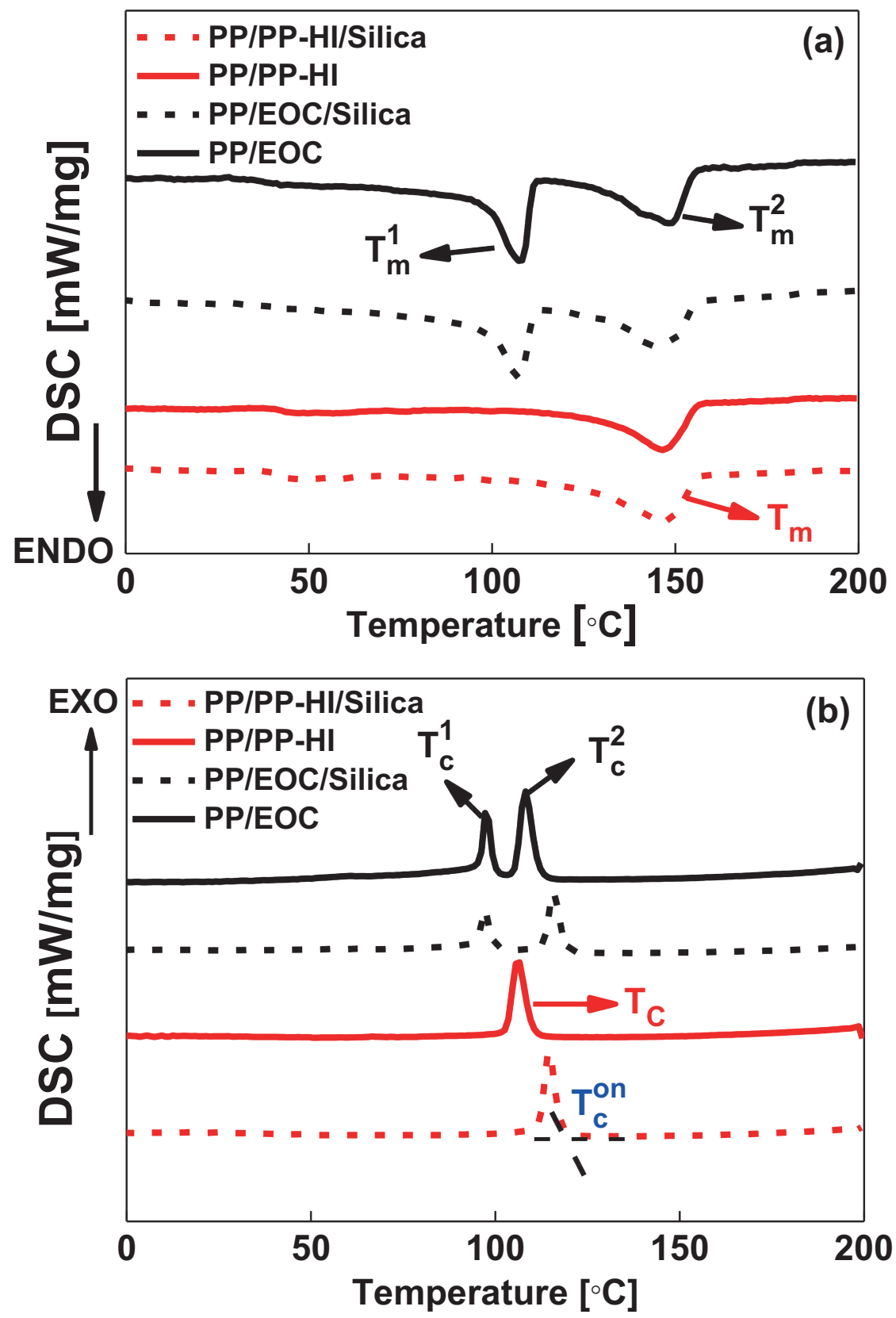

Figure 7.5 Melting (a) and crystallization (b) curves of PP/EOC, PP/PP-HI blends and PP/EOC/Silica, $\mathrm{PP} / \mathrm{PP}-\mathrm{HI} /$ Silica nanocomposites. 
According to previous results [11], the two peaks belong to the EOC (Peak 1) and PP phase (Peak 2), respectively, while there is only one single peak observed in the PP/PP-HI blend and the PP/PP-HI/Silica composite. Moreover, we have seen that the onset crystallization temperature of the $\mathrm{PP} / \mathrm{EOC}$ blend is higher than the one of the PP/PP-HI. This is caused by EOC acting as heterogeneous nucleating agent, accelerating the crystallization process in the PP/EOC blend [15]. The crystallinity of the PP/EOC blend is also higher than the one of the PP/PP-HI blend, as seen in Table 7.1.

Regarding the effect of silica addition as shown in Table 7.1, no significant melting temperature changes are observed after the incorporation of silica for any of the polymer blends. However, the incorporation of silica increased the crystallization temperature values by about 8 to $9{ }^{\circ} \mathrm{C}$. This is due to the nucleation effect of the nanofiller [16]. For the PP/EOC based composite, two crystallization peaks are observed. The addition of silica has no influence on $\mathbf{T}_{\mathbf{c}}^{\mathbf{1}}$, the crystallization peak temperature of the EOC phase, but increases the $\mathbf{T}_{\mathbf{c}}^{2}$ measured for the PP phase by about $8^{\circ} \mathrm{C}$. The different effects of silica in the two polymer matrices are a result of the distribution of silica: it is evenly distributed in the PP/PP-HI composite, while in the PP/EOC composite the silica is mostly located in the PP phase only. Therefore, the presence of silica changed only the $\mathbf{T}_{\mathbf{c}}^{2}$ of the PP phase, but has no effect on the $\mathbf{T}_{\mathbf{c}}^{\mathbf{1}}$ of the EOC phase in the PP/EOC composite.

It can be seen that the crystallinity of the unfilled PP/EOC blend is higher than the one of the unfilled $\mathrm{PP} / \mathrm{PP}-\mathrm{HI}$ blend. There is a significant reduction of crystallinity in both nanocomposites compared to the unfilled polymer blends [17]. When introducing silica into a polymer matrix, this brings about a large interface between silica and the polymer matrix. The interaction between silica and polymer decreases the chain mobility in the interface. This leads to lower chain mobility inhibiting crystallization and, as a consequence, less and smaller crystals are formed [18].

It is also interesting to note that silica increased the crystallization temperature (DSC results, Figure 7.5), but decreased the crystallinity of both polymer matrices (Table 7.1). Such a behavior was also observed by other authors $[17,18,19]$. This stems from the double function of nanosilica in a polymer matrix $[18,19]$ :

(i) Silica acts as a nucleating agent, which accelerates the process of non-isothermal crystallization.

(ii) The polymer interacts with the silica surface, the more as the surface area of the filler is rather large. This adsorption blocks the movement of crystallizable molecular chain segments and thus disturbs crystallization.

\subsubsection{Polarized Optical Microscope (POM)}

Figure 7.6 shows the polarized light micrographs of the PP/EOC and PP/PP-HI blends as well as the $\mathrm{PP} / \mathrm{EOC} /$ Silica and PP/PP-HI/Silica nanocomposites. For both unfilled blends, the size of the spherulites in the PP/PP-HI blend is bigger than in the PP/EOC samples. This results from a nucleating effect of the EOC [15] in the PP/EOC blend, as also seen in the DSC results in Figure 7.5, which will induce smaller spherulites. From the DSC results, it can be concluded that the crystallinity of the PP/EOC blend is higher than the one of PP/PP-HI. Higher crystallinity together with smaller crystals results in a larger amorphouscrystalline interface in the PP/EOC blend than in the PP/PP-HI blend. This difference in the amorphouscrystalline interface is expected to influence the dielectric properties, which will be discussed in Section 7.3.7 and 7.3.8. 
The addition of nanosilica significantly decreases the spherulite size: no bright spherulites are visible in the POM images of the nanocomposites. Most likely that the crystal size of the spherulites is too small to be visible in the nanocomposites, especially in the one of PP/PP-HI/silica. This is in line with the DSC results, indicating that nanosilica acts as nucleating agent. Furthermore, we have observed that the presence of silica homogenized the distribution of the spherulites in the polymer, while for unfilled blends, the spherulites have different sizes and are not evenly distributed in the polymer (Figure 7.6). It is noticeable
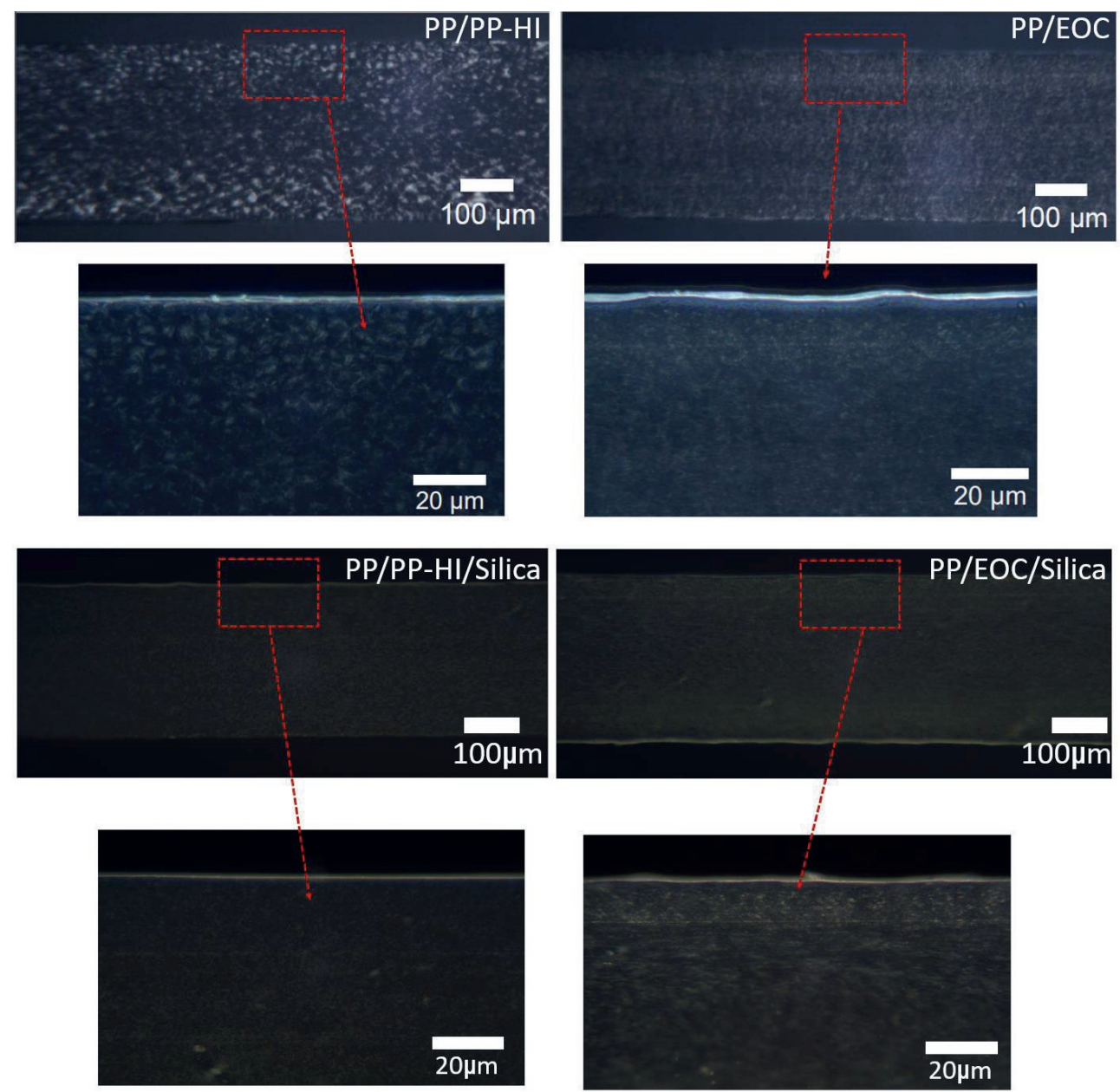

Figure 7.6 POM images of PP/EOC, PP/PP-HI blends and PP/EOC/Silica, PP/PP-HI/Silica nanocomposites.

that there is also a difference in morphology between PP/PP-HI/silica and PP/EOC/silica. This is due to the effect of the different silica distribution and dispersion in both polymeric matrices. In case of PP/PP$\mathrm{HI} / \mathrm{silica}$, the silica is evenly distributed in the polymer, leading to an uniformly distributed nucleating agent (silica) and evenly distributed spherulite. Differently, the silica is only located in PP phase in the sample of $\mathrm{PP} / \mathrm{EOC} /$ silica, causing a heterogeneous distribution of spherulites. As it is known that the 
crystalline-amorphous area interfacial area affects the dielectric properties, the changes the spherulite size and distribution are expected to influence the dielectric properties.

\subsubsection{Thermogravimetric Analysis (TGA)}

The thermal stability was studied by TGA in a synthetic air atmosphere. The mass loss kinetics recorded
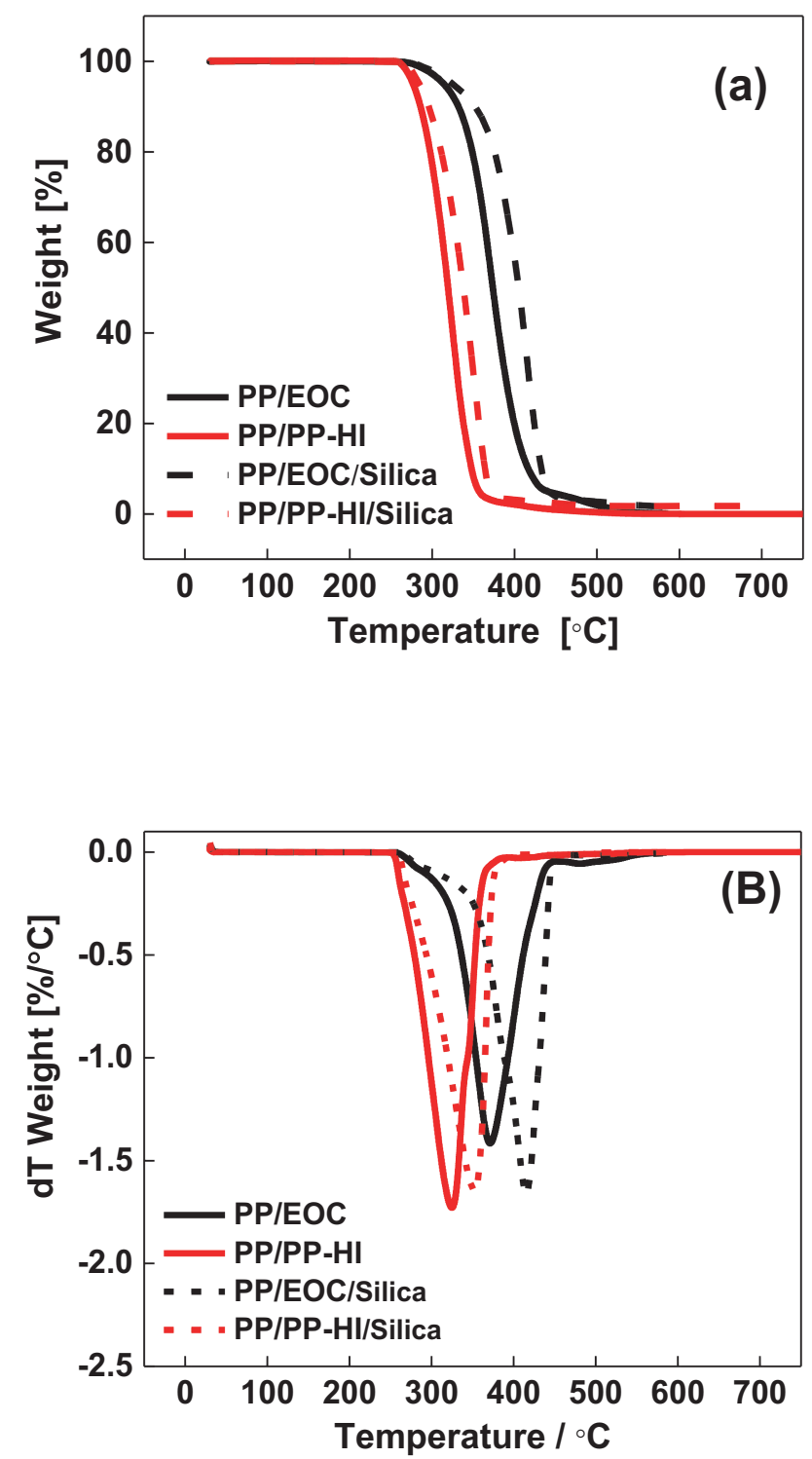

Figure 7.7 TGA profile (a) and the derivative (b) of PP/EOC, PP/PP-HI blends and PP/EOC/Silica, $\mathrm{PP} / \mathrm{PP}-\mathrm{HI} /$ Silica nanocomposites. 
during heating are presented in the left graph of Figure 7.7, and their corresponding derivative (DTGA) results are shown in the right graph of the same figure. The peaks of the DTGA indicating the highest decomposition rate are given in Table 7.2.

During the thermal degradation, a single step degradation is obvious for all samples. Thermal decomposition of the unfilled PP/EOC blend occurs at higher temperatures than of the unfilled PP/PP-HI blend. This is possibly due to the higher thermal stability of EOC compared to polypropylene [20], that increases the overall thermal stability of the PP/EOC blend.

The incorporation of nanosilica causes a shift of the DTGA peak to a higher temperature in both polymer matrices, which indicates that nanosilica can improve their thermal stability. Analogous behaviour has been reported in literature [21-24]. During the thermal degradation of PP, volatile hydrocarbon products are generated through a radical degradation process, and the weight loss is proportional to the amount of volatile products generated. The degradation mechanism is mainly based on chemical bond scission starting from the polymer chain ends. The same behaviour is expected for the blends [22]. The role of a nanofiller in enhancing the thermal stability of the PP/PP-HI/Silica composite is ascribed to the weight loss retarding effect of a nanofiller: the volatile products (especially the polar volatiles and oxidized volatiles such as $\mathrm{H}_{2} \mathrm{O}_{2}$, ketons, alcohols) can be physically or chemically adsorbed onto the nanofiller surface, thus leading to their delayed release [25], resulting in an effective delay of mass loss described as improved thermal stability [21].

Table 7.2 Peak decomposition temperature TD of PP/EOC, PP/PP-HI and PP/EOC/Silica, PP/PPHI/Silica nanocomposites.

\begin{tabular}{|c|c|c|c|c|}
\hline Sample & PP/EOC & PP/EOC/Silica & PP/PP-HI & PP/PP-HI/Silica \\
\hline $\mathrm{T}_{\mathrm{D}}\left({ }^{\circ} \mathrm{C}\right)$ & 370.4 & 416.4 & 324.2 & 350.7 \\
\hline
\end{tabular}

\subsubsection{Dynamic Mechanical Analysis (DMA)}

The dynamic mechanical behaviour was investigated by means of a DMA in order to verify the compatibility and dynamic transitions. The time dependence of the storage and loss modulus as well as the loss factor are shown in Figure 7.8.

The storage modulus $\left(E^{\prime}\right)$ is associated with the elastic energy stored in the polymer and influenced by polymer morphology changes, for example, by the crystalline phase [26]. The storage modulus $\mathrm{E}^{\prime}$ of $\mathrm{PP} / \mathrm{EOC}$ is higher than the one of the PP/PP-HI blend, which is attributed to its higher crystallinity (Table 7.1): crystallites act as polymer network nodes and thus contribute to the reinforcement. The addition of nanosilica decreased the storage modulus of both polymer matrices, which is a direct consequence of the crystallinity reduction.

The loss modulus $\left(\mathrm{E}^{\prime \prime}\right)$ peak temperature ranges indicate the corresponding transition zones [27]. Figure 7.8 (b) shows, that there is a broad peak, Peak 1 , between $-80^{\circ} \mathrm{C}$ and $150{ }^{\circ} \mathrm{C}$, with the maximum around $5.7^{\circ} \mathrm{C}$ in the $\mathrm{PP} / \mathrm{EOC}$ as well as the $\mathrm{PP} / \mathrm{EOC} /$ silica sample. In addition, there are two peaks noticed at $33.7^{\circ} \mathrm{C}($ Peak 2$)$ and $50^{\circ} \mathrm{C}($ Peak 3$)$, which are overlapping with the broad Peak 1. Peak 1 (at $-5.7^{\circ} \mathrm{C}$ ) and 
Peak $2\left(\right.$ at $-33.7^{\circ} \mathrm{C}$ ) are the glass transition peaks of the PP and EOC amorphous phase, respectively. The presence of these two peaks indicates that the miscibility between PP and EOC is low, which is corresponding to the conclusions based on the SEM pictures showing a co-continuous two-phase morphology. Peak 3 at $50{ }^{\circ} \mathrm{C}$ is attributed to melting of fragmentary crystals [28] and the molecular movements of the polyolefin with weak crystallization ability [29]. In the PP/PP-HI and PP/PP-HI/Silica samples, only one narrow and sharp Peak 4 is present around $-22.7^{\circ} \mathrm{C}$, which proves that the miscibility between PP and PP-HI is high. In general, nanosilica addition has only a very slight effect on the dynamic transitions of polymers, but decreases the peak intensity of the loss modulus. This stems from the decreased crystallinity, which results in more mobile polymer chains in the amorphous phase.

The loss factor results from various polymer chain relaxation processes. All measured samples show three relaxation peaks: $\beta, \alpha 1, \alpha 2$. Addition of silica does not have any influence on these relaxation transitions. The $\beta$ relaxation is associated with the relaxation of the amorphous polymer stemming from increased segmental movement of the macromolecules, which is also called glass transition [30]. The $\alpha$ relaxation [31] is associated with molecular motion within the crystals [32]. In our case, the $\alpha 1$ peak is related to the molecular motion within the weak crystals (imperfect crystal formation), and the $\alpha 2$ peak is caused by melting of the crystalline phase of the polymer blends.
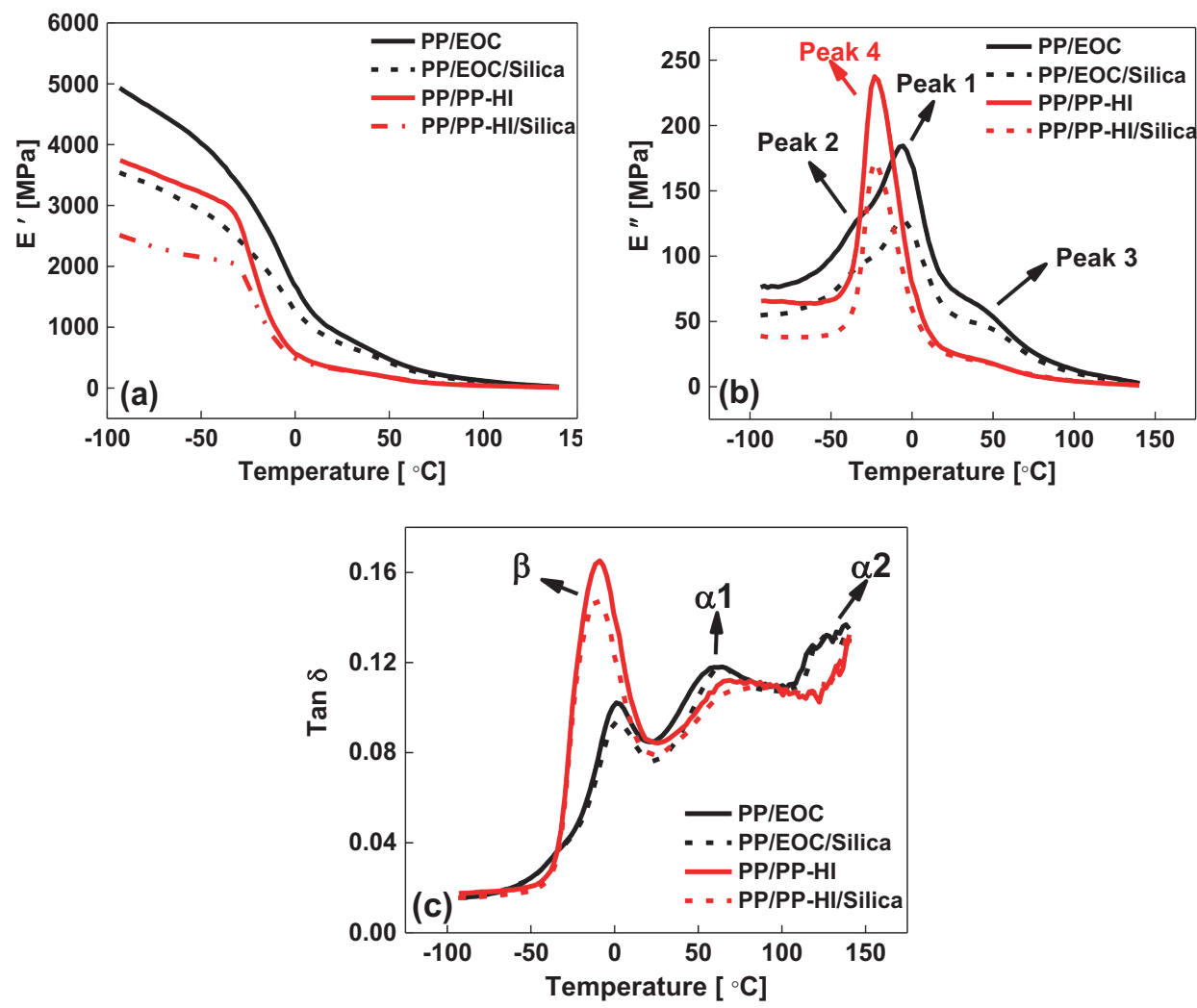

Figure 7.8 DMA curves of the PP/EOC, PP/PP-HI blends and PP/EOC/Silica, PP/PP-HI/Silica nanocomposites: (a) storage modulus vs temperature; (b) loss modulus vs temperature; (c) loss factor vs temperature. 


\subsubsection{Thermally Stimulated Depolarization Current (TSDC)}

Thermally Stimulated Depolarization Current (TSDC) measurements of the PP/EOC, PP/PP-HI blends and the PP/EOC/Silica, PP/PP-HI/Silica nanocomposites are shown in Figure 7.9. The charge trap level distribution curve is shown in the right graph of Figure 7.9. Comparing the unfilled PP/EOC and PP/PPHI blends, both of them show one complete peak and one incomplete peak, associated with release of trapped charges. The position of the full peak at $55^{\circ} \mathrm{C}$ of the PP/PP-HI sample is corresponding to a trap level of $0.98 \mathrm{eV}$, which is lower than the one of the PP/EOC sample $\left(1.02 \mathrm{eV}\right.$ at $\left.69^{\circ} \mathrm{C}\right)$. The incomplete peaks of PP/PP-HI and PP/EOC are starting from a temperature of $93{ }^{\circ} \mathrm{C}$ and $100{ }^{\circ} \mathrm{C}$, respectively, until the maximum measured temperature range. The measured TSDC results also show that the depolarization current density of the unfilled PP/PP-HI sample is lower than the one of the unfilled PP/EOC sample. PP is a semi-crystalline polymer, with a large interface between the crystalline and amorphous phases. Due to the discontinuity at the interface between amorphous and crystalline areas, some chemical and/or physical disorder in chain alignment will be present at the interface, by which the charge carrier traps are formed [2]. It is reported that the interface between a crystalline and an amorphous phase is considered as the main factor for the formation of charge carrier traps [7, 33]. For the unfilled PP/PP-HI sample, crystallinity is lower (Table 7.1), but crystals are larger (Figure 7.5) compared to the PP/EOC sample, resulting in less interface area between the crystalline and amorphous phases in PP/PP-HI. Hence, the trap density of the $\mathrm{PP} / \mathrm{PP}-\mathrm{HI}$ sample is lower than the one of the PP/EOC sample. In addition, the interface between PP and EOC [34] resulting from their low miscibility may also contribute to the higher trap density in the PP/EOC sample compared to the homogenous PP/PP-HI sample. The higher main trap level of the unfilled PP/EOC blend $(1.02 \mathrm{eV})$ compared to the level of the unfilled PP/PP-HI blend $(0.98 \mathrm{eV})$ is probably due to the presence of the EOC phase. The separate phases formed by EOC and PP and the new type of crystals (orthorhombic PE (110) and PE (200)) introduced by the EOC phase creates physical and chemical disorder in the PP/EOC matrix. These physical and chemical disorders are defects, which act as charge traps. For these two unfilled blends, it is also interesting to note that the complete (P1) and the incomplete peak (P2) in the TSDC graph are corresponding to the $\alpha 1$ and $\alpha 2$ relaxation in the DMA results, respectively, as shown in Figure 7.10 (a) and (b). This indicates that the charge releasing process is associated with the $\alpha$ relaxation, which is the chain motion related to the constrained amorphous chain mobility and also the crystalline melting. It implies that the charges might mostly be located at the interface between crystalline and amorphous phases and intra-crystal areas in the unfilled blends, as shown in Figure 7.11.

In case of the nanocomposites, the addition of nanosilica significantly changes the trap distribution for both, PP/EOC and PP/PP-HI polymer matrices, as shown in Figure 7.8. There is one main peak (Pmain), one small peak $\mathrm{P} 1$ and one uncompleted peak $\mathrm{P} 2$ of the depolarization current, with a similar peak position for both, PP/EOC/Silica and PP/PP-HI/Silica nanocomposites. Moreover, there is no direct correlation between the TSDC charge relaxation and DMA chain relaxation peaks, which is different from the unfilled blend.

Based on our previous study [35], we could conclude that addition of APTES modified silica can introduce a new deep trap level at $1.13 \mathrm{eV}$. It is identical with the trap location of Pmain at a trap level of $1.13 \mathrm{eV}$ and $1.14 \mathrm{eV}$ for the PP/EOC/Silica and PP/PP-HI/Silica nanocomposites, respectively. The nanosilica was modified by a silane containing a polar amine group, which brings new energy states into the composites, resulting in the deep trap formation (Pmain). The presence of the silica nanoparticles creates electrical 

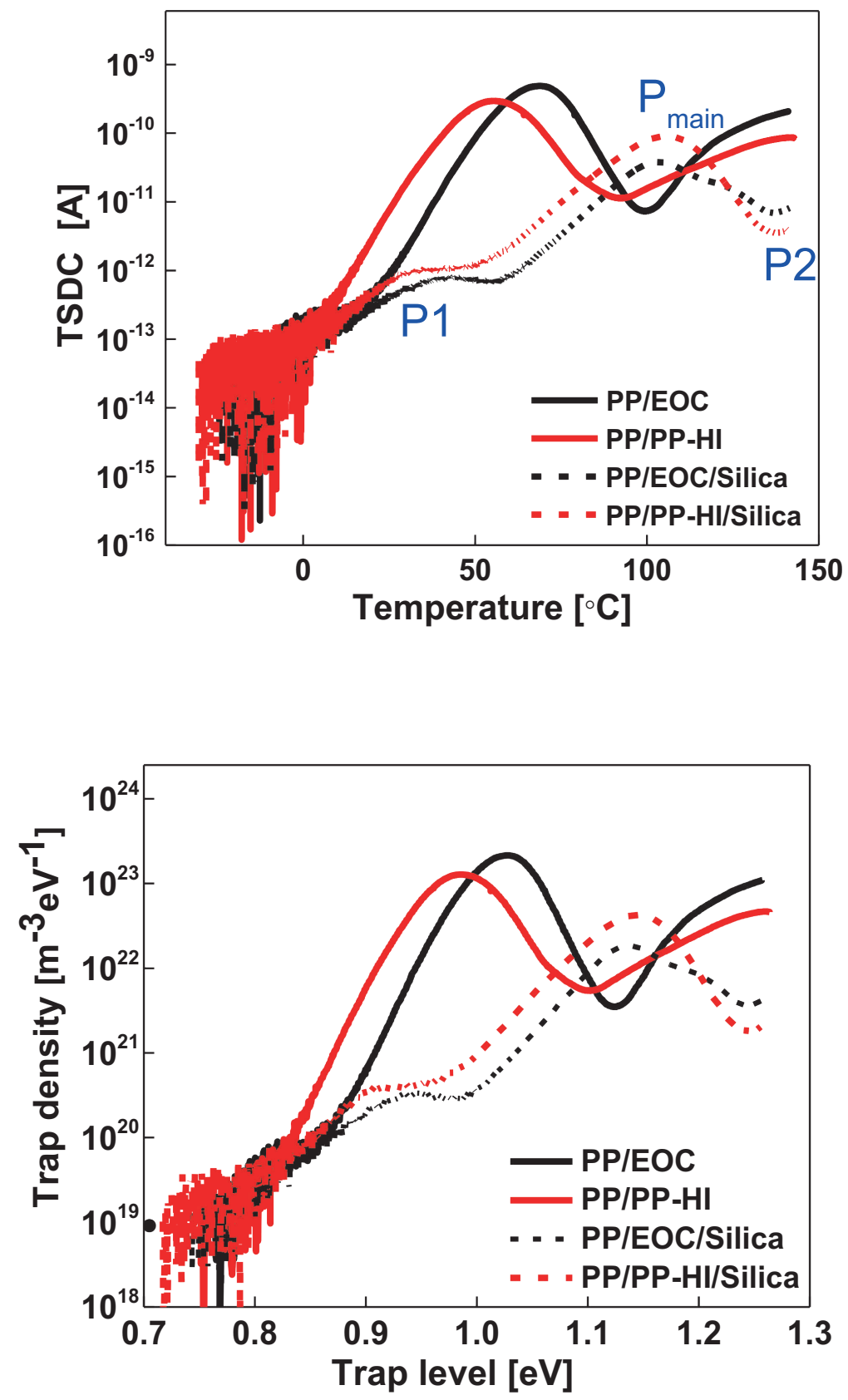

Figure 7.9 TSDC curve (left) and calculated trap level distribution (right) of the PP/EOC, PP/PP-HI blends and PP/EOC/Silica, PP/PP-HI/Silica nanocomposites. 

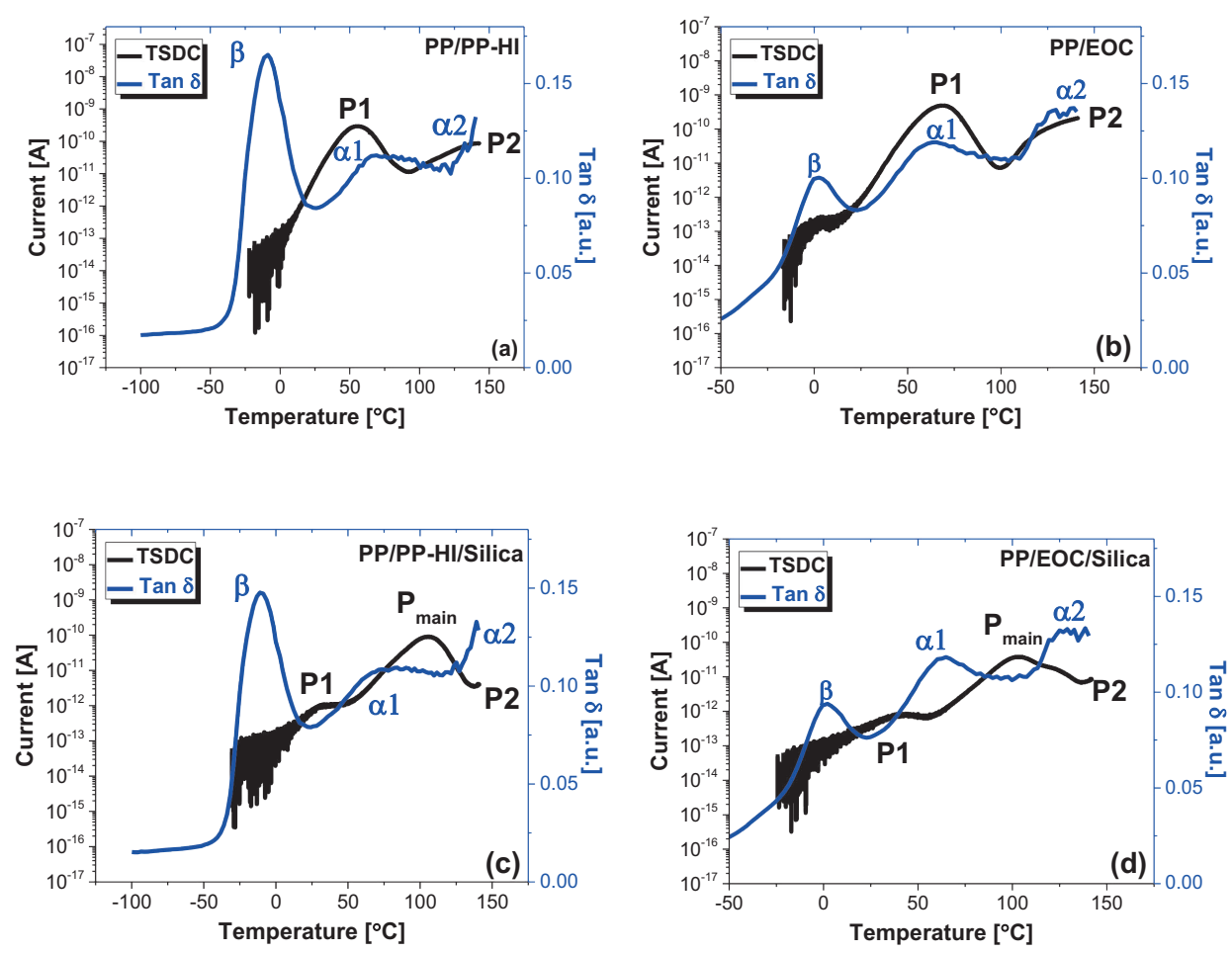

Figure 7.10 Correlation between TSDC depolarization current and DMA relaxation curve of unfilled PP/PP-HI (a), PP/EOC blend (b); PP/PP-HI/Silica (c); PP/EOC/Silica (d).

defect centres inducing local electric fields, which further contribute to accumulation of the charge carriers at the filler-polymer interface [36]. Moreover, the interface between nanofiller and polymer matrix is considered to be the place of charge trap location [37]. Consequently, the newly introduced peak Pmain is most likely related to the interface between the silica and polymer matrix.

Peak P2 starts at a temperature of around $140{ }^{\circ} \mathrm{C}$ in both nanocomposites, and the trap level of this peak is above $1.25 \mathrm{eV}$. The appearance of $\mathrm{P} 2$ after silica incorporation was reported in literature [38], where the authors also found that addition of nanosilica introduces very deep charge traps at a temperature around $140-160{ }^{\circ} \mathrm{C}$, which results from the silica itself acting as the deep traps. It is also reported that silanol groups and especially residual water molecules adsorbed on the silica surface exhibit strong attractive forces to electrons, withdrawing them from the polymer matrix [39]. As a consequence, the silica itself is able to create traps to attract charges; an indication that indeed silica itself created the deepest charge trap P2. However, in the current study, we have also noticed that:

1) The charge trap location of $\mathrm{P} 2$ around $140{ }^{\circ} \mathrm{C}$ is the beginning of the melting process of $\mathrm{PP}$ in the $\mathrm{PP} / \mathrm{EOC}$ and the PP/PP-HI blends; 
2) The nanosilica in the PP/EOC blend is only located in the PP phase, and evenly distributed in the PP/PP-HI blend;

3) The nanosilica acts as nucleating agent and changes the crystal morphology (as shown in Figure 7.5) of both polymer blends. Therefore, it is reasonable to deduct that the P2 peak might stem from

a) the interface between nanosilica and the crystalline PP phase in PP/EOC/Silica and PP/PP-HI/Silica composites, or

b) the changed crystal morphology in both composites caused by the nanosilica.

The small peak P1 in the nanocomposites could be related to chain relaxation $(\alpha 1)$ as shown in Figure 7.10, since they have similar starting temperature. It indicates that there might still be a little amount of charges, which are located at the interface between the amorphous and crystalline phases of the polymer. Due to the fact that the P1 peak intensity is much smaller than the one of Pmain, the nanosilica effect still dominates the charge relaxation process in the nanocomposites, with the nanofiller introducing deeper charge traps into the polymer blends. This is illustrated in Figures 7.11 and 7.12, in which the location of the trap sites is changed significantly due to the nanosilica incorporation.

In nanocomposites, the interface between nanofiller and polymer matrix is considered to be the main region of charge trap location [37]. In our nanocomposite, the trap density of Pmain in the PP/PP-HI/Silica composite is $4.39 \times 10^{22} \mathrm{~m}^{-3} \mathrm{eV}^{-1}$, which is higher than the value of this peak in the $\mathrm{PP} / \mathrm{EOC} /$ Silica composite $\left(1.84 \times 10^{22} \mathrm{~m}^{-3} \mathrm{eV}^{-1}\right)$. This is related to micron-sized clusters of nanoparticles and the physical defects caused by them [40]. As seen in Figure 7.1, the nanosilica is mainly located in the PP phase in the $\mathrm{PP} / \mathrm{EOC} /$ Silica composite, while it is evenly distributed in the PP/PP-HI/Silica composite. The PP/EOC and PP/PP-HI ratios are 55/45, therefore the silica in the PP/EOC blend has roughly only half the volume to be distributed in compared to the PP/PP-HI blend. The actual concentration of nanosilica in the PP phase is thus roughly two times higher in the former blend, resulting in lower filler-cluster distance and a stronger tendency to agglomerate in the PP/EOC/Silica system than in the PP/PP-HI/Silica composite. These larger silica units feature a relatively lower silica-polymer interface in the PP/EOC/Silica sample than in the PP/PP-HI/Silica sample.

There are two possible explanations for the much lower trap density of the nanocomposites in comparison to the unfilled blends:

1) The interface area between the nanosilica and polymer matrix is smaller than the interface between the crystalline and amorphous phase in the unfilled composites;

2) The deep traps introduced in the nanocomposites by the addition of silica immobilize charges. Consequently, the traps create a local electric field during poling inhibiting further charge injection, which leads to less injected charges in nanocomposites. As a result, lower TSDC values are measured and lower charge trap densities are calculated in both nanocomposites in comparison to the unfilled blends. 

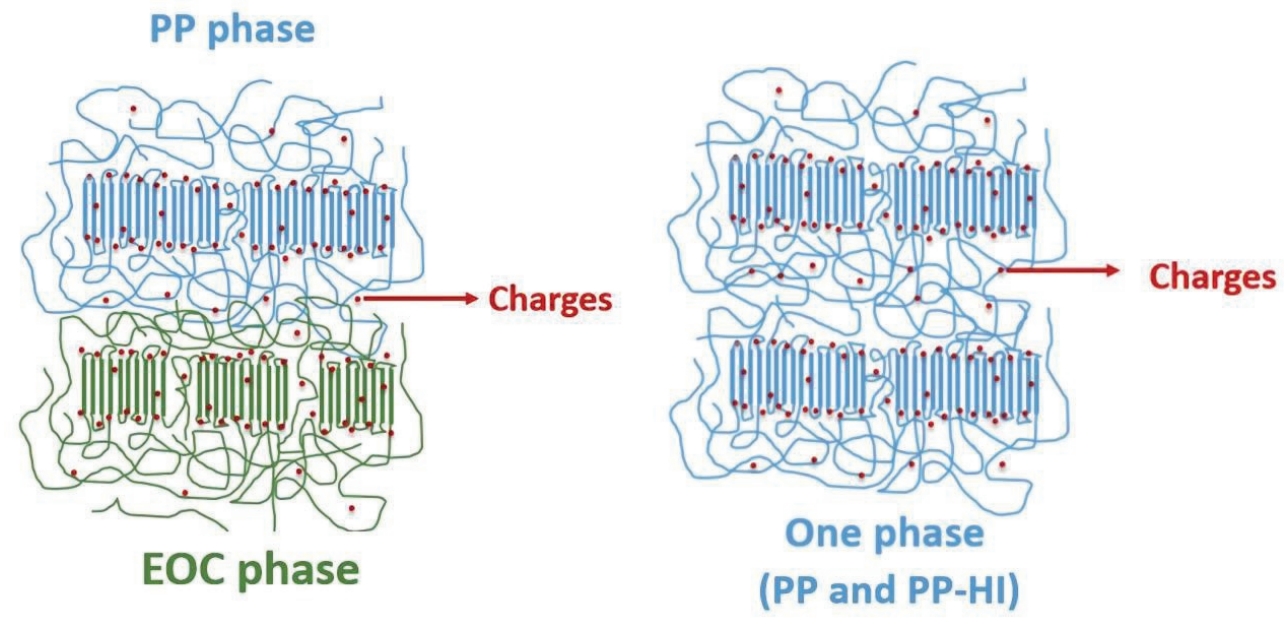

Figure 7.11 Schemes of the most of charges trapping site location in the PP/EOC blend (left) and PP/PP$\mathrm{HI}$ blend (right) - the space between lamellae is filled with the amorphous polymer.

PP phase

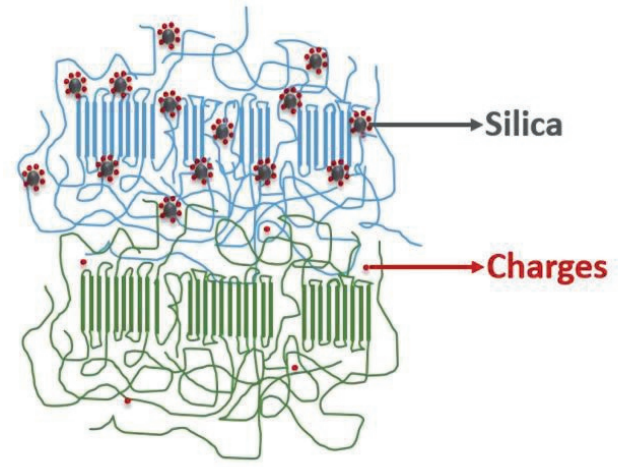

EOC phase

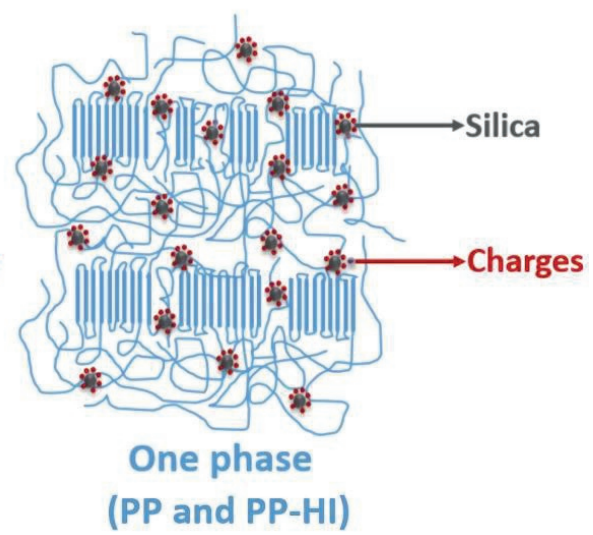

(PP and PP-HI)

Figure 7.12 Schemes of the most of the charges trapping sites of the Pmain peak in the PP/EOC/Silica (left) and PP/PP-HI/Silica nanocomposites (right). 


\subsubsection{Pulsed Electro Acoustic (PEA) Analysis}

To further investigate the space charge accumulation, PEA tests were performed. Space charge patterns of all samples are shown in Figure 7.13. The red and blue color represent the positive and negative charge densities, respectively. The upper electrode corresponds to the anode, while the lower is the cathode. Color bars represent the scale of charge density. The horizontal axis represents time (both polarization and depolarization) in seconds (s), the vertical axis represents the thickness of the tested sample in meters (m). The amount of space charge detected immediately after the beginning of the depolarization phase (Voltoff) is shown in Table 7.3. For the unfilled blends, the charge packets travel through the test specimens. However, it is obvious that the PP/PP-HI blend exhibited a lower amount of heterocharge (charge with opposite polarity to the electrode it is closer to) than PP/EOC. Table 7.3 also shows that the space charge accumulation for PP/PP-HI is lower than for PP/EOC. This might be due to the low compatibility between $\mathrm{PP}$ and EOC resulting in the generation of physical defects in the PP/EOC matrix [41]. Besides, the depth of the unfilled PP/EOC (1.02 eV) is slightly lower than the one of PP/PP-HI ( $0.98 \mathrm{eV})$, as shown in Figure 7.9. In general, deeper traps can hinder charge injection causing a reduction of it; therefore also trap density should be considered. The amount of charge is not only related to the trap depth, but also to trap density. Trap density for the PP/EOC sample is higher than for the PP/PP-HI sample, and this can actually result in a higher degree of charge injection, despite of a deeper trap level. Additionally, the charge injection is also related to the temperature (the poling phase is $70{ }^{\circ} \mathrm{C}$ and $60^{\circ} \mathrm{C}$ for TSDC and PEA, respectively) and electric field ( $3 \mathrm{kV}$ and $30 \mathrm{kV}$ for TSDC and PEA, respectively). The amount of space charge is a sum of these complex factors.

The addition of silica significantly decreases the presence of charge packets and reduces the amount of heterocharges trapped near the cathode, as shown in the bottom part of Figure 7.13. Table 7.3 also shows that addition of nanosilica decreases space charge accumulation of the PP/EOC and PP/PP-HI blends.

Table 7.3 The amount of space charge after Volt-off in the PP/EOC, PP/PP-HI blends and $\mathrm{PP} / \mathrm{EOC} /$ Silica, PP/PP-HI/Silica nanocomposites.

\begin{tabular}{|c|c|c|c|c|}
\hline Sample & PP/EOC & $\begin{array}{c}\text { PP/EOC } \\
\text { /Silica }\end{array}$ & PP/PP-HI & $\begin{array}{c}\text { PP/PP-HI } \\
\text { /Silica }\end{array}$ \\
\hline $\begin{array}{c}\text { Amount of space } \\
\text { charge after Volt- } \\
\text { off }\left(\mathrm{C} / \mathrm{m}^{3}\right)\end{array}$ & 3.3 & 1.5 & 2.7 & 0.6 \\
\hline
\end{tabular}

Furthermore, focus should be put on the depolarization phase of space charge profiles in Figure 7.13. The experimental results confirm what was observed from TSDC measurements as shown in Figure 7.8 for both, the unfilled blends as well as the composites. Charges generally deplete slower in the nanocomposites than in the unfilled blends due to the presence of deep charge traps introduced by the addition of silica. Materials featuring deeper traps can be considered as not suitable for HVDC cable applications, since the charge storage can result in maintaining the field deformation induced by space charge, in case the latter is accumulated for longer times, leading to faster degradation of the insulating material [36]. However, in our case, the overall lower amount of space charge accumulation can counterbalance the effect of slower 
charge depletion. Thus, overall improvement of the long-term dielectric performance for this material can be expected.

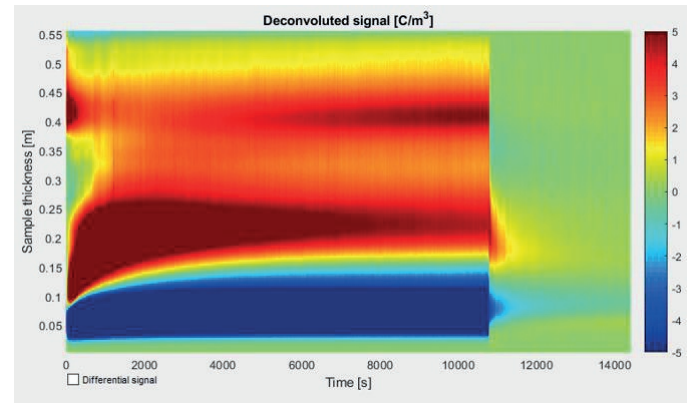

$\mathrm{PP} / \mathrm{EOC}$

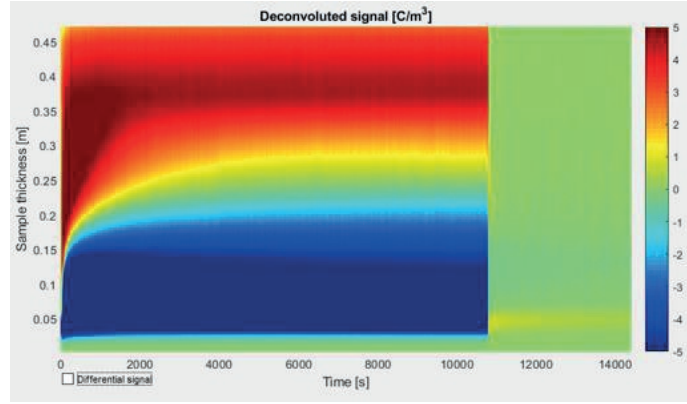

$\mathrm{PP} / \mathrm{PP}-\mathrm{HI}$

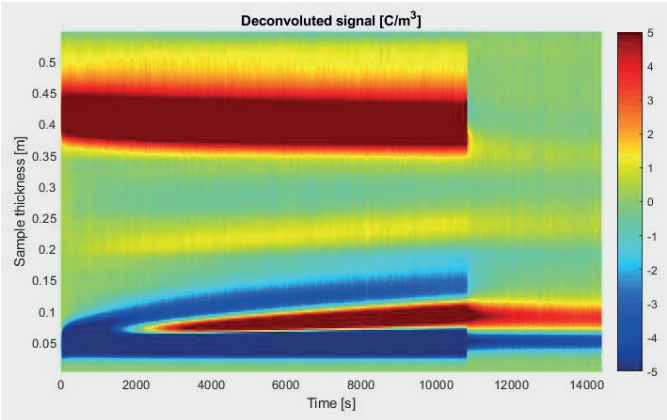

$\mathrm{PP} / \mathrm{EOC} /$ Silica

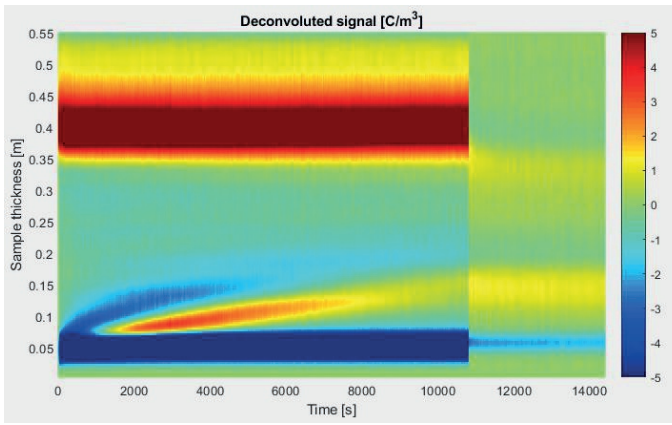

$\mathrm{PP} / \mathrm{PP}-\mathrm{HI} / \mathrm{Silica}$

Figure 7.13 Space charge profiles of PP/EOC, PP/PP-HI blends and PP/EOC/Silica, PP/PP-HI/Silica nanocomposites. 


\subsubsection{Conductivity}

The conductivity test was performed at elevated temperature $\left(60^{\circ} \mathrm{C}\right)$ under an electric field of $30 \mathrm{kV} / \mathrm{mm}$, and the results are shown in Figure 7.14. It should be noted that those results are arising from measurements taken after the polarization current reached full steady state.

Previously it was discussed that the addition of silica could decrease the conductivity of polymer nanodielectrics due to lower charge mobility resulting from the introduction of deeper traps. However, the opposite effect was observed in this study. The addition of the silica resulted in a higher conductivity than the unfilled blends exhibit. This result can be explained by electrical conduction processes being closely related to the motion of polymer chains [37]. In our case, adding nanosilica decreases the crystallinity of the polymer matrix, resulting in a higher amount of amorphous chains in the nanocomposites. These chains are more mobile. Consequently, the conductivity of the nanocomposites is slightly higher than the one of the unfilled blends.

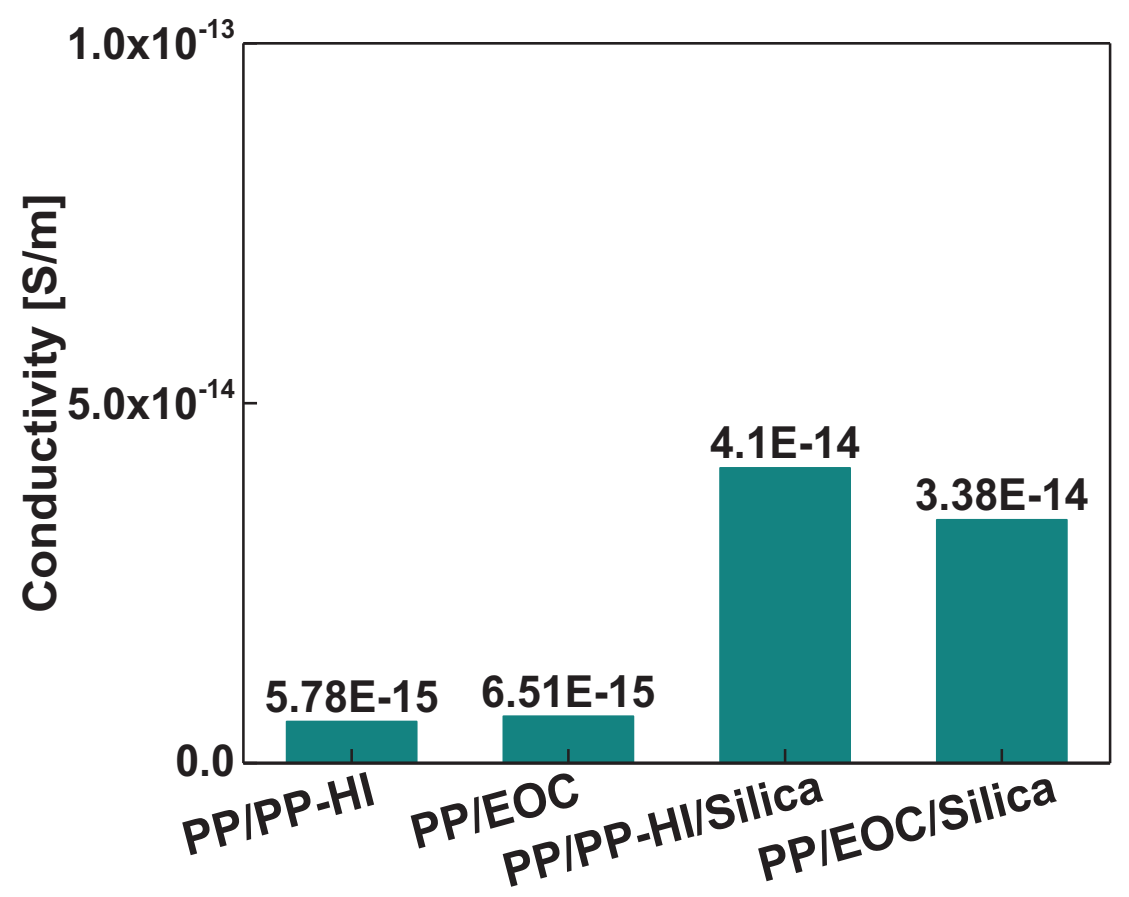

Figure 7.14 Conductivity of the PP/EOC, PP/PP-HI blends and the PP/EOC/Silica, PP/PP-HI/Silica nanocomposites

\subsection{Conclusions}

The morphological, mechanical and dielectric properties of PP/EOC and PP/PP-HI blends as well as $\mathrm{PP} / \mathrm{EOC} /$ Silica and PP/PP-HI/Silica nanocomposites were studied. SEM images showed that there is a co- 
continuous phase separation in the PP/EOC blend, while only one smooth phase in PP/PP-HI blend was visible. This shows that PP and EOC have a significantly lower miscibility than PP and PP-HI. It consequently leads to two melting and two crystallization peaks in the PP/EOC and PP/EOC/Silica samples, while only one melting and crystallization peak in the PP/PP-HI and PP/PP-HI/Silica samples were recorded. The nanosilica was selectively located in the PP phase in the PP/EOC/Silica composite, but evenly dispersed in the whole matrix of the PP/PP-HI/Silica. TGA results showed that the thermal weight loss of PP/PP-HI is lower at a certain temperature than of the loss of the PP/EOC blend. Adding nanosilica increase the thermal stability of both polymer blends. The crystallinity and storage modulus of the PP/EOC blend are higher compared to the PP/PP-HI material, as shown by DSC and DMA results, respectively. The addition of nanosilica resulted in a decrease in both, crystallinity and storage modulus of the polymer blends.

The DSC results showed that the nanosilica acts as nucleating agent, which increased the crystallization temperature of both polymer blends. DMA curves presented three different polymer transition peaks: $\beta$ (glass transition), $\alpha 1$ (crystal rearrangement) and $\alpha 2$ (melting) transition peaks. The addition of nanosilica did not affect the polymer transition peak position. TSDC results showed that the trap density of the $\mathrm{PP} / \mathrm{EOC}$ blend is higher than the one of PP/PP-HI, and that the trap depth of PP/EOC is deeper compared to PP/PP-HI. Nanosilica can introduce deep traps in both polymer blends and decrease the space charge accumulation shown by PEA results. However, the presence of nanosilica increased the conductivity of both polymer blends. Overall, the PP/PP-HI blend exhibited better performance than the PP/EOC blend. PP/PP-HI filled with $2 \%$ of APTES modified silica nanocomposite showed a potential for PP based HVDC cable insulation application in terms of decreased space charge accumulation.

Acknowledgments: This project has received funding from the European Union's Horizon 2020 research and innovation program under grant agreement No 720858.

The authors would like to thank the ECIU Researcher Mobility Fund for supporting this research work.

The authors also would like to thank Evonik Industries for providing a free silica sample. 


\section{References}

[1] Zhou Y, Dang B, Wang H, Liu J, Li Q, Hu J, He J. Polypropylene-based ternary nanocomposites for recyclable high-voltage direct-current cable insulation. Composites Science and Technology. 2018;165:168-74.

[2] Gao Y, Li J, Yuan Y, Huang S, Du B. Trap distribution and dielectric breakdown of isotactic polypropylene/propylene based elastomer with improved flexibility for DC cable insulation. IEEE Access. 2018;6:58645-61.

[3] Huang X, Zhang J, Jiang P, Tanaka T. Material progress toward recyclable insulation of power cables part 2: Polypropylene-based thermoplastic materials. IEEE Electrical Insulation Magazine. 2019;36(1):8-18.

[4] Meng P, Zhou Y, Yuan C, Li Q, Liu J, Wang H, Hu J, He J. Comparisons of different polypropylene copolymers as potential recyclable HVDC cable insulation materials. IEEE Transactions on Dielectrics and Electrical Insulation. 2019;26(3):674-80.

[5] Hosier IL, Cozzarini L, Vaughan AS, Swingler SG. Propylene based systems for high voltage cable insulation applications. Journal of Physics: Conference Series. 2009;183(1):012015.

[6] Green CD, Vaughan AS, Stevens GC, Pye A, Sutton SJ, Geussens T, Fairhurst MJ. Thermoplastic cable insulation comprising a blend of isotactic polypropylene and a propylene-ethylene copolymer. IEEE Transactions on Dielectrics and Electrical Insulation. 2015;22(2):639-48.

[7] Dang B, He J, Hu J, Zhou Y. Large improvement in trap level and space charge distribution of polypropylene by enhancing the crystalline - amorphous interface effect in blends. Polymer International. 2016;65(4):371-9.

[8] Avgeropoulos, G. N., F. C. Weissert, P. H. Biddison, and G. G. A. Bohm. Heterogeneous blends of polymers. Rheology and morphology. Rubber Chemistry and Technology. 1976;49(1): 93-104.

[9] Gao Y, Li J, Han T, Yuan Y, Huang S, Liu Y. Temperature and trap distribution dependence of electrical tree growth characteristics in polypropylene/elastomer blends for recyclable cable insulation. IET Science, Measurement \& Technology. 2019;13(5):755-65.

[10] Zhou Y, He J, Hu J, Dang B. Surface-modified MgO nanoparticle enhances the mechanical and direct-current electrical characteristics of polypropylene/polyolefin elastomer nanodielectrics. Journal of Applied Polymer Science. 2016;133(1).

[11] He X, Rytöluoto I, Anyszka R, Mahtabani A, Saarimäki E, Lahti K, Paajanen M, Dierkes W, Blume A. Surface Modification of Fumed Silica by Plasma Polymerization of Acetylene for PP/POE Blends Dielectric Nanocomposites. Polymers. 2019;11(12):1957.

[12] Du BX, Hou ZH, Li ZL, Li J. Temperature dependent space charge and breakdown strength of PP/ULDPE/graphene nanocomposites for HVDC extruded cable insulation. IEEE Transactions on Dielectrics and Electrical Insulation. 2019;26(3):876-84.

[13] Zare Y, Garmabi H., Nonisothermal crystallization and melting behavior of PP/nanoclay/CaCO3 ternary nanocomposite," Journal of Applied Polymer Science. 2012;124(2):12251233.

[14] Mirabella F. M., Bafna A., Determination of the crystallinity of polyethylene/-olefin copolymers by thermal analysis: Relationship of the heat of fusion of $100 \%$ polyethylene crystal and the density, Journal of Polymer Science. B, Polymer Physics. 2002;40(15):16371643.

[15] Ying J, Liu S, Guo F, Zhou X, Xie X. Non-isothermal crystallization and crystalline structure of PP/POE blends. Journal of Thermal Analysis and Calorimetry. 2008:91(3):723-31 
[16] Dang B, Li Q, Zhou Y, Hu J, He J. Suppression of elevated temperature space charge accumulation in polypropylene/elastomer blends by deep traps induced by surface-modified $\mathrm{ZnO}$ nanoparticles. Composites Science and Technology. 2017;153:103-10.

[17] Chrissafis K, Paraskevopoulos KM, Pavlidou E, Bikiaris D. Thermal degradation mechanism of HDPE nanocomposites containing fumed silica nanoparticles. Thermochimica Acta. 2009;485(12):65-71.

[18] Jiasheng Q, Pingsheng H. Non-isothermal crystallization of HDPE/nano-SiO 2 composite. Journal of Materials Science. 2003;38(11):2299-304.

[19] Gopakumar TG, Lee JA, Kontopoulou M, Parent JS. Influence of clay exfoliation on the physical properties of montmorillonite/polyethylene composites. Polymer. 2002;43(20):5483-91.

[20] Babu RR, Singha NK, Naskar K. Effects of mixing sequence on peroxide cured polypropylene (PP)/ethylene octene copolymer (EOC) thermoplastic vulcanizates (TPVs). Part. I. Morphological, mechanical and thermal properties. Journal of Polymer Research. 2010;17(5):657-71.

[21] Wang B, Huang HX. Effects of halloysite nanotube orientation on crystallization and thermal stability of polypropylene nanocomposites. Polymer Degradation and Stability. 2013;98(9):1601-8.

[22] Lim JW, Hassan A, Rahmat AR, Wahit MU. Morphology, thermal and mechanical behavior of polypropylene nanocomposites toughened with poly (ethylene-co-octene). Polymer International. 2006;55(2):204-15.

[23] Allen NS, Edge M. Fundamentals of polymer degradation and stabilization. Springer Science \& Business Media; 1992; pp 5.

[24] Chrissafis K, Paraskevopoulos KM, Stavrev SY, Docoslis A, Vassiliou A, Bikiaris DN. Characterization and thermal degradation mechanism of isotactic polypropylene/carbon black nanocomposites. Thermochimica Acta. 2007;465(1-2):6-17.

[25] Zanetti M, Camino G, Reichert P, Mülhaupt R. Thermal behaviour of poly (propylene) layered silicate nanocomposites. Macromolecular Rapid Communications. 2001;22(3):176-80.

[26] Batista NL, Olivier P, Bernhart G, Rezende MC, Botelho EC. Correlation between degree of crystallinity, morphology and mechanical properties of PPS/carbon fiber laminates. Materials Research. 2016;19(1):195-201.

[27] Dai S, Ye L, Hu G. Preparation and properties of PP/PC/POE blends. Polymers for Advanced Technologies. 2010;21(4):279-89.

[28] Tsi, Hung-Yi, Wen-Chin Tsen, Yao-Chi Shu, Fu-Sheng Chuang, and Chien-Chung Chen. Compatibility and characteristics of poly (butylene succinate) and propylene-co-ethylene copolymer blend. Polymer Testing. 2009; 28 (8): 875-885

[29] Ji-ru YI, Xiao-lin XI, Xing-ping ZH, Hua-min ZH, De-qun LI. Dynamic mechanical behavior and prediction for PP/POE blends. Chemical Research in Chinese Universities. 2009;25(4):573-8.

[30] Mohanty S, Nayak SK. Dynamic-mechanical and thermal characterization of polypropylene/ethylene-octene copolymer blend. Journal of Applied Polymer Science. 2007;104(5):3137-44.

[31] Luyt AS, Dramićanin MD, Antić Ž, Djoković V. Morphology, mechanical and thermal properties of composites of polypropylene and nanostructured wollastonite filler. Polymer Testing. 2009;28(3):348-56.

[32] Boyd RH. Relaxation processes in crystalline polymers: experimental behaviour-a review. Polymer. 1985;26(3):323-47. 
[33] Wang X, He HQ, Tu DM, Lei C, Du QG. Dielectric properties and crystalline morphology of low density polyethylene blended with metallocene catalyzed polyethylene. IEEE Transactions on Dielectrics and Electrical Insulation. 2008;15(2):319-26.

[34] Du BX, Xu H, Li J, Li Z. Space charge behaviors of PP/POE/ZnO nanocomposites for HVDC cables. IEEE Transactions on Dielectrics and Electrical Insulation. 2016;23(5):3165-74.

[35] He X, Rytöluoto I, Anyszka R, Mahtabani A, Saarimäki E, Lahti K, Paajanen M, Dierkes W, Blume A. Silica surface-modification for tailoring the charge trapping properties of $\mathrm{PP} / \mathrm{POE}$ based dielectric nanocomposites for HVDC cable application. IEEE Access. 2020.

[36] Ma D, Hugener TA, Siegel RW, Christerson A, Mårtensson E, Önneby C, Schadler LS. Influence of nanoparticle surface modification on the electrical behaviour of polyethylene nanocomposites. Nanotechnology. 2005;16(6):724.

[37] Zhang C, Zha JW, Yan HD, Li WK, Wen YQ, Dang ZM. Effects of trap density on space charge suppression of block polypropylene/A12O3 composite under high temperature. IEEE Transactions on Dielectrics and Electrical Insulation. 2018;25(4):1293-9.

[38] Gao M, Yang J, Zhao H, He H, Hu M, Xie S. Preparation methods of polypropylene/nanosilica/styrene-ethylene-butylene-styrene composite and its effect on electrical properties. Polymers. 2019;11(5):797.

[39] Saiz F, Quirke N. The excess electron in polymer nanocomposites. Physical Chemistry Chemical Physics. 2018;20(43):27528-38.

[40] Zhou Y, Yuan C, Li C, Meng P, Hu J, Li Q, He J. Temperature dependent electrical properties of thermoplastic polypropylene nanocomposites for HVDC cable insulation. IEEE Transactions on Dielectrics and Electrical Insulation. 2019;26(5):1596-604.

[41] Gao Y, Li J, Li Y, Yuan YQ, Huang SH, Du BX. Effect of elastomer type on electrical and mechanical properties of polypropylene/elastomer blends. In International Symposium on Electrical Insulating Materials (ISEIM). Toyohashi, Sep. 2017. p. 574-577. 
194 | P a g e 


\section{Chapter 8}

\section{PP/PP-HI/silica nanocomposites for HVDC cable insulation: Are silica clusters beneficial for space charge accumulation?}

This chapter has been published in Polymer Testing.

He, X., Rytöluoto, I., Seri, P., Anyszka, R., Mahtabani, A., Naderiallaf, H., Niittymäki, M., Saarimäki, E., Mazel, C., Perego, G. and Lahti, K., Paajanen, M., Dierkes, W. and Blume, A., 2021. PP/PPHI/Silica nanocomposites for HVDC cable insulation: Are silica clusters beneficial for space charge accumulation?. Polymer Testing, p.107186.

DOI: https://doi.org/10.1016/j.polymertesting.2021.107186 
PP/PP-HI/silica nanocomposites for HVDC cable insulation: Are silica clusters beneficial for space charge accumulation?

\author{
Xiaozhen $\mathrm{He}^{\mathrm{a}}$, Ilkka Rytöluoto ${ }^{\mathrm{b}}$, Paolo Seri ${ }^{\mathrm{c}}$, Rafal Anyszka ${ }^{\mathrm{a}}$, Amirhossein Mahtabani ${ }^{\mathrm{a}}$, \\ Hadi Naderiallaf ${ }^{c}$, Minna Niittymäki ${ }^{\mathrm{d}}$, Eetta Saarimäki ${ }^{\mathrm{b}}$, Christelle Mazel ${ }^{\mathrm{e}}$, Gabriele Perego ${ }^{\mathrm{e}}$, \\ Kari Lahti ${ }^{\mathrm{d}}$, Mika Paajanen ${ }^{\mathrm{b}}$, Wilma Dierkes ${ }^{\mathrm{a},{ }^{*}}$, Anke Blume ${ }^{\mathrm{a}}$ \\ " University of Twente, Faculty of Engineering Technology, Department of Mechanics of Solids, Surfaces \& Systems (MS3), Chair of Elastomer Technology and \\ Engineering, Enschede, the Netherlands \\ ${ }^{b}$ VTT Technical Research Centre of Finland Ltd, Tampere, Finland \\ "University of Bologna, Department of Electrical, Electronic and Information Engineering "Guglielmo Marconi", Bologna, Italy \\ ${ }^{d}$ Tampere University, High Voltage Engineering, Tampere, Finland \\ 'Nexans Research Center, 29 Rue Pré Gaudry, 69007, Lyon, France
}

\title{
A B S T R A C T
}

New potential High Voltage Direct Current (HVDC) cable insulation materials based on nanocomposites are developed in this study. The nanocomposites are produced by blending of polypropylene (PP), propyleneethylene copolymer (PP-HI) and a modified fumed silica (A-silica) in a concentration of 1 and $2 \mathrm{wt} \%$. The Asilica is successfully modified with (3-aminopropyl)triethoxysilane (APTES) via a solvent-free method, as proven by infrared spectroscopy, thermogravimetry and transmission electron microscope mapping.

A-silica in the polymer matrix acts as a nucleating agent resulting in an increase of the crystallization temperature of the polymers and a smaller crystal size. Moreover, the silica addition modified the crystals morphology of the unfilled PP/PP-HI blend. The composite containing A-silica with $2 \mathrm{wt} \%$ contains bigger-size silica clusters than the composite filled with $1 \mathrm{wt} \%$. The composite with the higher A-silica concentration shows lower space charge accumulation and a lower charge current value. Besides, much deeper traps and lower trap density are observed in the composite with $2 \mathrm{wt} \% \mathrm{~A}$-silica addition compared to the one with a lower concentration. Surprisingly, the presence of silica clusters with dimensions of more than $200 \mathrm{~nm}$ exhibit a positive effect on reducing the space charge accumulation. However, the real cause of this improvement might be due to change of the electron distribution stemming from the amine-amine hydrogen bond formation, or the change of the chain mobility due to the presence of occluded polymer macromolecules constrained inside the high structure silica clusters. Both phenomena may lead to a higher energetic barrier of charge de-trapping, thus increasing the depth of the charge traps.

\author{
Keywords: \\ HVDC insulation \\ PP/PP-HI blend \\ Space charge accumulation \\ Nanocomposites \\ Fumed silica
}




\subsection{Introduction}

The development of the High Voltage Direct Current (HVDC) technology did grow rapidly in the recent decades, owing to its lower cost and lower loss over long distance power transmission than High Voltage Alternating Current (HVAC) technology. This makes the HVDC profitable for integrating it in the remote renewable energy sources network such as wind or solar energy, and for efficiently transmitting electricity to the regions of demand. One of the important elements of the HVDC system is the cable insulation material. In order to further improve the power transmission capacity and increase the voltage of the HVDC transmission, it is crucial to develop new insulation materials for the next generation HVDC cable applications exhibiting low space charge accumulation and electrical conductivity [1-3]. One of the most promising approaches in the insulation material field is the development of dielectric nanocomposites, in which the electrical properties are tuned by addition of selected nano-sized fillers. To characterize the role of a nanofiller in the dielectric nanocomposite, one can consider the following effects:

i) Introducing large interfacial areas between their surface and the polymeric matrix: these areas may act as charge scattering centres resulting in an improvement of breakdown strength and voltage endurance [4]. Also, most charge trapping sites are located at the interfacial zone, which has a significant influence on the charge-transport behaviour and the space charge accumulation of the nanocomposites [5]

ii) Introducing deep traps: the interface area around the nanofiller show significant charge trapping in nanocomposites. Free-moving charges are trapped by the introduced deep traps in the high electric field and increase the electrostatic barrier reducing further charge injection. Hence, the space charge accumulation is suppressed by addition of the selected nanofillers. [6,7]

iii) Modifying the composite morphology with an increase of the loading of a nano-filler like alumina: this way the polymer lamella density increases resulting in a larger interface region, which act as electrical tree propagation channels. Hence, the electrical tree grows like zigzag and becomes bushy, which contributes to slowing down electrical tree propagation. [8];

iv) Tailoring the charge trap distribution: by nanofillers with different surface functionalization the charge trap distribution of the composites can be tailored and thus influence the charge transportation and space charge accumulation. Polyolefin composites containing nanosilica surface-modified with an unpolar silane for example show a higher charge trap density with the same level of trap depth, while nanosilica modified with a polar silane introduces deep charge traps with a low trap density. [9];

v) Increasing the potential barrier for hole tunneling: the addition of common nanofillers with certain band gaps to PE or PP increases the hole tunneling barrier in the amorphous phase of the polymer matrix, and thus leads to the consequent reduction in the conductivity and space charge accumulation. [10]

Regarding the base polymer selection driven by sustainability, recyclability of the material is recommended. Due to the crosslinked nature of the state-of-the-art crosslinked polyethylene (XLPE), effective recycling is challenging. Moreover, residues of the crosslinking agent are found to induce space 
charge accumulation and generally degrade the dielectric properties of the XLPE insulating materials. Therefore, development of a recyclable insulation material becomes indispensable. Polypropylene based materials, which do not need the crosslinking process due to the significantly higher melting temperature compared to PE exhibit a great potential for the next generation insulation application $[11,12]$.

As XLPE and PP, both are unipolar, it is profitable to apply an unpolar nanofiller in order to obtain good compatibility and dispersion. [13]. However, in order to introduce deep traps and suppress space charge accumulation [14], some polar functional groups are also beneficial to be grafted on the nanofiller surface. Therefore, finding the right balance between good polymer/filler compatibility and introduction of polar functional groups on the nanofiller surface is a challenge.

In our work, we selected polypropylene/poly(propylene-co-ethylene) as the insulation polymer blend and a fumed silica modified with polar 3-aminopropyltriethoxysilane as the nanofiller to develop a recyclable dielectric nanocomposite. Besides measuring the composite properties, it was studied whether nanofiller clusters larger than $100 \mathrm{~nm}$ can be profitable for the dielectric performance.

\subsection{Materials and Experimental Methods}

\subsubsection{Materials}

Fumed silica (Aerosil 200, Evonik, Germany) was selected as nanofiller due to its low water content and branched structure. 3-Aminopropyltriethoxysilane (APTES) was purchased from Sigma Aldrich, The Netherlands. Trifluoroacetic acid used as a catalyst was delivered by Sigma Aldrich, The Netherlands.

The silica modification procedure was as follows: Unmodified silica (20 g) was put into a sealed glass jar and mixed with 3-aminopropyltriethoxysilane (3.6 g) and catalysts, trifluoroacetic acid (0.4 g) and water $(0.6 \mathrm{~g})$, at room temperature for 24 hours. After the modification, the silica was put in a vacuum oven for another 24 hours in order to remove unreacted residuals.

For compounding, polypropylene (PP) (55 wt.\%) and poly(propylene-co-ethylene) (PP-HI) (45 wt.\%) were melt-mixed using a KraussMaffei Berstorff (Germany) ZE25A x 49D UTX twin-screw extruder (cylinder temperature range of $195-230^{\circ} \mathrm{C}$ and screw speed $85 \mathrm{rpm}$ ) with the surface-modified silica (1 or $2 \mathrm{wt.} \%$ ) and antioxidants $(0.3 \mathrm{wt} . \%)$. The obtained granulated compound was extruded into cast films by a Brabender (Germany) Plasticorder single screw extruder with a T-die and a calendaring system operated at $80^{\circ} \mathrm{C}$. A cast film with a thickness of 300-400 $\mu \mathrm{m}$ was obtained.

\subsubsection{Characterization Methods}

Silica characterization: Silica samples for FT-IR were prepared with spectroscopic grade KBr, and FTIR spectra were recorded using a Perkin Elmer (USA) Spectrum 100 FT-IR Spectrometer in Diffuse Reflectance Mode (DRIFT). The spectra were recorded at a resolution of $4.0 \mathrm{~cm}^{-1}$ and averaged over 128 scans from $4000-400 \mathrm{~cm}^{-1}$. The silica samples were also analysed using thermogravimetric analysis (TGA, TA 550) to quantitatively check the degree of modification. The test was done by heating the sample from $20{ }^{\circ} \mathrm{C}$ to $850{ }^{\circ} \mathrm{C}$ with a heating rate of $20{ }^{\circ} \mathrm{C} / \mathrm{min}$ under air atmosphere. The morphology of the silica and elemental mapping were observed by transmission microscopy (Philips, the Netherlands, CM300ST-FEG Transmission Electron Microscope 300 kV). 
Composite characterization: Scanning electron microscopy (SEM) was carried out with a Zeiss (Germany) MERLIN HR-SEM. The cast films were broken in liquid nitrogen, and the cross section was used without further treatment for SEM observation. The DSC test was done by cooling down the sample to $-50{ }^{\circ} \mathrm{C}$ and maintaining it at $-50^{\circ} \mathrm{C}$ for 5 mins, then heating up to $200{ }^{\circ} \mathrm{C}$ and maintaining it at $200{ }^{\circ} \mathrm{C}$ for another 5 mins, followed by cooling down to room temperature with a rate of $3{ }^{\circ} \mathrm{C} / \mathrm{min}$ under nitrogen atmosphere to mimic the TSDC measurements conditions. Polarized light microscopy (POM, Meiji, Japan, Ml 8530 microscope) was done on the cross section of the microtomed samples with a thickness of $30 \mu \mathrm{m}$. X-ray diffraction (XRD, Philips, the Netherlands, X'Pert 1 X-ray diffractometer) were carried on the surface of the compound film. A film was scanned under the $2 \theta$ diffraction angle from $5^{\circ}$ to $37^{\circ}(0.05 \%$ seconds). Dynamic mechanical analysis (DMA, Netzsch/Gabo, Germany, Eplexor 2500) was carried out on the compound film by heating the sample from -100 to $140{ }^{\circ} \mathrm{C}$ $\left(2{ }^{\circ} \mathrm{C} / \mathrm{min}\right.$ ) with a frequency of $1 \mathrm{~Hz}$ (static strain $0.2 \%$ and dynamic strain $0.1 \%$ ).

The polarization current density and thermally stimulated depolarization current (TSDC) were tested on gold-coated compound films. These were firstly coated with gold $(10 \mathrm{~nm})$ under high vacuum to serve as the two electrodes. A prepared sample was firstly heated up to $70{ }^{\circ} \mathrm{C}$ and stabilized for $3 \mathrm{~min}$, then an electrical field was applied for $20 \mathrm{~min}$. During this process, the polarization current versus time were recorded. The sample was quickly cooled down to $-50{ }^{\circ} \mathrm{C}$ and stabilized for another 5 min while the voltage was still on. Afterwards, the sample was shorted-circuited for $1 \mathrm{~min}$ in order to remove the fast polarization. Finally, the voltage was removed and the sample was linearly heated up to $150{ }^{\circ} \mathrm{C}$ with the heating rate of $3{ }^{\circ} \mathrm{C} / \mathrm{min}$ and the depolarization current versus temperature was recorded. Space charge measurements were done by a pulsed electroacoustic (PEA) test at $60^{\circ} \mathrm{C}$ under a DC electric field of 30 $\mathrm{kV} / \mathrm{mm}$ for 3 hours; 1 hour depolarization without electrical field was also included.

\subsection{Results and Discussion}

\subsubsection{Silica Characterization}

Figure 8.1 shows the DRIFT IR spectrums of the reference (Ref.) and APTES modified silicas (A-silica). The broad absorption band around $1222 \mathrm{~cm}^{-1}$ for both samples represents the Si-O-Si bond stretching from the silica siloxane main structure. The band at $3747 \mathrm{~cm}^{-1}$ originates from the isolated silanol (Si$\mathrm{O}-\mathrm{H})$ groups on the silica surface. It can be used as a measurement of the silica surface modification due to its high reactivity toward silanes [15] and its predominant concentration on the fumed silica surface in comparison to geminal and vicinal silanols. Its concentration reaches $80 \%$ of all silanol moieties, measured at a temperature above $400{ }^{\circ} \mathrm{C}$ [16]. Before modification, there is an obvious sharp band present at $3747 \mathrm{~cm}^{-1}$, while the band vanishes in the A-silica spectrum. This indicates that APTES reacts with silanol groups on the silica surface, consuming in particular the isolated Si-O-H groups. Furthermore, the bands at $3300 \mathrm{~cm}^{-1}, 2979 \mathrm{~cm}^{-1}, 1679 \mathrm{~cm}^{-1}$ and $1405 \mathrm{~cm}^{-1}$ appear [17] due to the N-H stretching, $\mathrm{C}-\mathrm{H}$ stretching and $\mathrm{N}-\mathrm{H}$ bending, respectively, stemming from the APTES silane structure, thus proving that the APTES grafting was successful. 


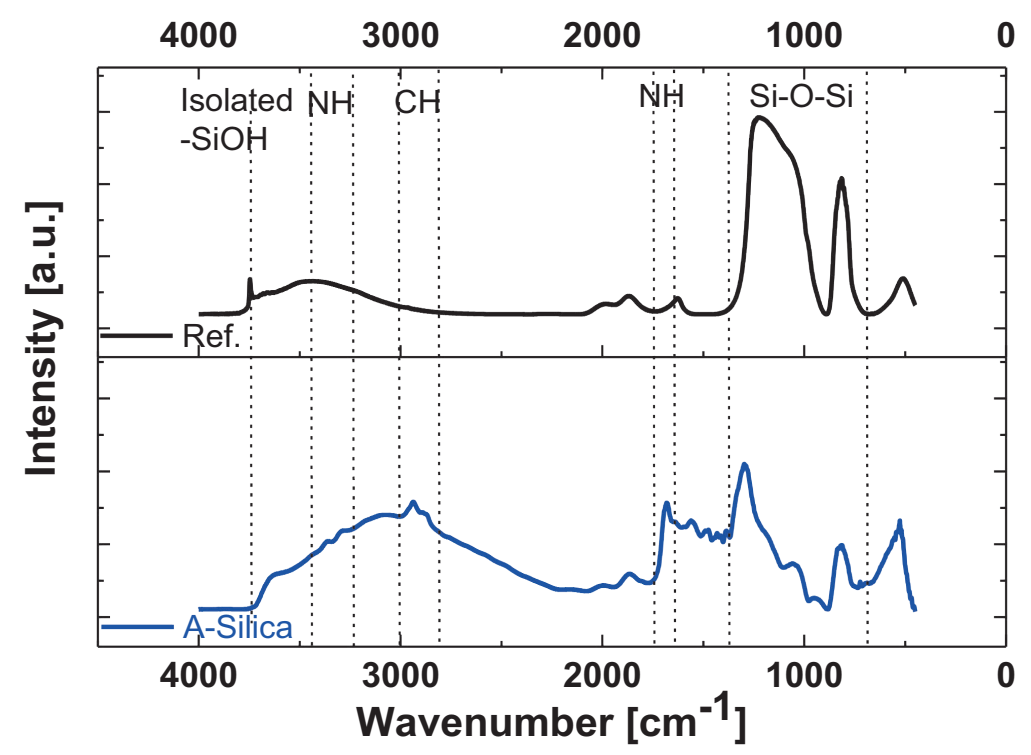

Figure 8.1 DRIFT IR spectrum of reference silica (Ref.) and APTES modified silica (A-silica).

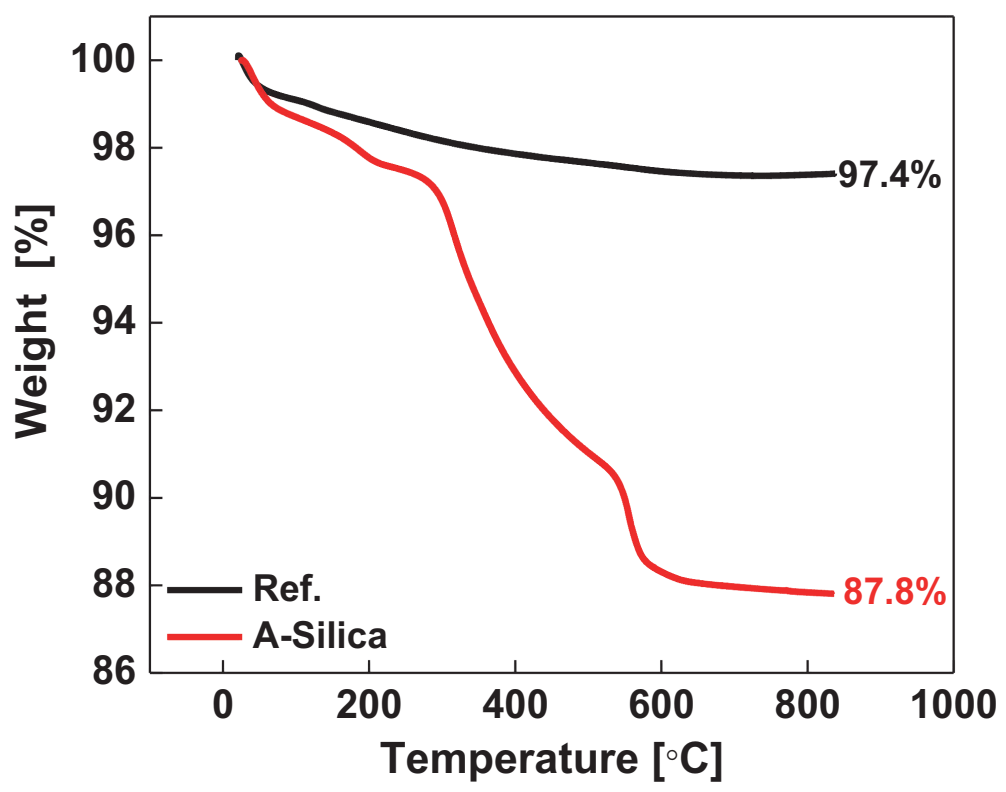

Figure 8.2 Mass loss versus temperature of the reference (Ref.) and APTES-modified silica (A-silica). 
In order to quantitatively investigate the silica surface modification, TGA measurements were done and results are shown in Figure 8.2. The A-silica shows a higher weight loss (12.2 wt.\%) than the Ref. silica (2.7 wt.\% originating from the loss of the grafted organic groups. This gives further evidence that APTES is grafted on the silica surface successfully. Figure 8.3 shows a schematic reaction mechanism of the silane being grafted onto the A-silica surface. The mechanism of the silica modification is based on alkoxy group hydrolysis, which further react with silanol groups on the silica surface. The APTES has three alkoxy groups, which not only react with the silica, but also condense with each other, forming oligomerized structures. Therefore, there is a layer of condensed APTES covering the silica surface, which contributes to the high weight loss of the TGA result.

In order to investigate the morphology of the studied silicas, TEM was performed and results are shown in Figure 8.4. The roughness of the silica surface was noticed to be different before and after the modification. In case of the Ref. silica, the surface is smooth and showing primary particles forming a cluster. While the surface of the A-silica is noticeably rougher owing to the modification and the silane oligomerization that covers the silica surface, without visible separation of the primary particles. It is reported in literature that the modification of silica results in a highly nanorough surface [18]. Hence, the change in surface morphology of the silica is another indication of successful APTES grafting onto the silica surface.
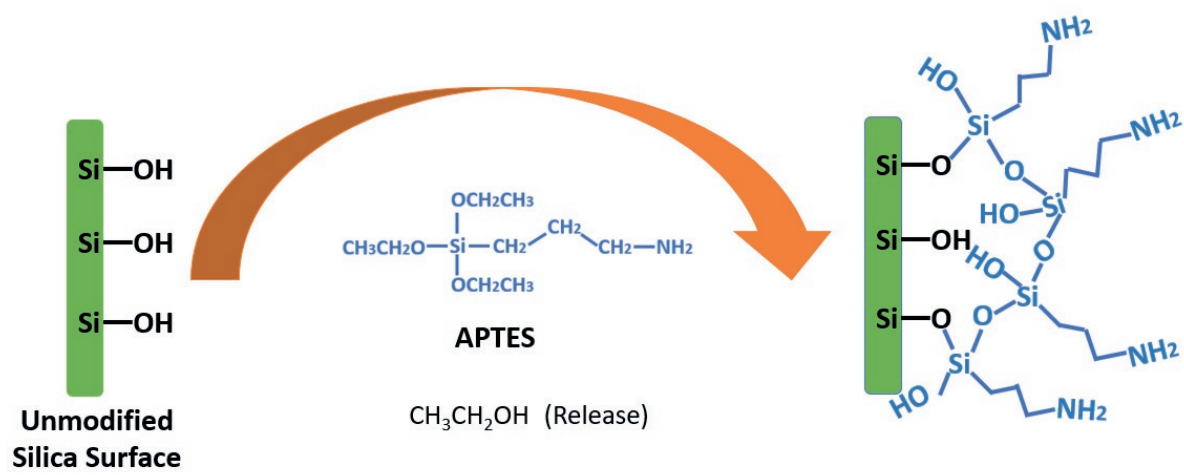

\section{Modified APTES-Silica Surface}

Figure 8.3 Schematic diagram of APTES grafting onto the silica surface.

To further visualize the silica surface modification, HR-TEM elemental mapping was performed, as shown in the Figure 8.5. The distribution of silicon (A, D) and carbon (B, E) of Ref. and A-silica are shown. For both silica types, a silica-rich area mapping is shown in Figure 8.5 (A and D), which is mostly coming from the bulk of the silica and does not show a significant difference between these two samples. However, there is a big difference in the carbon mapping (Figure 8. 5 B, E): While carbon only vaguely is mapped as shown in the Ref. sample (Figure $8.5 \mathrm{~B}$ ), a carbon-rich area is presented in Asilica (Figure 8.5 E), which is stemming from the carbon in the APTES chemical structure. This further proves successful silica modification. Furthermore, in Figure 8. C and D, the elemental mapping of Ref. barely shows any carbon (in green) on Ref. silica bulk (in red), whereas, the elemental mapping of A- 
silica (Figure $8.5 \mathrm{~F}$ ) reveals that there is carbon layer (in green) evenly covering the silica (in red) surface. It is also evidenced that the silane distribution is very homogeneous on the A-silica surface.
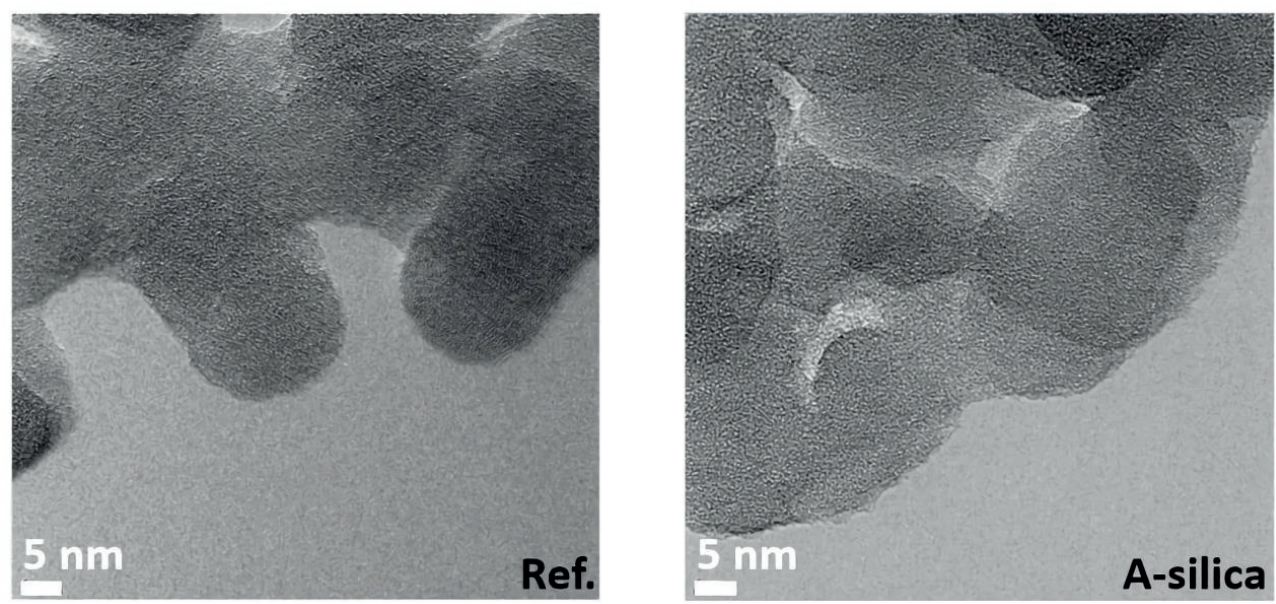

Figure 8.4 TEM image of Ref. and A-silica.

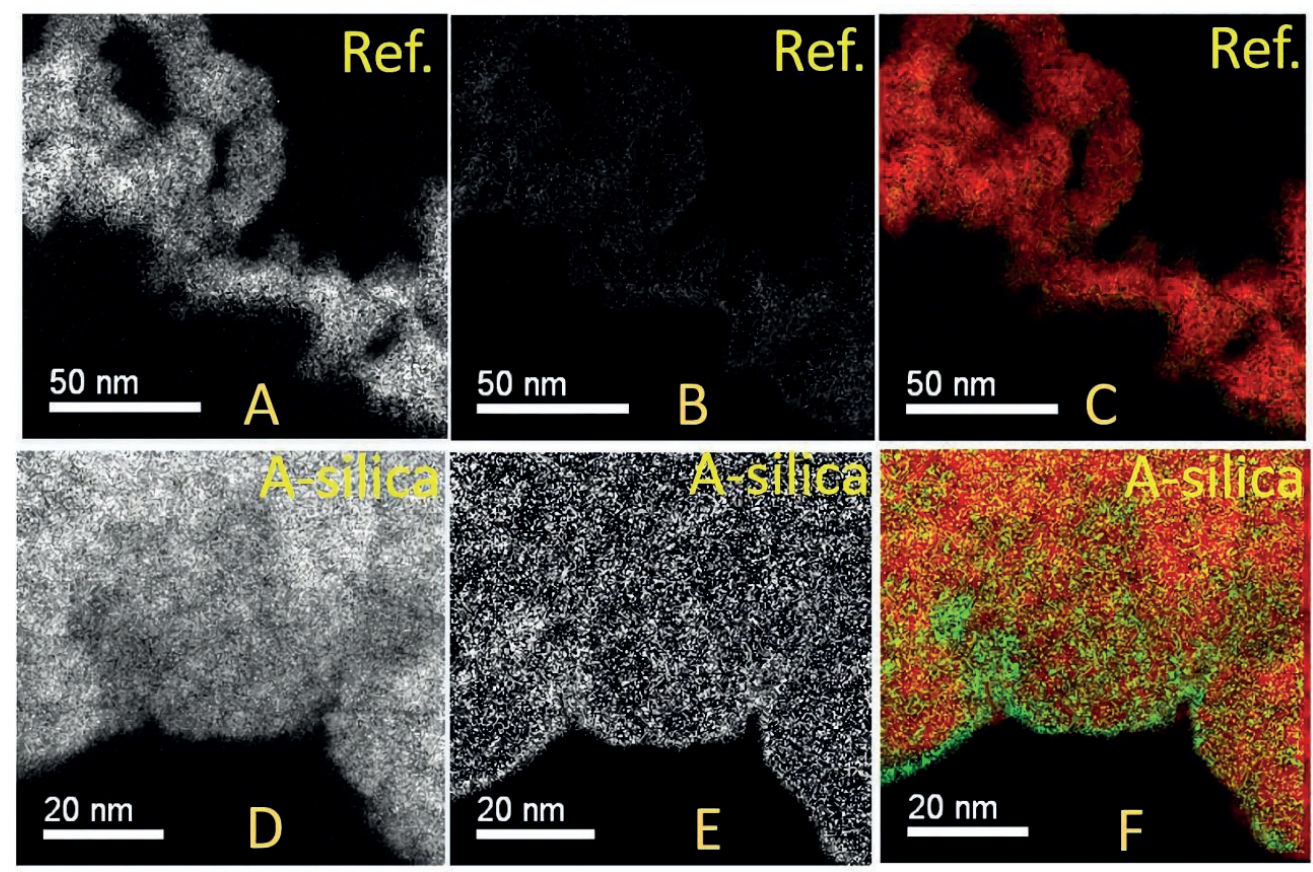

Figure 8.5 TEM images and corresponding elemental mapping of Ref. and A-silica: A, D - silicon mapping; B, E - carbon mapping. C, F - silica elemental mapping (red: silicon; green: carbon). 


\subsubsection{Nanocomposites Characterization}

\subsubsection{Morphology and Silica Dispersion}

The polymer blend morphology is considered to be a very important factor for the performance of nanodielectrics. A polymer blend of good compatibility may show a higher electrical breakdown strength and better mechanical properties than the one with low compatibility [19, 20]. The dispersion and distribution of a nanofiller in a polymer matrix are of general concern due to their large effect on the final performance of the nanocomposites. In the field of nanodielectrics, it is agreed that the large interface between a nanofiller and polymeric matrix plays an important role. Hence, the dispersion and distribution of the nanofiller affects the interfacial area and influence the dielectric performance of nanocomposites. Therefore, SEM was performed to study the morphology of the polymer blend and the dispersion of A-silica, as shown in Figure 8.6.

There is only one smooth polymer phase present in the unfilled PP/PP-HI blend. It indicates that PP and PP-HI are miscible with each other. This is most likely caused by a high content of polypropylene blocks in the PP-HI, improving its chemical compatibility with PP. Possibly also co-crystallization between the two polymers takes place since they form similar crystallites (Figure 8.7). Regarding the dispersion of the silica: agglomerates are present in both nanocomposites. There are three possible reasons for the formation of agglomerates:

1) Due to the high surface area of the silica nanofiller particles, there is a high tendency to reduce their surface energy by forming strong links of physical and chemical nature, which is the driving force to form large agglomerates [21];

2) Hydrogen bond formation through unreacted silanol groups on the silica surface and the amine groups originating from the ATPES grafted onto the silica surface. These hydrogen bonds bind the silica particles together;

3) APTES modified silica still has a polar nature which is not compatible with the unpolar polymeric matrix. Therefore, silica tends to agglomerate together. Moreover, we also notice that the filler dispersion and distribution in the sample with $1 \mathrm{wt} . \%$ silica is better than when $2 \mathrm{wt} . \%$ was added. With the increase of the silica concentration to $2 \mathrm{wt}$. \%, the average distance between silica particles decreases from $1102 \mathrm{~nm}$ (1 wt. \% silica) to $686 \mathrm{~nm}$ ( $2 \mathrm{wt}$. \% silica). Hence, more and bigger agglomerates (200$500 \mathrm{~nm}$ ) are formed. In comparison, the concentration of $1 \mathrm{wt} . \%$ of A-silica resulted in a reduced cluster size $(30-150 \mathrm{~nm})$, as shown in the histogram in Figure 8.6. This can be explained by a higher amount of silica resulting in a higher probability that broken clusters agglomerate during mixing to form bigger clusters. 

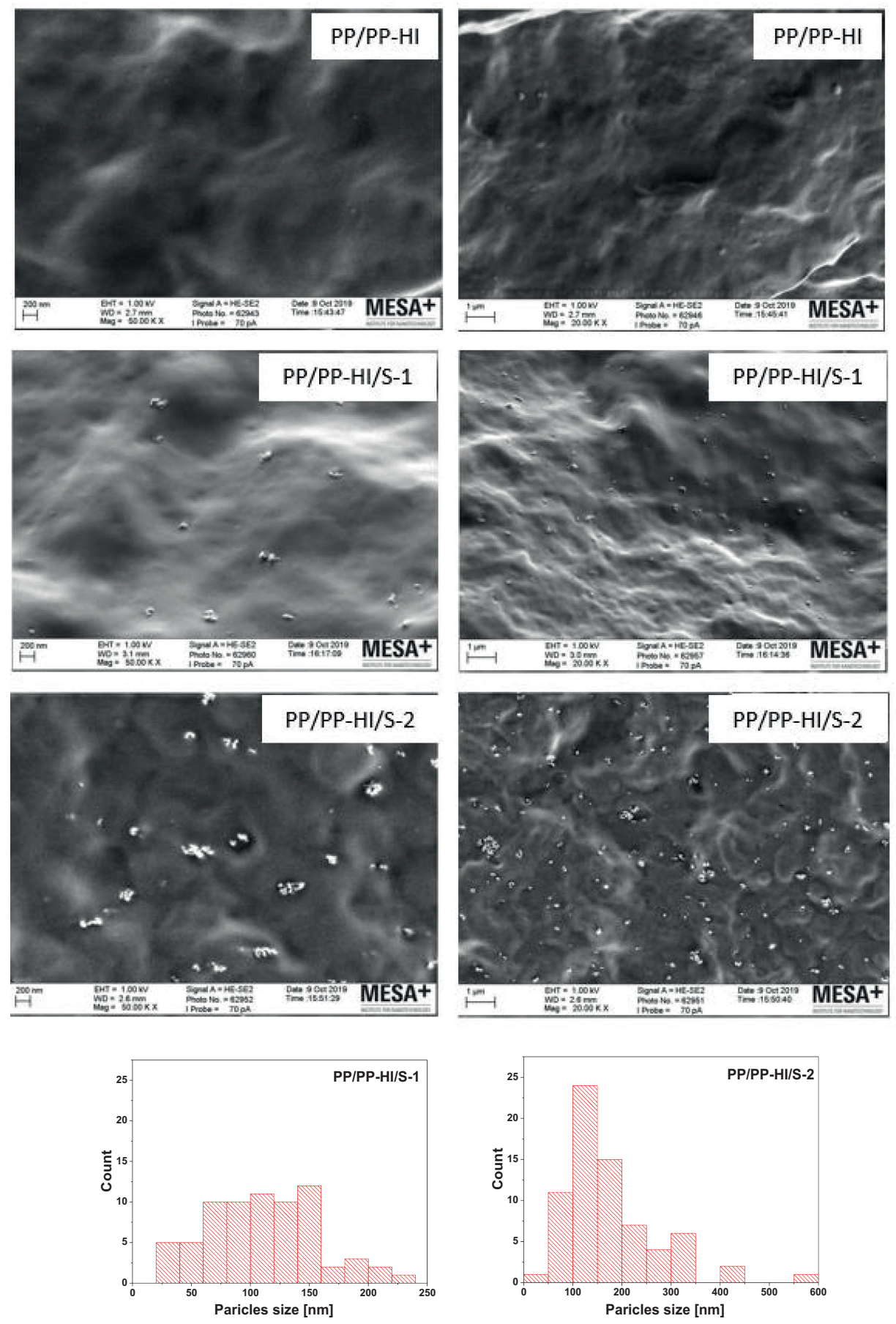

Figure 8.6 SEM images of the unfilled PP/PP-HI blend and the nanocomposites filled with $1 \mathrm{wt} . \%$ (PP/PP-HI/S-1) or 2 wt.\% of A-silica (PP/PP-HI/S-2) (Left column: 50k, right column: 20K) and the histogram of the silica cluster size distribution. 


\subsubsection{Crystallization Behaviour}

It is reported that the crystallization behaviour of semi-crystalline polymers is affected by adding nanofillers, [22] and that the changed crystallization behaviour can influence electrical insulation properties [23]. Therefore, the crystallization behaviour was studied by means of DSC, XRD and POM, as shown in Figure 8.7.

In Figure 8.7 (a), only one melting peak at around $146{ }^{\circ} \mathrm{C}$ for all three samples is visible, which also indicates good miscibility between the PP and PP-HI phases. There is no influence of silica addition on the melting peak as presented in Figure 8.7 (a). The crystallization behaviour is also shown in Figure 8.7 (b). The onset of the crystallization temperature is shifted to higher temperatures by addition of silica regardless of its concentration, which indicates that the silica acts as a nucleating agent, which we observed in earlier work as well [9].

Based on the XRD results as shown in Figure 8.7 (c), (the test was done on the surface of the cast film) there are the same type of crystals present in all samples. The main diffraction peaks at $2 \Theta=13.9^{\circ}$, $16.7^{\circ}, 18.2^{\circ}, 20.9^{\circ}, 21.8^{\circ}$ and $25.3^{\circ}$ correspond to $\alpha(101), \alpha(040), \alpha(130), \alpha(111 / 040), \alpha(060)$, respectively. Adding the A-silica does not change the crystal type, as they present the same diffraction peaks with the same location.

Furthermore, crystallinity is decreased by A-silica addition, as calculated and shown in Figure 8.7 (c). The higher the silica concentration, the smaller the crystalline phase. This is partially evidenced by the XRD results showing a visible decrease of the intensity of the $\alpha(040)$ and $\alpha(060)$ diffraction peaks. Silica is added to the polymer blend in molten state. During cooling, the silica clusters acts as a defect that disturbs the chain ordering process, which results in a lower degree of crystallinity. Compared with 1 wt. $\%, 2$ wt.\% silica addition results in more clusters of larger size, which further block the chain orientation to form crystals. Moreover, the silica acts as nucleating agent, which indicates good interfacial interaction between silica and polymers. During crystallization from the molten state, the immobilized polymer chains located on the silica surface are less likely to crystallize, and thus contribute to lower crystallinity [24]. With 2 wt.\% silica addition, there is a higher interfacial area between the silica and the polymer blend. Consequently, there are more immobile chains constrained by the silica surface. As a result, adding more silica leads to lower crystallinity. However, this reduction is not linearly correlated with the silica concentration, most likely due to a much more pronounced agglomeration of the silica when its content rises to $2 \mathrm{wt} . \%$ (Figure 8.6). Due to the presence of the bigger clusters, the expected higher silica-polymer interface is relatively reduced, and thus the effect of the crystalline phase decrease is less visible.

Thus the question arises: How is it possible that silica is acting as a nucleating agent, but also decreasing the crystallinity of the polymer? Although the nucleating effect in general contributes to higher crystallinity of the polymer [25], we propose that there are simultaneous effects which lead to decrease of the crystallinity:

1) The process conditions: the cooling gradient (temperature difference over time) upon extrusion of the molten polymer and formation of cast film on the calendaring system, which dictate the effective time 

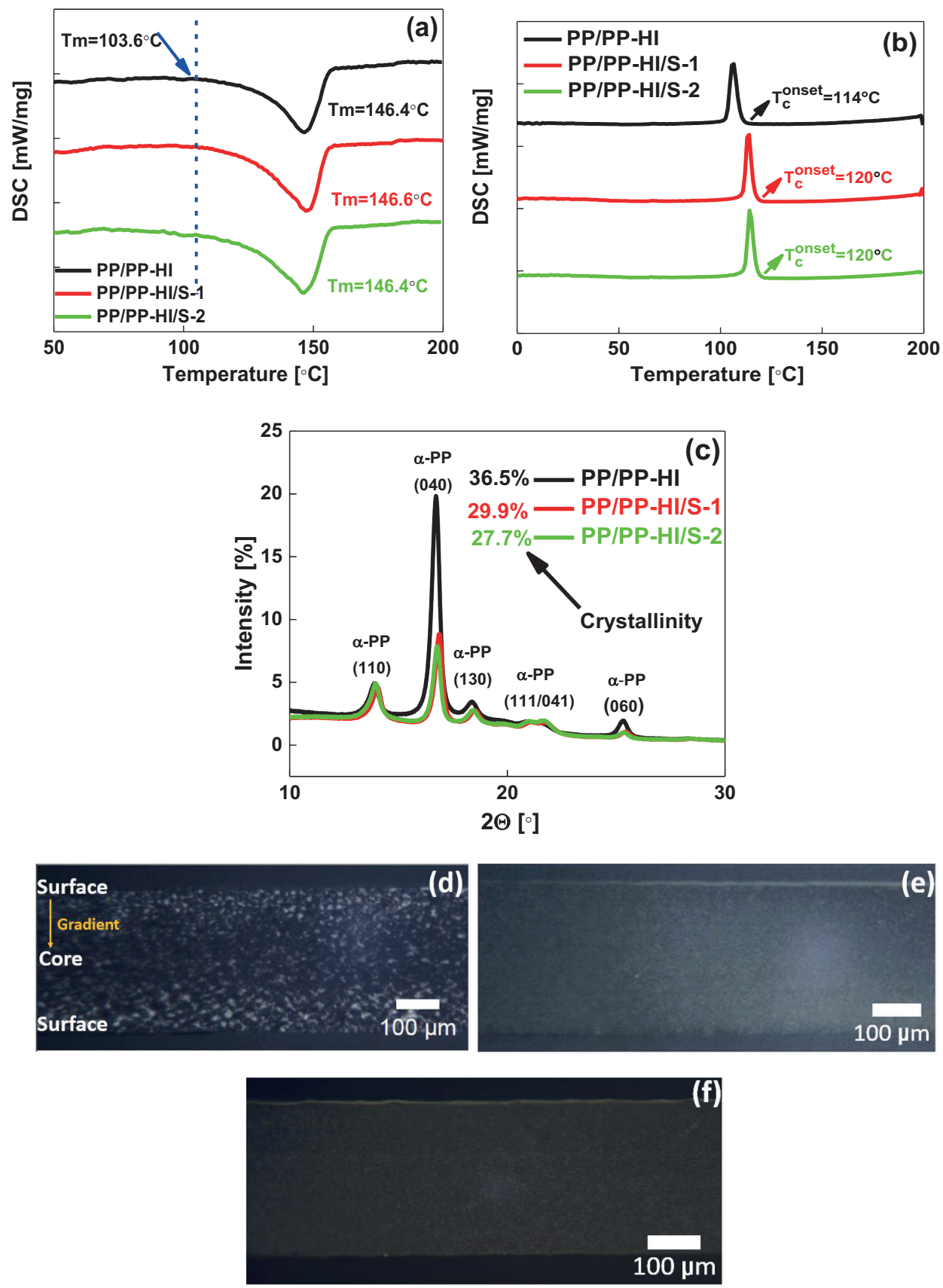

Figure 8.7 DSC heating (a) and cooling (b) curves; XRD results of the unfilled PP/PP-HI blend and the nanocomposites filled with $1 \mathrm{wt} . \%$ or $2 \mathrm{wt} . \%$ of modified silica (c); POM cross-sectional images of the unfilled PP/PP-HI blend (d); the PP/PP-HI/S-1 (e) and the PP/PP-HI/S-2 (f) nanocomposites. 
for the crystal growth. Therefore, the current process conditions might not be optimal resulting in a short effective time, leading to a less crystals formation.

2) The silica clusters act as structural "defects", which block the polymer chains arranging during their crystallization;

3) During crystallization in the molten state, the silica surface immobilized polymer chains are less likely to crystallize, and thus do not contribute to crystallinity [24];

4) The increased spherulite disorder with a higher amount of the nucleating agent retards the crystal growth. [26]

5) The "competing" effect arising from neighbouring nucleation sites for high amount of the nucleating agent might also hinder the crystal formation. More growing spherulites facilitate more intercrystallite interphase hosting the amorphous polymer chains.

To further study the crystallization behaviour, POM was performed on microtomed cast film cross sections of unfilled PP/PP-HI blends and PP/PP-HI samples filled with $1 \mathrm{wt} . \%$ silica or $2 \mathrm{wt} . \%$ of silica, as shown in Figures 8.7 (d), (e) and (f), respectively. A spherulitic crystalline gradient morphology with a mean spherulite size of ca. $5 \mu \mathrm{m}$ is visible in the unfilled PP/PP-HI sample (Figure 8.7 (d)). The spherulite density is higher and the average spherulite size is lower on both the surface layers in comparison to the core region in the unfilled blend, presenting a gradient morphology from the surface towards the inner core of the sample. This effect arises from the processing conditions, most notably the cooling gradient during cast film extrusion, affecting the crystallization process. During the cast film extrusion, as the molten compound exits from the T-die and is extruded on the calendering system chill roll, the cooling rate of the surface layers of the cast film is much higher in comparison to that within the inner core. The temperature gradient from the surface of the sample to the inner core results in spherulitic crystalline gradient morphology.

Interestingly, silica addition significantly changed the crystalline morphology. The nanocomposites do not appear to exhibit gradient type crystalline morphology. Assuming that spherulites are formed, their size in the nanocomposite is too small to be detected and characterized via POM. The formation of small spherulites is due to the nucleating effect of silica, which is in line with the DSC results, as shown in Figure 8.7 (b). The amount of the crystalline phase (principally related with the brightness in the POM images) appears to be lower in the nanocomposites than in the unfilled blend, which is consistent with the XRD results, as shown in Figure 8.7 (c).

\subsubsection{Relaxation Behavior}

The temperature dependence of the storage modulus, loss modulus and hysteresis (loss factor $-\tan \delta$ ) are presented in Figure 8.8. It is a well-known effect that addition of a nanofiller increases the storage modulus of a polymer due to the stiffing effect. The stiffing effect contains two part: 1) the stiff filler and 2) the constrained polymer by the nanofiller forming stiff phase [24], due to the filler polymer interaction. However, in our study, the storage modulus is lower for the silica-filled nanocomposites, and it is much lower in case of $2 \mathrm{wt} . \%$ silica filled sample compared to the one containing $1 \mathrm{wt} . \%$. This is probably due to a dual effect of silica: on one hand, adding silica stiffs the composite, on the other 
hand, it decreases the crystalline content thus decreases the modulus. Both the effects are competitive to each other; the decrease of the dynamic storage modulus by adding silica is an overlay of both effects. Regarding to the composite containing $2 \mathrm{wt} . \%$ silica with a relatively low crystallinity compared to the material with $1 \mathrm{wt} . \%$ silica, the storage modulus decreased more significantly. This might be due to the higher amount and larger silica cluster size. It is likely that the reinforcing potential of the silica is low due to its low amount and its clusters act as local discontinuities which decreases the crystallinity, leading to a lower modulus.
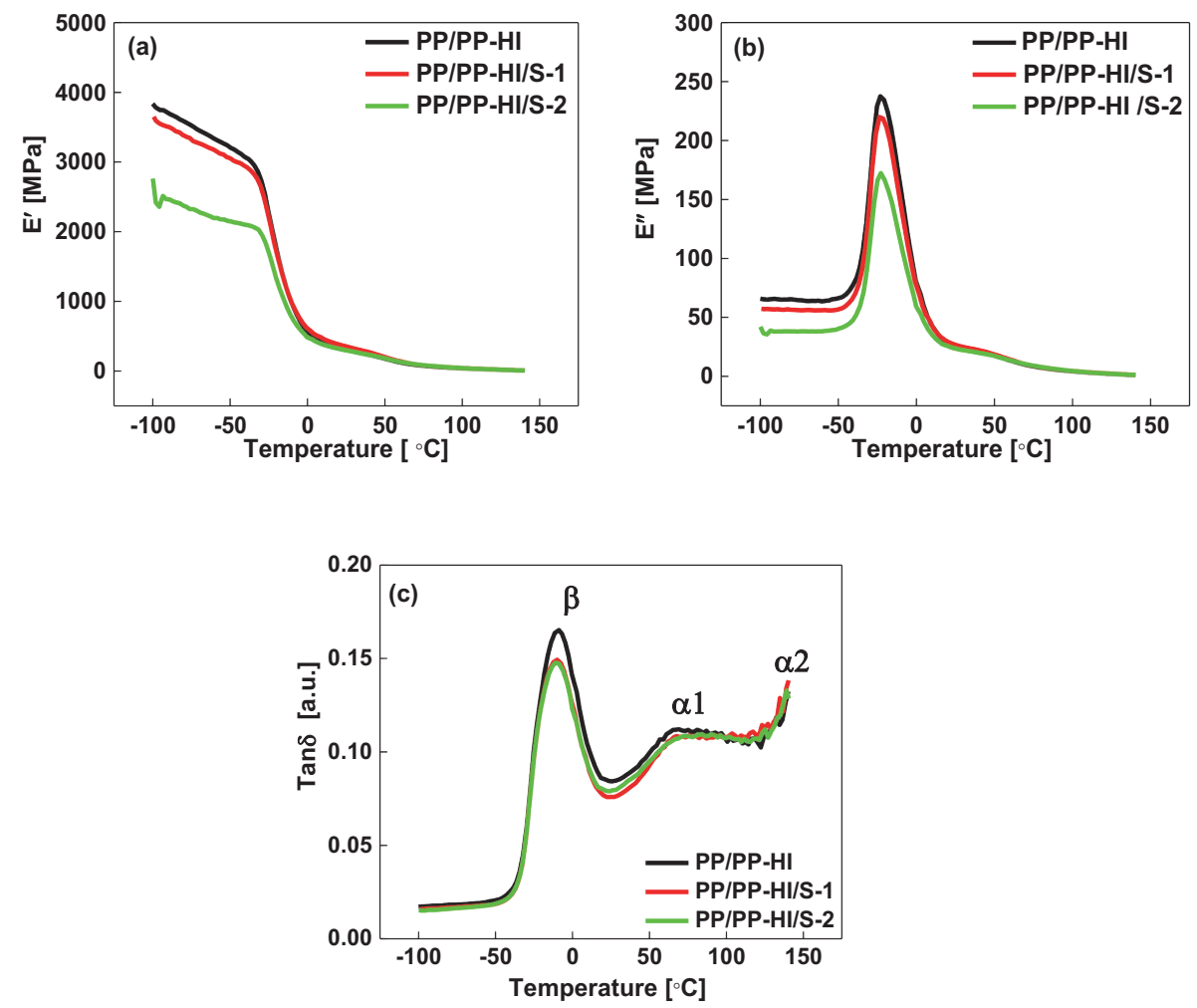

Figure 8.8 Storage modulus (a), loss modulus (b) and relaxation behavior (c) temperature dependence of the unfilled PP/PP-HI blend and the nanocomposites filled with $1 \mathrm{wt} . \%$ or $2 \mathrm{wt} . \%$ the modified silica.

The loss modulus peak represents the glass transition $(\mathrm{Tg})$ of the polymer. There is only one peak in Figure 8.8 (b), and it is obvious that the temperature of the glass transition peak does not change by addition of the nanofiller. However, the intensity of the peak is reduced by adding the nanofiller, and addition of $2 \mathrm{wt} . \%$ of A-silica reduces the peak intensity over proportionally compared to the influence of 1 wt. $\%$ of A-silica. This might be due to the immobile chains constrained by the nanofiller. It is reported that the "immobilized" matrix associated with the interface between filler and polymer does not contribute to energy loss [27]. Hence, less mobile amorphous chain leads to a reduced $\beta$ peak 
intensity. Additionally, the higher amount of A-silica causes relatively more "immobilized" matrix, due to the relatively more constrained rubber chains, leading to a lower amount of the polymer contributing to the energy loss.

Three relaxation peaks $(\beta, \alpha 1, \alpha 2)$ correspond to the different transition phases as shown in Figure 8.8 (c). The $\beta$ peak is attributed to the glass transition temperature of the amorphous polymer phase. The $\alpha 1$ peak is related to motions of chain units which lie within the crystalline region of the polymer [28]. The $\alpha 2$ peak is related to melting of the crystalline phase of the polymer. There is no influence of silica addition on the $\beta$ peak, $\alpha 1$ and $\alpha 2$ peaks.

\subsubsection{Space Charge Accumulation and Charging Current Density}

Space charge accumulation and conductivity are both major dielectric properties affecting HVDC cable insulation performance under electro-thermal stress. Higher space charge accumulation may lead to accelerated aging and ultimately insulation failure. A higher conductivity of an insulation material would potentially lead to accelerated thermal instabilities and premature breakdown. The influence of nanofillers on these properties are shown in Figure 8.9.

In case of the unfilled blend, strong homocharge injection is noticed in the sample, close to the vicinity of both electrodes. This distribution is possibly correlated to the spherulitic crystalline gradient morphology presented in the POM images (Figure 8.7 (d)) due to the resulting conductivity gradient present in the bulk of the material. In the surface region of the unfilled blend, the spherulite size is large, which shortens the conduction paths along the inter-spherulite boundaries, and consequently more charges are injected into the unfilled blend. However, in the case of the nanocomposites, the space charge distribution inside the sample is different. A clear negative and positive charge separation can be observed in the vicinity of the cathode and anode, respectively. The mobility of those charge packets is reduced by increasing the concentration of the A-silica. The amount of space charge detected at the end of the polarization period is shown in Table 8.1. There is a significant amount of space charge $\left(3.0 \mathrm{C} / \mathrm{m}^{3}\right)$ stored in the unfilled PP/PP-HI blend, while the addition of $1 \mathrm{wt} . \%$ of A-silica suppresses it to nearly half of that amount $\left(1.6 \mathrm{C} / \mathrm{m}^{3}\right)$. The addition of $2 \mathrm{wt} . \%$ of A-silica results in a further significant reduction $\left(0.6 \mathrm{C} / \mathrm{m}^{3}\right)$.

Depletion of space charge is also different in all three samples. In case of the unfilled PP/PP-HI blend, the charge is drained very quickly, while the A-silica addition reduces this tendency. This indicates that the trap depth in these three samples is different. This result is in line with the trap distribution estimation obtained via TSDC measurements (Figure 8.10), in which addition of the silica results in the formation of deeper traps, resulting in slower depletion (relaxation) of trapped charge.

The unfilled blend with the highest initial charging current (during the poling phase of the TSDC measurement) shows a slow polarization behaviour with a gradually decreasing current, which results from the superposition of polarization and leakage current (Figure 8.9(d)). The initial part of the charging current of the nanocomposites is much lower than the one of the unfilled blend, and they show very fast polarization to reach saturation, which might indicate less space charge formation. This is in line with the PEA results. It is reported that the initial drop can also be explained by the formation of charges in the vicinity of the electrodes due to the presence of nanoparticles, which leads to a higher charge injection barrier, and thus a lower charging current. [29]. Furthermore, the current recorded for 


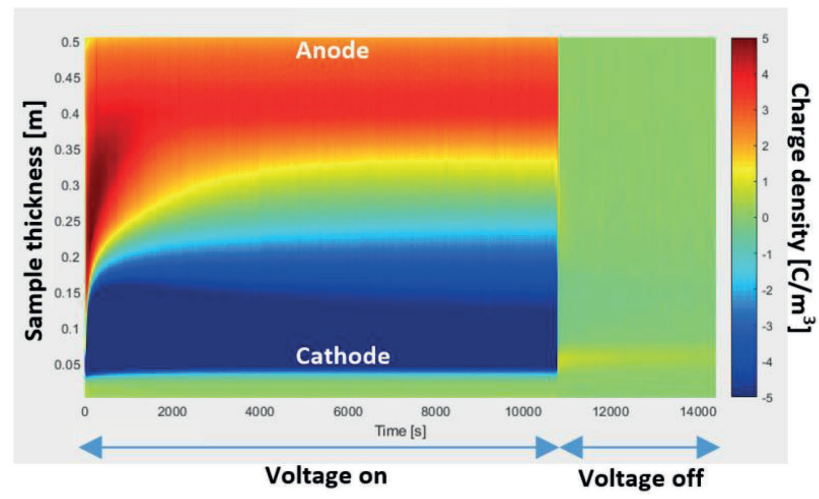

(a)

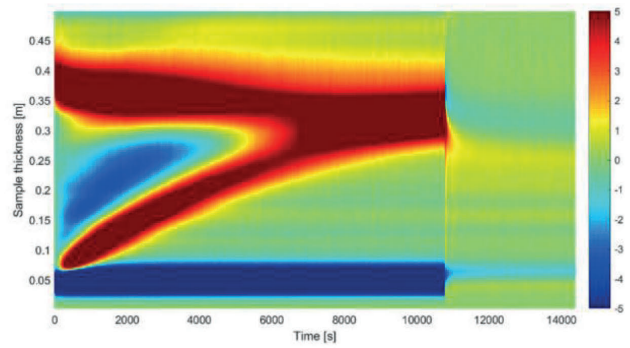

(b)

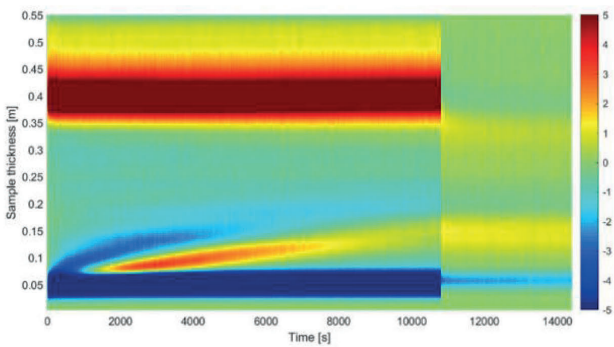

(c)

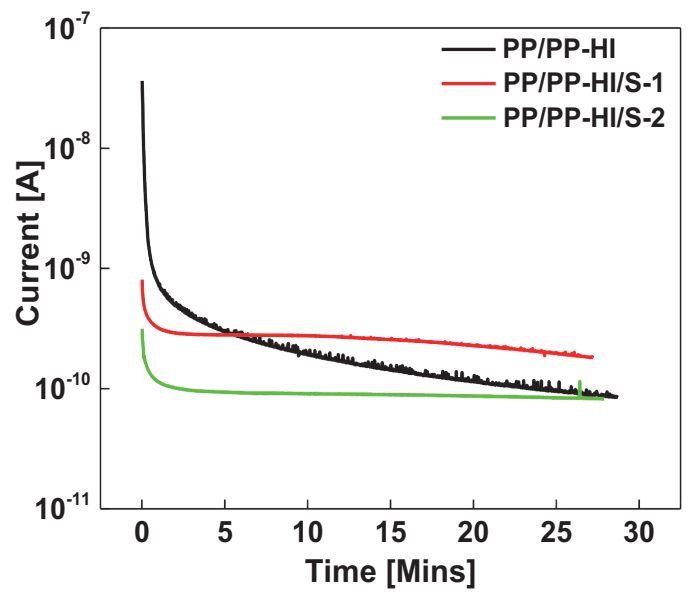

(d)

Figure 8.9 Space charge profile of unfilled PP/PP-HI blend (a), the nanocomposite filled with 1 wt.\% A-silica (b) and filled with 2 wt.\% A-silica (c), current density versus time during poling in the TSDC test (d). 
the PP/PP-HI/S-2 composite is lower than the one for the PP/PP-HI/S-1. Therefore, from the PEA and charging current results during the poling phase, $2 \mathrm{wt} . \%$ of A-silica gives the best outcome and shows a promising trend for HVDC cable application in terms of the lowest space charge accumulation (under $30 \mathrm{kV} / \mathrm{mm}$ ) and low current density (under $3 \mathrm{kV} / \mathrm{mm}$ ).

Table 8.1 Amount of space in the samples charge after Volt-off.

\begin{tabular}{|c|c|c|c|}
\hline Sample & PP/PP-HI & PP/PP-HI/S-1 & PP/PP-HI/S-2 \\
\hline $\begin{array}{c}\text { Amount of space charge after Volt- } \\
\text { off }\left[\mathrm{C} / \mathrm{m}^{3}\right]\end{array}$ & 3.0 & 1.6 & 0.6 \\
\hline
\end{tabular}

\subsubsection{Trapping and De-trapping Behaviour}

In order to gain a better understanding of the space charge and conductivity behaviour of the samples, the trap distribution was studied by means of TSDC and the results are shown in Figure 8.10. There are

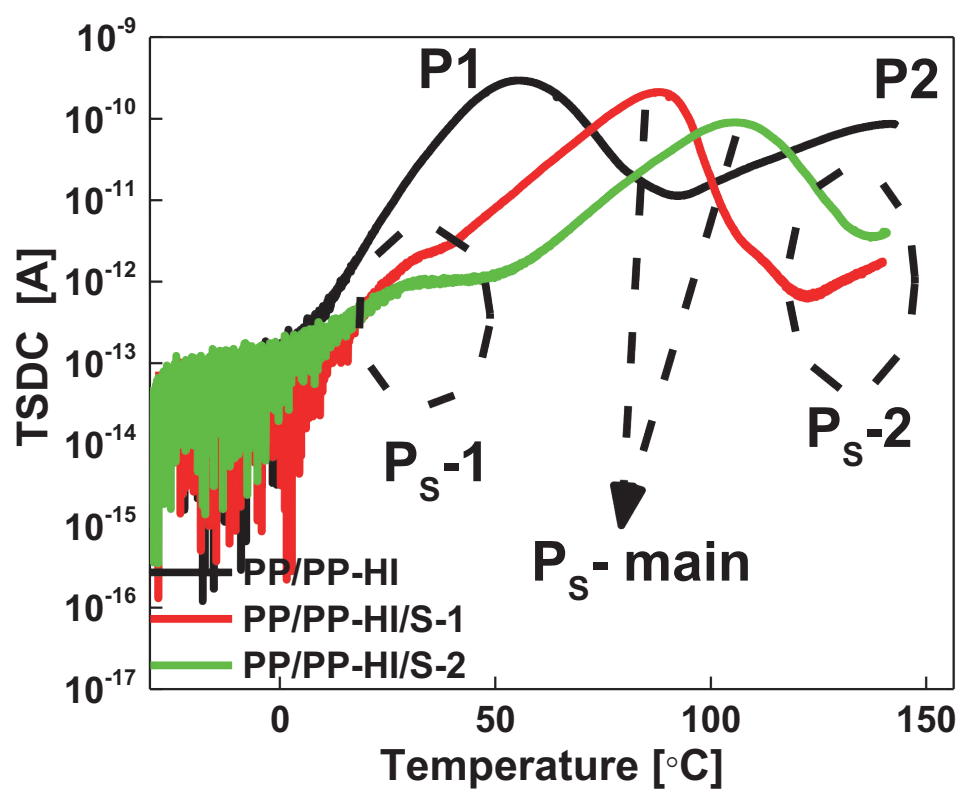

Figure 8.10 TSDC curve of the unfilled PP/PP-HI blend and of the nanocomposites filled with $1 \%$ (PP/PP-HI/S-1) or 2\% A-silica (PP/PP-HI/S-2). 

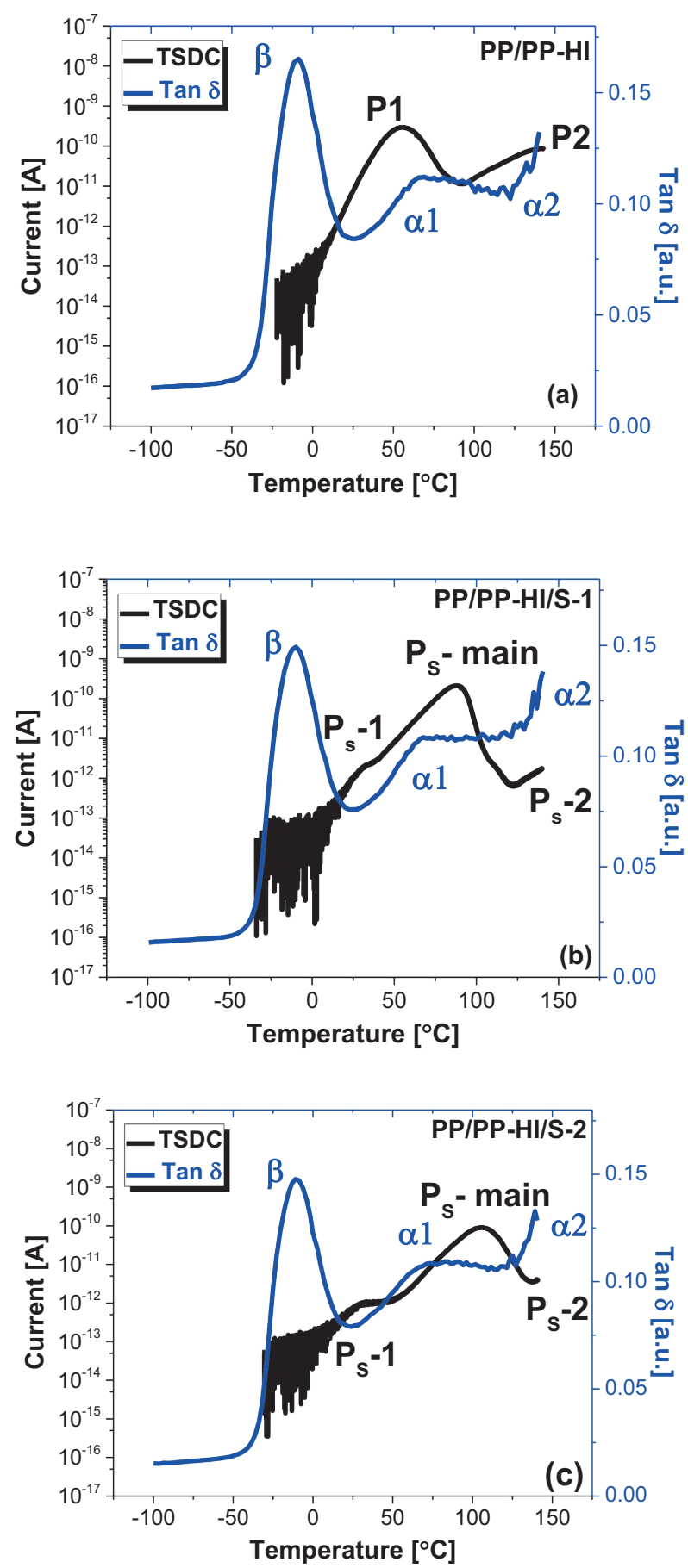

Figure 8.11 The relationship between TSDC and DMA relaxation peak of the unfilled PP/PP-HI blend (a) and the nanocomposite filled with 1 wt.\% (b) or 2 wt.\% A-silica (c). 
two prominent TSDC peaks (P1 and P2) present in the unfilled PP/PP-HI blend. The silica addition suppressed the first peak ( $\left.\mathrm{P}_{\mathrm{S}}-1\right)$ compared to the P1 peak in the unfilled blend, and introduced an additional main deep trap ( $\mathrm{P}_{\mathrm{S}}$-main) peak and another much deeper (incomplete) peak $\mathrm{P}_{\mathrm{S}}-2$ in place of the $\mathrm{P} 2$ of the unfilled blend. Moreover, the TSDC peak intensities are reduced for the nanocomposites compared to the peaks of the unfilled blend. Additionally, the addition of 2 wt.\% A-silica introduces much deeper traps than $1 \mathrm{wt} . \%$. The deeper trap indicates lower charge mobility. This is in line with the PEA results (Figure 8.9), in which the charge depletion rate during depolarization is lower for the nanocomposites than for the unfilled blend, and the depletion rate is much slower when $2 \mathrm{wt} . \%$ A-silica was added compared to 1 wt.\%.

In the case of the unfilled blend, the first peak is located around $50{ }^{\circ} \mathrm{C}$ with the temperature from $25^{\circ} \mathrm{C}$ to $100^{\circ} \mathrm{C}$ and the second peak is above $100{ }^{\circ} \mathrm{C}$, which is identical with the $\alpha 1$ and $\alpha 2$ relaxation transition peaks (DMA results), respectively, as shown in Figure 8.11 (a). It should be stressed that there is a different frequency used for DMA $(1 \mathrm{~Hz})$ and TSDC (about $10^{-4} \mathrm{~Hz}$ ) measurements. Figure 8.11 shows the general relationship between the charge de-trapping (TSDC) and molecular relaxations (DMA) observed in corresponding temperature ranges. It is not meant to directly compare the peak temperature positions (related to trap depth) from TSDC with the one observed in the DMA measurements $(\tan \delta)$. Nevertheless, the coincidence between the P1, P2 peaks from TSDC with the $\alpha 1$ and $\alpha 2$ relaxation transition peaks from DMA reveals that the P1 peak is derived from the charge release related to the chain motions which are restricted in the crystalline phase, and the P2 peak is stemming from the charge released during melting of the crystals. This is line with literature [30]. The above mentioned evidence revealed that the charges are stored at the interface between the amorphous and crystalline phases and within the crystalline structure for the unfilled blend. This also gives an explanation of the PEA pattern (Figure 8.9 (a)) of the unfilled blend, which shows the same pattern as the crystal distribution in the POM image (Figure 8.7 (d)), which is due to the fact that the charges are trapped around the crystals.

However, for the nanocomposites such an accurate correlation between the TSDC peaks and the chain relaxation peaks in the DMA spectra, as shown in Figure 8.11 (b, c), cannot be found. Silica addition did not change the $\alpha 1$ and $\alpha 2$ relaxation peaks (Figure 8.8 (b)), only changed the TSDC charge release peaks temperature range (Figure 8.10). This indicates that the charge location in the nanocomposites might be not be the same as for the unfilled blend. Based on literature, in nanodielectrics the interface between nanofiller and polymeric matrix plays an important role in terms of suppressing space charge accumulation. It was investigated for example that adding $\mathrm{ZnO}$ as nanofiller introduces deep traps, which will capture the charge injected from the electrodes, and the trapped charge near the electrodes in turn increases the barrier against charge injection [31]. Therefore, we deduce that most of the charge might be located at the interface between the silica surface and polymer matrix. Hence, the new interface between silica and polymer act as deep traps and suppresses trap density. We observed 3 TSDC peaks ( $\mathrm{P}_{\mathrm{S}}$-1, $\left.\mathrm{P}_{\mathrm{S}}-\mathrm{main}, \mathrm{P}_{\mathrm{S}}-2\right)$ originating from the nanocomposites. In a previous study, we found that the main trap level $\left(\mathrm{P}_{\mathrm{S}}\right.$-main) is related to the interface between silica and polymer, and that the trap level and trap density depend on the type of surface functionalization (with different chemical moieties) and surface properties [9]. The very small amount of traps type $\mathrm{P}_{\mathrm{s}}-1$ is related to the polymer chain relaxation $\alpha 1$, similarly to $\mathrm{P} 1$ from the unfilled blend. Therefore, these traps correspond to the chain motion which are restricted in the crystalline phase. Regarding the deepest traps $\mathrm{P}_{\mathrm{S}}-2$ located at $120^{\circ} \mathrm{C}$ and $140{ }^{\circ} \mathrm{C}$ for $\mathrm{PP} / \mathrm{PP}-\mathrm{HI} / \mathrm{S}-1$ and PP/PP-HI/S-2, respectively, there are three possible explanations: 
1) Melting of the PP/PP-HI blend occurs in the range of $110{ }^{\circ} \mathrm{C}$ to $160{ }^{\circ} \mathrm{C}$, so the $\mathrm{P}_{\mathrm{S}}-2$ traps are in the range of the polymer melting. Consequently, these traps might still be related to melting of the crystals. However, the $\mathrm{P}_{\mathrm{S}}-2$ traps of the nanocomposites are deeper than the $\mathrm{P} 2$ traps of the unfilled blend, which might be a result of the changed crystal size and morphology by addition of the nanosilica, as proven by POM images in Figures 8.7 (d, e and f);

2) Since A-silica acts as nucleating agent, it induces crystal grow resulting in possible changes of the interface between the silica and the induced crystalline phase. This might possibly also to contribute to deep trap $\mathrm{P}_{\mathrm{S}}-2$ formation in the nanocomposites;

3) Based on the literature [32], the deepest traps introduced by silica addition might be directly related to the silica itself. It is stated that since the temperature of the deepest trap release is higher than the melting temperature of the polymer, the silica itself might form the deepest traps in the nanocomposites.

When comparing these three trap levels $\left(\mathrm{P}_{\mathrm{S}}-1, \mathrm{P}_{\mathrm{S}}\right.$-main, $\left.\mathrm{P}_{\mathrm{S}}-2\right)$, the $\mathrm{P}_{\mathrm{S}}$-main peak, which is related to the interface between the silica and polymer, is predominant. This shows, that most of the charges are located in the interface between the A-silica and the polymer matrix.

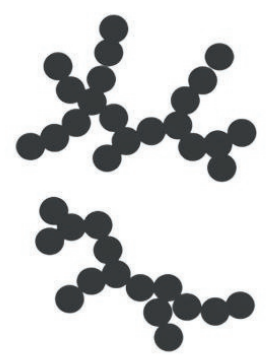

\section{Small cluster of silica}

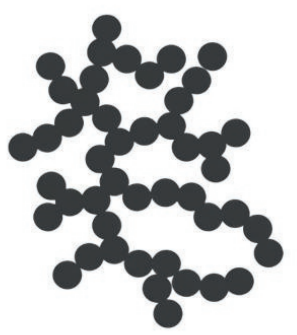

Large cluster of silica

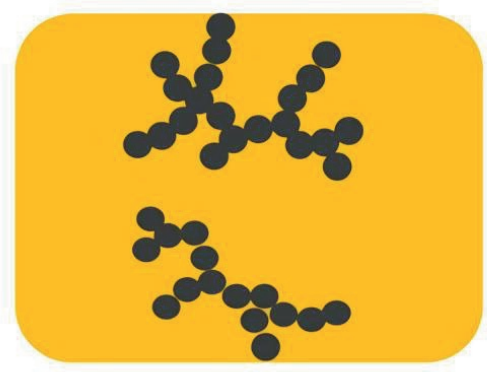

Small cluster (grey) in the polymeric matrix (yellow).

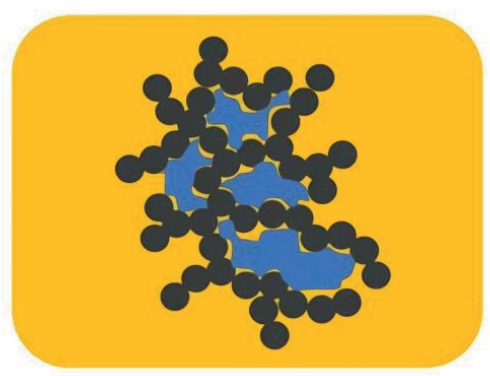

\section{Large cluster (grey) in the polymeric matrix (yellow). The blue region indicates polymer highly constrained within the silica cluster.}

Figure 8.12 Schematic diagram of small and large silica clusters and their interaction with polymer macromolecules. 
Consequently, another question is raised. Why does the addition of 1 wt. $\%$ or 2 wt. $\%$ of A-silica result in a different main peak level (Ps-main)? The 3-aminopropyltriethoxysilane is located on the A-silica surface, introducing polar amine groups as shown in Figure 8.3. The amine moiety has an electrically active dipole nature, which could attract a travelling electron to the silica surface, which might be one of the reasons that the charges are located in the interface between silica and polymer. This could lead to formation of a charge barrier around the silica clusters, which would prevent further charge from injection. As a result, the lower space charge accumulation and lower conductivity are exhibited by the nanocomposites. Furthermore, a higher silica concentration gives a stronger barrier. Consequently, less injected charge results in lower trap density, which also leads to much lower space charge accumulation for the sample with $2 \mathrm{wt} . \%$ of A-silica compared to the one with $1 \mathrm{wt} . \%$.

Furthermore, regarding the dispersion of A-silica (Figure 8.6), there are more silica clusters with larger dimensions and higher structures formed in the sample containing $2 \mathrm{wt} . \%$ A-silica. The deeper Ps-main traps recorded for the PP/PP-HI/S-2 nanocomposite may come from the tightly constrained polymer chains inside of the cluster, as shown in Figure 8.12. A tightly constrained polymer chain is less mobile. When the charges are trapped at the interface between the highly constrained polymer and silica, which has a very high energy potential, the charge cannot move or de-trap easily. Furthermore, the immobile trapped charge might form another barrier based on the repelling force suppressing further charge injection, which results in low trap density, low space charge accumulation and also low current density.

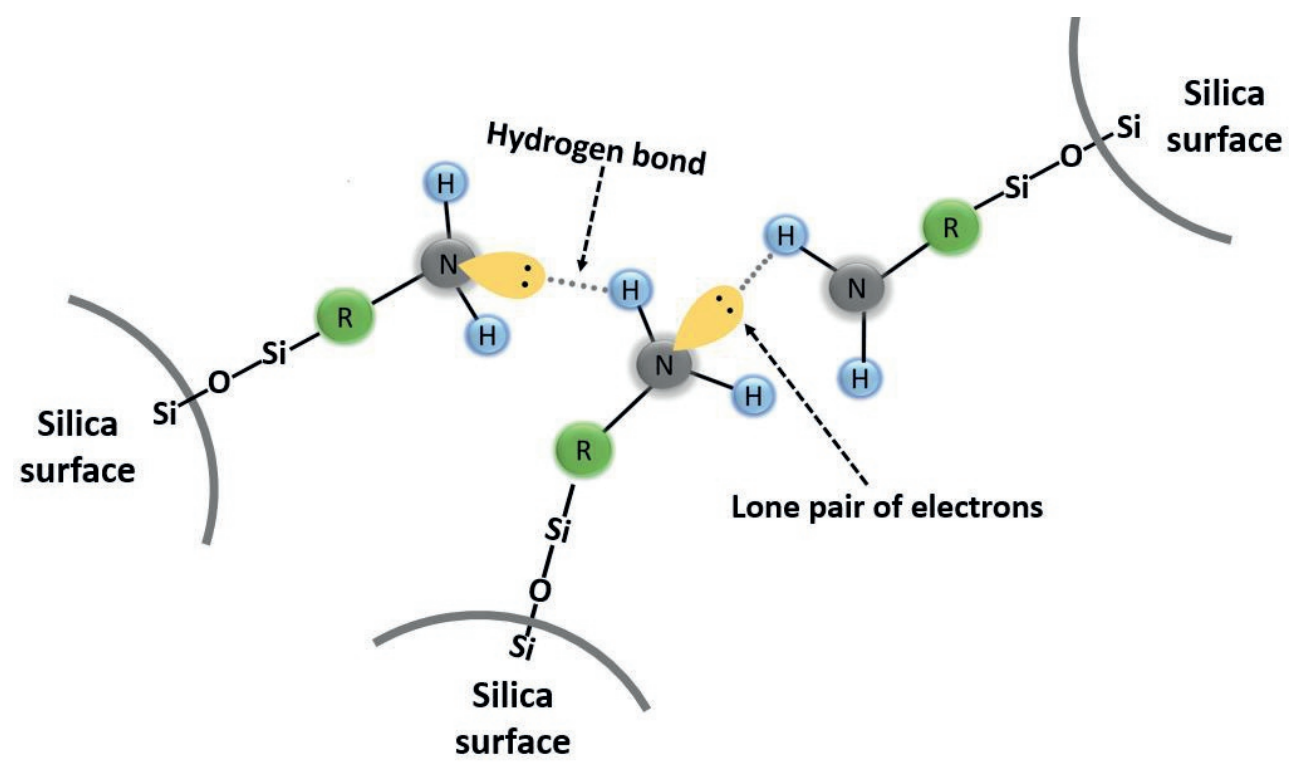

Figure 8.13 Schematic diagram of amine-amine hydrogen bond formation inside a silica cluster.

Another possibility of the higher trap level with $2 \%$ silica addition than $1 \%$ might stem from amineamine hydrogen bonding, as shown in Figure 8.13. Based on our previous study [9], nitrogen in amino groups plays an important role for the deep traps in the polymer matrix. Once there are more silica 
clusters of larger size and higher structure present, more hydrogen bonds are formed between amino groups. As a result, the changed cloud of electrons around nitrogen may induce deeper traps visible in the PP/PP-HI/S-2 peak in comparison to PP/PP-HI/S-1. Furthermore, if charges trapped around one amino group get enough energy to de-trap, it is highly likely that they will be easily trapped by a neighbouring amino group. This leads to a lower mobility of the charges.

\subsubsection{In-depth Discussion of the Dielectric Performance}

Based on the POM results in Figure 8.7 (d, e, f), there is a pronounced gradient of morphology from the surface towards the inner core region in the unfilled blend, while it does not appear as clearly in the nanocomposites. This is due to the nucleating effect of the nanosilica, resulting in smaller spherulites and their uniform distribution. However, although the nanosilica changed the sample morphology, it did not change the melting temperature of the blend (see DSC results in Figures 8.7 (a, b), consequently no effect on the $\alpha 1$ and $\alpha 2$ chain relaxation is observed in the DMA results in Figure 8.8.

In general, morphology as well as presence of additives (e.g., silica) in composites are both able to influence the TSDC charge trapping distribution, hence influencing charge injection, space charge accumulation and conductivity. However, previous work [33] indicates that morphology changes (different spherulite distribution altered by the different compounding techniques) do not significantly change the TSDC charging trap distribution, but they do change the space charge accumulation based on the PEA pattern.

The above observation indicates that the morphology does not influence the values of the TSDC and DMA. But it does influence the charge transportation and space charge accumulation. This is coherent with PEA results.

For an unfilled blend, there is a correlation between the $\alpha 1$ and $\alpha 2$ chain relaxation (DMA results) and the two current peaks from TSDC, as shown in Figure 8.11, which gives an indication that most of the charge is located at the amorphous-crystalline interface. The charges may move through the amorphouscrystalline interface at the surface and diffuse to the inner core. However, due to the broader spherulite size distribution observed for the unfilled blend than for the silica-filled composites, the conduction path along the spherulite boundaries is much shorter for the unfilled blend. Hence, in the latter case it is easier to diffuse the charge into the bulk of the unfilled blend. This can be further proven by the PEA pattern (Figures $8.9(\mathrm{a}, \mathrm{b}, \mathrm{c}))$ : For the unfilled blend, a strong homocharge injection is noticed in the sample, in the vicinity of both electrodes. This distribution can be correlated with the spherulitic crystalline gradient morphology presented in the POM images, and may result in a conductivity gradient in the bulk of the material.

Differently, there is no such correlation in the nanocomposite sample, as the charge trapping near the electrode-polymer interface becomes more pronounced due to increased density of traps brought by the nanosilica. This is also related to the crystalline morphology (spherulite size) observed in POM images. It is assumed that most of the charge is located at the filler-polymer interface. Due to the high specific area of the nanosilica, it brings a large interface into the polymer matrix. With the addition of the surface treatment, the amine group on the A-silica surface creates a deep trap at the filler-polymer interface. Additionally, the conduction path along the spherulite boundaries becomes more tedious for the nanocomposites due to the nucleating effect of silica, large specific area of the silica and the surface 
functionalization. The injected charges can be trapped by the deep traps on the surface region, which can hinder further charge injection to the bulk of the sample. This can be verified by the PEA pattern (Figure $8.9(\mathrm{a}, \mathrm{b}, \mathrm{c})$ ). The deeper traps reduce charge injection near the electrodes, and less charge can diffuse to the bulk of the sample.

Therefore, all the results can be correlated together to prove that the A-silica changes the morphology of the sample and generates the deep traps into the sample, which changes the charge location and alter the space charge accumulation.

Besides, although 2 wt. \% of A-silica shows larger size clusters than 1 wt. \% of A-silica as shown in Figure 8.6, the nanocomposite with 2 wt. \% of A-silica still feature lower space charge accumulation than the one filled with 1 wt. \% of A-silica: This is due to the much deeper traps introduced by 2 wt. \% of A-silica. There are two hypothesis to explain the large cluster effect:

1) The deeper charge traps are formed due to the constrained polymer chain in the structure of $2 \mathrm{wt} \%$ of A-silica, as shown in Figure 8.12. When the charges are trapped at the interface between the highly constrained polymer and silica, which has a very high energy potential, the charge cannot move or detrap easily, contributing to a deeper apparent trap in the sample filled with 2 wt. \% of A-silica.

2) The deeper charge traps are formed due to the hydrogen bond formation between amine groups within the silica cluster, as shown in Figure 8.13. If charges trapped around one amino group get enough energy to de-trap, it is highly likely that they will be easily trapped by a neighboring amino group. This leads to a lower mobility of the charges, leading to a deeper apparent trap in the sample filled with $2 \mathrm{wt}$ \% of A-silica.

Consequently, a charge trapped in the deeper trap is immobile, which might form another barrier based on the repelling electrostatic force suppressing further charge injection, which finally results in low trap density, low space charge accumulation and also low current density.

\subsection{Conclusions}

In this paper, the development of an HVDC insulation material based on PP/PP-HI/A-silica nanocomposites was presented. A-silica was obtained by modifying fumed silica with 3aminopropyltriethoxysilane (APTES) through a solvent-free silane-silica modification method. The APTES-silica is found to act as nucleating agent, improving the homogeneity crystal distribution in the polymer blend. A-silica addition decreases crystallinity, resulting in a decreased storage modulus. It also introduces deeper traps into the polymeric matrix thanks to the amine groups grafted on its surface. The A-silica addition also changed the charge trapping locations, where for the unfilled blend the charges are mostly located in the crystalline interface and the amorphous-crystalline interface, while for the nanocomposites, charges are mostly located in the A-silica-polymer interface. Although A-silica has a polar nature and is thus not compatible with the polymeric matrix, a higher concentration ( $2 \mathrm{wt.} \%$ ) of A-silica still benefits the performance of the material by reducing its space charge accumulation and current density. This indicates that the cluster formation with a proper surface functionalization may be 
beneficial for the dielectric properties in terms of reducing space charge accumulation and apparent conductivity.

\section{Acknowledgement}

This project has received funding from the European Union's Horizon 2020 research and innovation program under grant agreement No 720858.

The authors also would like to thank Evonik Industries for providing a free silica sample. 


\section{Reference}

[1] Tanaka, T. and Imai, T., 2013. Advances in nanodielectric materials over the past 50 years. IEEE Electrical Insulation Magazine, 29(1), pp.10-23. doi: 10.1109/MEI.2013.6410535.

[2] Thomas, J., Joseph, B., Jose, J.P., Maria, H.J., Main, P., Ali Rahman, A., Francis, B., Ahmad, Z. and Thomas, S., 2019. Recent advances in cross-linked polyethylene-based nanocomposites for high voltage engineering applications: A critical review. Industrial \& Engineering Chemistry Research, 58(46), pp.20863-20879.

[3] Li, C., Hu, J., Lin, C. and He, J., 2016. The control mechanism of surface traps on surface charge behavior in alumina-filled epoxy composites. Journal of Physics D: Applied Physics, 49(44), p.445304.

[4]Roy, M., Nelson, J.K., MacCrone, R.K. and Schadler, L.S., 2007. Candidate mechanisms controlling the electrical characteristics of silica/XLPE nanodielectrics. Journal of Materials Science, 42(11), pp.3789-3799.

[5] Zhou, Y., He, J., Hu, J. and Dang, B., 2016. Surface-modified MgO nanoparticle enhances the mechanical and direct-current electrical characteristics of polypropylene/polyolefin elastomer nanodielectrics. Journal of Applied Polymer Science, 133(1).

[6] Han, B., Chang, J., Song, W., Sun, Z., Yin, C., Lv, P. and Wang, X., 2019. Study on Micro Interfacial Charge Motion of Polyethylene Nanocomposite Based on Electrostatic Force Microscope. Polymers, 11(12), p.2035.

[7] Zha, J.W., Wang, Y., Wang, S.J., Zheng, M.S., Bian, X. and Dang, Z.M., 2019. Space charge suppression in environment-friendly PP nanocomposites by employing freeze-dried $\mathrm{MgO}$ with foam nanostructure for high-voltage power cable insulation. Applied Physics Letters, 114(25), p.252902.

[8] Alapati, S., Meledath, J.T. and Karmarkar, A., 2014. Effect of morphology on electrical treeing in low density polyethylene nanocomposites. IET Science, Measurement \& Technology, 8(2), pp.60-68.

[9] He, X., Rytöluoto, I., Anyszka, R., Mahtabani, A., Saarimäki, E., Lahti, K., Paajanen, M., Dierkes, W. and Blume, A., 2020. Silica surface-modification for tailoring the charge trapping properties of PP/POE based dielectric nanocomposites for HVDC cable application. IEEE Access. 8, pp. 8771987734. doi: 10.1109/ACCESS.2020.2992859.

[10] Lewis, T.J., 2014. Charge transport in polyethylene nano dielectrics. IEEE transactions on dielectrics and electrical insulation, 21(2), pp.497-502. doi: 10.1109/TDEI.2013.004173.

[11] Zhou, Y., Dang, B., Wang, H., Liu, J., Li, Q., Hu, J. and He, J., 2018. Polypropylene-based ternary nanocomposites for recyclable high-voltage direct-current cable insulation. Composites Science and Technology, 165, pp.168-174.

[12] G. C. Montanari, P. Seri, M. Ritamäki, K. Lahti, I. Rytöluoto and M. Paajanen, 2018"Performance of nanoparticles in the electrical behavior of DC capacitor films," 2018 12th International Conference on the Properties and Applications of Dielectric Materials (ICPADM), Xi'an, pp. 41-44, doi: 10.1109/ICPADM.2018.8401024. 
[13] Rao, B.N. and Nandakumar, V.S., 2019. A study on the dielectric response of polyethylene-metal oxide nanocomposites for electrical insulation in HVDC cables. Materials Today: Proceedings, 18, pp.994-1005

[14] Mahtabani, A., Rytöluoto, I., Anyszka, R., He, X., Saarimäki, E., Lahti, K., Paajanen, M., Dierkes, W. and Blume, A., 2020. On the Silica Surface Modification and Its Effect on Charge Trapping and Transport in PP-Based Dielectric Nanocomposites. ACS Applied Polymer Materials, 2(8), pp.31483160 .

[15] B. McCool, L. Murphy, and C.P. Tripp, 2006. A simple FTIR technique for estimating the surface area of silica powders and films. Journal of colloid and interface science, 295(1), pp, 294-298,

[16] Zhuravlev, L.T., 2000. The surface chemistry of amorphous silica. Zhuravlev model. Colloids and Surfaces A: Physicochemical and Engineering Aspects, 173(1-3), pp.1-38.

[17] Wilfong, W.C., Srikanth, C.S. and Chuang, S.S., 2014. In situ ATR and DRIFTS studies of the nature of adsorbed $\mathrm{CO} 2$ on tetraethylenepentamine films. ACS applied materials \& interfaces, 6(16), pp.13617-13626.

[18] Rezaei, S., Manoucheri, I., Moradian, R., \& Pourabbas, B. 2014. One-step chemical vapor deposition and modification of silica nanoparticles at the lowest possible temperature and superhydrophobic surface fabrication. Chemical Engineering Journal, 252, pp. 11-16.

[19] Gao, Y., Li, J., Yuan, Y., Huang, S. and Du, B., 2018. Trap distribution and dielectric breakdown of isotactic polypropylene/propylene based elastomer with improved flexibility for DC cable insulation. IEEE Access, 6, pp.58645-58661.

[20] Seri, Paolo. "Electrical properties of nanostructured polypropylene: a matter of morphology?." JICABLE (2019). doi 10.6092/unibo/amsacta/6304

[21] Fakirov, S., 2020. Polymer nanocomposites: Why their mechanical performance does not justify the expectation and a possible solution to the problem?. Express Polymer Letters, 14(5), pp.436-466.

[22] Davachi, S.M., Heidari, B.S., Sahraeian, R. and Abbaspourrad, A., 2019. The effect of nanoperlite and its silane treatment on the crystallinity, rheological, optical, and surface properties of polypropylene/nanoperlite nanocomposite films. Composites Part B: Engineering, 175, p.107088.

[23] Yang, J., Gao, M., Zhao, H., Liu, S., Hu, M. and Xie, S., 2019. Space charge characteristics of polypropylene modified by rare earth nucleating agent for $\beta$ crystallization. Materials, 12(1), p.42.

[24] Pedrazzoli, D., Pegoretti, A. and Kalaitzidou, K., 2016. Interfacial interactions in silica-reinforced polypropylene nanocomposites and their impact on the mechanical properties. Polymer Composites, 37(7), pp.2018-2026.

[25] Liu, J.H., Cai, J.H., Tang, X.H., Weng, Y.X. and Wang, M., 2020. Achieving highly crystalline rate and crystallinity in Poly (1-lactide) via in-situ melting reaction with diisocyanate and benzohydrazine to form nucleating agents. Polymer Testing, 81, p.106216.

[26] Alapati, S., Meledath, J.T. and Karmarkar, A., 2014. Effect of morphology on electrical treeing in low density polyethylene nanocomposites. IET Science, Measurement \& Technology, 8(2), pp.60-68. 
[27] Sumita, M., Tsukihi, H., Miyasaka, K., \& Ishikawa, K. (1984). Dynamic mechanical properties of polypropylene composites filled with ultrafine particles. Journal of Applied Polymer Science, 29(5), 1523-1530. doi:10.1002/app.1984.070290506

[28] Laredo, E., Suarez, N., Bello, A., de Gáscue, B.R., Gomez, M.A. and Fatou, J.M.G., 1999. $\alpha, \beta$ and $\gamma$ relaxations of functionalized HD polyethylene: a TSDC and a mechanical study. Polymer, 40(23), pp.6405-6416.

[29] Pallon, L.K.H., Hoang, A.T., Pourrahimi, A.M., Hedenqvist, M.S., Nilsson, F., Gubanski, S., Gedde, U.W. and Olsson, R.T., 2016. The impact of $\mathrm{MgO}$ nanoparticle interface in ultra-insulating polyethylene nanocomposites for high voltage DC cables. Journal of Materials Chemistry A, 4(22), pp.8590-8601.. doi:10.1039/c6ta02041k

[30] Mizutani, T., Suzuoki, Y. and Ieda, M., 1977. Thermally stimulated currents in polyethylene and ethylene-vinyl-acetate copolymers. Journal of Applied Physics, 48(6), pp.2408-2413.

[31] Dang, B., Li, Q., Zhou, Y., Hu, J. and He, J., 2017. Suppression of elevated temperature space charge accumulation in polypropylene/elastomer blends by deep traps induced by surface-modified $\mathrm{ZnO}$ nanoparticles. Composites Science and Technology, 153, pp.103-110.

[32] Gao, M., Yang, J., Zhao, H., He, H., Hu, M. and Xie, S., 2019. Preparation methods of polypropylene/nano-silica/styrene-ethylene-butylene-styrene composite and its effect on electrical properties. Polymers, 11(5), p.797.

[33] Rytöluoto, I., Saarimäki, E., Pelto, J., Paajanen, M., He, X., Anyszka, R., Mahtabani, A., Dierkes, W., Seri, P., Naderiallaf, H. and Lahti, K., Feasibility of Mini-Scale Injection Molding for ResourceEfficient Screening of PP-Based Cable Insulation Nanocomposites. 
$222 \mid \mathrm{P}$ a g e 


\section{Chapter 9 \\ Joining good dispersion with tailored charge trapping in nanodielectrics by hybrid functionalization on silica}

This chapter has been submitted in E-Polymers.

He, X., Rytöluoto, I., Anyszka, R., Mahtabani, A., Niittymäki, M., Saarimäki, E., Mazel, C., Perego, G. and Lahti, K., Dierkes, W, Blume, A. 2021. Joining good dispersion with tailored charge trapping in nanodielectrics by hybrid functionalization on silica. 


\title{
Joining good dispersion with tailored charge trapping in nanodielectrics by hybrid functionalization on silica
}

\author{
Xiaozhen $\mathrm{He}^{1}$, Ilkka Rytöluoto ${ }^{2}$, Rafal Anyszka ${ }^{1}$, Amirhossein Mahtabani ${ }^{1}$, Minna Niittymäki ${ }^{3}$, \\ Eetta Saarimäki ${ }^{2}$, Christelle Mazel ${ }^{4}$, Gabriele Perego ${ }^{4}$, Kari Lahti ${ }^{3}$, Mika Paajanen ${ }^{2}$, Wilma \\ Dierkes $^{1 *}$, Anke Blume ${ }^{1}$ \\ 1 University of Twente, Faculty of Engineering Technology, Department of Mechanics of Solids, \\ Surfaces \& Systems (MS3), Chair of Elastomer Technology and Engineering, 7522 NB, Enschede, \\ The Netherlands
}

2 VTT Technical Research Centre of Finland Ltd, FI-33720, Tampere, Finland

3 Tampere University, High Voltage Engineering, 33100, Tampere, Finland

4 Nexans Research Center, 29 rue Pré Gaudry, 69007 Lyon, France

* Correspondence to: Wilma Dierkes (E-mail: w.k.dierkes@utwente.nl)

\begin{abstract}
The fumed silica filled polypropylene (PP) based nanodielectrics were studied in this paper. In order not only to improve the dispersion of the silica, but also to introduce the deep charge traps into the polymeric matrix, five types of modified silicas were manufactured with different surface modification agents. The modified silicas' surface comprise of an inner layer and surface layer. The inner layer contains a polar urethane group for tailoring the charge trap properties of the PP/PP-HI (propylene-ethylene copolymer) nanocomposites, while the surface layer consists of a hydrocarbon (ethyl-, tert-butyl-, cyclopentyl-, phenyl- or naphthalenyl-moiety) in order to gain the good dispersion of the silica in the unpolar polymer blend. Scanning electron microscopy pictures proved that these tailored silicas show a much better dispersion than the unmodified one. Thermally stimulated depolarization current measurements revealed the ability of the silica to introduce deep charge traps with low trap density. The trap depth distribution also depends on the unpolar surface layer consisting of the hydrocarbons. Among these five differently modified silicas, introduction of the one with a surface layer consisting of tert-butyl moieties resulted in the lowest charge injection and the lowest charge current in the nanocomposite, proving good dielectric performance. Additionally, this silica exhibits a good dispersion in the polymeric matrix, indicating a promising performance for nanodielectric application.
\end{abstract}

Keywords: fumed silica, surface functionalization, charge trap distribution, nanodielectrics 


\subsection{Introduction}

The term "nanodielectrics" describes a nanocomposite designed for maximized dielectric performance. The definition of a "nanocomposite" describes a composite filled with nanoparticles, of which at least one dimension is lower than $100 \mathrm{~nm}$. The nanodielectrics designed for application in high voltage fields require high electrical insulation properties to effectively fulfill this task. Early studies with promising results were published in 2002 by J. K. Nelson and his co-authors [1]. They reported that the nanoparticles can delay the charge transportation and reduce the space charge accumulation, which indicates the potential of the nanoparticles for dielectric applications. Since then, nanodielectrics have attracted an ever-increasing attention [2,3]. Subsequently, a lot of effort was spent on understanding the effect of these fillers in nanodielectrics $[4,5]$. The large interfacial area between the nanofiller surface and polymer matrix is widely accepted to be one of the most important factors that greatly impact the performance of nanodielectrics. The nanofiller not only alters the polymer chain dynamics and the chain mobility at the interface region [6], but also brings various chemical functional groups into the interfacial region, tailoring the charge trapping properties of the nanocomposite [7].

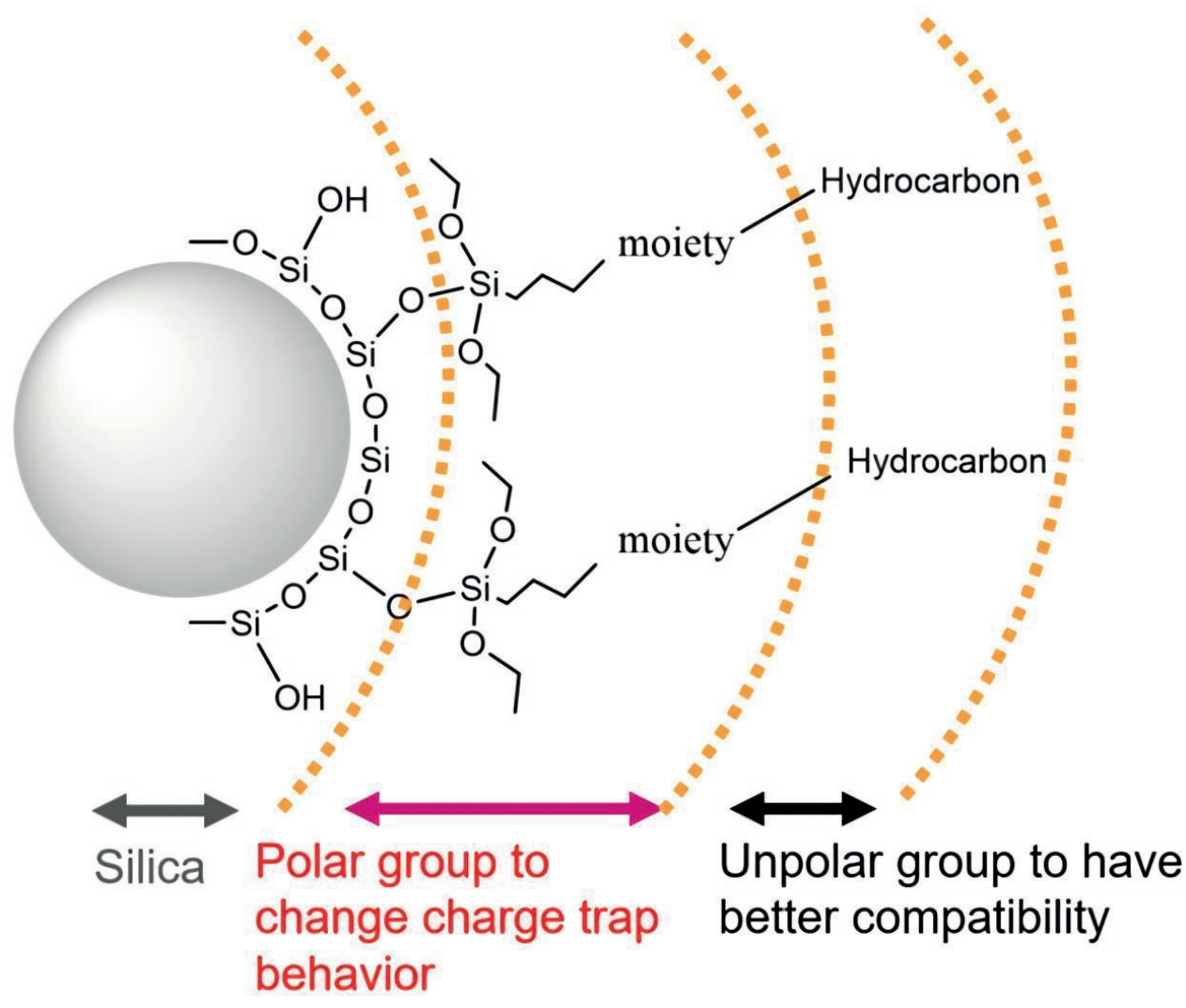

Figure 9.1 Scheme of the designed surface-functionalized silica. 
Good adhesion between the well dispersed nanofiller and the polymer matrix is considered to be the most important feature for HVDC cable insulation materials [8]. During the installation of a high voltage cable, the cable is under mechanical stress and strain. Poor nanofiller-polymer adhesion or presence of nanofiller aggregates might cause formation of cavities under strain, which can lead to microcrack formation and subsequently mechanical failure. This might also affect the dielectric performance of the nanocomposite. Besides, introducing a polar functional group on the nanofiller surface can provide a positive effect on the dielectric performance due to the introduced deep charge traps [9].

In general, the commonly most used polymers for HVDC insulation are crosslinked polyethylene (XLPE) and polypropylene (PP). Due to the crosslinked nature of the XLPE, it is not recyclable. Driven by the focus on sustainability, PP is a potential candidate for replacing XLPE in the future due to its recyclable character. However, XLPE and PP are both unpolar polymers. Considering the need of a good dispersion of a nanofiller in an unpolar polymeric matrix, an unpolar surface functionalization is required $[8,10]$. The challenge is to get the best of both worlds: improved the dispersion of the nanofiller by an unpolar surface functionalization as well as introduction of deep traps and tailoring of the charge trapping properties by a polar modification.

In this study, an approach is used that may provide the best balance of these improvements: designing a surface-functionalized silica as shown in Figure 9.1. The inner layer consists of polar groups (containing nitrogen) in order to tailor the charge trap distribution of the nanocomposite, while the surface layer is comprised of unpolar groups (hydrocarbon moieties) in order to improve the dispersion of the silica in the polymeric matrix. The polymer used in this study is a polypropylene/propylene-ethylene copolymer (PP/PP-HI) blend.<smiles>CCO[Si](CCCN=O)(OCC)OCC</smiles>

3-(Triethoxysilyl)propyl isocyanate

TEPI

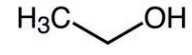

Ethanol

E<smiles>CC(C)(C)O</smiles>

Tert butanol

TB

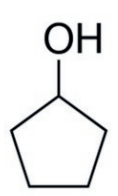

Cyclopentanol

CP

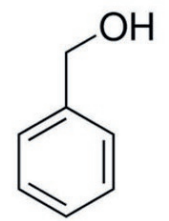

Phenyl methanol PM<smiles>OCc1cccc2ccccc12</smiles>

1-Naphthalene methanol NM1

Figure 9.2 Chemical structures of the selected silane and alcohols. 


\subsection{Experimental}

\subsubsection{Preparation of the Nanocomposites}

\subsubsection{Silane Synthesis}

In order to realize the surface structure sketched in Figure 9.1, an isocyanate silane and different alcohols were selected for the synthesis of the new silane, which will react with silica, as shown in Figure 9.2. The reason to choose an isocyanate silane is that it can introduce deep traps into the polymeric matrix as was shown in the earlier study [11]. The isocyanate group is an electrophile, thus an electron acceptor, and is very reactive towards alcohols, which are nucleophiles and donate an electron pair. As a result, they form a urethane bridge to link both molecules as shown in Figure 9.3 [12].

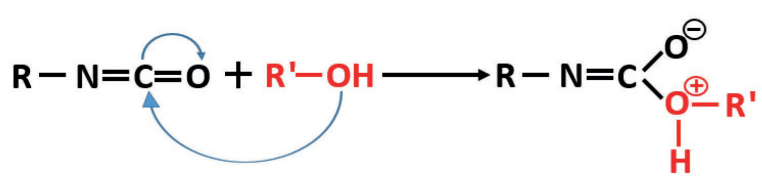

Intermediate

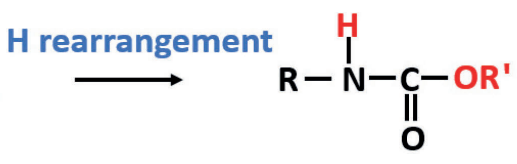

Urethane

Figure 9.3 Reaction of an isocyanate with alcohol.

\subsubsection{Reaction of 3-(Triethoxysilyl)propyl Isocyanate Silane (TEPI) with Alcohols}

TEPI and the different alcohols (equimolar ratios) were mixed in a glass flask. The flask was carefully sealed in order to avoid humidity. It was put into a laboratory oven at $70{ }^{\circ} \mathrm{C}$ for a specified number of days as given in Table 9.1, until all isocyanate groups had fully reacted with the alcohol. This was detected by the disappearance of the isocyanate group band via FTIR. The reaction schemes of all alcohols are shown in Figure 9.4. All alcohols and TEPI were bought from Sigma Aldrich, Netherlands. The purities were $99.8 \%$ (ethanol), $99.5 \%$ (tert. butanol), $99 \%$ (cyclopentanol), $99.8 \%$ (phenyl methanol), $98 \%$ of (1-naphthalene methanol) $95 \%$ of TEPI.

Table 9.1 Reaction parameters.

\begin{tabular}{|c|c|c|c|c|c|}
\hline \multicolumn{5}{|c|}{$20 \mathrm{~g}$ of TEPI } \\
\hline Alcohol & E & TB & CP & PM & NM1 \\
\hline Weight $(\mathrm{g})$ & 3.7 & 6.0 & 5.6 & 8.7 & 12.8 \\
\hline $\begin{array}{c}\text { Reaction time at } 70^{\circ} \mathrm{C} \\
\text { (days) }\end{array}$ & 7 & 25 & 8 & 10 & 35 \\
\hline
\end{tabular}




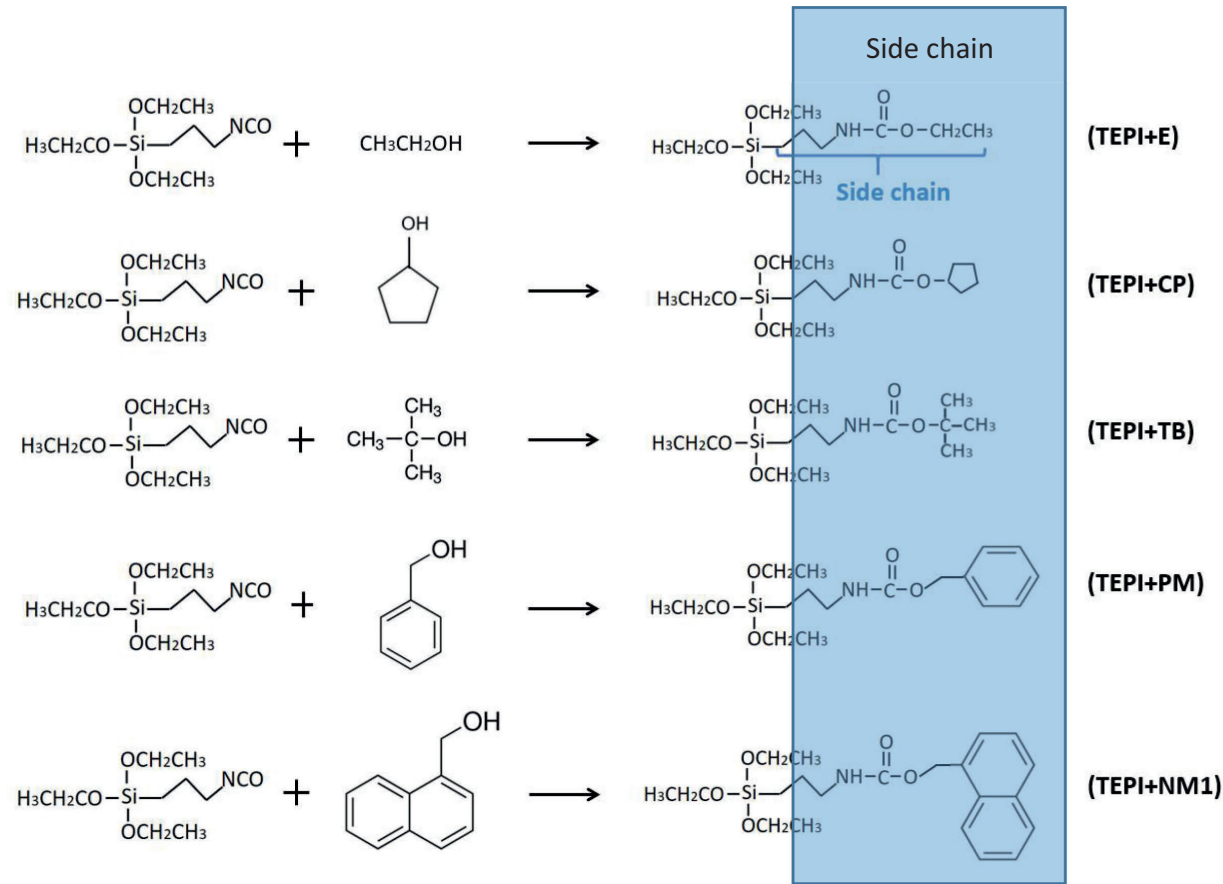

Figure 9.4 Scheme of reactions of TEPI with different alcohols.

\subsubsection{Silica Surface Modification}

As a result of the reaction of TEPI with the alcohols, five new types of silanes were obtained. They are denoted as TEPI+E, TEPI+TB, TEPI+CP, TEPI + PM, TEPI + NM1, see Figure. 9.4. The procedure of solvent-free modification of the silica was as follows: $20 \mathrm{~g}$ of fumed silica and one of the silanes were mixed and stirred in a glass flask with deionized water $(0.6 \mathrm{~g})$ and trifluoroacetic acid $(0.4 \mathrm{~g}$, from Abcr $\mathrm{GmbH})$ as a catalyst for 24 hours at room temperature. Then the flask was placed in a vacuum oven for 24 hours at $80^{\circ} \mathrm{C}$ in order to remove all unreacted components. The ingredients are shown in Table 9.2.

Table 9.2 Ingredients for the modification process.

\begin{tabular}{|c|c|c|c|c|}
\hline & Silane/g & Silica/g & Water/g & Acid/g \\
\cline { 1 - 2 } TEPI+E & 4.78 & & & \\
\cline { 1 - 2 } TEPI+TB & 5.24 & \multirow{2}{*}{20} & \multirow{2}{*}{0.6} & 0.4 \\
\cline { 1 - 2 } TEPI+CP & 5.24 & & & \\
\hline TEPI+PM & 5.79 & & & \\
\hline TEPI+NM1 & 6.61 & & & \\
\hline
\end{tabular}




\subsubsection{Nanocomposites Manufacturing}

The nanocomposites were produced by mixing PP and PP-HI in a ratio of 55:45, with 2 wt.\% of a nanosilica and 0.3 wt.\% of antioxidant in the Haake MiniLab Rheomix CTW5 twin screw extruder. Two types of antioxidants were used: $0.15 \mathrm{wt} . \%$ of a phenolic one for preventing thermal oxidation of PP at higher temperatures, and $0.15 \mathrm{wt} \%$ of a phosphite processing stabilizer. The extruder setting temperature was $230{ }^{\circ} \mathrm{C}$, while the screw speed was $100 \mathrm{rpm}$ and the mixing time was 4 mins. After the mixing, the compounds were immediately transferred to the Haake MiniJet Pro Piston Injection Molding System (pressure 960 bar, mold temperature $60{ }^{\circ} \mathrm{C}$, injection + hold time $40 \mathrm{~s}$ ) to prepare the final thin film of a size of $26 \times 26 \mathrm{~mm}$ and a thickness of $0.5 \mathrm{~mm}$.

\subsubsection{Characterization Techniques}

\subsubsection{Fourier-Transform Infrared Spectroscopy of the Silanes}

The reaction of TEPI with the alcohols was firstly analyzed by Fourier-Transform Infrared Spectroscopy (FTIR, Perkin Elmer Spectrum 100) equipped with an Attenuated Total Reflectance unit in the range of 650 to $4000 \mathrm{~cm}^{-1}$ in order to detect if the reaction is completed, indicated by the disappearance of the isocyanate band. This took in general a few days.

\subsubsection{Diffuse Reflectance Infrared Fourier-Transform Spectroscopy of the Silicas}

Diffuse reflectance infrared Fourier-Transform Spectroscopy (DRIFTS) was performed on the unmodified reference silica and the modified silicas in order to prove the successful silica modification with the silanes. Silica was mixed with $\mathrm{KBr}$ for scanning. The spectra were recorded using a Perkin Elmer Spectrum 100 FT-IR Spectrometer in Diffuse Reflectance Mode (DRIFT). The spectra were recorded at a resolution of $4.0 \mathrm{~cm}^{-1}$ and averaged over 128 scans from $4000-400 \mathrm{~cm}^{-1}$.

\subsubsection{Thermogravimetric Analysis (TGA) of the Silicas}

TGA was performed using a TA 550 thermogravimetric analyzer (TA Instruments). The measurements were performed in an atmosphere of synthetic air with a heating rate of $10{ }^{\circ} \mathrm{C} / \mathrm{min}$. The temperature range was ramped from ambient temperature up to $850^{\circ} \mathrm{C}$.

\subsubsection{Scanning Electron Microscopy (SEM) of the Nanocomposites}

The nanocomposite samples were firstly broken in liquid nitrogen to obtain a cross section. This cross section without any further treatment or coating (in order to preserve the original morphology of the samples) was investigated by SEM (Zeiss MERLIN HR-SEM). In order to get insight into the particle size distribution, the SEM micrographs were analyzed with the software Image J (version 1.53a, supplied by Wayne Rasband, USA). The measurement of the particle size was done manually by drawing a line with the scale. Three images of each samples were measured in order to get the particle size distribution. 


\subsubsection{Thermally Stimulated Depolarization Current of the Nanocomposites}

Thermally Stimulated Depolarization Current (TSDC) was applied on the nanocomposites to study their charge trapping properties. The samples were firstly gold coated by circular electrodes to a thickness of $100 \mathrm{~nm}$ and a diameter of $16 \mathrm{~mm}$ on both sides. The TSDC test was done by the following steps:

1 - The sample was heated up to $70{ }^{\circ} \mathrm{C}$ and then kept at this temperature for $5 \mathrm{~min}$.

2 - A DC electric field $(3 \mathrm{kV} / \mathrm{mm})$ was applied for 20 mins.

3 - The sample was quickly cooled down to $-50{ }^{\circ} \mathrm{C}$ under the DC electric field and stabilized for 5 mins.

4 - The DC electric field was removed and the sample was short-circuited for $3 \mathrm{~min}$ at $-50{ }^{\circ} \mathrm{C}$.

5 - The sample was heated up to $130^{\circ} \mathrm{C}$ in a linear mode $\left(3^{\circ} \mathrm{C} / \mathrm{min}\right)$. At the same time, the setup recorded the depolarization current.

\subsection{Results and discussion}

\subsubsection{Fourier-Transform Infrared Spectroscopy (FTIR) of the Silanes}

In order to track the progress of the reaction of TEPI and the alcohols, FTIR was performed. Based on the mechanism of the reaction as shown in Figure 9.3, the isocyanate group will vanish during the reaction.

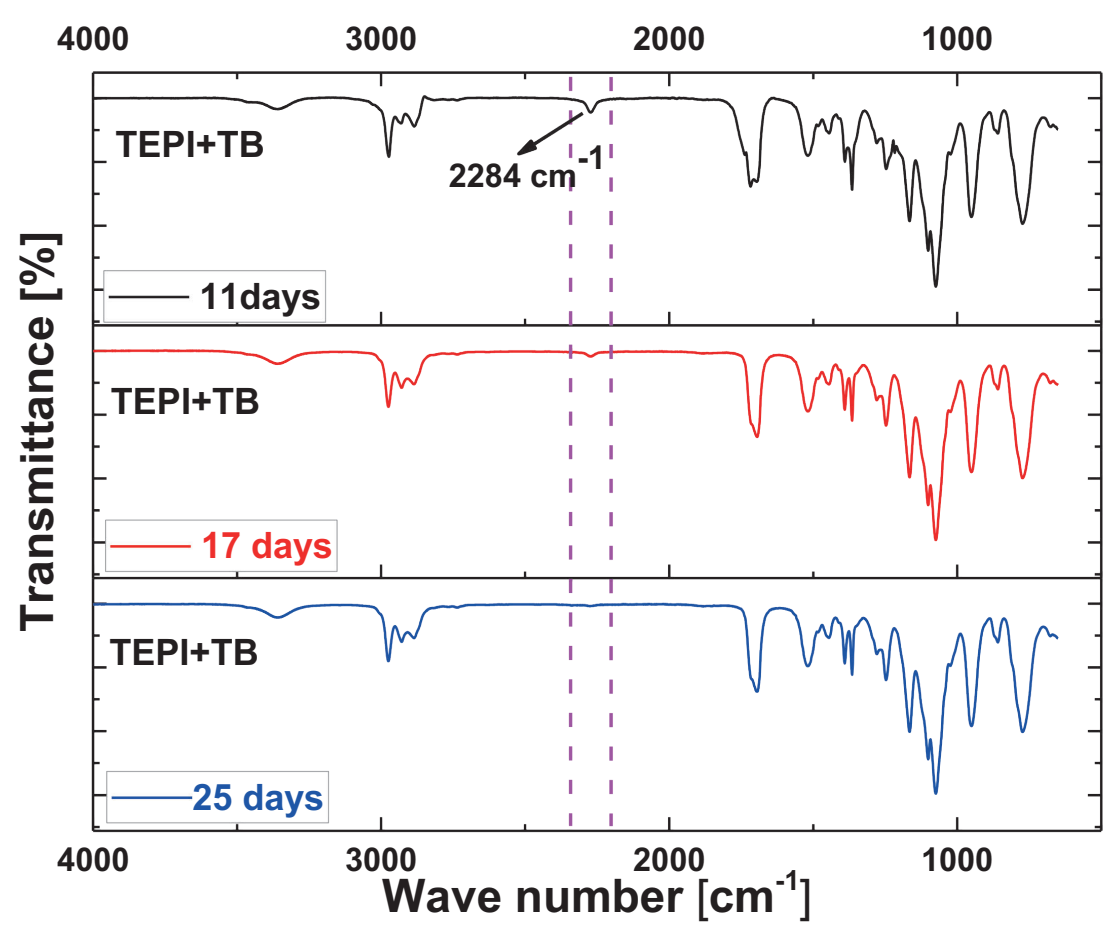

Figure 9.5 FTIR spectra of the mixture of TEPI and TB after 11, 17 and 25 days. 
Therefore, the isocyanate band is the indicator to determine whether the reaction is completed or not. An example is presented in Figure 9.5: the spectra show the change of the isocyanate band at $2284 \mathrm{~cm}^{-}$ ${ }^{1}$ [13] with the time for the reaction of TEPI and TB. It shows that after 11 days, there is still a signal at $2284 \mathrm{~cm}^{-1}$. With increasing time it gradually disappears until after 25 days the band is vanished, indicating that the reaction is completed. All final spectra are shown in Figure 9.6. The isocyanate band vanished in all cases as an evidence that the new silane was synthesized.

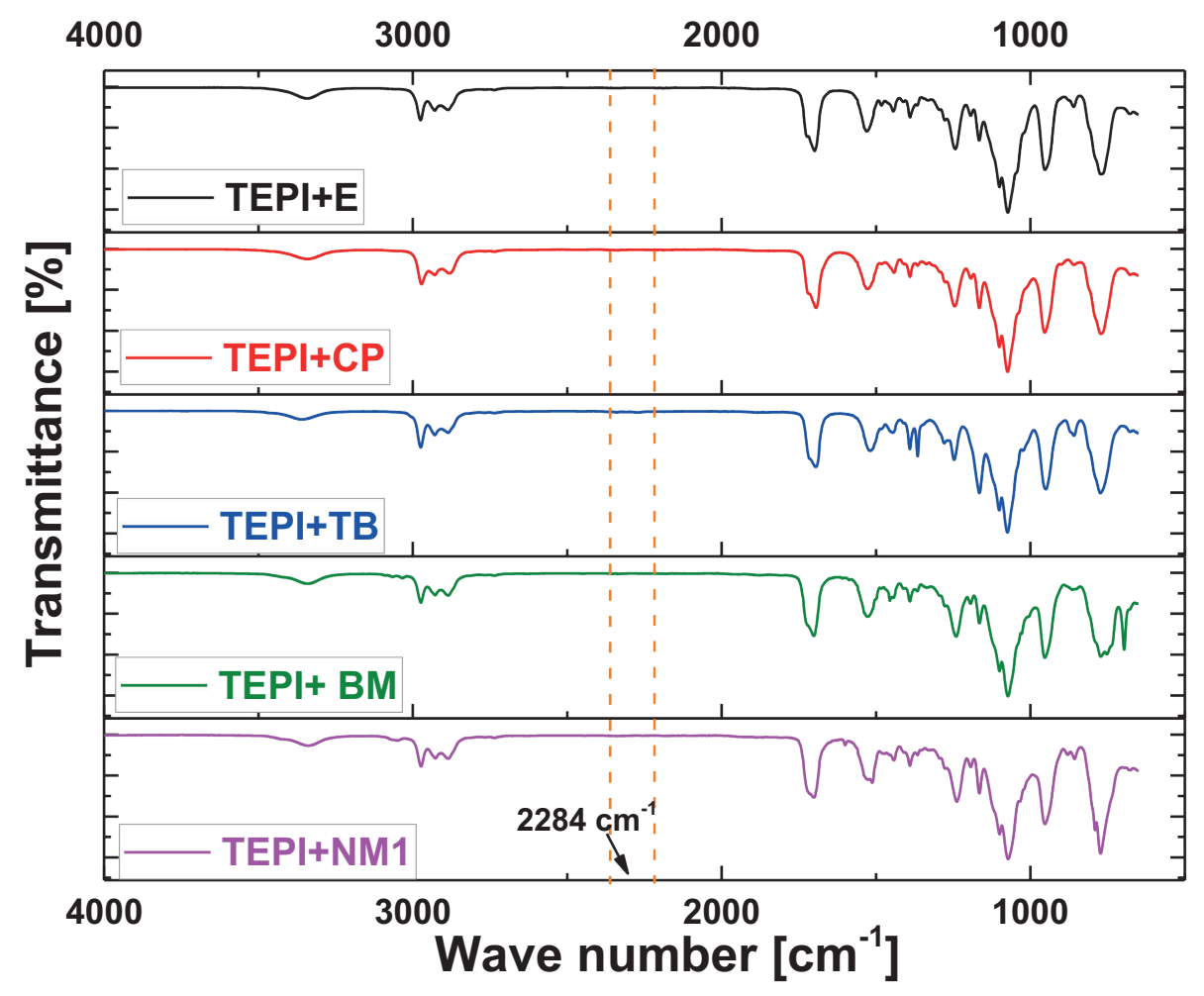

Figure 9.6 FTIR spectra of all synthesized silanes.

\subsubsection{Diffuse reflectance infrared Fourier-Transform Spectroscopy (DRIFTS) of the Silicas}

In order to prove that the silica was modified by the synthetized silanes, DRIFTS was performed, and the results are shown in Figure 9.7. All spectra show the broad absorbance band at a wavenumber of $1112 \mathrm{~cm}^{-1}$, resulting from the silica bulk structure of Si-O-Si stretching [14]. Regarding the reference silica: the sharp band at wavenumber $3700 \mathrm{~cm}^{-1}$ originates from the isolated silanol groups (-Si-OH), which is the indicator to determine the silica modification. This is because the isolated silanol groups on silica surface are the most reactive group and will react with the alkoxy groups from the silanes. This band vanished in all spectra of the modified silicas, proving that the silicas were successfully modified. In addition, the bands of the urethane group showed up after the silica modification. The bands at 1700 $\mathrm{cm}^{-1}$ and $1533 \mathrm{~cm}^{-1}$ are stemming from the stretching of $\mathrm{C}=\mathrm{O}$ and bending of $\mathrm{N}-\mathrm{H}$ [15] bonds in the 
urethane group, respectively. A band resulting from the stretching of $-\mathrm{NH}\left(3343 \mathrm{~cm}^{-1}\right)$ in the urethane group also appeared in the modified silicas. Moreover, the $-\mathrm{CH}$ stretching bands from hydrocarbon chains appear in the range from $2970 \mathrm{~cm}^{-1}$ to $2880 \mathrm{~cm}^{-1}$. The absorbance bands of both, urethane and alkyl groups, further prove that the silica is successfully modified by the synthetized silanes. Additionally, one can also observe that the bands of the urethane and alkyl moieties are weak in the spectrum of TEPI+NM1, which indicates a lower modification level than for the other modified silicas.

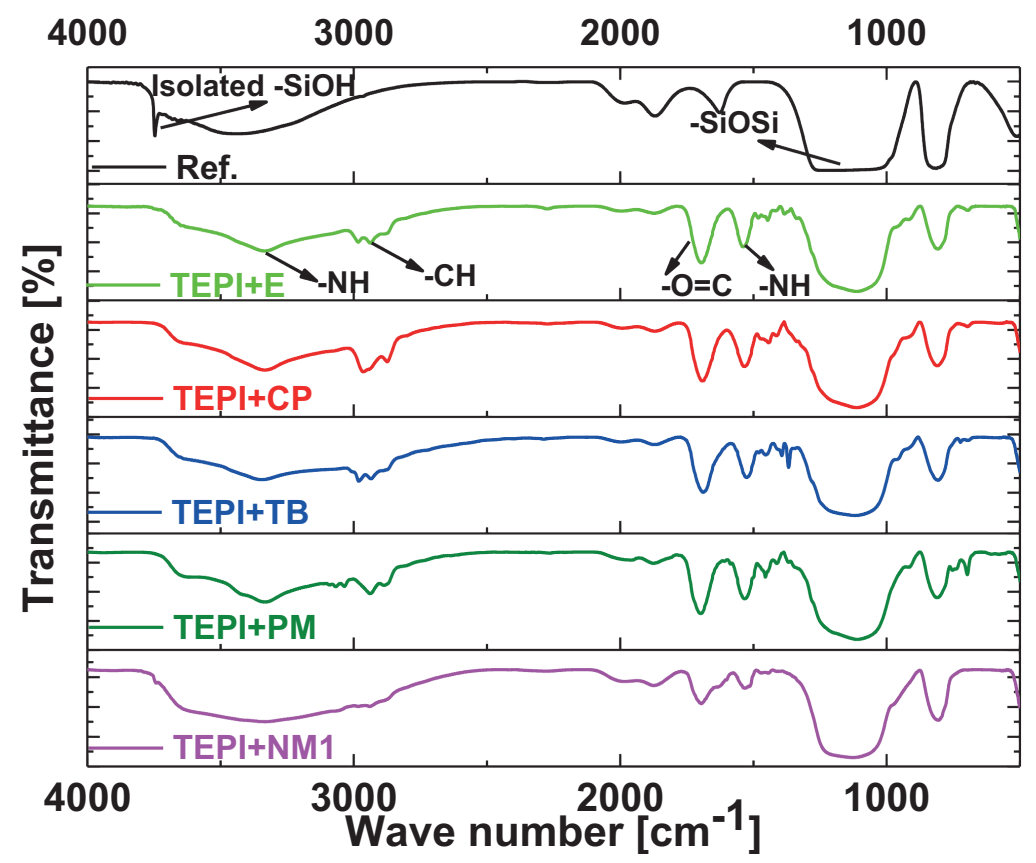

Figure 9.7 DRIFTS spectra of the reference (Ref.) and modified silicas.

\subsubsection{Thermogravimetrical Analysis (TGA) of Silicas}

To further study the modification of the silica surface, TGA was performed in order to quantitatively investigate the deposition level. The TGA results are presented in Figure 9.8. It is clearly shown that the surface modified silicas exhibit higher weight losses than the reference silica. The weight loss of the modified silicas is ascribed to the removal of the organic molecules from the silica surface, indicating the successful modification of the silica with the synthetized silanes.

In order to further investigate the degree of modification of the five designed silicas, the molar amount of the grafted molecules was calculated based on Equation (9.1), similar to [11].

$$
\text { Grafted amount }(\mathrm{mmol} / \mathrm{g})=\frac{10^{3} \mathrm{~W}}{\mathrm{M}(100-\mathrm{W})}
$$


$\mathrm{W}$ is the weight loss (wt.\%) of the modified silica measured by TGA. M is the molecular weight of the side chain of the synthetized silanes $(130 \mathrm{~g} / \mathrm{mol}$ - side chain of TEPI+E; $154 \mathrm{~g} / \mathrm{mol}$ - side chain of TEPI+CP; $158 \mathrm{~g} / \mathrm{mol}$ - side chain of TEPI+TB; $192 \mathrm{~g} / \mathrm{mol}$ - side chain of TEPI+PM; $232 \mathrm{~g} / \mathrm{mol}$ - side chain of TEPI+NM1, the side chains of the new silanes are indicated in Figure 9.4).
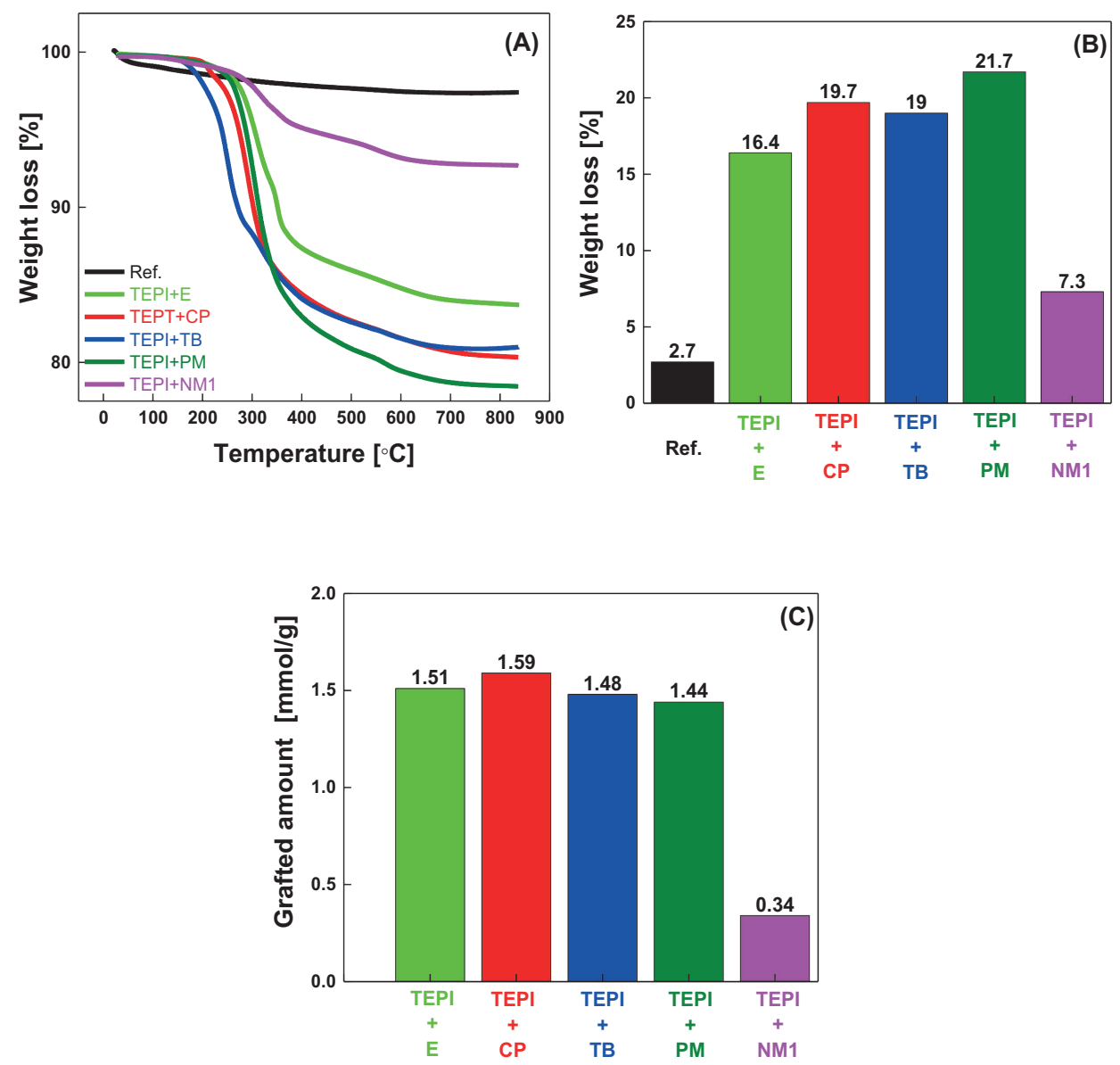

Figure 9.8 TGA curves of the reference silica and modified silicas (A), weight loss up to a temperature of $850^{\circ} \mathrm{C}(\mathrm{B})$, molar grafting effectiveness of the silanes $(\mathrm{C})$.

Almost no weight loss below a temperature of $200{ }^{\circ} \mathrm{C}$ was measured for any of the five modified silicas, which means that there is very less water or unreacted ethoxy groups present in the modified silicas. Therefore, we assumed that all weight loss of the modified silicas stems from the removal of the organic side chain $\left(-\mathrm{CH}_{2}-\mathrm{CH}_{2}-\mathrm{CH}_{2}-\mathrm{NHCO}\right.$-hydrocarbon) of the silanes. The calculated values of the grafted amount of the silane are shown in Figure 9.8 (C). It is clearly seen that TEPI+E, TEPI+CP, TEPI+TB, and TEPI+PM show a similar grafting density due to a similar molecular mobility of the silanes during 
the modification. However, the TEPI+NM1 sample shows a very low grafting density due to the high molecular weight and potentially high intermolecular interactions (due to the flat molecule structure of the naphthalene and П-П stacking) of the silane leading to a low molecular mobility, resulting in a low degree of the modification. Despite the different weight loss and related grafted densities of the modified silicas, it can still be concluded that the designed structure of the silicas (Figure 9.9) was successfully realized through the solvent free modification.

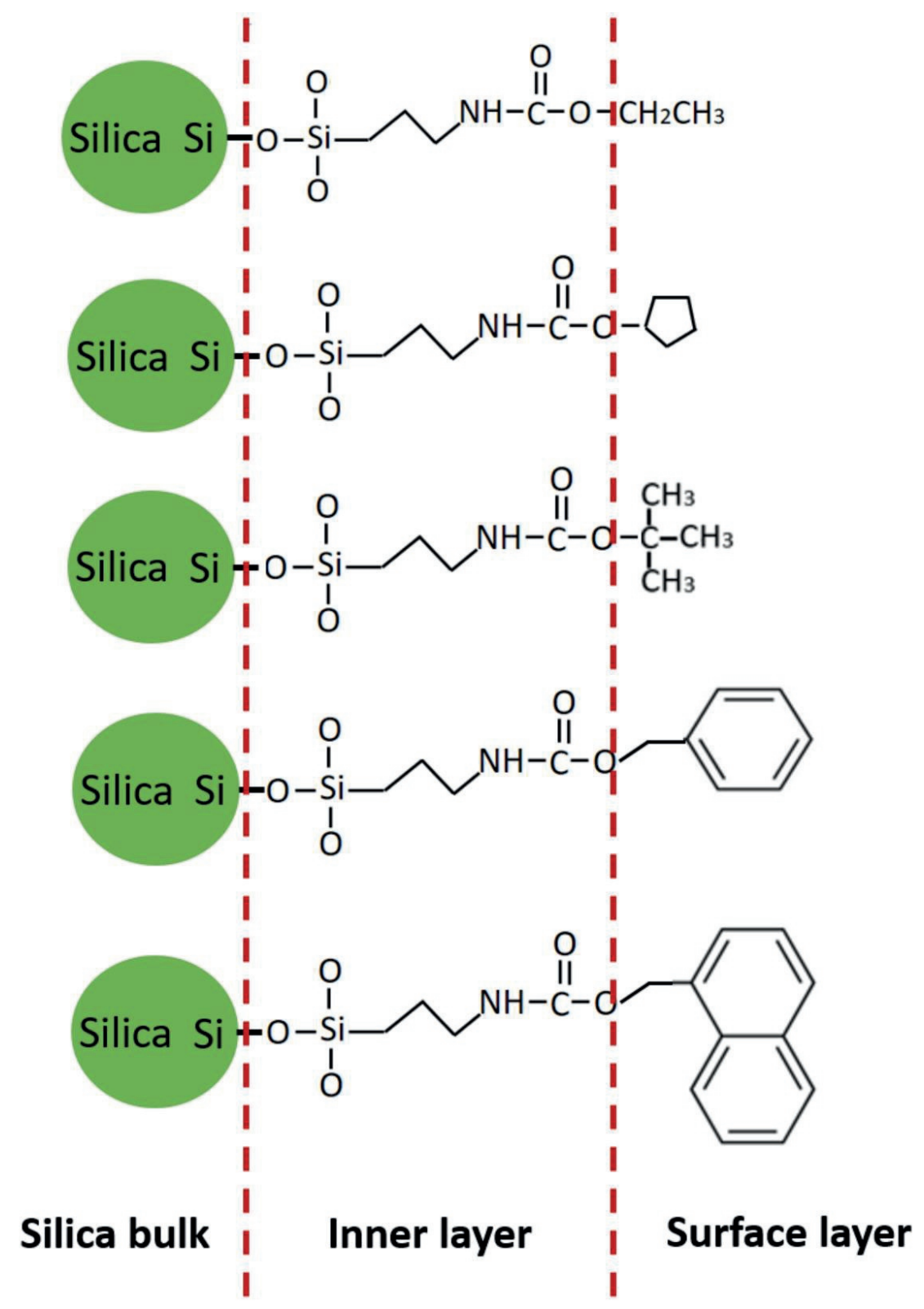

Figure 9.9 Chemical structure of the surface modified silicas. 


\subsubsection{Scanning Electronic Microscopy (SEM) of the Nanocomposites}

After modification of silica with the designed silanes (inner layer + surface layer), the nanocomposites were prepared through mini-injection molding. One of the reasons to modify silica by the designed structures was to improve the compatibility with the polymer resulting in a better dispersion of the silica in the polymeric matrix. Therefore, SEM was performed on the cross-sections of the nanocomposites
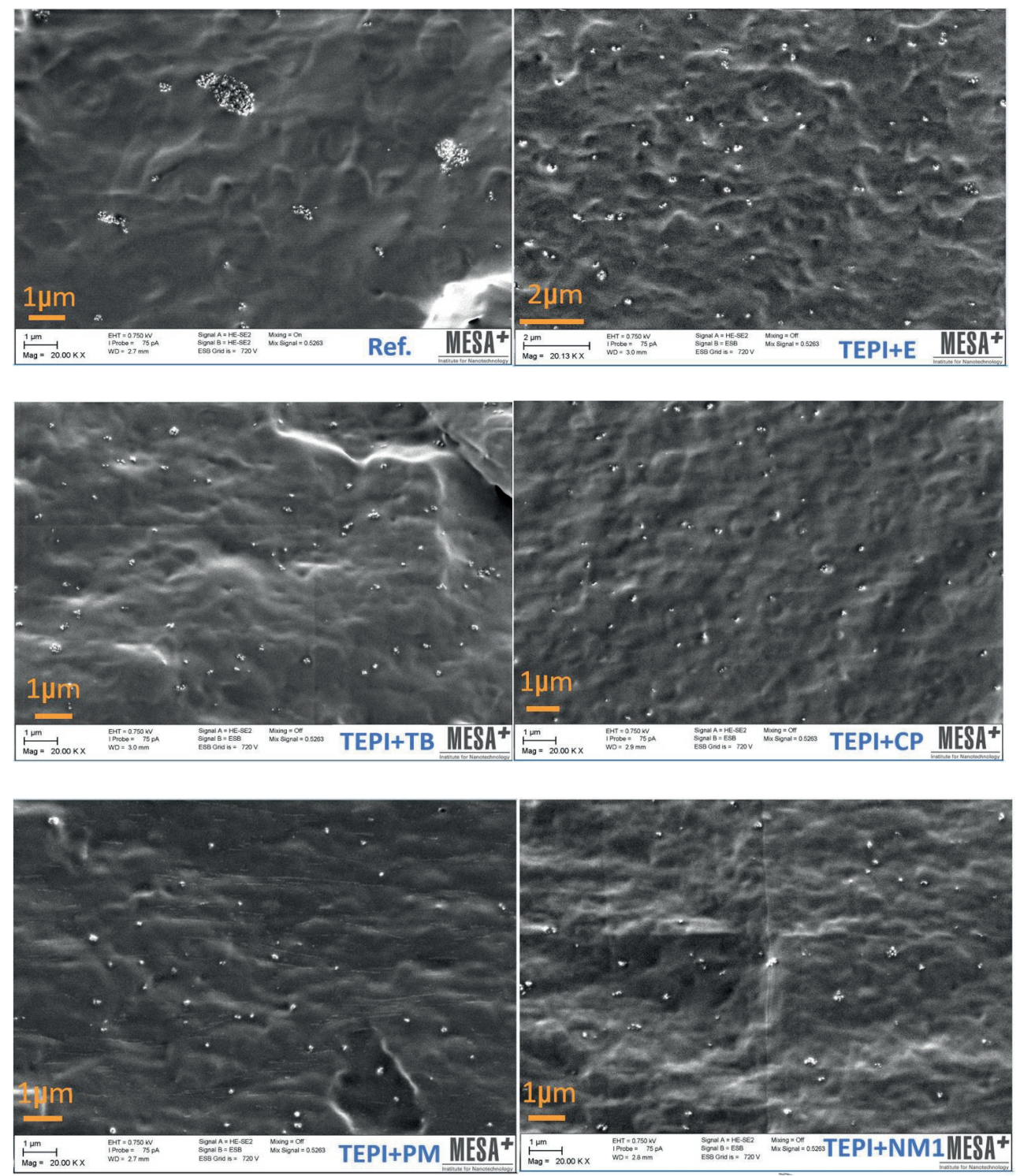

Figure 9.10 SEM images of nanocomposites filled with reference silica or modified silicas.

filled with the reference and modified silicas, as shown in Figure 9.10. In the nanocomposite filled with the reference silica, large size clusters are present. This is due to the fact that the reference silica has a 

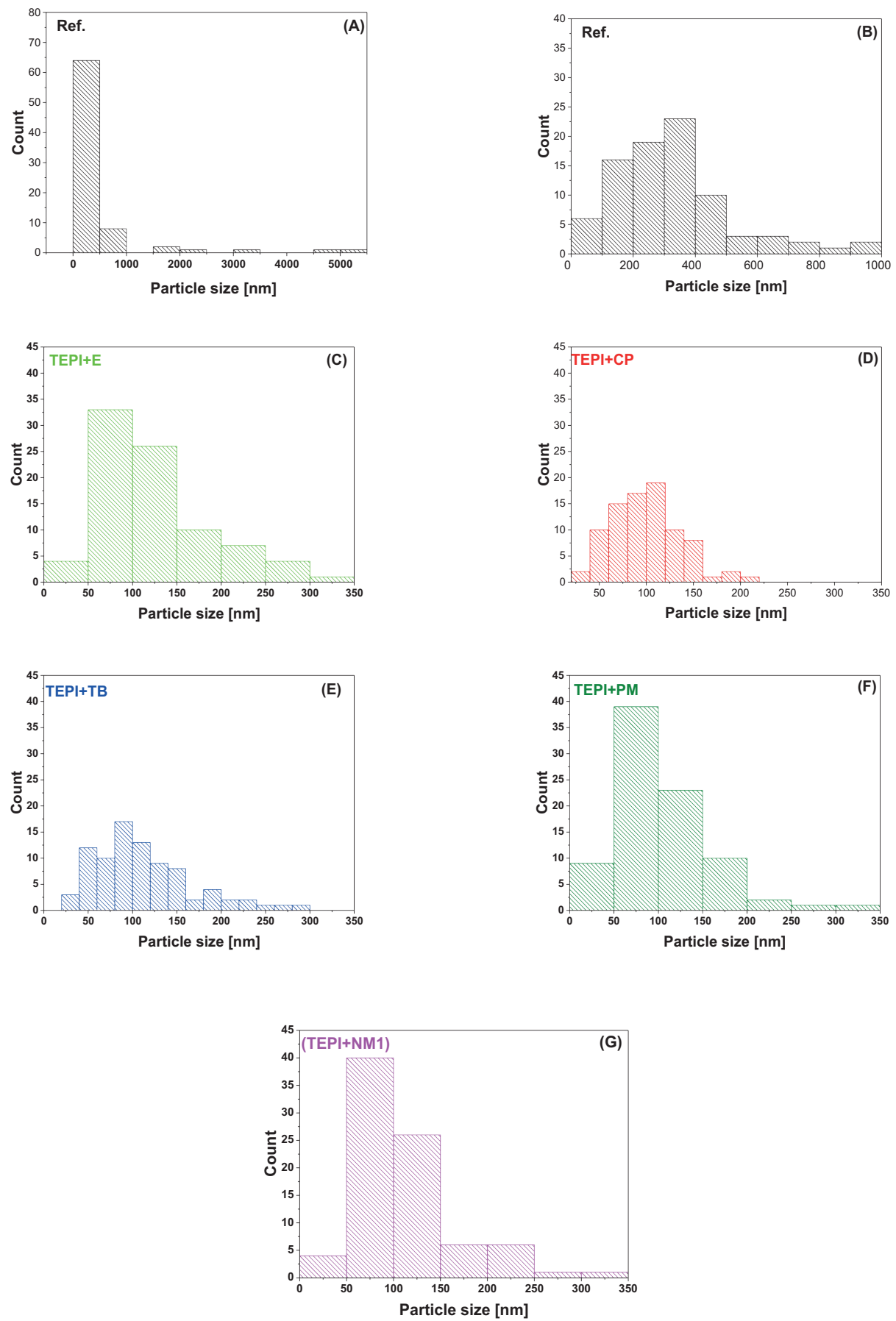

Figure 9.11 Histograms of the cluster size distribution for the reference silica and the modified silicas in the polymer matrix. 
very polar surface and is therefore not compatible with the polymer matrix exhibiting an unpolar nature. The nanocomposites filled with the modified silicas clearly show a much better dispersion of the filler, without large clustering, than the one filled with the reference silica. In order to get insight into the particle size distribution, several SEM micrographs were analysed with the software Image J (version 1.53a, supplied by Wayne Rasband, USA). The histograms of the particle size distributions are shown in Figure 9.11. The reference silica shows a broad size distribution in a range of 80 to $5000 \mathrm{~nm}$, and most clusters are above $300-400 \mathrm{~nm}$ in diameter. For the modified silicas, smaller size clusters as well as a narrower size distribution are seen. The mean size of the modified silica clusters is around $100 \mathrm{~nm}$ in diameter. This proves that the modification improves the nanosilica dispersion and distribution in the polymer matrix. Although the polar urethane group is introduced on the silica surface, the surface layer of the modified silica with the unpolar hydrocarbon group improves the compatibility of the silica with the unpolar polymer matrix.

\subsubsection{Thermally Stimulated Depolarization Current (TSDC)}

In order to compare the effect of the reference silica and the modified silicas on the charge trapping properties of the PP/PP-HI polymer blend TSDC measurements were performed and the results are presented in Figure 9.12. The calculated trap depth and trap density is shown in Figure 9.13 and the method used is shown in Chapter 4 Supplementary Material S1. The relaxation of the trapped charges contributes to the current during the thermal depolarization process. The TSDC temperature and current intensity are associated with the trap depth and density, respectively. The TSDC main peak temperatures and current intensities of the composites are shown in Table 9.3. Compared to the reference silica, the TSDC peak temperature of the composites filled with the modified silicas move to higher values, indicating deeper charge traps. This is due to the introduced polar moieties in the inner layer of the modified silicas. The samples filled with the designed silicas also show a lower TSDC peak intensity, indicating a lower trap density. Among all the samples, the sample filled with TEPI+NM1 shows the highest TSDC peak temperature (the deepest charge traps), while the sample with TEPI+TB exhibits the lowest TSDC current intensity (the lowest trap intensity).

In addition, it is also found that TEPI+TB and TEPI+E both exhibit quite different effect on the charge trap distribution of the composites as shown in Figure 9.14, even though, TEPI+TB is similar to TEPI+E from a chemical point of view. This is due to the two effects:

1) The shielding effect: tert-butyl groups exhibit a better shielding effect than the ethyl groups [16]. The shielding effect can hinder the charge move to the silica surface, which contributes to the less charges accessible to traps on the silica surface. This leads to a lower apparent trap density in the sample filled with TEPI+TB in comparison to the one containing TEPI+E.

2) The formation of deep traps: there is small amount of the deep traps located at energy of $1.2 \mathrm{eV}$ in the samples filled with TEPI+TB as shown in Figure 9.14. The charges trapped inside these traps cannot de-trap easily and a local electrical field is formed, as a result, hindering the further charges injection into the sample. Consequently, a lower apparent trap density is presented for the sample filled with TEPI+TB than TEPI+E. 


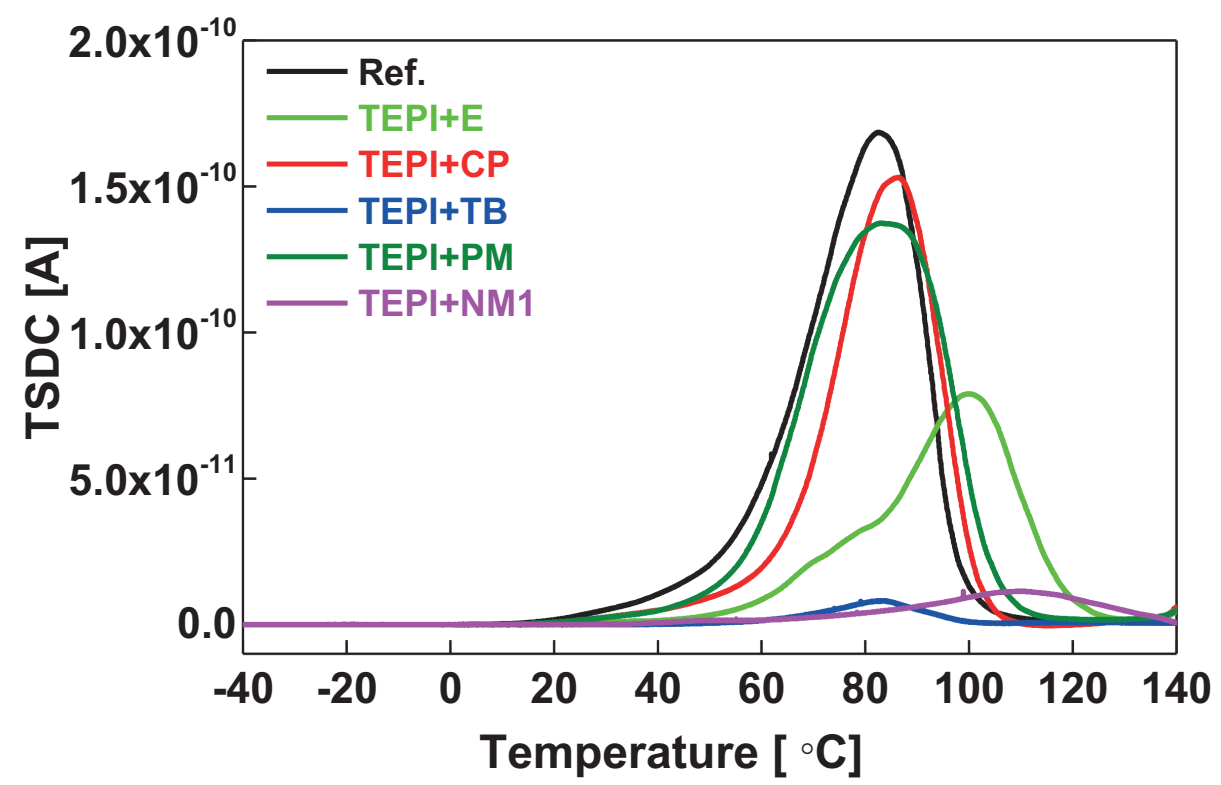

Figure 9.12 TSDC spectra of the nanocomposites filled with the reference silica and the modified silicas.

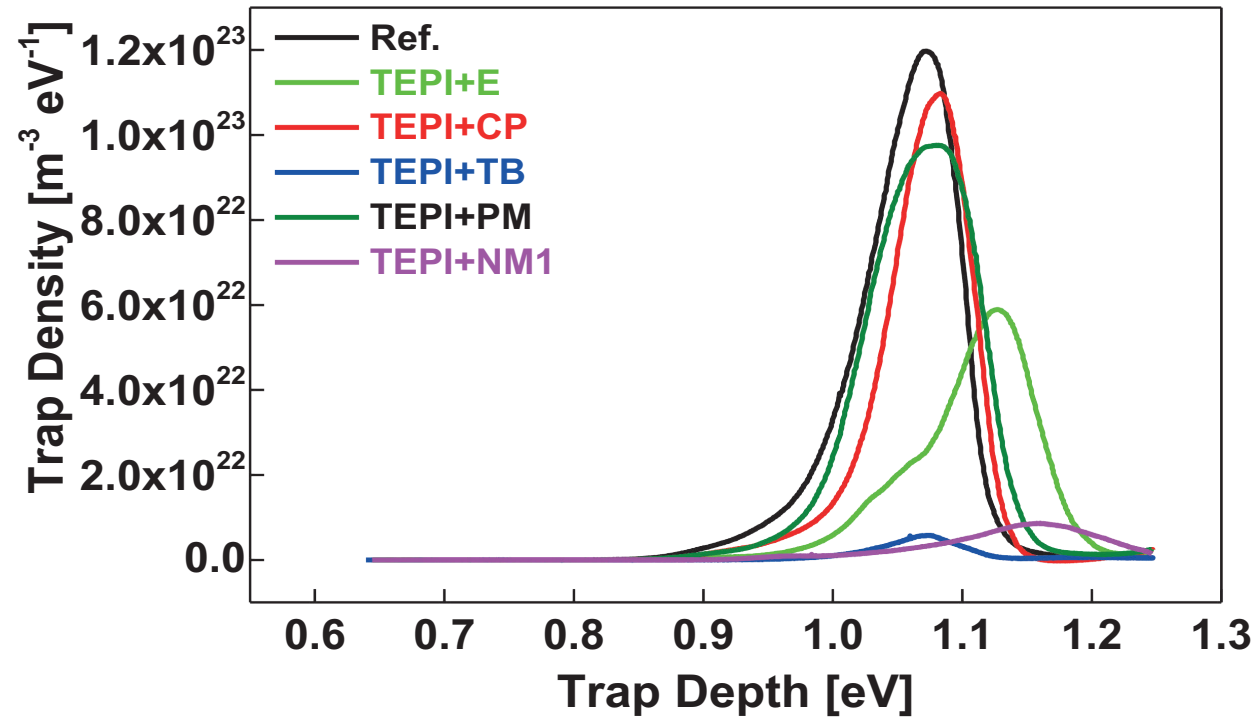

Figure 9.13 Calculated trap density and depth of the nanocomposites filled with the reference silica and the modified silicas. 
Table 9.3 TSDC main peak temperature and peak intensity.

\begin{tabular}{|c|c|c|c|c|c|c|}
\hline & Ref. & TEPI+E & TEPI+TB & TEPI+CP & TEPI+PM & TEPI+NM1 \\
\hline $\begin{array}{c}\text { TSDC peak } \\
\text { temperature }\left({ }^{\circ} \mathrm{C}\right)\end{array}$ & 82.6 & 100.1 & 83.1 & 86.1 & 83.2 & 110.3 \\
\hline $\begin{array}{c}\text { TSDC peak } \\
\text { intensity (A) }\end{array}$ & $1.88 \mathrm{E}-10$ & $0.79 \mathrm{E}-10$ & $0.08 \mathrm{E}-10$ & $1.53 \mathrm{E}-10$ & $1.37 \mathrm{E}-10$ & $0.11 \mathrm{E}-10$ \\
\hline
\end{tabular}

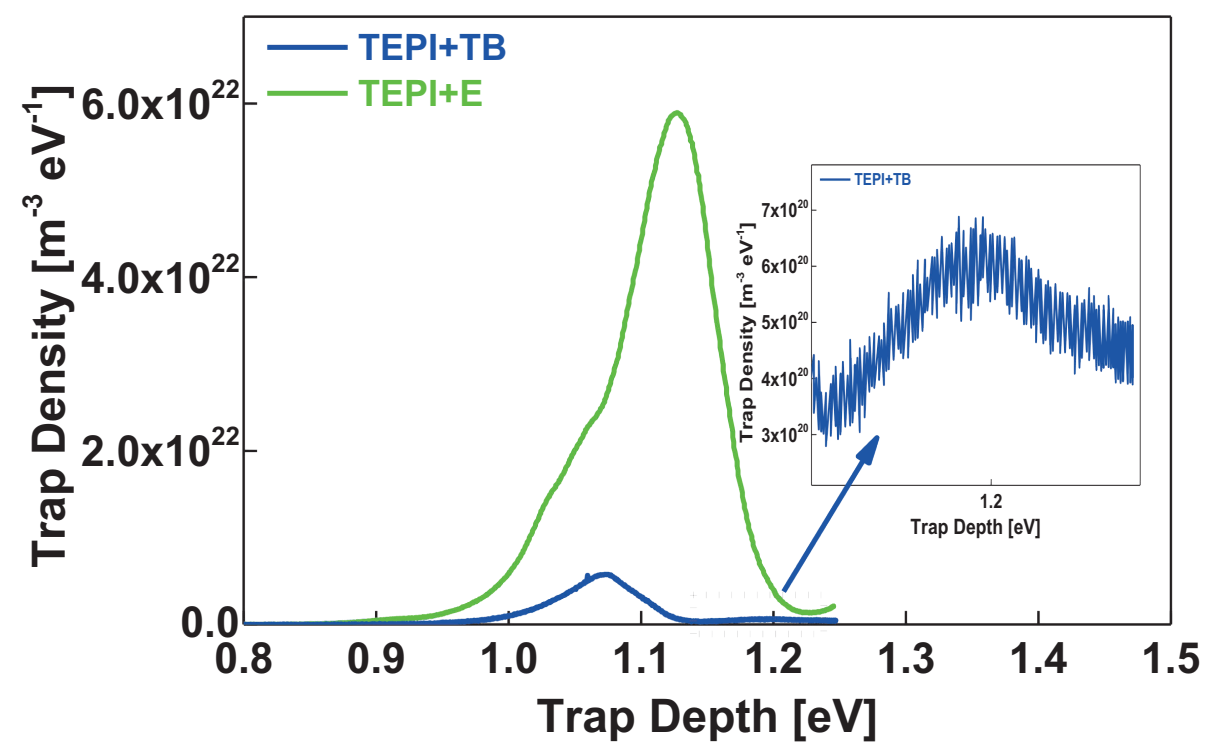

Figure 9.14 TSDC spectra of the nanocomposites filled with the TEPI+E and the TEPI+TB.

In order to further study the TSDC results, the amount of injected charges during polarization and the released charges during the depolarization were calculated by integrating the area under the current versus time curves. The calculated results are presented in Figure 9.15. With regard to the injected charges, the samples filled with the reference, TEPI $+\mathrm{CP}$ and TEPI $+\mathrm{PM}$ silica all show a similar amount of injected charges, while the samples filled with TEPI+E, TEPI+TB and TEPI+NM1 show a lower amount. In case of released charges, the samples present the same trend as for injected charges. The injected charges are related to the charge trap distribution in the sample. Although we have observed a deeper trap with lower intensity in all samples filled with the designed silicas compared to the one containing the reference silica, the changes for the sample filled with TEPI $+\mathrm{CP}$ and TEPI+PM are small, their charge trap depth is only slightly deeper and the trap density is slightly lower than the reference composite. In case of TEPI+TB, the peak intensity is much lower than the one of the reference composite, and it also contains a small amount of the deep traps as shown in Figure 9.14. Both contributing to the low number of injected charges. For the TEPI+E and TEPI+NM1 composites, the TSDC peak temperature is $17.5^{\circ} \mathrm{C}$ and $27.7^{\circ} \mathrm{C}$ higher than for the sample containing the reference silica. This means that the traps present in the samples filled with TEPI+E and TEPI+NM1 are much deeper than the traps in the reference composite. In our previous study, we have observed that a sample with deep traps can 
block further charge injection, resulting in a low amount of injected charges [11]. The charges caught by the deep traps are not easily de-trapped and require additional energy support. The trapped charge will repel the further charge injection from the electrode. Therefore, the amount of injected charges to the samples filled with the TEPI+E and TEPI+NM1 is low due to the deep traps introduced by the corresponding silicas. In addition, the low TSDC peak intensity indicating low trap density also contributes to the low amount of injected charges for the TEPI+E and TEPI+NM1 silica filled samples.

In summary, charge trap depth and density, both can influence charge injection. The samples with very deep traps show a low level of injected charges, see the samples filled with TEPI+E or TEPI+NM1. Besides, a sample with less deep traps but with very low trap density can also result in low charge injection, as in the case of the sample filled with the TEPI+TB modified silica.

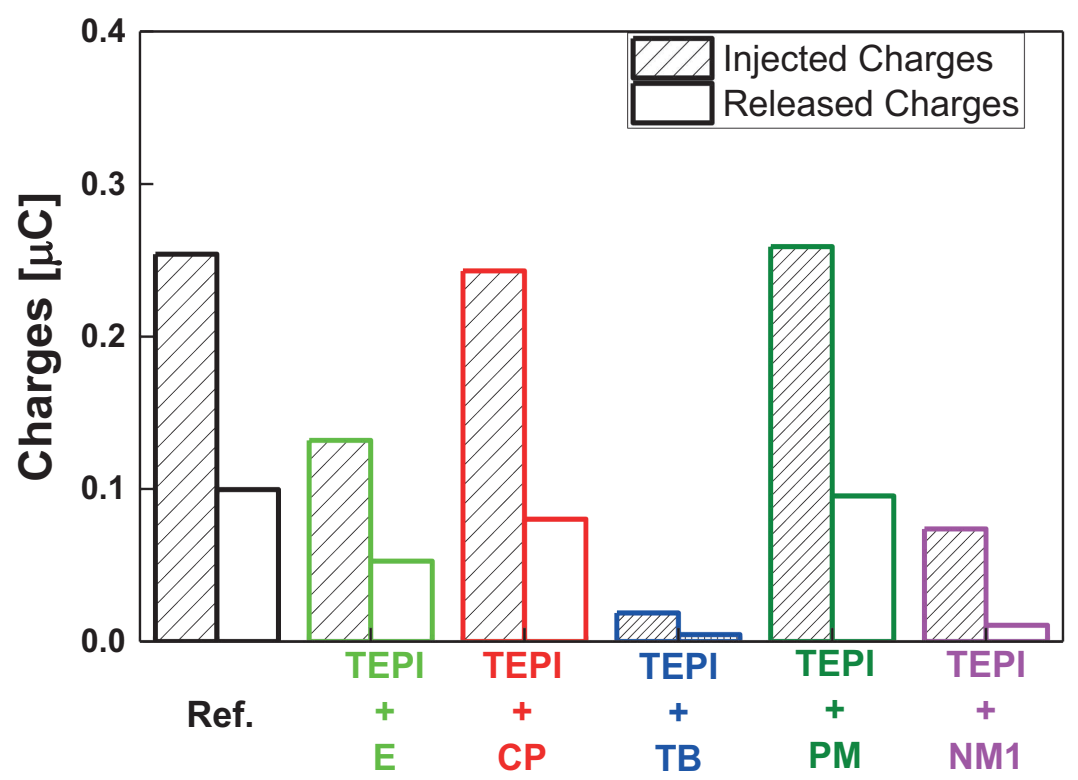

Figure 9.15 Calculated amount of injected and released charges.

In order to estimate the conductivity of the studied nanocomposites, the charging current density during isothermal polarization is measured and presented in Figure 9.16. Although it is clear that not all the samples reached the steady state DC conductivity current due to the short poling phase (20 mins), the difference of the charging current behaviour between all the studied samples is apparent. The initial current value is related to the fast polarization mechanisms. After the initial current transient, the slowly decaying current over time results from many factors [17]: 1) electrode polarization 2) dipole orientation 3) charge storage leading to trapped space charge effects 4) tunnelling of the charge carriers from the electrodes and 5) hopping of the charge carriers through localized states. Taking into consideration of the same electrode for all the tests and the same non-polor polymer, the electrode polarization and dipole orientation can be negligible to compare all the samples. Hence, the absolute current level and the decay 
rate of the charging current over time can convey the charge storage (indicating the occupied trap density) and charge mobility (indicating the trap depth) in the samples.

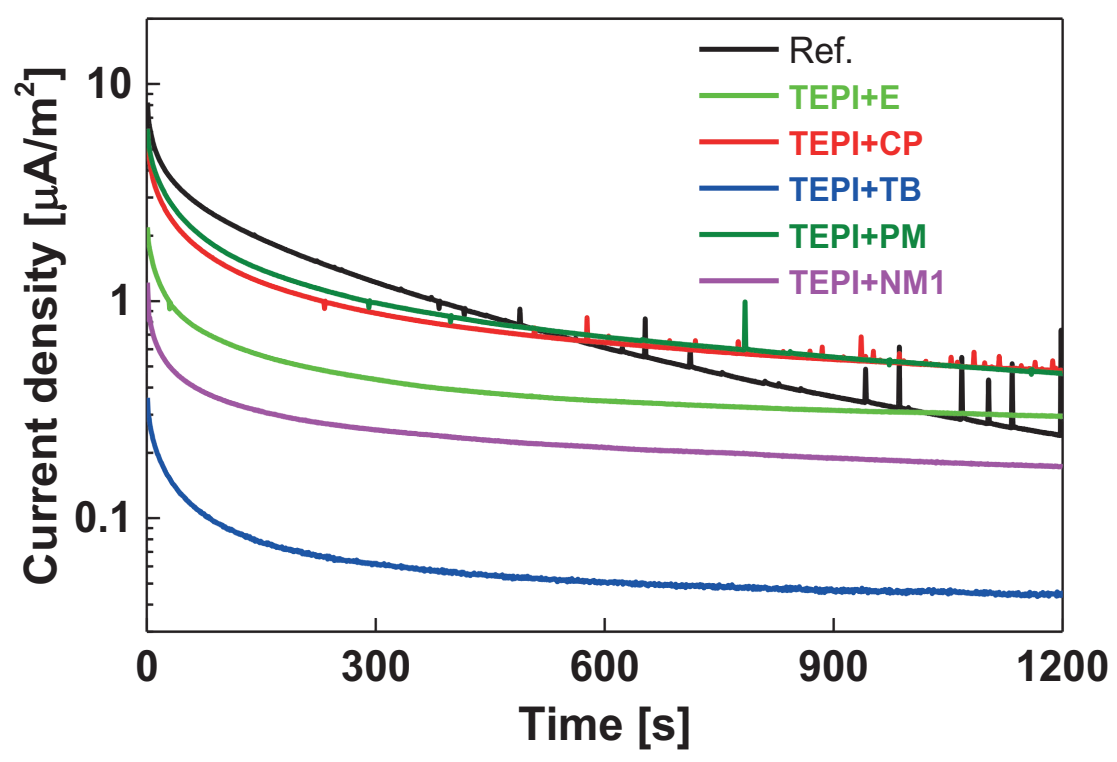

Figure 9.16 Charging current density during the isothermal polarization process.

The sample filled with the reference silica exhibits a slow current decrease (decay) behaviour and higher current density than the other samples measured in the 20 mins poling time, implying a high amount of occupied traps or shallower trap depth (proving by the Figure 9.13). In case of the composites filled with the modified silicas, especially, for the TEPI-TB sample, a rapid decay behaviour and very low current density indicate rapid trapping, low number of trap site and build-up of homocharge in deep traps. The deep traps can hinder the charge injection from the electrode, causing a current level indicating a low amount of the occupied trap density of this sample as well.

Nevertheless, among all these samples, the sample filled with the TEPI+TB modified silica exhibits a very low current density, indicating a low transient conductivity characteristics. It should be stressed that this test was only done at low electrical field at $70{ }^{\circ} \mathrm{C}$ and it cannot represent the electrical behavior under high electrical field and other temperature. However, this preliminary results already provide an valuable information that these innovative modified silica TEPI+TB presents a good dispersion as seen in Figure 9.10 and 9.11 and the lowest amount of charge injection (Figure 9.14) under $3 \mathrm{kv} / \mathrm{mm}$ at $70{ }^{\circ} \mathrm{C}$, indicating is a potential candidate for the HVDC cable insulation application. To further prove the applicability of this silica, more studies under high electrical fields and different temperature are necessary. 


\subsection{Conclusions}

Five designed surface-functionalized silicas were successfully synthetized. The designed silicas and the unmodified reference silica were compounded with the polypropylene/ propylene-ethylene copolymer to produce the nanocomposites via injection molding.

The modified silicas exhibit a better dispersion than the unmodified reference one. The designed silicas introduce deeper traps with a lower trap density than the reference filler in the nanocomposite. However, the charge trap depth level and densities are different for the silica composites containing the modified silicas. The sample filled with silica modified with 3-(triethoxysilyl)propyl isocyanate silane (TEPI) + CP (cyclopentanol) and TEPI + PM (phenyl methanol) show a trap distribution close to the one of the reference sample. As a result, the amount of injected charges and the charging current densities of these three composites are similar. In contrast to this, the TEPI + E (ethanol) and TEPI + NM1 (1 - naphthalene methanol) modified silicas introduce much deeper traps than the reference silica, leading to a low current density and low amount of charge injection. In case of the sample filled with the TEPI + TB (tert butanol), it shows the lowest charging current density and smallest charge injection, indicating low conductivity and space charge injection, probably due to the low trap density and a small amount of deep traps in the sample under $3 \mathrm{kV} / \mathrm{mm}$ at $70{ }^{\circ} \mathrm{C}$. With additionally the good dispersion of the TEPI+TB modified silica, the sample filled with this silica is a potential candidate for application in HVDC cable insulation material. As mentioned before, the TSDC test and current density are done only at low electrical field. Wide characterizations on difference elelctrical field and temperature including breakdown test, aging, space charge measurement are needed to evaluate overall performance of $\mathrm{HV}$ insulation material in the future.

\section{Acknowledgements}

The authors also would like to thank Evonik Industries for providing a free silica sample.

\section{Research funding}

This project has received funding from the European Union's Horizon 2020 research and innovation program under grant agreement No 720858 .

\section{Author contribution}

Xiaozhen He: Formal analysis, Investigation, Resources, Writing - Original Draft, Writing - Review \& Editing, Visualization, Ilkka Rytöluoto: Investigation, Writing - Review \& Editing, Rafal Anyszka: Writing - Review \& Editing, Amirhossein Mahtabani: Formal analysis, Minna Niittymäki: Investigation, Eetta Saarimäki: Investigation, Christelle Mazel: Resources, Gabriele Perego: Resources, Kari Lahti: Writing - Review \& Editing, Mika Paajanen: Project administration, Wilma Dierke: Supervision, Writing - Review \& Editing, Anke Blume: Supervision.

\section{Conflict of interest}

Authors state no conflict of interest. 


\section{References}

[1] Nelson JK, Fothergill JC, Dissado LA. and Peasgood W. Towards an understanding of nanometric dielectrics. In Annual report conference on electrical insulation and dielectric phenomena IEEE. 2002 Oct 20-24. Mexico. pp. 295-298.

[2] Zhou Y, Dang B, Wang H, Liu J, Li Q, Hu J. et al. Polypropylene-based ternary nanocomposites for recyclable high-voltage direct-current cable insulation. J. Compos. Sci., 2018;165:168-174.

[3] Pourrahimi, A.M., Pallon, L.K., Liu, D., Hoang, T.A., Gubanski, S., Hedenqvist, M.S., et al. Polyethylene nanocomposites for the next generation of ultralow-transmission-loss HVDC cables: Insulation containing moisture-resistant $\mathrm{MgO}$ nanoparticles. ACS Appl. Mater. Interfaces, 2016;8(23):14824-14835.

[4] Roy, M., Nelson, J.K., MacCrone, R.K., Schadler, L.S., Reed, C.W. and Keefe, R. Polymer nanocomposite dielectrics-the role of the interface. IEEE Trans Dielectr Electr Insul, 2005;12(4):629643.

[5] Green, C. and Vaughan, A. Nanodielectrics-How much do we really understand? IEEE Electr. Insul. Mag, 2008;24(4):6-16.

[6] Min, D., Cui, H., Hai, Y., Li, P., Xing, Z., Zhang, C. and Li, S. Interfacial regions and network dynamics in epoxy/POSS nanocomposites unravelling through their effects on the motion of molecular chains. J. Compos. Sci., 2020;199:108329.

[7] Roy, M., Nelson, J.K., MacCrone, R.K. and Schadler, L.S. Candidate mechanisms controlling the electrical characteristics of silica/XLPE nanodielectrics. J. Mater. Sci., 2007; 42(11):3789-3799.

[8] Liu, D., Pourrahimi, A.M., Olsson, R.T., Hedenqvist, M.S. and Gedde, U.W. Influence of nanoparticle surface treatment on particle dispersion and interfacial adhesion in low-density polyethylene/aluminium oxide nanocomposites. Eur. Polym. J, 2015;66:67-77.

[9] Mahtabani, A., Rytöluoto, I., Anyszka, R., He, X., Saarimäki, E., Lahti, K., Paajanen, M., Dierkes, W. and Blume, A. On the Silica Surface Modification and Its Effect on Charge Trapping and Transport in PP-Based Dielectric Nanocomposites. ACS Appl. Polym. Mater., 2020;2(8):3148-3160.

[10] He, X., Rytöluoto, I., Anyszka, R., Mahtabani, A., Saarimäki, E., Lahti, K., et al. Surface modification of fumed silica by plasma polymerization of acetylene for PP/POE blends dielectric nanocomposites. Polymers, 2019;11(12):1957.

[11] He, X., Rytöluoto, I., Anyszka, R., Mahtabani, A., Saarimäki, E., Lahti, K., et al. Silica surfacemodification for tailoring the charge trapping properties of PP/POE based dielectric nanocomposites for HVDC cable application. IEEE Access. 2020; 8: 87719-87734

[12] Bacaloglu, R., Cotarca, L., Marcu, N. and Tölgyi, S. Kinetics and mechanism of isocyanate reactions. II. Reactions of Aryl Isocyanates with Alcohols in the presence ob tertiary amines. J. Prakt. Chem., 1988;330(4):530-540.

[13] Xu, L., Li, C. and Ng, K.S. In-situ monitoring of urethane formation by FTIR and Raman spectroscopy. J. Phys. Chem. A, 2000;104(17):3952-3957. 
[14] Huang, J., Zhang, L., Tang, Z., Wu, S. and Guo, B. Reprocessable and robust crosslinked elastomers via interfacial CN transalkylation of pyridinium. J. Compos. Sci., 2018;168:320-326.

[15] Nouri, N., Rezaei, M., Sofla, R.L.M. and Babaie, A. Synthesis of reduced octadecyl isocyanatefunctionalized graphene oxide nanosheets and investigation of their effect on physical, mechanical, and shape memory properties of polyurethane nanocomposites. . J. Compos. Sci., 2020:108170.

[16] Kawamura, A., Ueno, S., Takai, C., Takei, T., Razavi-Khosroshahi, H. and Fuji, M. Effect of steric hindrance on surface wettability of fine silica powder modified by n-or t-butyl alcohol. Advanced Powder Technology, 2017;28(10): 2488-2495.

[17] Gupta DD, Brockley RS. A study of'absorption currents' in low-density polyethylene. Journal of Physics D: Applied Physics. 1978;11(6):955. 


\section{Chapter 10}

Summary 
In order to meet the growing electricity demand and reduce $\mathrm{CO}_{2}$ emissions (mostly produced by burning fossil fuels), "green energy" production and its long-distance transport from remote locations have to be further developed. To transport the green energy efficiently with low electrical loss and low construction cost, the development of high performance HVDC technology is required. As a contribution to this, the present study is focused on the next generation HVDC insulation nanocomposites based on a thermoplastic polypropylene (PP) matrix containing tailored nanosilica. The latter was surface functionalized in order to improve its dispersion in the polymeric matrix, to tailor the charge trapping distribution and to reduce the space charge accumulation of the PP based nanocomposites.

Fumed silica was used as nanofiller in this study, and the charge trapping properties of the nanocomposites were tailored through different surface modifications via plasma or solvent free modification. Plasma modification is an effective way to perform chemical particle surface modifications. With acetylene as precursor, a hydrocarbon layer was deposited on the silica surface which improves the filler dispersion in the polymeric matrix by better matching the polarity of the polymer. However, the improved dispersion of the silica did not provide a significant improvement of the charge current density or significantly change the charge trap distribution of the nanocomposites.

Another effective and environmentally friendly approach for silica surface modification by a solvent free method with an acid as catalyst and conducted at room temperature. Nine types of modified silicas with silanes differing in polarity and number of alkoxy groups were successfully obtained via the solvent free modification. Silicas modified with all unpolar silanes showed better dispersion in the polymeric matrix than the polar ones; however, the unpolar silicas did not significantly alter the trap depth and only slightly increased the trap density of the nanocomposites. In contrast, polar silicas introduced deeper charge traps, and the trap depth and density depended on the functional groups of the modifying agents: silanes containing nitrogen atoms introduced the deepest traps and exhibited the lowest charge injection into the nanocomposites, which is positive for space charge reduction.

In order to conduct a thorough investigation of the performance of the nanocomposites, composite samples were prepared with the best performing polar silica, modified with 3aminopropyltriethoxysilane (APTES), as well as the best unpolar silica modified with trimethylethoxysilane. The unpolar silica showed a negative effect on space charge accumulation of the nanocomposites. Although the polar silica exhibited poor dispersion, it significantly decreased the space charge accumulation due to the deep trap introduction. Besides, it was noticed that adding either silica, polar or unpolar, improved the thermal stability of the composites. The silica acts as nucleating agent increasing the crystallization temperature, but usually decreasing the crystallinity of the polymer blend, resulting in a lower storage modulus of the nanocomposites. Silica addition also altered the charge trapping location in the nanocomposites. For the unfilled polymer, the charge trap sites are mostly located at the crystalline-amorphous interfaces, while for the nanocomposites most of the charges are preferably accumulated in the silica-polymer interface and inside silica clusters.

The effect of different concentrations of polar silica was also studied. The composite containing a polar silica in a concentration of $2 \mathrm{wt} . \%$ contains bigger-sized silica clusters than the nanocomposite filled with $1 \mathrm{wt} . \%$ of the silica. Interestingly, in the case of the polar silica, the nanocomposite with a higher silica concentration shows lower space charge accumulation and a lower charging current due to much deeper traps and lower trap density. This improvement might be due to a change of the charge mobility stemming from: 
1) Amine-amine hydrogen bond formation: If a charge trapped around one amino group gets enough energy to de-trap, it is highly likely that it will be easily trapped by a neighbouring amino group again, leading to a lower mobility of the charges.

2) Change of chain mobility due to the presence of occluded polymer macromolecules constrained inside the silica clusters.

Both phenomena may lead to a higher energetic barrier of charge de-trapping, thus increasing the depth of the charge traps.

Two polymer blends with different miscibility were compared: an immiscible PP/EOC (polypropylene/ethylene-co-octene) and a miscible PP/PP-HI (polypropylene/ethylene-co-propylene) blend. The filler used for this study was APTES-modified silica. The comprehensive performance of the $\mathrm{PP} / \mathrm{PP}-\mathrm{HI}$ composite is better than the one of the PP/EOC composite due to a better polymer miscibility and flexibility, as well as lower conductivity and space charge accumulation. Due to the immiscibility of PP and EOC, phase separation occurred in these samples, while there is only one smooth phase in PP/PP-HI sample. The properties of the polymer blend also influence the location of the silica: In the immiscible PP/EOC blend, the silica is only located in the PP phase due to the lower viscosity of PP compared to EOC. In the miscible PP/PP-HI blend, the silica is evenly distributed within the whole polymeric matrix. In general, APTES-silica introduces deep traps in the polymer blends and suppresses space charge accumulation. Overall, the PP/PP-HI/APTES-silica composite exhibited the most promising dielectric performance (lowest space charge accumulation), which is beneficial for the HVDC cable insulation application.

Although the polar APTES-silica addition gave the best results, its dispersion is not satisfactory. Therefore, a further improvement of the compatibility of silica and polymer while maintaining good dielectric performance is needed. Hence, a newly designed modified silica was proposed. The structure of this silica comprises:

i) a polar group: nitrogen-containing urethane, with the purpose to tailor the charge trap behaviour, and

ii) an unpolar hydrocarbon moiety: ethyl, tert-butyl, cyclopentyl, phenyl or naphthyl, with the purpose to improve the compatibility between the silica and polymeric matrix.

This study showed that the five modified silica's with an outer layer made of hydrocarbon moieties all result in a good dispersion in the PP matrix. The silica with the inner layer composed of the urethane moieties can additionally lead to a tailored charge trap distribution of the nanocomposites. Among all five modified silicas, the one based on a tert. butyl group as the outer layer showed the lowest charge injection and the lowest charge current in the nanocomposite. These performance improvements indicate that such a newly designed and modified nanosilica is a potential candidate for HVDC cable insulation nanocomposites.

However, as this research focuses on improving the nanoparticle dispersion within the PP based polymer matrix and reducing the space charge accumulation via nanoparticle surface functionalization, additional tests are needed get a complete picture of the suitability of these materials for HVDC cable insulation. Other aspects of importance such as short term and long term breakdown performance, dielectric loss, thermal conductivity, aging performance, and other properties have to be investigated in-depth; this was beyond the scope of the present study. 
The newly designed and modified silica is a promising development to further improve the high performance of PP based insulation nanocomposites. Still remaining challenges are

i) to control the thickness of the two layers (inner and outer) in the modification process;

ii) to elucidate the influence of the thickness of the layers on silica dispersion and dielectric performance of the nanocomposite.

Meeting these challenges will be a big step forward in the development of these nanocomposites for HVDC cable insulation materials. 


\section{Samenvatting}

Om aan de groeiende vraag naar elektriciteit en de eis voor verlaging van $\mathrm{CO}_{2}$ emissies te voldoen (met name door gebruik van fossiele bronnen), moeten zowel de productie van 'groene energie' alsmede het transport over langere afstanden tussen opwekking en gebruik verbeterd worden. Om elektrische energie efficiënt en tegen lage kosten te kunnen transporteren, is technologie gebaseerd op hoogspanningsgelijkstroom (HVDC) de aangewezen manier. Deze studie levert een bijdrage aan het verder ontwikkelen van deze technologie door met behulp van elektrisch isolerende nanocomposieten op basis van een matrix van thermoplastisch polypropyleen (PP) met nanosilica als functioneel additief de elektrische verliezen te verminderen. Om dit nanosilica beter dispergeerbaar te maken in de polymeermatrix, om de 'charge trapping' te optimaliseren en de ruimteladingsverdeling te verlagen is het gefunctionaliseerd.

De voor deze studie gekozen nanovulstof is pyrogene silica, en de 'charge trapping'-eigenschappen zijn door middel van verschillende oppervlakte-modificaties geoptimaliseerd: plasma coating en een oplossingsmiddelvrije chemische modificatie. Plasma technologie is een effectieve manier om de oppervlakte-chemie van deeltjes op maat te maken. Met acetyleen als monomeer wordt een koolwaterstof-laag aangebracht ter verbetering van de compatibiliteit en, door een verlaging van oppervlakte-polariteit, de dispersie in PP. De verbeterde dispersie van gemodificeerde silica heeft niet, zoals verwacht, een duidelijke verbetering in de elektrische ladingsstroomdichtheid gebracht, noch een duidelijke invloed op de 'charge trap' verdeling van het nanocomposiet gehad.

Een andere milieuvriendelijke methode om het silica oppervlak te functionaliseren is een oplossingsmiddelvrije modificatie bij kamertemperatuur met gebruik van zuur als katalysator. Middels deze methode zijn negen gemodificeerde silica types, verschillend in polariteit en aantal alkoxy groepen, gesynthetiseerd. Silica's met een apolaire oppervlaktelaag resulteerden in een betere dispersie dan de polaire varianten, maar het inbrengen van laag polaire silica's in de polymeer-matrix heeft geen verandering qua 'trap'-diepte teweeg gebracht, alleen een kleine verhoging van de 'trap' dichtheid. Daarentegen geven polaire silica's diepere 'charge traps' waarbij de 'trap' dichtheid en diepte afhankelijk zijn van het type functionele groep van de monomeren. Silanen met een stikstof groep veroorzaken de diepste 'traps' en de laagste ladingsinjectie in de nanocomposieten, met een verlaging van ruimtelading als positief gevolg.

Om meer inzicht te krijgen in het potentieel van deze nanocomposieten, zijn deze gemaakt met zowel de best presterende polaire silica gemodificeerd met 3-aminopropyltriethoxysilaan (APTES), alswel als met de beste apolaire silica met trimethylethoxysilaan als monomeer. Dit laatstgenoemde apolaire silica had een negatief effect op ruimtelading, terwijl het polaire silica de ruimtelading verlaagd door meer diepe 'traps', ondanks het feit dat de dispersie minder goed is dan voor het apolaire silica. Daarnaast verbeterd het toevoegen van silica in beide gevallen de thermische stabiliteit van de nanocomposieten. 
De aanwezigheid van silica bevordert de nucleatie van kristallen in de nanocomposieten, verhoogt de kristallisatietemperatuur maar verlaagd de kristalliniteit, met als gevolg een lagere opslagmodulus. Silica beïnvloed daarnaast de locatie van de 'charge traps': in het polymeermengsel zonder silica zijn de 'charge traps' met name te vinden in het grensvlak tussen amorfe en kristallijne gebieden; in de nanocomposiet zijn de 'traps' gelocaliseerd in het grensvlak tussen silica en polymeer en in silica clusters.

Het effect van silica concentratie is eveneens onderzocht: het nanocomposiet met $2 \mathrm{wt} . \%$ silica bevat grotere clusters dan het materiaal met maar $1 \mathrm{wt} . \%$ silica. Interessant is dat voor het polaire silica een hogere concentratie resulteert in een lagere ruimteladingsverdeling en een lagere elektrische ladingsvereffeningsstroom, veroorzaakt door diepere 'traps' en lagere 'trap' dichtheid. Deze effecten zouden veroorzaakt kunnen zijn door een gewijzigde ladingsmobiliteit door:

1) Amine-amine waterstofbruggen: Als een lading, die gelokaliseerd is in de buurt van een amino groep, genoeg energie heeft om vrij te komen, is de kans groot dat deze lading door een andere 'trap' weer ingevangen wordt. Dit leidt tot een lagere mobiliteit.

2) Lagere mobiliteit van polymeerketens door verankering in de clusterstructuur van silica.

Beide fenomenen kunnen tot een hogere barrière leiden voor het vrijkomen van ladingen en daardoor de 'traps' voor ladingen verdiepen.

Twee polymeerblends met verschil qua mengbaarheid werden vergeleken: de niet-mengbare polymeren $\mathrm{PP} / \mathrm{EOC}$ (polypropyleen/ethyleen-co-octeen), en de mengbare polymeren PP/PP-HI (polypropyleen/ethyleen-co-propyleen). Het voor deze studie gebruikte silica is met APTES gemodificeerd. Globaal is de prestatie van het PP/PP-HI nanocomposiet beter vergeleken met de eigenschappen van de PP/EOC blend. Dit kan verklaart worden met de betere mengbaarheid en lagere ruimteladingsverdeling met een lagere elektrische geleiding als gevolg. In het PP/EOC mengsel wordt de fasenscheiding veroorzaakt door het gebrek aan mengbaarheid van PP en EOC; het PP/PP-HI mengsel vormd een homogene fase. Dit verschil in fasenscheiding beïnvloed ook de locatie van het silica: In de niet-mengbare PP/EOC blend is het silica te vinden in de PP fase, omdat deze een lagere viscositeit heeft dan EOC. Daarentegen is in de mengbare blend van PP en PP-HI het silica gelijk verdeelt over de hele matrix. Over het algemeen introduceert APTES-silica diepe 'traps' in de polymeerblends en onderdrukt ruimteladingsverdeling. Het PP/PP-HI/APTES-silica nanocomposiet heeft de laagste ruimteladingsverdeling en presteert daardoor beter dan het PP/EOC composiet. Dit zou dus de betere keuze zijn voor kabelisolatie.

Ondanks dat de dispersie is niet optimaal is, geeft het polaire APTES-silica de beste resultaten qua dielektrische eigenschappen. Verdere verbetering van de mengbaarheid van silica en deze polymeren 
met behoud van de goede dielektrische eigenschappen is wenselijk. Om dit te bewerkstelligen is een compleet nieuwe manier van oppervlaktemodificatie ontwikkeld. Deze modificatie houdt in:

i) Een polair deel: een urethaan-groep, die dus ook een stikstof-atoom bevat en daardoor de 'charge trap' verdeling bepaald; en

ii) Een apolaire koolwaterstof-verbinding gesynthetiseerd uit ethyl-, tert. butyl-, cyclopentyl-, phenyl- of nafthyl-groepen, om de compatibiliteit met de matrix te verbeteren.

Alle silica's met een apolaire buitenlaag verbeteren de dispersie van deze vulstof in de polymeer-matrix, en door de urethaan groep kan de 'charge trap' verdeling van de nanocomposieten verbeterd worden. Van deze vijf silica's leidt het met een tert. butylgroep gemodificeerde type tot de laagste ladingsinjectie en elektrische ladingsvereffeningsstroom. Deze resultaten geven aan, dat een nanocomposiet met een vergelijkbare modificatie een veelbelovend materiaal is voor HVDC kabel isolatie.

Om dat nader te kunnen beoordelen zijn tests nodig van eigenschappen zoals korte en lange termijn falen, diëlektrische verliezen, thermische geleiding, verouderingsgedrag. Deze vielen helaas buiten deze studie.

De ontwikkeling van een zodanig gemodificeerd silica is een veelbelovende ontwikkeling om de prestaties van isolerende nanocomposieten op basis van PP verder te verbeteren. De uitdagingen voor de verdere ontwikkeling zijn:

i) De dikte van de twee lagen te controleren,

ii) De invloed van de laagdikte op de dispersie van silica en de diëlektrische eigenschappen van de nanocomposiet te bepalen.

Als al deze vragen beantwoordt zijn, is een grote stap genomen richting ontwikkeling van op maat gemaakte nanocomposieten voor HVDC kabel isolatie materialen. 
252 | P a g e 


\section{List of Publications}

\section{Journal Articles}

1. He, X.. Rytöluoto, I., Anyszka, R., Mahtabani, A., Saarimäki, E., Lahti, K., Paajanen, M., Dierkes, W. and Blume, A., 2019. Surface modification of fumed silica by plasma polymerization of acetylene for PP/POE blends dielectric nanocomposites. Polymers, 11(12), pp. 1957.

2. He, X. Rytöluoto, I., Anyszka, R., Mahtabani, A., Saarimäki, E., Lahti, K., Paajanen, M., Dierkes, W. and Blume, A., 2020. Silica surface-modification for tailoring the charge trapping properties of PP/POE based dielectric nanocomposites for HVDC cable application. IEEE Access, 8, pp.87719-87734.

3. He, X. Seri, P., Rytöluoto, I., Anyszka, R., Mahtabani, A., Naderiallaf, H., Niittymäki, M., Saarimäki, E., Mazel, C., Perego, G. and Lahti, K., Paajanen, M., Dierkes, W. and Blume, A. 2021. Dielectric Performance of Silica-Filled Nanocomposites Based on Miscible (PP/PP-HI) and Immiscible (PP/EOC) Polymer Blends. IEEE Access, 9, pp.15847-15859.

4. He, X. Rytöluoto, I., Seri, P., Anyszka, R., Mahtabani, A., Naderiallaf, H., Niittymäki, M., Saarimäki, E., Mazel, C., Perego, G. and Lahti, K., Paajanen, M., Dierkes, W. and Blume, A. 2021. PP/PP-HI/Silica nanocomposites for HVDC cable insulation: Are silica clusters beneficial for space charge accumulation? Polymer Testing, pp.107186.

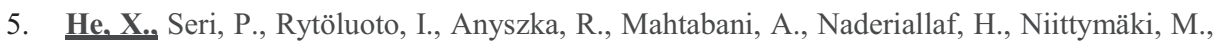
Saarimäki, E., Mazel, C., Perego, G. and Lahti, K., Paajanen, M., Dierkes, W. and Blume, A. The influence of nanosilica surface functionalization on $\mathrm{PP} / \mathrm{EOC}$ cast thin film nanocomposites for HVDC cable application: micromorphology, dynamic and dielectric properties. Surface and Interfaces (2021, Under Review)

6. He, X. Rytöluoto, I., Seri, P., Anyszka, R., Mahtabani, A., Naderiallaf, H., Niittymäki, M., Saarimäki, E., Mazel, C., Perego, G. and Lahti, K., Paajanen, M., Dierkes, W. and Blume, A. Designing core-shell surface-structured silicas for HVDC cable insulation nanodielectrics . EPolymers (2021, Under Review)

7. He, X. Rytöluoto, I., Anyszka, R., Mahtabani, A., Saarimäki, E., Lahti, K., Paajanen, M., Dierkes, W. and Blume, A. APTES-plasma functionalization on fumed silica and its effect on PP based dielectric nanocomposites. (2021, In Preparation)

\section{Full Text Conference Publications}

1. He, X. Seri, P., Rytöluoto, I., Anyszka, R., Mahtabani, A., Naderiallaf, H., Saarimäki, E., Niittymäki, M., Mazel, C., Perego, G. and Lahti, K., 2020, July. Influence of polar and unpolar 
silica functionalization on the dielectric properties of PP/POE nanocomposites. In 2020 IEEE 3rd International Conference on Dielectrics (ICD) (pp. 229-232). IEEE.

2. He, X. Mahtabani, A., Rytöluoto, I., Saarimäki, E., Lahti, K., Paajanen, M., Anyszka, R., Dierkes, W. and Blume, A., 2019, April. Surface modification of fumed silica by dry silanization for PP-based dielectric nanocomposites. In 2019 2nd International Conference on Electrical Materials and Power Equipment (ICEMPE) (pp. 254-259). IEEE.

3. He, X.. Rytöluoto, I., Mahtabani, A., Saarimäki, E., Lahti, K., Paajanen, M., Anyszka, R., Dierkes, W. and Blume, A., 2019, August. Surface Modification of Fumed Silica by Dry Silanization for PP/POE-based Dielectric Nanocomposites. In Proceedings of the Nordic Insulation Symposium (No. 26, pp. 32-36).

\section{Collaborations}

1. Mahtabani, A., Rytöluoto, I., Anyszka, R., He. X.. Saarimäki, E., Lahti, K., Paajanen, M., Dierkes, W. and Blume, A., 2020. On the Silica Surface Modification and Its Effect on Charge Trapping and Transport in PP-Based Dielectric Nanocomposites. ACS Applied Polymer Materials, 2(8), pp.3148-3160.

2. Mahtabani, A., La Zara, D., Anyszka, R., He. X. Paajanen, M., van Ommen, R., Dierkes, W. and Blume, A., 2021. Gas Phase Modification of Silica Nanoparticles in a Fluidized Bed: Tailored Deposition of Aminopropylsiloxane. Langmuir. 37(15),pp.4481-4492.

3. Anyszka, R., He, X. Mahtabani, A., Dierkes, W.K. and Blume, A., 2019, August. Effective surface functionalization of fumed silica with NBR telechelic oligomer for high-voltage polyolefin-based dielectric composites. In Proceedings of the Nordic Insulation Symposium (No. 26, pp. 118-122).

4. Anyszka, R., He. X. Mahtabani, A., Rytöluoto, I., Saarimäki, E., Lahti, K., Paajanen, M., Dierkes, W. and Blume, A., 2020, July. Silica surface modification with liquid rubbers \& functional groups for new polyolefin-based dielectric nano-composites. In 2020 IEEE 3rd International Conference on Dielectrics (ICD) (pp. 213-216). IEEE.

5. Suraci, S.V., He. X. Chaudhary, S., Wang, X., Anyszka, R., Mahtabani, A., Fabiani, D., Andritsch, T. and Vaughan, A., 2020. Assessment of the chemical and electrical properties of nano structured polyethylene with antioxidant-grafted nanosilica. In 2020 IEEE 3rd International Conference on Dielectrics (ICD) IEEE.

6. Mahtabani, A., He. X. Rytöluoto, I., Lahti, K., Paajanen, M., Saarimäki, E., Anyszka, R., Dierkes, W. and Blume, A., 2019, April. Effect of silica modification on charge trapping behavior of PP blend/silica nanocomposites. In 2019 2nd International Conference on Electrical Materials and Power Equipment (ICEMPE) (pp. 241-245). IEEE.

7. Mahtabani, A., Rytöluoto, I., Anyszka, R., Saarimäki, E., He. X.. Lahti, K., Paajanen, M., Dierkes, W. and Blume, A., 2020, July. Silica Functionalization: How Does it Affect Space Charge Accumulation in Nanodielectrics Under DC?. In 2020 IEEE 3rd International Conference on Dielectrics (ICD) (pp. 281-284). IEEE. 
8. Blume, A., Jin, J., Mahtabani, A., He. X.. Kim, S. and Andrzejewska, Z., 2019, September. New Structure Proposal for Silane Modified Silica. In International Rubber Conference, IRC 2019.

9. Mathabani, A., Rytöluoto, I., He. X. Saarimäki, E., Lahti, K., Paajanen, M., Anyszka, R., Dierkes, W. and Blume, A., 2019, August. Solution modified fumed silica and its effect on charge trapping behavior of PP/POE/silica nanodielectrics. In Proceedings of the Nordic Insulation Symposium (No. 26, pp. 129-133).

10. Rytöluoto, I., Saarimäki, E., Pelto, J., Paajanen, M., He. X. Anyszka, R., Mahtabani, A., Dierkes, W., Seri, P., Naderiallaf, H. and Lahti, K., Feasibility of Mini-Scale Injection Molding for Resource-Efficient Screening of PP-Based Cable Insulation Nanocomposites. In 2020 IEEE 3rd International Conference on Dielectrics (ICD) . IEEE

11. Montanari, G.C., Seri, P., Naderiallaf, H., Blume, A., Mahtabani, A., He, X. Perego, G., Mazel, C., Paajanen, M. and Karttunen, M., 2019, June. Adding nanofillers in polymeric insulating materials: so far so good? The case of polypropylene for DC cables. In 2019 IEEE Electrical Insulation Conference (EIC) (pp. 477-480). IEEE.

12. Montanari, G.C., Seri, P., Paajanen, M., Lahti, K., Rytöluoto, I., Ritamäki, M., Blume, A.,

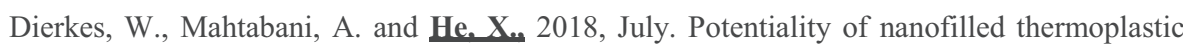
insulation for DC cables and capacitors. In 2018 IEEE 2nd International Conference on Dielectrics (ICD) (pp. 1-4). IEEE.

\section{Conference Presentations and Posters}

1. Presentation: Surface Modification of Fumed Silica by Dry Silanization for PP/POE-based Dielectric Nanocomposites. In 2019 Proceedings of the Nordic Insulation Symposium.

2. Presentation: Influence of polar and unpolar silica functionalization on the dielectric properties of PP/POE nanocomposites. In 2020 IEEE 3rd International Conference on Dielectrics (ICD).

3. Poster: Surface modification of fumed silica by dry silanization for PP-based dielectric nanocomposites. In 2019 2nd International Conference on Electrical Materials and Power Equipment (ICEMPE).

4. Poster: Surface Modification of Nano-silica by Plasma Polymerization. 2018 Dutch Polymer Day. Lunteren, The Netherlands. 
256 | P a g e 


\section{Acknowledgement}

On $30^{\text {th }}$ of January 2017, I started my journey and the adventure of studying abroad by stepping on the flight from Qingdao, China, to Amsterdam, the Netherlands. After long and excited traveling, I have finally arrived in Enschede, the Netherlands, on $31^{\text {st }}$ of January 2017, where my Ph.D. life began. After 4 years and 4 months joyful bumpy ride, I have finished my thesis and passed the committee's assessment. I am approaching to the end chapter of my precious Ph.D. life. It is difficult to say goodbye, as this memorable Ph.D. experience is the wonderful time in my life. I will cherish it and let it encourage me for my future career. Along the journey, there are important people, without who I will never be able to finish my study. Therefore, I would like to express my heartwarming gratitude to all of you.

My deep gratitude to my dear supervisor, Prof. dr. Wilma Dierkes.

During the past four years, I have written countless "thank you very much" via email, but it is still not enough to express my sincere gratitude to you. You are not only motivating me, inspiring me, encouraging me, but also guiding me through many difficulties as a beacon during my research path, allowing me always to see the light from other end of the tunnel. Without your constant support and kind help, I could not have finished the 6 manuscripts for journal publications. I thank you for your patience with me. The most touching thing, which gives me the drive and the momentum for carrying on each of the research day, is that you told me that YOU TRUST ME. I thank you for giving me the freedom to balance my work and life well. I thank you for giving me the confidence by trusting my work and working performance. I have enjoyed every of our discussions and the enlightenments that you have given me. At last, but not the least, "thank you very much!" again.

I thank dear Prof. dr. Anke Blume for accepting me as a Ph.D. candidate.

I still remember that the first sentence in our first email correspondence, which is "Your CV looks promising to me." I deeply appreciate you for providing me the interview opportunity and offering me this Ph.D. position. Back then, it is not just a simple job offer which allowed me to study abroad. But it helped me to walk out of the dark period my life in 2016. It opened a complete new and happy chapter of my life here. I can never say enough thank you for this. Your wisdom and guidance leading us to explore and unveil the truth in the scientific world. I also thank you for your continuous supports and encouragements which helped our Gridable project running smoothly.

I would also like to thank dear Prof. dr. Jacques Noodermeer for teaching us to have rigorous attitude towards science and critical attitude towards our own work.

I feel lucky to have one of the best teachers in the Netherlands in our group. You are not only having prefund professional knowledge, but also critical attitude towards science. I have learned so much from you during my Ph.D. study. These valuable experiences will be condensed along my future academic career and help me to be better and better. I thank you for your kindness, patience with me and constructive advice and suggestion for my work.

I would like to express my gratitude towards the committees' members.

Dear Prof. dr. S. Luding, Prof. dr. A.J.A. Winnubst, Prof. dr. G. Chen, Prof. dr. R. Gerhard, Prof. dr. K. Lahti and Dr. E. Sarlin, I thank you for accepting to be the committees' members to evaluate my Ph.D. 
work. I also thank you for taking your time from your busy schedule to read my thesis and providing the valuable suggestion and comments!

I am grateful to have Dr. Rafal Anyska and future Dr. Amirhossein Mahtabani as colleagues.

We are Gridable Teams! You have been giving me endless encouragement and valuable help. Looking back this past four years, we have been working together, facing challenges and difficulties together. We have managed to solve the problems one by one together. Amir, I miss our back to back working order. Rafal, I miss the time when you are still in UT (University of Twente). I want to express my sincere gratitude to both of you. Without you, I could not have finished my work in time. I am sure that our friendship will last forever. I thank you again and I wish you to be happy in each of single day.

I want to thank my colleagues, my officemates and my friend for your company.

I thank you all for filling up my Ph.D. period with a lot of joy and happiness. I am so lucky to be one of the members in our excellent ETE group. My special thanks to Neven, I thank you for writing down "chill" on the palm at my first day at UT. It has been heartwarming until now. I thank Marzieh for inviting me for dinners and for helping me look for accommodation during my first few months at UT. I thank Akansha for your company with me, and it was a nice time when we always stayed together. For Arqam and Sun, you are always so generously in helping me, and I thank you for sharing your research and life experience to me. Dear Hans, thank you for being my Dutch colleague and helped me to complete the smenvatting. I also want to thank dear Ceciel for your kind help and continuous support. I thank Sir. Dires for helping me to assemble and refurbish plasma setup. I thank you for being there to spend a lot of time and efforts to make it work again, as nobody has used the equipment for the past 10 years. I also would like to express my gratitude to Binbin as well. I thank you for helping me to adapt the study and work life at Enschede. Dear Dr. Peter Morshuis, although I am not your direct student, I am so glad to meet you during summer school. You have provided me valuable technical discussion and encouraged me to become better and better. I also thank you for helping me gain confidence to move forward.

I want to also thank the Gridable consortium for our effective collabrations.

Great Gridable! I would like to extend my sincere gratitude to all project partners. I particularly appreciate the constant support and invaluable discussion with Dr. Ilkka Rytöluoto from VTT in Finland, Dr. Paolo Sari from Univiersity of Bologna in Italy, Prof. Kari. Lahti from Tampere university in Finland. I would also like thank Etta Saarimäki (VTT) and Minna Niittymäki (Tampere University) for your effort and time spending on preparing the compound and performing measurements for me. Dear Dr. Mika Paajanen (VTT), thank you for being the Gridable leader. Your leadership secured our collaboration and encouraged us to work in harmony. We have worked together and helped each other. And we are finally approaching to the end of this wonderful project. I cannot only thank you, but also gratitude you for providing more opportunities for our project partners. I am proud of being part of the Gridable project!

I want to thank my lovely families.

The day is coming. Baba, Mama, after 24 years of continuous study, I am finally going to graduate with a Doctorate degree. It is pity that you both cannot join my defense at Enschede, the Netherlands. I know that you will be proud of me. I would like to thank my mother-in-law as well. I thank you for coming to the Netherlands to help me looking after my son. Without you and my Baba, Mama's selfless dedication, 
I could not finish my work on time. I want to thank my dear sister, Liqin Ren, for being my family in the Netherlands! I appreciate you very much for your kindness and the help during the past four years. To my wonderful son, Shen, you are such a cute and sweet little soul full of happiness and surprises. I love you so much as being such a precious gift in my life. I thank my dear husband, Dr. Wei Ya, for being there, always and forever!

To whom I may missed here but also important, I thank you for being part of my life and I wish you to have a wonderful and productive life. 


ISBN 978-90-365-5186-1 